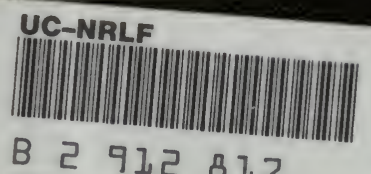

\& 4 r 


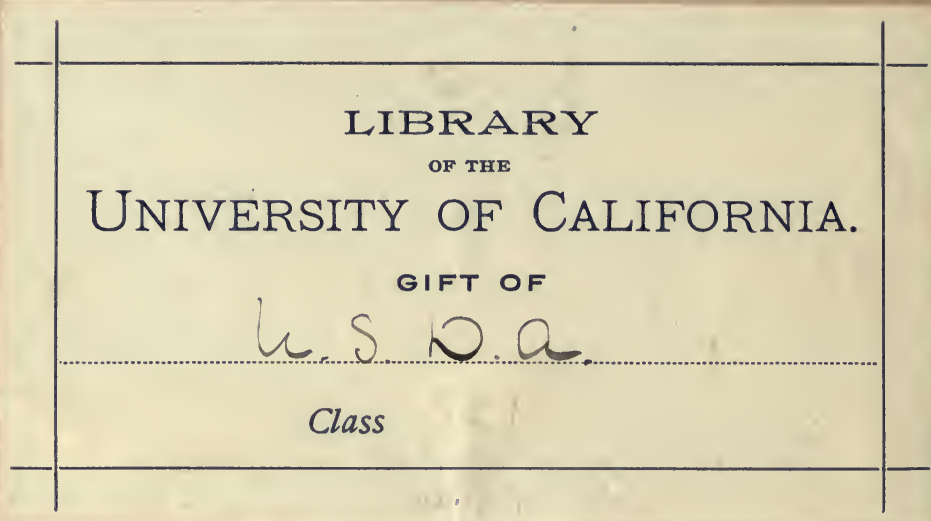




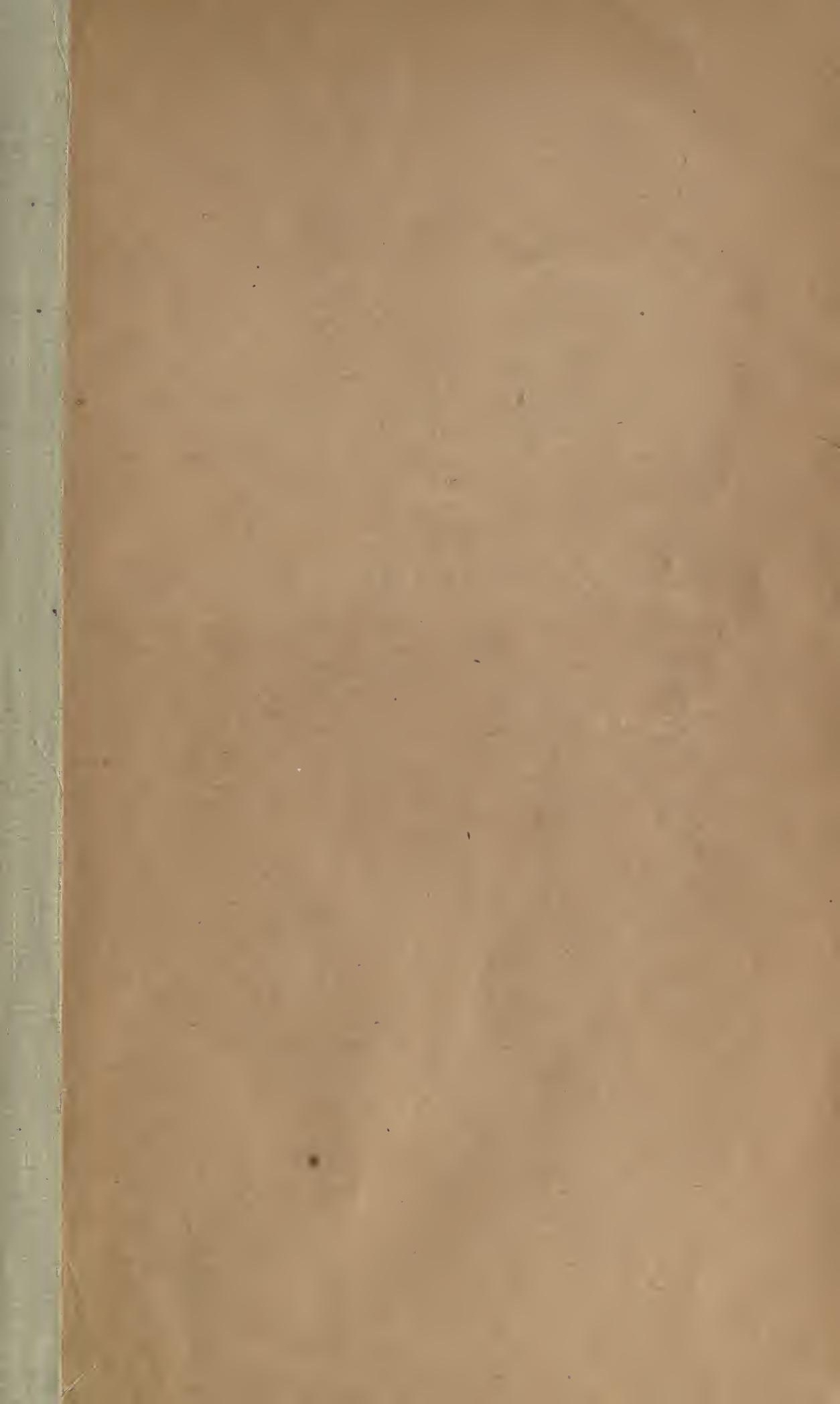




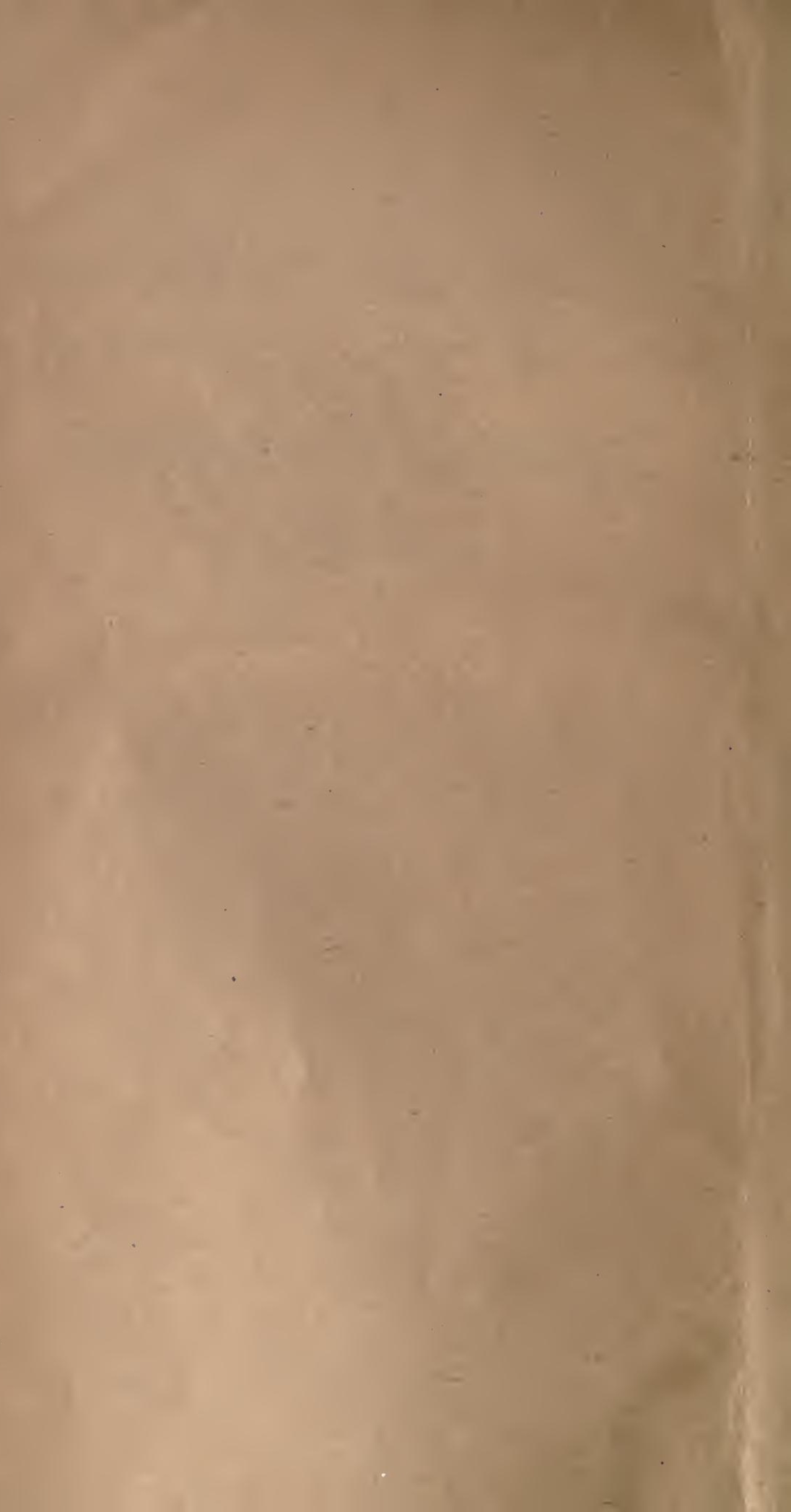

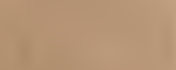



Digitized by the Internet Archive in 2007 with funding from Microsoft Corporation 




\section{U. FS 1761906}

\section{U. S. DEPARTMENT OF AGRICULTURE,}

BUREAU OF CHEMISTRY - BULLETIN No. 84, PART II.

H. W. WTLEY, CHIEF OF BUREAU.

\section{INFLLENCE OF POOD PRISERVITIIILS AND ARTHFOLAL COLORS ON DIGLSTION AND HEALT'H.}

\section{II.-SALICYLIC ACID AND SALICYLATES.}

BY H. W. WHEEY, N. D.,

WITH THE COLLABORATION OF W. D. BIGELOW, CHIEF OF THE DIVISION OF FOODS, F. C. WEBER, AND OTHERS.

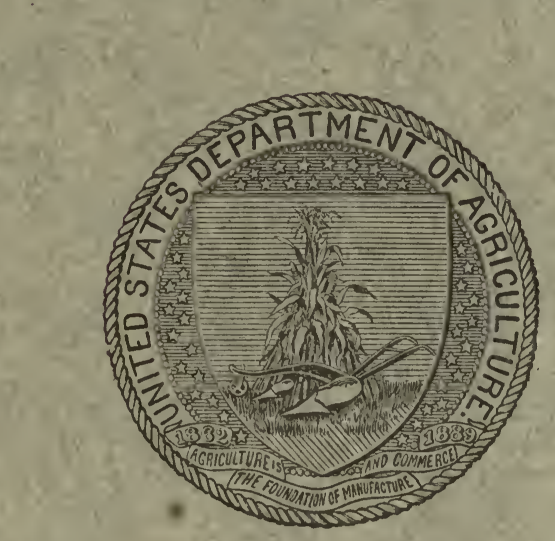

WASHINGTON:

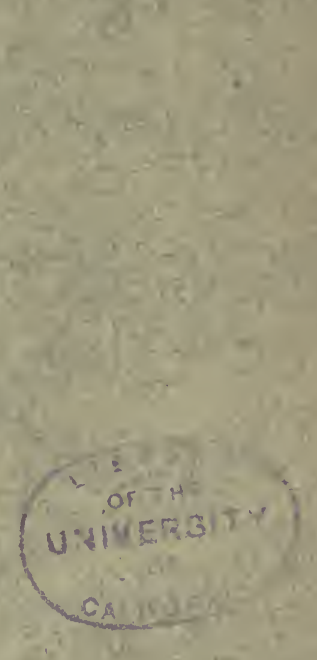

GOVERNMENT PRINTING OFFICE.

1906 . 



\section{U. S. DEPARTMENT OF AGRICULTURE,}

BUREAU OF CHEMISTRY-BULLETIN No. 84, PART II.

H. W. WILEY, CHIEF OF BUREAU.

\section{INFLENCE OF F00D PRRSERVATIVES ADD. ARTIFICIAL COLORS ON DIGESTION AND HELLTH.}

\section{II.-SALICYLIC ACID AND SALICYLATES.}

By H. W. WILEY, N. D.,

WITH THE COLLABORATION OF W. D. BIGELOW, CHIEF OF THE DIVISION OF FOODS, F. C. WEBER, AND OTHERS.

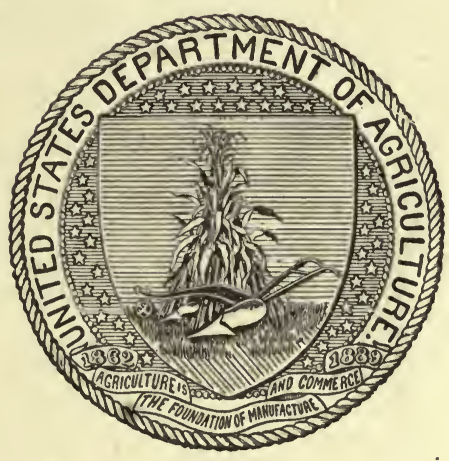

WASHINGTON:

GOVERNMENT PRINTING OFFICE.

1906 . 



\section{LETTER OF TRANSMITTAL.}

United States Department of Agriculture, Bureau of Chemistry, -

Washington, D. C., April 28, 1906.

SIR: I beg to submit for your inspection and approval the results of the investigations which have been made in this Bureau to determine the effect of salicylic acid and salicylates upon digestion and health. The work is a continuation in plan of that described in Part I of Bulletin 84, devoted to boric acid and borax. I recommend that the report be published as Part II of Bulletin 84 . Respectfully,

Hon. James WiLson,

H. W. WILEY,

Chief of Bureau.

Secretary of Agriculture. 



\section{CONTENTS.}

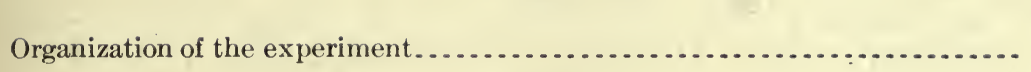

SERIES VI.

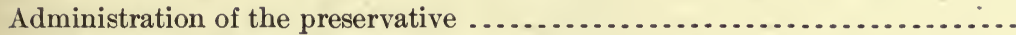

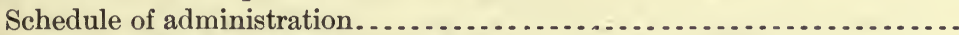

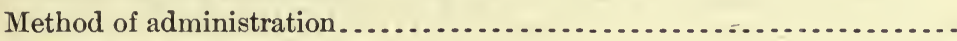

Quantity of the preservative ......................................

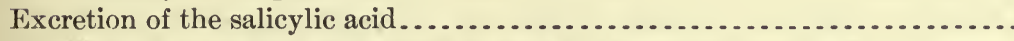

Method for determining salicylic acid in the urine..................

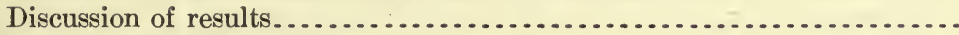

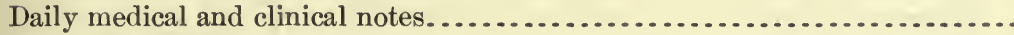

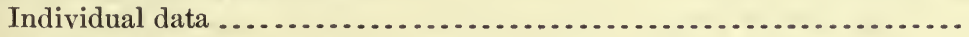

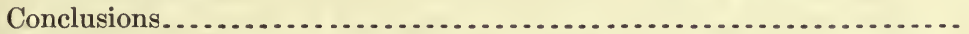

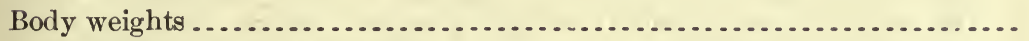

Variations in body weights ................................ 505

Ratio of food weight to body weight........................... 508

Microscopical examination of the blood ........................... 520

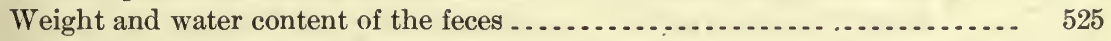

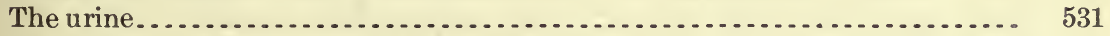

Volume, specific gravity, and total solids ..................... 531

Individual data ..... . . . . . . . . . . . .

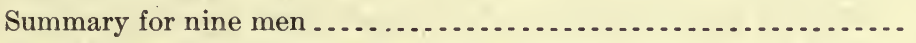

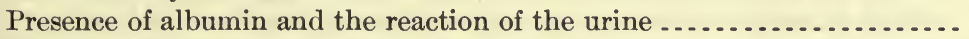

Ratio of sulphur, sulphates, and phosphoric acid to nitrogen excreted in the urine

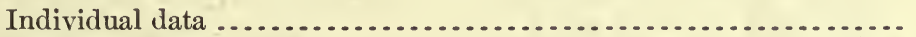

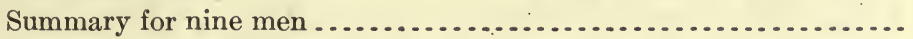

Changes in the relative quantities of sulphur compounds excreted in the

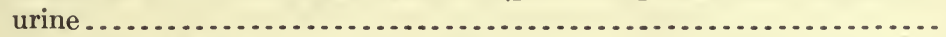

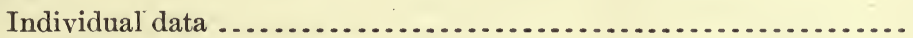

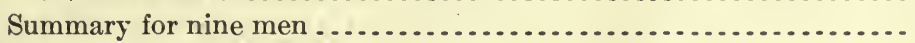

Microscopical examination of the urine.........................

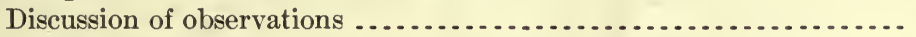

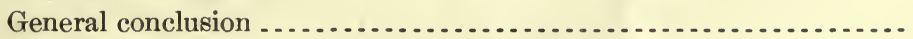

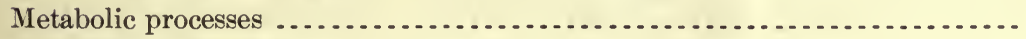

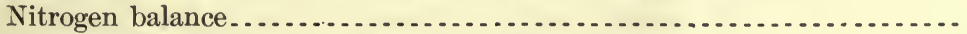

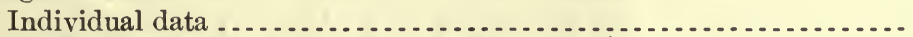

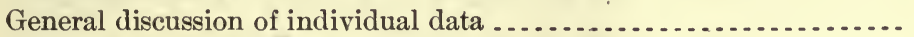

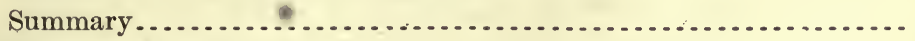

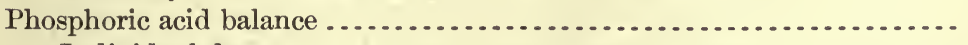

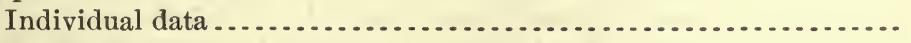

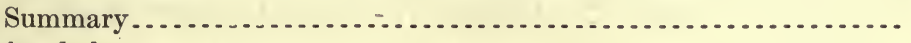

Sulphur balance............

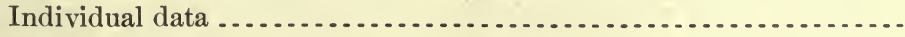

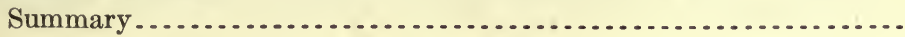


Metabolic processes-Continued. Page.

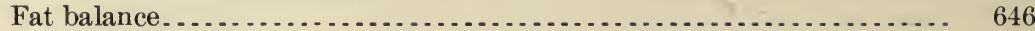

Individual data ................................................. 646

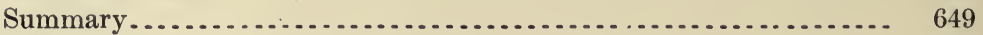

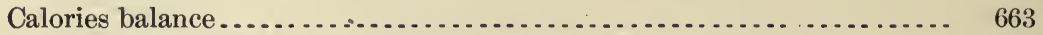

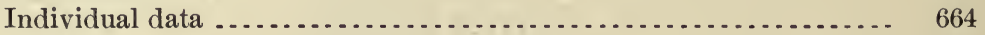

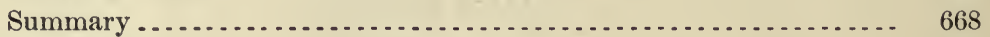

Solids balance.................................................... 681

Individual data ............................................ 681

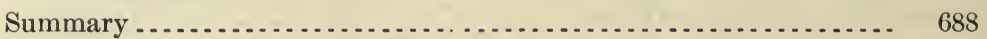

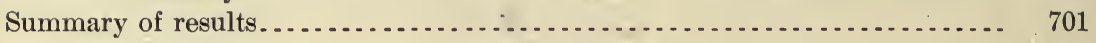

SERIES XI.

The effect of salicylic acid and sodium salicylate upon the nitrogenous elements

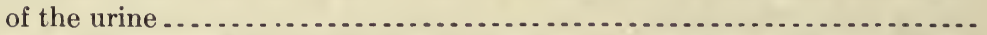

Preliminary study for the determination of the ration and methods of analysis

The ration

Analytical results. . . . .

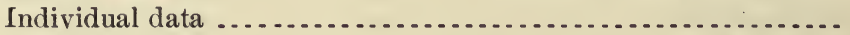

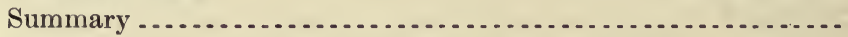

Methods of analysis employed and comparison of results ...........

Urea determinations ....................................

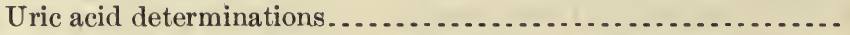

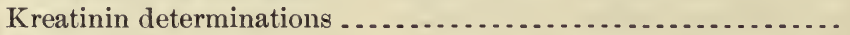

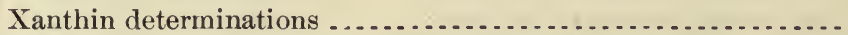

Special study of the distribution of the nitrogenous constituents of the urine as affected by the preservatives

Introduction

Schedule of administration of the preservative..................

Supplemental study of the presence of albumin and the reaction of

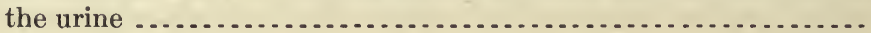

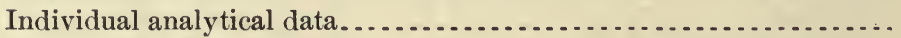

Summary for Nos. 1 and 2 ......................................

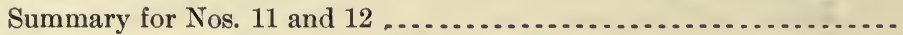

General summary ......................................... 737

The use of small quantities of the preservative........................ 754

General conclusions .............................................. 757

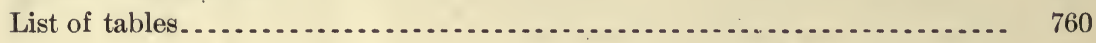

\section{ILLUSTRATIONS.}

Fig. 1. Daily and average body weights for Series VI, Nos. 1-8 ...........

2. Daily and average body weights for series VI, Nos. 9-12, and sum-

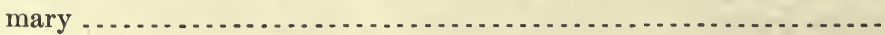

3. Graphic chart, representing the comparative influences of foods and preservatives 


\section{INFLUENCE OF FOOD PRESERVATIVES AND ARTI- FICIAL COLORS ON DIGESTION AND HEALTH.}

\section{II.-SALICYLIC ACID AND SALICYLATES.}

\section{ORGANIZATION OF THE EXPERIMENT.}

The researches to determine the effect of salicylic acid upon digestion and health were carried on in the same manner as those described in the first part of this bulletin on boric acid and borax. Some few changes were made in the methods of manipulation, sampling, and analysis in order to simplify the process and to save time. Instead of the analysis being made upon each daily sample of the food or feces, a careful study of composite samples for the various periods was made and the analysis of the composite sample for the period accepted as a true representative composition of the food or excrement during that period. It was found also, in so far as the labor and time were concerned, that it was just as convenient to have all twelve of the subjects under observation at once as to divide them into squads of six each and alternate the periods of observation of each squad with periods of rest. For example, in the analysis of the bread for the tables the daily samples were composited and the analyses made for twelve persons as easily as for six. The same is true for each article of diet and for the analysis of the excrementitious material. By this arrangement the analysts were able to complete the analytical work during the periods of recreation and to devote more time to the classification and tabulation of the data. The burden of the analytical work was thus diminished one-half, while its accuracy and efficiency were not sacrificed in any respect, but on the contrary rather increased.

The analyses of the foods and feces were made in the Division of Foods under the supervision of W. D. Bigelow; the conduct of the food table, the study of the body weights, and the urinalyses were under the charge of F. C. Weber, and the microscopic tests were conducted by B. J. Howard.

Owing to other arrangements the surgeons in connection with the Marine Hospital Service found themselves unable to give the 
time necessary to the regular inspection of the physical state of the young men under observation, and this inspection was undertaken by the Chief of the Bureau. A thorough physical and medical examination was given to each applicant before his admission to the table, in order to be certain that those admitted to the experimental work were in a perfectly sound physical state and for at least one year previous to the beginning of the experimental work had not suffered from any severe illness. In this respect the same means were employed and the same care exercised as in the experiment with borax, the squad having been chosen, thoroughly examined as described, and placed upon the permanent diet for about a week before the regular period of observation began, on October 19, 1903.

The main experiment on salicylic acid is referred to as Series VI, following Series V of Part I on boric acid, while Series XI is a supplemental, special study conducted at a later date to determine certain effects on the urine, the importance of which had been suggested by the work of Series VI. It will be noted from the schedules following that the work of Series VI began on October 19, 1903, and was completed on December 7, a period of fifty days, excluding the preparatory work, which began about October 15. Series XI consisted of a preliminary study of three men from March 29, 1905, to April 9, and a special study of four men from April 27 to May 16, a total of thirtytwo days under observation. 


\section{SERIES VI.}

\section{ADMINISTRATION OF THE PRESERVATIVE.}

\section{SCHEDULE OF ADMINISTRATION.}

In Table I are given the dates of the periods and subperiods of the observations, which are useful in case their duration is not repeated in the text. In Table II are given the data showing the administration of the salicylic acid for each of the periods and subperiods and the quantities given to each individual daily.

TABLE I.-Dates of periods and subperiods in Series VI.

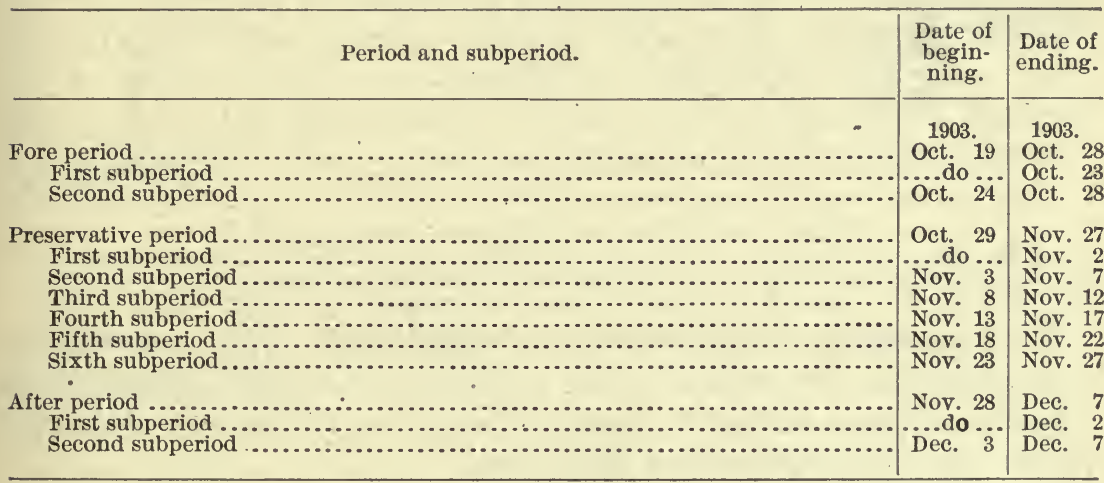

TABLE II.-Schedule of administration of preservative, Series VI.

IN TABLETS.

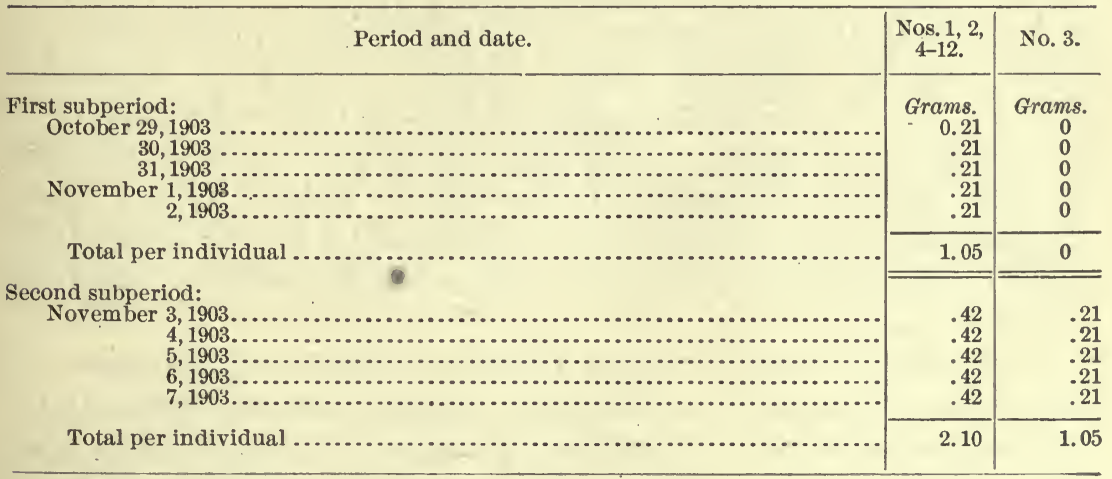


TABLE II.-Schedule of administration of preservative; Series VI-Continued. IN CAPSULES.

\begin{tabular}{|c|c|c|}
\hline Period and date. & $\begin{array}{c}\text { Nos. } 1,2 \\
4-12 .\end{array}$ & No. 3. \\
\hline 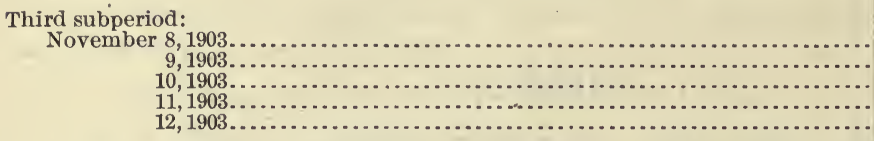 & $\begin{array}{r}\text { Grams. } \\
0.74 \\
.74 \\
.74 \\
.74 \\
.74\end{array}$ & $\begin{array}{r}\text { Grams. } \\
0.42 \\
.42 \\
.42 \\
.42 \\
.42\end{array}$ \\
\hline Total per individual ...................... & 3.70 & 2.10 \\
\hline 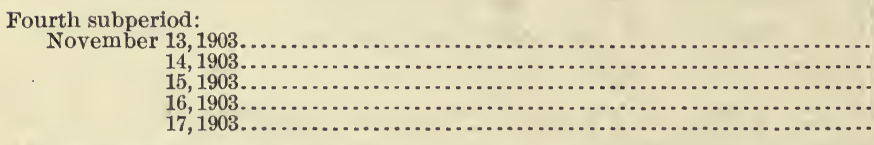 & $\begin{array}{l}1.2 \\
1.2 \\
1.2 \\
1.2 \\
1.2\end{array}$ & $\begin{array}{l}.8 \\
.8 \\
.8 \\
.8 \\
.8\end{array}$ \\
\hline Total per individual ................ & 6.0 & 4.0 \\
\hline 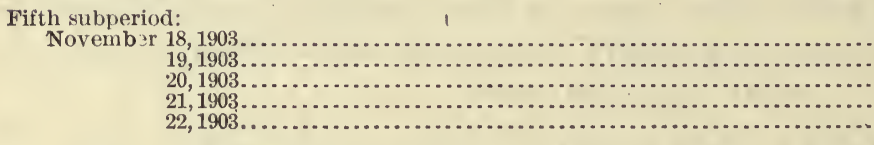 & $\begin{array}{l}1.6 \\
1.6 \\
1.6 \\
1.6 \\
1.6\end{array}$ & $\begin{array}{l}1.2 \\
1.2 \\
1.2 \\
1.2 \\
1.2\end{array}$ \\
\hline . Total per individual ..... & 8.0 & 6.0 \\
\hline 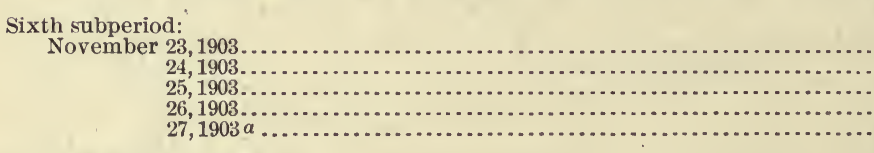 & $\begin{array}{l}2.0 \\
2.0 \\
2.0 \\
2.0 \\
2.0\end{array}$ & $\begin{array}{l}1.6 \\
1.6 \\
1.6 \\
1.6 \\
1.6\end{array}$ \\
\hline 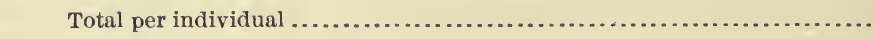 & 10.0 & 8.0 \\
\hline Total per individual for entire preservative period $\ldots . . \ldots \ldots \ldots \ldots \ldots \ldots . . . . . . .$. & 30.85 & 21.15 \\
\hline
\end{tabular}

aNo. 6 -took no preservative on November 27 , making his total dose for the sixth subperiod 8 grams instead of 10 , and the total for the entire preservative period 28.85 grams.

The only notable variation in the administration of the preservative occurred in the case of No. 3, who, on account of a slight indisposition which developed during the fore period, did not begin to take the preservative until the second subperiod.

\section{METHOD OF ADMINISTRATION.}

The preservative was administered in two forms, considered to be most convenient, namely, in tablets and in capsules. Objections have been urged against this manner of administering the preservative, and it has even been stated in some criticisms of the borax experiment that the use of this method is sufficient ground for the rejection of all the data collected relative to the injurious effects of the preservative upon the metabolic processes, because of the alleged irritant effects of preservatives so administered as compared with the effects produced by the same bodies as found in the foods themselves as purchased on the market.

It is hardly necessary to call attention to the futility of such an objection. Were the preservatives employed poisonous bodies, in the ordinary sense of the term, producing a direct mechanical effect upon the membranes of the mouth, esophagus, and stomach; there might be some ground for criticising their ingestion in the form of tablets or 
capsules. In the case of the preservatives employed, however, which in the quantities used produced no such effects, the objections are entirely groundless. A preservative administered in this way at the time of the meals, as was always the case, is at once thoroughly incased in the food, is rapidly mixed with the contents of the stomach during the process of digestion, and could not in any way exert any injurious effect by reason of the form of its administration. Moreover, this method of exhibition in connection with injection into the blood is one commonly followed in medical and pharmacological experiments.

QUANTITY OF THE PRESERVATIVE.

It will be seen that the quantities subjected vary from 210 milligrams a day at the beginning of the preservative period to as much as 2 grams a day at its close, the object in this arrangement being to determine progressively the limit of toleration for ordinary medicinal doses. With a substance whose activity is as limited as that of salicylic acid, it is evident that it would be impossible within any reasonable time to secure any idea of its physiological effect by administering mere traces of the reagent. On the other hand, the quantity used, namely, 30.85 grams, over a period of thirty days, an average of a gram a day, while not excessive, is sufficient to permit of a study of the effects of this substance upon the metabolic processes.

\section{EXCRETION OF THE SALICYLIC ACID.}

Tracing the history of salicylic acid in the organism is a somewhat difficult procedure. Soon after the exhibition of salicylic acid it or its derivatives appears in the urine, and it is evident that the kidney is the principal excretory organ. Owing to the changes in the composition of the salicylic acid resulting in the formation of salicyluric acid and other decomposition products, a comparison of the amounts excreted in the urine with the quantity given is a difficult operation. Table III shows in milligrams the quantity of salicylic acid administered and the amount thereof recovered in the urine. In the course of five days after the cessation of the administration of the salicylic acid nothing but a mere trace was found in the urine. In fact, in some cases only traces were left after four days.

In this connection attention is called to the difficulties attending the exact determination of salicylic acid and the products obtained therefrom in its passage through the body. The method used in these investigations for determining salicylic acid is as follows.

\section{METHOD FOR DETERMINING SALICYLIC ACID IN THE URINE.}

Make alkaline with sodium hydrate 25 or 50 cubic centimeters of urine, according to the amount of salicylic acid administered, and evaporate, with the addition of a little sand, to a thick sirup. Rub this mass with a pestle, after adding 50 cubic centimeters of 98-99 per 
cent alcohol, decant the alcohol on a filter paper and repeat the extraction five or six times. Transfer the precipitate to a filter and wash until no test for salicylic acid can be obtained by evaporating 10 cubic centimeters of the extract to dryness, taking up with petroleum spirit and testing in the usual way.

Evaporate the extracts to free them from alcohol, take up with water, acidify, extract with ether, and evaporate until free from ether. Dissolve the salicylic acid in hot water and make up to a definite volume at room temperature and make up aliquot portions of this solution to 100 cubic centimeters in Nessler's jars.

Add 5 cubic centimeters of a 0.5 per cent ferric alum solution to one of these jars and mix thoroughly, noting the depth of color. Make up a set of standards from a solution containing 0.1 milligram per cubic centimeter of salicylic acid so that they approximately match the color developed in the test just described. That is, if the color developed approximates 1.2 milligrams make up the standards so that they will contain $1.18,1.20$, and 1.22 milligrams of salicylic acid. Then compare a new solution of the sample with these samples, making the comparisons immediately after adding the ferric alum solution, as the color fades rapidly. The comparisons should be made in triplicate and are accurate to 0.02 of a milligram.

Blanks run by adding salicylic acid to normal urines averaged 95 per cent of the acid recovered. Extracts of urines passed during the preservative period were heated to $156^{\circ}$ to volatilize the salicylic acid and the residues weighed as salicyluric acid. In every case the residues were so small as to be negligible.

The samples of feces tested gave no indication of the presence of salicylic acid.

TABLE III.-Salicylic acid ingested and recovered in urine, Series VI.

\begin{tabular}{|c|c|c|c|c|c|c|c|c|}
\hline \multirow[b]{2}{*}{ Period. } & \multicolumn{2}{|c|}{ No. 1.} & \multicolumn{2}{|c|}{ No. 2.} & \multicolumn{2}{|c|}{ No. 3.} & \multicolumn{2}{|c|}{ No. 4.} \\
\hline & $\begin{array}{l}\text { ठ } \\
\text { \&̊ } \\
\text { م }\end{array}$ & 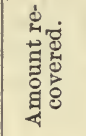 & $\begin{array}{l}0 \\
0 \\
0 \\
-1\end{array}$ & 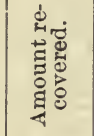 & 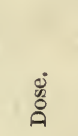 & 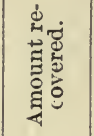 & $\begin{array}{l}\dot{0} \\
\text { के } \\
\text { ᄋ }\end{array}$ & 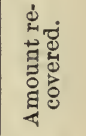 \\
\hline $\begin{array}{l}\text { Preservative period: } \\
\text { First subperiod, Oct. } 29-\text { Nov. } 2 . . \\
\text { Second subperiod, Nov. } 3-7 . \ldots . \\
\text { Third subperiod, Nov. } 8-12 \ldots \ldots \\
\text { Fourth subperiod, Nov. } 13-17 \ldots \\
\text { Fifth subperiod, Nov. } 18-22 \ldots \ldots \\
\text { Sixth subperiod, Nov. } 23-27 \ldots \ldots\end{array}$ & $\begin{array}{r}\text { Mgs. } \\
1,050 \\
2,100 \\
3,700 \\
6,000 \\
8,000 \\
10,000\end{array}$ & $\begin{array}{r}\text { Mgs. } \\
315 \\
785 \\
1,115 \\
2,787 \\
3,267 \\
4,990\end{array}$ & $\begin{array}{r}\text { Mgs. } \\
1,050 \\
2,100 \\
3,700 \\
6,000 \\
8,000 \\
10,000\end{array}$ & $\begin{array}{r}\text { Mgs. } \\
0 \\
695 \\
1,311 \\
2,992 \\
4,398 \\
4,945\end{array}$ & $\begin{array}{l}\text { Mgs. } \\
0 \\
1,050 \\
2,100 \\
4,000 \\
6,000 \\
8,000\end{array}$ & $\begin{array}{r}\text { Mgs. } \\
0 \\
314 \\
508 \\
1,769 \\
2,710 \\
3,386\end{array}$ & $\begin{array}{r}\text { Mgs. } \\
1,050 \\
2,100 \\
3,700 \\
6,000 \\
8,000 \\
10,000\end{array}$ & $\begin{array}{r}\text { Mgs. } \\
526 \\
575 \\
1,980 \\
2,759 \\
3,993 \\
5,010\end{array}$ \\
\hline $\begin{array}{l}\text { Total, Oct. } 29-\text { Nov. } 27 \ldots \ldots \ldots . \\
\text { Average per day .............. }\end{array}$ & $\begin{array}{r}30,850 \\
1,028\end{array}$ & $\begin{array}{r}13,259 \\
442\end{array}$ & $\begin{array}{r}30,850 \\
1,028\end{array}$ & $\begin{array}{r}14,341 \\
478\end{array}$ & $\begin{array}{r}21,150 \\
846\end{array}$ & $\begin{array}{r}8,687 \\
290\end{array}$ & $\begin{array}{r}30,850 \\
1,028\end{array}$ & $\begin{array}{r}14,843 \\
495\end{array}$ \\
\hline 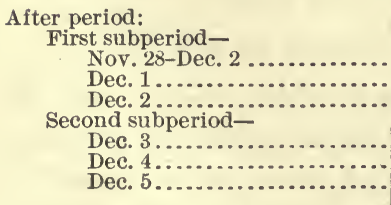 & $\begin{array}{l}0 \\
0 \\
0\end{array}$ & 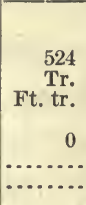 & $\begin{array}{l}0 \\
0 \\
0\end{array}$ & $\begin{array}{r}180 \\
\text { Good tr. } \\
\text { Ft. tr. } \\
\text { Ft. tr. } \\
0 \\
\cdots\end{array}$ & $\begin{array}{l}0 \\
0 \\
0\end{array}$ & $\begin{array}{r}129 \\
\text { Ft. tr. } \\
0 \\
0 \\
0 \\
\cdots\end{array}$ & $\begin{array}{l}0 \\
0 \\
0\end{array}$ & $\begin{array}{r}359 \\
\text { Tr. } \\
0 \\
0 \\
0 \\
\ldots .\end{array}$ \\
\hline
\end{tabular}


TABLE III.-Salicylic acid ingested and recovered in urine, Series VI-Continued.

\begin{tabular}{|c|c|c|c|c|c|c|c|c|}
\hline \multirow{2}{*}{ Period. } & \multicolumn{2}{|c|}{ No. 5.} & \multicolumn{2}{|c|}{ No. 6.} & \multicolumn{2}{|c|}{ No. 7.} & \multicolumn{2}{|c|}{ No. 8.} \\
\hline & $\begin{array}{l}\dot{8} \\
\text { o } \\
0\end{array}$ & 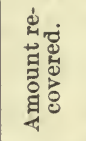 & $\begin{array}{l}\dot{0} \\
\text { in } \\
0\end{array}$ & 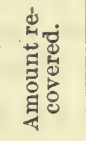 & $\begin{array}{l}\dot{0} \\
\dot{8} \\
\stackrel{8}{\circ}\end{array}$ & 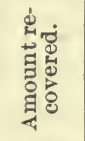 & $\begin{array}{l}\dot{0} \\
80 \\
0 \\
0\end{array}$ & 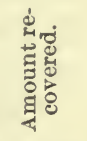 \\
\hline $\begin{array}{l}\text { Preservative period: } \\
\text { First subperiod, Oct. } 29-\text { Nov. } 2 . . \\
\text { Second subperiod, Nov. 3-7.... } \\
\text { Third subperiod, Nov. 8-12 } \ldots \text {. } \\
\text { Fourth subperiod, Nov. 13-17... } \\
\text { Fifth subperiod, Nov. 18-22.... } \\
\text { Sixth subperiod, Nov. } 23-27 \ldots \ldots\end{array}$ & $\begin{array}{r}\text { Mgs. } \\
1,050 \\
2,100 \\
3,700 \\
6,000 \\
8,000 \\
10,000\end{array}$ & $\begin{array}{r}\text { Mgs. } \\
302 \\
439 \\
1,947 \\
3,606 \\
4,422 \\
4,853\end{array}$ & $\begin{array}{l}\text { Mgs. } \\
1,050 \\
2,100 \\
3,700 \\
6,000 \\
8,000 \\
8,000\end{array}$ & $\begin{array}{r}\text { Mgs. } \\
513 \\
471 \\
1,070 \\
2,516 \\
3,636 \\
4,301\end{array}$ & $\begin{array}{r}M g 8 . \\
1,050 \\
2,100 \\
3,700 \\
6,000 \\
8,000 \\
10,000\end{array}$ & $\begin{array}{r}M g 8 . \\
276 \\
635 \\
1,548 \\
2,346 \\
3,567 \\
3,897\end{array}$ & $\begin{array}{r}M g 8 . \\
1,050 \\
2,100 \\
3,700 \\
6,000 \\
8,000 \\
10,000\end{array}$ & $\begin{array}{r}M g 8 . \\
281 \\
750 \\
1,431 \\
2,323 \\
3,892 \\
4,926\end{array}$ \\
\hline 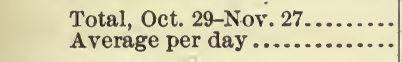 & $\begin{array}{r}30,850 \\
1,028\end{array}$ & $\begin{array}{r}15,569 \\
519\end{array}$ & $\begin{array}{r}28,850 \\
962\end{array}$ & $\begin{array}{r}12,507 \\
417\end{array}$ & & $\begin{array}{r}12,269 \\
406 \\
\end{array}$ & & $\begin{array}{r}13,603 \\
453\end{array}$ \\
\hline 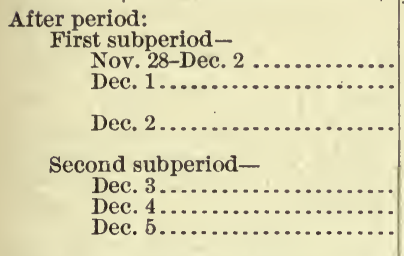 & $\begin{array}{l}0 \\
0 \\
0\end{array}$ & $\begin{array}{l}194 \\
\text { Tr. }\end{array}$ & $\begin{array}{l}0 \\
0 \\
0\end{array}$ & $\begin{array}{l}\text { Very } \\
\text { ft. tr. } \\
\text { Very } \\
\text { ft. tr. } \\
\quad 0\end{array}$ & $\begin{array}{l}0 \\
0 \\
0\end{array}$ & $\begin{array}{r}97 \\
\text { Tr. } \\
\text { Ft. tr. }\end{array}$ & $\begin{array}{l}0 \\
0 \\
0\end{array}$ & $\begin{array}{r}\text { Tr. } \\
\text { Ft. tr. } \\
0\end{array}$ \\
\hline & \multicolumn{2}{|c|}{ No. 9.} & \multicolumn{2}{|c|}{ No. 10.} & \multicolumn{2}{|c|}{ No. 11.} & \multicolumn{2}{|c|}{ No. 12.} \\
\hline Period. & $\begin{array}{l}\dot{8} \\
8 \\
8\end{array}$ & 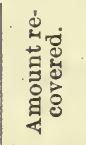 & ஓं & 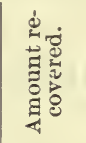 & $\begin{array}{l}\dot{0} \\
8 \\
8 \\
0\end{array}$ & 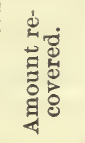 & ஹं & 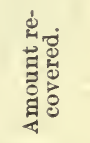 \\
\hline $\begin{array}{l}\text { Preservative period: } \\
\text { First subperiod, Oct. } 29-\text { Nov. } 2 . . \\
\text { Second subperiod, Nov. } 3-7 \ldots \ldots \\
\text { Third subperiod, Nov. } 8-12 \ldots \ldots \\
\text { Fourth subperiod, Nov. } 13-17 \ldots . \\
\text { Fifth subperiod, Nov. } 18-22 \ldots \ldots \\
\text { Sixth subperiod, Nov. } 23-27 \ldots \ldots\end{array}$ & $\begin{array}{r}\text { Mgs. } \\
1,050 \\
2,100 \\
3,700 \\
6,000 \\
8,000 \\
10,000\end{array}$ & $\begin{array}{r}\text { Mgs. } \\
212 \\
820 \\
1,496 \\
2,785 \\
3,993 \\
5,060\end{array}$ & $\begin{array}{r}M g s . \\
1,050 \\
2,100 \\
3,700 \\
6,000 \\
8,000 \\
10,000\end{array}$ & $\begin{array}{r}\text { Mgs. } \\
392 \\
892 \\
858 \\
2,399 \\
3,778 \\
4,250\end{array}$ & $\begin{array}{r}\text { Mgs. } \\
1,050 \\
2,100 \\
3,700 \\
6,000 \\
8,000 \\
10,000\end{array}$ & $\begin{array}{r}M g s . \\
360 \\
638 \\
1,361 \\
2,459 \\
3,624 \\
5,160\end{array}$ & $\begin{array}{r}\text { Mgs. } \\
1,050 \\
2,100 \\
3,700 \\
6,000 \\
8,000 \\
10,000\end{array}$ & $\begin{array}{r}\text { Mgs. } \\
267 \\
600 \\
1,705 \\
2,597 \\
3,660 \\
4,976\end{array}$ \\
\hline $\begin{array}{l}\text { Total, Oct. } 29-\text { Nov. } 27 . \ldots \ldots \ldots \\
\text { Average per day } . . . \ldots \ldots \ldots \ldots\end{array}$ & $\begin{array}{r}30,850 \\
1,028 \\
\end{array}$ & $\begin{array}{r}14,366 \\
479\end{array}$ & $\begin{array}{r}30,850 \\
1,028\end{array}$ & $\begin{array}{r}12,569 \\
419\end{array}$ & $\begin{array}{r}30,850 \\
1,028\end{array}$ & $\begin{array}{r}13,602 \\
453\end{array}$ & $\begin{array}{r}30,850 \\
1,028\end{array}$ & $\begin{array}{r}13,805 \\
460\end{array}$ \\
\hline 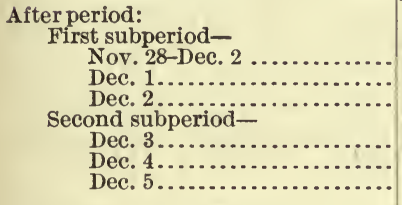 & $\begin{array}{l}0 \\
0 \\
0\end{array}$ & $\begin{array}{r}310 \\
\text { Tr. } \\
\text { Ft. tr. }\end{array}$ & $\begin{array}{l}0 \\
0 \\
0\end{array}$ & $\begin{array}{l}124 \\
\text { Tr. } \\
\text { Tr. }\end{array}$ & $\begin{array}{l}0 \\
0 \\
0\end{array}$ & $\begin{array}{r}231 \\
\text { Tr. } \\
0\end{array}$ & $\begin{array}{l}0 \\
0 \\
0\end{array}$ & $\begin{array}{l}215 \\
\text { Tr. } \\
\text { Tr. }\end{array}$ \\
\hline
\end{tabular}

\section{DISCUSSION OF RESULTS.}

As before stated, the quantities obtained by analysis represent 95 per cent of the actual quantities of salicylic acid in the urine. The tests as applied show that no weighable quantities of salicyluric acid are present in the urine.

The individual data show in the case of No. 1 a little over one-third of the salicylic acid recovered in the urine and the same is true of No. 2. A little more than one-third is recovered in the case of No. 3. 
Almost one-half is recovered in the case of No. 4, a little over one-half in the case of No. 5, a little less than one-half in the case of No. 6, a little over one-third in the case of No. 7, almost one-half in the case of Nos. 8 and 9, a little over a third in the case of No. 10, and less than one-half in the cases of Nos. 11 and 12.

The summary for 12 men shows that 44.47 per cent of the salicylic acid administered was recovered, an average of 443 milligrams out of 996. If the correction indicated by the blanks be made, a total average recovery of 46.8 per cent of salicylic acid unchanged is indicated. The balance of the salicylic acid is apparently changed into salicyluric acid or other combinations or remains stored in the body.

The difference in composition between salicylic acid and salicyluric acid is shown by the following formula:

$$
\begin{aligned}
& \text { Salicylic acid, } \mathrm{C}_{7} \mathrm{H}_{6} \mathrm{O}_{3}=\mathrm{OH} . \mathrm{C}_{6} \mathrm{H}_{4} \cdot \mathrm{CO}_{2} \mathrm{H} \text {. } \\
& \text { Salicyluric acid, } \mathrm{C}_{9} \mathrm{H}_{9} \mathrm{NO}_{4}=\mathrm{OH} \cdot \mathrm{C}_{6} \mathrm{H}_{4} \cdot \mathrm{CO} . \mathrm{NH} . \mathrm{CH}_{2} \cdot \mathrm{CO}_{2} \mathrm{H} \text {. }
\end{aligned}
$$

It is stated by most authorities that salicylic acid when administered internally is found in the urine partly as salicyluric acid. This body is separated from salicylic acid by the volatilization process described. The quantities found in our experiments by this method were not weighable.

\section{DAILY MEDICAI AND CLINICAL NOTES.}

\section{INDIVIDUAL DATA.}

$$
\text { No. 1.-J. H. S. }
$$

On the first day of the fore period the subject's weight was 53.45 kilograms; temperature, $98.4^{\circ}$; pulse, two observations, 76 and 80. His health was excellent and his physical condition without a flaw. No variations of note in physical condition occurred on the succeeding days of the fore period. The temperature on the final day of the fore period, October 28 , was $98.2^{\circ}$ and $98.6^{\circ}$, two observations; the pulse, 70 and 80 , and the weight 53.36 kilograms. The average weight for the entire fore period was 53.38 kilograms, and the daily variations were small.

On the first day of the first preservative subperiod (October 29 to November 2) the temperature was a little below the normal, two observations being $98.1^{\circ}$ and $97.8^{\circ}$, and the pulse 68 and 70 per minute, respectively. The second day the temperature was normal. No marked variation in condition was observed during the first preservative subperiod except a slight decrease in weight, the average weight for this period being 53.25 kilograms.

The second preservative subperiod began on November 3 and closed November 7. The temperature was slightly below the normal on November 4 , reaching $97.9^{\circ}$ on the first observation and $98.4^{\circ}$ on the second. On the 5 th the temperature was still slightly below the nor- 
mal, but the health of the subject was good. On November 6 No. 1 complained of not having his appetite satisfied by his meals and stated that he was hungry, but his health was good. The feeling of lack of sufficient food continued on the following day, and there was a slight increase in the temperature, three observations having been made, registering $98.2^{\circ}, 99.8^{\circ}$, and $99^{\circ}$, respectively. The rapidity of the pulse was also increased, having registered 82 at $6.20 \mathrm{p}$. m. There was a continued, though slight, falling off in weight during this subperiod, the average weight being 53.15 kilograms.

The third preservative subperiod began on November 8 and closed on November 12. On the first day of this subperiod the subject still complained of being hungry. His health was good, but the feeling of hunger was pronounced on the following day, when the subject entered upon his notes, "Don't get enough to eat," and on the following day registered the observation, "Could eat more." The feeling of hunger continued during the whole of the third preservative subperiod. The average weight decreased to 53.05 kilograms.

The fourth preservative subperiod began on November 13 and closed on the 17th. There was a slight increase of temperature noticed at the second observation on the 13th, the thermometer registering $99.4^{\circ}$ and the pulse registering 84 beats per minute. The feeling of hunger continued through the fourth subperiod, but no other notable variation in the condition of the subject was observed. The .weight throughout this subperiod remained practically constant, the average weight being 53.06 kilograms.

The fifth preservative subperiod began on November 18 and ended on the $22 \mathrm{~d}$. The feeling of hunger still persisted, and on the 19th the subject complained of feeling as though there were a lump in the stomach, accompanied by continued belching. His temperature was below the normal, the two observations being $97.7^{\circ}$ and $97.4^{\circ}$, respectively. The feeling of disturbance in the stomach and of hunger continued on the 20th, but the temperature was restored to normal. On the 21st the patient described himself as feeling uncomfortable in the region of the stomach and still hungry. On the $22 \mathrm{~d}$ he was very hungry, and the feeling as of a lump in the stomach continued. There was a continued loss of weight during this subperiod, the average for the subperiod being 52.78 kilograms.

The sixth preservative subperiod began on November 23 and ended on November 27. On the $23 \mathrm{~d}$ the patient described his symptoms as "Very hungry," with a gnawing feeling in the stomach. The same symptoms were also reported for the 24th and 25th of November. The average weight for the sixth preservative subperiod was 52.62 kilograms and that of the entire preservative period 52.99 kilograms. Judged by the daily chart, the permanent symptom connected with the preservative period in the case of No. 1 was a feeling of hunger, 
although the ration was exactly that given in the fore period, when no sense of hunger was experienced, accompanied during the latter preservative subperiods with a feeling of distress in the stomach and some belching.

The first after subperiod began on November 28 and ended on December 2. On the first day of the after period the subject complained of a slight cold and sore throat. There was no perceptible rise in temperature, however. The cold continued in a mild degree on the 29th, but the temperature was slightly below the normal. The temperature was normal on the 30 th, with slight cold continuing. The feeling of hunger had disappeared and did not return on December 1 and 2. The average weight for the first after subperiod was 52.42 kilograms.

The second after subperiod began on December 3 and ended December 7. A slight cold was reported on the $3 \mathrm{~d}$, with temperature slightly below the normal. On the 4 th the slight cold continued, with almost normal temperature. On the 5 th the second observation of temperature was slightly above normal, $99^{\circ}$, with the pulse at 80 . On the 6 th all symptoms of cold had disappeared and no sense of hunger was experienced. On the 7 th the temperature was normal and the hunger symptom occurred after dinner. The average weight for the second after subperiod was 52.31 kilograms, and the mean weight for the entire after period 52.37 kilograms.

$$
\text { No. 2. -W. P. }
$$

At the beginning of the fore period, on November 19, the temperature was slightly above 98 , the pulse 73 beats per minute, and the weight of the body 67.9 kilograms. On Tuesday, October 20, there was a slight rise in temperature, but no other symptoms of a derangement of the normal processes appeared. The temperature was normal on the 21st, and no variation from the normal was observed on the following days, except an occasional rise in temperature after the dinner hour. Throughout the whole of the fore period, with very few exceptions, this tendency to an increased temperature after dinner was quite apparent. The temperature before dinner was perhaps slightly below the normal, the arerage being only a little above $98^{\circ}$. The average weight for the first fore subperiod was 68.43 kilograms and for the second subperiod 68.21 kilograms. The final weight on the last day of the fore period, October 28, was 67.72, a total loss of 180 grams. The average weight for the entire fore period, however, was 68.32 kilograms.

The preservative period began on October 29 , on which day there was a slight diminution in the normal temperature and a slight increase of weight, the first weighing being 68.05 kilograms. On the second 
day of the first preservative subperiod the temperature was slightly above the normal, but no other symptoms of abnormality were observed. This increase in temperature also continued during the 31st, and on November 1 the temperature was again normal and the weight had increased to 68.35 kilograms, followed by a fall on November 2 to 67.85 kilograms. The average weight for the first preservative subperiod was 68.01 kilograms.

The second preservative subperiod began on November 3 with normal temperature and a weight of 68.05 kilograms. On the 5th of November No. 2 was reported as feeling well with the exception of a slight headache. There was also a slight depression of temperature after dinner in this case instead of before. On the 7th of November No. 2 described himself as "hungry as a bear." .Temperature and respiration, however, remained normal. The weight of the body had fallen on November 7 to 67.60 kilograms, the average weight for this subperiod being 67.84 , a slight decrease as compared with the average for the preceding subperiod.

The third preservative subperiod began on November 8 with all symptoms normal. On the 9th No. 2 described himself as still hungry, and on the 10th as feeling all right. On the 11th he was normal, and the hungry feeling had disappeared. The average weight was 67.80 , theoretically a very slight decrease.

The fourth preservative subperiod began on the 13th of November with no unfavorable symptoms. On November 14 No. 2 described himself as feeling a little feverish and as having a bad headache in the morning. On the 15 th of November the headache continued all day, but the subject was not ill. The headache disappeared on the 16th. This subperiod closed on the following day with an average weight of 67.65 kilograms, the average decrease continuing.

On the 18th, the first day of the fifth preservative subperiod, there were decided pains in the stomach, and the temperature fluctuated somewhat, but there were no marked symptoms of fever. The weight on this day was 67.63 kilograms. The condition of No. 2 remained normal from this time until the beginning of the sixth preservative subperiod, on November 23, when he had nausea during the whole afternoon. After dinner on the 24 th he returned to the dining room after a short absence complaining of very severe burning pains in the stomach, and was evidently quite ill and in considerable distress. After meals on November 25 there were burning sensations in the stomach which lasted for a greater or less length of time, and the weight decreased to 67.11 kilograms. On the.26th the patient was feeling better, could eat more, and the appetite was fairly good. On the 27th extreme sensitiveness in the stomach was manifested, and there was a slight increase of temperature at the dinner hour. The

7656-No. 84, pt $2-06-2$ 
preservative period closed November 27 , showing an average weight of 67.66 kilograms, a slight decrease as compared with the fore period.

The after period began with a weight of 67 kilograms, temperature and pulse normal. No. 2 speedily regained his normal feelings, although on the 30th he had considerable headache and again felt sensations of nausea. These feelings passed away on the 1st of December and an increased appetite was developed, although the weight continued to fall. On December 2 the patient complained of being still hungry after having eaten three normal meals during the day.

The first day of the second after subperiod was characterized by unpleasant sensations, nausea, and general discomfort during the afternoon. This continued on the following day. The symptoms were better on the 5th of December, the nausea having entirely passed away at that time. Only normal symptoms were exhibited from that time until the end of the after period on December 7. At this time the subject was perfectly well, temperature and pulse normal, but continued to lose weight, as the last recorded weight was 66.65 kilograms and the average for the after period 66.79 .

$$
\text { No. 3.-C. P. }
$$

This subject at the time of the beginning of the fore period was in good physical condition, although of a rather small stature, having a weight of 52.78 kilograms, normal pulse and temperature. There was a slight rise of temperature on the 21st of October, and also a considerable quickening of the pulse, showing a decidedly feverish condition. This indisposition developed to such an extent that No. 3 did not report for observation at the table until October 26. On the 26th the fever still continued, the pulse was above the normal, and his weight had fallen to 50.54 kilograms. On the 28th of October there had been some improvement in the subject's condition and his pulse and temperature were only slightly above the normal. Owing to this illness the analytical data of the fore period are without value and the time of the first preservative subperiod, October 29 to November 2, became, in the case of No. 3, the fore period, at the beginning of which the temperature and pulse were normal and the weight 50.34 kilograms. No medical symptoms of any significance were developed during the fore period. The pulse and temperature remained practically normal and the weight had increased at the end of the period to 50.43 kilograms, the average for the period being 50.42. During the first preservative subperiod (November 3-7 in the case of this subject) the pulse and temperature were normal and the weight slightly increased, the average being 50.73 kilograms. The appetite of No. 3 increased very markedly during this subperiod and he was still hungry after the three regular rations had been eaten. A slight cold developed on the 5th of November, but was not of any consequence. At the end of this sub- 
period the patient was in good condition, but was still complaining of hunger. His weight had increased to 50.80 kilograms and the pulse and temperature were normal.

During the second preservative subperiod some pain was felt in the shoulder, but evidently this bore no definite relation to the diet. Complaint was made on December 9 of a very empty feeling in the stomach; otherwise the subject was feeling well. This condition continued throughout this subperiod, with a general feeling that the amount of ration eaten was not sufficient to supply the appetite, and the subject complained frequently of being hungry.

The third preservative subperiod (November 13-17) began with pulse and temperature normal. The weight had risen to 51.15 kilograms. Complaint was made during the first day of severe pains in the stomach. This continued during the next day, but the subject still complained of being hungry. On the 15th severe pains were felt in the shoulder, but normal conditions were restored on the 16th. On the 17 th the pains in the stomach returned, but the other conditions were normal, and at the end of this subperiod the weight of the subject was 51.14 kilograms; but the average weight was only 50.96 kilograms.

The fourth preservative subperiod (November 18-22) began with marked symptoms of hunger after the usual rations were eaten. On the 19 th constipation was marked and the pains in the stomach continued. On the 20 th report was made of very severe pains in the stomach, continuing during the night, and the constipation continued markedly. The general condition was better on the 21st, but the constipation continued. On the $22 \mathrm{~d}$ the subject was belching all the time with acidity of the stomach and indigestion. This subperiod closed with the subject in rather an unfavorable condition, but with an increased average weight, i. e., 51.14 kilograms.

The fifth preservative. subperiod (November 23-27) began with normal pulse and temperature, but with continued belching, indigestion, and heartburn, which rendered the subject very uncomfortable. On the 24th these symptoms were ameliorated to a certain degree and he was feeling reasonably well. The symptoms returned, however, with renewed vigor on the 25 th, with continued burning sensation in the throat and stomach, especially after meals, and continuing for about two hours. The weight on this day was slightly increased, being 51.54 kilograms, the average weight for the fifth subperiod being 51.31 kilograms. The end of the entire preservative period found the patient in reasonably good condition, with a strong appetite, pulse and temperature normal, and weight 51.60 kilograms, while the average weight for the period was 50.93 kilograms.

The first after subperiod began with a rapidly restored normal condition, which continued until December 1 , when the patient was attacked with another severe cold, the temperature having risen at the dinner 
period to $102^{\circ}$. The appetite, however, was not impaired. The ill feelings arising from the cold were increased on the $2 \mathrm{~d}$ of December, although the temperature had fallen somewhat. The weight at the close of the subperiod on this day was 51.54 kilograms; the average for the subperiod was 51.57 kilograms.

The second after subperiod began with both temperature and pulse slightly above the normal, pains in the chest, and the patient under treatment by Doctor Perry, of the Public Health and Marine-Hospital Service. The patient was decidedly better on December 4, and the temperature and pulse were normal. A slight headache developed on the 5th of December, but with no other abnormal symptoms. On the 6th the patient was feeling perfectly well, and the same is true of the 7 th, the close of the after period. On this date the temperature and pulse were normal and the weight was 51.55 kilograms. The average weight for the after period was 51.66 kilograms, showing a gain in weight throughout the observation, in connection with which the subject's state of convalescence must be remembered.

No. 4. - F. E. B.

No. 4 proved to be one of the best subjects,under observation, being very steady in his habits and very careful in the entry of data intrusted to him. At the beginning of the fore period No. 4 was in excellent physical condition, normal in every respect and having a weight of 61.08 kilograms. There were no deviations from the normal during the entire fore period, at the end of which the weight was 60.62 kilograms, the average weight for the period being 60.73 kilograms. During the first of the preservative subperiods there were no symptoms showing abnormality, except perhaps a slight increase in the appetite. The weight on the last day of the first preservative subperiod was 60.23 kilograms, and the average weight 60.53 kilograms.

During the second preservative subperiod the symptoms remained normal, but there was an increased desire for food, although the ration was not changed. At the end of this subperiod the weight was slightly greater than at the beginning, namely, 60.82 kilograms, and no marked symptoms of any abnormal condition had been developed. The average weight had also increased slightly, to 60.59 kilograms.

The third preservative subperiod began with a keen appetite, normal pulse and temperature. On the 9 th of November the patient complained of being ravenously hungry, although his weight had not sensibly diminished, remaining at 60.60 kilograms. On the 10 th the feeling of hunger at the end of the day seemed to be appeased and the subject expressed himself as having had enough to eat. The weight remained almost constant, namely, 60.64 kilograms. This subperiod ended with normal symptoms, a good appetite, but no 
unusual feeling of hunger, and with a weight of 60.51 kilograms. There was an increase in the average weight to 60.62 kilograms.

The fourth preservative subperiod began with a good appetite and no unfavorable symptoms. At the end of the period there was a slight increase in weight, namely, to 60.90 kilograms, and the average weight also increased to 60.85 kilograms.

The fifth preservative subperiod began with a good physical condition, normal in every respect. At the close of this period the normal conditions were still maintained, with good appetite but not excessive hunger, and the weight had risen to 61 kilograms, though the average weight slightly decreased, being 60.81 kilograms.

During the sixth preservative subperiod the patient complained of a slight headache but still had a fairly good appetite, though not so keen as during the earlier preservative subperiods. The average weight for the entire preservative period was 60.67 kilograms, a slight decrease as compared with the fore period average.

At the close of the first after subperiod normal conditions still continued, but the weight had fallen to 60.35 kilograms, though showing an average of 60.42 kilograms. The second after subperiod began with normal conditions, which continued unchanged to the end of the period, when the pulse and temperature were normal, and the weight was 60.38 kilograms and the average 60.43 kilograms. The average weight for the entire after period was 60.43 kilograms, showing a continued loss in weight throughout the experiment.

$$
\text { No. 5. - G. E. C. }
$$

No. 5 began the fore period in good physical condition, with temperature and pulse slightly below the normal. His weight was 59.43 kilograms. No unfavorable symptoms were developed during the fore period, the physical condition of the subject remaining practically unchanged. During the whole observation there seemed to be a slight normal depression of the temperature, which, however, was not due to any unusual cause. At the end of the fore period No. 5 was in excellent physical condition, with temperature slightly below the normal. The weight had risen to 59.94 kilograms, the average weight for the entire fore period being 59.76 kilograms.

The first preservative subperiod was passed without any unusual symptoms, except the continued slight depression of the temperature until November 2, when a slight headache was developed which continued during the whole day. The second preservative subperiod passed without any. unusual phenomena until the 7 th of November, when a remarkably strong appetite persisted after the last meal of the day. The weight of the subject on this date was 59.83 kilograms, the average weight for the subperiod being 60.07 kilograms. The third preservative subperiod passed without incident until November 10, when the 
patient felt that his appetite was satisfied with the usual rations. On the 12th of November, the appetite still remaining normal, the subject was not feeling well, having a slight tendency to diarrhea. The beginning of the fourth preservative subperiod found the patient feeling drowsy, but still with a normal appetite. No other abnormal symptoms were developed during this subperiod.

During the fifth preservative subperiod the patient remained in a normal condition until the 20th of November, when symptoms of indigestion or uneasiness in the region of the stomach appeared, but the appetite was not noticeably disturbed. These feelings passed away on the following day and the subject remained normal during the rest of that period. During the sixth preservative subperiod the patient continued normal and at the end of the preservative period was in good condition, weighing 59.52 kilograms. The average weight for the entire preservative period, however, was 59.71 kilograms, a slight decrease as compared with the fore period.

The patient was in good condition at the beginning of the after period and no unfavorable symptoms of any kind were developed; the appetite was normal and the food appeared to suit the demands of the appetite. At the end of the after period the pulse and temperature were normal and the weight was 59.29 kilograms, that being also the average for the entire period, thus showing a slight continued decrease in weight.

$$
\text { No. 6. }- \text { L. M. S. }
$$

This subject began the fore period in normal condition as respects both temperature and pulse and with a weight of 58.12 kilograms. No unusual symptoms developed during the fore period excepting perhaps a slight tendency to constipation. At the end of the fore period the patient's condition remained normal but with a slight loss of weight, which had fallen to 57.48 kilograms. The average weight for the fore period, however, was somewhat higher, i. e., 58.06 kilograms.

During the preservative period the condition of the subject remained normal without any notable symptoms during the first and second subperiods until the 5th of November, when complaint was made of a feeling of hunger after the usual rations of the day had been consumed. There was a gradual but not marked loss of weight, and the subject continued to complain at times of leaving the table very hungry. The appetite remained good during the third preservative subperiod, and on the 9th of November a slight cold in the head developed without any marked increase in temperature. There was a considerable fall in weight on that date, which was ascribed to a little unusual exercise. A slight cold continued on the 10th of November, but otherwise the subject was feeling well and the appetite was still keen, 
the feeling of hunger continuing during the next day. On November 12 , at 3.40 p. m., the patient was seized with cramps in the abdomen but not of a very severe character. At the beginning of the fourth preservative subperiod the feeling of hunger had disappeared, but the slight cold in the head continued without notable rise of temperature. The cold continued on the $14 \mathrm{th}$, with the feeling of uneasi ness in the stomach, which feeling disappeared on the 15th, the cold continuing but not in a disturbing degree. Normal conditions were restored on the 16th. On the 17th the subject was still hungry after taking the usual meals and had an uneasy feeling in the stomach. During the fifth preservative subperiod the feeling of hunger persisted. A tendency to diarrhea developed on the 19th, and there was some discomfort from indigestion. A little cold persisted during the following days but not of any serious nature, and the sensation of hunger continued. The sixth preservative subperiod began with pains in the stomach and abdomen but with the feeling of hunger continuing. On the 24 th the pains in the stomach were marked, especially after meals; the slight cold continued but without notable increase of temperature. Although still hungry on the 25th, there was a feeling as of a lump in the stomach and other unpleasant sensations, described as "unusual" feelings in the stomach. This indisposition continued on the 26th, and the temperature was slightly below the normal. At $6.45 \mathrm{p} . \mathrm{m}$. of this day considerable nausea developed, and most of the dinner was vomited. A cold and sore throat were complained of on the 27th, the last day of the preservative period. The temperature on the afternoon of this day was considerably above the normal, and the weight was 56.51 kilograms, the average weight for the entire preservative period being 56.94 kilograms, a decided decrease.

At the beginning of the after period the temperature was normal, but a slight sore throat persisted which continued also on the following three days. The feeling of hunger also was reported at times during this period. In the second after subperiod there was a-slight headache and some continued cold, but the cold in general was better. The weight continued to diminish. On the 5th of December normal symptoms were fully reestablished in every particular, but there was a slight feeling of indigestion after dinner. Eight grains of quinine had been taken in the preceding two or three days. On December 6 normal symptoms and normal appetite were reestablished, and these continued until the end of the after period, at which time the-weight was 55.80 kilograms.

To summarize, the average weight of No. 6 for the fore period was 58.06 kilograms; for the preservative period, 56.94 kilograms; and for the after period, 55.87 kilograms, showing a loss of 1.12 kilograms in the preservative period from the fore period and 1.07 kilograms in 
the after period from the preservative period, a total loss during the experiment of 2.19 kilograms, in connection with which, of course, the cold from which the subject suffered must be remembered.

$$
\text { No. \%. -J. N. B. }
$$

At the beginning of the fore period the weight of No. 7 was 68.3 kilograms, and the temperature and pulse were normal. He was in excellent physical condition, no organic disease of any of the vital organs was found, and he had had no serious illness for a long period. The first fore subperiod passed without any unusual incident. At the beginning of the second subperiod the weight had fallen to 67.90 kilograms, but no other disturbances were noted. At the close of the fore period the weight was 67.55 kilograms and the average weight for the fore period 67.91 kilograms.

The preservative period opened with the subject in good condition, though somewhat inclined to indulge in vivid imagination and to discover symptoms of trouble which did not exist. No unusual symptoms were manifested during the first preservative subperiod, but at the close of the second subperiod the symptoms of hunger appeared, the subject not feeling that the rations, which were entirely sufficient to satisfy his hunger during the fore period, were enough. On November 7 he first complained of indigestion, and at the beginning of the third preservative subperiod was feeling reasonably well. During November 9 a very bad headache was developed, which lasted during the afternoon, and on the following day there was an uneasy sensation in the stomach. The general appearance of the subject at this time was not as good as at first, but no specific complaints were made. During the night of November 12 the subject was feeling quite ill and was not well throughout the following day. On the 14th the symptoms of malaise had largely disappeared, but not entirely. Headache developed again on the 15th, and the feeling of hunger seemed to be accentuated. Insomnia was complained of on the night of the 15th, and the statement was made that for two preceding nights difficulty in sleeping, especially in the early morning hours, had been experienced. Insomnia continued during the $17 \mathrm{th}$, and the headache also persisted during that day. On the 19th symptoms of indigestion were clearly manifested, especially in the afternoon, but the sensation of hunger still continued. On the 20th of November the symptoms of indigestion increased, and the subject was feeling very ill. There seemed also to be a slight depression of the temperature. On the 21 st the weight fell below 67 kilograms. The indigestion became more pronounced, and the headache also continued. The appetite failed on the $22 \mathrm{~d}$, and some difficulty was experienced in taking the full ration. At the beginning of the sixth preservative subperiod the weight 
had fallen to 66.80 kilograms and the illness of the preceding days had passed away; the appetite was restored and a sensation of hunger was complained of after the regular meals. This feeling of hunger continued for several days, and on the 26th the illness before complained of again appeared but passed away on the following day.

The subject started on the after period with a weight of 66.90 kilograms, pulse and temperature normal, but complaining of constipation. On the 29th he felt quite well, but during the succeeding days there was a continued loss of weight, although the regular rations selected at the fore period were eaten and the subject felt well. In the second part of the after period the weight fell below 66 kilograms, but the subject was feeling well. This condition of health was continued to the end of the after period. 'The weight on the last day of the after period was 66.83 kilograms and the average weight for the after period was 66.34 kilograms.

Comparing the average weights of the three periods, we have for the fore period 67.91 kilograms, for the preservative period 67.28 kilograms, and for the after period 66.34 kilograms.

$$
\text { No. 8.-W. C. L. }
$$

No. 8 was a somewhat peculiar subject, very conscientious and very attentive to every detail, but much inclined at all times to. imagine that he had some specific or general trouble. The data which were obtained with No. 8, therefore, are of peculiar interest. He was in a very good physical condition at the time of the beginning of the experimental work and in the physical examination revealed no lesion of any vital organ, nor had he suffered from any severe disease for a period of more than a year. His weight at the beginning of the fore period was 61.30 kilograms and his temperature and pulse were normal. He entered the preservative period weighing 60.65 kilograms. On the 1st of November a slight headache was developed early in the morning, which continued until the afternoon, when, after the subject had slept for forty minutes, it disappeared. An unpleasant feeling in the abdomen was experienced at the same time. It should be stated here that during the whole of the observation No. 8 took regularly a given quantity of laxative, administered in such a way that it could not interfere with any of the observations, inasmuch as it was the same during all the periods. A feeling of languor was experienced on the 4 th of November, and it was reported as having been experienced for several preceding days. A slight headache was noted on the 5th of November, but it passed away after breakfast; the subject felt tired on waking. A sore knee with which he entered the preservative period gave him some little trouble at this time, due, as he explained, to hitting it accidentally. On the 8th of November a slight tendency 
to diarrhea was developed, but this was followed on the succeeding day by a period of constipation. Abdominal pains were experienced on the night of November 10, but in general the subject was feeling better. There was pain in the stomach on the night of the 11th, but the feeling of languor and indispesition to exertion had passed away. A feeling of weight in the stomach was also noted. Abdominal pains, though slight, continued on the 12th. On the 13th a feeling of indigestion was experienced, but it passed away within a short time. A small quantity of food was lost by belching on the 14th, but not to exceed a spoonful. On the 15 th the subject was feeling quite well again, and this condition continued until the 18th, when a slight headache developed with a tendency to diarrhea. Slight headache was also experienced on the 19th and the bowels continued loose. On the 20th the subject felt exceedingly well, although there was a very slight headache after dinner. This favorable condition continued until the $22 \mathrm{~d}$ and then was interrupted only by a slight headache with a tendency to drowsiness. Headache continued on November 23 and 24 , with slight pains in the stomach and abdomen and with a drowsy feeling coming on early in the evening. A slight abdominal disturbance was noticed on the $25 \mathrm{th}$, while a slight headache continued on the $26 \mathrm{th}$ and on the afternoon of the $27 \mathrm{th}$. The subject closed the preservative period with a weight of 60.13 kilograms and feeling reasonably well. His average weight for the whole preservative period was 60.62 kilograms, and for the fore period 61.20 kilograms.

The first day of the after period the headache continued, but the subject felt much better after luncheon. The second day the subject felt well, except for a slight feeling of uneasiness in the stomach. Headache came on about an hour before dinner with pains in the back and some weariness. No unpleasant feelings were experienced during the 30 th, but a slight feeling of laziness or drowsiness. A slight cold developed on December 1 , and the temperature at dinner was $99.6^{\circ}$. Some symptoms of this cold had been manifested for several days before, but no account had been made of it until this day. Slight pains in the back were experienced on the $2 \mathrm{~d}$ of December, but otherwise the subject was feeling well. Some little headache was experienced on the $3 \mathrm{~d}$ of December. An abscess formed on a tooth at this time which kept the subject awake during the night of the 4 th of December and he felt correspondingly ill during the day. The abscess was opened on the 5th of December and the subject felt much better, but slept only half the night. On the 6 th all bad symptoms had disappeared. The subject closed the after period feeling in excellent condition in spite of the trouble which he had had with his tooth. The final weight on the last day of the after period was 59.87 kilograms, and his average weight during the after period was 59.84 kilograms, showing a progressive loss in weight. 
No. 9.-G. W. L.

The weight of No. 9 at the beginning of the fore period was 62.10 kilograms. The temperature was very slightly above the normal and the rate of pulsation 84 per minute. The general physical condition was good. This subject had suffered from no serious disease within a year and had a long history of good health. Physical examination disclosed all the organs in sound state and performing their normal functions. There was but little variation in the condition of No. 9 during the fore period. The pulsation remained at about the original rate, which was slightly above normal. The average weight for the fore period was 62.25 kilograms, but the weight on the last day of the fore period was exactly that of the first day, namely, 62.10 kilograms.

No. 9 entered the preservative period in good condition. The first day there was a slight increase of temperature, almost 1 degree, but not sufficient to indicate a fever. This condition passed away, and on the second day the usual normal conditions were restored. The first preservative subperiod passed without any unusual incident. No. 9 did not, as most of the others had done, complain of being hungry during this period. Normal conditions were continued until November 3, when a slight cold was noticed, with an increase of temperature of about three-fourths of a degree. This condition continued on the following day, a slight degree of fever being manifested, with headache and a feeling of drowsiness during the day. These symptoms evidently were to be attributed to the cold rather than to the preservative. The conditions were very much improved on the 5th instant; in fact, with the exception of a slight cold in the head, the conditions were normal. The slight cold continued but without any inconvenience on the 6th instant, and the temperature and pulsation were normal. At this time there was a loss of weight amounting to about half a kilogram. This loss of weight was due in part to a rather larger evacuation than usual. Conditions were practically normal on the 7th with the exception of a slight continuation of the cold. All conditions were reported as favorable on November 9, during the second preservative subperiod, but a dislike for mutton and lamb was expressed when these meats were served. There was a slight increase of the cold on November 10, but without any unpleasant results. During the night of the 10th a restless condition which interfered to some extent with sleep developed, and on November 12 the cold was worse, but without any increase of temperature or other unfavorable symptoms.

No. 9 entered the fourth preservative subperiod feeling reasonably well and weighing 61.90 kilograms, almost the same as at the beginning of the period. There was a complaint of slight blurring of the vision during the previous day, and though the cold in the head was 
better, a pain in the region of the kidneys developed. This did not persist, however, and on the following day No. 9 was in good condition. Some pain in the eyes was noticed on November 17.

The fifth preservative subperiod found No. 9 with exactly the same weight as at the beginning of the fore period, namely, 62.10 kilograms. He complained of coughing slightly at night and of some nocturnal perspiration, but this was of -an entirely unimportant character.

At the beginning of the sixth preservative subperiod No. 9 weighed 62.70 kilograms and was in excellent condition, feeling better even than at any period during the progress of the experiment. The appetite was excellent, and on November 26 the subject felt hungry after dinner.

At the end of the preservative period, namely, November 27, No. 9 weighed 62.40 kilograms and was feeling in excellent condition. The average weight for the entire preservative period was 62.22 kilograms, as compared with 62.25 , the average of the fore period.

The beginning of the after period found No. 9 in excellent condition, and he passed through the entire after period without any incident worthy of attention, save that he complained of hunger on every day of the first subperiod, but made no such complaint during the second subperiod.

The weight of No. 9 at the end of the after period was 62.30 kilograms, temperature and pulse normal, and all the functions of the body apparently properly discharged. This case is quite in contrast with most of the others. First, in the fact that no unpleasant symptoms were observed even with the largest doses of salicylic acid; and, second, that the feeling of hunger, which was so commonly manifested during the first periods of the administration of the salicylic acid in the other cases, was not noticed in this, but the symptoms of hunger developed decidedly after the cessation of the doses of the salicylic acid.

Judged, therefore, simply by the medical history no unfavorable symptoms of any kind were noticed during the whole course of observation.

$$
\text { No. 10. }- \text { R. D. D. }
$$

No. 10 began the fore period weighing 57.10 kilograms. Physical examination revealed no defects in any of the organs of the body. There had been no previous disease of any consequence within a year and no tendency to any organic disturbances was found. The fore period passed without any incident worthy of record. The temperature and pulse remained normal during the entire time. At the close of the fore period the weight of No. 10 was 56.99 kilograms and all the functions of the body were normally discharged. The average weight for the entire fore period was 56.91 kilograms. 
At the beginning of the preservative period the weight of No. 10 was 56.95 kilograms; temperature and pulsation normal. During the first preservative subperiod he did not report any unusual hunger.

During the second subperiod he remained in excellent condition, without any apparent disturbance of any of the functions of the body. A slight cold developed at the beginning of the third preservative subperiod on November 8, but it was of no consequence and passed away on the following day.

At the beginning of the fourth subperiod the weight of No. 10 was 57.32 kilograms. A feeling of fullness was reported by the subject on that day. There was a slight depression of temperature amounting to about three-tenths of a degree. No unfavorable symptoms were manifested during the rest of this period.

At the beginning of the fifth preservative subperiod the weight of No. 10 was 57.26 kilograms and he was in excellent condition.

At the beginning of the sixth subperiod the weight of No. 10 was 57.10 kilograms, the temperature and pulse normal, and he was feeling very well. At the close of the sixth preservative subperiod the weight of No. 10 was 57.10 kilograms and no unfavorable symptoms had developed. No. 10 did not even complain of the hunger which was a characteristic symptom in most of the other cases. The average weight of No. 10 during the preservative period was 57.33 kilograms.

The subject entered the after period in excellent condition and on the second day complained of being hungry. He again complained of hunger on December 1.

At the beginning of the second after subperiod his weight was 56.30 kilograms and he still complained of being hungry. On December 4 No. 10 was taken ill and was unable to appear at his meals. On the 5th he reported for duty with a temperature about 1 degree above the normal and with a pulse of 90 per minute. He complained of feeling weak, his stomach was out of order, and his tongue coated. His condition was somewhat improved on the following day, but he did not feel entirely well. On the 7th the temperature and pulse were normal again and No. 10 reported himself as feeling well. The subject's weight on the last day of the after period was 52.2 kilograms, having lost about 4 kilograms from his illness.

It does not appear that in the above case the administration of the salicylic acid had any apparent effect either in increasing or decreasing the appetite or affecting in any way the ordinary functions of the organs of the body.

$$
\text { No. 11.-A. F. M. }
$$

The weight of No. 11 at the beginning of the fore period was 65.46 kilograms. His temperature and pulse were normal. During the whole of the fore period No. 11 remained in excellent condition and no variations of any consequence in his condition were noted. His 
weight on the last day of the fore period was 64.95 kilograms and his average weight for the period 65.36 kilograms.

He entered upon the preservative period in excellent physical condition. There were no unfavorable symptoms developed during the first preservative subperiod nor was there any complaint of hunger. A feeling of hunger was reported after dinner on November 7 , the end of the second subperiod, the usual amount of food not having satisfied the craving.

The third preservative subperiod found No. 11 in excellent condition, weighing 64.89 kilograms, and with normal pulse and temperature. During the night of November 9 he was restless and had a slight headache throughout the following day. On the 11th decided symptoms of indigestion developed, but without disturbing the temperature or pulse. The subject was feeling better on November 12 and entered the fourth preservative subperiod in fairly good condition, weighing 64.85 kilograms and with normal pulse and temperature. These normal conditions continued during the whole of the fourth preservative subperiod.

The weight of No. 11 at the beginning of the fifth subperiod was 64.27 kilograms. Slight symptoms of indigestion appeared at this time, especially after luncheon. No. 11 was feeling better on the following day and no further unfavorable symptoms were reported until November 22, when a slight headache was noticed.

At the beginning of the sixth preservative subperiod No. 11 weighed 64.03 kilograms and was suffering from a slight febrile attack, the temperature rising to $100^{\circ}$ and the pulsation to 90 . Headache persisted during the day, with a fever following and general weakness. Symptoms of a sore throat were developed and slight headache persisted during the following day, but the temperature was rormal. On the evening of the 24th he indulged in unusual exercise, but without discomfort. On the 25th No. 11 was again feeling in excellent condition, and this continued until the close of the preservative period. His average weight during the preservative period was 64.59 kilograms.

No. 11 entered the after period weighing 63.98 kilograms and with a normal temperature and pulse. The appetite increased during the after period, and on December 2 No. 11 complained of hunger after dinner. The second after subperiod passed without any unusual incident. No. 11 feft remarkably well during this time and weighed at its close 63.52 kilograms. His average weight for the entire after period was 63.57 kilograms. In the case of No. 11, as is seen, there were no very marked symptoms, though in the absence of any cold or influenza of any description the feelings of headache and indigestion which were developed from time to time could justly be attributed to the preservative that was administered. These symptoms, however, in no case were very serious nor did they cause any lasting discomfort. 
No. 12.- R. B. R.

No. 12 was one of the very best men of the class. His weight at the beginning of the fore period was 69.50 kilograms; his temperature and pulse were normal. No unfavorable symptoms of any description were developed during the entire fore period and his average weight was 69.70 kilograms.

No. 12 entered the preservative period in excellent condition, weighing 69.80 kilograms, and with normal pulse and temperature. No unfavorable symptoms were recorded during the first preservative subperiod. A good deal of mental work was required on November 3 , probably incident to his studies. The amount of exercise taken on November 5 was less than usual, No. 12 having experienced a feeling of drowsiness on that day with a sensation of fullness in the head and a slight headache which persisted during the entire day. He was nervous during the night of the 5th, but there was some improvement in his feelings during the 6 th. On this date also there were symptoms of a slight cold in the head and the feeling of fullness in the head continued. There was also a loss of appetite, and the urine discharged during the day was more cloudy than usual. The loss of appetite continued during the following day, but otherwise No. 12 was feeling very much better.

The third preservative subperiod began with a severe headache which lasted all day, but there was some improvement in the appetite. He complained of feeling very hungry at dinner time. The headache and other unfavorable symptoms had passed away on the 9th, and the feeling of hunger was increased. On the 10th, however, the appetite failed again partially, and symptoms of a bad cold were manifested, but without any marked increase in temperature or pulsation.

No. 12 was better on November 11, but on the following day he was not so well. He had a feeling of fatigue, although he had not taken any more than the usual amount of exercise, and the symptoms of hunger persisted.

The fourth preservative subperiod was marked by a slight loss of appetite and a headache which continued during the day. Otherwise No. 12 was feeling very well. On the 14 th he was extremely nervous, and had not slept well during the previous night. The headache and a sense of dryness in the mouth and throat continued throughout the day, and the headache persisted during the following day with a loss of appetite. The headache continued during the day of November 16 , but otherwise the symptoms, were more favorable and the subject improved. The unfavorable symptoms had passed away on the 17th, and a feeling of hunger was manifested.

The fifth preservative subperiod found No. 12 in the possession of a good appetite and without any indisposition of any kind. The restless condition, however, returned on the night of the 18th and lasted 
throughout the night, but did not interfere with the appetite the following day. On the 20th No. 12 was in excellent condition again. The feeling of hunger continued on the $22 \mathrm{~d}$ of November with a headache which lasted all day, preceded by a restless night, No. 12 reporting that he had very little sleep the night before.

The sixth preservative subperiod found No. 12 in good condition, weighing 70.15 kilograms and with normal temperature and pulse. During the remainder of this subperiod he felt very well, and the close of the preservative period found him in excellent condition, weighing 70.22 kilograms and with normal temperature and pulse. The average weight for the entire preservative period was 70.03 kilograms, an increase as compared with the average weight of the fore periods, 69.70 kilograms.

In the beginning of the after period No. 12 weighed 69.95 kilograms, with a temperature just a trifle below the normal and with normal pulsation.

During the first day of the after period No. 12 experienced a considerable degree of hunger, which was not entirely satisfied by the dinner. This feeling of hunger continued for two or three days, but with no other unfavorable symptoms. No. 12 continued to feel better during the whole of the after period and at its close weighed 69.8 kilograms. His average weight during the after period was 69.87 kilograms, a slight increase over that of the fore period, but a slight decrease as compared with the preservative period.

From the above description it is seen that No. 12 suffered very little during the administration of the preservative save from headache and fullness in the head and sometimes a disposition to drowsiness. The feeling of hunger, which was very marked in this case, was especially pronounced in the after period, and this, while similar to two or three cases, is quite different from the experience of most of the other subjects.

\section{CONCLUSIONS.}

A general review of the medical data shows in some instances decidedly unfavorable symptoms' attending the use of the salicylic acid, while in a minority of cases no symptoms of a distinctly unfavorable character can be attributed to the use of the preservative. In the majority of cases there was a strong feeling of hunger developed during the administration of the preservative, especially during its early stages, although the rations served were not less in quantity and were of the same quality as those which were provided during the fore period. In at least three of the cases-perhaps four-the feeling of hunger which was developed was noticeable chiefly during the after period. The general conclusion, therefore, judged by the medical history of all the cases considered as a whole, is that in some instances, 
and probably the majority, the administration of the preservative developed unfavorable conditions, while in the minority of the cases it seemed to have practically little effect one way or the other upon those who consumed it.

\section{BODY WEIGHTS.}

\section{VARIATIONS IN BODY WEIGHTS.}

In order to bring this important factor into prominence and to render a comparison easier, the changes in body weights of the individuals, daily and by periods, are expressed in graphic form in figs. 1 and 2 , and the general average for nine men, by periods, is also given. The data for total and average weights, by periods, on which the lines of average weight in the charts are based may be found in Table IV, on the relation between the food weights and body weights, under which head these data are discussed in greater detail. The daily variations are shown only by the broken lines, of which the straight lines give the average.

It is seen that No. 1 experienced a marked loss of weight during the preservative period and a greater loss during the after period. In the case of No. 2, the same condition exists, but in a still more marked degree. The case of No. 3 is especially interesting. This subject at the opening of the experiment weighed 52.78 kilograms; but after the second day of the fore period was taken ill with the grippe and was confined to his room until October 26. On his return to the table his weight had decreased to 50.54 kilograms. A new ration was selected as suited to his condition of convalescence and calculated to restore normal conditions. The graphic chart shows that there was a slight tendency throughout the preservative period toward a gain in weight, amounting as a whole throughout the preservative period of 25 days to 0.51 kilogram, but when the preservative was withdrawn the weight suddenly rises and the average for the after period shows a very marked increase, amounting to a gain of 0.73 kilogram for the ten days of that period. It is certainly indicated in this instance that the preservative inhibited to a marked degree the assimilation of the food, as a ration had been allowed which would meet the needs of the body in repairing and building up tissue after a short illness.

In the case of No. 4 the weight during the preservative period was almost identical with that of the fore period, but there was a slight loss of weight during the after period. The same conditions obtain in the case of No. 5. No. 6 shows an extreme case of very decided loss of weight amounting to about 1 kilogram in both the preservative and the after periods. No. 7 loses more than half a kilogram in the preservative period and about a kilogram in the after period. No. 8 lost 0.58 kilogram in the preservative period and 0.78 kilogram 


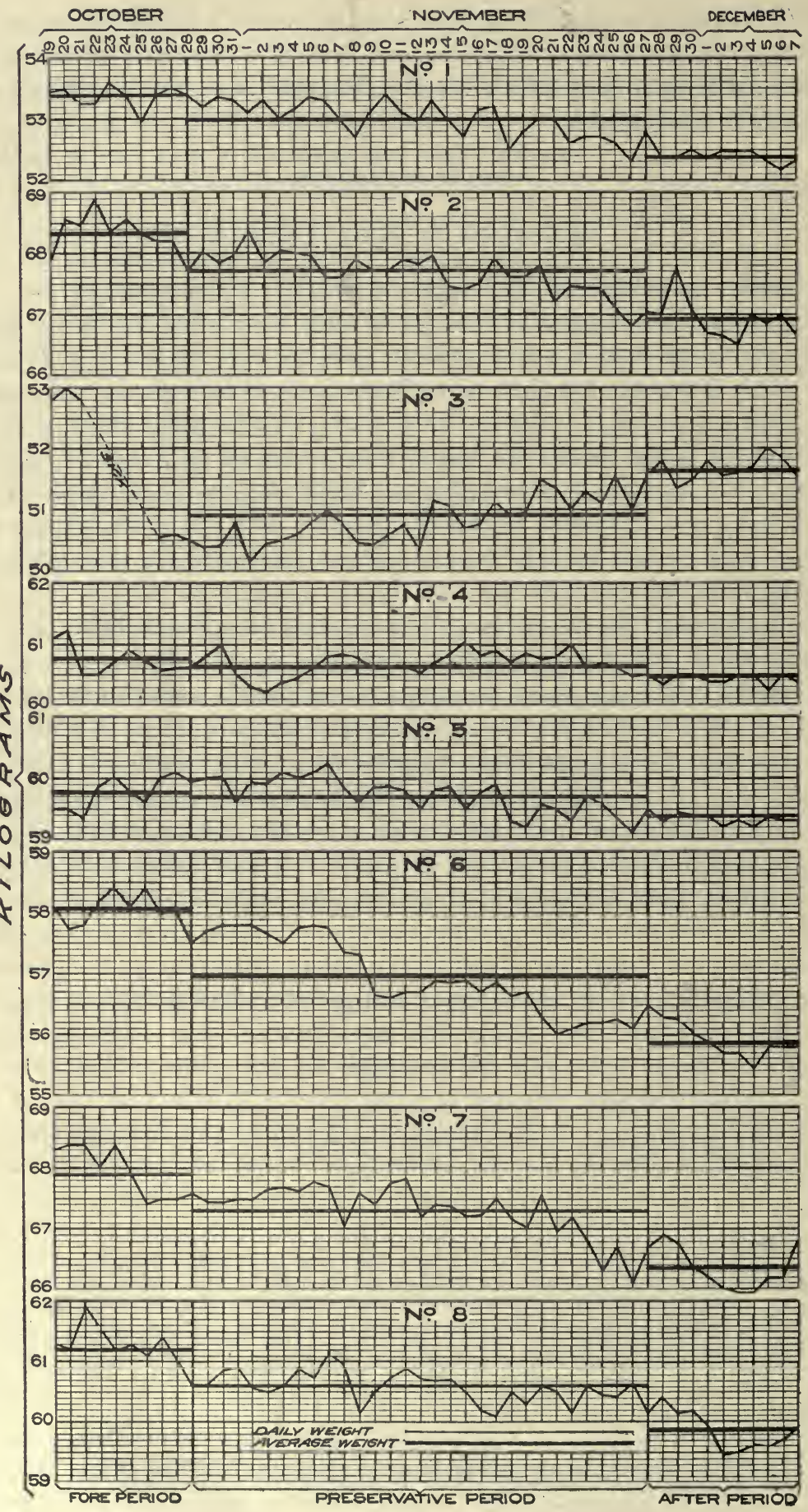

FIG. 1.-Daily and average body weights for Series VI, Nos. 1-8. 
in the after period. In the case of No. 9 the weight remains practically constant throughout the whole series, there being only a tendency to decrease in weight, which was more pronounced in the after period. No. 10 showed a considerable increase in weight during the preservative period and a slight loss during the after period covered by his illness, the average weight for the after period being practically the same as that of the fore period. It will be observed, however, that Nos. 3,9 , and 10 are excluded from the general summary, the data in

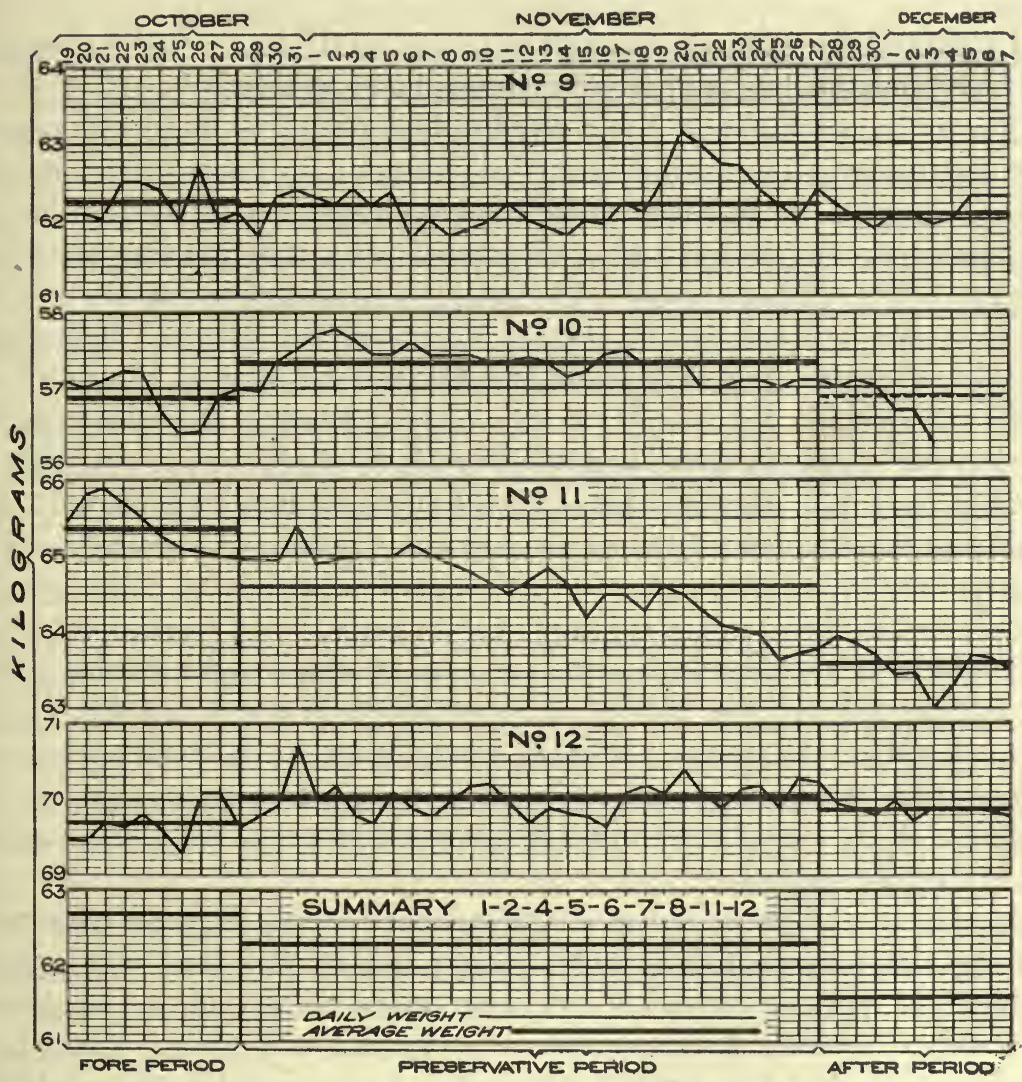

FIG. 2.-Daily and average body weights for Series VI, Nos. 9-12, and summary.

these cases, owing to variations introduced by sickness and other external causes, not being considered strictly comparable. No. 11 shows a considerable loss of weight during the preservative period, amounting to 0.77 kilogram, and a still more marked loss during the after period, of 1.03 kilograms. No. 12 shows a slightly increased weight during the preservative period, 0.33 kilogram, and a very slight loss in the after period, resulting in a slight gain for the entire series, i. e., 0.17 kilogram. 
The data showing the average effect produced on the nine men completing the series are plotted in the chart shown in fig. 2 . It is evident that this effect is toward a loss of weight during the preservative period and an increased loss during the after period. In other words, the larger relative loss produced during the after period is due doubtless to the momentum, as it may be called, of the effects produced during the preservative period.

RATIO OF FOOD WEIGHT TO BODY WEIGHT.

In Table IV are shown the results of the study made of the quantity of food consumed as compared with the body weight.

The average weight of No. 1 for the fore period is 53.38 kilograms, for the preservative period 52.99 kilograms, and for the after period 52.37 kilograms. It is seen that there is a slight loss of weight during the preservative period and a slightly greater loss during the after period. The average weight of moist food administered during the fore period is $2,427 \mathrm{grams}$ and of dry food $467 \mathrm{grams}$. The daily weight of moist food consumed is 4.55 per cent and of the dry food 0.87 per cent of the weight of the body. During the preservative period the average daily quantity of moist food consumed is 2,421 and of dry food 474 grams. The moist food consumed daily is 4.57 per cent and the dry food 0.89 per cent of the weight of the body. During the after period the quantity of moist food consumed daily is 2,345 grams and of dry food 476 grams. The moist food consumed is 4.48 per cent and the dry food 0.91 per cent of the weight of the body. It will be noticed that the quantity of dry food consumed during the various periods is almost constant, being least in the fore period and greatest in the after period. The loss of weight therefore can not be ascribed to any diminution of the quantity of food, the latter having been slightly increased.

In the case of No. 2 the average quantity of moist food consumed during the fore period is 2,669 grams and of dry food 604 grams, being 3.91 per cent and 0.88 per cent, respectively, of the weight of the body. During the preservative period the quantity of moist food consumed by No. 2 is 2,908 grams and of dry food 598 grams, being 4.30 and 0.88 per cent, respectively, of the weight of the body. During the after period the average quantity of moist food consumed by No. 2 is 2,876 grams and of dry food 612 grams daily, being 4.30 and 0.91 per cent, respectively, of the weight of the body.

The average weight of No. 2 during the fore period is 68.32 , during the preservative period 67.66 , and during the after period 66.91 kilograms. There is thus seen a progressive loss of weight which continued through the after, period, although the quantity of food remained practically the same, but was diminished by a few grams 
during the preservative period and increased by a few grams auring the after period.

The data for No. 3 are only fragmentary, as has already been explained, and therefore are of little value for experimental purposes. During the second subperiod of the fore period the quantity of moist food consumed daily by No. 3 is 2,610 grams, equivalent to 530 grams of dry material, or 5.18 and 1.05 per cent, respectively, of the weight of the body. For five subperiods of the preservative period the weight of moist food consumed daily is 2,568 grams, equivalent to 592 grams of the dry material, corresponding to 5.04 and 1.16 per cent, respectively, of the weight of the body. During the after period the quantity of moist food daily consumed is 2,524 grams, equivalent to 621 grams of dry food, 4.89 and 1.20 per cent, respectively, of the body weight. The average weight of. No. 3 during his fore period (five days) is 50.42 kilograms, during the five subperiods of the preservative period 50.93 kilograms, and during the after period 51.66 kilograms. No. 3 therefore presents a progressive gain in weight and also an increased quantity of dry food consumed. The explanation of this condition in connection with the illness of the subject has already been discussed under body weights.

The average weight of moist food consumed daily by No. 4 in the fore period is 2,311 grams, equivalent to 535 grams of dry material, these data corresponding to 3.80 and 0.88 per cent, respectively, of the weight of the body. For the preservative period the average quantity of moist food consumed daily is 2,378 grams, equivalent to 556 grams of dry material, corresponding to 3.92 and 0.92 per cent, respectively, of the weight of the body. For the after period the quantity of moist food consumed daily is $2,408 \mathrm{grams}$, corresponding to 569 grams of dry material, equivalent to 3.98 and 0.94 per cent, respectively, of the weight of the body. The mean weight of No. 4 for the fore period is 60.73 , for the preservative period 60.67 , and for the after period 60.43 kilograms. The loss of weight is practically negligible during the preservative period, and becomes a small though noticeable quantity for the after period, notwithstanding the slightly increased quantity of food.

The quantity of moist food consumed daily by No. 5 during the fore period is 2,357 grams, corresponding to 558 grams of dry material. These data represent 3.94 and 0.93 per cent, respectively, of the body weight. For the entire preservative period the average daily quantity of moist food consumed is 2,346 grams, equivalent to 563 grams of dry material and corresponding to 3.93 and 0.94 per cent, respectively, of the weight of the body. During the after period the average daily quantity of food consumed is $2,382 \mathrm{grams}$, corresponding to $56 \pm$ grams of dry material, equivalent to 4.01 and 
0.95 per cent, respectively, of the weight of the body. The average weight of No. 5 for the fore period is 59.76 , for the preservative period 59.71, and for the after period 59.33 kilograms. There is scarcely any change of weight between the fore period and the preservative period, but a decided loss during the after period, in spite of a slightly increased quantity of food.

The average quantity of moist food consumed daily during the fore period by No. 6 is 2,289 grams, corresponding to 537 grams of dry material, equivalent to 3.94 and 0.92 per cent, respectively, of the weight of the body. During the preservative period the mean daily quantity of moist food consumed is 2,372 grams, corresponding to 556 grams of dry material, equivalent to 4.17 and 0.98 per cent, respectively, of the weight of the body. During the after period the quantity of moist food consumed daily is 2,275 grams, corresponding to 561 grams of dry material, equivalent to 4.07 and 1 per cent, respectively of the weight of the body. The mean daily weight of No. 6 during the fore period is 58.06, for the preservative period 56.94, and for the after period 55.87 kilograms. These data show a very decided loss of weight during the preservative period, although the quantity of dry food consumed was somewhat greater than in the fore period. This loss of weight was continued through the after period and the total decrease was considerable, namely 2.19 kilograms. The amount of dry food consumed increased throughout the series.

The quantity of moist food consumed daily by No. 7 during the fore period is 2,203 grams, corresponding to 481 grams of dry material, equivalent to 3.24 and 0.71 per cent, respectively, of the weight of the body. During the preservative period the daily quantity of moist food consumed is 2,333 grams, corresponding to 482 grams of dry material, equivalent to 3.47 and 0.72 per cent, respectively, of the weight of the body. During the after period the quantity of moist food consumed daily by No. 7 is 2,261 grams, corresponding to 488 grams of dry material, equivalent to 3.41 and 0.74 per cent, respectively, of the weight of the body. The average weight of No. 7 during the fore period is 67.91 , during the preservative period 67.28 , and during the after period 66.33 kilograms. There is thus observed a considerable loss of weight during the preservative period as compared with the fore period (630 grams), and an increased loss of weight during the after period (950 grams). 'The respective quantities of food consumed are almost the same for the three periods. There is, however, an increase of 7 grams of dry food daily in the after period over the fore period, and of 6 grams daily in the after period over the preservative period. This continued loss of weight, attended as it is with a slight increase of the weight of food consumed, can only be attributed to the persistent effects of the preservative upon the metabolic processes. 
The average quantity of moist food consumed daily by No. 8 during the fore period is 3,338 grams, corresponding to 531 grams of dry material, equivalent to 5.45 and 0.87 per cent, respectively, of the weight of the body. During the preservative period the average daily quantity of moist food consumed by No. 8 is 3,408 grams, corresponding to 575 grams of dry material, equivalent to 5.62 and 0.95 per cent, respectively, of the weight of the body. During the after period the quantity of moist food consumed daily by No. 8 is 2,966 grams, corresponding to 587 grams of dry material, equivalent to 4.96 and 0.98 per cent, respectively, of the weight of the body. The average weight of No. 8 during the fore period is 61.20 , during the preservative period 60.62 , and during the after period 59.84 kilograms. 'The data here show also a progressive decrease in weight which was continued through the after period, although both in the preservative period and in the after period the quantity of dry material consumed is greater than in the fore period. This continued depressing action of the preservative on the metabolic processes as a whole, shown in decreased weight, is quite as marked in the case of No. 8, if not more so, as in that of No. 7, for although the actual decrease in weight is slightly less, the amount of dry food taken by No. 8 was appreciably greater, namely, for No. 8 an average daily increase of 56 grams, as compared with an increase of only 7 grams for No. 7 .

The average quantity of moist food consumed daily by No. 9 is 2,833 grams, corresponding to 634 grams of dry material, equivalent to 4.55 and 1.02 per cent, respectively, of the weight of the body. During the preservative period the average quantity of moist food consumed daily by No. 9 is 2,867 grams, corresponding to 624 grams of dry material, equivalent to 4.61 and 1 per cent, respectively, of the weight of the body. The average daily quantity of moist food consumed by No. 9 during the after period is 2,579 grams, corresponding to 623 grams of dry material, equivalent to 4.15 and 1 per cent, respectively, of the weight of the body. The average weight of No. 9 during the fore period is 62.25 , during the preservative period 62.22 , and during the after period 62.11 kilograms. These data show no marked change in the weight of the body during the entire progress of the observation, though the tendency is toward a decrease.

The average daily quantity of moist food consumed by No. 10 is 2,710 grams, corresponding to 660 grams of dry material, equivalent to 4.76 and 1.16 per cent, respectively, of the weight of the body. No. 10 consumed daily during the preservative period 3,029 grams of moist food, corresponding to 676 grams of dry material, equivalent to 5.28 and 1.18 per cent, respectively, of the weight of the body. During the after period No. 10 was ill during the second subperiod and the data for the first subperiod are therefore taken as the average for the whole after period. These data show that No. 10 in the after period 
consumed daily 2,897 grams of moist food, corresponding to 659 grams of dry material, equivalent to 5.09 and 1.16 per cent, respectively, of the weight of the body. The average weight of No. 10 during the fore period is 56.91, during the preservative period 57.33, and during the after period 56.90 kilograms. These data show a considerable increase in weight during the administration of the preservative and a slight loss during the after period, doubtless due to the illness occurring at that time.

The quantity of moist food consumed daily by No. 11 during the fore period is 3,013 kilograms, corresponding to 621 kilograms of dry material, and equivalent to 4.61 and 0.95 per cent, respectively, of the weight of the body. During the preservative period No. 11 consumed daily 2,997 grams of moist food, corresponding to 622 grams of dry material, equivalent to 4.64 and 0.96 per cent, respectively, of the weight of the body. During the after period No. 11 consumed daily 3,074 grams of moist food, corresponding to 615 grams of dry material, and equivalent to 4.84 and 0.97 per cent, respectively, of the weight of the body. The average weight of No. 11 during the fore period is 65.36 , during the preservative period 64.59 , and during the after period 63.56 kilograms. These data show a decided tendency toward a decrease in the body weight, although the amount of food remained practically the same in the fore and preservative periods; this tendency continued during the after period, and is not explained by the slight decrease in the weight of dry food consumed in that period, namely, 6 grans per day.

The average daily quantity of moist food consumed by No. 12 during the fore period is 2,806 grams, corresponding to 660 grams of the dry material, and equivalent to 4.03 and 0.95 per cent, respectively, of the weight of the body. During the preservative period No. 12 consumed daily 2,788 grams of moist food, corresponding to 653 grams of dry material, and equivalent to 3.98 and 0.93 per cent, respectively, of the weight of the body. During the after period No. 12 consumed daily 2,628 grams of moist food, corresponding to 640 grams of dry material, and equivalent to 3.76 and 0.92 per cent, respectively, of the weight of the body. The average weight of No. 12 during the fore period is 69.70 , during the preservative period 70.03, and during the after period 69.87 kilograms. These data show a slight increase of weight during the preservative period, which increase was somewhat diminished during the after period, leaving the average weight for that period 170 grams greater than the average for the fore period. It is also to be noted that in this case the amount of dry food decreased an average of 7 grams a day in the preservative period, and 13 grams additional in the after period, presenting a very slight tendency in the opposite direction to that manifested in the majority of cases, namely, an increase in dry food and a decrease in weight. This might indicate 
that the condition of No. 12 was such that the salicylic acid administered had a medicinal value, and shows the fallacy of depending on individual results in such a study. These figures, whatever their explanation, receive full weight in the summary for nine men given in Table V. The data for the three men excluded, Nos. 3, 9, and 10, while given in detail, are not included in the averages of the summary for the reason that illness on the part of No. 3 rendered the data in his case not strictly comparable, and certain marked irregularities in the balances for Nos. 9 and 10 could only be explained on the basis that they had violated the rules of observation and so invalidated the data.

The data for the nine men, averaged by periods, are as follows:

Body weight for the fore period, 62.71 kilograms; moist food consumed, 2,601 grams; dry food, 555 grams; mean percentage of body weight represented by the moist food, 4.15 ; by the dry food, 0.88 .

For the entire preservative period the average weight of the nine men is 62.27 kilograms; the amount of moist food consumed daily, 2,661 grams; the amount of dry food, 564 grams; the average percentage of the body weight represented by the moist food is 4.27 , and by the dry food, 0.91 .

In the after period the average daily weight for the nine men is 61.61 kilograms; the amount of moist foor, 2,579 grams; the amount of dry food, 568 grams; the average percentage of the weight of the body represented by the moist food is 4.19 , and by the dry food, 0.92 .

This summary shows that the average body weight declined consistently throughout the experiment, the average total loss of weight being 1.1 kilograms. The quantity of dry food consumed, however, gradually increased, rising from 555 grams for the fore period, to 564 in the preservative period, and to 568 in the after period, an average daily increase of 9 grams during the preservative period and 4 grams additional during the after period. These data show a distinct tendency as a whole on the part of the preservative to diminish the weight of the body notwithstanding an increase in dry food consumed.

The graphic presentations of the variations in body weight as discussed above and given in the tables are to be found in figs. 1 and 2. 
TABLE IV.-Amount of moist and dry food consumed, expressed as percentage of body weight, Series VI.

[Averages are per day.]

\begin{tabular}{|c|c|c|c|c|c|c|c|c|c|c|}
\hline \multirow{3}{*}{ Period. } & \multicolumn{5}{|c|}{ No. 1.} & \multicolumn{5}{|c|}{ No. 2.} \\
\hline & \multirow[t]{2}{*}{$\begin{array}{c}\text { Body } \\
\text { weight. }\end{array}$} & \multicolumn{2}{|c|}{ Weight of food. } & \multicolumn{2}{|c|}{$\begin{array}{c}\text { Average } \\
\text { daily ratio } \\
\text { of food } \\
\text { weight to } \\
\text { body weight. }\end{array}$} & \multirow[t]{2}{*}{$\begin{array}{l}\text { Body } \\
\text { weight. }\end{array}$} & \multicolumn{2}{|c|}{ Weight of food. } & \multicolumn{2}{|c|}{$\begin{array}{c}\text { Average } \\
\text { daily ratio } \\
\text { of food } \\
\text { weight to } \\
\text { body weight. }\end{array}$} \\
\hline & & Dry. & Moist. & Drý. & Moist. & & Dry. & Moist. & Dry. & Moist. \\
\hline Fore period. & & & & & & & & & & \\
\hline $\begin{array}{l}\text { First subperiod: } \\
\quad \text { Total.................. } \\
\text { A verage } . . . . . .\end{array}$ & $\begin{array}{r}\text { Kilos. } \\
267.18 \\
53.44\end{array}$ & $\begin{array}{r}\text { Grams. } \\
2,394 \\
479\end{array}$ & $\begin{array}{r}\text { Grams. } \\
12,335 \\
2,467\end{array}$ & $\begin{array}{l}\text { P. ct. } \\
0.90 \\
\ldots \ldots\end{array}$ & $\begin{array}{l}P_{i .62} . \\
4.62\end{array}$ & $\begin{array}{l}\text { Kilos. } \\
342.16 \\
68.43\end{array}$ & $\begin{array}{r}\text { Grams. } \\
2,999 \\
600\end{array}$ & $\begin{array}{r}\text { Grams. } \\
13,346 \\
2,669\end{array}$ & $\begin{array}{l}\text { P. } c t \text {. } \\
0.88\end{array}$ & $\begin{array}{l}\text { P. ct. } \\
\quad 3.90\end{array}$ \\
\hline 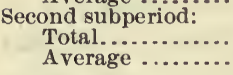 & $\begin{array}{r}266.58 \\
53.32\end{array}$ & $\begin{array}{r}2,275 \\
455\end{array}$ & $\begin{array}{r}11,930 \\
2,386\end{array}$ & .85 & 4.48 & $\begin{array}{r}341.05 \\
68.21\end{array}$ & $\begin{array}{r}3,040 \\
608\end{array}$ & $\begin{array}{r}13,348 \\
2,670\end{array}$ & .89 & 3.91 \\
\hline $\begin{array}{l}\text { Entire fore period: } \\
\text { Total................ } \\
\text { Average .......... }\end{array}$ & $\begin{array}{r}533.76 \\
53.38\end{array}$ & $\begin{array}{r}4,669 \\
467\end{array}$ & $\begin{array}{r}24,265 \\
2,427\end{array}$ & .87 & 4.55 & $\begin{array}{r}683.21 \\
68.32\end{array}$ & $\begin{array}{r}6,039 \\
604\end{array}$ & $\begin{array}{r}26,694 \\
2,669\end{array}$ & .88 & 3.91 \\
\hline Preservative period. & & & & & & & & & & \\
\hline $\begin{array}{l}\text { First subperiod: } \\
\text { Total................ } \\
\text { Average .......... }\end{array}$ & $\begin{array}{r}266.26 \\
53.25\end{array}$ & $\begin{array}{r}2,360 \\
472\end{array}$ & $\begin{array}{r}12,258 \\
2,452\end{array}$ & .89 & 4.60 & $\begin{array}{r}340.05 \\
68.01\end{array}$ & $\begin{array}{r}2,925 \\
585\end{array}$ & $\begin{array}{r}14,552 \\
2,910\end{array}$ & .86 & 4.28 \\
\hline $\begin{array}{l}\text { Second subperiod: } \\
\text { Total............. } \\
\text { Average }\end{array}$ & $\begin{array}{r}265.77 \\
53.15\end{array}$ & $\begin{array}{r}2,338 \\
468\end{array}$ & $\begin{array}{r}12,385 \\
2,477\end{array}$ & .88 & 4.66 & $\begin{array}{r}339.18 \\
67.84\end{array}$ & $\begin{array}{r}2,937 \\
587\end{array}$ & $\begin{array}{r}14,656 \\
2,931\end{array}$ & .87 & 4.32 \\
\hline $\begin{array}{l}\text { Third subperiod: } \\
\text { Total........... } \\
\text { Average ...... }\end{array}$ & $\begin{array}{r}265.25 \\
53.05\end{array}$ & $\begin{array}{r}2,340 \\
468\end{array}$ & $\begin{array}{r}11,934 \\
2,387\end{array}$ & .88 & 4.50 & $\begin{array}{r}339.02 \\
67.80\end{array}$ & $\begin{array}{r}501 \\
2,996 \\
599\end{array}$ & $\begin{array}{r}14,553 \\
2,911\end{array}$ & .88 & 4.29 \\
\hline $\begin{array}{l}\text { Fourth subperiod: } \\
\text { Total............. } \\
\text { Average }\end{array}$ & $\begin{array}{r}265.81 \\
53.06\end{array}$ & $\begin{array}{r}2,305 \\
461\end{array}$ & $\begin{array}{r}12,273 \\
2,455\end{array}$ & .87 & 4.63 & $\begin{array}{r}338.23 \\
67.65\end{array}$ & $\begin{array}{r}3,009 \\
602\end{array}$ & $\begin{array}{r}14,391 \\
2 ; 878\end{array}$ & .89 & 4.25 \\
\hline $\begin{array}{l}\text { Fifth subperiod: } \\
\text { Total............. } \\
\text { Average ....... }\end{array}$ & $\begin{array}{r}263.88 \\
52.78\end{array}$ & 2,568 & $\begin{array}{r}11,830 \\
, 366\end{array}$ & .97 & 4.48 & 337.69 & 3,100 & 14,814 & .92 & 4.39 \\
\hline 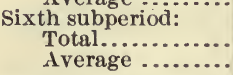 & $\begin{array}{r}263.10 \\
52.62\end{array}$ & $2, ? 15$ & $\begin{array}{r}11,943 \\
2,389\end{array}$ & .88 & 4.54 & $\begin{array}{r}335.75 \\
67.15\end{array}$ & $\begin{array}{r}2,963 \\
593\end{array}$ & $\begin{array}{r}14,264 \\
2,853\end{array}$ & .88 & 4.25 \\
\hline $\begin{array}{l}\text { Entire preservative } \\
\text { period: } \\
\text { Total.............. } \\
\text { Average .......... }\end{array}$ & $\begin{array}{r}1,589.57 \\
52.99\end{array}$ & $\begin{array}{r}14,226 \\
474\end{array}$ & $\begin{array}{r}72,623 \\
2,421\end{array}$ & .89 & 4. 57 & $\begin{array}{r}2,029.92 \\
67.66\end{array}$ & $\begin{array}{r}17,930 \\
598\end{array}$ & $\begin{array}{r}87,230 \\
2,908\end{array}$ & $\begin{array}{l}.88 \\
\ldots .\end{array}$ & $\begin{array}{r}4.30 \\
\ldots . .\end{array}$ \\
\hline After period. & & & & & & & & & & \\
\hline $\begin{array}{l}\text { First subperiod: } \\
\text { Total............ } \\
\text { Average }\end{array}$ & $\begin{array}{r}262.08 \\
52.42\end{array}$ & $\begin{array}{r}2,325 \\
465\end{array}$ & $\begin{array}{r}11,895 \\
2,379\end{array}$ & .89 & 4.54 & $\begin{array}{r}335.12 \\
67.02\end{array}$ & $\begin{array}{r}3,021 \\
604\end{array}$ & $\begin{array}{r}14,069 \\
2,814\end{array}$ & .90 & 4.20 \\
\hline $\begin{array}{l}\text { Second subperiod: } \\
\text { Total.............. } \\
\text { Average .............. }\end{array}$ & $\begin{array}{r}261.57 \\
52.31\end{array}$ & $\begin{array}{r}2,435 \\
487\end{array}$ & $\begin{array}{r}11,558 \\
2,312\end{array}$ & .93 & 4.42 & $\begin{array}{r}333.95 \\
66.79\end{array}$ & $\begin{array}{r}3,094 \\
619\end{array}$ & $\begin{array}{r}14,692 \\
2,938\end{array}$ & .93 & 4.40 \\
\hline 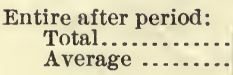 & $\begin{array}{r}523.65 \\
52.37\end{array}$ & $\begin{array}{r}4,760 \\
476\end{array}$ & $\begin{array}{r}23,453 \\
2,345\end{array}$ & .91 & 4.48 & $\begin{array}{r}669.07 \\
66.91\end{array}$ & $\begin{array}{r}6,115 \\
612\end{array}$ & $\begin{array}{r}28,761 \\
2,876\end{array}$ & .91 & 4.30 \\
\hline
\end{tabular}


TABLE IV.-Amount of moist and dry food, consumed, expressed as percentage of body weight, Series VI-Continued.

[Averages are per day.]

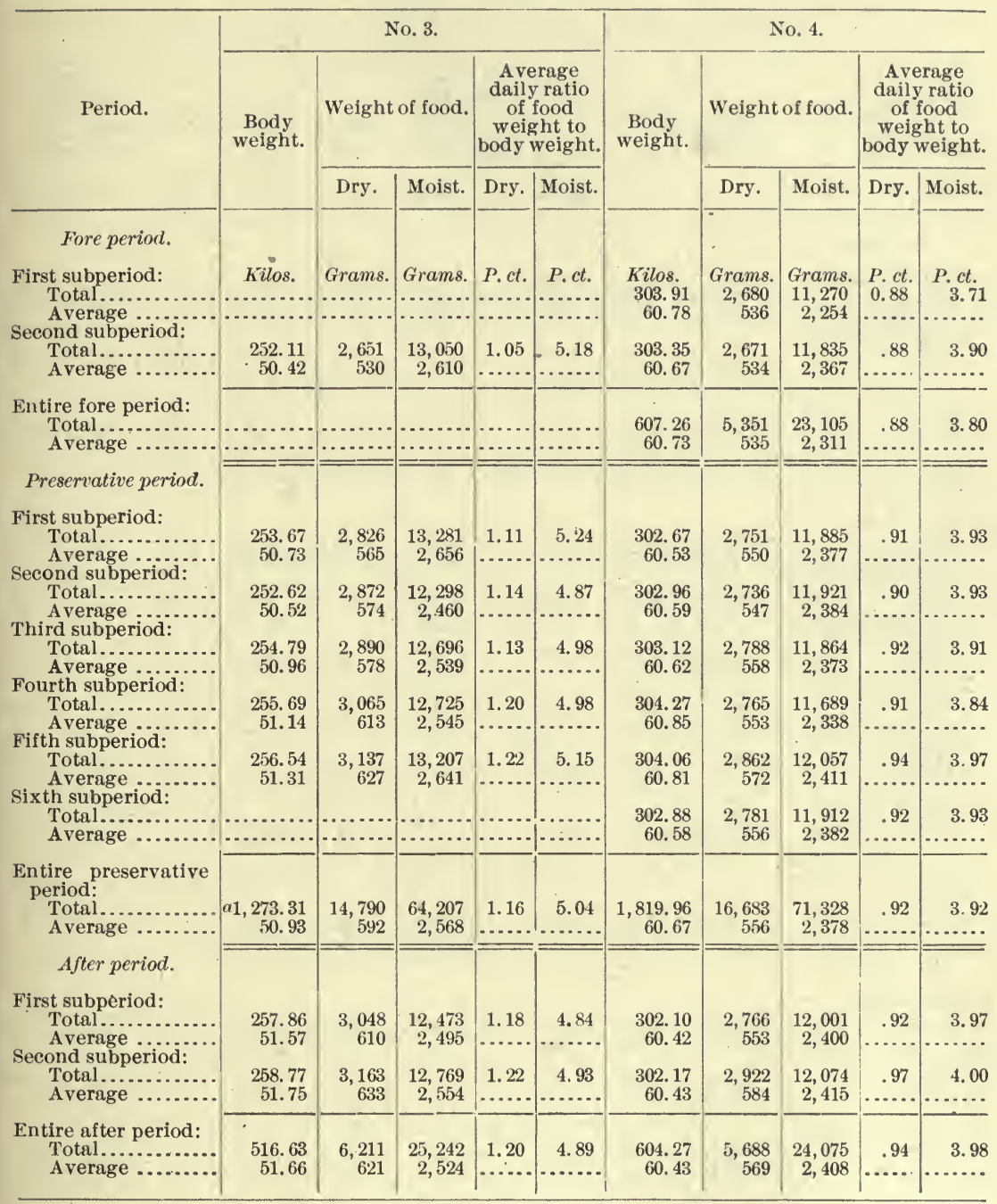

$a$ No. 3 had only five preservative subperiods. 
TABLE IV.-Amount of moist and dry food consumed, expressed as percentage of body weight, Series VI-Continued.

[Averages are per day.]

\begin{tabular}{|c|c|c|c|c|c|c|c|c|c|c|}
\hline \multirow{3}{*}{ Period. } & \multicolumn{5}{|c|}{ No. 5.} & \multicolumn{5}{|c|}{ No. 6.} \\
\hline & \multirow[t]{2}{*}{$\begin{array}{c}\text { Body } \\
\text { weight. }\end{array}$} & \multicolumn{2}{|c|}{ Weight of food. } & \multicolumn{2}{|c|}{$\begin{array}{c}\text { Average } \\
\text { daily ratio } \\
\text { of food } \\
\text { weight to } \\
\text { body weight. }\end{array}$} & \multirow[t]{2}{*}{$\begin{array}{c}\text { Body } \\
\text { weight. }\end{array}$} & \multicolumn{2}{|c|}{ Weight of food. } & \multicolumn{2}{|c|}{$\begin{array}{l}\text { Average } \\
\text { daily ratio } \\
\text { of food } \\
\text { weight to } \\
\text { body weight. }\end{array}$} \\
\hline & & Dry. & Moist. & Dry. & Moist. & & Dry. & Moist. & Dry. & Moist. \\
\hline Fore period. & & & & & & & & & & \\
\hline $\begin{array}{l}\text { First subperiod: } \\
\text { Total............... } \\
\text { Average ......... }\end{array}$ & $\begin{array}{r}\text { Kilos. } \\
298.15 \\
59.63\end{array}$ & $\begin{array}{r}\text { Grams. } \\
2,824 \\
565\end{array}$ & $\begin{array}{r}\text { Grams. } \\
11,689 \\
2,338\end{array}$ & $\begin{array}{l}\text { P. ct. } \\
0.95\end{array}$ & $\begin{array}{l}\text { P. ct. } \\
3.92\end{array}$ & $\begin{array}{r}\text { Kilos. } \\
290.32 \\
58.06\end{array}$ & $\begin{array}{r}\text { Grams. } \\
2,631 \\
526\end{array}$ & $\begin{array}{r}\text { Grams. } \\
11,885 \\
2,377\end{array}$ & $\begin{array}{l}\text { P. ct. } \\
0.91\end{array}$ & $\begin{array}{l}\text { P. ct. } \\
4.09\end{array}$ \\
\hline Second subperiod: & & & 2, 030 & & & 08.00 & & 2,377 & $\cdots \cdots$ & (n...... \\
\hline $\begin{array}{l}\text { Total....................... } \\
\text { A verage.... }\end{array}$ & $\begin{array}{r}299.45 \\
59.89\end{array}$ & $\begin{array}{r}2,758 \\
552\end{array}$ & $\begin{array}{r}11,885 \\
2,377\end{array}$ & $\begin{array}{r}.92 \\
\cdots . . \\
\end{array}$ & $\begin{array}{r}3.97 \\
\cdots\end{array}$ & $\begin{array}{r}290.28 \\
58.06\end{array}$ & $\begin{array}{r}2,736 \\
547\end{array}$ & $\begin{array}{r}11,000 \\
2,200\end{array}$ & $\begin{array}{r}.94 \\
\cdots \cdots\end{array}$ & $\begin{array}{r}3.79 \\
\cdots\end{array}$ \\
\hline $\begin{array}{l}\text { Entire fore period: } \\
\quad \text { Total............ } \\
\text { Average........ }\end{array}$ & $\begin{array}{r}597.60 \\
59.76 \\
\end{array}$ & $\begin{array}{r}5,582 \\
558 \\
\end{array}$ & $\begin{array}{r}23,574 \\
2,357 \\
\end{array}$ & $\begin{array}{r}.93 \\
\therefore \\
\end{array}$ & $\begin{array}{r}3.94 \\
\cdots \cdots \\
\end{array}$ & $\begin{array}{r}580.60 \\
58.06 \\
\end{array}$ & $\begin{array}{r}5,367 \\
537 \\
\end{array}$ & $\begin{array}{r}22,885 \\
2,289 \\
\end{array}$ & $\begin{array}{r}.92 \\
\cdots \\
\end{array}$ & $\begin{array}{l}3.94 \\
\cdots \\
\end{array}$ \\
\hline \multicolumn{11}{|l|}{ Preservative period. } \\
\hline $\begin{array}{l}\text { First subperiod: } \\
\text { Total............. } \\
\text { Average........ }\end{array}$ & $\begin{array}{r}299.45 \\
59.89\end{array}$ & $\begin{array}{r}2,867 \\
573\end{array}$ & $\begin{array}{r}11,872 \\
2,374\end{array}$ & .96 & $\begin{array}{r}3.96 \\
\cdots\end{array}$ & $\begin{array}{r}288.73 \\
57.75\end{array}$ & $\begin{array}{r}2,602 \\
520\end{array}$ & $\begin{array}{r}11,412 \\
2,282\end{array}$ & .90 & $\begin{array}{l}3.95 \\
\cdots . .\end{array}$ \\
\hline $\begin{array}{l}\text { Second subperiod: } \\
\text { Total........................ }\end{array}$ & & & & & & & & 2,202 & & \\
\hline $\begin{array}{l}\text { Average....................... } \\
\text { Third subperiod: }\end{array}$ & $\begin{array}{r}300.33 \\
60.07\end{array}$ & $\begin{array}{r}2,753 \\
551\end{array}$ & $\begin{array}{r}11,798 \\
2,360\end{array}$ & .92 & 3.93 & $\begin{array}{r}288.19 \\
57.64\end{array}$ & $\begin{array}{r}2,721 \\
544\end{array}$ & $\begin{array}{r}11,693 \\
2,339\end{array}$ & .94 & 4.06 \\
\hline $\begin{array}{l}\text { Third subperiod: } \\
\quad \text { Total................... }\end{array}$ & 298.56 & 2,787 & 11,596 & .93 & & 283.91 & & & & \\
\hline $\begin{array}{l}\text { A verage........... } \\
\text { rourth subperiod: }\end{array}$ & 59.71 & 557 & 2,319 & $\begin{array}{c}.93 \\
\cdots \cdot .\end{array}$ & $\begin{array}{r}3.88 \\
\cdots . . .\end{array}$ & $\begin{array}{r}283.91 \\
56.78\end{array}$ & $\begin{array}{r}2,745 \\
549\end{array}$ & $\begin{array}{r}11,960 \\
2,393\end{array}$ & $\begin{array}{r}97 \\
.9\end{array}$ & $\begin{array}{l}4.21 \\
\ldots . .\end{array}$ \\
\hline $\begin{array}{l}\text { Tourth subperiod: } \\
\text { Total................. } \\
\text { Average }\end{array}$ & 298.84 & 2,804 & 11,538 & 94 & 3,86 & 284.22 & 2.778 & & & 300 \\
\hline $\begin{array}{l}\text { A verage........ } \\
\text { Fifth subperiod: }\end{array}$ & 59.77 & 561 & 2,308 & & $\begin{aligned} & 0.80 \\
\cdots & . . .2\end{aligned}$ & $\begin{array}{r}284.22 \\
56.84\end{array}$ & 556 & $\begin{array}{r}11,343 \\
2,269\end{array}$ & .98 & 3.99 \\
\hline $\begin{array}{l}\text { Fifth subperiod: } \\
\text { Total............... }\end{array}$ & 296.77 & 2,862 & 11.822 & .96 & 3.98 & 281.77 & 2,874 & 12,185 & 1.02 & 4. 32 \\
\hline $\begin{array}{l}\text { Average............ } \\
\text { Sixth subperiod: }\end{array}$ & 59.35 & 572 & 2,364 & & $\cdots \cdots$ & 56.35 & 575 & 2,437 & & (....... \\
\hline 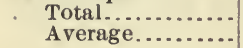 & $\begin{array}{r}297.33^{\circ} \\
59.47\end{array}$ & 2,814 & 11,756 & .95 & 3.95 & 281.28 & 2,960 & 12,571 & 1.05 & 4. 47 \\
\hline \multirow{2}{*}{\multicolumn{11}{|c|}{$\begin{array}{l}\text { Entire preservative } \\
\text { period: }\end{array}$}} \\
\hline & & & & & & & & & & \\
\hline 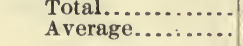 & $\begin{array}{r}1,791.28 \\
59.71\end{array}$ & $\begin{array}{r}16,887 \\
563\end{array}$ & $\begin{array}{r}70,382 \\
2,346\end{array}$ & .94 & 3.93 & $1,708.10$ & 16,680 & 71,169 & .98 & 4. 17 \\
\hline \multirow{2}{*}{\multicolumn{11}{|c|}{ After period. }} \\
\hline & & & & & & & & & & \\
\hline $\begin{array}{r}\text { Total......................... } \\
\text { Average.... }\end{array}$ & 296.82 & 2,791 & 11,904 & .94 & 4. 01 & 280.10 & 2,761 & 11,673 & .99 & 4. 17 \\
\hline $\begin{array}{l}\text { Average.......... } \\
\text { Second subperiod: }\end{array}$ & & & 2,001 & & & & & 2,335 & & - \\
\hline $\begin{array}{l}\text { Total } \ldots . . . . . \\
\text { Average............. }\end{array}$ & $\begin{array}{r}296.43 \\
59.29\end{array}$ & $\begin{array}{r}2,850 \\
570\end{array}$ & 11, 911 & .96 & 4.02 & 278.56 & 2,848 & 11,077 & 1.02 & 3. 98 \\
\hline Entire after & & & & & & & & & $\cdots$ & \\
\hline $\begin{array}{l}\text { Total... } \\
\text { Averag }\end{array}$ & 593.25 & 5,641 & 23,815 & .95 & 4. 01 & 558.66 & 5,609 & 22,750 & 1.00 & 4. 07 \\
\hline & & & & & & & 561 & & & \\
\hline
\end{tabular}


TABLE IV.-Amount of moist and dry food consumed, expressed as percentage of body weight, Series VI-Continued.

[Averages are per day.]

\begin{tabular}{|c|c|c|c|c|c|c|c|c|c|c|}
\hline \multirow{3}{*}{ Period. } & \multicolumn{5}{|c|}{ No. 7.} & \multicolumn{5}{|c|}{ No. 8.} \\
\hline & \multirow[t]{2}{*}{$\begin{array}{c}\text { Body } \\
\text { weight. }\end{array}$} & \multicolumn{2}{|c|}{ Weight of food. } & \multicolumn{2}{|c|}{$\begin{array}{l}\text { Average } \\
\text { daily ratio } \\
\text { of food } \\
\text { weight to } \\
\text { body weight. }\end{array}$} & \multirow[t]{2}{*}{$\begin{array}{c}\text { Body } \\
\text { weight. }\end{array}$} & \multicolumn{2}{|c|}{ Weight of food. } & \multicolumn{2}{|c|}{$\begin{array}{c}\text { Average } \\
\text { daily ratio } \\
\text { of food } \\
\text { weight to } \\
\text { body weight }\end{array}$} \\
\hline & & Dry. & Moist. & Dry. & Moist. & & Dry. & Moist. & Dry. & Moist. \\
\hline Fore period. & & & & & & & - & & & \\
\hline $\begin{array}{l}\text { First subperiod: } \\
\text { Total............... } \\
\text { Average.......... }\end{array}$ & $\begin{array}{r}\text { Kilos. } \\
341.27 \\
68.25\end{array}$ & $\begin{array}{r}\text { Grams. } \\
2,317 \\
463\end{array}$ & $\begin{array}{r}\text { Grams. } \\
11,644 \\
2,329\end{array}$ & $\begin{array}{l}\text { P. ct. } \\
0.68\end{array}$ & $\begin{array}{l}\text { P. ct. } \\
\text { 3. } 41\end{array}$ & $\begin{array}{r}\text { Kilos. } \\
307.10 \\
61.42\end{array}$ & $\begin{array}{r}\text { Grams. } \\
2,667 \\
533\end{array}$ & $\begin{array}{l}\text { Grams. } \\
17,169 \\
3,434\end{array}$ & $\begin{array}{l}\text { P. ct. } \\
0.87\end{array}$ & $\begin{array}{l}\text { P. ct. } \\
5.59\end{array}$ \\
\hline econd subperiod: & 0 & & 2,329 & & & 61.42 & & 3,434 & - ...... & $\ldots \ldots$ \\
\hline 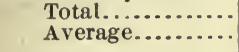 & $\begin{array}{r}337.86 \\
67.57\end{array}$ & $\begin{array}{r}2,490 \\
498\end{array}$ & $\begin{array}{r}10,386 \\
2,077\end{array}$ & $\begin{array}{r}.74 \\
\cdots\end{array}$ & $\begin{array}{r}3.07 \\
\ldots \ldots\end{array}$ & $\begin{array}{r}304.88 \\
60.98\end{array}$ & $\begin{array}{r}2,644 \\
529\end{array}$ & $\begin{array}{r}16,214 \\
3,243\end{array}$ & $\begin{array}{r}.87 \\
\ldots .8\end{array}$ & $\begin{array}{r}5.32 \\
\ldots .\end{array}$ \\
\hline $\begin{array}{l}\text { Entire fore period: } \\
\text { Total............... } \\
\text { Average........ }\end{array}$ & $\begin{array}{r}679.13 \\
67.91\end{array}$ & $\begin{array}{r}4,807 \\
481\end{array}$ & $\begin{array}{r}22,030 \\
2,203 \\
\end{array}$ & $\begin{array}{r}.71 \\
\cdots \cdots\end{array}$ & $\begin{array}{r}3.24 \\
\cdots \cdots\end{array}$ & $\begin{array}{r}611.98 \\
61.20\end{array}$ & $\begin{array}{r}5,311 \\
531\end{array}$ & $\begin{array}{r}33,383 \\
3,338\end{array}$ & $\begin{array}{r}.87 \\
\cdots \cdots\end{array}$ & $\begin{array}{r}5.45 \\
\ldots \ldots .\end{array}$ \\
\hline Preservative period. & & & & & & & & & & \\
\hline $\begin{array}{l}\text { First subperiod: } \\
\quad \text { Total............ }\end{array}$ & 337.53 & 2,376 & 11,771 & .70 & 3.49 & 304.55 & 2,847 & 18,140 & .93 & 5.96 \\
\hline riod: & & 475 & 2,354 & ....... & ........ & & 569 & 3,628 & ...... & ...... \\
\hline $\begin{array}{l}\text { Total... } \\
\text { Average }\end{array}$ & $\begin{array}{r}337.88 \\
67.58\end{array}$ & $\begin{array}{r}2,402 \\
480\end{array}$ & $\begin{array}{r}11,335 \\
2,267\end{array}$ & .71 & 3.35 & $\begin{array}{r}304.34 \\
60.87\end{array}$ & $\begin{array}{r}2,777 \\
555\end{array}$ & $\begin{array}{r}17,766 \\
3,553\end{array}$ & .91 & $\begin{array}{l}5.84 \\
\ldots . .\end{array}$ \\
\hline Third subp & & & & & & & & & & \\
\hline $\begin{array}{l}\text { Tetal.. } \\
\text { Average }\end{array}$ & $\begin{array}{r}337.82 \\
67.56\end{array}$ & $\begin{array}{r}2,396 \\
479\end{array}$ & $\begin{array}{r}12,093 \\
2,419\end{array}$ & .71 & 3.58 & $\begin{array}{r}303.06 \\
60.61\end{array}$ & 2,760 & $\begin{array}{r}16,646 \\
3,329\end{array}$ & .91 & 5.49 \\
\hline Fourth subperiod: & & & & & & & & & & . \\
\hline $\begin{array}{l}\text { Total.................. } \\
\text { Average...... }\end{array}$ & $\begin{array}{r}336.68 \\
67.34\end{array}$ & $\begin{array}{r}2,431 \\
486\end{array}$ & $\begin{array}{r}11,839 \\
2,368\end{array}$ & .72 & 3.52 & $\begin{array}{r}302.15 \\
60.43\end{array}$ & $\begin{array}{r}2,827 \\
565\end{array}$ & $\begin{array}{r}17,357 \\
3,471\end{array}$ & .94 & 5.74 \\
\hline Fifth & & & 2,000 & & & & & & & \\
\hline (......... & $\begin{array}{r}335.8 \\
67.1\end{array}$ & $\begin{array}{r}2,434 \\
487\end{array}$ & $\begin{array}{r}11,517 \\
2,303\end{array}$ & .72 & 3.43 & 302.08 & 3,008 & 17,177 & 1.00 & 5.69 \\
\hline Sixth subperiod: & & & & & & & & 0,800 & $\cdots$ & \\
\hline $\begin{array}{l}\text { Total................... } \\
\text { Average..... }\end{array}$ & $\begin{array}{r}332.62 \\
66.52\end{array}$ & $\begin{array}{r}2,435 \\
487\end{array}$ & $\begin{array}{r}11,429 \\
2,286\end{array}$ & .73 & 3.44 & $\begin{array}{r}302.33 \\
60.47\end{array}$ & $\begin{array}{r}3,040 \\
608\end{array}$ & $\begin{array}{r}15,162 \\
3,032\end{array}$ & $\begin{array}{c}1.01 \\
\cdots \ldots\end{array}$ & $\begin{array}{l}5.02 \\
\ldots . .\end{array}$ \\
\hline $\begin{array}{l}\text { Entire preservative } \\
\text { period }\end{array}$ & & & & & & & & & & \\
\hline & $2,018.38$ & 14,474 & 69,984 & .72 & 3.47 & $1,818.51$ & 17,259 & 102,248 & .95 & 5.62 \\
\hline & & 482 & & 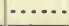 & & & 575 & & $\ldots$ & $\cdots$ \\
\hline After period. & & & & & & & & & & \\
\hline $\begin{array}{l}\text { First subperiod: } \\
\text { Total.......... } \\
\text { Average..... }\end{array}$ & $\begin{array}{r}332.20 \\
66.44\end{array}$ & $\begin{array}{r}2,395 \\
479\end{array}$ & $\begin{array}{r}11,026 \\
2,205\end{array}$ & & 3.32 & $\begin{array}{r}300.12 \\
60.02\end{array}$ & $\begin{array}{r}2,807 \\
561\end{array}$ & $\begin{array}{r}15,347 \\
3,069\end{array}$ & .94 & 5.11 \\
\hline $\begin{array}{l}\text { Second su } \\
\text { Total }\end{array}$ & & 2,488 & & .75 & 3.50 & & 3058 & & 103 & \\
\hline & 66.23 & 498 & $\begin{array}{r}11,001 \\
2,316\end{array}$ & & 0.00 & $\begin{array}{r}298.20 \\
59.65\end{array}$ & $\begin{array}{r}3,008 \\
612\end{array}$ & $\begin{array}{r}14,810 \\
2,863\end{array}$ & $\begin{array}{l}1.05 \\
\cdots . . .\end{array}$ & $\begin{array}{r}4.80 \\
\cdots . .\end{array}$ \\
\hline $\begin{array}{l}\text { Entire after period: } \\
\text { Total............... } \\
\text { Average........ }\end{array}$ & $\begin{array}{r}663.34 \\
66.33\end{array}$ & $\begin{array}{r}4,883 \\
488\end{array}$ & $\begin{array}{r}22,607 \\
2,261\end{array}$ & .74 & $\begin{array}{c}3.41 \\
\ldots . .2\end{array}$ & $\begin{array}{r}598.38 \\
59.84\end{array}$ & $\begin{array}{r}5,865 \\
587\end{array}$ & $\begin{array}{r}29,660 \\
2,966\end{array}$ & .98 & 4.96 \\
\hline
\end{tabular}


TABLE IV.-Amount of moist and dry food consumed, expressed as percentage of body weight, Series VI-Continued.

[Averages are per day.]

\begin{tabular}{|c|c|c|c|c|c|c|c|c|c|c|}
\hline \multirow{3}{*}{ Period. } & \multicolumn{5}{|c|}{ No. 9.} & \multicolumn{5}{|c|}{ No. 10.} \\
\hline & \multirow[t]{2}{*}{$\begin{array}{l}\text { Body } \\
\text { weight. }\end{array}$} & \multicolumn{2}{|c|}{ Weight of food. } & \multicolumn{2}{|c|}{$\begin{array}{c}\text { Average } \\
\text { daily ratio } \\
\text { of food } \\
\text { weight to } \\
\text { body weight. }\end{array}$} & \multirow[t]{2}{*}{$\begin{array}{c}\text { Body } \\
\text { weight. }\end{array}$} & \multicolumn{2}{|c|}{ Weight of food. } & \multicolumn{2}{|c|}{$\begin{array}{c}\text { Average } \\
\text { daily ratio } \\
\text { of food } \\
\text { weight to } \\
\text { body weight. }\end{array}$} \\
\hline & & Dry. & Moist. & Dry. & Moist. & & Dry. & Moist. & Dry. & Moist. \\
\hline \multicolumn{11}{|l|}{ Fore period. } \\
\hline $\begin{array}{l}\text { First subperiod: } \\
\text { Total............. } \\
\text { Average ......... }\end{array}$ & $\begin{array}{l}\text { Kilos. } \\
311.25 \\
62.25\end{array}$ & Grams. & $\begin{array}{l}\text { Grams. } \\
13,035\end{array}$ & $\begin{array}{l}\text { P. ct. } \\
1.01\end{array}$ & $\begin{array}{l}\text { P. ct. } \\
4.19\end{array}$ & $\begin{array}{l}\text { Kilos. } \\
285.63\end{array}$ & $\begin{array}{r}\text { Grams. } \\
3,286\end{array}$ & Grams. & $\begin{array}{l}\text { P.ct. } \\
1.15\end{array}$ & $\begin{array}{l}\text { P. ct. } \\
\text { 4. } 73\end{array}$ \\
\hline Second subperiod: & & & 2,607 & & & 57.13 & & 2,702 & & \\
\hline Total................. & $\begin{array}{r}311.20 \\
62.24\end{array}$ & $\begin{array}{r}3,178 \\
636\end{array}$ & $\begin{array}{r}15,299 \\
3,060\end{array}$ & $\begin{array}{l}1.02 \\
\cdots \ldots\end{array}$ & $\begin{array}{r}4.92 \\
\hdashline . . .\end{array}$ & $\begin{array}{r}283.47 \\
56.69\end{array}$ & $\begin{array}{r}3,310 \\
662\end{array}$ & $\begin{array}{r}13,586 \\
2,717\end{array}$ & $\begin{array}{l}1.17 \\
\ldots \ldots\end{array}$ & $\begin{array}{r}4.79 \\
\cdots\end{array}$ \\
\hline $\begin{array}{l}\text { Entire fore period: } \\
\text { Total.............. } \\
\text { Average ........ }\end{array}$ & $\begin{array}{r}622.45 \\
62.25 \\
\end{array}$ & $\begin{array}{r}6,335 \\
634\end{array}$ & $\begin{array}{r}28,334 \\
2,833 \\
\end{array}$ & $\begin{array}{l}1.02 \\
\cdots \ldots\end{array}$ & $\begin{array}{r}4.55 \\
\cdots \cdots\end{array}$ & $\begin{array}{r}569.10 \\
56.91\end{array}$ & $\begin{array}{r}6,596 \\
660\end{array}$ & $\begin{array}{r}27,096 \\
2,710\end{array}$ & $\begin{array}{l}1.16 \\
\cdots \cdots\end{array}$ & $\begin{array}{l}4.76 \\
\ldots \ldots\end{array}$ \\
\hline \multicolumn{11}{|l|}{ Preservative period. } \\
\hline $\begin{array}{l}\text { First subperiod: } \\
\text { Total............... } \\
\text { Average ........ }\end{array}$ & $\begin{array}{r}310.98 \\
62.20\end{array}$ & $\begin{array}{r}3,117 \\
623\end{array}$ & $\begin{array}{r}15,963 \\
3,193\end{array}$ & $\begin{array}{l}1.00 \\
\cdots \cdots\end{array}$ & 5.13 & $\begin{array}{r}287.26 \\
57.45\end{array}$ & $\begin{array}{r}3,417 \\
683\end{array}$ & $\begin{array}{r}16,629 \\
3,326\end{array}$ & 1.19 & 5.79 \\
\hline $\begin{array}{l}\text { Second subperiod: } \\
\text { Total............. }\end{array}$ & 310.73 & 3,130 & 15,383 & 101 & 4. 95 & 0.00 & & & & \\
\hline Average ............ & 62.15 & $\begin{array}{r}-100 \\
626\end{array}$ & $\begin{array}{r}10,000 \\
3,077\end{array}$ & 1.01 & 4.90 & $\begin{array}{r}287.60 \\
57.52\end{array}$ & $\begin{array}{r}3,392 \\
678\end{array}$ & $\begin{array}{r}15,939 \\
3,188\end{array}$ & 1.18 & 5.54 \\
\hline \multicolumn{11}{|l|}{ Third subperiod: } \\
\hline Total.................. & $\begin{array}{r}309.90 \\
61.98\end{array}$ & $\begin{array}{r}3,129 \\
626\end{array}$ & $\begin{array}{r}14,015 \\
2,803\end{array}$ & 1.01 & 4.52 & $\begin{array}{r}287.06 \\
57.41\end{array}$ & $\begin{array}{r}3,300 \\
660\end{array}$ & $\begin{array}{r}15,424 \\
3,085\end{array}$ & 1.15 & 5.37 \\
\hline $\begin{array}{l}\text { Fourth subperiod: } \\
\text { Total.... }\end{array}$ & & & & & & & & 0,0 & & \\
\hline $\begin{array}{l}\text { Total................. } \\
\text { Average }\end{array}$ & $\begin{array}{r}309.86 \\
61.97\end{array}$ & $\begin{array}{r}3,146 \\
629\end{array}$ & $\begin{array}{r}13,552 \\
2,710\end{array}$ & $\begin{array}{l}1.02 \\
\ldots . .\end{array}$ & 4.37 & $\begin{array}{r}286.65 \\
57.33\end{array}$ & $\begin{array}{r}3,337 \\
667\end{array}$ & $\begin{array}{r}13,827 \\
2,765\end{array}$ & 1.16 & 4.82 \\
\hline $\begin{array}{l}\text { Fifth subperiod: } \\
\text { Total }\end{array}$ & & & & & & & & & & \\
\hline $\begin{array}{l}\text { Total................... } \\
\text { Average }\end{array}$ & $\begin{array}{r}313.45 \\
62.69\end{array}$ & $\begin{array}{r}3,141 \\
628\end{array}$ & 13,850 & 1.00 & 4.42 & 285.96 & 3,468 & $\begin{array}{r}14,967 \\
0\end{array}$ & 1.21 & 5.23 \\
\hline \multirow{2}{*}{\multicolumn{11}{|c|}{$\begin{array}{l}\text { Sixth subperiod: } \\
\text { Total................. }\end{array}$}} \\
\hline & $\begin{array}{r}311.71 \\
62.34\end{array}$ & $\begin{array}{r}3,062 \\
612\end{array}$ & $\begin{array}{r}13,246 \\
2,649\end{array}$ & .98 & 4.25 & $\begin{array}{r}285.40 \\
57.08\end{array}$ & $\begin{array}{r}3,371 \\
674\end{array}$ & $\begin{array}{r}14,077 \\
2,815\end{array}$ & 1.18 & 4.93 \\
\hline \multirow{2}{*}{\multicolumn{11}{|c|}{$\begin{array}{l}\text { Entire preservative } \\
\text { period: }\end{array}$}} \\
\hline & & & & & & & & & & \\
\hline Total... & $\begin{array}{r}1,866.63 \\
62 . ? 2\end{array}$ & 18,725 & 86,009 & 1.00 & 4.61 & $1,719.93$ & 20,285 & 90,863 & 1.18 & 5.28 \\
\hline Average.. & & & & $-5+2+$ & & .35 & & & & \\
\hline \multicolumn{11}{|l|}{ After period. } \\
\hline $\begin{array}{l}\text { First subperiod: } \\
\text { Total........... } \\
\text { Average...... }\end{array}$ & $\begin{array}{r}310.27 \\
62.05\end{array}$ & $\begin{array}{r}3,113 \\
623\end{array}$ & $\begin{array}{r}12,852 \\
2,570\end{array}$ & 1.00 & 4.14 & $\begin{array}{r}284.50 \\
56.90\end{array}$ & $\begin{array}{r}3,293 \\
659\end{array}$ & $\begin{array}{r}14,484 \\
2,897\end{array}$ & 1.16 & 5.09 \\
\hline $\begin{array}{l}\text { Second subperiod: } \\
\text { Total........... }\end{array}$ & & & & & & & & & & \\
\hline $\begin{array}{l}\text { Total................ } \\
\text { A rerage }\end{array}$ & $\begin{array}{r}310.87 \\
62.17\end{array}$ & $\begin{array}{r}3,119 \\
624\end{array}$ & $\begin{array}{r}12,937 \\
2,587\end{array}$ & 1.00 & 4.16 & $a(284.50)$ & $(3,293)$ & $(14,484)$ & (1.16) & $(5.09)$ \\
\hline & & & & & & & & & & \\
\hline $\begin{array}{l}\text { Tre arter period: } \\
\text { Total.............. }\end{array}$ & 621.14 & 6,232 & 25,789 & 1.00 & 4.15 & $a(569.00)$ & $(6,586)$ & $(28,968)$ & (1.16) & $(5.09)$ \\
\hline & 62.11 & 623 & 2,579 & & & $(56.90)$ & $(659)$ & $(2,897)$ & & \\
\hline
\end{tabular}

$a$ Data observed for first subperiod only, owing to illness of subject during second subperiod. 
TABLE IV.-Amount of moist and dry food consumed, expressed as percentage of body weight, Series VI-Continued.

[Averages are per day.]

\begin{tabular}{|c|c|c|c|c|c|c|c|c|c|c|}
\hline \multirow{3}{*}{ Period. } & \multicolumn{5}{|c|}{ No. 11.} & \multicolumn{5}{|c|}{ No. 12.} \\
\hline & \multirow[t]{2}{*}{$\begin{array}{c}\text { Body } \\
\text { weight. }\end{array}$} & \multicolumn{2}{|c|}{ qVeight of food. } & \multicolumn{2}{|c|}{$\begin{array}{c}\text { Average } \\
\text { daily ratio } \\
\text { of food } \\
\text { weight to } \\
\text { body weight. }\end{array}$} & \multirow[t]{2}{*}{$\underset{\text { weight. }}{\text { Body }}$} & \multicolumn{2}{|c|}{ Weight of food. } & \multicolumn{2}{|c|}{$\begin{array}{l}\text { A verage } \\
\text { daily ratio } \\
\text { of food } \\
\text { weight to } \\
\text { body weight. }\end{array}$} \\
\hline & & Dry. & Moist. & Dry. & Moist. & & Dry. & Moist. & Dry. & Moist. \\
\hline \multicolumn{11}{|l|}{ Fore period. } \\
\hline $\begin{array}{l}\text { First subperiod: } \\
\text { Total.............. } \\
\text { Average ......... }\end{array}$ & $\begin{array}{r}\text { Kilos. } \\
328.24 \\
65.65\end{array}$ & $\begin{array}{r}\text { Grams. } \\
3,120 \\
624\end{array}$ & $\begin{array}{r}\text { Grams. } \\
15,481 \\
3,096\end{array}$ & $\begin{array}{l}\text { P. ct. } \\
0.95 \\
\ldots .\end{array}$ & $\begin{array}{c}P . c t . \\
4.72 \\
\cdots\end{array}$ & $\begin{array}{r}\text { Kilos. } \\
348.18 \\
69.64\end{array}$ & $\begin{array}{r}\text { Grams. } \\
3,247 \\
649\end{array}$ & $\begin{array}{r}\text { Grams. } \\
13,352 \\
2,670\end{array}$ & $\begin{array}{l}P . c t . \\
0.93 \\
\cdots . . .\end{array}$ & $\begin{array}{r}P . c t . \\
3.83\end{array}$ \\
\hline $\begin{array}{l}\text { Second subperiod: } \\
\text { Total............ } \\
\text { Average ....... }\end{array}$ & $\begin{array}{r}325.34 \\
65.07\end{array}$ & $\begin{array}{r}3,093 \\
619\end{array}$ & $\begin{array}{r}14,651 \\
2,930\end{array}$ & $\begin{array}{l}.95 \\
. . .\end{array}$ & $\begin{array}{r}4.50 \\
\ldots .\end{array}$ & $\begin{array}{r}348.78 \\
69.76\end{array}$ & $\begin{array}{r}3,356 \\
671\end{array}$ & $\begin{array}{r}14,70 \\
2,9\end{array}$ & $\begin{array}{l}.96 \\
-. . .\end{array}$ & 4.22 \\
\hline $\begin{array}{l}\text { Entire fore period: } \\
\text { Total............... } \\
\text { Average ........ }\end{array}$ & $\begin{array}{r}653.58 \\
6.3 .36\end{array}$ & $\begin{array}{r}6,213 \\
621\end{array}$ & $\begin{array}{r}30,132 \\
3,013\end{array}$ & .95 & 4.61 & $\begin{array}{r}696.96 \\
69.70\end{array}$ & $\begin{array}{r}6,603 \\
660\end{array}$ & $\begin{array}{r}28,061 \\
2,806\end{array}$ & $\begin{array}{r}.95 \\
\ldots . . . \\
\end{array}$ & 4.03 \\
\hline \multicolumn{11}{|l|}{ Preservative period. } \\
\hline $\begin{array}{l}\text { First subperiod: } \\
\text { Total............ } \\
\text { Average ....... }\end{array}$ & $\begin{array}{r}325.14 \\
65.03\end{array}$ & $\begin{array}{r}3,192 \\
638\end{array}$ & $\begin{array}{r}14,490 \\
2,898\end{array}$ & .98 & 4.46 & $\begin{array}{r}350.66 \\
70.13\end{array}$ & 3,274 & 14,331 & .93 & 4.09 \\
\hline isubperiod: & & & & & & & & & & \\
\hline $\begin{array}{l}\text { Total................. } \\
\text { Average }\end{array}$ & 325.11 & 3,087 & 15,369 & .95 & 4.73 & 349.30 & 3,252 & 13,435 & .93 & 3.85 \\
\hline period: & & 617 & & ...... & $\ldots$. & & 650 & & ...... & ....... \\
\hline age.................. & $\begin{array}{r}323.56 \\
64.71\end{array}$ & $\begin{array}{r}3,088 \\
618\end{array}$ & $\begin{array}{r}15,038 \\
3,002\end{array}$ & .95 & 4.64 & 350.10 & 3,237 & 13,982 & .92 & 3.99 \\
\hline eriod: & & & & & & & & & & . \\
\hline To & 322.77 & 3,089 & 14,870 & .96 & 4. 61 & 349.36 & 3,273 & 14,677 & .94 & 4.20 \\
\hline Fifth subperiod: & & & & & & & & & - & 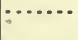 \\
\hline $\begin{array}{l}\text { Total............. } \\
\text { Average }\end{array}$ & $\begin{array}{r}321.78 \\
64.36\end{array}$ & $\begin{array}{r}3,143 \\
629\end{array}$ & $\begin{array}{r}14,476 \\
2,895\end{array}$ & .98 & 4.50 & $\begin{array}{r}350.70 \\
70.14\end{array}$ & $\begin{array}{r}3,326 \\
665\end{array}$ & $\begin{array}{r}13,800 \\
2,760\end{array}$ & .95 & 3.93 \\
\hline Sixth sub] & & & & & & & & & & \\
\hline Total. & 319.19 & 3,070 & 15,711 & .96 & 4.92 & 350.77 & 3,230 & 13,421 & .92 & 3.8 \\
\hline Average .......... & & 614 & & ....... & $\cdots \cdots \cdots$ & & 646 & & ....... & ... \\
\hline \multirow{2}{*}{\multicolumn{11}{|c|}{$\begin{array}{l}\text { Entire preservative } \\
\text { period: }\end{array}$}} \\
\hline & 1,937 & 18,669 & 89,924 & .96 & 4.64 & 2,100 & 19,592 & & .93 & 3.98 \\
\hline Average .......... & 64.59 & 622 & 2,997 & & & 70.03 & 653 & & & \\
\hline After period. & & & & & & & & & & \\
\hline \multicolumn{10}{|l|}{ First subperiod: } & 3.63 \\
\hline & 63.69 & 608 & & & & & 638 & & .81 & 0.00 \\
\hline $\begin{array}{l}\text { Second subp } \\
\text { Total.... }\end{array}$ & & & & & & & & & $0 ?$ & \\
\hline Average... & 63.44 & $\begin{array}{r}-111 \\
-\quad 622\end{array}$ & 3,035 & & $\begin{array}{l}4.18 \\
\ldots . . .\end{array}$ & $\begin{array}{r}69.85 \\
69.87\end{array}$ & $\begin{array}{r}3,208 \\
642\end{array}$ & $\begin{array}{r}13,012 \\
2,722\end{array}$ & .92 & 3.90 \\
\hline $\begin{array}{c}\text { Entire after period: } \\
\text { Total............... } \\
\text { Average ........ }\end{array}$ & $\begin{array}{r}635.64 \\
63.56\end{array}$ & $\begin{array}{r}6,150 \\
615\end{array}$ & $\begin{array}{r}30,739 \\
3,074\end{array}$ & .97 & $\begin{array}{c}4.84 \\
\ldots . . .\end{array}$ & $\begin{array}{r}698.70 \\
69.87\end{array}$ & $\begin{array}{r}6,396 \\
640\end{array}$ & $\begin{array}{r}26,284 \\
2,628\end{array}$ & .92 & 3.76 \\
\hline
\end{tabular}


TABLE V.-Summary for nine men, by periods, showing average daily ratio of food weight to body weight, Series VI.

[Averages are per man per day.]

\begin{tabular}{|c|c|c|c|c|c|}
\hline \multirow{2}{*}{ Period. } & \multirow{2}{*}{$\begin{array}{l}\text { Body } \\
\text { weight. }\end{array}$} & \multicolumn{2}{|c|}{ Weight of food. } & \multicolumn{2}{|c|}{$\begin{array}{l}\text { Average daily ratio } \\
\text { of food weight to } \\
\text { body weight. }\end{array}$} \\
\hline & & Dry. & Moist. & Dry. & Moist. \\
\hline Fore period. & & & & & \\
\hline $\begin{array}{l}\text { First subperiod: } \\
\quad \text { Total } \ldots \ldots \ldots \ldots \ldots \ldots\end{array}$ & $\begin{array}{r}\text { Kilograms. } \\
2,826.51 \\
62.81\end{array}$ & $\begin{array}{r}\text { Grams. } \\
24,879 \\
553\end{array}$ & $\begin{array}{r}\text { Grams. } \\
118,171 \\
2,626\end{array}$ & $\begin{array}{r}\text { Per cent. } \\
0.88\end{array}$ & $\begin{array}{l}\text { Per cent. } \\
4.18\end{array}$ \\
\hline Second subperiod: & & & & & \\
\hline $\begin{array}{l}\text { Total } \\
\text { Average.................. }\end{array}$ & $\begin{array}{r}2,817.57 \\
62.61\end{array}$ & $\begin{array}{r}25,063 \\
557\end{array}$ & $\begin{array}{r}115,958 \\
2,577\end{array}$ & .89 & $\begin{array}{r}4.12 \\
4 . . .\end{array}$ \\
\hline $\begin{array}{l}\text { Entire fore period: } \\
\quad \text { Total } \ldots \ldots \ldots \\
\text { Average } \ldots \ldots \\
\end{array}$ & $\begin{array}{r}5,644.08 \\
62.71 \\
\end{array}$ & $\begin{array}{r}49,942 \\
555 \\
\end{array}$ & $\begin{array}{r}234,129 \\
2,601 \\
\end{array}$ & .88 & $\begin{array}{r}4.15 \\
\cdots\end{array}$ \\
\hline Preservative period. & & & & & \\
\hline $\begin{array}{l}\text { First subperiod: } \\
\quad \text { Total } \ldots \ldots \ldots \ldots \\
\quad \text { Average } \ldots \ldots \ldots \\
\end{array}$ & $\begin{array}{r}2,815.04 \\
62.56\end{array}$ & $25, \frac{194}{560}$ & $\begin{array}{r}120,711 \\
2,682\end{array}$ & .89 & 4. 29 \\
\hline 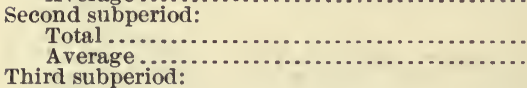 & $\begin{array}{r}2,813.06 \\
62.51\end{array}$ & 25,003 & $\begin{array}{r}120,358 \\
2,675\end{array}$ & .89 & $\begin{array}{l}4.28 \\
\text {..... }\end{array}$ \\
\hline 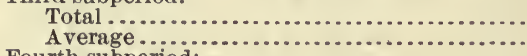 & $\begin{array}{r}2,804.40 \\
62.32\end{array}$ & $\begin{array}{r}25,137 \\
559\end{array}$ & $\begin{array}{r}119,641 \\
2,659\end{array}$ & .90 & $\begin{array}{l}4.27 \\
\ldots . .\end{array}$ \\
\hline $\begin{array}{l}\text { Fourth subperiod: } \\
\text { Total .................. }\end{array}$ & $\begin{array}{r}2,801.83 \\
62.27\end{array}$ & 25,281 & $\begin{array}{r}119,977 \\
2,666\end{array}$ & .90 & 4. 28 \\
\hline $\begin{array}{l}\text { Fifth subperiod: } \\
\text { Total }\end{array}$ & & 26,177 & 119,678 & .94 & 4.28 \\
\hline 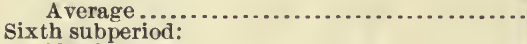 & 62.10 & 582 & 2,660 & & 10.0 \\
\hline 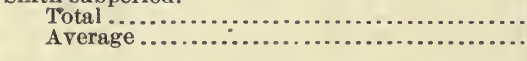 & $\begin{array}{r}2,785.25 \\
61.89\end{array}$ & $\begin{array}{r}25,608 \\
569\end{array}$ & $\begin{array}{r}118,169 \\
2,626\end{array}$ & .92 & 4.24 \\
\hline $\begin{array}{l}\text { Entire preservative period: } \\
\quad \text { Total } \ldots \ldots \ldots \ldots \\
\text { Average } \ldots \ldots \ldots \\
\end{array}$ & $\begin{array}{r}16,814.16 \\
62.27 \\
\end{array}$ & $\begin{array}{r}152,400 \\
564\end{array}$ & $\begin{array}{r}718,534 \\
2,661 \\
\end{array}$ & $\begin{array}{l}.91 \\
\cdots\end{array}$ & $\begin{array}{l}4.27 \\
\cdots\end{array}$ \\
\hline After period. & & & & & \\
\hline $\begin{array}{l}\text { First subperiod: } \\
\text { Total } \ldots \ldots \ldots \\
\text { Average } \ldots \ldots \ldots\end{array}$ & $\begin{array}{r}2,776.33 \\
61.70\end{array}$ & $\begin{array}{r}25,093 \\
558\end{array}$ & $\begin{array}{r}116,153 \\
2,581\end{array}$ & $\begin{array}{c}.90 \\
\cdots . . .\end{array}$ & $\begin{array}{r}4.18 \\
\cdots . . .\end{array}$ \\
\hline 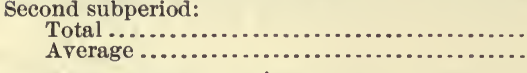 & $\begin{array}{r}2,768.63 \\
61.53\end{array}$ & $\begin{array}{r}26,014 \\
578\end{array}$ & $\begin{array}{r}115,991 \\
2,578\end{array}$ & $\begin{array}{r}.94 \\
\ldots . . .9 .\end{array}$ & $\begin{array}{r}4.19 \\
\cdots \ldots . .\end{array}$ \\
\hline 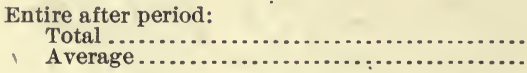 & $\begin{array}{r}5,544.96 \\
61.61\end{array}$ & $\begin{array}{r}51,107 \\
568\end{array}$ & $\begin{array}{r}232,144 \\
.2,579\end{array}$ & .92 & $\begin{array}{r}4.19 \\
\cdots \ldots .\end{array}$ \\
\hline
\end{tabular}

MICROSCOPICAL EXAMINATION OF THE BLOOD.

The blood was examined at stated intervals for corpuscles and hemoglobin, according to the methods described in the borax report. Two independent estimates were made by Messrs. B. J. Howard and C. P. Knight, and the average taken as the correct expression for the count, with the results shown in Table VI.

In the case of No. 1 there was a diminution in the number of red corpuscles and an increase in the number of white corpuscles during the administration of the preservative. After the withdrawal of the preservative the red corpuscles again increased in number and the 
white corpuscles decreased. There was a slight loss in color also during the administration of the preservative, which was partially regained in the after period.

In the case of No. 2 both the red and the white corpuscles increased during the administration of the preservative and both diminished during the after period. The color of the blood was also less intense during both the preservative period and the after period.

The data for No. 3 have no comparative value and are somewhat fragmentary, owing to the illness of the subject. Considered individually the figures show an increase in the number of red corpuscles during the preservative period and a slight decrease in the after period. The number of white corpuscles also increased during the preservative period and decreased in the after period. The color of the blood was less intense in the preservative period and partially recovered in the after period.

In the case of No. 4 there was a marked diminution both in the number of the red and the white corpuscles and in the intensity of the color during the administration of the preservative. There was a recovery in the number of both the red and the white corpuscles in the after period, but the color continued to fade.

In the case of No. 5 no comparison can be made with one of the tests, as it was lost. The remaining test shows a slight diminution in the number of red corpuscles and a very great diminution in the number of white corpuscles and also a very slight fading of the color. In the after period the red corpuscles diminished again very considerably in number. The white corpuscles increased but the color faded still more.

No. 6 showed a marked increase in the number of red corpuscles during the administration of the preservative, a slight loss in white corpuscles, and a slight increase in the intensity of the color. During the after period the number of red corpuscles very greatly diminished, the number of white corpuscles slightly increased, and the color diminished in intensity.

In the case of No. 7 the number of red corpuscles very markedly decreased during the administration of the preservative, the number of white corpuscles increased, and the color faded very perceptibly. During the after period the number of red corpuscles was again greatly diminished, the number of white corpuscles slightly diminished, and the color slightly increased, not reaching, however, that of the fore period.

In the case of No. 8 the number of red corpuscles increased during the administration of the preservative and the number of white corpuscles was almost doubled. The color of the blood was slightly decreased. There was a diminution of the red corpuscles during the 
after period, a very marked diminution of the white corpuscles, and a slight fading of the blood.

While Nos. 9 and 10 are excluded from the summary for the reasons already stated, the individual data may be considered. In the case of No. 9 the red corpuscles decreased and the white corpuscles increased in the preservative period, while both increased in the after period. The color of the blood slightly increased in the preservative period, but diminished very decidedly in the after period.

In the case of No. 10 the red corpuscles decreased, the white corpuscles increased, and the color increased in the preservative period. In the after period the red corpuscles again decreased, but very slightly, the white corpuscles continued to increase, and the color of the blood faded.

No. 11 showed an increase in the number of red corpuscles during. the administration of the preservative, an increase in the white corpuscles, and a very slight increase in the intensity of the color of the blood. There was a decrease in the number of red corpuscles in the after period, a slight increase in the number of white corpuscles, and a very slight increase in the intensity of color.

No. 12 showed a considerable increase in the number of red corpuscles, a decrease in the number of white corpuscles, and a marked increase in the intensity of color during the administration of the preservative. During the after period the number of red corpuscles diminished, the number of white corpuscles increased, and the intensity of color was notably diminished.

The summary of the number of corpuscles and the percentage of hemoglobin in the blood for the nine men, compared throughout the series, shows that the average number of red corpuscles for the fore period is 5,690,000; for the preservative period, 5,900,938; for the after period, $5,450,444$. The number of white corpuscles in the fore period is 10,609 , in the preservative period, 10,714 , and in the after period, 9,836. The intensity of color, measured by the hemoglobin, diminished regularly from the fore to the after period, being represented by 106.1 in the fore period, 104.7 in the preservative period, and 103.3 in the after period. There is an apparent tendency on the part of the preservative to increase the number of the red and the white corpuscles in the blood and at the same time to slightly diminish the intensity of the color. During the after period the number of both the red and the white corpuscles decreases and at the same time there is still another slight decrease in the intensity of the color. 
TABLE VI.-Averages, by periods, of corpuscles and hemoglobin in the blood, Series VI.

\begin{tabular}{|c|c|c|c|c|c|c|c|}
\hline \multirow[b]{2}{*}{ Period. } & \multirow[b]{2}{*}{ Date. } & \multicolumn{3}{|c|}{ No. 1.} & \multicolumn{3}{|c|}{ No. 2.} \\
\hline & & $\begin{array}{l}\text { Red corpus- } \\
\text { cles per } \\
\text { cubic milli- } \\
\text { meter. }\end{array}$ & $\begin{array}{l}\text { White } \\
\text { corpus- } \\
\text { cles per } \\
\text { cubicmil- } \\
\text { limeter. }\end{array}$ & $\begin{array}{l}\text { Hemo- } \\
\text { globin. }\end{array}$ & $\begin{array}{l}\text { Red corpus- } \\
\text { cles per } \\
\text { cubic milli- } \\
\text { meter. }\end{array}$ & $\begin{array}{l}\text { White } \\
\text { corpus- } \\
\text { cles per } \\
\text { cubicmil- } \\
\text { limeter. }\end{array}$ & $\begin{array}{l}\text { Hemo- } \\
\text { globin. }\end{array}$ \\
\hline $\begin{array}{l}\text { Fore period: } \\
\text { Estimate A. } \\
\text { Estimate B. }\end{array}$ & \multirow[t]{2}{*}{ Oct. $26-29$} & $\left\{\begin{array}{l}6,295,000 \\
5,835,000 \\
\end{array}\right.$ & $\begin{array}{l}8,593 \\
9,620 \\
\end{array}$ & $\begin{array}{l}109.2 \\
108.4\end{array}$ & $\begin{array}{l}5,745,000 \\
5,380,000\end{array}$ & $\begin{array}{l}12,796 \\
11,768\end{array}$ & $\begin{array}{r}108.6 \\
108.6 \\
\end{array}$ \\
\hline Mean..... & & $6,065,000$ & 9,107 & 108.8 & $5,562,500$ & 12,281 & 108.6 \\
\hline $\begin{array}{l}\text { Preservative period: } \\
\text { Estimate A } \ldots . . . \\
\text { Estimate B...... }\end{array}$ & \multirow[t]{2}{*}{ Nov.23-27 } & $\left\{\begin{array}{l}5,475,000 \\
5,545,000 \\
\end{array}\right.$ & $\begin{array}{r}9,196 \\
11,768\end{array}$ & $\begin{array}{l}104.9 \\
105.4\end{array}$ & $\begin{array}{l}6,825,000 \\
6,160,000 \\
\end{array}$ & $\begin{array}{l}14,127 \\
16,625 \\
\end{array}$ & $\begin{array}{l}104.7 \\
105.1\end{array}$ \\
\hline Mean ............. & & $5,510,000$ & 10,482 & 105.2 & $6,492,500$ & 15,376 & 104.9 \\
\hline \multirow[t]{2}{*}{$\begin{array}{l}\text { After period: } \\
\quad \text { Estimate A } \ldots \ldots \ldots \ldots \\
\text { Estimate B } \ldots \ldots \ldots \ldots\end{array}$} & \multirow[t]{2}{*}{ Dec. $\quad 5-7$} & $\begin{array}{l}6,660,000 \\
6,350,000\end{array}$ & $\begin{array}{l}6,426 \\
8,499\end{array}$ & $\begin{array}{l}105.6 \\
107.7\end{array}$ & $\begin{array}{l}5,320,000 \\
5,035,000\end{array}$ & $\begin{array}{l}6,039 \\
6,538\end{array}$ & $\begin{array}{l}98.5 \\
97.1\end{array}$ \\
\hline & & $6,505,000$ & 7,463 & 106.7 & $5,177,500$ & 6,289 & 97.8 \\
\hline \multirow[b]{2}{*}{ Period. } & \multirow[b]{2}{*}{ Date. } & \multicolumn{3}{|c|}{ No. 3.} & \multicolumn{3}{|c|}{ No. 4.} \\
\hline & & $\begin{array}{l}\text { Red corpus- } \\
\text { cles per } \\
\text { cubic milli- } \\
\text { meter. }\end{array}$ & $\begin{array}{l}\text { White } \\
\text { corpus- } \\
\text { cles per } \\
\text { cubicmil- } \\
\text { limeter. }\end{array}$ & $\begin{array}{c}\text { Hemo- } \\
\text { globin. }\end{array}$ & $\begin{array}{l}\text { Red corpus- } \\
\text { cles per } \\
\text { cubic milli- } \\
\text { meter. }\end{array}$ & $\begin{array}{c}\text { White } \\
\text { corpus- } \\
\text { cles per } \\
\text { cubicmil- } \\
\text { limeter. }\end{array}$ & $\begin{array}{l}\text { Hemo- } \\
\text { globin. }\end{array}$ \\
\hline $\begin{array}{l}\text { Fore period: } \\
\text { Estimate A. } \\
\text { Estimate B. }\end{array}$ & \multirow[t]{2}{*}{ Oct. $26-29$} & $\begin{array}{l}5,385,000 \\
4,705,000 \\
\end{array}$ & $\begin{array}{l}8,966 \\
9,340\end{array}$ & $\begin{array}{r}98.0 \\
\cdots \cdots \\
\end{array}$ & $\begin{array}{l}5,820,000 \\
5,232,000\end{array}$ & $\begin{array}{r}11,955 \\
8,686 \\
\end{array}$ & $\begin{array}{l}106.7 \\
106.4\end{array}$ \\
\hline Mean . & & $5,045,000$ & 9,153 & ........... & $5,526,000$ & 10,321 & 106.6 \\
\hline $\begin{array}{l}\text { Preservative period: } \\
\text { Estimate A } . . . . \\
\text { Estimate B } \ldots . . .\end{array}$ & \multirow[t]{2}{*}{ Nov.23-27 } & $\left\{\begin{array}{r}\because, 833,333 \\
\end{array}\right.$ & $\begin{array}{r}9,086 \\
10,087\end{array}$ & $\begin{array}{l}94.2 \\
93.7\end{array}$ & $\begin{array}{l}5,105,000 \\
5,095,000\end{array}$ & $\begin{array}{l}5,511 \\
8,032 \\
\end{array}$ & $\begin{array}{l}103.9 \\
102.5\end{array}$ \\
\hline Mean.... & & (............... & 9,587 & 94.0 & $5,100,000$ & 6,772 & 103.2 \\
\hline \multirow[t]{2}{*}{$\begin{array}{l}\text { After period: } \\
\quad \text { Estimate A } \ldots . . . \ldots . . \\
\text { Estimate B } \ldots \ldots \ldots\end{array}$} & \multirow[t]{2}{*}{ Dee. $5-7$} & $\begin{array}{l}5,660,000 \\
5,760,000 \\
\end{array}$ & $\begin{array}{l}5,706 \\
8,312\end{array}$ & $\begin{array}{l}97.0 \\
95.3\end{array}$ & $\begin{array}{l}5,288,000 \\
5,495,000\end{array}$ & $\begin{array}{l}10,834 \\
12,702\end{array}$ & $\begin{array}{l}100.6 \\
101.9\end{array}$ \\
\hline & & $5,710,000$ & 7,009 & 96.2 & $5,391,500$ & 11,768 & 101.3 \\
\hline \multirow[b]{2}{*}{ Period. } & \multirow[b]{2}{*}{ Date. } & \multicolumn{3}{|c|}{ No. 5.} & \multicolumn{3}{|c|}{ No. 6.} \\
\hline & & $\begin{array}{l}\text { Red corpus- } \\
\text { cles per } \\
\text { cubic milli- } \\
\text { meter. }\end{array}$ & \begin{tabular}{|} 
White \\
corpus- \\
cles per \\
cubicmil- \\
limeter.
\end{tabular} & $\begin{array}{l}\text { Hemo- } \\
\text { globin. }\end{array}$ & $\begin{array}{c}\text { Red corpus- } \\
\text { cles per } \\
\text { cubic milli- } \\
\text { meter. }\end{array}$ & \begin{tabular}{|c|} 
White \\
corpus- \\
cles per \\
cubicmil- \\
limeter.
\end{tabular} & $\begin{array}{l}\text { Hemo- } \\
\text { globin. }\end{array}$ \\
\hline $\begin{array}{l}\text { Fore period: } \\
\text { Estimate A. } \\
\text { Estimate B. }\end{array}$ & $\begin{array}{c}1903 . \\
\text { Oct, } 26-29\end{array}$ & $\left\{\begin{array}{l}6,010,000 \\
6,328,000 \\
\end{array}\right.$ & $\begin{array}{l}8,966 \\
8,173\end{array}$ & $\begin{array}{l}104.6 \\
106.9\end{array}$ & $\begin{array}{l}5,500,000 \\
5,725,000\end{array}$ & $\begin{array}{l}13,263 \\
12,702\end{array}$ & $\begin{array}{l}107.7 \\
107.3\end{array}$ \\
\hline Mean.... & & $6,169,000$ & 8,570 & 105.8 & $5,612,500$ & 12,983 & 107.5 \\
\hline $\begin{array}{l}\text { Preservative period: } \\
\text { Estimate A } \ldots . . . \\
\text { Estimate B...... }\end{array}$ & Nov. $23-27$ & $\left\{\begin{array}{r}6,110,000 \\
\text { iost. }\end{array}\right.$ & $\begin{array}{l}6,445 \\
6,912\end{array}$ & $\begin{array}{l}103.5 \\
105.9\end{array}$ & $\begin{array}{l}6,790,000 \\
6,400,000 \\
\end{array}$ & $\begin{array}{l}10,637 \\
13,917\end{array}$ & $\begin{array}{l}108.9 \\
108.1\end{array}$ \\
\hline Mean..... & & $\ldots \ldots \ldots$ & 6,679 & 104.7 & $6,595,000$ & 12,277 & 108.5 \\
\hline $\begin{array}{l}\text { After period: } \\
\text { Estimate A } \ldots . \\
\text { Estimate B } \ldots\end{array}$ & Dec. $5-7$ & $\left\{\begin{array}{l}5,255,000 \\
5,250,000 \\
\end{array}\right.$ & $\begin{array}{r}8,199 \\
10,834 \\
\end{array}$ & $\begin{array}{l}102.7 \\
102.4\end{array}$ & $\begin{array}{l}5,370,000 \\
5,230,000 \\
\end{array}$ & $\begin{array}{l}12,243 \\
15,318\end{array}$ & $\begin{array}{l}103.4 \\
107.0\end{array}$ \\
\hline Mean.... & & $5,252,500$ & 9,517 & 102.6 & $5,300,000$ & 13,781 & 105.2 \\
\hline
\end{tabular}


TaBle VI.-Averages, by periods, of corpuscles and hemoglobin in the blood, Series VIContinued.

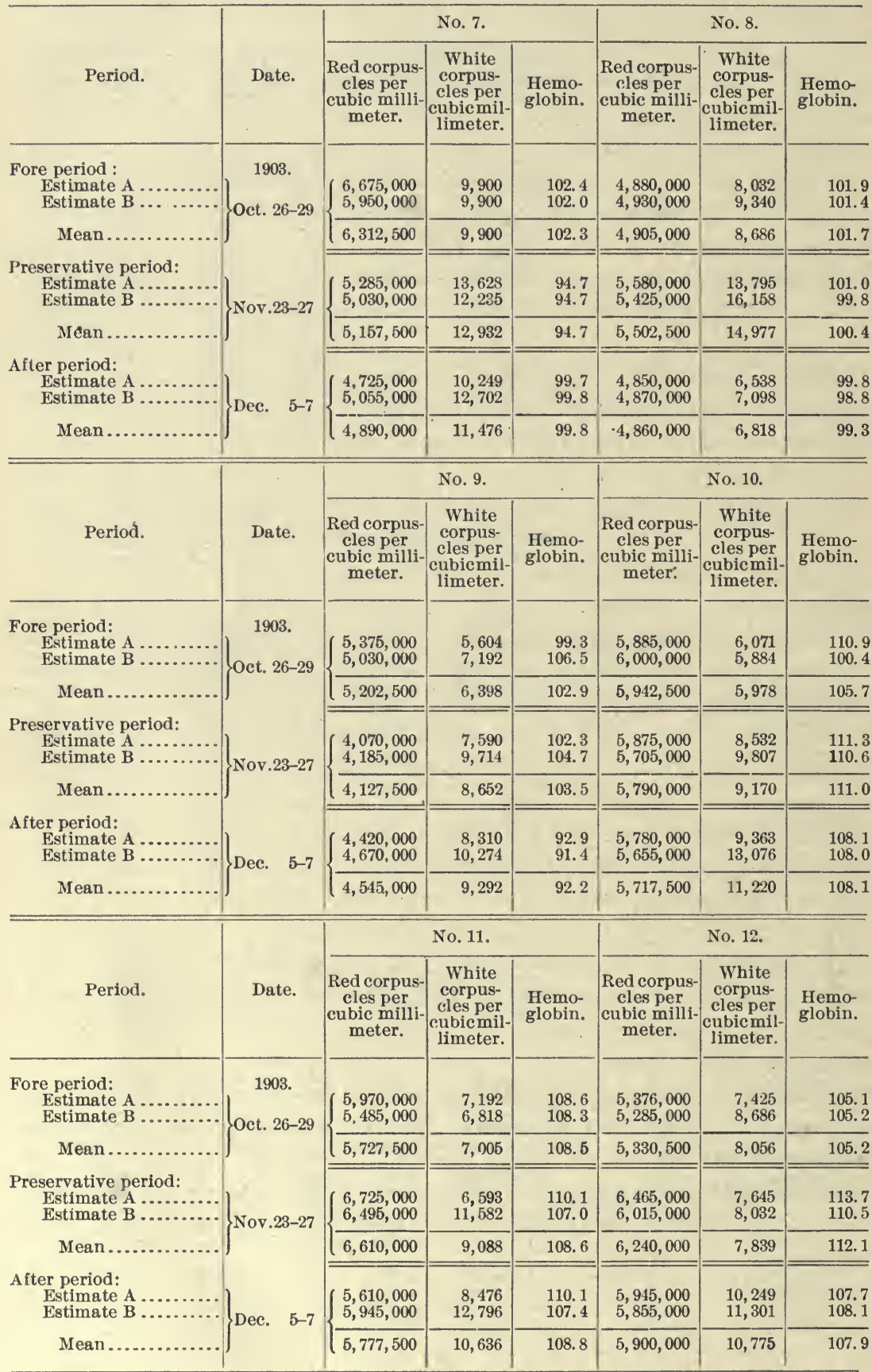


TABLE VI.-Average, by periods, of corpuscles and hemoglobin in the blood, Series VIContinued.

SUMMARY FOR NINE MEN (NOS. 3, 9, AND 10 EXCLUDED).

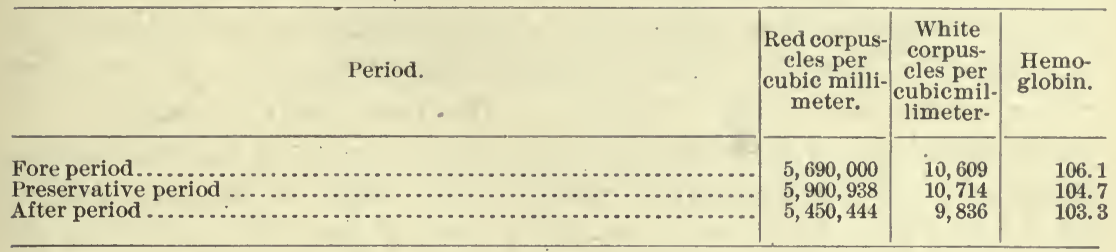

\section{WEIGHT AND WATER CONTENT OF THE FECES.}

In Table VII are given the data respecting the weight and water content of the feces for the individuals by periods, together with a summary of the data for the nine men compared throughout the series.

In the case of No. 1 the average content of water in the feces in the preservative period is markedly less than in the fore period, and there is a still further diminution in the after period. No. 2 also shows a diminution in the percentage of water in the feces in the preservative period, but this is restored in the after period to almost the same content as at the beginning. The weight of the dry feces is less both in the preservative period and in the after period than in the fore period, while in the case of No. 1 the weight of the dry feces is slightly less in the preservative period and greater in the after period. The percentage of water in the feces of No. 3 is greater in the preservative period than in either of the other periods. The actual weight of the dry feces is also greater. No. 4 shows a diminution in the percentage of water in the feces and a slight decrease in weight in the preservative period, and almost the same rate of diminution is continued in the after period. In No. 5 a like condition obtains as in the case of No. 4, but to a somewhat greater degree. In No. 6 there is also a slight diminution in the percentage of water, attended with a slight decrease in the dry weight, and these conditions continue in an increased degree in the after period. In No. 7 there is a marked decrease in the percentage of water in the feces in the preservative period, while in the after period there is a partial restoration to the condition of the fore period. There is a marked diminution in the weight of the dry feces in the preservative period, and the dry weight is greater in the after period than in the fore period. No. 8 also shows a diminution in the percentage of water in the feces in the preservative period, but an increase in the dry weight. In the after period the percentage of water is higher than in the fore period, and the dry weight is greater than in the preservative period and the fore period. In No. 9 there is also an increase in the percentage of water in the feces, and a marked increase in the weight in the preservative period. 
The percentage of water in the after period is slightly greater than in the preservative period but the weight is slightly less, but it is still greater than in the fore period. In No. 10 there is almost no difference in the percentage of water in the preservative period, but there is a marked decrease in the weight of the dry feces. In the after period there is a slight decrease in the percentage of water and a marked increase in the weight of the dry feces. No. 11 shows a marked increase in the percentage of water in the feces in the preservative period and a slight decrease in the weight of the dry feces. In the after period there is a smaller percentage of water in the feces than in the preservative period, and also a marked decrease in the weight of the dry feces. In No. 12 the percentage of water in the feces in the preservative period is slightly increased, but the dry weight of the feces very markedly diminishes. There is a decided decrease in the percentage of moisture in the after period, but a slight increase in the weight of the dry feces.

The summary for the nine men who can be compared throughout the series shows that the average daily weight of moist feces during the fore period is 89 grams and of the dry feces 22 grams, and the percentage of water therein 75.61. For the preservative period the average weight of the moist feces is 75 grams, the average weight of the dry feces 20 grams, and the percentage of moisture 73.83. These data show that the administration of the preservative has a distinet tendency to diminish both the percentage of water in the feces and also the quantity of dry feces excreted.

For the after period the average weight of the moist feces is 79 grams, the average weight of the dry feces 20 grams, and the percentage of moisture 74.12. The effect of the preservative therefore is to diminish the total quantity of the feces both wet and dry, the average amount of dry feces being 2 grams less during the preservative period than in the fore period. In the after period the weight of dry feces remains the same but there is an increase of 4 grams in the weight of moist feces, which is only 10 grams less than in the fore period. In general there appears to be a distinct influence of the preservative to diminish the weight of the solid matter excreted in the feces and also a tendency to decrease the quantity of water therein. While the latter effect is not marked, it is evident that salicylic acid in the quantities administered does not produce any tendency to diarrhea but rather the opposite effect. 
TABLE VII.-Weight and water content of feces, by periods, Series VI.

[Averages are per day.]

\begin{tabular}{|c|c|c|c|c|c|c|c|c|c|}
\hline \multirow[b]{2}{*}{ Period. } & \multicolumn{3}{|c|}{ No. 1.} & \multicolumn{3}{|c|}{ No. 2.} & \multicolumn{3}{|c|}{ No. 3.} \\
\hline & $\begin{array}{l}\text { Feces } \\
\text { moist. }\end{array}$ & $\begin{array}{l}\text { Water } \\
\text { infeces. }\end{array}$ & $\begin{array}{l}\text { Feces } \\
\text { dry. }\end{array}$ & $\begin{array}{l}\text { Feces } \\
\text { moist. }\end{array}$ & $\begin{array}{c}\text { Water } \\
\text { infeces. }\end{array}$ & $\begin{array}{l}\text { Feces } \\
\text { dry. }\end{array}$ & $\begin{array}{l}\text { Feces } \\
\text { moist. }\end{array}$ & $\begin{array}{l}\text { Water } \\
\text { in feces. }\end{array}$ & $\begin{array}{l}\text { Feces } \\
\text { dry. }\end{array}$ \\
\hline \multicolumn{10}{|l|}{ Fore period. } \\
\hline $\begin{array}{l}\text { First subperiod: } \\
\quad \text { Total } \ldots \ldots \ldots \ldots \ldots \ldots \\
\text { Average } \ldots \ldots \ldots \ldots \ldots\end{array}$ & $\begin{array}{r}\text { Grams. } \\
236 \\
47\end{array}$ & $\begin{array}{r}\mathrm{Per}^{*} \mathrm{ct} . \\
66.97 \\
\ldots\end{array}$ & $\begin{array}{r}\text { Grams. } \\
78 \\
16\end{array}$ & $\begin{array}{r}\text { Grams. } \\
453 \\
91\end{array}$ & $\begin{array}{c}\operatorname{Per} c t \\
71.74 \\
\end{array}$ & $\begin{array}{r}\text { Grams. } \\
128 \\
26\end{array}$ & Grams. & Per ct. & Grams. \\
\hline Second subperiod: & & & & & & & & & \\
\hline Total ${ }_{\text {Average }} \ldots \ldots \ldots$ & $\begin{array}{r}172 \\
34\end{array}$ & $\begin{array}{r}63.97 \\
\ldots \ldots . .\end{array}$ & $\begin{array}{l}62 \\
12\end{array}$ & $\begin{array}{r}492 \\
98\end{array}$ & $\begin{array}{r}71.15 \\
\cdots \cdots\end{array}$ & $\begin{array}{r}142 \\
28\end{array}$ & $\begin{array}{r}294 \\
59\end{array}$ & $\begin{array}{r}73.50 \\
\cdots\end{array}$ & $\begin{array}{l}78 \\
16\end{array}$ \\
\hline $\begin{array}{l}\text { Entire fore period: } \\
\quad \text { Total } \ldots \ldots \ldots \ldots \ldots \ldots \\
\quad \text { A verage } \ldots \ldots \ldots \ldots \ldots\end{array}$ & $\begin{array}{r}408 \\
41\end{array}$ & $\begin{array}{r}65.69 \\
\ldots \ldots \ldots \\
\end{array}$ & $\begin{array}{r}140 \\
14\end{array}$ & $\begin{array}{r}945 \\
95\end{array}$ & $\begin{array}{r}71.43 \\
\cdots \cdots \\
\end{array}$ & $\begin{array}{r}270 \\
27\end{array}$ & & & \\
\hline \multicolumn{10}{|l|}{ Preservative period. } \\
\hline $\begin{array}{l}\text { First subperiod: } \\
\text { Total ................ } \\
\text { Average ........... } \\
\text { Second subperiod: }\end{array}$ & $\begin{array}{r}172 \\
34\end{array}$ & $\begin{array}{r}63.87 \\
\cdots\end{array}$ & $\begin{array}{l}62 \\
12\end{array}$ & $\begin{array}{r}359 \\
72\end{array}$ & $\begin{array}{r}70.17 \\
\cdots\end{array}$ & $\begin{array}{r}107 \\
21\end{array}$ & $\begin{array}{r}327 \\
65\end{array}$ & $\begin{array}{r}80.76 \\
\cdots\end{array}$ & $\begin{array}{l}63 \\
13\end{array}$ \\
\hline 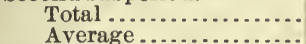 & 193 & 70.95 & 56 & 400 & 68.97 & 124 & 593 & 77.57 & 133 \\
\hline \multicolumn{10}{|l|}{ Third subperiod: } \\
\hline Total ................... & 172 & 63.36 & 63 & 419 & 68.74 & 131 & 212 & 71.74 & 60 \\
\hline Average ........ & 34 & .......... & 13 & 84 & & 26 & 42 & & 12 \\
\hline \multicolumn{10}{|l|}{ Fourth subperiod: } \\
\hline Average ................. & $\begin{array}{r}184 \\
37\end{array}$ & $\begin{array}{r}64.20 \\
\ldots \ldots\end{array}$ & $\begin{array}{l}66 \\
13\end{array}$ & $\begin{array}{r}464 \\
93\end{array}$ & $\begin{array}{r}70.25 \\
\ldots \ldots \ldots\end{array}$ & $\begin{array}{r}138 \\
28\end{array}$ & $\begin{array}{r}399 \\
80\end{array}$ & $\begin{array}{r}69.96 \\
\ldots \ldots . .\end{array}$ & $\begin{array}{r}120 \\
24\end{array}$ \\
\hline \multicolumn{10}{|l|}{$\begin{array}{l}\text { Fifth subperiod: } \\
\text { Total }\end{array}$} \\
\hline $\begin{array}{l}\text { Total ....................... } \\
\text { Average }\end{array}$ & $\begin{array}{r}192 \\
38\end{array}$ & 62.02 & 73 & 237 & 67.98 & 76 & 267 & 75.62 & 65 \\
\hline \multicolumn{10}{|l|}{$\begin{array}{l}\text { Sixth subperiod: } \\
\text { Total ............ }\end{array}$} \\
\hline $\begin{array}{l}\text { Total } \\
\text { Average } \ldots \ldots\end{array}$ & $\begin{array}{r}126 \\
25\end{array}$ & $\begin{array}{r}53.06 \\
\ldots .\end{array}$ & $\begin{array}{l}59 \\
12\end{array}$ & $\begin{array}{r}433 \\
87\end{array}$ & 69.52 & $\begin{array}{r}132 \\
26\end{array}$ & & & $\cdots \cdot$ \\
\hline \multirow{3}{*}{ 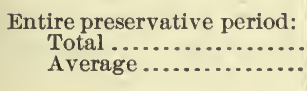 } & & & & & & & & & \\
\hline & 1,039 & 63.52 & 379 & 2,312 & 69.38 & 708 & a 1,798 & 75.47 & 441 \\
\hline & 35 & $\ldots \ldots \ldots$ & 13 & 77 & …...... & 24 & 72 & ............ & 18 \\
\hline \multicolumn{10}{|l|}{ After period. } \\
\hline \multicolumn{10}{|l|}{$\begin{array}{l}\text { First subperiod: } \\
\text { Total ......... }\end{array}$} \\
\hline $\begin{array}{l}\text { Average ........ } \\
\text { Second subperiod: }\end{array}$ & 57 & n....... & 22 & 69 & & 19 & & & \\
\hline \multicolumn{5}{|l|}{$\begin{array}{l}\text { Second subperiod: } \\
\text { Total }\end{array}$} & & & & 74.20 & 81 \\
\hline Average ......... & 46 & ......... & 17 & 80 & ......... & 24 & 63 & ......... & 16 \\
\hline $\begin{array}{l}\text { Entire after period: } \\
\text { Total } \ldots . . . . . . .\end{array}$ & 517 & 62.48 & 194 & 744 & 71.10 & 215 & & & \\
\hline & 52 & & 19 & 74 & & 22 & & & \\
\hline
\end{tabular}

$\alpha$ No. 3 had only five preservative subperiods. 
TABLE VII.-Weight and water content of feces, by periods, Series VI-Continued.

[Averages are per day.]

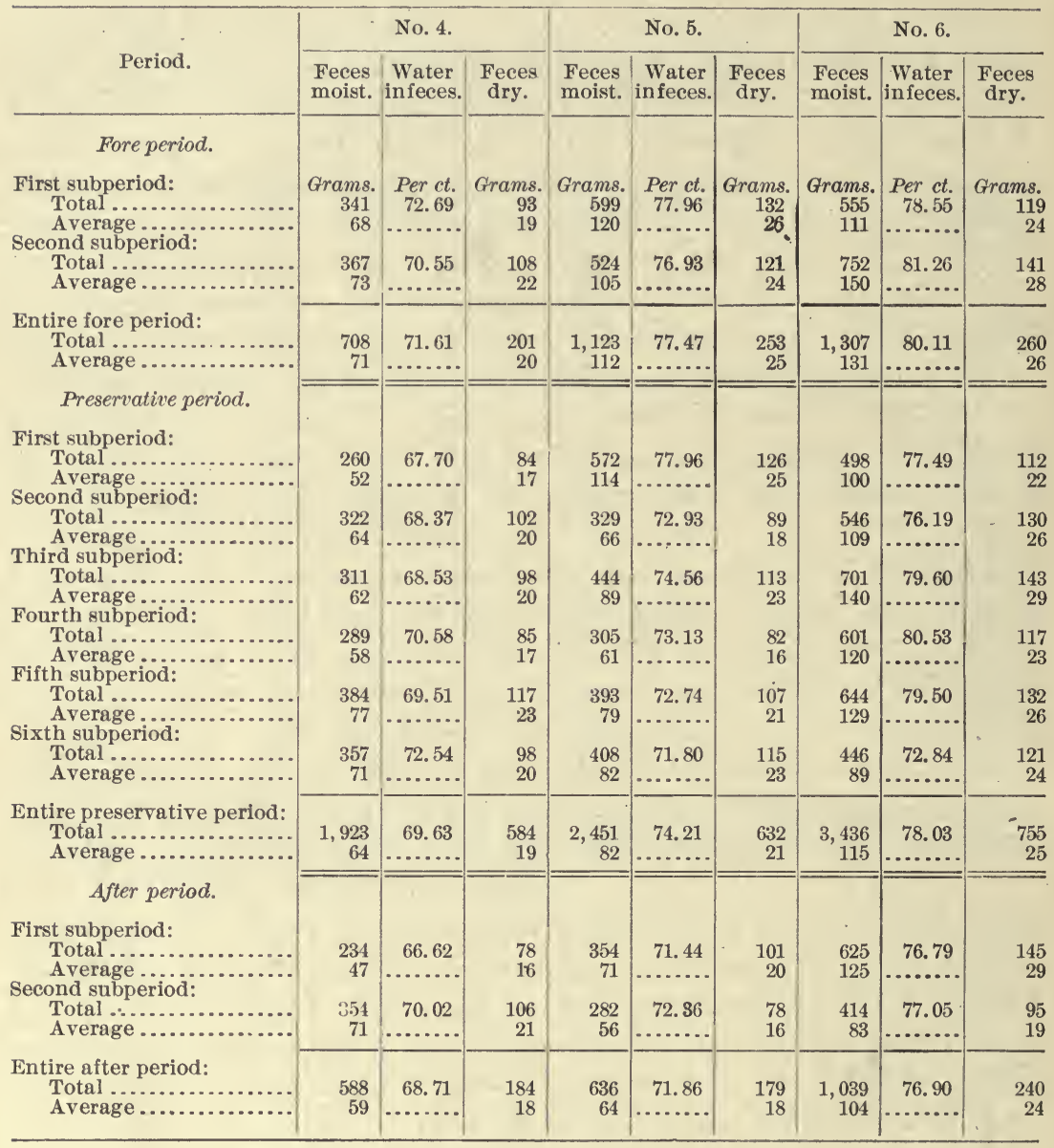


TABLE VII.-Weight and water content of feces, by periods, Series VI-Continued.

[Averages are per day.]

\begin{tabular}{|c|c|c|c|c|c|c|c|c|c|}
\hline \multirow[b]{2}{*}{. Period. } & \multicolumn{3}{|c|}{ No. 7.} & \multicolumn{3}{|c|}{ No. 8.} & \multicolumn{3}{|c|}{ No. 9.} \\
\hline & $\begin{array}{l}\text { Feces } \\
\text { moist. }\end{array}$ & $\begin{array}{l}\text { Water } \\
\text { infeces. }\end{array}$ & $\begin{array}{c}\text { Feces } \\
\text { dry. }\end{array}$ & $\begin{array}{l}\text { Feces } \\
\text { moist. }\end{array}$ & $\begin{array}{l}\text { Water } \\
\text { infeces. }\end{array}$ & $\begin{array}{c}\text { Feces } \\
\text { dry. }\end{array}$ & $\begin{array}{l}\text { Feces } \\
\text { moist. }\end{array}$ & $\begin{array}{l}\text { Water } \\
\text { infeces. }\end{array}$ & $\begin{array}{l}\text { Feces } \\
\text { dry. }\end{array}$ \\
\hline \multicolumn{10}{|l|}{ Fore period. } \\
\hline 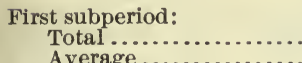 & Grams. & $\begin{array}{r}\text { Per ct. } \\
85.42\end{array}$ & $\begin{array}{r}\text { Grams. } \\
91\end{array}$ & $\begin{array}{r}\text { Grams. } \\
315\end{array}$ & $\begin{array}{r}\text { Per ct. } \\
76.82\end{array}$ & $\begin{array}{r}\text { Grams. } \\
73\end{array}$ & $\begin{array}{r}\text { Grams. } \\
208\end{array}$ & $\begin{array}{r}\text { Per ct. } \\
71.14\end{array}$ & $\begin{array}{r}\text { Grams. } \\
60\end{array}$ \\
\hline $\begin{array}{l}\text { Average .................. } \\
\text { Second subperiod: }\end{array}$ & 125 & $\cdots \cdots \cdots$ & 18 & 63 & ........ & 15 & 42 & $\cdots \cdots \cdots$ & 12 \\
\hline Total ............. & 212 & 75.95 & 51 & 415 & 77.34 & 94 & 277 & 65.74 & 95 \\
\hline Average & 42 & $\ldots \ldots \ldots$ & 10 & 83 & (n....... & 19 & 55 & …...... & 19 \\
\hline $\begin{array}{c}\text { Entire fore period: } \\
\text { Total ............ }\end{array}$ & $\begin{array}{r}836 \\
84\end{array}$ & $\begin{array}{r}83.01 \\
\cdots\end{array}$ & $\begin{array}{r}142 \\
14\end{array}$ & $\begin{array}{r}730 \\
73\end{array}$ & $\begin{array}{r}77.12 \\
\ldots \ldots\end{array}$ & $\begin{array}{r}167 \\
17\end{array}$ & $\begin{array}{r}485 \\
49\end{array}$ & $\begin{array}{r}68.04 \\
\ldots . . .\end{array}$ & $\begin{array}{r}155 \\
16\end{array}$ \\
\hline \multicolumn{10}{|l|}{ Preservative period. } \\
\hline $\begin{array}{l}\text { First subperiod: } \\
\text { Total } \ldots \ldots \\
\text { Average }\end{array}$ & $\begin{array}{r}259 \\
52\end{array}$ & 76.03 & $\begin{array}{l}62 \\
12\end{array}$ & 478 & 77.40 & 108 & .556 & & 159 \\
\hline $\begin{array}{l}\text { Average ......... } \\
\text { Second subperiod: }\end{array}$ & 52 & $\ldots \ldots$ & 12 & 96 & ........ & 22 & 111 & - $\ldots \ldots$ & 32 \\
\hline Total ............ & 205 & 76.60 & 48 & 466 & 75.53 & 114 & 427 & 75.20 & 106 \\
\hline $\begin{array}{l}\text { Average } \ldots . . . . . . . . . \\
\text { Third subperiod: }\end{array}$ & 41 & \multicolumn{5}{|c|}{ Third subperiod: } & 85 & $\cdots \ldots$ & 21 \\
\hline Total ............... & 254 & 74.82 & 64 & 235 & 68.93 & 73 & 380 & 71.56 & 108 \\
\hline Averag & 51 & & 13 & 47 & & 15 & 76 & & 22 \\
\hline $\begin{array}{l}\text { Fourth subp } \\
\text { Total }\end{array}$ & 214 & 78.49 & 46 & 511 & 77.71 & 114 & 275 & 71.66 & 78 \\
\hline Average ...... & 43 & ......... & 9 & 102 & ......... & 23 & 55 & ........ & 16 \\
\hline \multicolumn{10}{|l|}{ Fifth subperiod: } \\
\hline $\begin{array}{l}\text { Total } \\
\text { Average } \ldots \ldots\end{array}$ & $\begin{array}{r}271 \\
54\end{array}$ & $\begin{array}{r}75.26 \\
\ldots \ldots\end{array}$ & $\begin{array}{l}67 \\
13\end{array}$ & $\begin{array}{r}204 \\
41\end{array}$ & $\begin{array}{r}70.64 \\
\ldots\end{array}$ & $\begin{array}{l}60 \\
12\end{array}$ & $\begin{array}{l}522 \\
104\end{array}$ & 73.37 & $\begin{array}{r}139 \\
28\end{array}$ \\
\hline \multicolumn{10}{|l|}{ Sixth subperiod: } \\
\hline Total ............. & 119 & 72.22 & 33 & 425 & 78.37 & 92 & 364 & 68.98 & 113 \\
\hline Average & 24 & .......... & 7 & 85 & ........... & 18 & 73 & a....... & 23 \\
\hline & 1,322 & 75.79 & 320 & 2,319 & 75.81 & 561 & 2,524 & 72.15 & 703 \\
\hline & 44 & & 11 & 77 & & 19 & 84 & & 23 \\
\hline After period. & & & & & & & & & \\
\hline \multicolumn{10}{|l|}{ First subperiod: } \\
\hline & 53 & & 14 & 89 & & 21 & 31 & & 9 \\
\hline \multicolumn{10}{|l|}{ Second subperiod: } \\
\hline Average ............... & $\begin{array}{l}036 \\
111\end{array}$ & $\begin{array}{l}82.36 \\
\ldots \ldots . .\end{array}$ & $\begin{array}{l}98 \\
20\end{array}$ & $\begin{array}{l}630 \\
126\end{array}$ & $\begin{array}{r}83.66 \\
\ldots . . . .\end{array}$ & $\begin{array}{r}103 \\
21\end{array}$ & $\begin{array}{l}509 \\
102\end{array}$ & $\begin{array}{l}73.87 \\
\ldots . . . .\end{array}$ & $\begin{array}{r}133 \\
27\end{array}$ \\
\hline $\begin{array}{c}\text { Entire after period: } \\
\text { Total .......... }\end{array}$ & 820 & 79.51 & 168 & 1,076 & 80.58 & 209 & 662 & 72.96 & 179 \\
\hline Average & 82 & .......... & 17 & 108 & ......... & 21 & 66 & & 18 \\
\hline
\end{tabular}


TABLE VII. - Weight and water content of feces, by periods, Series VI-Continued.

[Averages are per day.]

\begin{tabular}{|c|c|c|c|c|c|c|c|c|c|}
\hline \multirow[b]{2}{*}{ Period. } & \multicolumn{3}{|c|}{ No. 10.} & \multicolumn{3}{|c|}{ No. 11.} & \multicolumn{3}{|c|}{ No. 12.} \\
\hline & $\begin{array}{l}\text { Feces } \\
\text { moist. }\end{array}$ & $\begin{array}{l}\text { Water } \\
\text { infeces. }\end{array}$ & $\begin{array}{l}\text { Feces } \\
\text { dry. }\end{array}$ & $\begin{array}{l}\text { Feces } \\
\text { moist. }\end{array}$ & $\begin{array}{l}\text { Water } \\
\text { infeces. }\end{array}$ & $\begin{array}{l}\text { Feces } \\
\text { dry. }\end{array}$ & $\begin{array}{l}\text { Feces } \\
\text { moist. }\end{array}$ & $\begin{array}{l}\text { Water } \\
\text { infeces. }\end{array}$ & $\begin{array}{l}\text { Feces } \\
\text { dry. }\end{array}$ \\
\hline Fore period. & & & & & & & & & \\
\hline $\begin{array}{l}\text { First subperiod: } \\
\text { Total .......... } \\
\text { Average ....... }\end{array}$ & $\begin{array}{r}\text { Grams. } \\
500 \\
100\end{array}$ & $\begin{array}{r}\text { Per et. } \\
75.00\end{array}$ & $\begin{array}{r}\text { Grams. } \\
125 \\
25\end{array}$ & $\begin{array}{r}\text { Grams. } \\
450 \\
90\end{array}$ & $\begin{array}{c}\text { Per ct. } \\
72.90\end{array}$ & $\begin{array}{r}\text { Grams. } \\
122 \\
24\end{array}$ & $\begin{array}{r}\text { Grams. } \\
585 \\
117\end{array}$ & $\begin{array}{l}\text { Per ct. } \\
74.88\end{array}$ & Grams. \\
\hline Second subperiod: & & & & & & & & & \\
\hline $\begin{array}{l}\text { Total ............. } \\
\text { Average ........ }\end{array}$ & $\begin{array}{r}312 \\
62\end{array}$ & $\begin{array}{c}72.43 \\
\cdots \cdots\end{array}$ & $\begin{array}{l}86 \\
17\end{array}$ & $\begin{array}{r}494 \\
99\end{array}$ & $\begin{array}{r}73.87 \\
\cdots \ldots . . .\end{array}$ & $\begin{array}{r}129 \\
26\end{array}$ & $\begin{array}{r}434 \\
87\end{array}$ & 71.22 & $\begin{array}{r}125 \\
25\end{array}$ \\
\hline $\begin{array}{l}\text { Entire fore period: } \\
\text { Total............ } \\
\text { Average...... }\end{array}$ & $\begin{array}{r}812 \\
81\end{array}$ & $\begin{array}{r}74.01 \\
\cdots \cdots . . . \\
\end{array}$ & $\begin{array}{r}211 \\
21 \\
\end{array}$ & $\begin{array}{r}944 \\
94\end{array}$ & 73.41 & $\begin{array}{r}251 \\
25\end{array}$ & $\begin{array}{r}1,019 \\
102\end{array}$ & 73.31 & $\begin{array}{r}272 \\
27\end{array}$ \\
\hline Preservative period. & & & & & & & & & \\
\hline $\begin{array}{l}\text { First subperiod: } \\
\quad \text { Total ............ } \\
\text { Average ....... }\end{array}$ & $\begin{array}{r}496 \\
99\end{array}$ & 75.61 & $\begin{array}{r}121 \\
24\end{array}$ & $\begin{array}{r}489 \\
98\end{array}$ & 76.08 & $\begin{array}{r}117 \\
23\end{array}$ & $\begin{array}{r}328 \\
66\end{array}$ & 73.44 & $\begin{array}{l}87 \\
17\end{array}$ \\
\hline $\begin{array}{l}\text { Second subperiod: } \\
\text { Total ............ }\end{array}$ & 408 & 74. 29 & 105 & & 75.65 & & 341 & & \\
\hline AV & 82 & & $\begin{array}{r}21 \\
21\end{array}$ & $\begin{array}{l}000 \\
101\end{array}$ & 10.60 & 25 & $\begin{array}{r}341 \\
68\end{array}$ & 74.18 & 1 \\
\hline Third & & & & & & & & & \\
\hline $\begin{array}{l}\text { To } \\
\text { Al }\end{array}$ & $\begin{array}{r}410 \\
82\end{array}$ & 72.65 & $\begin{array}{r}112 \\
22\end{array}$ & $\begin{array}{l}529 \\
106\end{array}$ & 76.56 & $\begin{array}{r}124 \\
25\end{array}$ & $\begin{array}{l}576 \\
115\end{array}$ & 75. 35 & 142 \\
\hline Fourtl & & & & & & & & & \\
\hline $\begin{array}{l}\text { Tot } \\
\text { Av }\end{array}$ & $\begin{array}{r}380 \\
76\end{array}$ & 76.33 & $\begin{array}{l}90 \\
18\end{array}$ & $\begin{array}{r}457 \\
91\end{array}$ & 74.81 & $\begin{array}{l}115 \\
23\end{array}$ & $\begin{array}{r}357 \\
71\end{array}$ & 76.22 & $\begin{array}{l}85 \\
17\end{array}$ \\
\hline Fifth sul & & & & & & & & & \\
\hline $\begin{array}{l}\text { Total } \\
\text { Avere }\end{array}$ & $\begin{array}{r}308 \\
62\end{array}$ & 74.66 & $\begin{array}{l}78 \\
16\end{array}$ & $\begin{array}{r}473 \\
95\end{array}$ & 77.17 & $\begin{array}{r}108 \\
22\end{array}$ & $\begin{array}{r}492 \\
98\end{array}$ & 73.97 & $\begin{array}{r}128 \\
26\end{array}$ \\
\hline Sixth sub & L & & 37 & 465 & 73.98 & 121 & & 71.20 & \\
\hline Average & 25 & 70.40 & 7 & $\begin{array}{r}403 \\
93\end{array}$ & 10.90 & 24 & $\begin{array}{r}581 \\
67\end{array}$ & 1.20 & 9 \\
\hline 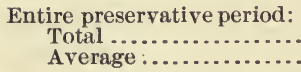 & $\begin{array}{r}2,127 \\
71\end{array}$ & $\begin{array}{l}74.47 \\
\cdots \ldots . .\end{array}$ & $\begin{array}{r}543 \\
18\end{array}$ & $\begin{array}{r}2,918 \\
97\end{array}$ & 75.74 & $\begin{array}{r}708 \\
24\end{array}$ & $\begin{array}{r}2,431 \\
81\end{array}$ & 74.21 & 62 \\
\hline After period. & & & & & & & & & \\
\hline $\begin{array}{l}\text { First subperiod: } \\
\text { Total ............... } \\
\text { Average ........ }\end{array}$ & $\begin{array}{r}457 \\
91\end{array}$ & 73.30 & $\begin{array}{r}122 \\
24\end{array}$ & $\begin{array}{r}466 \\
93\end{array}$ & 76.37 & $\begin{array}{r}110 \\
22\end{array}$ & $\begin{array}{r}416 \\
83\end{array}$ & 72.58 & 11 \\
\hline $\begin{array}{l}\text { Second subperiod: } \\
\quad \text { Total ................. } \\
\text { Average ......... }\end{array}$ & $\begin{array}{r}a(457) \\
(91)\end{array}$ & $\begin{array}{l}(73.30) \\
\ldots \ldots . . .\end{array}$ & $\begin{array}{r}(122) \\
(24)\end{array}$ & $\begin{array}{r}389 \\
78\end{array}$ & 72.48 & $\begin{array}{r}107 \\
21\end{array}$ & $\begin{array}{r}403 \\
81\end{array}$ & 71.21 & 116 \\
\hline $\begin{array}{l}\text { Entire after period: } \\
\text { Total ............... } \\
\text { Average .......... }\end{array}$ & $\begin{array}{r}a(914) \\
(91)\end{array}$ & (73. 30) & $\begin{array}{r}(244) \\
(24)\end{array}$ & $\begin{array}{r}855 \\
86\end{array}$ & $\begin{array}{c}74.62 \\
\cdots \ldots . .\end{array}$ & $\begin{array}{r}217 \\
22\end{array}$ & $\begin{array}{r}819 \\
82\end{array}$ & 71.92 & 230 \\
\hline
\end{tabular}

$a$ Dat $x$ ob: erved for subperiod only, owing to illness of subject during second period. 
Table VII.-Weight and water content of feces, by periods, Series VI-Continued.

[Averages are per day.]

SUMMARY FOR NINE MEN.

\begin{tabular}{|c|c|c|c|c|c|c|c|}
\hline Period. & $\begin{array}{l}\text { Feces } \\
\text { moist. }\end{array}$ & $\begin{array}{l}\text { Water } \\
\text { infeces. }\end{array}$ & $\begin{array}{l}\text { Fecess } \\
\text { dry. }\end{array}$ & Period. & $\begin{array}{l}\text { Feces } \\
\text { moist. }\end{array}$ & $\begin{array}{l}\text { Water } \\
\text { infeces. }\end{array}$ & $\begin{array}{l}\text { Feces } \\
\text { dry. }\end{array}$ \\
\hline Fore period. & \multirow{3}{*}{$\begin{array}{r}\text { Grams } \\
4,158 \\
92\end{array}$} & \multirow{3}{*}{$\begin{array}{l}\text { Per ct. } \\
76.36\end{array}$} & \multirow{3}{*}{$\begin{array}{r}\text { Grams } \\
983 \\
22\end{array}$} & $\begin{array}{l}\text { Preservative period-Con- } \\
\text { tinued. }\end{array}$ & \multirow{3}{*}{$\begin{array}{r}\text { Grams. } \\
3,290 \\
73\end{array}$} & \multirow{3}{*}{$\begin{array}{l}\text { Per ct. } \\
73.62\end{array}$} & \multirow{3}{*}{$\begin{array}{r}\text { Grams } \\
868 \\
19\end{array}$} \\
\hline $\begin{array}{l}\text { First subperiod: } \\
\text { Total......... }\end{array}$ & & & & Fifth subperiod: & & & \\
\hline $\begin{array}{l}\text { Average..... } \\
\text { Second subperio }\end{array}$ & & & & $\begin{array}{l}\text { Total .......... } \\
\text { Average.... }\end{array}$ & & & \\
\hline Total. & \multirow{2}{*}{$\begin{array}{r}3,862 \\
86\end{array}$} & \multirow[t]{2}{*}{.74 .81} & \multirow{2}{*}{$\begin{array}{r}973 \\
22\end{array}$} & Sixth subperiod: & \multirow{2}{*}{$\begin{array}{r}3,116 \\
69\end{array}$} & \multirow[b]{2}{*}{72.14} & \multirow{2}{*}{$\begin{array}{r}868 \\
19\end{array}$} \\
\hline Average & & & & Total & & & \\
\hline Entire fore period: & \multirow{2}{*}{$\begin{array}{r}8,020 \\
89\end{array}$} & \multirow{2}{*}{$\begin{array}{l}75.61 \\
\cdots \ldots . . .\end{array}$} & \multirow{2}{*}{$\begin{array}{r}1,956 \\
22\end{array}$} & Entire preservative pe- & & \multirow{3}{*}{$\begin{array}{l}73.83 \\
\cdots \cdots . . .\end{array}$} & \multirow{3}{*}{5,27} \\
\hline Average & & & & riod. & \multirow{2}{*}{$\begin{array}{r}20,151 \\
75\end{array}$} & & \\
\hline Preservative period. & \multirow{3}{*}{$\begin{array}{r}3,415 \\
76\end{array}$} & \multirow[b]{2}{*}{74.67} & \multirow{3}{*}{$\begin{array}{r}865 \\
19\end{array}$} & A verage. & & & \\
\hline $\begin{array}{l}\text { First subperiod: } \\
\text { Total............ }\end{array}$ & & & & After period. & \multirow{3}{*}{$\begin{array}{r}3,437 \\
76\end{array}$} & \multirow{3}{*}{73.00} & \multirow{3}{*}{$\begin{array}{c}928 \\
21\end{array}$} \\
\hline $\begin{array}{l}\text { Aver } \\
\text { Second } \mathrm{s}\end{array}$ & & \multirow[b]{2}{*}{73.57} & & $\begin{array}{r}\text { First subperio } \\
\text { Total..... }\end{array}$ & & & \\
\hline $\begin{array}{l}\text { Total } \\
\text { Aver }\end{array}$ & \multirow{2}{*}{$\begin{array}{r}3,307 \\
73\end{array}$} & & $\begin{array}{r}874 \\
19\end{array}$ & Äver & & & \\
\hline $\begin{array}{l}\text { Aver } \\
\text { Third su }\end{array}$ & & \multirow[b]{2}{*}{73.88} & \multirow{2}{*}{$\begin{array}{r}951 \\
21\end{array}$} & $\begin{array}{l}\text { Second sul } \\
\text { Total. }\end{array}$ & \multirow{2}{*}{$\begin{array}{r}3,657 \\
81\end{array}$} & & \multirow{2}{*}{$\begin{array}{r}908 \\
20\end{array}$} \\
\hline Tota & $\begin{array}{r}3,641 \\
81\end{array}$ & & & Average.... & & & \\
\hline Fourth subperiod: & \multirow{2}{*}{$\begin{array}{r}3,382 \\
75\end{array}$} & & & Entire after period: & \multirow[b]{2}{*}{$\begin{array}{r}7,094 \\
79\end{array}$} & \multirow[b]{2}{*}{74.12} & \multirow{2}{*}{$\begin{array}{r}1,836 \\
20\end{array}$} \\
\hline $\begin{array}{l}\text { Total..... } \\
\text { Average }\end{array}$ & & 74.93 & $\begin{array}{r}848 \\
19\end{array}$ & $\begin{array}{l}\text { Total ............... } \\
\text { Average....... }\end{array}$ & & & \\
\hline
\end{tabular}

\section{THE URINE.}

The importance of a study of the urine in connection with the ascertainment of any effects produced by the administration of salicylic acid and other preservatives is evident without comment. In the following tables are given the results of the observations obtained on the urine. The influence of any added preservative upon the volume of the urine and the amount of solids therein is quite significant, and these points were carefully studied. These studies were conducted by F. C. Weber.

\section{VOLUME, SPECIFIC GRAVITY, AND TOTAL SOLIDS.}

The data given in Table VIII relating to the total solids in the urine are calculated from the specific gravity, in harmony with the method used in the borax experiment, by the factor $0.245 .^{a}$

\section{INDIVIDUAL DATA.}

No. 1.

The volume of the urine is decreased during the preservative period and still further decreased in the after period. The specific gravity is increased in the preservative period and still further increased, by a very slight amount, in the after period. The increase in specific gravity is greater in proportion than the decrease in volume, since the weight of the total solids excreted is greater in the preservative period and still further increased, by a slight amount, in the after period. 


\section{No. 2.}

In this case the volume of the urine is increased and the specific gravity diminished in the preservative period. In the after period the volume of the urine is considerably diminished below the fore period, and its specific gravity is increased above that of the fore period and also of the preservative period. The weight of the total solids excreted is greater in the preservative period than in the fore period, and this weight is still further increased in the after period.

\section{No. 3.}

The data for No. 3 are somewhat fragmentary and show little change in the volume of the urine during the three periods. The specific gravity is slightly less in the preservative period, while in the after period it rises a very little above that of the fore period. The quantity of total solids is decreased during the preservative period, while in the after period the quantity is the same as in the fore period.

\section{No. 4 .}

In this case there is a slight increase in the volume of the urine in the preservative period and a still further slight increase in the after period. The specific gravity is high through all the periods, being slightly greater in the preservative and again slightly increased in the after period. The total quantity of solids excreted is greater in the preservative period and continues to increase in the after period.

\section{No. 5 .}

There is a slight increase in the total volume of the urine in the preservative period and a greater increase in the after period. The specific gravity is slightly higher in the preservative period and slightly lower in the after period than in the fore period. The total quantity of solid matter excreted in the urine is greater in the preservative period and still somewhat greater in the after period.

No. 6 .

There is a notable increase in the volume of the urine in the preservative period, but this increase is only partially maintained in the after period. The specific gravity of the urine is high and almost the same in the fore and preservative periods and slightly higher in the after period. The quantity of total solids excreted is notably greater in the preservative period, with a slight loss in weight in the after period.

No. $\%$.

There is a notable increase in the volume of the urine in the preservative period in this case, while in the after period the volume is only slightly greater than in the fore period. The specific gravity of 
the urine in the after period, while slightly greater than in the preservative period, is still far below that of the fore period. The total weight of solids excreted is, in this case, less in the preservative period than in the fore period and still less in the after period. No. 7 in this respect differs from five of the six cases already cited.

No. 8 .

In the case of No. 8 there is a slight decrease in the volume of the urine in the preservative period and a very notable decrease in the after period. The volume of the urine is very great in the case of No. 8 and the specific gravity correspondingly low, being slightly greater in the preservative period and notably greater in the after period than in the fore period. The quantity of solids excreted in the urine is slightly greater in the preservative period and still further increased in the after period.

No. 9.

The volume of urine in this case is greater in the preservative period and slightly greater in the after period than in the preservative period. The specific gravity is slightly higher in the preservative period, and in the after period it is almost the same as in the preservative period. The total weight of solids excreted is greater in the preservative period and almost the same in the after period as the preservative period.

\section{No. 10.}

There is a notable increase in the volume of the urine in this case in the preservative period, and the volume in the after period is almost identical with that of the preservative period. The specific gravity is less in the preservative period and still further diminished to a slight extent in the after period. The weight of the solids excreted is greater in the preservative period and almost the same in the after as in the preservative period.

No. 11 .

The volume of the urine in the ease of No. 11 is less in the preservative period and is almost the same in the after as in the preservative period. The specific gravity of the urine in the preservative period is increased and in the after period is only slightly less than in the preservative period. The total solids excreted are high and are slightly less in the preservative period and still further diminished in the after period.

No. 12 .

The volume of the urine in this case is notably diminished in the preservative period and increased over the preservative period in the after period, but not to the volume of the fore period. The specific 
gravity is notably higher in the preservative period, and in the after period it is still higher than in the fore period. The weight of the solids excreted is slightly greater in the preservative period and still further increased in the after period.

SUMMARY FOR NINE MEN.

The average effects produced upon the nine men who completed the observations show that the volume of the urine in the preservative period is almost the same as that of the fore period, being only 7 cubic centimeters greater. In the after period the volume is slightly less than in the fore period, being diminished by 47 cubic centimeters. The average specific gravity of the urine in the preservative period is slightly higher than in the fore period and in the after period is again slightly increased as compared with the preservative period. The quantity of solids excreted is increased in the preservative period and still further increased by half a gram a day in the after period.

The general effect therefore upon the urine is that no notable change is produced in the volume of the urine due to the administration of the preservative. There is, however, a distinct increase in the specific gravity of the urine under the administration of the preservative, due to a decided increase in the weight of the solids excreted. This effect is continued to a certain extent in the after period, during which even a larger quantity of solids is excreted than during the preservative period.

It is just to conclude from the above data that the administration of the salicylic acid increases the katabolic activities of the organs, resulting in an increased excretion of solid matters in the urine. 
SALICYLIC ACID AND SALICYLATES.

TABLE VIII.-Urine determinations-Volume, specific gravity, and total solids-Series VI.

[Averages are per day.]

\begin{tabular}{|c|c|c|c|c|c|c|c|c|c|}
\hline \multirow{2}{*}{ Period. } & \multicolumn{3}{|c|}{ No. 1.} & \multicolumn{3}{|c|}{ No. 2.} & \multicolumn{3}{|c|}{ No. 3.} \\
\hline & $\begin{array}{l}\text { Vol- } \\
\text { ume. }\end{array}$ & $\begin{array}{l}\text { Specific } \\
\text { gravity } \\
\text { at } \\
25^{\circ} / 25^{\circ} \\
\text { C. }\end{array}$ & $\begin{array}{c}\text { Total } \\
\text { solids } \\
\text { (factor } \\
0.245 \text { ). }\end{array}$ & $\begin{array}{l}\text { Vol- } \\
\text { ume. }\end{array}$ & $\begin{array}{c}\text { Specific } \\
\text { gravity } \\
\text { at } \\
25^{\circ} / 25^{\circ} \\
\text { C. }\end{array}$ & $\begin{array}{c}\text { Total } \\
\text { solids } \\
\text { (factor } \\
0.245 \text { ). }\end{array}$ & $\begin{array}{l}\text { Vol- } \\
\text { ume. }\end{array}$ & $\begin{array}{l}\text { Specific } \\
\text { gravity } \\
\text { at } \\
25^{\circ} / 25^{\circ} \\
\text { C. }\end{array}$ & $\begin{array}{l}\text { Total } \\
\text { solids } \\
\text { (factor } \\
0.245)\end{array}$ \\
\hline Fore period. & & & & & & & & & \\
\hline $\begin{array}{l}\text { First subperiod: } \\
\text { Total ........... } \\
\text { Average ....... }\end{array}$ & $\begin{array}{l}c c . \\
6,830 \\
1,366\end{array}$ & $\begin{array}{l}1.0161 \\
\ldots \ldots \ldots\end{array}$ & $\begin{array}{r}\text { Grams. } \\
269.4 \\
53.9\end{array}$ & $\begin{aligned} c c . \\
a 5,663 \\
1,133\end{aligned}$ & $\begin{array}{l}1.0254 \\
\ldots \ldots \ldots\end{array}$ & $\begin{array}{r}\text { Grams. } \\
352.4 \\
70.5\end{array}$ & $\begin{array}{l}c c . \\
\cdots \cdots \\
\cdots \cdots\end{array}$ & & \begin{tabular}{l} 
Grams. \\
\hdashline$\ldots$ \\
\hdashline
\end{tabular} \\
\hline Second subperiod: & & & & & & & & & \\
\hline $\begin{array}{l}\text { Total ......................... } \\
\text { Average }\end{array}$ & $\begin{array}{l}6,685 \\
1,337\end{array}$ & $\begin{array}{l}1.0187 \\
\cdots\end{array}$ & $\begin{array}{r}306.3 \\
61.3\end{array}$ & $\begin{array}{l}6,485 \\
1,297\end{array}$ & $\begin{array}{l}1.0192 \\
\ldots \ldots\end{array}$ & $\begin{array}{r}305.1 \\
61.0\end{array}$ & $\begin{array}{r}4,820 \\
964\end{array}$ & $\begin{array}{c}1.0213 \\
\cdots \ldots\end{array}$ & $\begin{array}{r}251.5 \\
50.3\end{array}$ \\
\hline $\begin{array}{l}\text { Entire fore period: } \\
\quad \text { Total } \ldots \ldots \\
\text { Average } \ldots \ldots\end{array}$ & $\begin{array}{r}13,515 \\
1,352\end{array}$ & 1.0174 & $\begin{array}{r}575.7 \\
57.6\end{array}$ & $\begin{array}{r}12,148 \\
1,215\end{array}$ & 1.0223 & $\begin{array}{r}657.5 \\
65.8\end{array}$ & & & \\
\hline Preservative period. & & & & & & & & & \\
\hline $\begin{array}{l}\text { First subperiod: } \\
\text { Total .............. } \\
\text { Average . }\end{array}$ & $\begin{array}{l}5,840 \\
1,168\end{array}$ & $\begin{array}{l}1.0210 \\
\cdots \ldots . .\end{array}$ & $\begin{array}{r}300.5 \\
60.1\end{array}$ & $\begin{array}{l}6,080 \\
1,216\end{array}$ & $\begin{array}{l}1.0207 \\
\cdots \ldots . .\end{array}$ & $\begin{array}{r}308.4 \\
61.7\end{array}$ & $\begin{array}{r}4,485 \\
897\end{array}$ & $\begin{array}{l}1.0123 \\
\cdots . . . . .\end{array}$ & $\begin{array}{r}135.2 \\
27.4\end{array}$ \\
\hline $\begin{array}{l}\text { Second subperiod: } \\
\text { Total } \ldots . . . \ldots \ldots\end{array}$ & & & & & & & & & \\
\hline Total.. & $\begin{array}{l}6,745 \\
1,349\end{array}$ & $\begin{array}{r}1.0190 \\
\ldots . \ldots .\end{array}$ & $\begin{array}{r}314.0 \\
62.8\end{array}$ & $\begin{array}{l}7,170 \\
1,434\end{array}$ & $\begin{array}{l}1.0188 \\
\ldots \ldots\end{array}$ & $\begin{array}{r}330.3 \\
66.1\end{array}$ & $\begin{array}{r}b 4,888 \\
978\end{array}$ & $\begin{array}{c}1.0213 \\
\ldots \ldots \ldots\end{array}$ & $\begin{array}{r}a 255.0 \\
51.0\end{array}$ \\
\hline $\begin{array}{l}\text { Third subp } \\
\text { Total. }\end{array}$ & & & & & & & & & \\
\hline & $\begin{array}{l}b 5,715 \\
.1,143\end{array}$ & $\begin{array}{c}1.0220 \\
\ldots \ldots \ldots\end{array}$ & $\begin{array}{r}a 307.9 \\
61.6\end{array}$ & $\begin{array}{l}5,960 \\
1,192\end{array}$ & 1.0231 & $\begin{array}{r}337.3 \\
67.5\end{array}$ & $\begin{array}{r}4,970 \\
994\end{array}$ & 1.0214 & $\begin{array}{r}260.6 \\
52.1\end{array}$ \\
\hline Fourt] & & & & & & & & & \\
\hline & 5,930 & 1.0216 & 313.8 & 5,645 & 1.0240 & 331.9 & 5,420 & 1. 0197 & 261.6 \\
\hline $\begin{array}{l}\text { Averag } \\
\text { Fifth subp }\end{array}$ & 1 & & & & & 66.4 & 1,084 & & 52.3 \\
\hline (n.............. & 6,050 & 1.0210 & 311.3 & 7,330 & 1.0204 & 366.4 & 5,130 & 1.0206 & 258 \\
\hline Sixth subpe & r & & & & $\cdots \cdot$ & & 1,026 & & \\
\hline $\begin{array}{l}\text { Total } \ldots \ldots \ldots \\
\text { Average } . . .\end{array}$ & $\begin{array}{l}5,940 \\
1,188\end{array}$ & $\begin{array}{l}1.0219 \\
\cdots\end{array}$ & $\begin{array}{r}318.7 \\
63.7\end{array}$ & $\begin{array}{l}7,065 \\
1,413\end{array}$ & $\begin{array}{l}1.0195 \\
\ldots \ldots\end{array}$ & $\begin{array}{r}337.6 \\
67.5\end{array}$ & & & \\
\hline $\begin{array}{l}\text { Entire preservative period: } \\
\text { Total } . . . \\
\text { Average } . . .\end{array}$ & $\begin{array}{r}36,220 \\
1,207\end{array}$ & 1.0211 & $\begin{array}{r}1,866.2 \\
62.2\end{array}$ & $\begin{array}{r}39,250 \\
1,308\end{array}$ & $\dddot{1.0211}$ & $\begin{array}{r}2,011.9 \\
67.3\end{array}$ & $\begin{array}{r}c 24,893 \\
996\end{array}$ & $\left|\begin{array}{c}1.0190 \\
\cdots \ldots\end{array}\right|$ & $\begin{array}{r}1,171.3 \\
46.8\end{array}$ \\
\hline After period. & & & & & & & & & \\
\hline $\begin{array}{l}\text { First subperiod: } \\
\text { Total ......... } \\
\text { Average..... }\end{array}$ & $\begin{array}{l}5,820 \\
1,164\end{array}$ & 1.0222 & $\begin{array}{r}316.5 \\
63.3\end{array}$ & $\begin{array}{l}5,630 \\
1,126\end{array}$ & 1.0248 & $\begin{array}{r}342.1 \\
68.4\end{array}$ & $\begin{array}{l}5,020 \\
1,004\end{array}$ & 1.0204 & $\begin{array}{r}250.9 \\
50.2\end{array}$ \\
\hline$\underset{T C}{\text { Secon }}$ & & & & & & & & & \\
\hline Average ............... & $\begin{array}{l}5,660 \\
1,132\end{array}$ & $\begin{array}{l}1.0227 \\
\ldots \ldots \ldots\end{array}$ & $\begin{array}{r}314.8 \\
63.0\end{array}$ & $\begin{array}{l}5,720 \\
1,144\end{array}$ & 1.0249 & $\begin{array}{c}348.91 \\
69.8\end{array}$ & $\begin{array}{r}4,410 \\
882\end{array}$ & $\begin{array}{l}1.0233 \\
\ldots \ldots \ldots\end{array}$ & $\begin{array}{r}251.9 \\
50.4\end{array}$ \\
\hline $\begin{array}{l}\text { Entire after period: } \\
\text { Total ............... } \\
\text { Average ........ }\end{array}$ & $\begin{array}{r}11,480 \\
1,148\end{array}$ & 1.0225 & $\begin{array}{r}631.3 \\
63.1\end{array}$ & $\begin{array}{r}11,350 \\
1,135\end{array}$ & 1.0249 & $\begin{array}{r}691.0 \\
69.1\end{array}$ & $\begin{array}{r}9,430 \\
943\end{array}$ & 1.0219 & $\begin{array}{r}502.8 \\
50.3\end{array}$ \\
\hline
\end{tabular}

$a$ Average added in order to complete record.

b Four-day composites; average added in each case in order to complete five-day period.

$c$ No. 3 only had five preservative subperiods. 
TABLE VIII.-Urine determinations-Volume, specific gravity, and total solids-Series VI-Continued.

[Averages are per day.]

\begin{tabular}{|c|c|c|c|c|c|c|c|c|c|}
\hline \multirow[b]{2}{*}{ - $\quad$ Period. } & \multicolumn{3}{|c|}{ No. 4.} & \multicolumn{3}{|c|}{ No. 5.} & \multicolumn{3}{|c|}{ No. 6.} \\
\hline & $\begin{array}{l}\text { Vol- } \\
\text { ume. }\end{array}$ & $\left|\begin{array}{c}\text { Specific } \\
\text { gravity } \\
\text { at } \\
25^{\circ} \% / 25^{\circ} \\
\text { C. }\end{array}\right|$ & $\begin{array}{c}\text { Total } \\
\text { solids } \\
\text { (factor } \\
0.245 \text { ). }\end{array}$ & $\begin{array}{l}\text { Vol- } \\
\text { ume. }\end{array}$ & $\begin{array}{c}\text { Specific } \\
\text { gravity } \\
\text { at } \\
25^{\circ} / 25^{\circ} \\
\text { C. }\end{array}$ & $\begin{array}{l}\text { Total } \\
\text { solids } \\
\text { (factor } \\
0.245 \text { ). }\end{array}$ & $\begin{array}{l}\text { Vol- } \\
\text { ume. }\end{array}$ & $\begin{array}{c}\text { Specific } \\
\text { gravity } \\
\text { at } \\
25^{\circ} / 25^{\circ} \\
\text { C. }\end{array}$ & $\begin{array}{c}\text { Total } \\
\text { solids } \\
\text { (factor } \\
0.245 \text { ). }\end{array}$ \\
\hline \multicolumn{10}{|l|}{ Fore period. } \\
\hline $\begin{array}{l}\text { First subperiod: } \\
\text { Total............. } \\
\text { Average......... }\end{array}$ & $\begin{array}{l}c c \\
4,995 \\
999\end{array}$ & $\begin{array}{c}1.0257 \\
\cdots\end{array}$ & $\begin{array}{r}\text { Grams. } \\
314.5 \\
62.9\end{array}$ & $\begin{array}{l}c c . \\
4,620 \\
924\end{array}$ & 1.0261 & $\begin{array}{r}\text { Grams. } \\
295.4 \\
59.1\end{array}$ & $\begin{array}{l}c c . \\
3,635 \\
727\end{array}$ & $\begin{array}{c}1.0272 \\
\ldots \ldots\end{array}$ & $\begin{array}{r}\text { Grams. } \\
242.2 \\
48.4\end{array}$ \\
\hline Second subperiod: & & & & & & & & & \\
\hline $\begin{array}{l}\text { Total ................. } \\
\text { Average }\end{array}$ & $\begin{array}{r}4,835 \\
967\end{array}$ & \begin{tabular}{|l}
1.0263 \\
$\cdots \ldots$
\end{tabular} & $\begin{array}{r}311.6 \\
62.3\end{array}$ & $\begin{array}{l}5,665 \\
1,133\end{array}$ & $\begin{array}{l}1.0199 \\
\cdots \ldots\end{array}$ & $\begin{array}{r}276.2 \\
55.3\end{array}$ & $\begin{array}{r}3,850 \\
770\end{array}$ & $\begin{array}{l}1.0267 \\
\cdots \ldots\end{array}$ & $\begin{array}{r}251.9 \\
50.4\end{array}$ \\
\hline $\begin{array}{l}\text { Entire fore period: } \\
\text { Total ............... } \\
\text { Average ........ }\end{array}$ & $\begin{array}{r}9,830 \\
983\end{array}$ & 1.0260 & $\begin{array}{r}626.1 \\
62.6\end{array}$ & $\begin{array}{r}10,285 \\
1,029\end{array}$ & 1.0230 & $\begin{array}{r}571.6 \\
57.2\end{array}$ & $\begin{array}{r}7,485 \\
749\end{array}$ & 1.0269 & $\begin{array}{r}494.1 \\
49.4\end{array}$ \\
\hline \multicolumn{10}{|l|}{ Preservative period. } \\
\hline $\begin{array}{l}\text { First subperiod: } \\
\text { Total ............. } \\
\text { Average......... }\end{array}$ & $\begin{array}{r}4,480 \\
896\end{array}$ & $\begin{array}{c}1.0291 \\
\ldots \ldots \ldots\end{array}$ & $\begin{array}{r}319.4 \\
53.9\end{array}$ & $\begin{array}{r}4,720 \\
944\end{array}$ & $\begin{array}{l}1.0257 \\
\ldots \ldots \ldots\end{array}$ & $\begin{array}{r}297.2 \\
59.4\end{array}$ & $\begin{array}{r}4,070 \\
814\end{array}$ & $\begin{array}{l}1.0273 \\
. \ldots \ldots\end{array}$ & $\begin{array}{r}272.2 \\
54.4\end{array}$ \\
\hline $\begin{array}{l}\text { Second subperiod: } \\
\text { Total ............ }\end{array}$ & 4,715 & 1.0286 & 330.4 & 5,785 & 1.0206 & 292.0 & 4,485 & 1.0250 & \\
\hline $\begin{array}{l}\text { Average } \ldots . . . . . \\
\text { Third subperiod: }\end{array}$ & 943 & .......... & 66.1 & 1,157 & (1.......... & 58.4 & 897 & $\begin{array}{l}1.0230 \\
\cdots \ldots . . .\end{array}$ & 54.9 \\
\hline $\begin{array}{l}\text { Thra subperiod: } \\
\text { Total ............. }\end{array}$ & 4,960 & 1.0273 & 331.7 & 5,410 & 1.0231 & 306.2 & $a 4,100$ & 1.0275 & 276.3 \\
\hline $\begin{array}{l}\text { Average ............. } \\
\text { Fourth subperiod: }\end{array}$ & 992 & a....... & 66.3 & 1,082 & .......... & 61.2 & 820 & ........ & 55.3 \\
\hline Total ............... & 5,415 & 1. 0261 & 346.2 & 5,900 & 1.0222 & 320.9 & $a 4,588$ & 1. 0273 & b 306.8 \\
\hline Fiîth subperiod: & 1,083 & n........ & & 1,180 & ......... & 64.2 & 918 & .......... & 61.4 \\
\hline $\begin{array}{l}\text { Total } \ldots . . . . . . . . \\
\text { Average } . . . . .\end{array}$ & $\begin{array}{l}5,470 \\
1,094\end{array}$ & 1.0246 & 329.7 & 5,765 & 1.0227 & $\begin{array}{r}320.6 \\
64.1\end{array}$ & $\begin{array}{r}4,545 \\
909\end{array}$ & 1.0273 & 304.0 \\
\hline $\begin{array}{l}\text { Sixth subperiod: } \\
\text { Total }\end{array}$ & & & & & & & & & \\
\hline Average ......................... & $\begin{array}{l}5,965 \\
1,193\end{array}$ & $\begin{array}{l}1.0243 \\
\ldots \ldots \ldots\end{array}$ & $\begin{array}{r}355.2 \\
71.0\end{array}$ & $\begin{array}{l}5,170 \\
1,034\end{array}$ & $\begin{array}{l}1.0250 \\
\cdots \ldots \ldots\end{array}$ & $\begin{array}{r}316.7 \\
63.3\end{array}$ & $\begin{array}{r}4,480 \\
896\end{array}$ & \begin{tabular}{|l}
1.0263 \\
$\ldots \ldots \ldots$
\end{tabular} & $\begin{array}{r}288.7 \\
57.7\end{array}$ \\
\hline 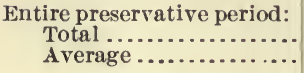 & $\begin{array}{r}31,005 \\
1,034\end{array}$ & 1.0266 & $\begin{array}{r}2,012.6 \\
67.1\end{array}$ & $\begin{array}{r}32,750 \\
1,092\end{array}$ & 1.0232 & $\begin{array}{r}1,853.6 \\
61.8\end{array}$ & $\begin{array}{r}26,268 \\
876\end{array}$ & 1.0268 & $\begin{array}{r}1,722.7 \\
57.4\end{array}$ \\
\hline After period. & & & & & & & & & \\
\hline 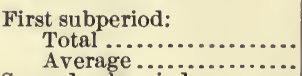 & $\begin{array}{l}5,445 \\
1,089\end{array}$ & $\begin{array}{l}1.0257 \\
\cdots \ldots . . .\end{array}$ & $\begin{array}{r}342.8 \\
68.6\end{array}$ & $\begin{array}{r}a 6,063 \\
1,213\end{array}$ & $\begin{array}{l}1.0210 \\
\ldots \ldots \ldots\end{array}$ & $\begin{array}{r}311.9 \\
62.4\end{array}$ & $\begin{array}{r}4,135 \\
827\end{array}$ & 1.0274 & $\begin{array}{r}277.6 \\
55.5\end{array}$ \\
\hline $\begin{array}{l}\text { Second subperiod: } \\
\text { Total ............. }\end{array}$ & 5,180 & 1.0280 & 355,3 & 5.095 & 1.0248 & 309.6 & 4,150 & & \\
\hline 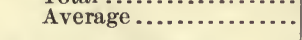 & 1,036 & & 71.1 & 1,019 & & 61.9 & 830 & 1.028. & $\begin{array}{r}28.6 \\
56.3\end{array}$ \\
\hline $\begin{array}{l}\text { Entire after period: } \\
\text { Total ............... } \\
\text { Average.......... }\end{array}$ & 10,625 & & 698.1 & 11,158 & & 621.5 & 8,285 & 1 & 559.2 \\
\hline & & 1.0209 & & & 1.0229 & 62.2 & & 1.026 & 5b. 9 \\
\hline
\end{tabular}

$a$ Four-day composites; average added in each case in order to complete five-day period. $b$ Average added in order to complete record. 
TABLE VIII.-Urine determinations-Volume, specific gravity, and total solids-Series $V I$-Continued.

[Averages are per day.]

\begin{tabular}{|c|c|c|c|c|c|c|c|c|c|}
\hline \multirow[b]{2}{*}{ Period. } & \multicolumn{3}{|c|}{ No. 7.} & \multicolumn{3}{|c|}{ No. 8.} & \multicolumn{3}{|c|}{ No. 9.} \\
\hline & $\begin{array}{l}\text { Vol- } \\
\text { ume. }\end{array}$ & $\begin{array}{c}\text { Specific } \\
\text { gravity } \\
\text { at } \\
25^{\circ} / 25^{\circ} \\
\text { C. }\end{array}$ & $\begin{array}{c}\text { Total } \\
\text { solids } \\
\text { (factor } \\
0.245 \text { ). }\end{array}$ & $\begin{array}{l}\text { Vol- } \\
\text { ume. }\end{array}$ & 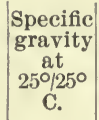 & $\begin{array}{l}\text { Total } \\
\text { solids } \\
\text { (factor } \\
0.245 \text { ). }\end{array}$ & $\begin{array}{l}\text { Vol- } \\
\text { ume. }\end{array}$ & $\begin{array}{c}\text { Specific } \\
\text { gravity } \\
\text { at } \\
25^{\circ} / 25^{\circ} \\
\text { C. }\end{array}$ & $\begin{array}{c}\text { Total } \\
\text { solids } \\
\text { (factor } \\
0.245 \text { ). }\end{array}$ \\
\hline \multicolumn{10}{|l|}{ Fore period. } \\
\hline $\begin{array}{l}\text { First subperiod: } \\
\text { Total ............ } \\
\text { Average........ }\end{array}$ & $\begin{array}{l}c c . \\
5,180 \\
1,036\end{array}$ & $\begin{array}{l}1.0228 \\
\cdots \ldots . . .\end{array}$ & $\begin{array}{r}\text { Grams. } \\
289.4 \\
57.9\end{array}$ & $\begin{array}{l}c c . \\
8,090 \\
1,618\end{array}$ & $\begin{array}{l}1.0128 \\
\cdots \ldots . .\end{array}$ & $\begin{array}{r}\text { Grams. } \\
253.7 \\
50.7\end{array}$ & $\begin{array}{l}c c . \\
5,515 \\
1,103\end{array}$ & $\begin{array}{l}1.0244 \\
\cdots . . .\end{array}$ & $\begin{array}{r}\text { Grams. } \\
329.7 \\
65.9\end{array}$ \\
\hline $\begin{array}{l}\text { Second subperiod: } \\
\text { Total .......... }\end{array}$ & 4,000 & 1.0295 & 289.1 & 10,925 & 1.0105 & 281.0 & 5.720 & 1.0238 & 333.5 \\
\hline 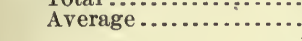 & 800 & 1.0290 & 57.8 & 2,185 & ......... & 56.2 & 1,144 & $\mid \begin{array}{ll}1.020 \\
\cdots\end{array}$ & 66.7 \\
\hline $\begin{array}{l}\text { Entire fore period: } \\
\quad \text { Total } \ldots \ldots \ldots \\
\text { Average } \ldots \ldots \\
\end{array}$ & $\begin{array}{r}9,180 \\
918\end{array}$ & 1.0262 & $\begin{array}{r}578.5 \\
57.9\end{array}$ & $\begin{array}{r}19,015 \\
1,902\end{array}$ & 1.0117 & $\begin{array}{r}534.7 \\
53.5\end{array}$ & $\begin{array}{r}11,235 \\
1,124\end{array}$ & 1.0241 & $\begin{array}{r}663.2 \\
66.3\end{array}$ \\
\hline \multicolumn{10}{|l|}{ Preservative period. } \\
\hline $\begin{array}{l}\text { First subperiod: } \\
\text { Total ....... }\end{array}$ & & & & & & & & & \\
\hline Average. & $\begin{array}{l}4,430 \\
886\end{array}$ & $\mid \begin{array}{c}1.0224 \\
\cdots \ldots \ldots\end{array}$ & $\begin{array}{r}243.1 \\
48.6\end{array}$ & $\begin{array}{l}9,300 \\
1,870\end{array}$ & 1.0113 & $\begin{array}{r}208.8 \\
51.8\end{array}$ & 1,152 & $\begin{array}{l}1.0264 \\
\ldots \ldots \ldots\end{array}$ & $\begin{array}{r}372.6 \\
74.5\end{array}$ \\
\hline $\begin{array}{l}\text { Second subp } \\
\text { Total ... }\end{array}$ & 5,340 & 1.0210 & 274.7 & 9,500 & 1.0119 & 277.0 & 5,580 & 1.0244 & 333.6 \\
\hline Ave & 1,068 & 1.0210 & 54.9 & 1,900 & 1.0110 & 55.4 & 1,116 & & 66.7 \\
\hline $\begin{array}{l}\text { Third su } \\
\text { Tota }\end{array}$ & 4. 960 & 1.0202 & 245.5 & 9.800 & & 2713 & & 10257 & $341 ?$ \\
\hline Aver & 992 & 1.0202 & 49.1 & 1,960 & 1.0110 & 54.3 & 084 & 1.0208 & $\begin{array}{r}68.2 \\
68.2\end{array}$ \\
\hline $\begin{array}{l}\text { Fourth } \\
\text { Tota }\end{array}$ & & & & & & & & & \\
\hline To & 5,560 & 1.0195 & 265.7 & a 8,800 & 1.0126 & 271.6 & 5,570 & 1.0268 & 365.7 \\
\hline Fifth sul & 112 & & & & & & & & 1 \\
\hline $\begin{array}{l}\text { Total } \\
\text { Averag }\end{array}$ & 4,820 & 1.0223 & 263.4 & 10,810 & 1.0114 & 301.9 & 5,275 & 1.0259 & 334.8 \\
\hline $\begin{array}{l}\text { Averag } \\
\text { Sixth subp }\end{array}$ & & .... & & & & & & & \\
\hline Total. & 6,495 & 1.0199 & 316.7 & 8,210 & 1.0146 & 293.7 & 6,775 & 1.0231 & 383.5 \\
\hline Average & 1,299 & $\cdots \ldots \ldots$ & 63.3 & 642 & . $\ldots \ldots \ldots$ & 58.7 & & ........... & 76.7 \\
\hline $\begin{array}{c}\text { Entire preservative period: } \\
\text { Total ................... }\end{array}$ & 31,605 & & $1,609.1$ & & & 1,674 & & & $2,131.4$ \\
\hline Average........... & 1,053 & 1.0209 & 53.6 & 1,882 & 1.0123 & & & 1.0254 & 71.0 \\
\hline After period. & & & & & & & & & \\
\hline $\begin{array}{l}\text { First sulpperiod: } \\
\text { Total ......... }\end{array}$ & 4,835 & 1.0205 & 242.8 & 9,720 & 1.0131 & 312.0 & 5,160 & 1.0268 & 338.8 \\
\hline $\begin{array}{l}\text { Av } \\
\text { econ }\end{array}$ & 967 & & 48.6 & 1,944 & & 62.4 & 1,032 & & 67.8 \\
\hline Total. & 4,745 & 1.0216 & 251.1 & 6,350 & 1.0186 & 289.4 & $a_{6}$, & 1.0243 & \\
\hline Average. & 949 & & 50.2 & 1,270 & & 57.9 & 1,279 & & 76.1 \\
\hline $\begin{array}{l}\text { Entire after period: } \\
\text { Total ............. }\end{array}$ & 9,580 & & 493.9 & 16,070 & & 601.4 & 11,554 & & 719.4 \\
\hline Average & 958 & 1.0211 & 49.4 & 1,607 & 1. 0159 & 60.1 & 1,155 & 1.0256 & 71.9 \\
\hline
\end{tabular}

$a$ Four-day composites; average added in each case in order to complete five-day record.

$7656-$ No. 84 , pt $2-06-5$ 
TABLE VIII.-Urine determinations-Volume, specific gravity, and total solids-Series $V I$-Continued.

[Averages are per day.]

\begin{tabular}{|c|c|c|c|c|c|c|c|c|c|}
\hline \multirow[b]{2}{*}{ Period. } & \multicolumn{3}{|c|}{ No. 10.} & \multicolumn{3}{|c|}{ No. 11.} & \multicolumn{3}{|c|}{ No. 12.} \\
\hline & $\begin{array}{l}\text { Vol- } \\
\text { ume. }\end{array}$ & $\begin{array}{c}\text { Specific } \\
\text { gravity } \\
\text { at } \\
25^{\circ} / 25^{\circ} \\
\text { C. }\end{array}$ & $\begin{array}{c}\text { Total } \\
\text { solids } \\
\text { (factor } \\
0.245 \text { ). }\end{array}$ & $\begin{array}{l}\text { Vol- } \\
\text { ume. }\end{array}$ & $\begin{array}{c}\text { Specific } \\
\text { gravity } \\
\text { at } \\
25^{\circ} / 25^{\circ} \\
\text { C. }\end{array}$ & $\begin{array}{c}\text { Total } \\
\text { solids } \\
\text { (factor } \\
0.245 \text { ). }\end{array}$ & $\begin{array}{l}\text { Vol- } \\
\text { ume. }\end{array}$ & $\begin{array}{c}\text { Specific } \\
\text { gravity } \\
\text { at } \\
25^{\circ} / 25^{\circ} \\
\text { C. }\end{array}$ & $\begin{array}{c}\text { Total } \\
\text { solids } \\
\text { (factor } \\
0.245 \text { ). }\end{array}$ \\
\hline \multicolumn{10}{|l|}{ Fore period. } \\
\hline $\begin{array}{l}\text { First subperiod: } \\
\text { Total ........... } \\
\text { Average ........ }\end{array}$ & $\begin{array}{l}c c . \\
4,240 \\
848\end{array}$ & $\begin{array}{l}1.0252 \\
\cdots \ldots . . .\end{array}$ & $\begin{array}{r}\text { Grams. } \\
261.8 \\
52.4\end{array}$ & $\begin{aligned} c c . \\
a 6,256 \\
1,251\end{aligned}$ & $\begin{array}{l}1.0234 \\
\ldots \ldots \ldots\end{array}$ & $\begin{array}{r}\text { Grams. } \\
358.6 \\
71.7\end{array}$ & $\begin{array}{l}c c . \\
6,180 \\
1,236\end{array}$ & $\begin{array}{l}1.0214 \\
\ldots . . . .\end{array}$ & $\begin{array}{r}\text { Grams. } \\
324.0 \\
64.8\end{array}$ \\
\hline Second subperiod: & & & & & ......... & & & & \\
\hline $\begin{array}{l}\text { Total } \ldots \ldots \ldots \\
\text { Average } \ldots \ldots\end{array}$ & $\begin{array}{r}4,640 \\
928\end{array}$ & 1.0240 & $\begin{array}{r}272.8 \\
54.6\end{array}$ & $\begin{array}{l}5,890 \\
1,178\end{array}$ & 1.0236 & $\begin{array}{r}340.6 \\
68.1\end{array}$ & 6,140 & 1.0204 & 306.9 \\
\hline & & & & & & & & & \\
\hline $\begin{array}{l}\text { Entire fore period: } \\
\text { Total ................... }\end{array}$ & 8,880 & & 534.6 & 12,146 & & 699.2 & 12,320 & & 630.9 \\
\hline Average ................ & 888 & 1.0246 & 53.5 & 1,215 & 1.0235 & 69.9 & 1,232 & 1. 0209 & 63.1 \\
\hline \multicolumn{10}{|l|}{ Preservative period. } \\
\hline \multicolumn{10}{|l|}{ First subperiod: } \\
\hline & 932 & & 52.2 & 1,160 & ......... & 74.8 & 954 & $1.02+2$ & 56.6 \\
\hline $\begin{array}{l}\text { Second subperiod: } \\
\text { Total .................. }\end{array}$ & & $10 ? 0 ?$ & & & & & & & \\
\hline Average......... & $\begin{array}{l}5,830 \\
1,166\end{array}$ & 1.0202 & 288.5 & 6,080 & 1.0238 & 354.5 & 5,170 & 1.0248 & 314.1 \\
\hline Third subperiod: & & & & 1,210 & & 10.9 & 1,034 & n....... & 62 \\
\hline $\begin{array}{l}\text { Total } \\
\text { Average } \ldots \ldots\end{array}$ & 5,955 & 1.0197 & 287.4 & 5,235 & 1.0256 & 328.3 & 5,840 & 1.0236 & 337.7 \\
\hline \multicolumn{10}{|l|}{ Fourth subperiod: } \\
\hline Total ........... & 5,330 & 1.0220 & 287.3 & 5,465 & 1.0254 & 340.1 & 5,410 & 1.0247 & 327.4 \\
\hline \multicolumn{10}{|l|}{ Fifth subperiod: } \\
\hline & 5,105 & 1.0222 & 277.7 & 5,330 & 1.0270 & 352.6 & 5,545 & 1.0250 & 339.6 \\
\hline $\begin{array}{l}\text { Average } \\
\text { Aixth }\end{array}$ & 1,021 & & 55.6 & 1,066 & ......... & 70.5 & 1,109 & & 67.9 \\
\hline $\begin{array}{l}\text { S1xth subpe } \\
\text { Total ... }\end{array}$ & 4,060 & $1: 0276$ & 274.5 & 5,575 & 1.0229 & 312.8 & 5,970 & 1.0244 & 356.9 \\
\hline Average ................ & 812 & (n....... & 54.9 & 1,115 & & 62.6 & 1,194 & ........ & 71.4 \\
\hline \multirow{3}{*}{$\begin{array}{r}\text { Entire preservative period: } \\
\text { Total } \ldots \ldots \\
\text { Average } \ldots \ldots \\
\end{array}$} & & & & & & & & & \\
\hline & 31,190 & ㅂ....... & $1,676.4$ & 33,485 & 부으. & $2,062.1$ & 32,705 & 10045 & $1,958.5$ \\
\hline & & 1.0222 & b.. 9 & 1,116 & 1.0252 & 68.7 & & 1.0245 & 60.3 \\
\hline \multicolumn{10}{|l|}{ After period. } \\
\hline \multicolumn{10}{|l|}{ First subperiod: } \\
\hline Total ............ & $\begin{array}{l}5,190 \\
1,038\end{array}$ & 1.0218 & $\begin{array}{r}277.2 \\
55.4\end{array}$ & $\begin{array}{l}5,765 \\
1,153\end{array}$ & 1.0228 & 322.0 & $\begin{array}{l}5,965 \\
1,193\end{array}$ & 1.0225 & 328.8 \\
\hline Second subperiod: & & & & & & & & & \\
\hline $\begin{array}{l}\text { Total... } \\
\text { Average }\end{array}$ & $\begin{array}{r}5,190 \\
\end{array}$ & 1.0218 & 277.2 & 5,630 & 1.0245 & 337.9 & 5,770 & 1. 0244 & 344.9 \\
\hline & & & & & & $\cdots$ & 1,104 & $\cdots$ & \\
\hline $\begin{array}{l}\text { Entire after period: } \\
\text { Total ............. }\end{array}$ & & & & & & & & & \\
\hline Average... & 1,038 & 1.0218 & 55.4 & 1,140 & 1.0247 & 66.0 & 1,174 & 1.0235 & 67.4 \\
\hline
\end{tabular}

$a$ Four-day composite; average added in order to complete five-day record. $b$ No. 10 out second after subperiod; average of first after subperiod used. 
TABLE VIII.-Urine determinations-Volume, specific gravity, and total solids, Series $V I$-Continued.

[Averages are per day.]

SUMMARY FOR NINE MEN.

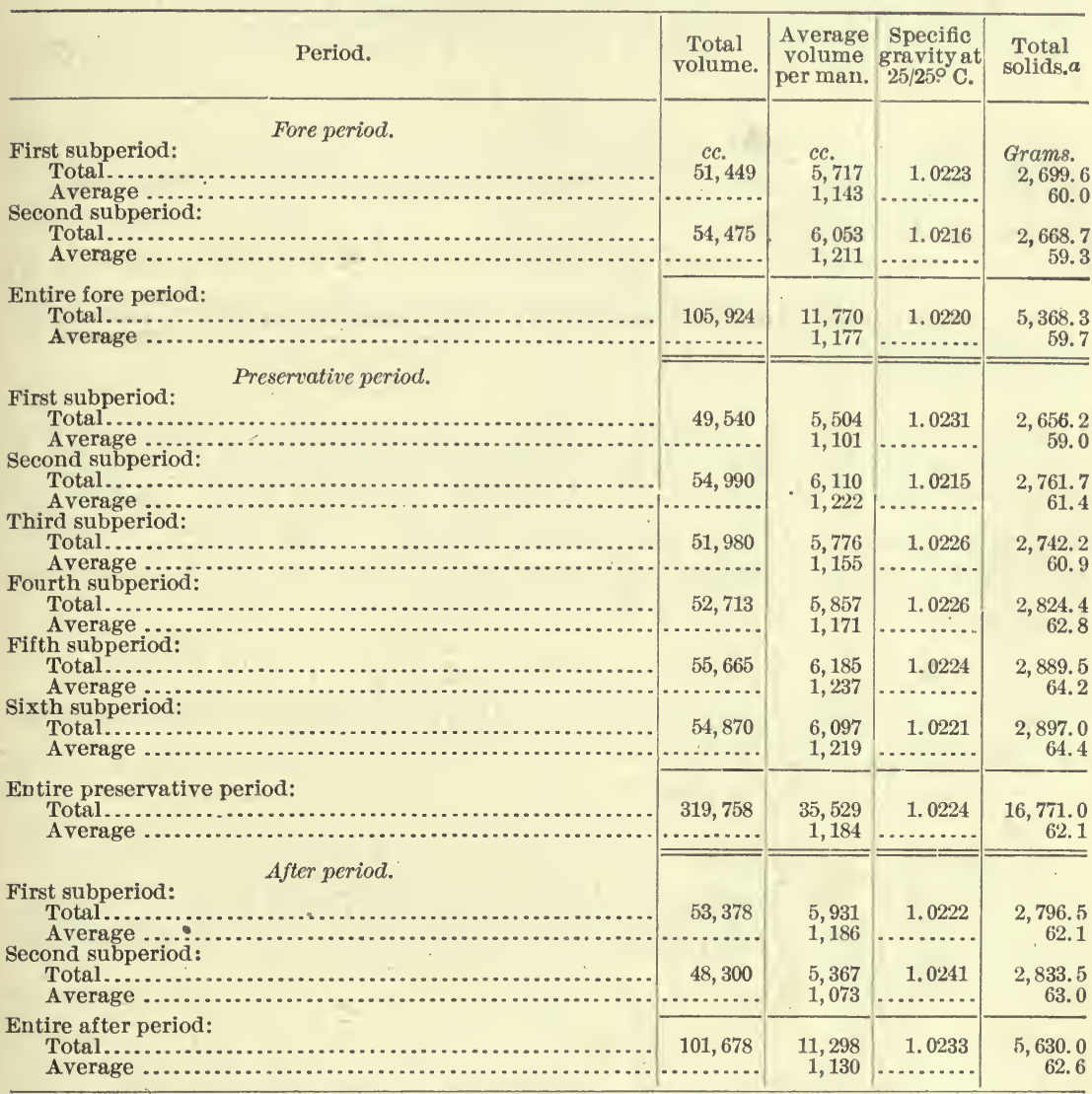

$a$ Average figures in this eolumn show the averages per man per day.

PRESENCE OF ALBUMIN AND THE REACTION OF THE URINE.

The urine was also examined for albumin, and its reaction was determined from time to time during the progress of the experiment. The quantity of albumin present at each test was not estimated, the examination being confined to ascertaining its presence. In Series VI, therefore, a comparison as to the relative quantity of albumin present in the three periods can not be made, as was done in Part I in the case of boric acid. The degree of acidity was determined in terms of standard alkali.

No. 1.

There is no albumin in the urine of No. 1 at the beginning of the fore period, but at the end there is a minute trace. There is no appearance of albumin during the preservative period. The reaction of the urine in this case is amphoteric in the fore period and was not determined in the other periods. 
No. 2.

A trace of albumin occurs in the urine of No. 2 during the whole period of observation. The reaction of the urine is amphoteric in the fore period, and it becomes acid in the preservative period.

No. 3.

No albumin is found in the urine of No. 3 during any period of observation. The acidity of the urine increases during the administration of the salicylic acid.

\section{No. 4 .}

The observations in the case of No. 4 are practically the same as in the case of No. 3 .

No. 5 .

There appear's to be no definite change produced in the case of No. 5 by the action of the salicylic acid either upon the small quantity of albumin present or upon the relative acidity. The urine is strongly acid during the fore period, and also strongly acid during the preservative period.

No. 6 .

The data in the case of No. 6 indicate practically the same absence of effect as those of No. 5 .

No. $\%$.

No albumin is observed in the case of No. 7 at any time during the observation. The reaction of the urine is alternately acid and amphoteric, and the preservative appears to have had no influence in determining the condition.

No. 8 .

No albumin appears in the case of the urine of No. 8 at any time. The reaction of the urine under the influence of the preservative becomes amphoteric. In this case there appears to have been a very slight influence on the part of the drug administered to diminish the acidity of the urine and to produce an amphoteric condition thereof.

No. 9 .

No albumin is present in the urine at any time of the observation. The reaction of the urine is amphoteric at one time during the fore period, but acid the rest of the time.

\section{No. 10.}

No albumin is found in this case at any time. During the administration of the salicylic acid the reaction of the urine changed from amphoteric to strongly acid. The apparent effect in this case is exactly the opposite of that produced in the case of No. 8 . 
No. 11 .

A very minute quantity of albumin is noticed in the urine during the administration of the salicylic acid. The reaction of the urine changed from amphoteric in the fore period to acid during the preservative period.

No. 12 .

No albumin occurs in the urine during the periods of observation. The reaction of the urine is changed from amphoteric in the fore period to strongly acid in the second preservative subperiod, becoming amphoteric again in the third preservative subperiod.

In so far as these data are concerned no effect upon the presence of albumin nor upon the relative acidity can be attributed to the administration of the salicylic acid. A more detailed study of these points, leading to more definite results, is to be found under Series XI, page 726 .

RATIO OF SULPHUR, SULPHATES, AND PHOSPHORIC ACID TO NITROGEN EXCRETED IN THE URINE.

In Table IX are found the data showing the ratio of sulphur, sulphates, and phosphates excreted to the nitrogen in the urine. The total weight of nitrogen excreted, the total weight of sulphur, the total weight of sulphur as $\mathrm{SO}_{3}$, and the total weight of phosphorus as $\mathrm{P}_{2} \mathrm{O}_{5}$ served as a basis for determining the ratios.

The object of this study was to ascertain whether or not the administration of salicylic acid changed in any definite way the ratio of these bodies to the total nitrogen excreted. When the ratio increases it shows that there is a loss in the quantity of the substance under consideration excreted in relation to the total nitrogen, and when the ratio decreases it shows that there is an increased quantity of that substance in relation to the total nitrogen.

INDIVIDUAL DATA.

No. 1 .

The data show a slight decrease in the ratio of sulphur to nitrogen and of sulphuric acid to nitrogen during the administration of the preservative, while the ratio of the phosphoric acid to the nitrogen remains unchanged. In the after period there is a still further decrease in the ratio of sulphur to nitrogen, while the ratio of sulphuric acid to nitrogen is restored to its original magnitude. There is a notable increase in the ratio of the phosphoric acid to nitrogen in the after period.

No. 2.

There is a marked decrease in the magnitude of the ratio of sulphur to nitrogen and of phosphoric acid to nitrogen in the preservative 
period, while the ratio of sulphuric acid to nitrogen remains unchanged. In the after period there is a still further slight decrease in the ratio of the sulphur to the nitrogen, while the ratio of phosphoric acid to nitrogen rises to a greater magnitude than in the fore period. The ratio of sulphuric acid to nitrogen remains unchanged.

No. 3.

The ratio of sulphur to nitrogen in the preservative period is the same as in the fore period. The ratio of sulphuric acid to nitrogen is the same in the preservative period as in the fore period. There is a slight decrease in the preservative period in the ratio of phosphoric acid to nitrogen. In the after period there is a notable decrease in the ratio of sulphur to nitrogen and a slight decrease in the ratio of sulphuric acid to nitrogen, and the ratio of phosphoric acid to nitrogen is higher than in the preservative period, but not so high as in the fore period. The fore period was, however, interrupted by the illness of the subject.

$$
\text { No. } 4 \text {. }
$$

A slight increase is noted in the ratio of sulphur and sulphuric acid in the preservative period and a slight decrease in the ratio of phosphoric acid. There is a further decrease in the sulphur and sulphuricacid ratios in the after period. There is, on the other hand, an increase in the phosphoric-acid ratio in this period.

No. 5 .

A notable decrease in the sulphur ratio is found in this case in the preservative period, while the ratios of sulphuric acid and phosphoric acid remain unchanged. In the after period there is a still further slight decrease in the sulphur ratio, no change in the sulphurı-acid ratio, and a notable increase in the phosphoric-acid ratio.

$$
\text { No. } 6 \text {. }
$$

A slight decrease of the sulphur and sulphuric-acid ratios is noticed in this case in the preservative period and a slight increase in the phosphoric-acid ratio. In the after period there is a notable decrease in the sulphur ratio, a still further slight decrease in the sulphuricacid ratio, and a slight increase in the phosphoric-acid ratio.

No. $\%$.

A notable decrease in the sulphur ratio is indicated in this case in the preservative period, a slight decrease in the sulphuric-acid ratio, and a heavy increase in the phosphoric-acid ratio. In the after period the sulphur ratio is restored in part to its original magnitude and the sulphuric-acid ratio exactly to its original magnitude. There is a very notable increase in the magnitude of the phosphoric-acid ratio. 
No. 8 .

There is noticed here for the first time a decided increase in the sulphur ratio in the preservative period, while the sulphuric-acid and phosphoric-acid ratios remain unchanged. In the after period there is a slight falling off in the sulphur ratio as compared with the preservative period, no change in the sulphuric-acid ratio, and a notable increase in the phosphoric-acid ratio.

No. 9.

There is a slight diminution in the magnitude of the sulphur and sulphuric-acid ratios in this case and a notable loss in the phosphoricacid ratio during the preservative period. In the after period the sulphur ratio is increased beyond its original magnitude, the sulphüric-acid ratio is restored to the figure for the fore period, and there is an increase in the magnitude of the phosphoric-acid ratio over the preservative period, but it does not quite reach the magnitude of the fore period.

No. 10 .

There is no change in the sulphur and sulphuric-acid ratios in the preservative period in this case and a slight diminution in the magnitude of the phosphoric-acid ratio. In the after period there is a slight loss in the magnitude of the sulphur and sulphuric-acid ratios and a notable increase in the phosphoric-acid ratio.

No. 11 .

The ratios of sulphur and sulphuric acid are smaller in this case in the preservative period and that of phosphoric acid larger. In the after period there is a still further diminution in the magnitude of the sulphur ratio over the preservative period, no change in that of the sulphuric acid, and a still further slight increase of ratio of the phosphoric acid.

No. 12 .

We have here the third instance of an increase in the magnitude of the sulphur. ratio in the preservative period, accompanied with a slight decrease in that of the sulphuric-acid ratio and the phosphoric-acid ratio. In the after period the sulphur ratio is slightly diminished as compared with that of the preservative period, the sulphuric-acid ratio remains unchanged, and there is a notable increase in the magnitude of the phosphoric-acid ratio.

SUMMARY FOR NINE MEN.

Combining into one expression the nine ratios which are complete and comparable, it is seen that there is a notable diminution in the ratio of sulphur and a slight diminution in the ratio of sulphuric acid 
to nitrogen in the preservative period. The ratio of phosphoric acid to nitrogen remains unchanged. The tendency to reduce the magnitude of the ratio of sulphur to nitrogen is maintained in the after period, whereas there is no further diminution of the magnitude of the ratio of sulphuric acid in that period. The ratio of the phosphoric acid, however, is very markedly increased in the after period. A general review of these data indicates that the administration of the salicylic acid tended to increase the relative excretion of sulphur to nitrogen, and that this tendency was continued in quite a marked degree in the after period. There is scarcely any effect produced by the salicylic acid upon the relative quantities of sulphuric acid and nitrogen excreted. There seems to be a marked tendency on the part of the preservative to produce a condition which diminishes the relative amount of phosphoric acid to nitrogen excreted, but this full effect is not shown until the after period.

In general, it may be said that the disturbing influence upon the relative quantities of these bodies excreted as compared with nitrogen is not very great, but that there is a marked tendency to disturb in a measurable degree the relative metabolic changes which the sulphur undergoes in relation to nitrogen as determined by the constitution of the urine. 
TABLE IX.-Urine determinations-Ratio of sulphur, sulphates, and phosphates to nitrogen-Series V1.

[Averages are per day.]

No. 1.

\begin{tabular}{|c|c|c|c|c|c|c|c|}
\hline \multirow{2}{*}{ Period. } & \multicolumn{4}{|c|}{ Quantity. } & \multicolumn{3}{|c|}{ Ratio. } \\
\hline & Nitrogen. & Sulphur. & $\mathrm{SO}_{3}$ & $\mathrm{P}_{2} \mathrm{O}_{5}$ & $\mathrm{~S}: \mathrm{N}$ & $\mathrm{SO}_{3}: \mathrm{N}$ & $\mathrm{P}_{2} \mathrm{O}_{5}: \mathrm{N}$ \\
\hline \multicolumn{8}{|l|}{ Fore period. } \\
\hline $\begin{array}{l}\text { First subperiod: } \\
\quad \text { Total } \ldots \ldots \ldots \ldots \ldots \ldots \\
\text { A verage } . \ldots \ldots\end{array}$ & $\begin{array}{r}\text { Grams. } \\
59.83 \\
11.97\end{array}$ & $\begin{array}{r}\text { Grams. } \\
4.002 \\
.800\end{array}$ & $\begin{array}{r}\text { Grams. } \\
8.674 \\
1.735\end{array}$ & $\begin{array}{r}\text { Gram.8. } \\
10.723 \\
2.145\end{array}$ & $\begin{array}{l}1: 15.0 \\
-\ldots . . .\end{array}$ & $1: 6.9$ & $\begin{array}{l}1: 5.6 \\
\cdots \cdots\end{array}$ \\
\hline 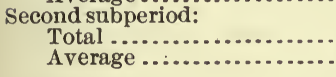 & $\begin{array}{l}67.99 \\
13.60\end{array}$ & $\begin{array}{r}4.252 \\
.850\end{array}$ & $\begin{array}{l}9.800 \\
1.960\end{array}$ & $\begin{array}{r}10.963 \\
2.193\end{array}$ & $\begin{aligned} 1: 16.0 \\
\cdots\end{aligned}$ & $\begin{array}{r}1: 6.9 \\
\cdots\end{array}$ & $\begin{array}{r}1: 6.2 \\
\cdots\end{array}$ \\
\hline 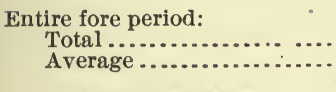 & $\begin{array}{r}127.82 \\
12.78\end{array}$ & $\begin{array}{r}8.254 \\
.825 \\
\end{array}$ & $\begin{array}{r}18.474 \\
-1.847 \\
\end{array}$ & $\begin{array}{r}21.686 \\
2.169 \\
\end{array}$ & $\begin{array}{r}1: 15.5 \\
\cdots \cdots \\
\end{array}$ & $\begin{array}{r}1: 6.9 \\
\cdots\end{array}$ & $\begin{array}{r}1: 5.9 \\
\cdots \cdots\end{array}$ \\
\hline \multicolumn{8}{|l|}{ Preservative period. } \\
\hline 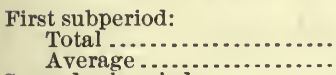 & $\begin{array}{l}66.40 \\
13.28\end{array}$ & $\begin{array}{r}4.415 \\
.883\end{array}$ & $\begin{array}{l}9.800 \\
1.960\end{array}$ & $\begin{array}{r}11.271 \\
2.254\end{array}$ & $\begin{aligned} 1: 15.0 \\
\cdots\end{aligned}$ & $1: 6.8$ & $\begin{array}{l}1: 5.9 \\
\ldots \ldots .\end{array}$ \\
\hline $\begin{array}{l}\text { Second subperiod: } \\
\text { Total } \ldots \ldots \ldots \ldots \ldots \ldots\end{array}$ & 69.68 & 4.654 & 10.239 & 12.343 & $1: 15.0$ & $1: 6.8$ & $1: 5.6$ \\
\hline Average ........ & 13.94 & .931 & 2.048 & 2.469 & & & 20.0 \\
\hline 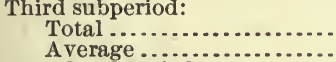 & $\begin{array}{r}a 68.95 \\
13.79\end{array}$ & $\begin{array}{r}4.376 \\
.875\end{array}$ & $\begin{array}{l}9.974 \\
1.995\end{array}$ & $\begin{array}{r}11.596 \\
2.319\end{array}$ & $1: 15.8$ & $1: 6.9$ & $1: 5.9$ \\
\hline Fourth subperiod: & & & & & & & ..... \\
\hline Average & $\begin{array}{l}68.55 \\
13.71\end{array}$ & $\begin{array}{r}4.720 \\
.944\end{array}$ & $\begin{array}{r}10.342 \\
2.068\end{array}$ & $\begin{array}{r}12.216 \\
2.443\end{array}$ & $\begin{array}{l}1: 14.5 \\
.\end{array}$ & $\begin{array}{l}1: 6.6 \\
\cdots . . . .\end{array}$ & $\begin{array}{l}1: 5.6 \\
\ldots \ldots\end{array}$ \\
\hline $\begin{array}{l}\text { Fifth subperiod: } \\
\quad \text { Total } \ldots \ldots \ldots \\
\text { Average }\end{array}$ & 71.33 & 4.429 & 10.539 & 11. 737 & $1: 16.1$ & $1: 6.8$ & $1: 6.1$ \\
\hline $\begin{array}{l}\text { Average } \\
\text { Sixth subperiod: }\end{array}$ & 14.27 & .886 & 2.108 & 2.347 & & & . \\
\hline $\begin{array}{l}\text { Total } \\
\text { Average } . \ldots \ldots \\
.\end{array}$ & $\begin{array}{l}74.07 \\
14.81\end{array}$ & $\begin{array}{r}4.621 \\
.924\end{array}$ & $\begin{array}{r}10.502 \\
2.100\end{array}$ & $\begin{array}{r}11.345 \\
2.269\end{array}$ & $\begin{array}{r}1: 16.0 \\
\cdots\end{array}$ & $\begin{array}{l}1: 7.1 \\
\cdots \cdots\end{array}$ & $\begin{array}{l}1: 6.5 \\
\cdots \cdots\end{array}$ \\
\hline $\begin{array}{l}\text { Entire preservative period: } \\
\text { Total } \ldots . . . \\
\text { A.verage } \ldots \ldots \\
\end{array}$ & $\begin{array}{r}418.98 \\
13.97\end{array}$ & $\begin{array}{r}27.215 \\
.907 \\
\end{array}$ & $\begin{array}{r}61.396 \\
2.047 \\
\end{array}$ & $\begin{array}{r}70.508 \\
2.350\end{array}$ & $\begin{array}{r}1: 15.4 \\
\cdots\end{array}$ & $\begin{array}{l}1: 6.8 \\
\cdots \cdots\end{array}$ & $\begin{array}{l}1: 5.9 \\
\cdots \cdots\end{array}$ \\
\hline After period. & & & & & & & \\
\hline 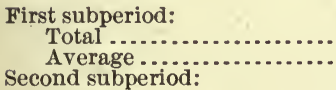 & $\begin{array}{l}69.61 \\
13.92\end{array}$ & $\begin{array}{r}4.580 \\
.916\end{array}$ & $\begin{array}{r}10.150 \\
2.030\end{array}$ & $\begin{array}{r}10.418 \\
2.084\end{array}$ & $\begin{array}{r}1: 15.2 \\
\ldots \ldots\end{array}$ & $\begin{array}{l}1: 6.9 \\
\cdots\end{array}$ & $\begin{array}{l}1: 6.7 \\
\cdots\end{array}$ \\
\hline 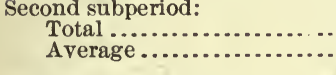 & $\begin{array}{l}70.86 \\
14.17\end{array}$ & $\begin{array}{r}4.771 \\
.954\end{array}$ & $\begin{array}{r}10.279 \\
2.056\end{array}$ & $\begin{array}{r}10.924 \\
2.185\end{array}$ & $\begin{array}{r}1: 14.9 \\
\ldots \ldots\end{array}$ & $\begin{array}{c}1: 6.9 \\
\ldots . . .\end{array}$ & $\begin{array}{c}1: 6.5 \\
\ldots \ldots .\end{array}$ \\
\hline $\begin{array}{l}\text { Entire after period: } \\
\quad \text { Total ........................ } \\
\text { Average................. }\end{array}$ & $\begin{array}{r}140.47 \\
14.05\end{array}$ & $\begin{array}{r}9.351 \\
.935\end{array}$ & $\begin{array}{r}20.429 \\
2.043\end{array}$ & $\begin{array}{r}21.342 \\
2.134\end{array}$ & $\begin{array}{r}1: 15.0 \\
\cdots\end{array}$ & $\begin{array}{c}1: 6.9 \\
\ldots \ldots \ldots\end{array}$ & $\begin{array}{c}1: 6.6 \\
\cdots \ldots .\end{array}$ \\
\hline
\end{tabular}


TABLE IX.-Urine determinations-Ratio of sulphur, sulphates, and phosphates to nitrogen-Series VI-Continued.

[Averages are per day.]

No. 2 .

\begin{tabular}{|c|c|c|c|c|c|c|c|}
\hline \multirow{2}{*}{ Period. } & \multicolumn{4}{|c|}{ Quantity. } & \multicolumn{3}{|c|}{ Ratio. } \\
\hline & Nitrogen. & Sulphur. & $\mathrm{SO}_{3}$ & $\mathrm{P}_{2} \mathrm{O}_{5}$ & $\mathrm{~S}: \mathrm{N}$ & $\mathrm{SO}_{3}: \mathrm{N}$. & $\mathrm{P}_{2} \mathrm{O}_{5}: \mathrm{N}$ \\
\hline \multicolumn{8}{|l|}{ Fore period. } \\
\hline $\begin{array}{l}\text { First subperiod: } \\
\quad \text { Total } \ldots \ldots \ldots \ldots \ldots \ldots \ldots \\
\text { Average.................... }\end{array}$ & $\begin{array}{r}\text { Grams. } \\
85.34 \\
17.07\end{array}$ & $\begin{array}{r}\text { Grams. } \\
5.448 \\
1.090\end{array}$ & $\begin{array}{r}\text { Grams. } \\
12.016 \\
2.408\end{array}$ & $\begin{array}{r}\text { Grams. } \\
16.025 \\
3.205\end{array}$ & $1: 15.7$ & $1: 7.1$ & $1: 5.3$ \\
\hline $\begin{array}{l}\text { Second subperiod: } \\
\text { Total }\end{array}$ & & & & & & & \\
\hline 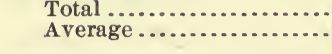 & $\begin{array}{l}72.37 \\
14.47\end{array}$ & $\begin{array}{r}4.643 \\
.929 \\
\end{array}$ & $\begin{array}{r}10.454 \\
2.091\end{array}$ & $\begin{array}{r}14.267 \\
2.853\end{array}$ & $\begin{array}{c}1: 15.6 \\
\cdots\end{array}$ & $\begin{array}{r}1: 6.9 \\
\cdots\end{array}$ & $\begin{array}{l}1: 5.1 \\
\cdots\end{array}$ \\
\hline 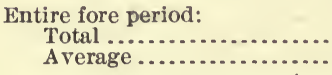 & $\begin{array}{r}157.71 \\
15.77\end{array}$ & $\begin{array}{r}10.091 \\
1.009\end{array}$ & $\begin{array}{r}22.470 \\
2.247\end{array}$ & $\begin{array}{r}30.292 \\
3.029\end{array}$ & $\begin{array}{l}1: 15.6 \\
\ldots \ldots\end{array}$ & $\begin{array}{c}1: 7.0 \\
\cdots \cdots\end{array}$ & $\begin{array}{l}1: 5.2 \\
\cdots\end{array}$ \\
\hline \multicolumn{8}{|l|}{ Preservative period. } \\
\hline $\begin{array}{l}\text { First subperiod: } \\
\text { Total } \ldots . . . \ldots \ldots \ldots \\
\text { Average ......... }\end{array}$ & $\begin{array}{l}71.87 \\
14.37\end{array}$ & $\begin{array}{r}4.621 \\
.924\end{array}$ & 9.971 & 14. 714 & $1: 15.6$ & $1: 7.2$ & $1: 4.9$ \\
\hline Second subperiod: & & & & & & (n). & ....... \\
\hline 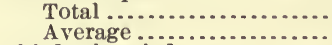 & 72.49 & 4. 517 & 10.181 & 16. 061 & $1: 16.0$ & $1: 7.1$ & $1: 4.5$ \\
\hline \multicolumn{8}{|l|}{ Third subperiod: } \\
\hline 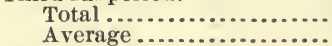 & 77.12 & 5.108 & 10.835 & 15. 734 & $1: 15.1$ & $1: 7.1$ & $1: 4.9$ \\
\hline $\begin{array}{l}\text { Average } \\
\text { Fourth subperiod: }\end{array}$ & & .022 & 167 & 3.147 & & $\cdots \cdot \cdots \cdot$ & $\therefore \cdots$ \\
\hline Total ................... & 71.30 & 4.516 & 10.940 & 14. 903 & $1: 15.8$ & $1: 6.5$ & $1: 4.8$ \\
\hline \multirow{2}{*}{\multicolumn{8}{|c|}{$\begin{array}{l}\text { Fifth subperiod: } \\
\text { Total } . . . . . . . .\end{array}$}} \\
\hline & 82.10 & 5.212 & 11. 596 & 15. 979 & $1: 15.8$ & $1: 7.1$ & $1: 5.1$ \\
\hline \multicolumn{8}{|l|}{$\begin{array}{l}\text { Sixth subperiod: } \\
\text { Total }\end{array}$} \\
\hline 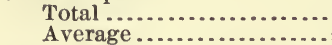 & 76.80 & 5.574 & 11.092 & 14. 271 & $1: 13.8$ & $1: 6.9$ & $1: 5.4$ \\
\hline Average & & & & & & .... & \\
\hline Entire preservative period: & 451.68 & 29.548 & 64.615 & 91. 662 & $1: 15.3$ & $1: 7.0$ & $1: 4.9$ \\
\hline Average ...................... & & .985 & 2.154 & 3.055 & & ......... & \\
\hline \multicolumn{8}{|l|}{ After period. } \\
\hline \multicolumn{8}{|l|}{ First subperiod: } \\
\hline $\begin{array}{l}\text { Total } \ldots . . . \ldots \ldots \\
\text { Average } . . . . \ldots \ldots\end{array}$ & $\begin{array}{l}77.92 \\
15.58\end{array}$ & $\begin{array}{l}5.123 \\
1.025\end{array}$ & $\begin{array}{r}11.170 \\
2.234\end{array}$ & $\begin{array}{r}14.075 \\
2.815\end{array}$ & $1: 15.2$ & $1: 7.0$ & $1: 5.5$ \\
\hline \multicolumn{8}{|l|}{$\begin{array}{l}\text { Second subperiod: } \\
\text { Total }\end{array}$} \\
\hline Total $\ldots \ldots \ldots$ & & 5.268 & 11.360 & 14.986 & $1: 15.2$ & $1: 7.0$ & $1: 5.3$ \\
\hline Average........... & 15.99 & 1.054 & 2.272 & 2.997 & & …... & …... \\
\hline $\begin{array}{l}\text { Entire after period: } \\
\text { Total ............... }\end{array}$ & 157.89 & 10. 391 & 22.530 & 29.061 & $1: 15.2$ & $1: 7.0$ & $1: 5.4$ \\
\hline Average........ & 15. 79 & 1.039 & 2.253 & 2.906 & & & \\
\hline
\end{tabular}


TABLE IX.-Urine determinations-Ratio of sulphur, sulphates, and phosphates to nitrogen-Series VI-Continued.

[Averages are per day.]

No. 3.

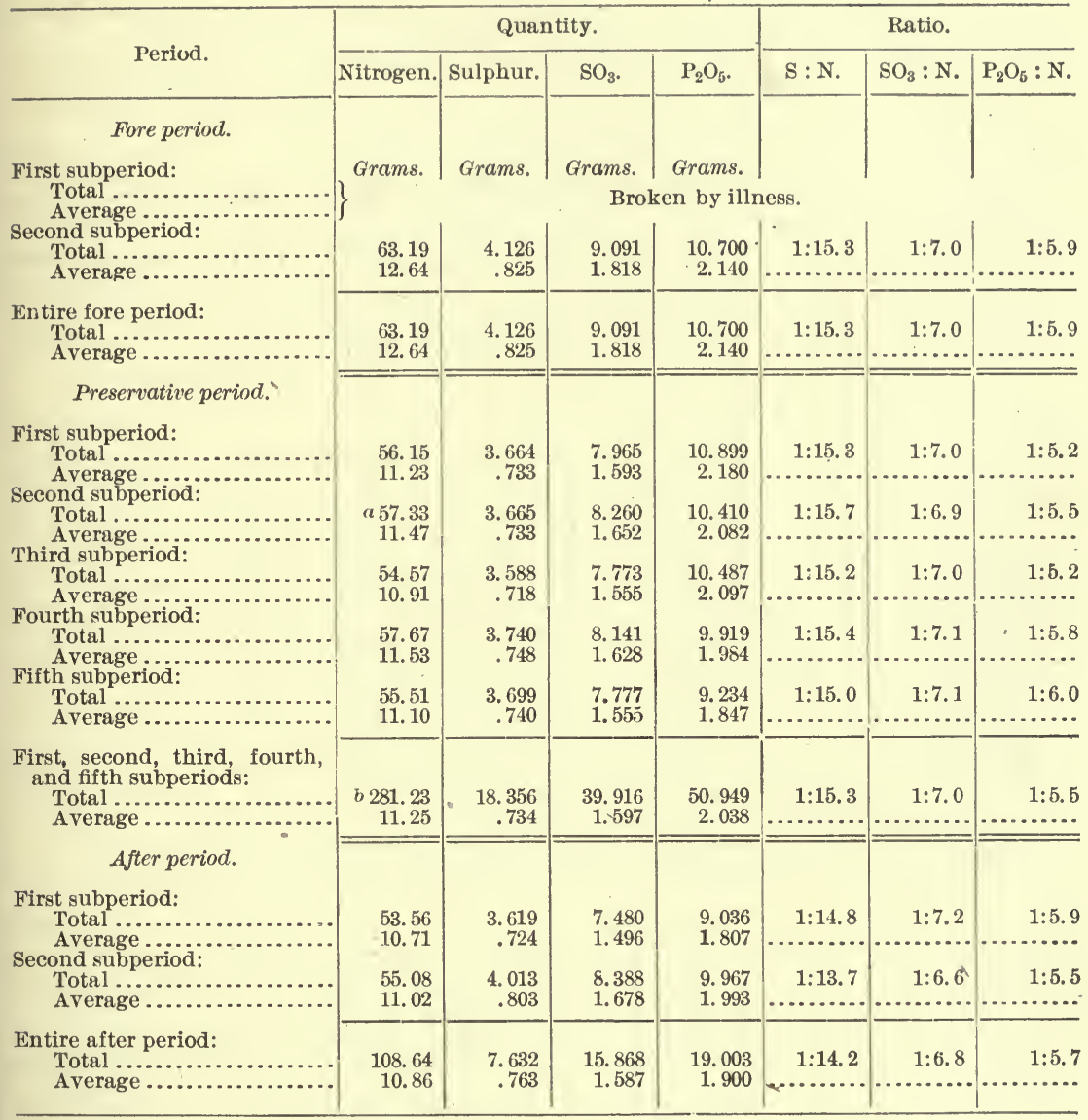

$a$ One day's average added in order to complete record.

$b$ No. 3 had only five preservative subperiods. 
TABLE IX.-Urine determinations-Ratio of sulphur, sulphates, and phosphates to nitrogen-Series VI-Continued.

[Averages are per day.]

No. 4.

\begin{tabular}{|c|c|c|c|c|c|c|c|}
\hline \multirow{2}{*}{ Period. } & \multicolumn{4}{|c|}{ Quantity. } & \multicolumn{3}{|c|}{ Ratio. } \\
\hline & Nitrogen. & Sulphur. & $\mathrm{SO}_{3}$. & $\mathrm{P}_{2} \mathrm{O}_{5}$ & $\mathrm{~S}: \mathrm{N}$. & $\mathrm{SO}_{3}: \mathrm{N}$ & $\mathrm{P}_{2} \mathrm{O}_{5}: \mathrm{N}$ \\
\hline Fore period. & & & & & & & \\
\hline 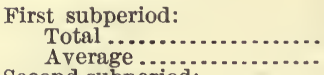 & $\begin{array}{r}\text { Grams. } \\
73.78 \\
14.76\end{array}$ & $\begin{array}{r}\text { Grams. } \\
\quad 4.905 \\
\quad .981\end{array}$ & $\begin{array}{r}\text { Grams. } \\
10.719 \\
2.144\end{array}$ & $\begin{array}{l}\text { Grams. } \\
12.787 \\
2.557\end{array}$ & 1:15. 0 & $1: 6.9$ & $1: 5.8$ \\
\hline $\begin{array}{l}\text { Second subperiod: } \\
\quad \text { Total ....................... } \\
\text { Average........................... }\end{array}$ & $\begin{array}{l}71.94 \\
14.39\end{array}$ & $\begin{array}{r}4.574 \\
.915 \\
\end{array}$ & $\begin{array}{r}10.724 \\
2.145\end{array}$ & $\begin{array}{r}14.505 \\
2.901\end{array}$ & $1: 15.7$ & $1: 6.7$ & $1: 5.0$ \\
\hline $\begin{array}{l}\text { Entire fore period: } \\
\quad \text { Total ............... } \\
\quad \text { Average ......... }\end{array}$ & $\begin{array}{r}145.72 \\
14.57 \\
\end{array}$ & $\begin{array}{r}9.479 \\
.948 \\
\end{array}$ & $\begin{array}{r}21.443 \\
2.144 \\
\end{array}$ & $\begin{array}{r}27.292 \\
2.729 \\
\end{array}$ & $\begin{array}{r}1: 15.4 \\
\cdots \cdots \cdots\end{array}$ & $\begin{array}{l}1: 6.8 \\
\cdots \ldots . .\end{array}$ & $\begin{array}{l}1: 5.3 \\
\cdots . . .\end{array}$ \\
\hline Preservative period. & & & & & & & \\
\hline $\begin{array}{l}\text { First subperiod: } \\
\text { Total .............. } \\
\text { Average....... } \\
\text { Second subperiod: }\end{array}$ & $\begin{array}{l}70.07 \\
14.01\end{array}$ & $\begin{array}{r}4.444 \\
.889\end{array}$ & $\begin{array}{r}10.268 \\
2.054\end{array}$ & $\begin{array}{r}13.843 \\
2.769\end{array}$ & 1:15.8 & $1: 6.8$ & 1:5.1 \\
\hline $\begin{array}{l}\text { Second subperiod: } \\
\text { Total .............. } \\
\text { Average ........ }\end{array}$ & $\begin{array}{l}74.59 \\
14.92\end{array}$ & $\begin{array}{r}4.724 \\
.945\end{array}$ & $\begin{array}{r}10.675 \\
2.135\end{array}$ & $\begin{array}{r}15.277 \\
3.055\end{array}$ & $1: 15.8$ & $1: 7.0$ & 1:4.9 \\
\hline $\begin{array}{l}\text { Third subperiod: } \\
\text { Total ............... }\end{array}$ & 14.92 & 4.548 & 10.267 & 0.050 & $1 \cdot 151$ & 1.67 & 1.50 \\
\hline $\begin{array}{l}\text { Average ........ } \\
\text { Fourth subperiod: }\end{array}$ & 13.70 & .910 & 2.053 & 2.728 & $1: 10.1$ & 1.0 .6 & $1: 5.0$ \\
\hline 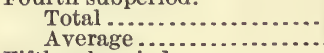 & $\begin{array}{l}72.07 \\
14.41\end{array}$ & $\begin{array}{r}4.559 \\
.912\end{array}$ & $\begin{array}{r}10.224 \\
2.045\end{array}$ & $\begin{array}{r}14.891 \\
2.978\end{array}$ & $1: 15.8$ & $1: 7.0$ & $1: 4.8$ \\
\hline $\begin{array}{l}\text { Fifth subperiod: } \\
\text { Total........... } \\
\text { Average....... }\end{array}$ & $\begin{array}{l}66.08 \\
13.22\end{array}$ & $\begin{array}{r}4.299 \\
.860\end{array}$ & $\begin{array}{l}9.726 \\
1.945\end{array}$ & $\begin{array}{r}13.402 \\
2.680\end{array}$ & 1:15. 4 & $1: 6.8$ & 1:4.9 \\
\hline 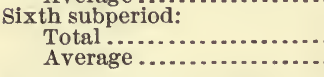 & $\begin{array}{l}71.94 \\
14.39\end{array}$ & $\begin{array}{r}4.766 \\
.953\end{array}$ & $\begin{array}{r}10.391 \\
2.078\end{array}$ & $\begin{array}{r}13.839 \\
2.768\end{array}$ & $1: 15.1$ & $1: 6.9$ & $\begin{array}{l}1: 5.2 \\
\cdots \cdots\end{array}$ \\
\hline 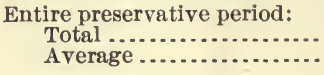 & $\begin{array}{r}423.25 \\
14.11 \\
\end{array}$ & $\begin{array}{r}27.340 \\
.911\end{array}$ & $\begin{array}{r}61.551 \\
2.052\end{array}$ & $\begin{array}{r}84.891 \\
2.830\end{array}$ & 1:15. 5 & $1: 6.9$ & $1: 5.0$ \\
\hline After period. & & & & & & & \\
\hline $\begin{array}{l}\text { First subperiod: } \\
\text { Total ............... } \\
\text { Average ......... }\end{array}$ & $\begin{array}{l}72.04 \\
14.41\end{array}$ & $\begin{array}{r}4.737 \\
.947\end{array}$ & $\begin{array}{r}10.618 \\
2.124\end{array}$ & $\begin{array}{r}12.959 \\
2.592\end{array}$ & 1:15. 2 & 1:6. 8 . & $1: 5.6$ \\
\hline 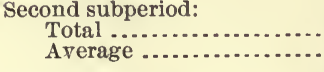 & $\begin{array}{l}74.44 \\
14.89\end{array}$ & $\begin{array}{r}4.848 \\
.970\end{array}$ & $\begin{array}{r}10.888 \\
2.178\end{array}$ & $\begin{array}{r}14.038 \\
2.808\end{array}$ & 1:15.4 & $1: 6.8$ & $1: 5.3$ \\
\hline 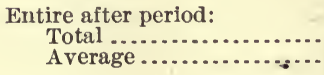 & $\begin{array}{r}146.48 \\
14.65\end{array}$ & $\begin{array}{r}9.585 \\
.958\end{array}$ & $\begin{array}{r}21.506 \\
2.151\end{array}$ & $\begin{array}{r}26.997 \\
2.700\end{array}$ & 1:15. 3 & 1:6.8 & $1: 5.4$ \\
\hline
\end{tabular}


TABLE IX.-Urine determinations-Ratio of sulphur, sulphates, and phosphates to nitrogen-Series VI-Continued.

[Averages are per day.]

No. 5 .

\begin{tabular}{|c|c|c|c|c|c|c|c|}
\hline \multirow{2}{*}{ Period. } & \multicolumn{4}{|c|}{ Quantity. } & \multicolumn{3}{|c|}{ Ratio. } \\
\hline & Nitrogen. & Sulphur. & $\mathrm{SO}_{3}$ & $\mathrm{P}_{2} \mathrm{O}_{5}$. & $\mathrm{S}: \mathrm{N}$ & $\mathrm{SO}_{3}: \mathrm{N}$ & $\mathrm{P}_{2} \mathrm{O}_{5}: \mathrm{N}$ \\
\hline \multicolumn{8}{|l|}{ Fore period. } \\
\hline $\begin{array}{l}\text { First subperiod: } \\
\quad \text { Total ................. } \\
\text { Average.......... }\end{array}$ & $\begin{array}{r}\text { Grams. } \\
69.53 \\
13.91\end{array}$ & $\begin{array}{r}\text { Grams. } \\
4.565 \\
.913\end{array}$ & $\begin{array}{r}\text { Grams. } \\
9.933 \\
1.987\end{array}$ & $\begin{array}{r}\text { Grams. } \\
12.890 \\
2.578\end{array}$ & $1: 15.2$ & $1: 7.0$ & $1: 5.4$ \\
\hline Second subperiod: & & & & & & & \\
\hline $\begin{array}{l}\text { Total } \ldots \ldots \\
\text { Average }\end{array}$ & $\begin{array}{l}61.86 \\
12.37\end{array}$ & $\begin{array}{r}3.711 \\
.742\end{array}$ & $\begin{array}{l}8.588 \\
1.718\end{array}$ & $\begin{array}{r}11.896 \\
2.379\end{array}$ & $\begin{array}{r}1: 16.7 \\
\therefore . . .\end{array}$ & $\begin{array}{l}1: 7.2 \\
\ldots . .\end{array}$ & $1: 5.2$ \\
\hline $\begin{array}{l}\text { Entire fore period: } \\
\text { Total } \ldots . . . \ldots \ldots \\
\text { Average } \ldots \ldots \ldots \ldots\end{array}$ & $\begin{array}{r}131.39 \\
13.14\end{array}$ & $\begin{array}{r}8.276 \\
.828\end{array}$ & $\begin{array}{r}18.521 \\
1.852\end{array}$ & $\begin{array}{r}24.786 \\
2.479\end{array}$ & $\begin{array}{l}1: 15.9 \\
\cdots \cdots\end{array}$ & $\begin{array}{l}1: 7.1 \\
\cdots\end{array}$ & 1:5. 3 \\
\hline \multicolumn{8}{|l|}{ Preservative period. } \\
\hline $\begin{array}{l}\text { First subperiod: } \\
\text { Total } \ldots . . . . . . . \\
\text { A verage ......... }\end{array}$ & $\begin{array}{l}68.49 \\
13.70\end{array}$ & $\begin{array}{r}4.342 \\
.868\end{array}$ & $\begin{array}{l}9.733 \\
1.947\end{array}$ & $\begin{array}{r}12.791 \\
2.558\end{array}$ & $1: 15.8$ & $1: 7.0$ & $1: 5.4$ \\
\hline \multicolumn{8}{|l|}{ Average ............. } \\
\hline $\begin{array}{l}\text { Total } \ldots \ldots \ldots \\
\text { Average .................. }\end{array}$ & $\begin{array}{l}69.02 \\
13.80\end{array}$ & $\begin{array}{r}4.495 \\
899\end{array}$ & 9.545 & $\begin{array}{r}13.595 \\
2.719\end{array}$ & $1: 15.4$ & $1: 7.2$ & $1: 5.1$ \\
\hline \multicolumn{8}{|l|}{ Third subperiod: } \\
\hline Total ................ & $\begin{array}{l}67.90 \\
13.58\end{array}$ & 4. 566 & 9.760 & 13.579 & $1: 14.9$ & $1: 7.0$ & $1: 5.0$ \\
\hline \multicolumn{8}{|l|}{$\begin{array}{l}\text { Average } \\
\text { Fourth subperiod: }\end{array}$} \\
\hline $\begin{array}{l}\text { Total } \\
\text { Average.................... }\end{array}$ & $\begin{array}{l}69.38 \\
13.88\end{array}$ & 4. 632 & 9.841 & 13.216 & $1: 15.0$ & $1: 7.1$ & $1: 5.2$ \\
\hline \multicolumn{8}{|l|}{ Fifth subperiod: } \\
\hline $\begin{array}{l}\text { Total } \ldots . . . . . . \\
\text { Average } . . . .\end{array}$ & $\begin{array}{l}72.81 \\
14.56\end{array}$ & $\begin{array}{r}4.670 \\
.934\end{array}$ & $\begin{array}{r}10.216 \\
2.043\end{array}$ & $\begin{array}{r}13.087 \\
2.617\end{array}$ & $1: 15.6$ & $1: 7.1$ & $1: 5.6$ \\
\hline \multicolumn{8}{|l|}{ Sixth subperiod: } \\
\hline Total ............. & 68.66 & 4. 534 & 9.430 & 12.408 & $1: 15.1$ & $1: 7.3$ & $1: 5.5$ \\
\hline Avera & 13.73 & .907 & 1.886 & & & $\cdots$ & \\
\hline \multirow{2}{*}{ 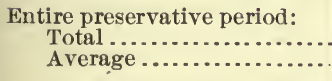 } & 416.26 & 27. 239 & 58.525 & 78.676 & $1: 15.3$ & $1: 7.1$ & $1: 5.3$ \\
\hline & & .908 & 1.951 & 2.623 & & .... & \\
\hline \multicolumn{8}{|l|}{ After period. } \\
\hline $\begin{array}{l}\text { First subperiod: } \\
\text { Total } \ldots . . . . . . . . \\
\text { Average......... }\end{array}$ & a 69.30 & 4. 516 & 9.373 & 11. 883 & $1: 15.3$ & $1: 7.4$ & $1: 5.8$ \\
\hline \multicolumn{8}{|l|}{ Second subperiod: } \\
\hline Total $\ldots \ldots \ldots \ldots$ & 70.67 & 4. 677 & 10.241 & 12,738 & 1:15. 1 & $1: 6.9$ & $1: 5.5$ \\
\hline Average ........ & & .935 & 2.048 & & ..... & $\cdots$ & \\
\hline \multirow{2}{*}{$\begin{array}{l}\text { Entire after period: } \\
\text { Total ............. } \\
\text { Average........ }\end{array}$} & 139.97 & 9.193 & 19.614 & 24.621 & $1: 15.2$ & $1: 7.1$ & $1: 5.7$ \\
\hline & 14.00 & .919 & 1.961 & 2.462 & & & \\
\hline
\end{tabular}

$a$ One day's average added in order to complete record. 
TABLE IX.-Urine determinations-Ratio of sulphur, sulphates, and phosphates to nitrogen-Series VI-Continued.

[Averages are per day.]

No. 6.

\begin{tabular}{|c|c|c|c|c|c|c|c|}
\hline \multirow{2}{*}{ Period. } & \multicolumn{4}{|c|}{ Quantity. } & \multicolumn{3}{|c|}{ Ratio. } \\
\hline & Nitrogen. & Sulphur. & $\mathrm{SO}_{3}$. & $\mathrm{P}_{2} \mathrm{O}_{5}$ & $\mathrm{~S}: \mathrm{N}$ & $\mathrm{SO}_{3}: \mathrm{N}$. & $\mathrm{P}_{2} \mathrm{O}_{5}: \mathrm{N}$ \\
\hline \multicolumn{8}{|l|}{ Fore period. } \\
\hline 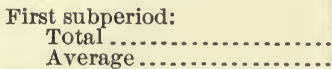 & $\begin{array}{r}\text { Grams. } \\
59.40 \\
11.88\end{array}$ & $\begin{array}{r}\text { Grams. } \\
4.038 \\
.808\end{array}$ & $\begin{array}{r}\text { Grams. } \\
9.080 \\
1.816\end{array}$ & $\begin{array}{r}\text { Grams. } \\
9.814 \\
1.963\end{array}$ & $1: 14.7$ & $1: 6.5$ & $1: 6.1$ \\
\hline Second subperiod: & & & & & & & \\
\hline 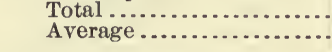 & $\begin{array}{l}57.71 \\
11.54\end{array}$ & $\begin{array}{r}3.796 \\
.759\end{array}$ & $\begin{array}{l}8.555 \\
1.711\end{array}$ & $\begin{array}{r}10.010 \\
2.002\end{array}$ & $\begin{array}{r}1: 15.2 \\
\cdots\end{array}$ & $\begin{array}{l}1: 6.7 \\
\cdots\end{array}$ & $\begin{array}{l}1: 5.8 \\
\cdots \cdots\end{array}$ \\
\hline $\begin{array}{l}\text { Entire fore period: } \\
\text { Total ........... }\end{array}$ & & 7.834 & 635 & 9.824 & $1: 14.9$ & $1: 6,6$ & 1.5 .9 \\
\hline Average ..................... & & .783 & 1.764 & 1.982 & ……... & 1.0 .0 & 1.5 .9 \\
\hline \multicolumn{8}{|l|}{ Preservative period. } \\
\hline \\
\hline & 65.93 & $\begin{array}{l}4.090 \\
818\end{array}$ & 9.621 & 11.315 & $1: 16.1$ & $1: 6.9$ & $1: 5.8$ \\
\hline \multicolumn{8}{|l|}{$\begin{array}{l}\text { Second subperiod: } \\
\text { Total ............... }\end{array}$} \\
\hline $\begin{array}{l}\text { Total ................ } \\
\text { Average........ }\end{array}$ & 68.49 & 4. 521 & 9.966 & 12. 020 & $1: 15.1$ & $1: 6.9$ & $1: 5.7$ \\
\hline \multicolumn{8}{|l|}{$\begin{array}{l}\text { Average ......... } \\
\text { Third subperiod; } \\
\text { Total ............. }\end{array}$} \\
\hline Total ............... & $a 70.76$ & 4.748 & 10. 660 & 11.275 & $1: 14.9$ & $1: 6.6$ & $1: 6.3$ \\
\hline \multicolumn{8}{|l|}{$\begin{array}{l}\text { Average } \\
\text { Fourth subperiod: } \\
\text { Total }\end{array}$} \\
\hline $\begin{array}{l}\text { Total } \ldots \ldots \\
\text { Average } \ldots\end{array}$ & a 73.26 & 5.065 & 11. 224 & 11.928 & $1: 14.5$ & $1: 6.4$ & $1: 6.1$ \\
\hline \multicolumn{8}{|l|}{$\begin{array}{l}\text { Average } \\
\text { Fifth subperiod: } \\
\text { Total } \ldots . . . . . . . . . . .\end{array}$} \\
\hline 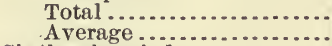 & $\begin{array}{l}74.36 \\
14.87\end{array}$ & $\begin{array}{r}4.800 \\
.960\end{array}$ & $\begin{array}{r}10.899 \\
2.180\end{array}$ & $\begin{array}{r}11.044 \\
2.269\end{array}$ & $1: 15.5$ & $1: 6.8$ & $1: 6.7$ \\
\hline \multicolumn{8}{|l|}{$\begin{array}{l}\text { Sixth subperiod: } \\
\text { Total }\end{array}$} \\
\hline $\begin{array}{l}\text { Total } \\
\text { Average } \ldots \ldots\end{array}$ & 53.40 & 4. 390 & 9.757 & 9.990 & 1:12.2 & $1: 5.5$ & $1: 5.3$ \\
\hline Averag & & .818 & & 1.998 & .............. & ...... & ...... \\
\hline \multicolumn{8}{|l|}{ Entire preservative period: } \\
\hline A verage........... & & .920 & 2.081 & 2.252 & (1.2...... & 1.0 .0 & 1.0 .0 \\
\hline \multicolumn{8}{|l|}{ After period. } \\
\hline \multicolumn{8}{|l|}{$\begin{array}{l}\text { First subperiod: } \\
\text { Total }\end{array}$} \\
\hline Total ............. & $\begin{array}{l}66.12 \\
13.22\end{array}$ & $\begin{array}{r}4.346 \\
.869\end{array}$ & $\begin{array}{l}9.444 \\
1.889\end{array}$ & $\begin{array}{l}9.180 \\
1.836\end{array}$ & $1: 15.2$ & $1: 7.0$ & $1: 7.2$ \\
\hline \multicolumn{8}{|l|}{$\begin{array}{l}\text { Second subperiod: } \\
\text { Total ........... }\end{array}$} \\
\hline $\begin{array}{l}\text { Total ................ } \\
\text { Average ......... }\end{array}$ & $\begin{array}{l}54.90 \\
10.98\end{array}$ & 4. 611 & 9.968 & 10.707 & 1:11. 9 & $1: 5.5$ & $1: 5.1$ \\
\hline Average & & $\cdot 922$ & & & ......... & ....... & ....... \\
\hline $\begin{array}{l}\text { Entire after period: } \\
\text { Total ............ }\end{array}$ & & 8.957 & & & $1: 13.5$ & $1: 6.2$ & $1: 6.1$ \\
\hline Avera & 12.10 & .896 & 1.941 & 1.989 & & & \\
\hline
\end{tabular}

$a$ One day's average added in order to complete record. 
TABLE IX.-Urine determinations-Ratio of sulphur, sulphates, and phosphates to nitrogen-Series VI-Continued.

[Averages are per day.]

No. 7.

\begin{tabular}{|c|c|c|c|c|c|c|c|}
\hline \multirow{2}{*}{ Period. } & \multicolumn{4}{|c|}{ Quantity. } & \multicolumn{3}{|c|}{ Ratio. } \\
\hline & Nitrogen. & Sulphur. & $\mathrm{SO}_{3}$. & $\mathrm{P}_{2} \mathrm{O}_{5}$. & S:N. & $\mathrm{SO}_{3}: \mathrm{N}$ & $\mathrm{P}_{2} \mathrm{O}_{5}: \mathrm{N}$. \\
\hline \multicolumn{8}{|l|}{ Fore period. } \\
\hline $\begin{array}{l}\text { First subperiod: } \\
\quad \text { Total } \ldots \ldots \ldots \ldots \\
\text { Average } \ldots \ldots \ldots\end{array}$ & $\begin{array}{r}\text { Grams. } \\
56.57 \\
11.31\end{array}$ & $\begin{array}{r}\text { Grams. } \\
3.756 \\
.751\end{array}$ & $\begin{array}{r}\text { Grams. } \\
7.956 \\
1.591\end{array}$ & $\begin{array}{r}\text { Grams. } \\
8.547 \\
1.709\end{array}$ & $1: 15.1$ & 1:7.1 & $1: 6.6$ \\
\hline 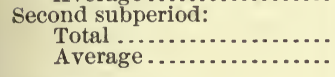 & $\begin{array}{l}63.00 \\
12.60\end{array}$ & $\begin{array}{r}4.280 \\
.856\end{array}$ & $\begin{array}{l}9.288 \\
1.858\end{array}$ & $\begin{array}{l}8.480 \\
1.696\end{array}$ & $1: 14.7$ & $1: 6.8$ & $1: 7.4$ \\
\hline 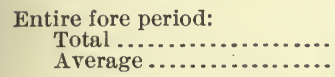 & $\begin{array}{r}119.57 \\
11.96\end{array}$ & $\begin{array}{r}8.036 \\
.804\end{array}$ & $\begin{array}{r}17.244 \\
1.724\end{array}$ & $\begin{array}{r}17.027 \\
1.703\end{array}$ & $1: 14.9$ & $1: 6.9$ & $1: 7.0$ \\
\hline \multicolumn{8}{|l|}{ Preservative period. } \\
\hline $\begin{array}{l}\text { First subperiod: } \\
\text { Total ........... } \\
\text { Average } . . . . .\end{array}$ & $\begin{array}{l}50.72 \\
10.14\end{array}$ & $\begin{array}{r}3.513 \\
.703\end{array}$ & $\begin{array}{l}7.425 \\
1.485\end{array}$ & $\begin{array}{l}7.354 \\
1.471\end{array}$ & $1: 14.4$ & $1: 6.8$ & $1: 6.9$ \\
\hline $\begin{array}{l}\text { Second subperiod: } \\
\text { Total............ } \\
\text { Average....... }\end{array}$ & $\begin{array}{l}56.98 \\
11.40\end{array}$ & $\begin{array}{l}4.085 \\
.817\end{array}$ & $\begin{array}{l}8.715 \\
1.743\end{array}$ & $\begin{array}{l}7.903 \\
1.581\end{array}$ & 1:13.9 & $1: 6.5$ & $1: 7.2$ \\
\hline $\begin{array}{l}\text { Third subperiod: } \\
\text { Total .......... } \\
\text { Average....... }\end{array}$ & $\begin{array}{l}51.24 \\
10.25\end{array}$ & $\begin{array}{r}3.368 \\
674\end{array}$ & $\begin{array}{l}7.341 \\
1.468\end{array}$ & $\begin{array}{l}6.597 \\
1.319\end{array}$ & $1: 15.2$ & $1: 7.0$ & $1: 7.8$ \\
\hline $\begin{array}{l}\text { Average ......... } \\
\text { Fourth subperiod: }\end{array}$ & & .674 & .468 & & & & \\
\hline Total ............... & $\begin{array}{r}49.15 \\
9.83\end{array}$ & $\begin{array}{r}3.564 \\
.713\end{array}$ & $\begin{array}{l}7.539 \\
1.508\end{array}$ & $\begin{array}{l}6.450 \\
1.290\end{array}$ & $1: 13.8$ & $1: 6.5$ & $1: 7.6$ \\
\hline $\begin{array}{l}\text { Fifth subperiod: } \\
\text { Total }\end{array}$ & 52.92 & 3,870 & & & & & \\
\hline $\begin{array}{l}\text { Total } \\
\text { Avera }\end{array}$ & 10.58 & $\begin{array}{r}3.080 \\
.774\end{array}$ & $\begin{array}{l}8.184 \\
1.637\end{array}$ & $\begin{array}{l}6.989 \\
1.398\end{array}$ & $1: 13.7$ & $1: 6.5$ & $1: 7.6$ \\
\hline $\begin{array}{l}\text { Sixth subperi } \\
\text { Total } \ldots . . .\end{array}$ & & 6.424 & & & $1 \cdot 10$ & & \\
\hline Average............. & 12.83 & 1.285 & 1.811 & 1.676 & 1.10 .0 & (1..1. & $\begin{array}{r}1: 7.7 \\
\ldots . . . .\end{array}$ \\
\hline $\begin{array}{l}\text { Entire preservative period: } \\
\text { Total ...................................... }\end{array}$ & $\begin{array}{r}325.18 \\
10.84\end{array}$ & $\begin{array}{r}24.824 \\
827\end{array}$ & $\begin{array}{r}48.258 \\
1.608\end{array}$ & 43.672 & $1: 13.1$ & $1: 6.7$ & $1: 7.4$ \\
\hline \multirow{2}{*}{\multicolumn{8}{|c|}{ After period. }} \\
\hline & & & & & & & \\
\hline $\begin{array}{l}\text { First subperiod: } \\
\text { Total ............. } \\
\text { Average ........ }\end{array}$ & $\begin{array}{l}52.80 \\
10.56\end{array}$ & $\begin{array}{l}3.650 \\
730\end{array}$ & 7. 697 & 5. 802 & $1: 14.5$ & $1: 6.9$ & $1: 9.1$ \\
\hline $\begin{array}{l}\text { Second subperiod: } \\
\text { Total ................. }\end{array}$ & & & & & & & \\
\hline Average....................... & $\begin{array}{l}56.09 \\
11.22\end{array}$ & $\begin{array}{r}3.891 \\
.778\end{array}$ & $\begin{array}{l}8.180 \\
1.636\end{array}$ & $\begin{array}{r}6.596 \\
\quad 1.319\end{array}$ & $1: 14.4$ & $1: 6.9$ & $1: 8.5$ \\
\hline $\begin{array}{l}\text { Entire after period: } \\
\text { Total ............... } \\
\text { Average......... }\end{array}$ & $\begin{array}{r}108.89 \\
10.89\end{array}$ & $\begin{array}{r}7.541 \\
.754\end{array}$ & $\begin{array}{r}15.877 \\
1.588\end{array}$ & $\begin{array}{r}12.398 \\
1.240\end{array}$ & 1:14.4 & $1: 6.9$ & $1: 8.8$ \\
\hline & & & & & & & \\
\hline
\end{tabular}


TABLE IX. - Urine determinations-Ratio of sulphur, sulphates, and phosphates to nitrogen-Series VI-Continued.

[Averages are per day.]

No. 8 .

\begin{tabular}{|c|c|c|c|c|c|c|c|}
\hline \multirow{2}{*}{ Period. } & \multicolumn{4}{|c|}{ Quantity. } & \multicolumn{3}{|c|}{ Ratio. } \\
\hline & Nitrogen. & Sulphur. & $\mathrm{SO}_{3}$ & $\mathrm{P}_{2} \mathrm{O}_{5}$ & $\mathrm{~S}: \mathrm{N}$ & $\mathrm{SO}_{3}: \mathrm{N}$ & $\mathrm{P}_{2} \mathrm{O}_{5}: \mathrm{N}$ \\
\hline \multicolumn{8}{|l|}{ Fore period. } \\
\hline $\begin{array}{l}\text { First subperiod: } \\
\text { Total ............ } \\
\text { Average......... }\end{array}$ & $\begin{array}{r}\text { Grams. } \\
52.91 \\
10.58\end{array}$ & $\begin{array}{r}\text { Grams. } \\
3.689 \\
.738\end{array}$ & $\begin{array}{r}\text { Grams. } \\
7.653 \\
.1 .531\end{array}$ & $\begin{array}{r}\text { Grams. } \\
8.818 \\
1.764\end{array}$ & $\begin{array}{l}1: 14.3 \\
\cdots \ldots . .\end{array}$ & $\begin{array}{l}1: 6.9 \\
\ldots \ldots \ldots\end{array}$ & $\begin{array}{l}1: 6.0 \\
\cdots . . .\end{array}$ \\
\hline Second subperiod: & & & & & & & \\
\hline $\begin{array}{l}\text { Total } \ldots \ldots \ldots \\
\text { Average }\end{array}$ & $\begin{array}{l}55.50 \\
11.10\end{array}$ & $\begin{array}{r}3.988 \\
.798\end{array}$ & $\begin{array}{l}8.019 \\
1.604\end{array}$ & $\begin{array}{l}8.740 \\
1.748\end{array}$ & $1: 13.9$ & $1: 6.9$ & $1: 6.4$ \\
\hline $\begin{array}{l}\text { Entire fore period: } \\
\text { Total ............ } \\
\text { Average ......... }\end{array}$ & $\begin{array}{r}108.41 \\
10.84\end{array}$ & $\begin{array}{r}7.677 \\
.768\end{array}$ & $\begin{array}{r}15.672 \\
1.567\end{array}$ & $\begin{array}{r}17.558 \\
1.756\end{array}$ & $\begin{array}{c}1: 14.1 \\
\ldots \ldots\end{array}$ & $\begin{array}{c}1: 6.9 \\
\cdots \cdots\end{array}$ & $\begin{array}{l}1: 6.2 \\
\cdots . . .\end{array}$ \\
\hline \multicolumn{8}{|l|}{ Preservative period. } \\
\hline $\begin{array}{l}\text { First subperiod: } \\
\text { Total ............. } \\
\text { A verage........ }\end{array}$ & $\begin{array}{l}52.73 \\
10.55\end{array}$ & $\begin{array}{r}3.441 \\
.688\end{array}$ & $\begin{array}{l}7.611 \\
1.522\end{array}$ & $\begin{array}{l}8.882 \\
1.776\end{array}$ & $1 ; 15.3$ & $1: 6.9$ & $1: 5.9$ \\
\hline \multicolumn{8}{|l|}{ Second subperiod: } \\
\hline Total ................ & 54.44 & 3.610 & 7.885 & 9.500 & $1: 15.1$ & $1: 6.9$ & $1: 5.7$ \\
\hline \multicolumn{8}{|l|}{ Third subperiod: } \\
\hline Total .............. & 51.74 & 3.675 & 7.252 & 8.820 & $1: 14.1$ & $1: 7.1$ & $1: 5.9$ \\
\hline \multicolumn{5}{|l|}{ Fourth subperiod: } & (........... & ........ & ....... \\
\hline Total ................ & a 50.16 & 3. 731 & 7. 744 & 8.888 & $1: 13.4$ & $1: 6.5$ & $1: 5.6$ \\
\hline Áverage ........ & 10.03 & .746 & 1.549 & 1.778 & .......... & & ....... \\
\hline \multicolumn{8}{|l|}{ Fifth subperiod: } \\
\hline Average & 11.52 & .765 & 1.578 & 1.686 & & & ....... \\
\hline \multicolumn{3}{|l|}{ Sixth subperiod: } & & & $1: 14.1$ & $1: 6.6$ & $1: 7.2$ \\
\hline Average......... & 11.15 & .790 & 1.695 & 1.557 & ............ & ............ & ...... \\
\hline \multirow{2}{*}{ 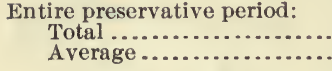 } & 322.44 & 22. 223 & 46.856 & 52.305 & $1: 14.5$ & $1: 6.9$ & $1: 6.2$ \\
\hline & 10.75 & .741 & 1.562 & 1.744 & & $\cdots$ & \\
\hline \multicolumn{8}{|l|}{ After period. } \\
\hline \multicolumn{8}{|l|}{ First subperiod: } \\
\hline A verage...$\ldots \ldots \ldots$ & $\begin{array}{l}60.36 \\
12.07\end{array}$ & $\begin{array}{r}4.209 \\
.842\end{array}$ & $\begin{array}{l}8.534 \\
1.707\end{array}$ & $\begin{array}{l}7.387 \\
1.477\end{array}$ & $\begin{array}{l}1: 14.3 \\
\ldots . . . \ldots .\end{array}$ & $1: 7.1$ & $1: 8.2$ \\
\hline \multicolumn{8}{|l|}{ Second subperiod: } \\
\hline $\begin{array}{l}\text { Total } \ldots . . . . . . . . . \\
\text { Average }\end{array}$ & $\begin{array}{l}59.18 \\
11.84\end{array}$ & $\begin{array}{r}4.166 \\
.833\end{array}$ & $\begin{array}{l}8.788 \\
1.758\end{array}$ & $\begin{array}{l}7.938 \\
1.588\end{array}$ & $\bullet 1: 14.2$ & $1: 6.7$ & $1: 7.5$ \\
\hline \multirow{2}{*}{\multicolumn{8}{|c|}{$\begin{array}{c}\text { Entire after period: } \\
\text { Total } \ldots . . . \ldots \ldots \ldots\end{array}$}} \\
\hline & 119.54 & 8.375 & 17.322 & 15. 325 & $1: 14.3$ & $1: 6.9$ & $1: 7.8$ \\
\hline A verage......... & 11.95 & .838 & 1.732 & 1.533 & & & \\
\hline
\end{tabular}


TABLE IX.-Urine determinations-Ratio of sulphur, sulphates, and phosphates to nitrogen-Series VI-Continued.

[Averages are per day.]

No. 9.

\begin{tabular}{|c|c|c|c|c|c|c|c|}
\hline \multirow{2}{*}{ Period. } & \multicolumn{4}{|c|}{ Quantity. } & \multicolumn{3}{|c|}{ Ratio. } \\
\hline & Nitrogen. & Sulphur. & $\mathrm{SO}_{3}$ & $\mathrm{P}_{2} \mathrm{O}_{5}$. & $\mathrm{S}: \mathrm{N}$ & $\mathrm{SO}_{3}: \mathrm{N}$ & $\mathrm{P}_{2} \mathrm{O}_{5}: \mathrm{N}$ \\
\hline Fore period. & & & & & & & - \\
\hline $\begin{array}{l}\text { First subperiod: } \\
\quad \text { Total ............. }\end{array}$ & Grams. & Grams. & Grams. & Grams. & $1: 15.0$ & $1: 6.0$ & $1: 5.9$ \\
\hline $\begin{array}{l}\text { Average ............................. } \\
\text { Second subperiod: }\end{array}$ & 14.05 & .934 & 2.333 & 2.371 & $\cdots \cdots$ & ….... & \\
\hline $\begin{array}{l}\text { Total } \ldots \ldots \ldots \\
\text { Average } . \ldots \ldots\end{array}$ & $\begin{array}{l}67.15 \\
13.43\end{array}$ & $\begin{array}{r}4.547 \\
.909\end{array}$ & $\begin{array}{r}11.354 \\
2.271\end{array}$ & $\begin{array}{r}10.410 \\
2.082\end{array}$ & $\begin{array}{r}1: 14.8 \\
2\end{array}$ & $\begin{array}{l}1: 5.9 \\
\cdots \cdots\end{array}$ & $1: 6.5$ \\
\hline 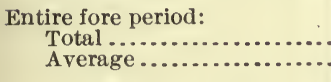 & $\begin{array}{r}137.41 \\
13.74 \\
\end{array}$ & $\begin{array}{r}9.218 \\
.9^{\prime 22}\end{array}$ & $\begin{array}{r}23.017 \\
2.302\end{array}$ & $\begin{array}{r}22.267 \\
2.227\end{array}$ & $\begin{array}{r}1: 14.9 \\
\cdots\end{array}$ & $\begin{array}{r}1: 6.0 \\
\cdots\end{array}$ & $\begin{array}{l}1: 6.2 \\
\cdots \cdots\end{array}$ \\
\hline Preservative period. & & & 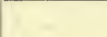 & - & & & \\
\hline 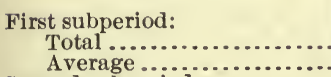 & $\begin{array}{l}72.12 \\
14.42\end{array}$ & $\begin{array}{r}4.948 \\
.990\end{array}$ & $\begin{array}{r}12.355 \\
2.471\end{array}$ & $\begin{array}{r}11.750 \\
2.350\end{array}$ & $\begin{array}{l}1: 14.6 \\
\cdots \cdots \cdots\end{array}$ & $\begin{array}{l}1: 5.8 \\
\ldots \ldots . .\end{array}$ & $1: 6.1$ \\
\hline Second subperiod: & & & & & & & \\
\hline $\begin{array}{l}\text { Total } \\
\text { Average........................ }\end{array}$ & $\begin{array}{l}66.12 \\
13.22\end{array}$ & $\begin{array}{r}4.570 \\
.914\end{array}$ & $\begin{array}{r}11.411 \\
2.282\end{array}$ & $\begin{array}{r}11.997 \\
2.399\end{array}$ & $\begin{aligned} 1: 14.5 \\
\ldots \ldots \ldots\end{aligned}$ & $\begin{array}{l}1: 5.8 \\
\ldots \ldots\end{array}$ & $1: 5.5$ \\
\hline Third subperiod: & & & & & & & \\
\hline $\begin{array}{l}\text { Total } \ldots . . . . . \\
\text { Average } . . . .\end{array}$ & $\begin{array}{l}69.10 \\
13.82\end{array}$ & $\begin{array}{r}4.780 \\
.956\end{array}$ & $\begin{array}{r}11.936 \\
2.387\end{array}$ & $\begin{array}{r}12.249 \\
2.450\end{array}$ & $1: 14.5$ & $1: 5.8$ & $1: 5.6$ \\
\hline Fourth subperiod: & 10.02 & & & & & & \\
\hline Total $1 . . .$. & 67.84 & 4.567 & 11.404 & 13. 981 & $1: 14.9$ & $1: 5.9$ & $1: 4.9$ \\
\hline $\begin{array}{l}\text { Average } \\
\text { Fifth subperiod: }\end{array}$ & 13.57 & .913 & 2.281 & 2.796 & $\cdots \cdots, \cdots$ & $\cdots \cdots \cdots$ & $\cdots$ \\
\hline 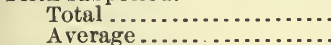 & 63.35 & 4. 072 & 10.168 & 10. 919 & $1: 15.6$ & $1: 6.2$ & $1: 5.8$ \\
\hline Sixth subperiod: & 12.07 & .814 & 2.054 & 2.100 & & & \\
\hline $\begin{array}{l}\text { Total } \\
\text { Average } \ldots\end{array}$ & $\begin{array}{l}73.44 \\
14.69\end{array}$ & $\begin{array}{r}4.864 \\
973\end{array}$ & 12.145 & 12.805 & $1: 15.1$ & $1: 6.0$ & $1: 5.7$ \\
\hline 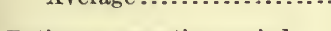 & & & & & & ..... & \\
\hline 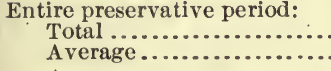 & $\begin{array}{r}411.97 \\
13.73\end{array}$ & $\begin{array}{r}27.801 \\
.927\end{array}$ & $\begin{array}{r}69.419 \\
2.314\end{array}$ & $\begin{array}{r}73.701 \\
2.457\end{array}$ & $\begin{array}{r}1: 14.8 \\
\ldots\end{array}$ & $\begin{array}{l}1: 5.9 \\
\cdots \cdots \cdots\end{array}$ & $\begin{array}{l}1: 5.6 \\
\cdots\end{array}$ \\
\hline After period. & & & & & & & \\
\hline 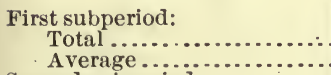 & $\begin{array}{l}64.45 \\
12.89\end{array}$ & $\begin{array}{r}4.345 \\
.869\end{array}$ & $\begin{array}{r}10.849 \\
2.170\end{array}$ & $\begin{array}{r}10.423 \\
2.085\end{array}$ & $\begin{aligned} 1: 14.8 \\
\cdots\end{aligned}$ & $\begin{array}{l}1: 5.9 \\
1 . .\end{array}$ & $1: 6.2$ \\
\hline $\begin{array}{l}\text { Second subperiod: } \\
\quad \text { Total ................ } \\
\text { Average .......... }\end{array}$ & $\begin{array}{l}70.20 \\
14.04\end{array}$ & $\begin{array}{r}4.578 \\
.916\end{array}$ & $\begin{array}{r}11.431 \\
2.286\end{array}$ & $\begin{array}{r}12.531 \\
2.506\end{array}$ & $1: 15.3$ & $1: 6.1$ & $1: 5$. \\
\hline $\begin{array}{l}\text { Entire after period: } \\
\text { Total .............. } \\
\text { Average ......... }\end{array}$ & $\begin{array}{r}134.65 \\
13.47\end{array}$ & $\begin{array}{r}8.923 \\
.892\end{array}$ & $\begin{array}{r}22.280 \\
2.228\end{array}$ & $\begin{array}{r}22.954 \\
2.295\end{array}$ & $\begin{array}{r}1: 15.1 \\
\cdots \ldots \ldots\end{array}$ & $1: 6.0$ & $1: 5.9$ \\
\hline
\end{tabular}

7656-No. 84, pt $2-06-6$ 
TABLE IX.-Urine determinations-Ratio of sulphur, sulphates, and phosphates to nitrogen-Series VI-Continued.

[Averages are per day.]

No. 10.

\begin{tabular}{|c|c|c|c|c|c|c|c|}
\hline \multirow{2}{*}{ Period. } & \multicolumn{4}{|c|}{ Quantity. } & \multicolumn{3}{|c|}{ Ratio. } \\
\hline & Nitrogen. & Sulphur. & $\mathrm{SO}_{3}$. & $\mathrm{P}_{2} \mathrm{O}_{5}$. & $\mathrm{S}: \mathrm{N}$ & $\mathrm{SO}_{3}: \mathrm{N}$ & $\mathrm{P}_{2} \mathrm{O}_{5}: \mathrm{N}$ \\
\hline \multicolumn{8}{|l|}{ Fore period. } \\
\hline $\begin{array}{l}\text { First subperiod: } \\
\quad \text { Total } \ldots \ldots \ldots \ldots \ldots \\
\text { Average } . . . . . . . . . . .\end{array}$ & $\begin{array}{r}\text { Grams. } \\
63.81 \\
12.76\end{array}$ & $\begin{array}{r}\text { Grams. } \\
4.215 \\
.843\end{array}$ & $\begin{array}{r}\text { Grams. } \\
10.525 \\
2.105\end{array}$ & $\begin{array}{r}\text { Grams. } \\
10.727 \\
2.145\end{array}$ & $\begin{array}{l}1: 15.1 \\
\cdots \ldots \ldots\end{array}$ & $\begin{array}{l}1: 6.1 \\
-\ldots . . .\end{array}$ & $\begin{array}{l}1: 5.9 \\
-\ldots .\end{array}$ \\
\hline Second subperiod: & 12.00 & & & & & & 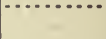 \\
\hline $\begin{array}{l}\text { Total } \\
\text { Average } \ldots \ldots\end{array}$ & $\begin{array}{l}62.78 \\
12.56\end{array}$ & $\begin{array}{r}4.301 \\
.860\end{array}$ & $\begin{array}{r}10.740 \\
2.148\end{array}$ & $\begin{array}{r}11.600 \\
2.320\end{array}$ & $1: 14.6$ & $1: 5.8$ & $1: 3 . .4$ \\
\hline $\begin{array}{l}\text { Entire fore period: } \\
\quad \text { Total } \ldots \ldots \ldots \\
\quad \text { Average }\end{array}$ & $\begin{array}{r}126.59 \\
12.66\end{array}$ & $\begin{array}{r}8.516 \\
.852\end{array}$ & $\begin{array}{r}21.265 \\
2.126\end{array}$ & $\begin{array}{r}22.327 \\
2.233\end{array}$ & $\begin{array}{l}1: 14.9 \\
2 \ldots \ldots\end{array}$ & $\begin{array}{l}1: 6.0 \\
\cdots\end{array}$ & $\begin{array}{l}1: 5.7 \\
\cdots \cdots\end{array}$ \\
\hline \multicolumn{8}{|l|}{ Preservative period. } \\
\hline $\begin{array}{l}\text { First subperiod: } \\
\quad \text { Total } \ldots \ldots \ldots \ldots \ldots \\
\quad \text { Average } \\
\end{array}$ & $\begin{array}{l}61.96 \\
12.39\end{array}$ & $\begin{array}{r}3.997 \\
.799\end{array}$ & $\begin{array}{l}9.981 \\
1.996\end{array}$ & $\begin{array}{r}10.851 \\
2.170\end{array}$ & $\begin{array}{l}1: 15.5 \\
\ldots \ldots \ldots\end{array}$ & $1: 6.2$ & $\begin{array}{l}1: 5.7 \\
\ldots \ldots\end{array}$ \\
\hline Second subperiod: & & & & & & & \\
\hline $\begin{array}{c}\text { Total } \ldots \ldots \ldots \\
\text { Average } . . .\end{array}$ & $\begin{array}{l}71.36 \\
14.27\end{array}$ & $\begin{array}{r}4.786 \\
.957\end{array}$ & $\begin{array}{r}11.951 \\
2.390\end{array}$ & $\begin{array}{r}12.768 \\
2.554\end{array}$ & $1: 14.9$ & $1: 6.0$ & $1: 5.6$ \\
\hline Third subperiod: & & & & & & & \\
\hline Total $\ldots . . . .$. & 67.35 & 4. 073 & 10.170 & 11.612 & $1: 16.5$ & $1: 6.6$ & $1: 5.8$ \\
\hline $\begin{array}{l}\text { Average ............ } \\
\text { Fourth subperiod: }\end{array}$ & 13.47 & .815 & 2.034 & 2.322 & (......... & …..... & ....... \\
\hline 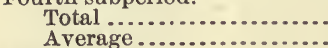 & $\begin{array}{l}61.61 \\
12.32\end{array}$ & $\begin{array}{r}4.264 \\
.853\end{array}$ & $\begin{array}{r}10.647 \\
2.129\end{array}$ & $\begin{array}{r}11.513 \\
2.303\end{array}$ & $1: 14.4$ & $1: 5.8$ & $1: 5.4$ \\
\hline Fifth subperiod: & & & & & & & \\
\hline $\begin{array}{l}\text { Total } \ldots \ldots \ldots \\
\text { Average } . . .\end{array}$ & $\begin{array}{l}64.37 \\
12.87\end{array}$ & $\begin{array}{r}4.135 \\
.827\end{array}$ & $\begin{array}{r}10.325 \\
2.065\end{array}$ & $\begin{array}{r}10.823 \\
2.165\end{array}$ & $1: 15.6$ & $1: 6.2$ & $1: 5.9$ \\
\hline Sixth subperiod: & & & & & & & \\
\hline $\begin{array}{l}\text { Total } \\
\text { Average } \ldots \ldots\end{array}$ & $\begin{array}{r}47.99 \\
9.60\end{array}$ & $\begin{array}{r}3.934 \\
.787\end{array}$ & $\begin{array}{l}9.823 \\
1.965\end{array}$ & $\begin{array}{l}9.744 \\
1.949\end{array}$ & $1: 12.2$ & $1: 4.9$ & $\dddot{1}: 4.9$ \\
\hline 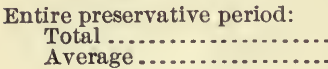 & $\begin{array}{r}374.64 \\
12.49\end{array}$ & $\begin{array}{r}25.189 \\
.840\end{array}$ & $\begin{array}{r}62.897 \\
2.097\end{array}$ & $\begin{array}{r}67.311 \\
2.244\end{array}$ & $\begin{array}{r}1: 14.9 \\
\end{array}$ & $\begin{array}{l}1: 6.0 \\
\cdots\end{array}$ & $\begin{array}{l}1: 5.6 \\
\cdots\end{array}$ \\
\hline After period. & & & & & & & \\
\hline 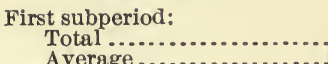 & 59. 74 & 4. 033 & 10.070 & 9.498 & $1: 14.8$ & $1: 5.9$ & $1: 6.3$ \\
\hline 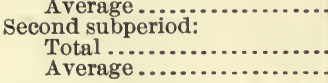 & Sick. & Sick. & Sick. & Sick. & & & $\cdots$ \\
\hline $\begin{array}{l}\text { Entire after period: } \\
\text { Total } \ldots \ldots \ldots \\
\text { Average } \ldots \ldots \ldots\end{array}$ & $\begin{array}{l}59.74 \\
11.95\end{array}$ & $\begin{array}{l}4.033 \\
.807\end{array}$ & $\begin{array}{r}10.070 \\
2.014\end{array}$ & $\begin{array}{l}9.498 \\
1.900\end{array}$ & $1: 14.8$ & $1: 5.9$ & $1: 6.3$ \\
\hline & & & & & & & \\
\hline
\end{tabular}


TABLE IX.-Urine determinations-Ratio of sulphur, sulphates, and phosphates to nitrogen-Series VI-Continued.

[Averages are per day.]

No. 11 .

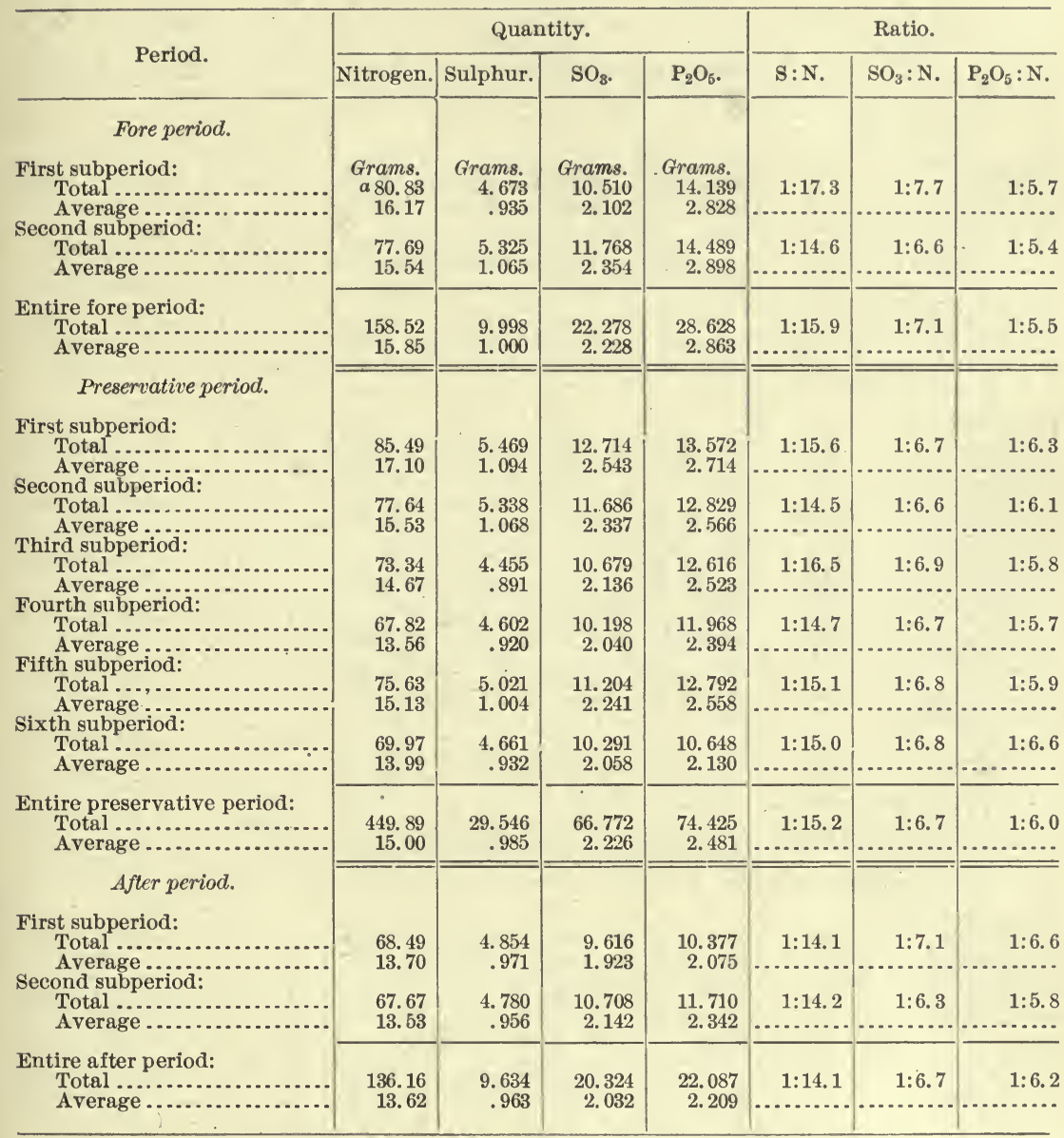

$a$ One day's a verage added in order to complete record. 
TABLE IX.-Urine determinations-Ratio of sulphur, sulphates, and phosphates to nitrogen-Series VI-Continued.

[Averages are per day.]

No. 12 .

\begin{tabular}{|c|c|c|c|c|c|c|c|}
\hline \multirow{2}{*}{ Period. } & \multicolumn{4}{|c|}{ Quantity. } & \multicolumn{3}{|c|}{ Ratio. } \\
\hline & Nitrogen. & Sulphur. & $\mathrm{SO}_{3}$ & $\mathrm{P}_{2} \mathrm{O}_{5}$. & $\mathrm{S}: \mathrm{N}$ & $\mathrm{SO}_{3}: \mathrm{N}$ & $\mathrm{P}_{2} \mathrm{O}_{5}: \mathrm{N}$ \\
\hline \multicolumn{8}{|l|}{ Fore period. } \\
\hline $\begin{array}{l}\text { First subperiod: } \\
\text { Total .................. } \\
\text { Average }\end{array}$ & $\begin{array}{r}\text { Grams. } \\
76.69\end{array}$ & Grams. & Grams. & Grams. & $1: 14.8$ & $1: 6.7$ & $1: 6.0$ \\
\hline $\begin{array}{l}\text { Average ............... } \\
\text { Second subperiod: }\end{array}$ & 15.34 & 1.033 & 2.296 & 2.546 & & & -.. \\
\hline $\begin{array}{l}\text { Total } \ldots . . . \\
\text { Average } \ldots \ldots\end{array}$ & $\begin{array}{l}72.02 \\
14.40\end{array}$ & $\begin{array}{r}4.918 \\
.984\end{array}$ & $\begin{array}{r}10.487 \\
2.097\end{array}$ & $\begin{array}{r}12.955 \\
2.591\end{array}$ & $\begin{array}{l}1: 14.6 \\
2\end{array}$ & $\begin{array}{l}1: 6.9 \\
\cdots \cdots\end{array}$ & $\begin{array}{l}1: 5.6 \\
\cdots\end{array}$ \\
\hline $\begin{array}{l}\text { Entire fore period: } \\
\quad \text { Total } \ldots \ldots \ldots \ldots \ldots \\
\text { Average } . . . \ldots \ldots\end{array}$ & $\begin{array}{r}148.71 \\
14.87\end{array}$ & $\begin{array}{r}10.084 \\
1.008\end{array}$ & $\begin{array}{r}21.969 \\
2.197\end{array}$ & $\begin{array}{r}25.686 \\
2.569\end{array}$ & $\begin{array}{l}1: 14.7 \\
\cdots \cdots\end{array}$ & $\begin{array}{l}1: 6.8 \\
\ldots \ldots . .\end{array}$ & $\begin{array}{r}1: 5.8 \\
\cdots\end{array}$ \\
\hline \multirow{2}{*}{\multicolumn{8}{|c|}{ Preservative period. }} \\
\hline & & & & & & & \\
\hline \multicolumn{8}{|l|}{ First subperiod: } \\
\hline $\begin{array}{l}\text { Average......... } \\
\text { Second subperiod: }\end{array}$ & 13.01 & .847 & 1.937 & 2.318 & .......... & ........ & ....... \\
\hline \multicolumn{8}{|l|}{ Second subperiod: } \\
\hline Average......... & $\begin{array}{l}75.17 \\
15.03\end{array}$ & $\begin{array}{l}5.129 \\
1.026\end{array}$ & $\begin{array}{r}11.364 \\
2.273\end{array}$ & $\begin{array}{r}13.235 \\
2.647\end{array}$ & $1: 14.7$ & $1: 6.6$ & $1: 5.7$ \\
\hline \multicolumn{8}{|l|}{ Third subperiod: } \\
\hline 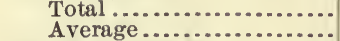 & 74.75 & 4.906 & 10.769 & 14.425 & $1: 15.2$ & $1: 6.9$ & $1: 5.2$ \\
\hline \multicolumn{8}{|l|}{$\begin{array}{l}\text { Fourth subperiod: } \\
\text { Total }\end{array}$} \\
\hline Total .................. & 70.01 & 4.734 & 10.625 & 12.930 & $1: 14.8$ & $1: 6.6$ & $1: 5.4$ \\
\hline \multicolumn{8}{|l|}{ Fifth subperiod: } \\
\hline Total ........... & 74.47 & 5.173 & 11. 279 & 12. 088 & 1:14.4 & $1: 6.6$ & $1: 6.2$ \\
\hline \multicolumn{8}{|l|}{ Sixth subperiod: } \\
\hline Total ............................. & 75.28 & 4.973 & 11.092 & 13.015 & $1: 15.1$ & $1: 6.8$ & $1: 5.8$ \\
\hline A verage...$\ldots \ldots \ldots \ldots \ldots$ & 15.06 & .995 & 2.218 & 2.603 & $\therefore \ldots \ldots$. & & .............. \\
\hline \multirow{2}{*}{ 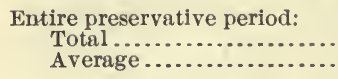 } & 434.74 & 29.151 & 64.812 & 77. 284 & $1: 14.9$ & $1: 6.7^{\prime}$ & $1: 5.6$ \\
\hline & 14.49 & .972 & 2.160 & 2.576 & & & \\
\hline After period. & 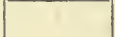 & & & & & & \\
\hline \multicolumn{8}{|l|}{$\begin{array}{l}\text { First subperiod: } \\
\text { Total ........ }\end{array}$} \\
\hline $\begin{array}{l}\text { Total } \ldots . . . . . . . . \\
\text { Average........ }\end{array}$ & 73.67 & 4.999 & 11.023 & 11.870 & $1: 14.7$ & $1: 6.7$ & $1: 6.2$ \\
\hline \multicolumn{8}{|l|}{$\begin{array}{l}\text { Second subperiod: } \\
\text { Total }\end{array}$} \\
\hline Total .............. & 76.80 & 5.164 & 11. 321 & 12. 694 & $1: 14.9$ & $1: 6.8$ & $1: 6.1$ \\
\hline Average........ & 15.36 & 1.033 & 2.264 & 2.539 & & & \\
\hline \multirow{2}{*}{$\begin{array}{c}\text { Entire after period: } \\
\text { Total ............. } \\
\text { Average........ }\end{array}$} & 150.47 & 10.163 & 22.344 & 24.564 & $1: 14.8$ & $1: 6.7$ & $1: 6.1$ \\
\hline & 15.05 & 1.016 & 2.234 & 2.456 & & & \\
\hline
\end{tabular}


TABLE IX.-Urine determinations-Ratio of sulphur, sulphates, and phosphates to nitrogen-Series VI-Continued.

[Averages are per man per day.]

Summary for nine men.

\begin{tabular}{|c|c|c|c|c|c|c|c|}
\hline \multirow{2}{*}{ Period. } & \multicolumn{4}{|c|}{ Quantity. } & \multicolumn{3}{|c|}{ Ratio. } \\
\hline & Nitrogen. & Sulphur. & $\mathrm{SO}_{3}$. & $\mathrm{P}_{2} \mathrm{O}_{5}$ & $\mathrm{~S}: \mathrm{N}$ & $\mathrm{SO}_{3}: \mathrm{N}$ & $\mathrm{P}_{2} \mathrm{O}_{5}: \mathrm{N}$ \\
\hline Fore period. & & & & & & & \\
\hline $\begin{array}{l}\text { First subperiod: } \\
\text { Total ............ } \\
\text { Average ........ }\end{array}$ & $\begin{array}{r}\text { Grams. } \\
614.88 \\
13.66\end{array}$ & $\begin{array}{r}\text { Grams. } \\
40.242 \\
.894\end{array}$ & $\begin{array}{r}\text { Grams. } \\
88.023 \\
1.956\end{array}$ & $\begin{array}{r}\text { Grams. } \\
106.474 \\
2.366\end{array}$ & $1: 15.3$ & $1: 7.0$ & $1: 5.8$ \\
\hline $\begin{array}{l}\text { Second subperiod: } \\
\text { Total ............... } \\
\text { Average....... }\end{array}$ & $\begin{array}{r}600.08 \\
13.33\end{array}$ & $\begin{array}{r}39.487 \\
.877 \\
\end{array}$ & $\begin{array}{r}87.683 \\
1.949 \\
\end{array}$ & $\begin{array}{r}106.305 \\
2.362\end{array}$ & 1:15.2 & $1: 6.8$ & $1: 5.6$ \\
\hline $\begin{array}{l}\text { Entire fore period: } \\
\text { 'Total ............. } \\
\text { Average......... }\end{array}$ & $\begin{array}{r}214.96 \\
13.50 \\
\end{array}$ & $\begin{array}{r}79.729 \\
.886 \\
\end{array}$ & $\begin{array}{r}175.706 \\
1.952 \\
\end{array}$ & $\begin{array}{r}212.779 \\
2.364 \\
\end{array}$ & $\begin{array}{l}1: 15.2 \\
\cdots \cdots\end{array}$ & $\begin{array}{c}1: 6.9 \\
\cdots \ldots . .\end{array}$ & $1: 5.7$ \\
\hline Preservative period. & & & & & & & \\
\hline $\begin{array}{l}\text { First subperiod: } \\
\quad \text { Total ............. } \\
\text { Average........ }\end{array}$ & $\begin{array}{r}596.76 \\
13.26\end{array}$ & $\begin{array}{r}38.571 \\
.857\end{array}$ & $\begin{array}{r}86.826 \\
1.929\end{array}$ & $\begin{array}{r}105.333 \\
2.341\end{array}$ & $1: 15.5$ & $1: 6.9$ & $1: 5.7$ \\
\hline $\begin{array}{l}\text { Second subperiod: } \\
\text { Total............. } \\
\text { Average ........ }\end{array}$ & $\begin{array}{r}618.50 \\
13.74\end{array}$ & $\begin{array}{r}41.073 \\
.913\end{array}$ & $\begin{array}{r}90.256 \\
2.006\end{array}$ & $\begin{array}{r}112.763 \\
2.506\end{array}$ & $1: 15.1$ & 1:6.9 & $1: 5.5$ \\
\hline $\begin{array}{l}\text { Third subperiod: } \\
\text { Total ................ }\end{array}$ & $\begin{array}{r}604.30 \\
13.43\end{array}$ & $\begin{array}{l}39.750 \\
883\end{array}$ & $\begin{array}{r}87.537 \\
1.945\end{array}$ & 108. 282 & $1: 15.2$ & $1: 6.9$ & $1: 5.6$ \\
\hline $\begin{array}{l}\text { Fourth subperiod: } \\
\text { Total ..................... }\end{array}$ & & .88 & 1.940 & 2.406 & $1: 14.7$ & $1: 6.7$ & $1: 5.5$ \\
\hline $\begin{array}{l}\text { Average } \\
\text { Fifth subperiod: }\end{array}$ & 13.1 & $\begin{array}{r}.892 \\
.892\end{array}$ & 1.977 & 2.386 & & 1.0 .1 & 1:0.0 \\
\hline $\begin{array}{l}\text { Total } \ldots \ldots \ldots \ldots \\
\text { Average } . . . \ldots \ldots\end{array}$ & $\begin{array}{r}627.32 \\
13.94\end{array}$ & $\begin{array}{r}41.301 \\
.918\end{array}$ & $\begin{array}{r}91.534 \\
2.034\end{array}$ & $\begin{array}{r}105.550 \\
2.346\end{array}$ & $1: 15.2$ & $1: 6.9$ & $1: 5.9$ \\
\hline $\begin{array}{l}\text { Sixth subperiod: } \\
\quad \text { Total } \ldots \ldots \ldots \ldots \ldots \\
\text { Average ....................... }\end{array}$ & $\begin{array}{r}610.04 \\
13.56\end{array}$ & $\begin{array}{r}43.892 \\
.975\end{array}$ & $\begin{array}{r}90.082 \\
2.002\end{array}$ & $\begin{array}{r}101.678 \\
2.259\end{array}$ & $\begin{array}{l}1: 13.9 \\
1: 13 \ldots\end{array}$ & $1: 6.8$ & $1: 6.0$ \\
\hline 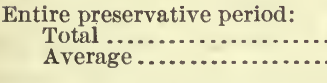 & $\begin{array}{r}3,648.62 \\
13.51 \\
\end{array}$ & $\begin{array}{r}244.710 \\
.906 \\
\end{array}$ & $\begin{array}{r}535.212 \\
1.982 \\
\end{array}$ & $\begin{array}{r}640.996 \\
2.374\end{array}$ & $\begin{array}{r}1: 14.9 \\
\ldots \ldots \ldots\end{array}$ & $1: 6.8$ & $\begin{array}{r}1: 5.7 \\
\cdots \cdots \\
\end{array}$ \\
\hline After period. & & & & & & & \\
\hline $\begin{array}{l}\text { First subperiod: } \\
\quad \text { Total } \ldots \ldots \ldots \ldots \ldots \\
\text { Average } \ldots \ldots \ldots \ldots\end{array}$ & $\begin{array}{r}610.31 \\
13.57\end{array}$ & $\begin{array}{r}41.014 \\
.911\end{array}$ & $\begin{array}{r}87.625 \\
1.947\end{array}$ & $\begin{array}{r}93.951 \\
2.088\end{array}$ & $\begin{array}{r}1: 14.9 \\
\ldots \ldots \ldots\end{array}$ & 1::7.0 & $1: 6.5$ \\
\hline 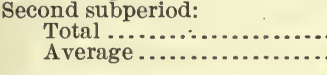 & $\begin{array}{r}610.58 \\
13.57\end{array}$ & $\begin{array}{r}42.176 \\
.937\end{array}$ & $\begin{array}{r}91.733 \\
2.039\end{array}$ & $\begin{array}{r}102.331 \\
2.274\end{array}$ & $1: 14.3$ & $1: 6.7$ & $1: 6.0$ \\
\hline $\begin{array}{l}\text { Entire after period: } \\
\quad \text { Total ............... } \\
\text { Average ......... }\end{array}$ & $\begin{array}{r}1,220.89 \\
13.57\end{array}$ & $\begin{array}{r}83.190 \\
.924\end{array}$ & $\begin{array}{r}179.358 \\
1.993\end{array}$ & $\begin{array}{r}196.282 \\
2.181\end{array}$ & $1: 14.7$ & $1: 6.8$ & $1: 6.2$ \\
\hline
\end{tabular}

CHANGES IN THE RELATIVE QUANTITIES OF SULPHUR COMPOUNDS EXCRE'TED IN THE URINE.

The changes which the urine may undergo in respect of its relative content of sulphur compounds are of great physiological importance.

For the purpose of determining the extent of any such changes an elaborate study was made of the total sulphur, sulphates, and other sulphur compounds excreted in the urine. To this end not only was the total sulphur determined, but also the amount occurring naturally as sulphates and that occurring as ethereal compounds of sulphur or as neutral sulphur. The data were compared with the respective quantities of sulphur administered in the food and the ratio of ethereal 
to inorganic sulphates determined as well as the ratio of the sulphur in different forms to the nitrogen in the urine. The particular object in view was to determine whether or not the total quantity of sulphur in the urine was affected by the administration of the preservative and whether the kinds of sulphur, as related to each other, were changed in any definite proportions. This required the determination of the total nitrogen in the urine, the total sulphur, total sulphur as $\mathrm{SO}_{3}$, neutral sulphur as $\mathrm{SO}_{3}$, total sulphuric acid as $\mathrm{SO}_{3}$, ethereal sulphates as $\mathrm{SO}_{3}$, inorganic sulphates as $\mathrm{SO}_{3}$, the ratio of the ethereal sulphates to the inorganic sulphates and the calculation of the percentage relations of the various kinds of sulphur to the total sulphur, namely, the percentage of neutral sulphur as $\mathrm{SO}_{3}$, total sulphur as $\mathrm{SO}_{3}$, and ethereal sulphates as $\mathrm{SO}_{3}$.

The importance of the urine as an index of changes in metabolic activity is fully realized and, without minimizing the importance of the constitution of the feces or of the other excretions, it is sufficient to call attention to the greater magnitude of the urine excretions and to their greater significance in relation to the metabolized products of the food elements.

The individual and summarized data on the relation of the preformed sulphates to the ethereal sulphates and neutral sulphur are given in Table $\mathrm{X}$.

INDIVIDUAL DATA.

No. 1.

Inasmuch as the quantities of sulphur in the food vary slightly in the different periods, it is best to base the discussion upon the percentage of the total sulphur occurring under the various forms rather than upon the ratios alone. The quantities excreted, however, and the ratios which have been determined are stated in the table, so that full information respecting the whole matter may be available. In the case of No. 1 the percentage of sulphur occurring in the neutral state is diminished in the preservative period, while it is very notably increased in the after period. The percentage of total sulphur occurring as sulphates is slightly increased in the preservative period and notably diminished in the after period. The percentage of total sulphur occurring as ethereal sulphates is increased in the preservative period and somewhat diminished in the after period, but not to the minimum of the fore period. The percentage of total sulphur as inorganic sulphates is the same in the preservative period as in the fore period and is slightly diminished in the after period.

No. 2.

In the case of No. 2 there is an increase in the percentage of neutral sulphur in the preservative period and a still further increase in the after period. This is attended with a decrease in the percentage of 
total sulphates both in the preservative and after periods. There is also a decrease in the ethereal sulphur in the preservative period and this decrease is continued in the after period. The percentage of inorganic sulphates is diminished in the preservative period and still further diminished in the after period.

\section{No. 3.}

The neutral sulphur is increased in the preservative period and very notably increased in the after period, while the percentage of total sulphates decreases in both periods. The ethereal sulphur is very low in this case and slightly higher in the preservative period than in either the fore or after period. The percentage of inorganic sulphur decreases slightly in the preservative period and very notably in the after period:

\section{No. 4.}

There is a slight increase in the neutral sulphur in the preservative period and a notable increase therein in the after period, with corresponding inverse changes in the percentage of sulphur as sulphates. The percentage of ethereal sulphates is increased in the preservative period, with a slight loss in the after period. The inorganic sulphates show a loss in the preservative period and a still further slight loss in the after period.

No. 5 .

There is a notable increase in this case of the neutral sulphur in the preservative period and a still further slight increase in the after period, with corresponding inverse changes in the total sulphur as sulphates. The percentage of ethereal sulphates is slightly diminished in the preservative period and still further diminished in the after period. The percentage of inorganic sulphates is notably diminished in the preservative period and again slightly decreased in the after period.

No. 6 .

The percentage of neutral sulphur is slightly diminished in the preservative period, but very notably increased in the after period, with correspending inverse changes in the percentage of total sulphates present. The percentage of ethereal sulphates remains practically unchanged throughout the three periods. There is a slight increase in the percentage of inorganic sulphates in the preservative period and a notable decrease therein in the after period.

No. $\%$.

This case shows an extraordinary increase in the neutral sulphur in the preservative period. This increase is nearly all lost in the after period, where the percentage is only slightly greater than the fore 
period. There are corresponding inverse changes in the percentage of total sulphates present. The percentage of ethereal sulphates is slightly increased in the preservative period, but falls in the after period to a lower number than in the fore period. The percentage of inorganic sulphates is somewhat low to begin with, but there is a very great loss in the preservative period which is not quite wholly restored in the after period.

\section{No. 8 .}

There is a notable loss in this case in the percentage of neutral sulphur in the preservative period, and this loss is partially restored in the after period, with corresponding inverse changes in the percentage of total sulphates present. There is a slight increase in the percentage of ethereal sulphates in the preservative period, but in the after period the number falls below that of the fore period. The percentage of inorganic sulphates is very low, being slightly greater in the preservative and after periods than in the fore period.

No. 9.

There is an increase in the percentage of neutral sulphur in the preservative period, which is partially lost in the after period, with corresponding inverse changes in the percentage of total sulphates present. In the percentage of ethereal sulphates there is a loss in the preservative period, and this loss is further increased in the after period. There is but little change in the percentage of inorganic sulphur present in the three periods, a slight decrease occurring in the preservative period and an increase in the after period.

No. 10.

There is an increase in the percentage of neutral sulphur in the preservative period in this case and a corresponding decrease in the total sulphates. There is also a slight increase in the percentage of ethereal sulphates and a decrease in the percentage of inorganic sulphates. The data for the after period are incomplete.

No. 11.

There is a decrease in the percentage of neutral sulphates in this case in the preservative period and a very large increase in the after period, with corresponding inverse changes in the percentage of total sulphates present. The ethereal sulphates are remarkably low in this case and the percentage is slightly higher in the preservative period than in either of the others. There is a slight increase in the percentage of inorganic sulphates in the preservative period and a notable loss in the after period. 
No. 12.

In this case there is a decrease in the percentage of neutral sulphur in the preservative period, while in the after period the loss is partially restored, with corresponding inverse changes in the percentage of total sulphates. There is little difference in the percentage of ethereal sulphates in the fore and preservative periods and a notable loss in the after period. The percentage of inorganic sulphates is slightly increased in the preservative period and is almost the same in the after as in the preservative period.

SUMMARY FOR NINE MEN.

It is seen that the average quantity of sulphur exhibited in the foods is greater in the preservative period than in the fore period, and decreases in the after period (Table XVII, p. 645). This fact must be taken into consideration in the study of the table in regard to the actual weight of the different kinds of sulphur found in the urine, and also in connection with the ratio of the ethereal to the inorganic sulphates. This ratio, it is seen, in general is almost $1: 11$, being slightly less in the preservative period than in either of the others. The percentage of the total sulphur occurring as neutral sulphur is 0.7 greater in the preservative period than in the fore period, and the increase is much more marked in the after period, amounting to 1.3 per cent. There is a corresponding decrease in the percentage of total sulphates, since the neutral sulphur and the total sulphates make up the whole quantity of sulphur. The ethereal sulphates and the inorganic sulphates, expressed as $\mathrm{SO}_{3}$, together make up the total sulphates. It is seen that there is a slight increase in the percentage of ethereal sulphates in the preservative period, while in the after period the percentage of ethereal sulphates falls slightly below that of the fore period. There is a slight decrease in the inorganic sulphates in the preservative period, and a still further decrease of about the same magnitude occurs in the after period.

A general summary of the data shows that the administration of the salicylic acid produces a well-marked tendency to increase the percentage of neutral sulphur with a corresponding decrease of total sulphates during the administration of the preservative, and that this tendency is continued, as might well be expected, in the after period. On the other hand, the administration of the salicylic acid appears to have had no notable effect in disturbing the relative percentages of ethereal sulphates and the inorganic sulphates in the urine. It must be admitted, therefore, in the light of these data, that the principal disturbing effect of the preservative has been upon the relative proportion of neutral sulphur excreted. 
TABLE X.-Urine determinations-Ratio of preformed sulphates to ethereal sulphates and neutral sulphur-Series VI.

[Averages are per day.]

No. 1.

\begin{tabular}{|c|c|c|c|c|c|c|c|c|c|c|c|}
\hline \multirow[b]{2}{*}{ Period. } & \multirow[b]{2}{*}{ 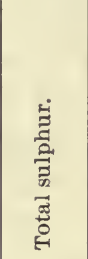 } & \multirow{2}{*}{ 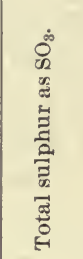 } & \multirow{2}{*}{ 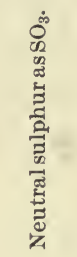 } & \multirow[b]{2}{*}{ 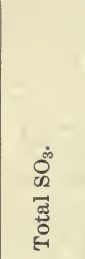 } & \multirow{2}{*}{ 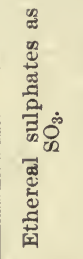 } & \multirow{2}{*}{ 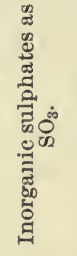 } & \multirow{2}{*}{ 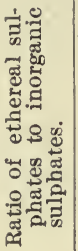 } & \multicolumn{4}{|c|}{$\begin{array}{l}\text { Results expressed in per } \\
\text { cent of total sulphur } \\
\text { in terms of } \mathrm{SO}_{3} \text {. }\end{array}$} \\
\hline & & & & & & & & 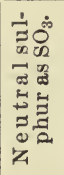 & 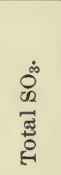 & 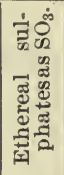 & 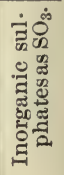 \\
\hline $\begin{array}{l}\text { Fore period. } \\
\text { First subperiod: } \\
\text { Total } \ldots . . . . . . . . \\
\text { Average........ }\end{array}$ & $\begin{array}{r}\text { Grams. } \\
4.002 \\
.800\end{array}$ & $\begin{array}{r}\text { Grams. } \\
9.993 \\
1.998\end{array}$ & $\begin{array}{r}\text { Grams. } \\
1.319 \\
.263\end{array}$ & $\begin{aligned} \text { Grams. } \\
8.674 \\
1.735\end{aligned}$ & $\begin{array}{r}\text { Grams. } \\
0.867 \\
.173\end{array}$ & $\begin{array}{r}\text { Grams. } \\
7.807 \\
1.562\end{array}$ & $1: 9.0$ & $\begin{array}{l}\text { P. ct. } \\
13.2\end{array}$ & $\begin{array}{l}\text { P. ct. } \\
86.8\end{array}$ & $\begin{array}{r}\text { P. } c t \text {. } \\
8.7\end{array}$ & $\begin{array}{r}\text { P. ct. } \\
78.1\end{array}$ \\
\hline $\begin{array}{l}\text { Second subperiod: } \\
\text { Total ............. } \\
\text { Average ........ }\end{array}$ & $\begin{array}{r}4.252 \\
.850\end{array}$ & $\begin{array}{r}10.617 \\
2.122\end{array}$ & $\begin{array}{l}.817 \\
.162\end{array}$ & $\begin{array}{l}9.800 \\
1.960\end{array}$ & $\begin{array}{r}.989 \\
.198 \\
\end{array}$ & $\begin{array}{l}8.811 \\
1.762\end{array}$ & $1: 8.9$ & 7.7 & 92.3 & 9.3 & 83.0 \\
\hline $\begin{array}{l}\text { Entire fore period: } \\
\text { Total } \\
\text { Average } . . . . . . . .\end{array}$ & $\begin{array}{r}8.254 \\
.825\end{array}$ & $\begin{array}{r}20.610 \\
2.060\end{array}$ & $\begin{array}{r}2.136 \\
.213 \\
\end{array}$ & $\begin{array}{r}18.474 \\
1.847 \\
\end{array}$ & $\begin{array}{r}1.856 \\
.186\end{array}$ & $\begin{array}{r}16.618 \\
1.661\end{array}$ & $1: 9.0$ & 10.4 & 89.6 & 9.0 & 80.6 \\
\hline Preservative period. & & & & & & & & & & & \\
\hline $\begin{array}{l}\text { First subperiod: } \\
\text { Total .............. } \\
\text { Average ....... }\end{array}$ & $\begin{array}{r}4.415 \\
.883\end{array}$ & $\begin{array}{r}11.024 \\
2.205\end{array}$ & $\begin{array}{r}1.224 \\
.245\end{array}$ & $\begin{array}{l}9.800 \\
1.960\end{array}$ & $\begin{array}{r}1.069 \\
.214\end{array}$ & $\begin{array}{l}8.731 \\
1.746\end{array}$ & $1: 8.2$ & 11.1 & 88.9 & 9.7 & 79.2 \\
\hline $\begin{array}{l}\text { lit subperiod: } \\
\text { tal ............. } \\
\text { erage }\end{array}$ & $\begin{array}{r}4.654 \\
.931\end{array}$ & $\begin{array}{r}11.621 \\
2.325\end{array}$ & $\begin{array}{r}1.382 \\
.277\end{array}$ & $\begin{array}{r}10.239 \\
2.048\end{array}$ & $\begin{array}{l}.992 \\
.198\end{array}$ & $\begin{array}{l}9.247 \\
1.850\end{array}$ & $1: 9.3$ & 11.9 & 88.1 & 8.5 & 79.6 \\
\hline $\begin{array}{r}\text { Third } \\
\text { To }\end{array}$ & $\begin{array}{r}4.376 \\
.875\end{array}$ & $\begin{array}{r}10.927 \\
2.185\end{array}$ & $\begin{array}{l}.953 \\
.191\end{array}$ & $\begin{array}{l}9.974 \\
1.995\end{array}$ & $\begin{array}{r}1.040 \\
.208\end{array}$ & $\begin{array}{l}8.934 \\
1.787\end{array}$ & $1: 8.6$ & 8.7 & 91.3 & 9.5 & 81.8 \\
\hline 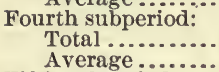 & $\begin{array}{r}4.720 \\
.944\end{array}$ & $\begin{array}{r}11.786 \\
2.357\end{array}$ & $\begin{array}{r}1.444 \\
.289\end{array}$ & $\begin{array}{r}10.342 \\
2.068\end{array}$ & $\begin{array}{r}1.091 \\
.218\end{array}$ & $\begin{array}{l}9.251 \\
1.850\end{array}$ & $1: 8.5$ & 12.3 & 87.7 & 9.3 & 78.5 \\
\hline $\begin{array}{r}\text { Fifth subperiod: } \\
\quad \text { Total } \ldots . . . . . . . . \\
\text { Average............ }\end{array}$ & $\begin{array}{r}4.429 \\
.886\end{array}$ & $\begin{array}{r}11.059 \\
2.212\end{array}$ & $\begin{array}{l}.520 \\
.104\end{array}$ & $\begin{array}{r}10.539 \\
2.108\end{array}$ & $\begin{array}{r}1.162 \\
.232\end{array}$ & $\begin{array}{l}9.377 \\
1.875\end{array}$ & $1: 8.1$ & 4.7 & 95.3 & 10.5 & 84.8 \\
\hline 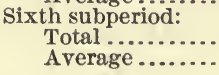 & $\begin{array}{r}4.621 \\
.924\end{array}$ & $\begin{array}{r}11.539 \\
2.308\end{array}$ & $\begin{array}{r}1.037 \\
.207\end{array}$ & $\begin{array}{r}10.502 \\
2.100\end{array}$ & $\begin{array}{r}1.283 \\
.257\end{array}$ & $\begin{array}{l}9.219 \\
1.844\end{array}$ & $1: 7.2$ & 9.0 & 91.0 & 11.1 & 79.9 \\
\hline $\begin{array}{l}\text { Entire preservative } \\
\text { period: } \\
\text { Total ............. } \\
\text { Average........ }\end{array}$ & $\begin{array}{r}27.215 \\
.907 \\
\end{array}$ & $\begin{array}{r}67.956 \\
2.266\end{array}$ & $\begin{array}{r}6.560 \\
.219\end{array}$ & $\begin{array}{r}61.396 \\
2.047\end{array}$ & $\begin{array}{r}6.637 \\
.221\end{array}$ & $\begin{array}{r}54.759 \\
1.825\end{array}$ & $1: 8.3$ & 9.7 & 90.3 & 9.8 & 80.6 \\
\hline After period. & & & & & & & & & & & \\
\hline $\begin{array}{l}\text { First subperiod: } \\
\text { Total............ } \\
\text { Average ........ }\end{array}$ & $\begin{array}{r}4.580 \\
.916\end{array}$ & $\begin{array}{r}11.436 \\
2.287\end{array}$ & $\begin{array}{r}1.286 \\
.257\end{array}$ & $\begin{array}{r}10.150 \\
2.030\end{array}$ & $\begin{array}{r}1.100 \\
.220\end{array}$ & $\begin{array}{l}9.050 \\
1810\end{array}$ & $1: 8.2$ & 11.2 & 88.8 & 9.6 & 79.1 \\
\hline $\begin{array}{l}\text { Second subperiod: } \\
\text { Total ............... } \\
\text { Average........ }\end{array}$ & $\begin{array}{r}4.771 \\
.954\end{array}$ & $\begin{array}{r}11.913 \\
2.382\end{array}$ & $\begin{array}{r}1.634 \\
.326\end{array}$ & $\begin{array}{r}10.279 \\
2.056\end{array}$ & $\begin{array}{r}1.081 \\
.216\end{array}$ & $\begin{array}{l}9.198 \\
1.840\end{array}$ & $\begin{array}{l}1: 8.5 \\
\ldots . .\end{array}$ & 13.7 & 86.3 & 9.1 & 77.2 \\
\hline $\begin{array}{r}\text { Entire after period: } \\
\text { Total ............. } \\
\text { Average........ }\end{array}$ & $\begin{array}{r}9.351 \\
.935\end{array}$ & $\begin{array}{r}23.349 \\
2.335\end{array}$ & $\begin{array}{r}2.920 \\
.292\end{array}$ & $\begin{array}{r}20.429 \\
2.043\end{array}$ & $\begin{array}{r}2.181 \\
.218\end{array}$ & $\begin{array}{r}18.248 \\
1.825\end{array}$ & $1: 8.4$ & 12.5 & 87.5 & 9.3 & 78.2 \\
\hline
\end{tabular}


TABLE X.-Urine determinations-Ratio of preformed sulphates to ethereal sulphates and neutral sulphur-Series VI-Continued.

[Averages are per day.]

No. 2 .

\begin{tabular}{|c|c|c|c|c|c|c|c|c|c|c|c|}
\hline \multirow[b]{2}{*}{ Period. } & \multirow[b]{2}{*}{ 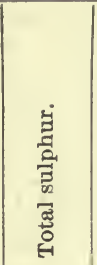 } & \multirow{2}{*}{ 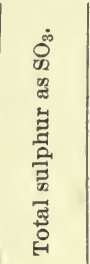 } & \multirow{2}{*}{ 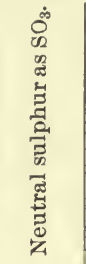 } & \multirow[b]{2}{*}{ 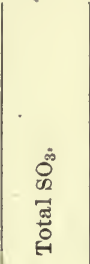 } & \multirow{2}{*}{ 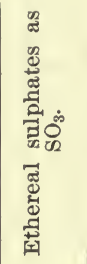 } & \multirow{2}{*}{ 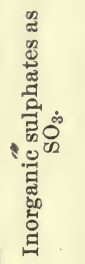 } & \multirow{2}{*}{ 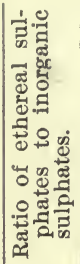 } & \multicolumn{4}{|c|}{$\begin{array}{l}\text { Results expressed in } \\
\text { per cent of total sul- } \\
\text { phur in terms of } \mathrm{SO}_{3} \text {. }\end{array}$} \\
\hline & & & & & & & & 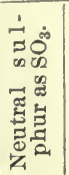 & 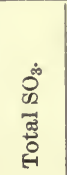 & 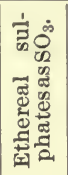 & 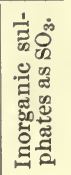 \\
\hline \multicolumn{12}{|l|}{ Fore period. } \\
\hline $\begin{array}{l}\text { First subperiod: } \\
\text { Total ............. } \\
\text { Average....... }\end{array}$ & $\begin{array}{r}\text { Grams. } \\
5.448 \\
1.090\end{array}$ & $\begin{array}{c}\text { Grams. } \\
13.604 \\
2.721\end{array}$ & $\begin{array}{r}\text { Grams. } \\
1.588 \\
.318\end{array}$ & $\begin{array}{r}\text { Grams. } \\
12.016 \\
2.403\end{array}$ & $\begin{array}{r}\text { Grams. } \\
1.235 \\
.247\end{array}$ & $\begin{array}{c}\text { Grams. } \\
10.781 \\
2.156\end{array}$ & $1: 8.7$ & $\begin{array}{l}\text { P.ct. } \\
11.7\end{array}$ & $\begin{array}{l}\text { P. ct. } \\
88.3\end{array}$ & $\begin{array}{r}P . c t . \\
9.1\end{array}$ & $\begin{array}{r}\text { P. } c t . \\
79.2\end{array}$ \\
\hline Second subperiod: & & & & & & & & & & & ...... \\
\hline Total ................. & $\begin{array}{r}4.643 \\
.929\end{array}$ & $\begin{array}{r}11.594 \\
2.320\end{array}$ & $\begin{array}{r}1.140 \\
229\end{array}$ & $\begin{array}{r}10.454 \\
2.091\end{array}$ & 1.154 & 9.300 & $1: 8.1$ & 9.8 & 90.2 & 10.0 & 80.2 \\
\hline Average......... & & & & & & & & $\cdots \cdots$ & $\cdots \cdot \cdot$ & ….... & $\cdots \cdots$ \\
\hline $\begin{array}{c}\text { Entire fore period: } \\
\text { Total ............ }\end{array}$ & 10.091 & 25.198 & 2.728 & 22.470 & 2.389 & 20.081 & $1: 8.4$ & 10.8 & 89.2 & 9.5 & 79.7 \\
\hline Average ......... & 1.009 & 2.520 & .273 & 2.247 & .239 & 2.008 & $\ldots \ldots \ldots$ & $\ldots \ldots$ & $\ldots \ldots$ & $\ldots \ldots$ & $\cdots \cdots$ \\
\hline \multicolumn{12}{|l|}{ Preservative period. } \\
\hline $\begin{array}{l}\text { First subperiod: } \\
\text { Total ............. }\end{array}$ & 4. 621 & 11. 539 & 1.568 & 9.971 & 1.143 & 8.828 & $1: 7.7$ & 13.6 & 86.4 & 9.9 & 76.5 \\
\hline $\begin{array}{l}\text { Average ......... } \\
\text { Second subperiod: }\end{array}$ & .924 & 2.307 & .313 & 1.994 & .229 & 1.765 & ..... & ...... & ...... & 0 & ...... \\
\hline Total ............. & 4.517 & 11.279 & 1.098 & 10.181 & i. 018 & 9.163 & $1: 9.0$ & 9.7 & 90.3 & 9.0 & 81,2 \\
\hline $\begin{array}{l}\text { Averag } \\
\text { hird subp }\end{array}$ & .903 & 2.255 & .219 & 2.036 & .204 & 1.832 & & $\cdots \cdot \cdot$ & $\cdots \cdot \cdot$ & & $\begin{array}{r}81.2 \\
\ldots . . .\end{array}$ \\
\hline $\begin{array}{l}\text { Third subpe } \\
\text { Total .. }\end{array}$ & 5.108 & 12.755 & 1.920 & & 1.120 & 9.715 & & & & & \\
\hline Average .............. & 1.022 & 2.552 & .385 & 2.167 & $\begin{array}{r}1.224 \\
.224\end{array}$ & $\begin{array}{l}9.715 \\
1.943\end{array}$ & $1: 8.7$ & 15.1 & 84.9 & 8.8 & 76.2 \\
\hline $\begin{array}{l}\text { Fourth subperiod: } \\
\text { Total ........... }\end{array}$ & & 276 & & & & & & & $\cdots \cdots$ & ...... & $\ldots \ldots$ \\
\hline Average...... & $\begin{array}{r}4.516 \\
.903\end{array}$ & $\begin{array}{r}11.276 \\
2.255\end{array}$ & $\begin{array}{l}.336 \\
.067\end{array}$ & $\begin{array}{r}10.940 \\
2.188\end{array}$ & $\begin{array}{r}1.061 \\
.212\end{array}$ & $\begin{array}{l}9.879 \\
1.976\end{array}$ & $1: 9.3$ & 3.0 & 97.0 & 9.4 & 87.6 \\
\hline $\begin{array}{l}\text { Fifth subperiod: } \\
\text { Total ........ }\end{array}$ & $5.212^{\circ}$ & & & & & 1.970 & & & ..... & .... & ....... \\
\hline Total. & $\begin{array}{l}5.212 \\
1.042\end{array}$ & $\begin{array}{r}13.014 \\
2.602\end{array}$ & $\begin{array}{r}1.418 \\
.283\end{array}$ & $\begin{array}{r}11.596 \\
2.319\end{array}$ & $\begin{array}{l}1.180 \\
236\end{array}$ & 10.416 & $1: 8.8$ & 10.9 & 89.1 & 9.1 & 80.0 \\
\hline $\begin{array}{l}\text { xth subpe } \\
\text { Total. }\end{array}$ & & & & & .236 & 2.083 & & & ..... & $\cdots \cdot$ & ....... \\
\hline Average........... & $\begin{array}{l}5.574 \\
1.115\end{array}$ & $\begin{array}{r}13.918 \\
2.784\end{array}$ & $\begin{array}{r}2.826 \\
.566\end{array}$ & $\begin{array}{r}11.092 \\
2.218\end{array}$ & 1. 130 & $\begin{array}{l}9.962 \\
1.992\end{array}$ & $1: 8.8$ & 20.2 & 79.8 & 8.1 & 71.6 \\
\hline \multicolumn{12}{|l|}{$\begin{array}{l}\text { Entire preservative } \\
\text { period: }\end{array}$} \\
\hline & & 73.781 & 9. 166 & 64.615 & 6652 & & & 1) 4 & & 90 & \\
\hline Average ......... & .985 & 2.460 & .306 & 2.154 & .222 & $\begin{array}{r}1.932 \\
1.932\end{array}$ & $\begin{array}{l}1.8 .7 \\
\ldots . . .\end{array}$ & 12.4 & $\begin{array}{l}87.6 \\
\ldots \ldots . .\end{array}$ & 9.0 & $\begin{array}{r}78.6 \\
\ldots \ldots\end{array}$ \\
\hline After period. & & & & & & & & & & & \\
\hline \multicolumn{10}{|l|}{$\begin{array}{l}\text { First subperiod: } \\
\text { Total ............ }\end{array}$} & & 78.7 \\
\hline $\begin{array}{l}\text { Average ......... } \\
\text { Second subperiod: }\end{array}$ & & 2.559 & & 2.234 & .222 & 2.012 & & & & & $\ldots . .$. \\
\hline Total ............. & 5. 268 & 13. 154 & 1.794 & 11.360 & 1.155 & 10.205 & $1: 8.8$ & 13.6 & 86.4 & 8.8 & 77.6 \\
\hline Average & 1.054 & 2.632 & .360 & 2.272 & .231 & 2.041 & & $\cdots$ & $\cdots \cdot$ & $\cdots$ & $\cdots$ \\
\hline $\begin{array}{l}\text { Entire after period: } \\
\text { Total ........... }\end{array}$ & 10.391 & 25.946 & 3.416 & 22.530 & 2.264 & 20.266 & $1: 9.0$ & 13.2 & 86.8 & 8.7 & 78.1 \\
\hline Average.... & 1.039 & 2.594 & .341 & 2.253 & .226 & 2.027 & & & & & \\
\hline
\end{tabular}


TABLE X.-Urine determinations-Rutio of preformed sulphates to ethereal sulphates and neutral sulphur-Series VI-Continued.

[Averages are per day.]

No. 3.

\begin{tabular}{|c|c|c|c|c|c|c|c|c|c|c|c|}
\hline \multirow[b]{2}{*}{ Period. } & \multirow[b]{2}{*}{ 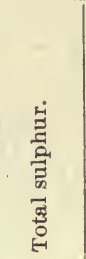 } & \multirow{2}{*}{ 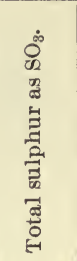 } & \multirow{2}{*}{ 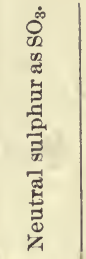 } & \multirow[b]{2}{*}{ 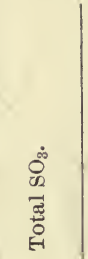 } & \multirow{2}{*}{ 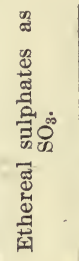 } & \multirow{2}{*}{ 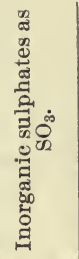 } & \multirow{2}{*}{ 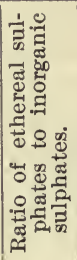 } & \multicolumn{4}{|c|}{$\begin{array}{l}\text { Results expressed in per } \\
\text { cent of total sulphur } \\
\text { in terms of } \mathrm{SO}_{3} \text {. }\end{array}$} \\
\hline & & & & & & & & 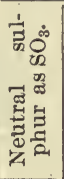 & 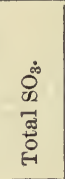 & 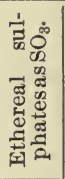 & 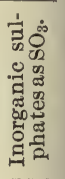 \\
\hline Fore period. & & & & & & & & & & & \\
\hline $\begin{array}{l}\text { First subperiod: } \\
\text { Total ............. } \\
\text { Average........ }\end{array}$ & Grams. & Grams. & Grams. & $\begin{array}{r}\text { Grams. } \\
\text { B }\end{array}$ & $\begin{array}{l}\text { Grams. } \\
\text { roken b }\end{array}$ & $\begin{array}{l}\text { Grams. } \\
\text { y illness. }\end{array}$ & & P.ct. & P.ct. & P.ct. & P.ct. \\
\hline $\begin{array}{l}\text { Second subperiod: } \\
\text { Total ............... } \\
\text { Average....... }\end{array}$ & $\begin{array}{r}4.126 \\
.825\end{array}$ & $\begin{array}{r}10.303 \\
2.060\end{array}$ & $\begin{array}{r}1.212 \\
.242\end{array}$ & $\begin{array}{l}9.091 \\
1.818\end{array}$ & $\begin{array}{r}0.670 \\
.134\end{array}$ & $\begin{array}{l}8.421 \\
1.684\end{array}$ & $\begin{array}{l}1: 12.6 \\
\ldots . . . . .\end{array}$ & $\begin{array}{c}11.8 \\
\ldots . .\end{array}$ & $\begin{array}{c}88.2 \\
\ldots \ldots\end{array}$ & 6.5 & $\begin{array}{r}81.7 \\
\ldots \ldots .\end{array}$ \\
\hline $\begin{array}{l}\text { Eutire foro period: } \\
\text { Total ............. } \\
\text { Average........ }\end{array}$ & $\begin{array}{r}4.126 \\
.825\end{array}$ & $\begin{array}{r}10.303 \\
2.060\end{array}$ & $\begin{array}{r}1.212 \\
.242\end{array}$ & $\begin{array}{l}9.091 \\
1.818\end{array}$ & $\begin{array}{r}0.670 \\
.134\end{array}$ & $\begin{array}{l}8.421 \\
1.684\end{array}$ & $\begin{array}{c}1: 12.6 \\
\ldots \ldots \ldots\end{array}$ & $\begin{array}{l}11.8 \\
\ldots . . .\end{array}$ & $\begin{array}{c}88.2 \\
\cdots . . .\end{array}$ & $\begin{array}{c}6.5 \\
\ldots . .\end{array}$ & 81.7 \\
\hline Preservative period. & & & & & & & & & & & \\
\hline $\begin{array}{l}\text { First subperiod: } \\
\text { Total ............ } \\
\text { Average........ }\end{array}$ & $\begin{array}{r}3.664 \\
.733\end{array}$ & $\begin{array}{l}9.149 \\
1.830\end{array}$ & $\begin{array}{r}1.184 \\
.237\end{array}$ & $\begin{array}{l}7.965 \\
1.593\end{array}$ & $\begin{array}{l}.520 \\
.104\end{array}$ & $\begin{array}{l}7.445 \\
1.489\end{array}$ & $\begin{array}{l}1: 14.3 \\
\ldots \ldots \ldots\end{array}$ & 12.9 & 87.1 & $5 . \dot{7}$ & 81.4 \\
\hline $\begin{array}{l}\text { Second s } \\
\text { Total } \\
\text { Aver }\end{array}$ & $\begin{array}{r}3.665 \\
.733\end{array}$ & $\begin{array}{l}9.151 \\
1.830\end{array}$ & $\begin{array}{l}.891 \\
.178\end{array}$ & $\begin{array}{l}8.260 \\
1.652\end{array}$ & $\begin{array}{l}.591 \\
.118\end{array}$ & $\begin{array}{l}7.669 \\
1.534\end{array}$ & 1:13.0 & 9.7 & $\begin{array}{l}90.3 \\
\ldots . .\end{array}$ & 6.5 & 83.8 \\
\hline $\begin{array}{c}\text { Third subperiod: } \\
\text { Total ............. } \\
\text { Average }\end{array}$ & $\begin{array}{r}3.588 \\
.718\end{array}$ & $\begin{array}{l}8.959 \\
1.793\end{array}$ & $\begin{array}{r}1.186 \\
237\end{array}$ & $\begin{array}{l}7.773 \\
1.555\end{array}$ & $\begin{array}{l}.676 \\
135\end{array}$ & $\begin{array}{l}7.097 \\
1.419\end{array}$ & $1: 10.5$ & 13.2 & 86.8 & 7.5 & 79.2 \\
\hline $\begin{array}{l}\text { Fourth subperiod: } \\
\text { Total .............. } \\
\text { Average....... }\end{array}$ & $\begin{array}{r}3.740 \\
.748\end{array}$ & $\begin{array}{l}9.339 \\
1.868\end{array}$ & $\begin{array}{r}1.198 \\
.240\end{array}$ & $\begin{array}{l}8.141 \\
1.628\end{array}$ & $\begin{array}{l}.656 \\
.131\end{array}$ & $\begin{array}{l}7.485 \\
1.497\end{array}$ & $1: 11.4$ & 12.8 & 87.2 & 7.0 & 80.1 \\
\hline $\begin{array}{l}\text { Fifth subperiod: } \\
\text { Total ............. } \\
\text { Average........ }\end{array}$ & $\begin{array}{r}3.699 \\
.740\end{array}$ & $\begin{array}{l}9.236 \\
1.848\end{array}$ & $\begin{array}{r}1.459 \\
.293\end{array}$ & $\begin{array}{l}7.777 \\
1.555\end{array}$ & $\begin{array}{l}.723 \\
.145\end{array}$ & $\begin{array}{l}7.054 \\
1.411\end{array}$ & $\begin{array}{c}1: 9.8 \\
\ldots .\end{array}$ & $\begin{array}{c}15.8 \\
\ldots \ldots .\end{array}$ & $\begin{array}{c}84.2 \\
\cdots \ldots . .\end{array}$ & $\begin{array}{l}7.8 \\
. . .\end{array}$ & $\begin{array}{r}76.4 \\
\ldots \ldots\end{array}$ \\
\hline $\begin{array}{l}\text { First, second, third, } \\
\text { fourth, and fifth } \\
\text { subperiods: } a \\
\text { Total ............. } \\
\text { Average........ }\end{array}$ & $\begin{array}{r}18.356 \\
.734\end{array}$ & $\begin{array}{r}45.834 \\
1.833\end{array}$ & $\begin{array}{r}5.918 \\
.237\end{array}$ & $\begin{array}{r}39.916 \\
1.597\end{array}$ & $\begin{array}{r}3.166 \\
.127\end{array}$ & $\begin{array}{r}36.750 \\
1.470\end{array}$ & $\begin{array}{c}1: 11.6 \\
\ldots \ldots \ldots\end{array}$ & $\begin{array}{c}12.9 \\
\ldots \ldots .\end{array}$ & $\begin{array}{c}87.1 \\
\cdots \ldots . .\end{array}$ & $\begin{array}{c}6.9 \\
\ldots . .\end{array}$ & $\begin{array}{r}80.2 \\
\ldots \ldots .\end{array}$ \\
\hline After period. & & & & & & & & & & & \\
\hline $\begin{array}{l}\text { First subperiod: } \\
\text { Total ............ } \\
\text { Average }\end{array}$ & $\begin{array}{r}3.619 \\
.724\end{array}$ & $\begin{array}{r}9.037 \\
1.807\end{array}$ & $\begin{array}{r}1.557 \\
311\end{array}$ & $\begin{array}{l}7.480 \\
1.496\end{array}$ & $\begin{array}{r}.532 \\
.106\end{array}$ & $\begin{array}{r}6.948 \\
1.390\end{array}$ & $1: 13.1$ & 17.2 & 82.8 & 5.9 & 76.9 \\
\hline $\begin{array}{l}\text { Second subperiod: } \\
\text { Total ............. } \\
\text { Average........ }\end{array}$ & $\begin{array}{r}4.013 \\
.803\end{array}$ & $\begin{array}{r}10.020 \\
2.005\end{array}$ & $\begin{array}{r}1.632 \\
.327\end{array}$ & $\begin{array}{l}8.388 \\
1.678\end{array}$ & $\begin{array}{l}.679 \\
.136\end{array}$ & $\begin{array}{l}7.709 \\
1.542\end{array}$ & $\begin{array}{l}1: 11.4 \\
\ldots \ldots . .\end{array}$ & $\begin{array}{c}16.3 \\
\cdots \cdots\end{array}$ & $\begin{array}{c}83.7 \\
\ldots . . .\end{array}$ & 6.8 & $\begin{array}{l}76.9 \\
\ldots . .\end{array}$ \\
\hline $\begin{array}{l}\text { Entire after period: } \\
\text { Total ............. } \\
\text { Average........ }\end{array}$ & $\begin{array}{r}7.632 \\
.763\end{array}$ & $\begin{array}{r}19.057 \\
1.906\end{array}$ & $\begin{array}{r}3.189 \\
.319\end{array}$ & $\begin{array}{r}15.868 \\
1.587\end{array}$ & $\begin{array}{r}1.211 \\
.121\end{array}$ & $\begin{array}{r}14.657 \\
1.466\end{array}$ & $1: 12.1$ & 16.7 & 83.3 & 6.4 & 76.9 \\
\hline
\end{tabular}

$a$ No. 3 had only five preservative subperiods. 
TABLE X.-Urine determinalions-Ratio of preformed sulphates to ethereal sulphates and neutral sulphur-Series VI-Continued.

[Averages are per day.]

No. 4.

\begin{tabular}{|c|c|c|c|c|c|c|c|c|c|c|c|}
\hline \multirow[b]{2}{*}{ Period. } & \multirow[b]{2}{*}{ 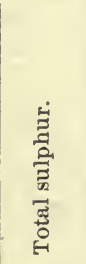 } & \multirow{2}{*}{ 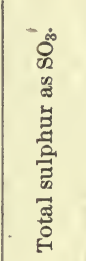 } & \multirow{2}{*}{ 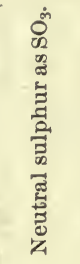 } & \multirow[b]{2}{*}{ 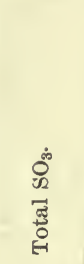 } & \multirow{2}{*}{ 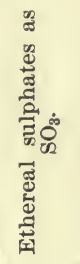 } & \multirow{2}{*}{ 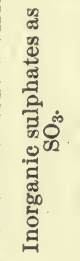 } & \multirow{2}{*}{ 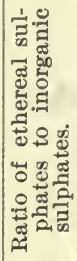 } & \multicolumn{4}{|c|}{$\begin{array}{l}\text { Results expressed in per } \\
\text { cent of total sulphur } \\
\text { in terms of } \mathrm{SO}_{3} \text {. }\end{array}$} \\
\hline & & & & & & & & 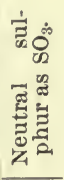 & 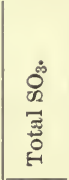 & 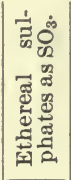 & 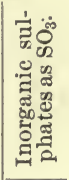 \\
\hline Fore period. & & & & & & & & & & & \\
\hline $\begin{array}{l}\text { First subperiod: } \\
\text { Total ............ } \\
\text { Average....... }\end{array}$ & $\begin{array}{r}\text { Grams. } \\
4.905 \\
.981\end{array}$ & $\begin{array}{r}\text { Grams. } \\
12.248 \\
2.450\end{array}$ & $\begin{array}{r}\text { Grams. } \\
1.529 \\
.306\end{array}$ & $\begin{array}{r}\text { Grams. } \\
10.719 \\
2.144\end{array}$ & $\begin{array}{r}\text { Grams. } \\
0.694 \\
.139\end{array}$ & $\begin{array}{r}\text { Grams } \\
10.025 \\
2.005\end{array}$ & $1: 14.4$ & $\begin{array}{l}P . c t . \\
12.5\end{array}$ & $\begin{array}{l}\text { P. ct. } \\
87.5 \\
. . . .\end{array}$ & P. ct. & $\begin{array}{l}\text { P. ct. } \\
81.9 \\
\text {. . . . }\end{array}$ \\
\hline Second subperiod: & & & & & & & & & & & \\
\hline 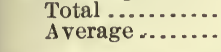 & $\begin{array}{r}4.574 \\
.915\end{array}$ & $\begin{array}{r}11.421 \\
2.285\end{array}$ & $\begin{array}{l}.697 \\
.140 \\
\end{array}$ & $\begin{array}{r}10.724 \\
2.145\end{array}$ & $\begin{array}{l}.803 \\
.161\end{array}$ & $\begin{array}{l}9.921 \\
1.984\end{array}$ & $\begin{array}{l}1: 12.4 \\
\cdots \cdots\end{array}$ & $\begin{array}{c}6.1 \\
\cdots \cdots\end{array}$ & $\begin{array}{l}93.9 \\
\cdots \ldots\end{array}$ & $\begin{array}{c}7.0 \\
\cdots \\
\cdots\end{array}$ & $\begin{array}{r}86.9 \\
\cdots\end{array}$ \\
\hline $\begin{array}{l}\text { Entire fore period: } \\
\text { Total .......... } \\
\text { Average ........ }\end{array}$ & $\begin{array}{r}9.479 \\
.948 \\
\end{array}$ & $\begin{array}{r}23.669 \\
2.367 \\
\end{array}$ & $\begin{array}{r}2.226 \\
.223 \\
\end{array}$ & $\begin{array}{r}21.443 \\
2.144 \\
\end{array}$ & $\begin{array}{r}1.497 \\
.150\end{array}$ & $\begin{array}{r}19.946 \\
1.994 \\
\end{array}$ & $\begin{array}{l}1: 13.3 \\
\cdots \ldots\end{array}$ & $\begin{array}{r}9.4 \\
\cdots \cdots \\
\end{array}$ & $\begin{array}{r}90.6 \\
\cdots \cdots \\
\end{array}$ & $\begin{array}{r}6.3 \\
\cdots \cdots \\
\end{array}$ & $\begin{array}{r}84.3 \\
\cdots \\
\end{array}$ \\
\hline Preservative period. & & & & & & & & & & & \\
\hline $\begin{array}{l}\text { First subperiod: } \\
\text { Total ........... } \\
\text { Average....... }\end{array}$ & $\begin{array}{r}4.444 \\
.889\end{array}$ & $\begin{array}{r}11.097 \\
2.220\end{array}$ & $\begin{array}{l}.829 \\
.166\end{array}$ & $\begin{array}{r}10.268 \\
2.054\end{array}$ & $\begin{array}{l}.905 \\
.181\end{array}$ & $\begin{array}{l}9.363 \\
1.873\end{array}$ & $\begin{array}{l}1: 10.3 \\
\ldots \ldots\end{array}$ & 7.5 & 92.5 & 8.2 & 84.4 \\
\hline Second subperiod: & & & & & & & & & & & \\
\hline $\begin{array}{l}\text { Total ............... } \\
\text { Average....... }\end{array}$ & $\begin{array}{l}4.724 \\
945\end{array}$ & 11.796 & 1. 121 & 10.675 & .783 & 9.892 & $1: 12.6$ & 9.5 & 90.5 & 6.6 & 83.9 \\
\hline Third subperiod: & .940 & 2.360 & .220 & 2.130 & .107 & 1.978 & & ....... & ........ & ....... & ........ \\
\hline $\begin{array}{l}\text { Total } \ldots \ldots \ldots \\
\text { Average } \ldots\end{array}$ & $\begin{array}{r}4.548 \\
.910\end{array}$ & $\begin{array}{r}11.356 \\
2.272\end{array}$ & $\begin{array}{r}1.089 \\
.219\end{array}$ & $\begin{array}{r}10.267 \\
2.053\end{array}$ & $\begin{array}{l}.789 \\
.158\end{array}$ & $\begin{array}{l}9.478 \\
1.895\end{array}$ & $\begin{array}{l}1: 12.0 \\
\ldots\end{array}$ & 9.6 & 90.4 & 6.9 & 83.5 \\
\hline Fourth subperiod: & & & & & & & & $\ldots$ & & . & 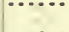 \\
\hline 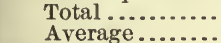 & $\begin{array}{l}4.559 \\
912\end{array}$ & 11.384 & 1. 160 & 10.224 & .758 & 9.466 & $1: 12.5$ & 10.2 & 89.8 & 6.7 & 83.2 \\
\hline Fifth subperiod: & .912 & 2.216 & .202 & 2.040 & .132 & 1.095 & & & & & $\cdots$ \\
\hline $\begin{array}{l}\text { Total } . . . . . . . \\
\text { Average........ }\end{array}$ & $\begin{array}{r}4.299 \\
860\end{array}$ & 10.735 & 1.009 & 9. 726 & .717 & 9.009 & $1: 12.6$ & 9.4 & 90.6 & 6.7 & 83.9 \\
\hline Sixth subperiod: & .860 & 2.147 & .202 & 1.945 & .143 & 1.802 & & ........ & $\cdots \ldots$ & ....... & ...... \\
\hline $\begin{array}{l}\text { Total } \\
\text { Average.............. }\end{array}$ & $\begin{array}{r}4.766 \\
.953\end{array}$ & $\begin{array}{r}11.901 \\
2.380\end{array}$ & $\begin{array}{r}1.510 \\
.302\end{array}$ & $\begin{array}{r}10.391 \\
2.078\end{array}$ & $\begin{array}{l}.865 \\
.173\end{array}$ & $\begin{array}{l}9.526 \\
1.905\end{array}$ & $1: 11.0$ & 12.7 & 87.3 & 7.3 & 80.0 \\
\hline & & & & & & & & ….... & $\cdots$ & 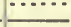 & $\cdots$ \\
\hline $\begin{array}{l}\text { Entire preservative } \\
\text { period: } \\
\text { Total ............. } \\
\text { Average ........ }\end{array}$ & $\begin{array}{r}27.340 \\
.911 \\
\end{array}$ & $\begin{array}{r}68.269 \\
2.276 \\
\end{array}$ & $\begin{array}{r}6.718 \\
.224\end{array}$ & $\begin{array}{r}61.551 \\
2.052 \\
\end{array}$ & $\begin{array}{r}4.817 \\
.161\end{array}$ & $\begin{array}{r}56.734 \\
1.891\end{array}$ & $\begin{array}{l}1: 11.8 \\
\cdots \ldots\end{array}$ & 9.8 & 90.2 & $\begin{array}{c}7.1 \\
\cdots \cdots \\
\end{array}$ & $\begin{array}{r}83.1 \\
\cdots \\
\end{array}$ \\
\hline After period. & & & & & & & & & & & \\
\hline $\begin{array}{l}\text { First subperiod: } \\
\text { Total ........... } \\
\text { Average ........ }\end{array}$ & $\begin{array}{r}4.737 \\
.947\end{array}$ & $\begin{array}{r}11.828 \\
2.365\end{array}$ & $\begin{array}{r}1.210 \\
.242\end{array}$ & $\begin{array}{r}10.618 \\
2.124\end{array}$ & $\begin{array}{r}.800 \\
160\end{array}$ & $\begin{array}{l}9.818 \\
1.964\end{array}$ & $1: 12.3$ & 10.2 & 89.8 & 6.8 & -83.0 \\
\hline Second subperiod: & & & & & & & & & & & \\
\hline Total ............ & $\begin{array}{r}4.848 \\
.970\end{array}$ & $\begin{array}{r}12.105 \\
2.421\end{array}$ & $\begin{array}{r}1.217 \\
.243\end{array}$ & $\begin{array}{r}10.888 \\
2.178\end{array}$ & $\begin{array}{l}.850 \\
.170\end{array}$ & $\begin{array}{r}10.038 \\
2.008\end{array}$ & $\begin{array}{l}1: 11.8 \\
\cdots \cdots\end{array}$ & $\begin{array}{l}10.1 \\
\cdots . . .\end{array}$ & $\begin{array}{l}89.9 \\
\cdots . . .\end{array}$ & 7.0 & $\begin{array}{r}82.9 \\
\cdots\end{array}$ \\
\hline $\begin{array}{l}\text { Entire after period: } \\
\text { Total ............. } \\
\text { Average........ }\end{array}$ & $\begin{array}{r}9.585 \\
.958\end{array}$ & $\begin{array}{r}23.934 \\
2.393\end{array}$ & $\begin{array}{r}2.428 \\
.243\end{array}$ & $\begin{array}{r}21.506 \\
2.151\end{array}$ & $\begin{array}{r}1.650 \\
.165\end{array}$ & $\begin{array}{r}19.856 \\
1.986\end{array}$ & $1: 12.0$ & 10.1 & 89.9 & 6.9 & $\begin{array}{r}83.0 \\
\cdots \cdots\end{array}$ \\
\hline
\end{tabular}


TABLE X.-Urine determinations-Ratio of preformed sulphates to ethereal sulphates and neutral sulphur-Series VI-Continued.

[Averages are per day.]

No. 5.

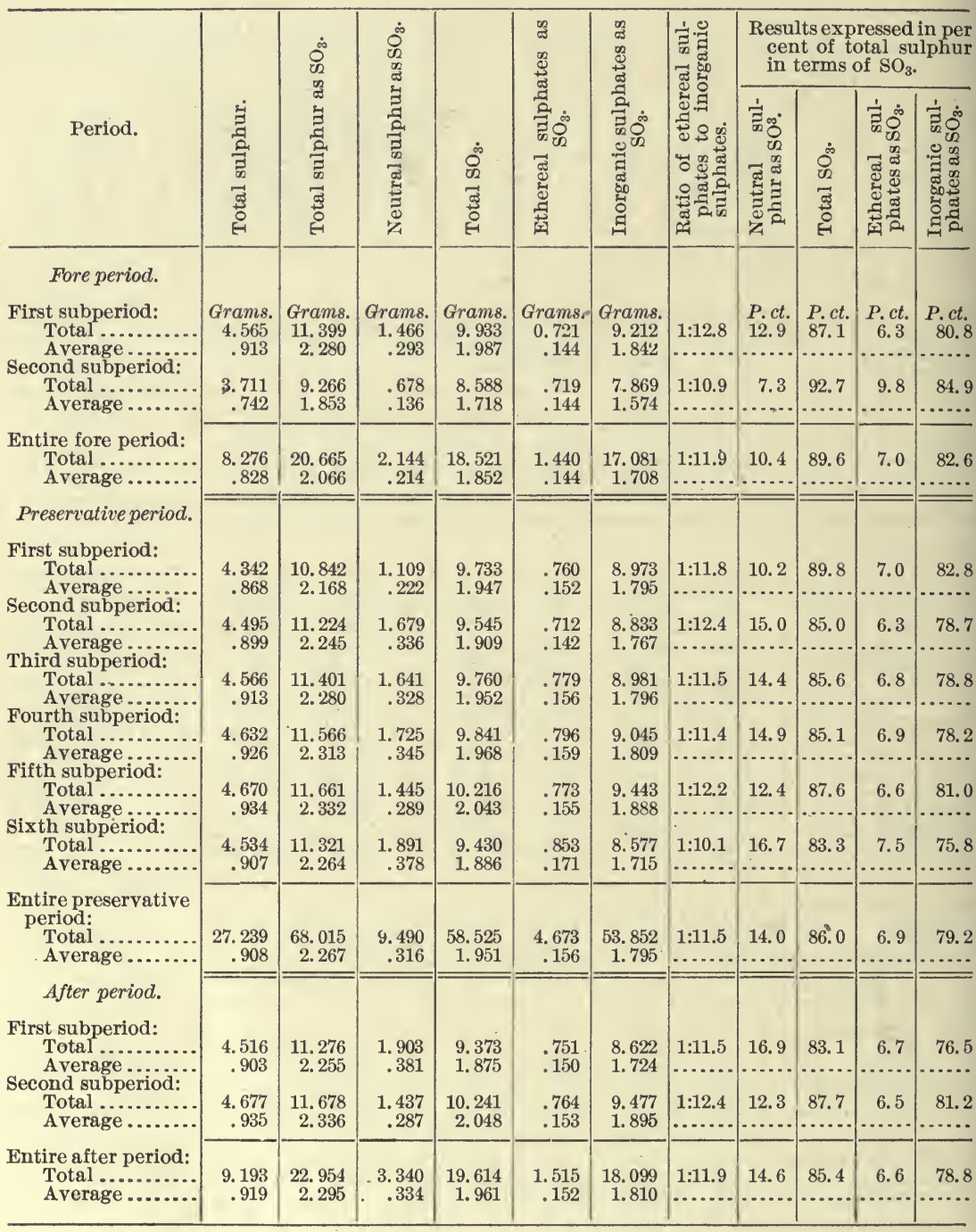


TABLE X.-Urine determinations-Ratio of preformed sulphates to ethereal sulphates and neutral sulphur-Series VI-Continued.

[Averages are per day.]

No. 6.

\begin{tabular}{|c|c|c|c|c|c|c|c|c|c|c|c|}
\hline \multirow[b]{2}{*}{ Period. } & \multirow[b]{2}{*}{ 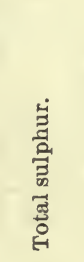 } & \multirow{2}{*}{ 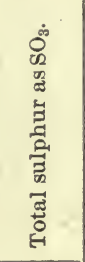 } & \multirow{2}{*}{ 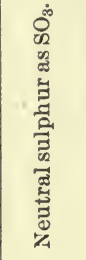 } & \multirow[b]{2}{*}{ 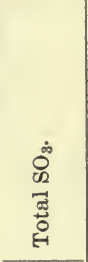 } & \multirow{2}{*}{ 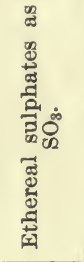 } & \multirow{2}{*}{ 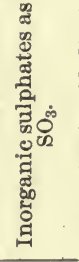 } & \multirow{2}{*}{ 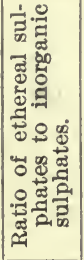 } & \multicolumn{4}{|c|}{$\begin{array}{l}\text { Results expressed in per } \\
\text { cent of total sulphur } \\
\text { in terms of } \mathrm{SO}_{3} \text {. }\end{array}$} \\
\hline & & & & & & & & 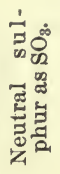 & 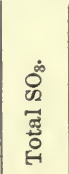 & 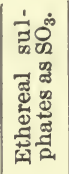 & 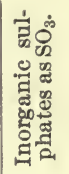 \\
\hline Fore period. & & & & & & & & & & & \\
\hline $\begin{array}{l}\text { First subperiod: } \\
\quad \text { Total ............ } \\
\text { Average........ }\end{array}$ & $\begin{array}{r}\text { Grams. } \\
4.038 \\
.808\end{array}$ & $\begin{array}{r}\text { Grams. } \\
10.083 \\
2.018\end{array}$ & $\begin{array}{r}\text { Grams. } \\
1.003 \\
.202\end{array}$ & $\begin{array}{c}\text { Grams. } \\
9.080 \\
1.816\end{array}$ & $\begin{array}{c}\text { Grams. } \\
0.622 \\
.124\end{array}$ & $\begin{array}{r}\text { Grams. } \\
8.458 \\
1.692\end{array}$ & $1: 13.6$ & $\begin{array}{l}P . \\
9.9\end{array}$ & $\begin{array}{l}\text { P. ct. } \\
90.1\end{array}$ & $\begin{array}{l}\text { P. ct. } \\
6.2\end{array}$ & $\begin{array}{l}\text { P. ct. } \\
83.9\end{array}$ \\
\hline Second subperiod: & .000 & 2.018 & & & & 1.092 & & .... & ...... & $\cdots$ & $\cdots \cdots$ \\
\hline $\begin{array}{l}\text { Total } \ldots . . . . \\
\text { Average.......... }\end{array}$ & $\begin{array}{r}3.796 \\
.759\end{array}$ & $\begin{array}{l}9.479 \\
1.895\end{array}$ & .924 & $\begin{array}{l}8.555 \\
1.711\end{array}$ & .585 & $\begin{array}{l}7.970 \\
1.594\end{array}$ & 1:13. 6 & 9.7 & 90.3 & 6.2 & 84.1 \\
\hline & & & & & & & & & & & \\
\hline $\begin{array}{l}\text { Entre iore period: } \\
\text { Total ............... } \\
\text { Average ....... }\end{array}$ & $\begin{array}{r}7.834 \\
.783\end{array}$ & $\begin{array}{r}19.561 \\
1.955\end{array}$ & $\begin{array}{r}1.926 \\
.191\end{array}$ & $\begin{array}{r}17.635 \\
1.764\end{array}$ & $\begin{array}{r}1.207 \\
.121\end{array}$ & $\begin{array}{r}16.428 \\
1.643\end{array}$ & $\begin{array}{l}1: 13.6 \\
\ldots \ldots \ldots\end{array}$ & 9.8 & $\begin{array}{l}90.2 \\
\cdots . . .\end{array}$ & 6.2 & 84.0 \\
\hline & & & & & & & & & & & 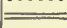 \\
\hline Preservative period. & & & & & & & & & & & \\
\hline $\begin{array}{l}\text { First subperiod: } \\
\text { Total ............ }\end{array}$ & 4.090 & 10.213 & .592 & 9.621 & .720 & 8.910 & $1: 12.4$ & 5.8 & 94.2 & 7.0 & 87.2 \\
\hline Average....... & .818 & 2.043 & .119 & 1.924 & .144 & 1. 780 & & & . & or & ...... \\
\hline $\begin{array}{l}\text { Second subperiod: } \\
\text { Total }\end{array}$ & 4.521 & 11.289 & & 9. 966 & & & & 11.7 & 883 & & 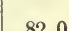 \\
\hline Average .......... & $\begin{array}{r}4.021 \\
.904\end{array}$ & $\begin{array}{l}11.289 \\
2.257\end{array}$ & $\begin{array}{r}1.523 \\
.264\end{array}$ & $\begin{array}{l}9.900 \\
1.993\end{array}$ & .142 & $\begin{array}{l}9.257 \\
1.851\end{array}$ & $\mid \begin{array}{l}1.13 .1 \\
\ldots \ldots\end{array}$ & 11.7 & 88.3 & 0.3 & $\begin{array}{l}82.0 \\
\ldots . . .\end{array}$ \\
\hline Third subperiod: & & & & & & & & & & & . \\
\hline $\begin{array}{l}\text { Total ............. } \\
\text { Average ........ }\end{array}$ & $\begin{array}{r}4.748 \\
.950\end{array}$ & $\begin{array}{r}11.856 \\
2.371\end{array}$ & $\begin{array}{r}1.196 \\
.239\end{array}$ & $\begin{array}{r}10.660 \\
2.132\end{array}$ & $\begin{array}{l}.698 \\
.140\end{array}$ & $\begin{array}{l}9.962 \\
1.992\end{array}$ & $1: 14.3$ & 10.0 & 90.0 & 5.9 & 84.0 \\
\hline Fourth subperiod: & & & & & & & & & & & $\cdots$ \\
\hline Total .............. & 5.065 & 12.647 & 1.123 & 11.524 & .688 & 10.836 & $1: 15.8$ & 8.9 & 91.1 & 5.4 & 85.7 \\
\hline 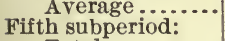 & 1.013 & 2.529 & .225 & 2.305 & .138 & 2.167 & & 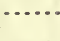 & $\cdots \cdots$ & ...... & $\cdots \cdots$ \\
\hline $\begin{array}{l}\text { Total } \ldots . . . . . \\
\text { Average .......... }\end{array}$ & $\begin{array}{r}4.800 \\
960\end{array}$ & $\begin{array}{r}11.986 \\
2.397\end{array}$ & $\begin{array}{r}1.087 \\
.217\end{array}$ & $\begin{array}{r}10.899 \\
2,180\end{array}$ & $\begin{array}{r}.700 \\
140\end{array}$ & $\begin{array}{r}10.199 \\
2.040\end{array}$ & $1: 14.6$ & 9.1 & 90.9 & 5.8 & 85.1 \\
\hline Sixth subperiod: & & 2.000 & .218 & 2.100 & & 2.010 & & & & & \\
\hline Total ............ & 4. 390 & 10.962 & 1. 205 & 9.757 & .735 & 9.022 & $1: 12.3$ & 11.0 & 89.0 & 6.7 & 82.3 \\
\hline Average.......... & .818 & 2.192 & .241 & 1.951 & .147 & 1.804 & $\begin{array}{lll}\cdots \cdots \\
\end{array}$ & …. & ........ & $\cdots \cdots$ & $\cdots \cdots$ \\
\hline $\begin{array}{l}\text { Entire preservative } \\
\text { period: }\end{array}$ & & & & & & & & & & & \\
\hline Total ............ & 27.614 & 68.953 & 6.526 & 62.427 & 4.250 & 58.177 & $1: 13.7$ & 9.5 & 90.5 & 6.2 & 84.4 \\
\hline Average .... & .920 & 2.298 & .218 & 2.081 & .142 & 1.939 & & ....... & ….... & $\cdots$ & $\ldots \ldots$ \\
\hline After period. & & & & & & & & & & & \\
\hline $\begin{array}{l}\text { First subperiod: } \\
\text { Total ......... }\end{array}$ & 4.346 & 10.852 & 1.408 & 9.444 & . 695 & 8.749 & $1: 12.6$ & 13.0 & 87.0 & 6.4 & 80.6 \\
\hline Average ...... & .869 & 2.170 & .281 & 1.889 & .139 & 1. 750 & & & & & ..... \\
\hline $\begin{array}{l}\text { second subperiod: } \\
\text { Total ............ }\end{array}$ & 4. 611 & 11.514 & 1.546 & 9.968 & .718 & 9.250 & 1:12.9 & 13.4 & 86.6 & 6.2 & 80.3 \\
\hline Average......... & .922 & 2.302 & .308 & 1.994 & .144 & 1.850 & & $\cdots$ & $\cdots, \cdots$ & $\ldots \ldots$ & $\cdots \cdots$ \\
\hline $\begin{array}{l}\text { Entire after period: } \\
\text { Total ............ }\end{array}$ & 8.957 & 22.366 & 2.954 & 19.412 & 1.413 & 17.999 & $1: 12.7$ & 13.2 & 86.8 & 6.3 & 80.5 \\
\hline Average ......... & .896 & 2.237 & .296 & 1.941 & .141 & 1.800 & & & & & \\
\hline
\end{tabular}


TABLE X.-Urine determinations-Ratio of preformed sulphates to ethereal sulphates and neutral sulphur-Series VI-Continued.

[Averages are per day.]

No. 7.

\begin{tabular}{|c|c|c|c|c|c|c|c|c|c|c|c|}
\hline \multirow[b]{2}{*}{ Period. } & \multirow[b]{2}{*}{ 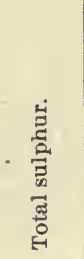 } & \multirow{2}{*}{ 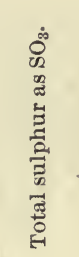 } & \multirow{2}{*}{ 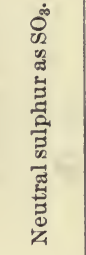 } & \multirow[b]{2}{*}{ 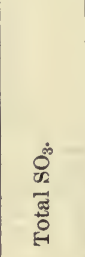 } & \multirow{2}{*}{ 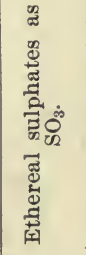 } & \multirow{2}{*}{ 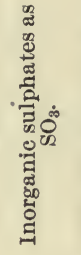 } & \multirow{2}{*}{ 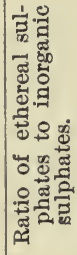 } & \multicolumn{4}{|c|}{$\begin{array}{l}\text { Results expressed in per } \\
\text { cent of total sulphur } \\
\text { in terms of } \mathrm{SO}_{3} \text {. }\end{array}$} \\
\hline & & & & & & & & 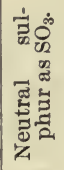 & 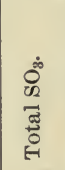 & 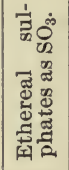 & 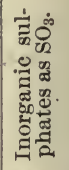 \\
\hline \multicolumn{12}{|l|}{ Fore period. } \\
\hline $\begin{array}{l}\text { First subperiod: } \\
\text { Total ............ } \\
\text { Average ........ }\end{array}$ & $\begin{array}{r}\text { Grams } \\
3.756 \\
.751\end{array}$ & $\begin{array}{r}\text { Grams. } \\
9.379 \\
1.875\end{array}$ & $\begin{array}{r}\text { Grams. } \\
1.42 \% \\
.284\end{array}$ & $\begin{array}{r}\text { Grams. } \\
7.956 \\
1.591\end{array}$ & $\begin{array}{r}\text { Grams. } \\
0.596 \\
.119\end{array}$ & $\begin{array}{l}\text { Grams. } \\
7.360 \\
1.472\end{array}$ & $\begin{array}{l}1: 12.3 \\
\cdots\end{array}$ & $\begin{array}{l}\text { P. ct. } \\
15.2 \\
\text {..... }\end{array}$ & $\begin{array}{l}\text { P. ct. } \\
84.8 \\
\text {... }\end{array}$ & $\begin{array}{r}\text { P. ct. } \\
6.4 \\
.\end{array}$ & $\begin{array}{r}\text { P. } c t . \\
78.5 \\
\cdots\end{array}$ \\
\hline Second subperiod: & & & & & & & & $\cdots \cdots$ & …. & $\cdots$ & ....... \\
\hline 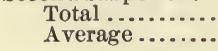 & $\begin{array}{r}4.280 \\
.856\end{array}$ & $\begin{array}{r}10.687 \\
2.137\end{array}$ & $\begin{array}{r}1.399 \\
.279\end{array}$ & $\begin{array}{l}9.288 \\
1.858\end{array}$ & $\begin{array}{l}.800 \\
.160\end{array}$ & $\begin{array}{l}8.488 \\
1.698\end{array}$ & $\begin{array}{l}1: 10.6 \\
\cdots\end{array}$ & $\begin{array}{c}13.1 \\
\cdots \cdots\end{array}$ & $\begin{array}{l}86.9 \\
\ldots . .\end{array}$ & 7.5 & $\begin{array}{l}79.4 \\
\cdots . .\end{array}$ \\
\hline $\begin{array}{l}\text { Entire fore period: } \\
\text { Total ............. } \\
\text { Average ....... }\end{array}$ & $\begin{array}{r}8.036 \\
.804\end{array}$ & $\begin{array}{r}20.066 \\
2.008\end{array}$ & $\begin{array}{r}2.822 \\
.284\end{array}$ & $\begin{array}{r}17.244 \\
1.724\end{array}$ & $\begin{array}{r}1.396 \\
.140\end{array}$ & $\begin{array}{r}15.848 \\
1.584\end{array}$ & $\begin{array}{l}1: 11.4 \\
\cdots \cdots\end{array}$ & $\begin{array}{l}14.1 \\
\cdots \cdots\end{array}$ & $\begin{array}{c}85.9 \\
\cdots\end{array}$ & $\begin{array}{r}6.9 \\
\ldots \ldots .\end{array}$ & 79.0 \\
\hline \multicolumn{12}{|l|}{ Preservative period. } \\
\hline $\begin{array}{l}\text { First subperiod: } \\
\text { Total ........... }\end{array}$ & 3.513 & 8.772 & 1.347 & 7.425 & .780 & 6.645 & 1: 8.5 & 15.4 & 84.6 & 8.9 & 75.8 \\
\hline od:... & .703 & .755 & .270 & 485 & .156 & 1. 329 & & & ....... & $\cdots \cdots$ & $\ldots .$. \\
\hline & 4.085 & 10.200 & 1. 485 & 8.715 & .742 & 7.973 & $1: 10.7$ & 14. 6 & 85.4 & 7.3 & 78.2 \\
\hline $\begin{array}{r}\text { Ave } \\
\text { hird st }\end{array}$ & .817 & 2.040 & .297 & & .148 & & & …... & ….... & ...... & $\cdots$ \\
\hline a.... & 3.368 & 8.410 & 1.069 & 7.341 & .709 & 6. 632 & 1: 9.4 & 12.7 & 87.3 & 8.4 & 78.9 \\
\hline period: & .674 & 1.683 & 215 & & .142 & & & ...... & $\cdots \cdots$ & $\ldots .$. & $\ldots .$. \\
\hline Total . & 3.564 & 8.899 & 1.360 & 7.539 & .656 & 6.883 & $1: 10.5$ & 15.3 & 84.7 & 7.4 & 77.3 \\
\hline $\begin{array}{l}\text { Aver } \\
\text { Fifth sul }\end{array}$ & .713 & 1.780 & .272 & 508 & .131 & 1.377 & & ..... & ....... & ....... & ...... \\
\hline Total & 3.870 & 9.663 & 1. 479 & 8.184 & .747 & 7.437 & $1: 10.0$ & 15.3 & 84.7 & 7.7 & 77.0 \\
\hline Ave & .774 & 1.933 & .296 & 637 & .149 & 1.488 & & & & $\ldots \ldots$ & \\
\hline & a 6.424 & 16.041 & 6.987 & & .766 & 8.288 & $1: 10.8$ & 43. 6 & 56.4 & 4.8 & 51.7 \\
\hline Average ......... & 1.285 & 3.209 & 1. 398 & & .153 & 1.658 & & ....... & ....... & $\mid \ldots \ldots$ & ....... \\
\hline \multirow{2}{*}{\multicolumn{12}{|c|}{$\begin{array}{l}\text { Entire preservative } \\
\text { period: } \\
\text { Total }\end{array}$}} \\
\hline & 24.824 & 61,986 & 13. 728 & & 4.400 & 43.858 & $1: 10.0$ & 22.1 & 77.9 & 7.1 & 70.8 \\
\hline & .827 & 2.065 & .457 & 1.608 & .147 & 1.461 & & & ....... & ..... & .... \\
\hline \multicolumn{12}{|l|}{ After period. } \\
\hline $\begin{array}{l}\text { First subperiod: } \\
\text { Total ......... }\end{array}$ & 3.650 & .114 & 1.417 & & .000 & 7.097 & $1: 11.8$ & 15.5 & 84.5 & 6.6 & 77.9 \\
\hline Âv & .730 & 1.823 & .284 & 1.539 & .120 & 1.419 & & $\cdots$ & ....... & & \\
\hline & 3.891 & 9.716 & 1.536 & 8.1 & .584 & & $1: 13.0$ & 15.8 & 84.2 & 6.0 & 78.2 \\
\hline Average... & .778 & 1.943 & .307 & 1.636 & .117 & 1.519 & & 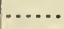 & ....... & $\ldots . .$. & 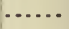 \\
\hline $\begin{array}{l}\text { Entire after period: } \\
\text { Total ............ }\end{array}$ & 7.541 & 18.830 & 2.953 & 15. 877 & 1.184 & 14. 693 & 1:12.4 & 15.7 & 84.3 & 6.3 & 78.0 \\
\hline Average. & .754 & 1.883 & .295 & 1.588 & .118 & 1.470 & & & & & \\
\hline
\end{tabular}

a. Unaccountably high. 
TABLE X.-Urine determinations-Ratio of preformed sulphates to ethereal sulphates and neutral sulphur-Series VI-Continued.

[Averages are per day.]

No. 8.

\begin{tabular}{|c|c|c|c|c|c|c|c|c|c|c|c|}
\hline \multirow[b]{2}{*}{ Period. } & \multirow[b]{2}{*}{ 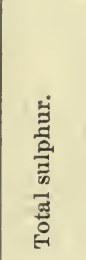 } & \multirow{2}{*}{ 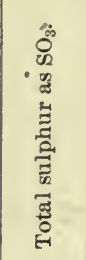 } & \multirow{2}{*}{ 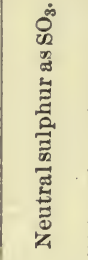 } & \multirow[b]{2}{*}{ 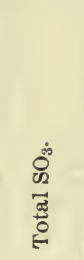 } & \multirow{2}{*}{ 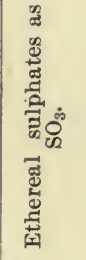 } & \multirow{2}{*}{ 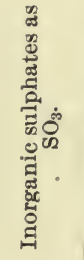 } & \multirow{2}{*}{ 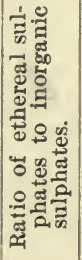 } & \multicolumn{4}{|c|}{$\begin{array}{l}\text { Results expressed in per } \\
\text { cent of total sulphur } \\
\text { in terms of } \mathrm{SO}_{3} \text {. }\end{array}$} \\
\hline & & & & & & & & 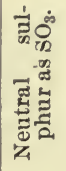 & 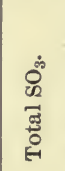 & 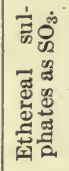 & 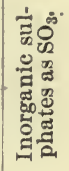 \\
\hline \multicolumn{12}{|l|}{ Fore period. } \\
\hline $\begin{array}{l}\text { First subperiod: } \\
\text { Total ............ } \\
\text { Average ......... }\end{array}$ & $\begin{array}{r}\text { Grams. } \\
3.689 \\
.738\end{array}$ & $\begin{array}{l}\text { Grams. } \\
9.211 \\
1.843\end{array}$ & $\begin{array}{r}\text { Grams. } \\
1.558 \\
.312\end{array}$ & $\begin{array}{l}\text { Grams. } \\
7.653 \\
1.531\end{array}$ & $\begin{array}{r}\text { Grams. } \\
0.938 \\
.188\end{array}$ & $\begin{array}{l}\text { Grams. } \\
6.715 \\
1.343\end{array}$ & $1: 1.2$ & $\begin{array}{l}\text { P. ct. } \\
16.9\end{array}$ & $\begin{array}{l}\text { P. ct. } \\
83.1\end{array}$ & $\begin{array}{l}\text { P. ct. } \\
10.2\end{array}$ & P.ct. \\
\hline Second subperiod: & & & & & & & & & $\cdots$ & $\cdots$ & \\
\hline $\begin{array}{l}\text { Total ................ } \\
\text { Average ....... }\end{array}$ & $\begin{array}{r}3.988 \\
.798\end{array}$ & $\begin{array}{l}9.958 \\
1.993\end{array}$ & $\begin{array}{r}1.939 \\
.389\end{array}$ & $\begin{array}{l}8.019 \\
1.604\end{array}$ & $\begin{array}{l}.940 \\
.188\end{array}$ & $\begin{array}{l}7.079 \\
1.416\end{array}$ & $\begin{array}{l}1: 7.5 \\
\ldots \ldots\end{array}$ & 19.5 & 80.5 & 9.4 & 71.0 \\
\hline atre I & & & & & & & - & & & & \\
\hline $\begin{array}{l}\text { Total ............... } \\
\text { Average }\end{array}$ & $\begin{array}{r}7.677 \\
.768\end{array}$ & $\begin{array}{r}19.169 \\
1.918\end{array}$ & $\begin{array}{r}3.497 \\
.351\end{array}$ & $\begin{array}{r}15.672 \\
1.567\end{array}$ & $\begin{array}{r}1.878 \\
.188\end{array}$ & $\begin{array}{r}13.794 \\
1.379\end{array}$ & $\begin{array}{l}1: 7.3 \\
\cdots . . .\end{array}$ & $\begin{array}{c}18.2 \\
\ldots . .\end{array}$ & 81.8 & 9.8 & \\
\hline \multicolumn{11}{|l|}{ Preservative period. } & \\
\hline First subperiod: & \multirow{3}{*}{$\begin{array}{r}3.441 \\
.688\end{array}$} & \multirow{3}{*}{$\begin{array}{l}8.592 \\
1.718\end{array}$} & \multirow{3}{*}{$\begin{array}{l}.981 \\
.196\end{array}$} & \multirow{3}{*}{$\begin{array}{l}7.611 \\
1.522\end{array}$} & \multirow{3}{*}{$\begin{array}{l}.907 \\
.181\end{array}$} & \multirow{3}{*}{$\begin{array}{l}6.704 \\
1.341\end{array}$} & & \multirow[b]{2}{*}{11.5} & \multirow[b]{2}{*}{88.5} & \multirow[b]{2}{*}{10.6 . } & \multirow[b]{2}{*}{78.0} \\
\hline Total & & & & & & & $1: 7.4$ & & & & \\
\hline Second subpe & & & & & & & & . & ..... & ....... & \\
\hline $\begin{array}{l}\text { Total .... } \\
\text { Average }\end{array}$ & 3.610 & 9.014 & 1. 129 & 7.885 & .922 & 6. 963 & $1: 7.6$ & 12.6 & 87.4 & 10.2 & 77.2 \\
\hline Third subperiod: & & & & & & & & -...... & ...... & ….... & $\cdots$ \\
\hline $\begin{array}{l}\text { Total } \\
\text { Avera }\end{array}$ & 3.675 & 9.176 & 1.924 & 7.252 & .892 & 6.360 & $1: 7.1$ & 21.0 & 79.0 & 9.7 & 69. \\
\hline Fverage ........ & .735 & & . 385 & 450 & .178 & 1.272 & & $\cdots \cdots$ & ..... & $\cdots$ & $\cdots$ \\
\hline $\begin{array}{l}\text { Total } \\
\text { Aver }\end{array}$ & 3.731 & 9.318 & 1.574 & 7.744 & .915 & 6.829 & $1: 7.5$ & 16.9 & 83.1 & 9.8 & 73.3 \\
\hline $\begin{array}{l}\text { Fifth subpe } \\
\text { Total.. }\end{array}$ & .746 & & .315 & & .18 & 1.366 & & ....... & ....... & ....... & $\cdots \cdots$ \\
\hline Total... & $\begin{array}{r}3.827 \\
.765\end{array}$ & $\begin{array}{l}9.556 \\
1.910\end{array}$ & $\begin{array}{r}1.665 \\
.332\end{array}$ & $\begin{array}{l}7.891 \\
1.578\end{array}$ & $\begin{array}{l}.940 \\
.188\end{array}$ & $\begin{array}{l}6.951 \\
1.390\end{array}$ & $1: 7.4$ & 17.4 & 82.6 & 9.8 & 72.7 \\
\hline $\begin{array}{r}\text { Sixth su } \\
\text { Tota }\end{array}$ & & & & & & & & & & & \\
\hline $\begin{array}{l}\text { Tota } \\
\text { Aver }\end{array}$ & $\begin{array}{l}3.949 \\
790\end{array}$ & & 1.388 & & .961 & & $1: 7.8$ & 14.1 & 85.9 & 9.7 & 76.2 \\
\hline & & & .218 & & .192 & 1.505 & $\cdots$ & $\cdots$ & -...... & -...... & ..... \\
\hline $\begin{array}{l}\text { Entire preservative } \\
\text { period: }\end{array}$ & & & & & & & & & & & \\
\hline $\begin{array}{l}\text { Total ... } \\
\text { Average }\end{array}$ & 22.233 & 55.518 & 8. 662 & $\begin{array}{r}46.856 \\
1562\end{array}$ & 5.537 & 41.319 & $1: 7.5$ & 15.6 & 84.4 & 10.0 & 74.4 \\
\hline (1) & & & .289 & & & & & ......... & …… & ….... & $\cdots \cdots$ \\
\hline After $p$ & & & & & & & & & & & \\
\hline $\begin{array}{l}\text { First subperiod: } \\
\text { Total ......... } \\
\text { Average ..... }\end{array}$ & 4. 209 & 10.510 & 1.976 & 8.534 & . 933 & 7. 601 & $1: 8.1$ & 18.7 & 81.2 & 8.9 & 72.3 \\
\hline $\begin{array}{l}\text { Averag } \\
\text { cond sul }\end{array}$ & .842 & 2.102 & .395 & 1.707 & .187 & 1.520 & & & 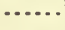 & & \\
\hline $\begin{array}{l}\text { Total } \\
\text { Avera }\end{array}$ & 4.166 & 10.402 & 1.614 & 8.788 & .826 & 7.962 & $1: 9.6$ & 15.5 & 84.5 & 7.9 & 76.5 \\
\hline Average ......... & .833 & 2.080 & .322 & & .165 & 1.593 & & . . . . . . & 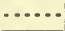 & ....... & ...... \\
\hline $\begin{array}{r}\text { Entire after period: } \\
\text { Total .............. } \\
\text { Average ....... }\end{array}$ & 8. 375 & 20.912 & 3.590 & & 1. 759 & 15.563 & $1: 8.8$ & 17.2 & 82.8 & 8.4 & 74.4 \\
\hline & .838 & & .360 & & .176 & & & & & & \\
\hline
\end{tabular}

7656-No. 84, pt 2-06 $\longrightarrow 7$ 
TABLE X.-Urine determinations-Ratio of preformed sulphates to ethereal sulphates and neutral sulphur-Series VI-Continued.

[Averages are per day.]

No.9.

\begin{tabular}{|c|c|c|c|c|c|c|c|c|c|c|c|}
\hline . & & $\overbrace{\infty}^{\infty}$ & 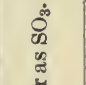 & & 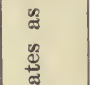 & 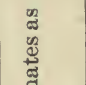 & 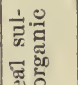 & $\begin{array}{r}\text { Resul } \\
\text { cen } \\
\text { in t }\end{array}$ & $\begin{array}{l}\text { tof } \\
\text { erm }\end{array}$ & fal & $\begin{array}{l}\text { nper } \\
\text { phur }\end{array}$ \\
\hline $\begin{array}{c}\text { Period. } \\
\qquad-\end{array}$ & 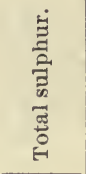 & 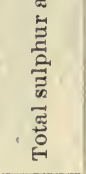 & 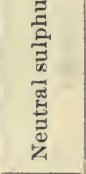 & 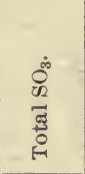 & 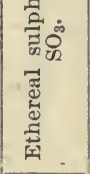 & 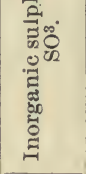 & 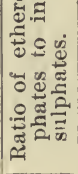 & 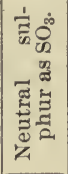 & 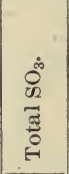 & 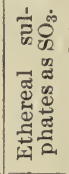 & 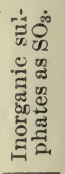 \\
\hline Fore period. & & & & & & & & & & & \\
\hline $\begin{array}{l}\text { First subperiod: } \\
\text { Total .............. } \\
\text { Average . }\end{array}$ & $\begin{array}{r}\text { Grams. } \\
4.671 \\
.934\end{array}$ & $\begin{array}{c}\text { Grams. } \\
11.663 \\
2.333\end{array}$ & $\begin{array}{r}\text { Grams. } \\
0.938 \\
.188\end{array}$ & $\begin{array}{l}\text { Grams. } \\
10.721 \\
2.144\end{array}$ & $\begin{array}{l}\text { Grams. } \\
\cdots \ldots \\
\cdots\end{array}$ & $\begin{array}{l}\text { Grams } \\
\cdots \ldots \ldots \\
\cdots \ldots\end{array}$ & & $\begin{array}{c}\text { P.ct. } \\
8.1\end{array}$ & $\begin{array}{l}P . \text { et. } \\
91.9\end{array}$ & $\begin{array}{l}\text { P.ct. } \\
\text {..... }\end{array}$ & $\begin{array}{l}\text { P. ct. } \\
\cdots . . . \\
\cdots\end{array}$ \\
\hline $\begin{array}{l}\text { Second subperiod: } \\
\text { Total ............. } \\
\text { Average ........ }\end{array}$ & $\begin{array}{r}4.547 \\
.909\end{array}$ & $\begin{array}{r}11.354 \\
2.271\end{array}$ & $\begin{array}{r}-818 \\
.164 \\
\end{array}$ & $\begin{array}{r}10.536 \\
2.107 \\
\end{array}$ & $\begin{array}{r}1.001 \\
.200\end{array}$ & $\begin{array}{l}9.535 \\
1.907\end{array}$ & $\begin{array}{l}1: 9.5 \\
\cdots . . .\end{array}$ & 7.2 & 92.8 & 8.8 & 84.0 \\
\hline $\begin{array}{l}\text { Entire fore period: } \\
\text { Total ............. } \\
\text { Average ....... }\end{array}$ & $\begin{array}{r}9.218 \\
.922 \\
\end{array}$ & $\begin{array}{r}23.017 \\
2.302 \\
\end{array}$ & $\begin{array}{r}1.757 \\
.176 \\
\end{array}$ & $\begin{array}{r}21.257 \\
2.126 \\
\end{array}$ & $\begin{array}{r}1.001 \\
.200 \\
\end{array}$ & $\begin{array}{l}9.535 \\
1.907\end{array}$ & $\begin{array}{l}1: 9.5 \\
\cdots \cdots \cdots \\
\end{array}$ & $\begin{array}{r}7.6 \\
\cdots \cdots \\
\end{array}$ & $\begin{array}{r}92.4 \\
\ldots \ldots . \\
\end{array}$ & $\begin{array}{r}8.8 \\
\ldots . . \\
\end{array}$ & $\begin{array}{r}83.6 \\
\cdots \cdots . . \\
\end{array}$ \\
\hline Preservative period. & & & & & & & & & & & \\
\hline 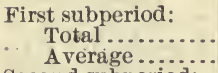 & $\begin{array}{r}4.948 \\
.593\end{array}$ & $\begin{array}{r}12.355 \\
2.471\end{array}$ & $\begin{array}{l}.881 \\
.176\end{array}$ & $\begin{array}{r}11.474 \\
2.295\end{array}$ & $\begin{array}{r}10.54 \\
2.11\end{array}$ & $\begin{array}{r}10.420 \\
2.084\end{array}$ & $\begin{array}{l}1: 9.9 \\
\ldots \ldots \ldots\end{array}$ & 7.1 & $\begin{array}{c}92.9 \\
\ldots \ldots .\end{array}$ & $\begin{array}{r}8.5 \\
\cdots . .\end{array}$ & $\begin{array}{r}84.4 \\
\ldots . . .\end{array}$ \\
\hline $\begin{array}{l}\text { Second subperiod: } \\
\text { Total............. } \\
\text { A verage......... }\end{array}$ & $\begin{array}{r}4.570 \\
.914\end{array}$ & $\begin{array}{r}11.411 \\
2.282\end{array}$ & $\begin{array}{r}1.255 \\
.251\end{array}$ & $\begin{array}{r}10.156 \\
2.031\end{array}$ & $\begin{array}{l}.798 \\
.160\end{array}$ & $\begin{array}{l}9.358 \\
1.872\end{array}$ & $\begin{array}{l}1: 11.7 \\
\cdots \ldots . .\end{array}$ & 11.0 & 89.0 & 7.0 & $\begin{array}{r}82.0 \\
\ldots \ldots\end{array}$ \\
\hline $\begin{array}{l}\text { Third st } \\
\text { Tots } \\
\text { Ave }\end{array}$ & $\begin{array}{r}4.780 \\
.956\end{array}$ & $\begin{array}{r}11.936 \\
2.387\end{array}$ & $\begin{array}{r}1.194 \\
.239\end{array}$ & $\begin{array}{r}10.742 \\
2.148\end{array}$ & $\begin{array}{l}.840 \\
.168\end{array}$ & $\begin{array}{l}9.902 \\
1.980\end{array}$ & $\begin{array}{l}1: 11.8 \\
\ldots \ldots . .\end{array}$ & $\begin{array}{l}10.0 \\
\cdots \cdots . .\end{array}$ & $\begin{array}{c}90.0 \\
\cdots: . .\end{array}$ & 7.0 & $\begin{array}{r}83.0 \\
\ldots \ldots .\end{array}$ \\
\hline $\begin{array}{l}\text { Fourth subperiod .. } \\
\text { Total ............. } \\
\text { Average....... }\end{array}$ & $\begin{array}{r}4.567 \\
.913\end{array}$ & $\begin{array}{r}11.404 \\
2.281\end{array}$ & $\begin{array}{r}1.200 \\
.240\end{array}$ & $\begin{array}{r}10.204 \\
2.041\end{array}$ & .886. & $\begin{array}{l}9.318 \\
1.864\end{array}$ & $1: 10.5$ & 10.5 & $\begin{array}{c}89.5 \\
\therefore \ldots . .\end{array}$ & $\begin{array}{c}7.8 \\
. . .\end{array}$ & $\begin{array}{r}81.7 \\
\ldots \ldots .\end{array}$ \\
\hline $\begin{array}{l}\text { Fifth sul } \\
\text { Tota } \\
\text { Aver }\end{array}$ & $\begin{array}{r}4.072 \\
. .814\end{array}$ & $\begin{array}{r}10.168 \\
2.034\end{array}$ & $\begin{array}{l}.821 \\
.164\end{array}$ & $\begin{array}{l}9.347 \\
1.869\end{array}$ & $\begin{array}{l}.818 \\
.164\end{array}$ & $\begin{array}{l}8.529 \\
1.706\end{array}$ & $1: 10.4$ & 8.1 & 91.9 & 8.0 & 83.9 \\
\hline $\begin{array}{l}\text { Sixth subperiod: } \\
\text { Total ............... } \\
\text { Average ........ }\end{array}$ & $\begin{array}{r}4.864 \\
.973\end{array}$ & $\begin{array}{r}12.145 \\
2.4: 9\end{array}$ & $\begin{array}{r}1.251 \\
.250\end{array}$ & $\begin{array}{r}10.894 \\
2.179\end{array}$ & $\begin{array}{r}949 \\
190\end{array}$ & $\begin{array}{l}9.945 \\
1.989\end{array}$ & $1: 10.5$ & $\begin{array}{l}10.3 \\
\ldots . . .\end{array}$ & \begin{tabular}{l}
89.7 \\
\hdashline$\ldots .$.
\end{tabular} & 7.8 & 81.9 \\
\hline $\begin{array}{l}\text { Entire preservative } \\
\text { period: } \\
\text { Total ............. } \\
\text { Average } . . . .\end{array}$ & $\begin{array}{r}27.801 \\
.927 \\
\end{array}$ & $\begin{array}{r}69.419 \\
2.314 \\
\end{array}$ & $\begin{array}{r}6.602 \\
.220 \\
\end{array}$ & $\begin{array}{r}62.817 \\
2.094\end{array}$ & $\begin{array}{r}5.345 \\
.178 \\
\end{array}$ & $\begin{array}{r}57.472 \\
1.916 \\
\end{array}$ & $1: 10.8$ & 9.5 & 90.5 & 7.7 & $\begin{array}{r}82.8 \\
\cdots \cdots \\
\end{array}$ \\
\hline After period. & & & & & & & & & & & \\
\hline $\begin{array}{l}\text { First subperiod: } \\
\text { Total ........... } \\
\text { Average ....... }\end{array}$ & $\begin{array}{r}4.345 \\
.869\end{array}$ & $\begin{array}{r}10.849 \\
2.170\end{array}$ & $\begin{array}{l}.911 \\
.182\end{array}$ & $\begin{array}{l}9.938 \\
1.988\end{array}$ & $\begin{array}{l}.789 \\
.158\end{array}$ & $\begin{array}{l}9.149 \\
1.830\end{array}$ & $1: 11.6$ & 8.4 & 91.6 & 7.3 & 84.3 \\
\hline $\begin{array}{l}\text { Second subperiod: } \\
\text { Total } \\
\text {.... A vérage } \\
\text { A }\end{array}$ & $\begin{array}{r}4.578 \\
.916\end{array}$ & $\begin{array}{r}11.431 \\
2.286\end{array}$ & $\begin{array}{l}.971 \\
.194\end{array}$ & $\begin{array}{r}10.460 \\
2.090\end{array}$ & $\begin{array}{l}.840 \\
.168\end{array}$ & $\begin{array}{l}9.620 \\
1.924\end{array}$ & $1: 11.5$ & 8.5 & 91.5 & 7.3 & 81.2 \\
\hline $\begin{array}{l}\text { Entire after period: } \\
\text { Total } \\
\text { Average }\end{array}$ & $\begin{array}{r}8.923 \\
.892\end{array}$ & $\begin{array}{r}22.280 \\
-2.228 \\
\end{array}$ & $\begin{array}{r}1.882 \\
.188\end{array}$ & $\begin{array}{r}20.398 \\
2.040\end{array}$ & $\begin{array}{r}1.629 \\
.163\end{array}$ & $\begin{array}{r}18.769 \\
1.877\end{array}$ & $1: 11.5$ & 8.4 & 91.6 & 7.3 & $\begin{array}{r}84.3 \\
\cdots \cdots\end{array}$ \\
\hline
\end{tabular}


TABLE X.-Urine determinations-Ratio of preformed sulphates to ethereal sulphates and neutral sulphur-Series VI-Continued.

[Averages are per day.]

No. 10.

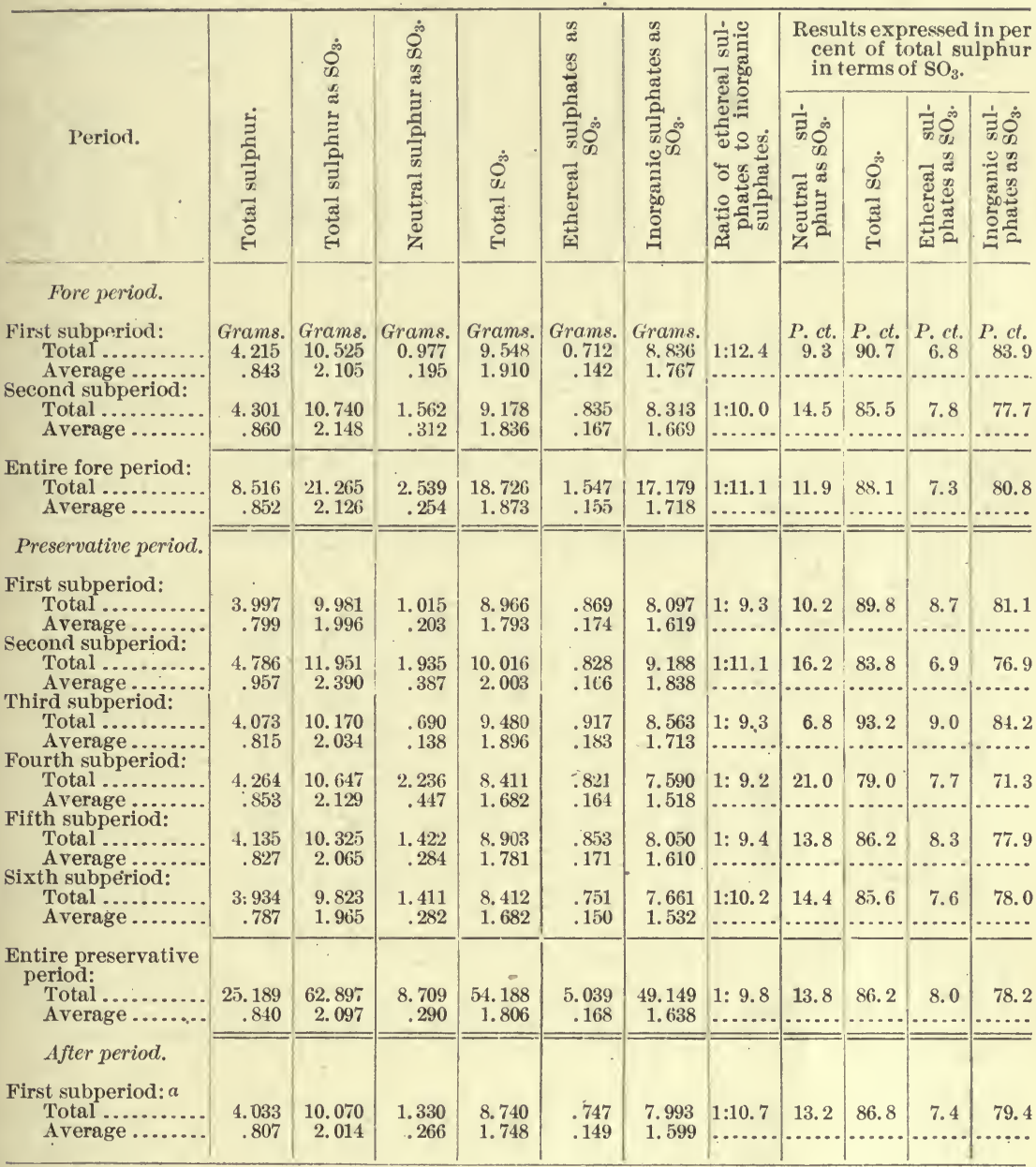

$a$ Sick in second subperiod. 
TABLE X.-Urine determinations-Ratio of preformed sulphates to ethereal sulphates and neutral sulphur-Series VI-Continued.

[Averages are per day.]

No. 11 .

\begin{tabular}{|c|c|c|c|c|c|c|c|c|c|c|c|}
\hline \multirow[b]{2}{*}{ Period. } & \multirow[b]{2}{*}{ 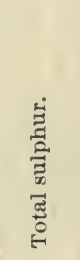 } & \multirow{2}{*}{ 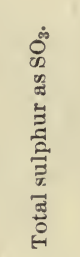 } & \multirow{2}{*}{ 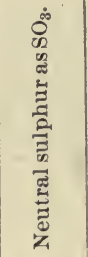 } & \multirow[b]{2}{*}{ 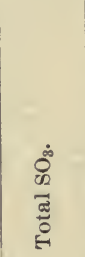 } & \multirow{2}{*}{ 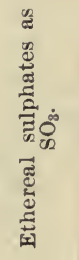 } & \multirow{2}{*}{ 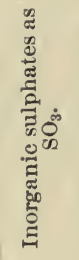 } & \multirow{2}{*}{ 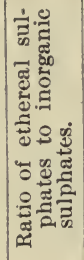 } & \multicolumn{4}{|c|}{$\begin{array}{l}\text { Results expressed in per } \\
\text { cent of total sulphur } \\
\text { in terms of } \mathrm{SO}_{3} \text {. }\end{array}$} \\
\hline & & & & & & & & 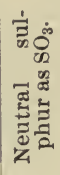 & 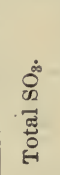 & 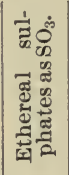 & 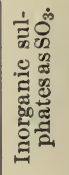 \\
\hline \multicolumn{12}{|l|}{ Fore period. } \\
\hline $\begin{array}{l}\text { First subperiod: } \\
\text { Total ............ } \\
\text { Average ........ }\end{array}$ & $\begin{array}{r}\text { Grams. } \\
4.673 \\
.935\end{array}$ & $\begin{array}{r}\text { Grams. } \\
11.668 \\
2.334\end{array}$ & $\begin{array}{r}\text { Grams. } \\
1.158 \\
.232\end{array}$ & $\begin{array}{r}\text { Grains. } \\
10.510 \\
2.102\end{array}$ & $\begin{array}{r}\text { Grams } \\
0.563 \\
.113\end{array}$ & $\begin{array}{r}\text { Grams. } \\
9.947 \\
1.989\end{array}$ & $1: 17.7$ & $\begin{array}{l}\text { P. ct. } \\
9.9\end{array}$ & $\begin{array}{l}P . \text { ct. } \\
90.1\end{array}$ & P. ct. & $\begin{array}{l}P_{\dot{8}} \text { ct. } \\
85.3 \\
. . .\end{array}$ \\
\hline Second subperiod: & & & & & & & & & & & \\
\hline $\begin{array}{l}\text { Total } \ldots . . . . . \\
\text { Average } . . . .\end{array}$ & $\begin{array}{l}5.325 \\
1.065\end{array}$ & $\begin{array}{r}13.297 \\
2.659\end{array}$ & $\begin{array}{r}1.529 \\
305\end{array}$ & $\begin{array}{r}11.768 \\
2.354\end{array}$ & .583 & 11. 185 & $1: 19.2$ & 11.5 & 88.5 & 4.4 & 84.1 \\
\hline $\begin{array}{l}\text { Entire fore period: } \\
\text { Total ............ } \\
\text { A verage ........ }\end{array}$ & $\begin{array}{l}9.998 \\
1.000\end{array}$ & $\begin{array}{r}24.965 \\
2.497\end{array}$ & $\begin{array}{r}2.687 \\
.269\end{array}$ & $\begin{array}{r}22.278 \\
2.228\end{array}$ & $\begin{array}{r}1.146 \\
.115\end{array}$ & $\begin{array}{r}21.132 \\
2.113\end{array}$ & $\begin{array}{l}1: 18.4 \\
\cdots \ldots\end{array}$ & 10.8 & 89.2 & 46 & 84.6 \\
\hline \multicolumn{12}{|l|}{ Preservative period. } \\
\hline $\begin{array}{l}\text { First subperiod: } \\
\text { Total .............. }\end{array}$ & 5. 469 & 13. 656 & .942 & 12.714 & .673 & 12.041 & $1: 17.9$ & 6.9 & 93.1 & 4.9 & 88.2 \\
\hline iod: & 1.094 & 2.732 & .189 & & .135 & 2.408 & & ..... & ...... & ....... & ...... \\
\hline (n... & 5. 339 & 13. 329 & 1. 643 & 11.686 & .517 & 11.169 & 1.21 .6 & 12.3 & 87.7 & 3.9 & 83.8 \\
\hline $\begin{array}{r}\text { Ave } \\
\text { Third si }\end{array}$ & 1.068 & 2.667 & .330 & & .103 & 2.234 & & 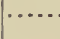 & ....... & $\ldots \ldots$ & (....... \\
\hline Total & 4. 455 & 11.124 & .445 & 10.679 & .628 & 10. 051 & $1: 16.0$ & 4. 0 & 96.0 & 5.6 & 90.4 \\
\hline $\begin{array}{r}\text { A } \\
\text { Fourt }\end{array}$ & 891 & 2. 225 & .089 & & .126 & 2.010 & & $\cdots \cdots$ & (...... & $\cdots \cdots$ & $\cdots \cdots$ \\
\hline & 4.602 & 11.491 & 1. 293 & 10.198 & .563 & & $1: 17.1$ & 11.3 & 88.7 & 4.9 & 83.8 \\
\hline $\begin{array}{r}\text { Av } \\
\text { Fifth sl }\end{array}$ & .920 & 2.297 & .257 & & .113 & & & & 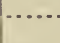 & & ....... \\
\hline Total & 5.021 & 12.537 & 1.333 & 11. 204 & .597 & 10.607 & $1: 17.8$ & 10.6 & 89.4 & 4.8 & 84.6 \\
\hline $\begin{array}{r}\text { Ave } \\
\text { Sixth su }\end{array}$ & 004 & 2.507 & .266 & & .119 & 2.122 & & $\cdots \cdots$ & & ...... & ....... \\
\hline Total .. & 4. 661 & 11. 639 & 1. 348 & 10. 291 & .613 & 9.678 & $1: 15.8$ & 11.6 & 88.4 & 5.3 & 83.2 \\
\hline Average......... & .932 & 2.327 & $: 269$ & & .123 & 1.935 & & $\cdots \cdots$ & ….. & 1 & $\ldots \ldots$ \\
\hline \multicolumn{12}{|l|}{$\begin{array}{l}\text { Entire preservative } \\
\text { period: }\end{array}$} \\
\hline Total ............ & 29.546 & 73.776 & 7. 004 & & 3.591 & & $1: 17.6$ & 9.4 & 90.6 & 4.9 & 85.6 \\
\hline & .985 & 2.460 & .234 & & .120 & 2.106 & & & & & $\cdots \ldots$ \\
\hline \multicolumn{12}{|l|}{ After period. } \\
\hline $\begin{array}{l}\text { First subpe } \\
\text { Total. }\end{array}$ & 1.854 & 12.120 & 2. $504^{\circ}$ & 9.616 & .519 & 9.097 & $1: 17.5$ & 20.7 & 79.3 & 4.3 & 75.1 \\
\hline & .971 & 2.425 & .502 & 1.923 & .104 & 1. 819 & & & …... & ..... & ...... \\
\hline To & .780 & 11.936 & 1. 228 & & .524 & & $1: 19.4$ & 10.3 & 89.7 & 4.4 & 85.3 \\
\hline Average........ & .000 & 2. 387 & .245 & 2.142 & .105 & 2.037 & & & & & ....... \\
\hline $\begin{array}{l}\text { Entire after period: } \\
\text { Total ............. }\end{array}$ & 9.634 & 24.056 & 3. 732 & 20.324 & 1. 043 & 19. 281 & $1: 18.5$ & 15.5 & 84.5 & 4.3 & 80.2 \\
\hline & & & .373 & 2.032 & .104 & 1.928 & & & & & \\
\hline
\end{tabular}


TABLE X. - Urine determinations-Ratio of preformed sulphates to ethereal sulphates and neutral sulphur-Series VI-Continued.

[Averages are per day.]

No. 12.

\begin{tabular}{|c|c|c|c|c|c|c|c|c|c|c|c|}
\hline \multirow[b]{2}{*}{ Period. } & \multirow[b]{2}{*}{ 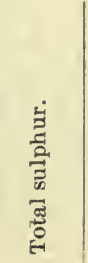 } & \multirow{2}{*}{ 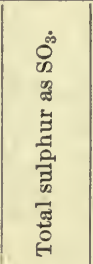 } & \multirow{2}{*}{ 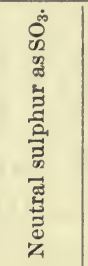 } & \multirow{2}{*}{ ○ึ } & \multirow{2}{*}{ 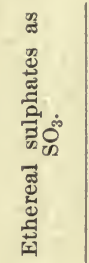 } & \multirow{2}{*}{ 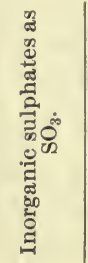 } & \multirow{2}{*}{ 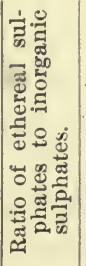 } & \multicolumn{4}{|c|}{$\begin{array}{l}\text { Results expressed as per } \\
\text { cent of total sulphur } \\
\text { in terms of } \mathrm{SO}_{3} \text {. }\end{array}$} \\
\hline & & & & & & & & 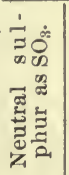 & 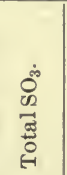 & 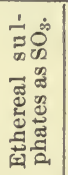 & 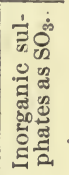 \\
\hline Fore period. & & & & & & & & & & & \\
\hline $\begin{array}{l}\text { First subperiod: } \\
\text { Total............ } \\
\text { Average........ }\end{array}$ & $\begin{array}{r}\text { Grams. } \\
5.166 \\
1.033\end{array}$ & $\begin{array}{c}\text { Grams. } \\
12.900 \\
2.579\end{array}$ & $\begin{array}{r}\text { Grams. } \\
1.418 \\
.283\end{array}$ & $\begin{array}{r}\text { Grams. } \\
11.482 \\
2.296\end{array}$ & $\begin{array}{r}\text { Grams. } \\
0.927 \\
.185\end{array}$ & $\begin{array}{r}\text { Grams. } \\
10.555 \\
2,111\end{array}$ & $1: 11.4$ & $\begin{array}{l}P . c t . \\
11.0\end{array}$ & $\begin{array}{l}P . c t . \\
89.0\end{array}$ & $\begin{array}{r}\text { P. } c t . \\
\div .2\end{array}$ & P. $c t .8$ \\
\hline $\begin{array}{l}\text { Average........ } \\
\text { Seeond subperiod: } \\
\text { Total ............. } \\
\text { Average........ }\end{array}$ & $\begin{array}{r}4.918 \\
.984\end{array}$ & $\begin{array}{r}12.280 \\
2.457\end{array}$ & $\begin{array}{r}1.793 \\
.360\end{array}$ & $\begin{array}{r}10.487 \\
2.097\end{array}$ & $\begin{array}{l}.976 \\
.195\end{array}$ & $\begin{array}{l}9.511 \\
1.902\end{array}$ & $\begin{array}{c}1: 9.7 \\
\cdots \ldots . .\end{array}$ & $\begin{array}{c}14.6 \\
\cdots \cdots . .\end{array}$ & $\begin{array}{c}85.4 \\
\cdots \ldots . .\end{array}$ & $\begin{array}{r}7.9 \\
\cdots \cdots \\
\end{array}$ & $\begin{array}{r}77.5 \\
\cdots \cdots \\
\end{array}$ \\
\hline $\begin{array}{l}\text { Entire fore period: } \\
\text { Total .......... } \\
\text { Average........ }\end{array}$ & $\begin{array}{r}10.084 \\
1.008\end{array}$ & $\begin{array}{r}25.180 \\
2.517 \\
\end{array}$ & $\begin{array}{r}3.211 \\
.320 \\
\end{array}$ & $\begin{array}{r}21.969 \\
2.197 \\
\end{array}$ & $\begin{array}{r}1.903 \\
.190 \\
\end{array}$ & $\begin{array}{r}20.066 \\
2.007 \\
\end{array}$ & $\begin{array}{c}1: 10.5 \\
\cdots \cdots\end{array}$ & $\begin{array}{r}12.8 \\
\ldots \ldots . \\
\end{array}$ & 87.2 & 7.6 & $\begin{array}{l}79.7 \\
\cdots \ldots .\end{array}$ \\
\hline Preservative period. & & & & & & & & 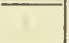 & & & \\
\hline $\begin{array}{l}\text { First subperiod: } \\
\text { Total .......... } \\
\text { Average...... }\end{array}$ & $\begin{array}{r}4.236 \\
.847\end{array}$ & $\begin{array}{r}10.577 \\
2.115\end{array}$ & .894 & $\begin{array}{l}9.683 \\
1.937\end{array}$ & $\begin{array}{l}.844 \\
.169\end{array}$ & $\begin{array}{l}8.839 \\
1.768\end{array}$ & $1: 10.5$ & 8.5 & 91.5 & 7.9 & 83.6 \\
\hline Seeond s & & & & & & 1.700 & & & & & .... \\
\hline 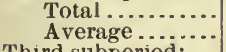 & $\begin{array}{l}5.129 \\
1.026\end{array}$ & $\begin{array}{r}12.807 \\
2.562\end{array}$ & $\begin{array}{r}1.443 \\
.289\end{array}$ & $\begin{array}{r}11.364 \\
2.273\end{array}$ & $\begin{array}{l}.879 \\
.176\end{array}$ & $\begin{array}{r}10.485 \\
2.097\end{array}$ & $\begin{array}{l}1: 11.9 \\
\ldots \ldots \ldots\end{array}$ & 11.3 & $\begin{array}{c}88.7 \\
\cdots \ldots .\end{array}$ & 6.8 & 81.9 \\
\hline $\begin{array}{r}\text { Third su } \\
\text { Tota }\end{array}$ & & 12.250 & 1.481 & 10.769 & .952 & 9.817 & $1: 10.3$ & & 87.9 & 78 & ..... \\
\hline & .981 & 2.450 & $\begin{array}{l}1.291 \\
.296\end{array}$ & 2.154 & .190 & 1.964 & & 12.1 & 87.9 & $\begin{array}{l}7.8 \\
\cdots\end{array}$ & $\begin{array}{r}80.1 \\
\ldots . . .\end{array}$ \\
\hline $\begin{array}{l}\text { od: } \\
\ldots \ldots \\
\ldots \ldots\end{array}$ & 4. 734 & 11.821 & 1. 496 & 10.625 & .887 & & $1: 11.0$ & 10.1 & 89.9 & 7.5 & 82.4 \\
\hline riod: & 5 & 2. 365 & .240 & & .177 & & & $\cdots, \cdots$ & -9 & -1 & ... \\
\hline & $\begin{array}{l}5.173 \\
1.035\end{array}$ & $\begin{array}{r}12.917 \\
2.584\end{array}$ & $\begin{array}{r}1.638 \\
.328\end{array}$ & $\begin{array}{r}11.279 \\
2.256\end{array}$ & $\begin{array}{l}.948 \\
.190\end{array}$ & $\begin{array}{r}10.331 \\
2.066\end{array}$ & $1: 10.9$ & 12.7 & 87.3 & 7.3 & 80.0 \\
\hline $\begin{array}{r}\text { Sixth sub } \\
\text { Total }\end{array}$ & 4.973 & 12,418 & 1.326 & 11092 & 081 & & & & & & $\cdots$ \\
\hline A verage ........... & .995 & 2.485 & .267 & 2. 218 & $\begin{array}{l}1.001 \\
.216\end{array}$ & $\begin{array}{r}10.011 \\
2.002\end{array}$ & $\begin{array}{c}1: 9.3 \\
\cdots \cdots\end{array}$ & $\begin{array}{c}10.7 \\
\cdots \cdots\end{array}$ & $\begin{array}{c}89.3 \\
\ldots \ldots .\end{array}$ & $\begin{array}{r}8.7 \\
\cdots . . .\end{array}$ & $\begin{array}{r}80.6 \\
\ldots . .\end{array}$ \\
\hline $\begin{array}{l}\text { Entire preservative } \\
\text { period: } \\
\text { Total .............. } \\
\text { Average........ }\end{array}$ & $\begin{array}{r}29.151 \\
.972 \\
\end{array}$ & $\begin{array}{r}72.790 \\
2.427 \\
\end{array}$ & $\begin{array}{r}7.978 \\
.267 \\
\end{array}$ & $\begin{array}{r}64.812 \\
2.160 \\
\end{array}$ & $\begin{array}{r}5.591 \\
.186\end{array}$ & $\begin{array}{r}59.221 \\
1.974\end{array}$ & $\begin{array}{l}1: 10.6 \\
\cdots \cdots\end{array}$ & 11.0 & 89.0 & 7. 7 & $\begin{array}{r}81.4 \\
\cdots \cdots \\
\end{array}$ \\
\hline After period. & & & & & & & & & & & \\
\hline $\begin{array}{l}\text { First subperiod: } \\
\text { Total ........ } \\
\text { Average ..... }\end{array}$ & $\begin{array}{l}4.999 \\
1.000\end{array}$ & $\begin{array}{r}12.483 \\
2.497\end{array}$ & $\begin{array}{r}1.460 \\
.292\end{array}$ & $\begin{array}{r}11.023 \\
2.205\end{array}$ & $\begin{array}{l}.907 \\
.181\end{array}$ & $\begin{array}{r}10.116 \\
2.024\end{array}$ & $1: 11.2$ & 11.7 & 88.3 & 7.3 & 81.0 \\
\hline $\begin{array}{l}\text { Seeond } \\
\text { Tot }\end{array}$ & & & & & & & & & & & \\
\hline 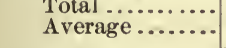 & $\begin{array}{l}5.164 \\
1.033\end{array}$ & $\begin{array}{r}12.895 \\
2.579\end{array}$ & $\begin{array}{r}1.574 \\
.315\end{array}$ & $\begin{array}{r}11.321 \\
2.264\end{array}$ & $\begin{array}{l}.871 \\
.174\end{array}$ & $\begin{array}{r}10.450 \\
2.090\end{array}$ & $1: 12.0$ & $\begin{array}{l}12.2 \\
\cdots \ldots\end{array}$ & $\begin{array}{l}87.8 \\
\cdots . . .\end{array}$ & 6.8 & $\begin{array}{r}81.0 \\
-. . .\end{array}$ \\
\hline $\begin{array}{l}\text { Entire after period: } \\
\text { Total ............. } \\
\text { Average........ }\end{array}$ & $\begin{array}{r}10.163 \\
1.016\end{array}$ & $\begin{array}{r}25.377 \\
2.537\end{array}$ & $\begin{array}{r}3.033 \\
.303\end{array}$ & $\begin{array}{r}22.344 \\
2.234\end{array}$ & $\begin{array}{r}1.778 \\
.178\end{array}$ & $\begin{array}{r}20.566 \\
2.056\end{array}$ & $1: 11.6$ & 12.0 & $\begin{array}{c}88.0 \\
\cdots \ldots . .\end{array}$ & 7.0 & 81.0 \\
\hline
\end{tabular}


TABLE X.-Urine determinations-Ratio of preformed sulphates to ethereal sulphates and neutral sulphur-Series VI-Continued.

[Averages are per man per day.]

Summary for nine men.

\begin{tabular}{|c|c|c|c|c|c|c|c|c|c|c|c|}
\hline \multirow[b]{2}{*}{ Period. } & \multirow[b]{2}{*}{ 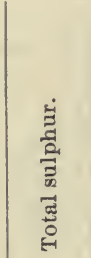 } & \multirow{2}{*}{ 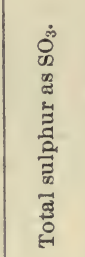 } & \multirow{2}{*}{ 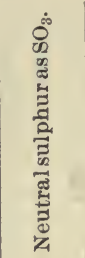 } & \multirow[b]{2}{*}{ 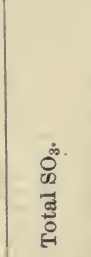 } & \multirow{2}{*}{ 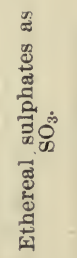 } & \multirow{2}{*}{ 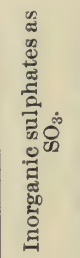 } & \multirow{2}{*}{ 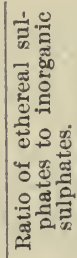 } & \multicolumn{4}{|c|}{$\begin{array}{l}\text { Results expressed in per } \\
\text { cent of total sulphur } \\
\text { in terms of } \mathrm{SO}_{3} \text {. }\end{array}$} \\
\hline & & & & & & & & 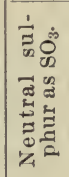 & 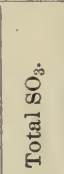 & 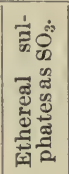 & 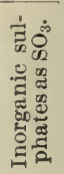 \\
\hline $\begin{array}{l}\text { Fore period. } \\
\text { First subperiod: } \\
\text { Total ............. } \\
\text { Average....... }\end{array}$ & $\begin{array}{l}\text { Grams. } \\
40.242 \\
.894\end{array}$ & $\begin{array}{r}\text { Grams. } \\
100.485 \\
2.233\end{array}$ & $\begin{array}{r}\text { Gramis. } \\
12.462 \\
.277\end{array}$ & $\begin{array}{r}\text { Grams. } \\
88.023 \\
1.956\end{array}$ & $\begin{array}{r}\text { Grams. } \\
7.163 \\
.159\end{array}$ & $\begin{array}{r}\text { Grams. } \\
80.860 \\
1.797\end{array}$ & $\begin{array}{l}1: 11.3 \\
\ldots \ldots \ldots\end{array}$ & $\begin{array}{l}\text { P.ct. } \\
12.4\end{array}$ & $\begin{array}{l}\text { P.ct. } \\
8 \overline{7} .6\end{array}$ & $\begin{array}{c}P . c t . \\
7.1\end{array}$ & $\begin{array}{r}P . c t . \\
80.5\end{array}$ \\
\hline $\begin{array}{r}\text { Second subperiod: } \\
\text { Total } \\
\text { Average.................... }\end{array}$ & $\begin{array}{r}39.487 \\
.878\end{array}$ & $\begin{array}{r}98.59 \dot{8} \\
2.191\end{array}$ & $\begin{array}{r}10.915 \\
.243\end{array}$ & $\begin{array}{r}87.683 \\
1.949\end{array}$ & $\begin{array}{r}7.549 \\
.168\end{array}$ & $\begin{array}{r}80.134 \\
1: 781\end{array}$ & $\begin{array}{l}1: 10.6 \\
\cdots \cdots\end{array}$ & 11.1 & 88.9 & 7.7 & 81.3 \\
\hline $\begin{array}{l}\text { Entire fore period: } \\
\text { Total ............. } \\
\text { Average........ }\end{array}$ & $\begin{array}{r}79.729 \\
.887 \\
\end{array}$ & $\begin{array}{r}199.083 \\
2.212 \\
\end{array}$ & $\begin{array}{r}23.377 \\
.260 \\
\end{array}$ & $\begin{array}{r}175.706 \\
1.952 \\
\end{array}$ & $\begin{array}{r}14.712 \\
.163 \\
\end{array}$ & $\begin{array}{r}160.994 \\
1.789 \\
\end{array}$ & \begin{tabular}{c}
$1: 10.9$ \\
\hdashline$\ldots \ldots$. \\
\end{tabular} & 11.7 & 88.3 & 7.4 & 80.9 \\
\hline Preservative period. & & & & & & & & & & & \\
\hline $\begin{array}{l}\text { First subperiod: } \\
\text { Total .............. } \\
\text { Average ......... }\end{array}$ & $\begin{array}{r}38.571 \\
.857\end{array}$ & $\begin{array}{r}96.311 \\
2.140\end{array}$ & $\begin{array}{r}9.485 \\
.211\end{array}$ & $\begin{array}{r}86.826 \\
1.929\end{array}$ & $\begin{array}{r}7.801 \\
.173\end{array}$ & $\begin{array}{r}79.025 \\
1.756\end{array}$ & $\begin{array}{l}1: 10.1 \\
\ldots \ldots . .\end{array}$ & 9.8 & 90.2 & 8.1 & 82.1 \\
\hline $\begin{aligned} \text { Secon } \\
\text { Tr } \\
\text { A }\end{aligned}$ & $\begin{array}{r}41.073 \\
.913\end{array}$ & $\begin{array}{r}102.560 \\
2.279\end{array}$ & $\begin{array}{r}12.304 \\
.273\end{array}$ & $\begin{array}{r}90.256 \\
2.006\end{array}$ & $\begin{array}{r}7.274 \\
.162\end{array}$ & $\begin{array}{r}82.982 \\
1.844\end{array}$ & & 12.0 & 88.0 & 7.1 & 80.9 \\
\hline $\begin{array}{r}\text { Third } \\
\text { Tf } \\
\text { A }\end{array}$ & $\begin{array}{r}39.750 \\
.883\end{array}$ & $\begin{array}{r}99.256 \\
2.206\end{array}$ & $\begin{array}{r}11.719 \\
.260\end{array}$ & $\begin{array}{r}87.537 \\
1.945\end{array}$ & $\begin{array}{r}7.607 \\
.169\end{array}$ & $\begin{array}{r}79.930 \\
1.776\end{array}$ & $1: 10.5$ & 11.8 & 88.2 & 7.7 & 80.5 \\
\hline $\begin{array}{l}\text { Fourth su } \\
\text { Total. } \\
\text { Avera }\end{array}$ & $\begin{array}{r}40.123 \\
.892\end{array}$ & $\begin{array}{r}100.187 \\
2.226\end{array}$ & $\begin{array}{r}11.210 \\
.249\end{array}$ & $\begin{array}{r}88.977 \\
1.977\end{array}$ & $\begin{array}{r}7.415 \\
.165\end{array}$ & $\begin{array}{r}81.562 \\
1.812\end{array}$ & & 11.2 & 88.8 & 7.4 & 81.4 \\
\hline $\begin{array}{r}\text { Fith sub } \\
\text { Total } \\
\text { Avera }\end{array}$ & $\begin{array}{r}41.301 \\
.918\end{array}$ & $\begin{array}{r}103.128 \\
\quad 2.292\end{array}$ & $\begin{array}{r}11.594 \\
.258\end{array}$ & $\begin{array}{r}91.534 \\
2.034\end{array}$ & $\begin{array}{r}7.764 \\
.173\end{array}$ & $\begin{array}{r}83.770 \\
1.862\end{array}$ & & 11.2 & 88.8 & 7.5 & 81.2 \\
\hline $\begin{array}{l}\text { Sixth subperiod: } \\
\text { Total ............ } \\
\text { Average......... }\end{array}$ & $\begin{array}{r}43.892 \\
\quad .975\end{array}$ & $\begin{array}{r}109.599 \\
2.436\end{array}$ & $\begin{array}{r}19.517 \\
.434\end{array}$ & $\begin{array}{r}90.082 \\
2.002\end{array}$ & $\begin{array}{r}8.287 \\
.184\end{array}$ & $\begin{array}{r}81.795 \\
1.818\end{array}$ & 1: 9.9 & 17.8 & 82.2 & 7.6 & 74.6 \\
\hline $\begin{array}{c}\text { Entire preservative } \\
\text { period: } \\
\text { Total............ } \\
\text { Average........ }\end{array}$ & $\begin{array}{r}244.710 \\
.906 \\
\end{array}$ & $\begin{array}{r}611.041 \\
2.263\end{array}$ & $\begin{array}{r}75.829 \\
.281 \\
\end{array}$ & $\begin{array}{r}535.212 \\
1.982 \\
\end{array}$ & $\begin{array}{r}46.148 \\
.171 \\
\end{array}$ & $\begin{array}{r}489.064 \\
1.811 \\
\end{array}$ & $\begin{array}{l}1: 10.6 \\
\cdots \ldots . .\end{array}$ & 12.4 & 87.6 & 7.6 & $\begin{array}{r}80.0 \\
\cdots . .\end{array}$ \\
\hline After period. & & & & & & & & & & & \\
\hline $\begin{array}{l}\text { First subperiod: } \\
\text { Total ........... } \\
\text { Average ..... }\end{array}$ & $\begin{array}{r}41.014 \\
.911\end{array}$ & $\begin{array}{r}102.413 \\
2.276\end{array}$ & $\begin{array}{r}14.788 \\
.329\end{array}$ & $\begin{array}{r}87.625 \\
1.947\end{array}$ & $\begin{array}{r}7.414 \\
.165\end{array}$ & $\begin{array}{r}80.211 \\
1.782\end{array}$ & 1:10.8 & 14.4 & 85.6 & 7.2 & 78.3 \\
\hline $\begin{array}{l}\text { Second subperiod: } \\
\text { Total ................ } \\
\text { Average .......... }\end{array}$ & $\begin{array}{r}42.176 \\
.937\end{array}$ & $\begin{array}{r}105.314 \\
2.340\end{array}$ & $\begin{array}{r}13.581 \\
.302\end{array}$ & $\begin{array}{r}91.733 \\
2.039\end{array}$ & $\begin{array}{r}7.373 \\
.164\end{array}$ & $\begin{array}{r}84.360 \\
1.875\end{array}$ & 1:11.4 & 12.9 & 87.1 & 7.0 & 80.1 \\
\hline $\begin{array}{r}\text { Entire after period: } \\
\text { Total ............. } \\
\text { Average ......... }\end{array}$ & $\begin{array}{r}83.190 \\
.924\end{array}$ & $\begin{array}{r}207.727 \\
2.308\end{array}$ & $\begin{array}{r}28.369 \\
.315\end{array}$ & $\begin{array}{r}179.358 \\
1.993\end{array}$ & $\begin{array}{r}14.787 \\
.164\end{array}$ & $\begin{array}{r}164.571 \\
1.829\end{array}$ & 1:11.1 & 13.7 & 86.3 & 7.1 & 79.2 \\
\hline
\end{tabular}

\section{MICROSCOPICAL EXAMINATION OF THE URINE.}

In giving the results of the microscopical examination of the urine (Table XI) the numerals are used to express approximately the frequency with which the various bodies named occur and have the following significance: None, 0 ; very few, 1 ; few, 2 ; fairly numerous, 3 ; numerous, 4 ; extremely numerous, 5 .

The dates given in the table represent the days on which the examinations were made, only one sample having been taken for each indi- 
vidual during the time specified. Each sample represented the urine collected during the twenty-four hours preceding its examination. The difficulty in keeping the sample and the time necessary for its examination made this method of procedure necessary.

DISCUSSION OF OBSERVATIONS.

Uric acid crystals and urates. - No crystals of uric acid were found during the observations, with the exception of one instance during the preservative period in the case of No. 9, when very few are recorded. Urates were also absent throughout the tests.

Crystals of calcium oxalate. - These crystals were found in all cases. In the case of No. 1 they were numerous in one instance in the after period; in the case of No. 2 they were numerous in two instances, once during the preservative period and once during the after period, and in the case of No. 11 they were found to be numerous once during the preservative period. The relative occurrence of these crystals during the three periods (determined by dividing the total for each period by the number of observations and multiplying by 100) indicates a marked tendency on the part of the preservative to increase the calcium oxalate crystals in the urine, and this tendency is continued to a more marked degree in the after period, the figures being 41.7 for the fore period, 85.4 for the preservative period, and 91.3 for the after period.

Crystalline phosphates. - No crystalline phosphates were present in the cases of Nos. $5,6,7,8$, and 9 , and in three other cases, namely, Nos. 3,4 , and 12 , only a very few were present in one or two instances. Nos. 1, 2; and 11 showed the largest numbers present, and they occurred principally in the preservative period, being numerous in one instance in the case of No. 1, extremely numerous in the case of No. 2, and fairly numerous in the case of No. 11. The figures giving the relative occurrence show a very slight increase during the preservative period, and a marked decrease in the after period, the figures for the three periods being $50,52.1$, and 39.1 , respectively.

Amorphous phosphates.-No amorphous phosphates were found in the cases of Nos. 5, 6, 7, 8, 9, and 12. The report for No. 1 shows that they were numerous in the fore period and at the first three examinations of the preservative period, were not present at all in the last observation of that period and the first one of the after period, but were extremely numerous at the last examination. Nos. 2 and 3 have no amorphous phosphates present in the fore and after periods, and only a very few at one observation of the preservative period. The report for No. 4 shows that they were fairly numerous in the fore period and were not again present. In the case of No. 10 they were numerous in the fore period and at the first observation in the preservative period, and did not appear again. In the case of No. 11 they did not occur until the after period, when the two observations 
shored them to be numerous and extremely numerous. The figures for the relative occurrence by periods would indicate a considerable reduction of these bodies during the preservative period, with an increase in the after period; but it must be remembered that these figures are used as representative in a general way of the mass expression of a condition and are not mathematically exact. In this case the individual variations do not seem to bear out the conclusion suggested by the average-certainly not to the extent indicated.

Epithelium cells. - These bodies were present in all cases, a few being present in the majority of observations, and occurring uniformly throughout the fore period except in the case of No. 12, where very few were indicated. During the preservative period the record for No. 2 shows numerous cells present in one observation, and in the other three examinations they were fairly numerous; in the case of No. 6 they were fairly numerons in one instance and a few were present at each of the other examinations. In the after period Nos. 2 and 6 continued to show the largest number present, one examination showing the cells to be numerous and one few in both cases. The figures on the relative occurrence in the three periods show that the number of epithelium cells present decreased during the preservative period and increased again to almost the same number as were present in the fore period after the preservative was withdrawn. The changes, however, were comparatively small, the figures reading 191.7, 183.3, and 191.3 for the fore, preservative, and after periods, respectively.

Lencocytes.-Leucocytes were present in the urine in all cases and at all observations with the exception of one in the fore period of No. 12 and the first observation of the preservative period of Nos. 2 and 10. In the large majority of cases few were reported, and they were fairly numerous only in one case, the last observation of the preservative period for No. 6. The figures for the relative occurrence show a decided tendency to increase the presence of these bodies during the preservative period, with a slight decrease in the after period.

Red blood cells.-No red blood cells were found at any time during the experiment.

Hyaline casts.-The hyaline casts are present in greater numbers than any of the other forms of casts observed. There are only three cases, however, in which more than a few are reported, namely, No. 2 , one case reported fairly numerous in the after period; No. 6, one case numerous in the after period; and No. 9, one case reported numerous at the close of the preservative period. In the case of No. 3 they were not present at all at four of the examinations, and for Nos. 2, 6, 7 , and 9 they are reported at each observation. The figures on the relative occurrence indicate a decided increase of these casts during the preservative period and a slight continued increase during the after period. 
Finely granular casts.-The finely granular casts were present to a much less extent than the hyaline casts, and the figures for relative occurrence, namely, 83.3, 56.3, and 39.1, indicate a continued decrease throughout the experiment. In the case of No. 3 they were not found at all, and in the case of No. 11 only once, when a very few are reported; for Nos. 7 and 10 very few were reported twice, and for Nos. 4, 8, and 12 three times. In only one instance, during the fore period of No. 1, were more than a few casts reported.

Coarsely granular casts. - These casts occur to a still less extent than the finely granular, as indicated by the figures for relative occurrence: Fore period, 41.7; preservative period, 35.4, and after period, 39.1. These figures show a slight tendency for the casts to decrease during the preservative period and increase in the after period. The individual figures show that these bodies did not occur at all in the case of Nos. $5,10,11$, and 12 , and were present to the greatest extent in the cases of Nos. 1,2 , and 9 .

Epithelial casts.-Epithelial casts were reported in only one instance, very few being observed in the case of No. 8 in the preservative period. Other forms of casts were reported also in one instance, namely, a report of very few in the case of No. 8 during the preservative period.

Mucous cylindroids. - These bodies were found for all individuals and at every observation taken. They were present to the greatest extent in the case of No. 6, being numerous throughout the preservative period and extremely numerous in the after period; only a few are reported in the fore period. Only in the cases of Nos. 5 and 7 are they reported as numerous, a few or a very few being reported in the other cases. The average figures given indicate a decided increase of these bodies in the preservative period and a slight decrease in the after period.

Mucous strands.-The presence of mucous strands was marked in all cases and in the cases of Nos. 6 and 7 they are recorded as being numerous or extremely numerous at every examination. The averages show the prevalence of this condition, being 183.3 for the fore period, 220.8 for the preservative period, and 243.5 for the after period, the increase in the after period being greater than in the preservative period.

\section{GENERAL CONCLUSION.}

A general view of the microscopical examination of the urine shows many instances in which thê specific effects observed can be attributed to the exhibition of the preservative.

The fact that the data as expressed by the figures represent only approximations must be remembered in reading the text on the tables, and also that the variations are evidently great from day to day and only a comparatively small number of observations were made. It is 
not possible therefore on the basis of the data submitted to infer more than that there is apparently a tendency on the part of the preservative to increase the number of these microscopic bodies appearing in the urine, about in the proportion indicated by the general summary, which shows a relative abundance of 68.3 for the fore period, 78.3 for the preservative period, and 79.4 for the after period.

TABLE XI.-Microscopical examination of the urine, Series VI.

[None, 0 ; very few, 1 ; few, 2 ; fairly numerous, 3 ; numerous, 4 ; extremely numerous, 5 .]

URIC-ACID CRYSTALS.

\begin{tabular}{|c|c|c|c|c|c|c|c|}
\hline \multirow{2}{*}{ No. } & \multirow{2}{*}{$\frac{\begin{array}{c}\text { Fore } \\
\text { period. }\end{array}}{\text { Oct. } 21-22 .}$} & \multicolumn{4}{|c|}{ Preservative period. } & \multicolumn{2}{|c|}{ After period. } \\
\hline & & Oet. 29-31. & Nov.5-9. & Nov. 12-14. & Nov. 19-21. & $\begin{array}{c}\text { Nov. 28- } \\
\text { Dec. } 1 .\end{array}$ & Dec. 3-5. \\
\hline 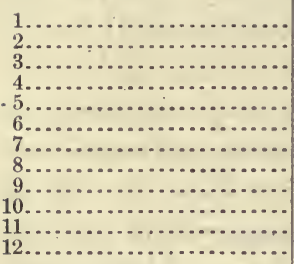 & $\begin{array}{l}0 \\
0 \\
0 \\
0 \\
0 \\
0 \\
0 \\
0 \\
0 \\
0 \\
0 \\
0\end{array}$ & $\begin{array}{l}0 \\
0 \\
0 \\
0 \\
0 \\
0 \\
0 \\
0 \\
0 \\
0 \\
0 \\
0\end{array}$ & $\begin{array}{l}0 \\
0 \\
0 \\
0 \\
0 \\
0 \\
0 \\
0 \\
1 \\
0 \\
0 \\
0\end{array}$ & $\begin{array}{l}0 \\
0 \\
0 \\
0 \\
0 \\
0 \\
0 \\
0 \\
0 \\
0 \\
0 \\
0\end{array}$ & $\begin{array}{l}0 \\
0 \\
0 \\
0 \\
0 \\
0 \\
0 \\
0 \\
0 \\
0 \\
0 \\
0\end{array}$ & $\begin{array}{l}0 \\
0 \\
0 \\
0 \\
0 \\
0 \\
0 \\
0 \\
0 \\
0 \\
0 \\
0\end{array}$ & $\begin{array}{l}0 \\
0 \\
0 \\
0 \\
0 \\
0 \\
0 \\
0 \\
0 \\
\because 0 \\
0\end{array}$ \\
\hline Total .................. & 0 & 0 & 1 & 0 & 0 & 0 & 0 \\
\hline Relative occurrence....... & 0 & \multicolumn{4}{|c|}{2.1} & \multicolumn{2}{|c|}{0} \\
\hline
\end{tabular}

URATES.

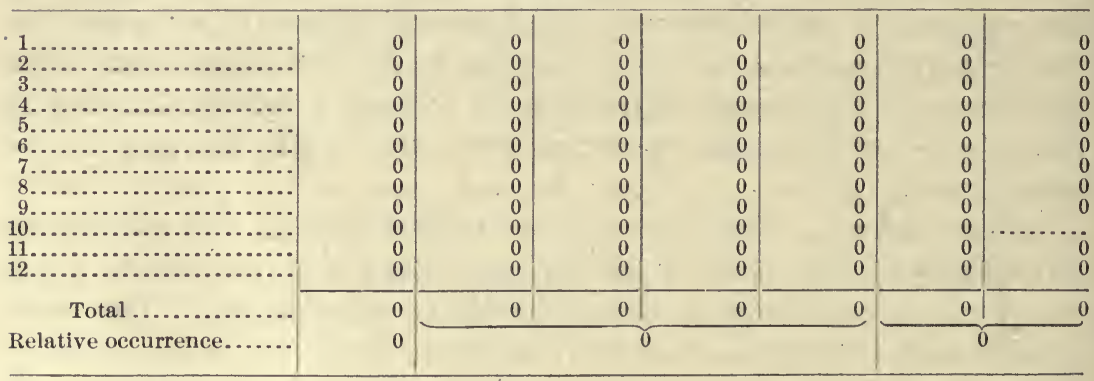

CALCIUM OXALATE CRYSTALS.

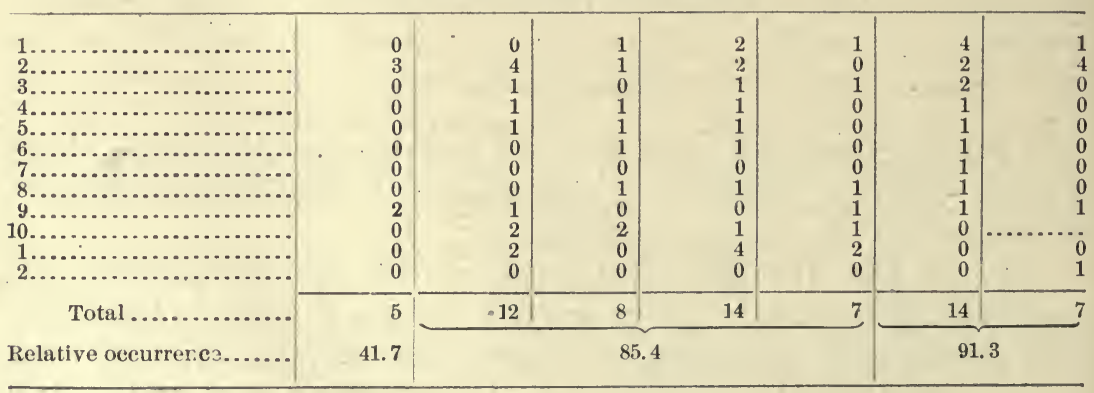


TABLE XI.-Microscopical examination of the urine, Series VI-Continued.

[None, 0 ; very few, 1 ; few, 2 ; fairly numerous, 3 ; numerous, 4 ; extremely numerous, 5.]

CRYSTALLINE PHOSPHATES.

\begin{tabular}{|c|c|c|c|c|c|c|c|}
\hline \multirow{2}{*}{ No. } & \multirow{2}{*}{$\frac{\begin{array}{c}\text { Fore } \\
\text { period. }\end{array}}{\text { Oct.21-22. }}$} & \multicolumn{4}{|c|}{ Preservative period. } & \multicolumn{2}{|c|}{ After period. } \\
\hline & & Oet.-29-31. & Nov. 5-9. & Nov. 12-14. & Nov. 19-21. & $\begin{array}{l}\text { Nov. 28- } \\
\text { Dec. } 1 .\end{array}$ & Dec. 3-5. \\
\hline 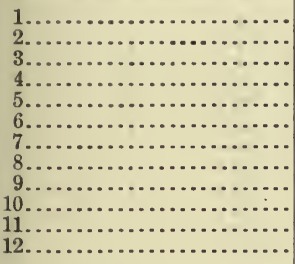 & $\begin{array}{l}0 \\
0 \\
1 \\
0 \\
0 \\
0 \\
0 \\
0 \\
0 \\
4 \\
1 \\
0\end{array}$ & $\begin{array}{l}2 \\
0 \\
0 \\
0 \\
0 \\
0 \\
0 \\
0 \\
4 \\
0 \\
0\end{array}$ & $\begin{array}{l}2 \\
5 \\
0 \\
0 \\
0 \\
0 \\
0 \\
0 \\
0 \\
1 \\
1 \\
0\end{array}$ & $\begin{array}{l}4 \\
1 \\
1 \\
0 \\
0 \\
0 \\
0 \\
0 \\
0 \\
0 \\
3 \\
0\end{array}$ & $\begin{array}{l}1 \\
0 \\
0 \\
0 \\
0 \\
0 \\
0 \\
0 \\
0 \\
0 \\
0 \\
0\end{array}$ & $\begin{array}{l}0 \\
0 \\
0 \\
0 \\
0 \\
0 \\
0 \\
0 \\
0 \\
3 \\
1 \\
0\end{array}$ & $\begin{array}{c}1 \\
1 \\
0 \\
1 \\
0 \\
0 \\
0 \\
0 \\
0 \\
1 \\
1 \\
1\end{array}$ \\
\hline Total .......... & 6 & 61 & 9 & 9 & 1 & 4 & 5 \\
\hline Relative occurrence....... & 50.0 & \multicolumn{4}{|c|}{52.1} & \multicolumn{2}{|c|}{39.1} \\
\hline
\end{tabular}

AMORPHOUS PHOSPHATES.

\begin{tabular}{|c|c|c|c|c|c|c|c|}
\hline 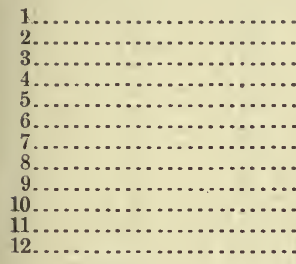 & $\begin{array}{r}4 \\
0 \\
0 \\
3 \\
0 \\
0 \\
0 \\
0 \\
0 \\
4 \\
0 \\
0\end{array}$ & $\begin{array}{l}4 \\
0 \\
0 \\
0 \\
0 \\
0 \\
0 \\
0 \\
0 \\
4 \\
0 \\
0\end{array}$ & $\begin{array}{l}4 \\
0 \\
1 \\
0 \\
0 \\
0 \\
0 \\
0 \\
0 \\
0 \\
0 \\
0\end{array}$ & $\begin{array}{l}4 \\
0 \\
0 \\
0 \\
0 \\
0 \\
0 \\
0 \\
0 \\
0 \\
0 \\
0\end{array}$ & $\begin{array}{l}0 \\
1 \\
0 \\
0 \\
0 \\
0 \\
0 \\
0 \\
0 \\
0 \\
0 \\
0\end{array}$ & $\begin{array}{l}0 \\
0 \\
0 \\
0 \\
0 \\
0 \\
0 \\
0 \\
0 \\
0 \\
4 \\
0\end{array}$ & $\begin{array}{l}5 \\
0 \\
0 \\
0 \\
0 \\
0 \\
0 \\
0 \\
0 \\
-5 \\
0\end{array}$ \\
\hline Total .................. & 11 & 8 & 5 & 4 & 1 & 4 & 10 \\
\hline Relative oceurrence....... & 91.7 & \multicolumn{4}{|c|}{37.5} & \multicolumn{2}{|c|}{60.9} \\
\hline
\end{tabular}

EPITHELIUM CELLS.

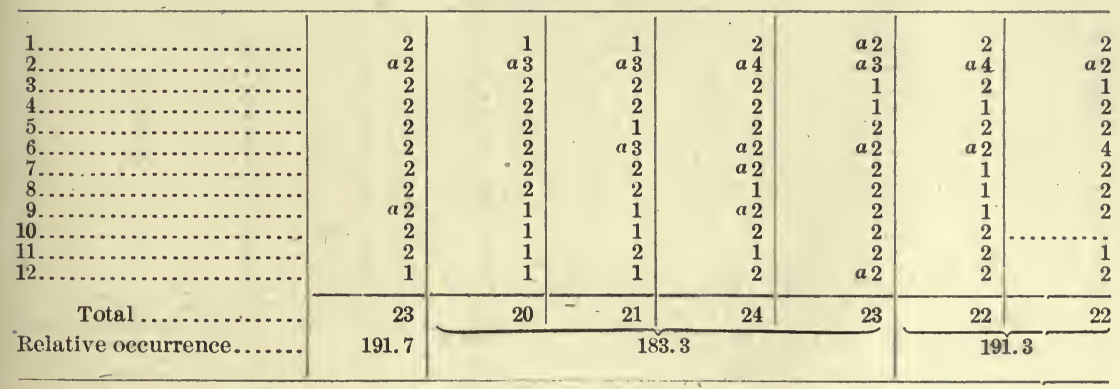

$a$ Some in sheets. 
TABLE XI.-Microscopical examination of the urine, Series VI-Continued.

[None, 0 ; very few, 1 ; few, 2; fairly numerous, 3 ; numerous, 4; extremely numerous, 5.] LEUCOCYTES.

\begin{tabular}{|c|c|c|c|c|c|c|c|}
\hline \multirow{2}{*}{ No. } & \multirow{2}{*}{$\begin{array}{c}\begin{array}{c}\text { Fore } \\
\text { period. }\end{array} \\
\text { Oct. } 21-22 .\end{array}$} & \multicolumn{4}{|c|}{ Preservative period. } & \multicolumn{2}{|c|}{ After period. } \\
\hline & & Oct. 29-31. & Nov. 5-9. & Nov. 12-14. & Nov. 19-21. & $\begin{array}{l}\text { Nov. } 28- \\
\text { Dec. } 1 .\end{array}$ & Dec. $3-5$. \\
\hline 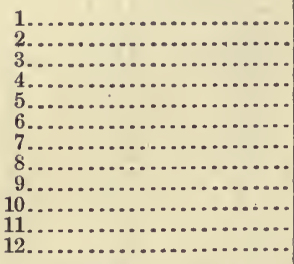 & $\begin{array}{l}1 \\
1 \\
1 \\
1 \\
2 \\
1 \\
2 \\
1 \\
1 \\
1 \\
1 \\
0\end{array}$ & $\begin{array}{l}1 \\
0 \\
1 \\
2 \\
2 \\
2 \\
2 \\
2 \\
2 \\
0 \\
2 \\
1\end{array}$ & $\begin{array}{r}1 \\
1 \\
1 \\
1 \\
2 \\
2 \\
a 2 \\
2 \\
2 \\
1 \\
1 \\
2 \\
2\end{array}$ & $\begin{array}{l}2 \\
2 \\
1 \\
2 \\
2 \\
2 \\
3 \\
2 \\
2 \\
2 \\
2 \\
2\end{array}$ & $\begin{array}{l}2 \\
2 \\
1 \\
2 \\
2 \\
2 \\
3 \\
2 \\
2 \\
2 \\
2 \\
2 \\
2 \\
1\end{array}$ & $\begin{array}{l}1 \\
2 \\
1 \\
1 \\
2 \\
2 \\
2 \\
2 \\
2 \\
2 \\
1 \\
1\end{array}$ & ? \\
\hline Total $\ldots \ldots \ldots \ldots \ldots$ & 13 & 17 & 19 & 24 & 23 & 19 & 18 \\
\hline Relative occurrence....... & 108.3 & \multicolumn{4}{|c|}{172.9} & \multicolumn{2}{|c|}{160.9} \\
\hline
\end{tabular}

RED BLOOD CELLS.

\begin{tabular}{|c|c|c|c|c|c|c|c|}
\hline 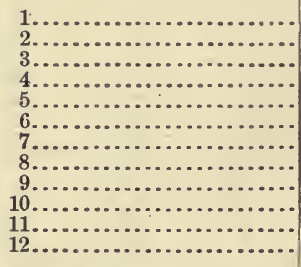 & $\begin{array}{l}0 \\
0 \\
0 \\
0 \\
0 \\
0 \\
0 \\
0 \\
0 \\
0 \\
0 \\
0\end{array}$ & $\begin{array}{l}0 \\
0 \\
0 \\
0 \\
0 \\
0 \\
0 \\
0 \\
0 \\
0 \\
0 \\
0\end{array}$ & $\begin{array}{l}0 \\
0 \\
0 \\
0 \\
0 \\
0 \\
0 \\
0 \\
0 \\
0 \\
0 \\
0\end{array}$ & $\begin{array}{l}0 \\
0 \\
0 \\
0 \\
0 \\
0 \\
0 \\
0 \\
0 \\
0 \\
0 \\
0\end{array}$ & $\begin{array}{l}0 \\
0 \\
0 \\
0 \\
0 \\
0 \\
0 \\
0 \\
0 \\
0 \\
0 \\
0\end{array}$ & $\begin{array}{l}0 \\
0 \\
0 \\
0 \\
0 \\
0 \\
0 \\
0 \\
0 \\
0 \\
0 \\
0\end{array}$ & $\begin{array}{l}0 \\
0 \\
0 \\
0 \\
0 \\
0 \\
0 \\
0 \\
0 \\
0 \\
0 \\
0\end{array}$ \\
\hline Total $\ldots \ldots \ldots \ldots \ldots$ & 0 & 0 & 0 & 0 & 0 & 0 & 0 \\
\hline Relative occurrence........ & 0 & & 0 & & & & \\
\hline
\end{tabular}

HYALINE CASTS.

\begin{tabular}{|c|c|c|c|c|c|c|c|}
\hline 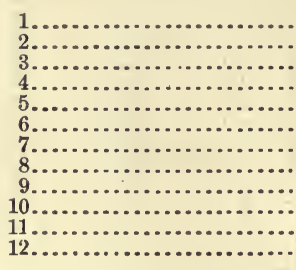 & $\begin{array}{l}2 \\
1 \\
0 \\
0 \\
2 \\
2 \\
1 \\
1 \\
1 \\
1 \\
0 \\
0\end{array}$ & $\begin{array}{l}1 \\
1 \\
0 \\
1 \\
2 \\
2 \\
1 \\
1 \\
2 \\
0 \\
1 \\
1\end{array}$ & $\begin{array}{r}0 \\
2 \\
0 \\
1 \\
1 \\
1 \\
-1 \\
1 \\
2 \\
0 \\
1 \\
1\end{array}$ & $\begin{array}{l}2 \\
2 \\
1 \\
2 \\
2 \\
2 \\
2 \\
1 \\
2 \\
1 \\
1 \\
2\end{array}$ & $\begin{array}{l}2 \\
2 \\
1 \\
0 \\
1 \\
2 \\
1 \\
1 \\
4 \\
2 \\
2 \\
2\end{array}$ & $\begin{array}{l}1 \\
3 \\
2 \\
1 \\
0 \\
2 \\
1 \\
0 \\
1 \\
0 \\
1 \\
1\end{array}$ & $\begin{array}{l}2 \\
2 \\
0 \\
2 \\
2 \\
4 \\
1 \\
1 \\
2 \\
1 \\
1\end{array}$ \\
\hline Total $\ldots \ldots \ldots \ldots$ & 11 & 13 & 11 & 20 & 20 & 13 & 19 \\
\hline Relative occurrence........ & 91.7 & \multicolumn{4}{|c|}{133.3} & \multicolumn{2}{|c|}{139.1} \\
\hline
\end{tabular}

$a$ Some in sheets. 
Table XI.-Microscopical examination of the urine, Series VI-Continued.

[None, 0 ; very few, 1 ; few, 2 ; fairly numerous, 3 ; numerous, 4 ; extremely numerous, 5 .]

FINELY GRANULAR CASTS.

\begin{tabular}{|c|c|c|c|c|c|c|c|}
\hline \multirow{2}{*}{ No. } & \multirow{2}{*}{$\begin{array}{c}\begin{array}{c}\text { Fore } \\
\text { period. }\end{array} \\
\text { Oct. } 21-22 .\end{array}$} & \multicolumn{4}{|c|}{ Preservative period. } & \multicolumn{2}{|c|}{ After period. } \\
\hline & & Oct. $29-31$. & Nov. 5-9. & Nov. 12-14. & Nov. 19-21. & $\begin{array}{l}\text { Nov. 28- } \\
\text { Dec.1. }\end{array}$ & Dec. 3-5. \\
\hline 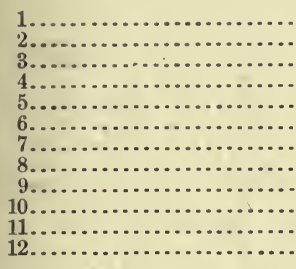 & $\begin{array}{l}3 \\
1 \\
0 \\
0 \\
1 \\
1 \\
2 \\
1 \\
0 \\
1 \\
0 \\
0 \\
0\end{array}$ & $\begin{array}{l}1 \\
1 \\
0 \\
1 \\
1 \\
2 \\
1 \\
1 \\
1 \\
0 \\
1 \\
1 \\
\end{array}$ & $\begin{array}{l}0 \\
0 \\
0 \\
1 \\
1 \\
0 \\
0 \\
1 \\
1 \\
0 \\
0 \\
0\end{array}$ & $\begin{array}{r}1 \\
1 \\
0 \\
0 \\
0 \\
1 \\
0 \\
1 \\
1 \\
1 \\
0 \\
1 \\
\end{array}$ & $\begin{array}{l}1 \\
1 \\
0 \\
0 \\
0 \\
0 \\
0 \\
0 \\
1 \\
1 \\
0 \\
1 \\
\end{array}$ & $\begin{array}{l}1 \\
2 \\
0 \\
0 \\
0 \\
1 \\
0 \\
0 \\
0 \\
0 \\
0 \\
0\end{array}$ & \\
\hline Total ................. & 10 & 11 & 4 & 7 & 5 & 4 & \\
\hline Relative occurrence........ & 83.3 & & & 56.3 & & & 9.1 \\
\hline
\end{tabular}

COARSELY GRANULAR CASTS.

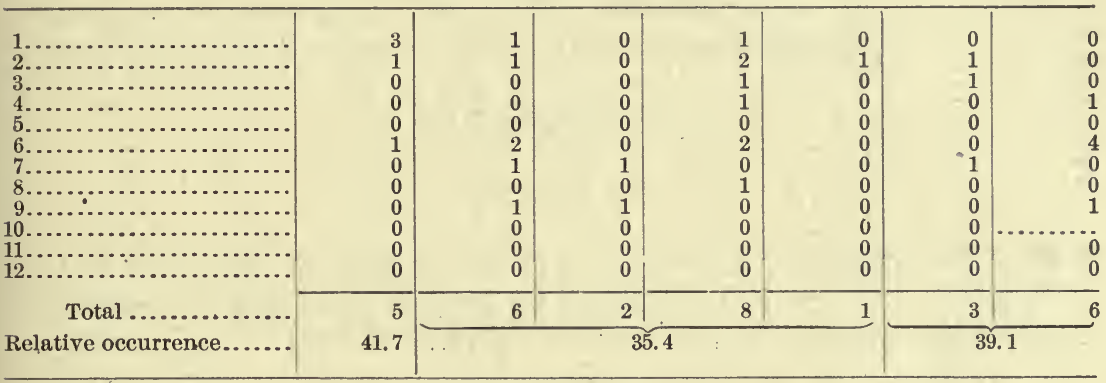

CASTS, EPITHELIAL.

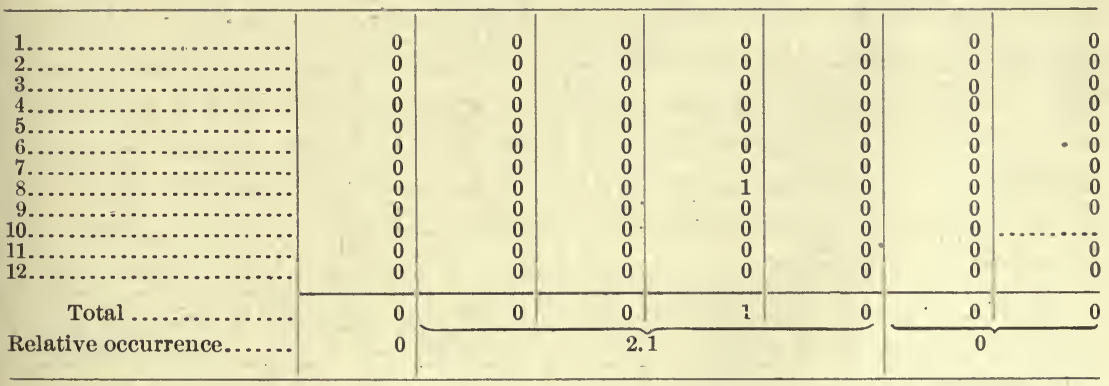


Table XI.-Microscopical examination of the urine, Series I'I-Continued.

[None, 0 ; very few, 1 ; few; 2 ; fairly numerous, 3 ; numerous, 4; extremely numerous, 5.]

OTHER FORMS OF CASTS.

\begin{tabular}{|c|c|c|c|c|c|c|c|}
\hline \multirow{2}{*}{ No. } & \multirow{2}{*}{$\begin{array}{c}\begin{array}{c}\text { Fore } \\
\text { period. }\end{array} \\
\text { Oct. } 21-22 .\end{array}$} & \multicolumn{4}{|c|}{ Preservative period. } & \multicolumn{2}{|c|}{ After period. } \\
\hline & & Oet. $29-31$. & Nov.5-9. & Nov.12-14. & Nov. 19-21. & $\begin{array}{l}\text { Nov. } 28- \\
\text { Dec. } 1 .\end{array}$ & Dec. 3-5. \\
\hline $\begin{array}{r}1 \\
1 \\
2 \\
3 \\
4 \\
4 \\
5 \\
6 \\
6 \\
7 \\
8 \\
8 \\
9 \\
9 \\
10 \\
11 \\
12 \\
12 \\
1\end{array}$ & $\begin{array}{l}0 \\
0 \\
0 \\
0 \\
0 \\
0 \\
0 \\
0 \\
0 \\
0 \\
0 \\
0\end{array}$ & $\begin{array}{l}0 \\
0 \\
0 \\
0 \\
0 \\
0 \\
0 \\
0 \\
0 \\
0 \\
0 \\
0\end{array}$ & $\begin{array}{l}0 \\
0 \\
0 \\
0 \\
0 \\
0 \\
0 \\
0 \\
0 \\
0 \\
0 \\
0\end{array}$ & $\begin{array}{l}0 \\
0 \\
0 \\
0 \\
0 \\
0 \\
0 \\
1 \\
0 \\
0 \\
0 \\
0\end{array}$ & $\begin{array}{l}0 \\
0 \\
0 \\
0 \\
0 \\
0 \\
0 \\
0 \\
0 \\
0 \\
0 \\
0\end{array}$ & $\begin{array}{l}0 \\
0 \\
0 \\
0 \\
0 \\
0 \\
0 \\
0 \\
0 \\
0 \\
0 \\
0\end{array}$ & \begin{tabular}{l}
0 \\
0 \\
0 \\
0 \\
0 \\
0 \\
0 \\
0 \\
0 \\
\hdashline \\
0 \\
0
\end{tabular} \\
\hline Total $\ldots \ldots \ldots$ & 0 & 0 & 0 & 1 & 0 & 0 & 0 \\
\hline Relative occurrence....... & 0 & \multicolumn{4}{|c|}{2.1} & \multicolumn{2}{|c|}{0} \\
\hline
\end{tabular}

MUCOUS CYLINDROIDS.

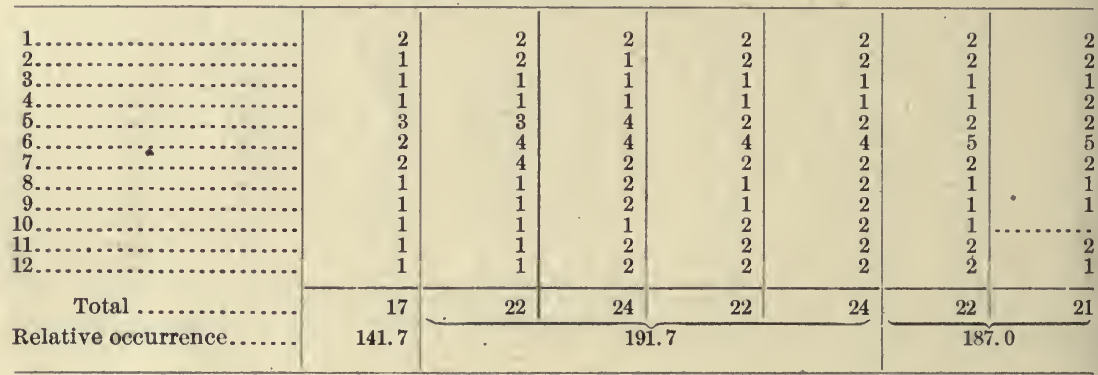

MUCOUS STRANDS.

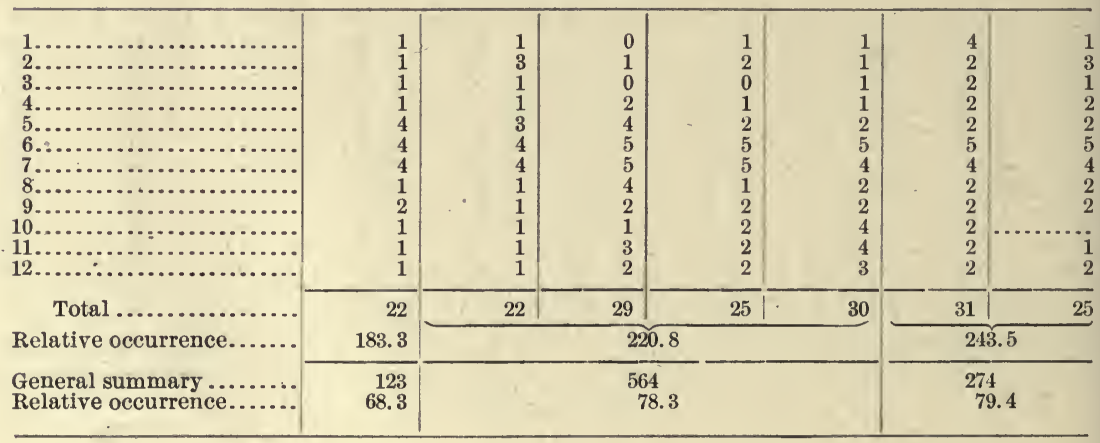

\section{METABOLIC PROCESSES.}

Having discussed the general chemical characteristics of the excreta in the preceding pages, we now turn to consider a special study of the metabolic processes. To this end the relative quantities of the different food elements ingested and those recovered in the excretions 
have been tabulated in such a way as to determine the effect, if any, of the added preservative upon the metabolic activities.

By reason of the imperfections' of the data of Nos. 3, 9, and 10 it has not been possible to consider the results obtained on these men in the general discussion. In order, however, that none of the data obtained may escape record, the analytical results have been tabulated and appear in the regular order for these three men. It is not likely in any case that, starting out with twelve men it will be possible to complete a study of this kind without the loss of some of the data. The figures for the three men which are excluded, had they been included in the general discussion, would not have changed to any appreciable extent the general results.

This general statement is made to cover all of the data recorded here for the study of the metabolic processes. The data collected relating to nitrogen, phosphoric acid, and sulphur are by far the most important as respects metabolic activities. In addition thereto, the balances and other data for fat, calories, and total solids have been appended. These are of less value, but probably will help to throw some light upon the general course of the investigation.

\section{NITROGEN BALANCE.}

INDIVIDUAL DATA.

The nitrogen balances are given in Table XIII (pp. 592 to 604) by periods and subperiods instead of by days, as in the case of the borax experiment. The same system having been followed as before, the repetition of the detail seemed cumbersome and unnecessary. Each subperiod covers five days, the exact dates being given in Table I (p. 481). The amount of nitrogen ingested, the amounts excreted in the feces and the urine, and the total amount excreted are expressed both in grams and in percentage, the balance being given in grams. For example, during the first subperiod of the fore period, extending over five days, the total nitrogen exhibited in the food of No. 1 is 71.56 grams, an average of 14.31 grams per day. The total nitrogen excreted in the feces covering this period is 3.76 grams, an average of 0.75 gram per day. The total nitrogen excreted in the urine during this period is 59.83 grams, an average of 11.97 grams per day. The total nitrogen excreted during the period in the feces and urine is 63.59 grams, an average of 12.72 grams per day. The total balance for the subperiod is 7.97 grams, an average of 1.59 grams per day. The total percentage of nitrogen excreted in the feces is 5.25 and in the urine 83.61 , and the total percentage of nitrogen excreted in both is 88.86. The totals and averages for each period and subperiod are presented in the same way. This explanation will sufficiently illustrate the principle on which the tables are constructed. 
No. 1.

The total nitrogen exhibited in the food of No. 1 in the fore period is $147.56 \mathrm{grams}$, an average of $14.76 \mathrm{grams}$ per day. Of this nitrogen 4.66 per cent is excreted in the feces and 86.62 per cent in the urine, and the total excreted in the feces and urine is 91.28 per cent. The average daily balance during the period is +1.29 grams.

For the whole preservative period the total quantity of nitrogen exhibited in the food is 436.16 grams, an average daily quantity of 14.54 grams. Of this quantity 18.42 grams are excreted in the feces and 418.98 grams in the urine; 4.22 per cent of the total amount excreted is found in the feces and 96.06 per cent in the urine. The nitrogen balance becomes a negative quantity, -0.04 gram per day. During the after period the quantity of nitrogen given in the food is 146.08 grams, or 14.61 grams per day. Of this quantity 6.31 per cent of nitrogen is excreted in the feces and 96.16 per cent in the urine, again showing a balance of -0.36 gram per day. In the case of No. 1 it is evident that the effect of the administration of the salicylic acid was to increase the metabolism of nitrogen. Not only was the quantity excreted in the feces diminished during the preservative period, but the quantity excreted in the urine was very greatly increased. During the after period the quantity of nonmetabolized nitrogen, that is, that appearing in the feces, was very largely increased, and the quantity of nitrogen in the urine was slightly increased. In the case of No. 1, therefore, a distinct influence is manifested on the part of the preservative to increase the output of nitrogen, especially of the metabolized nitrogen.

\section{No. 2.}

The total quantity of nitrogen exhibited during the fore period in the case of No. 2 is 164.70 grams, an average of 16.47 grams per day; 10.29 per cent of the nitrogen appeared in the feces and 95.76 per cent in the urine. The nitrogen balance is -1 gram per day, which represents an abnormal condition due to some cause not revealed in the analytical data. During the preservative period the total quantity of nitrogen exhibited in the food is $492.85 \mathrm{grams}$, with a duily average of 16.43 grams. The percentage of nitrogen excreted in the feces is 8.92 ; in the urine, 91.65 , and the nitrogen balance is a negative quantity of -0.09 . During the after period the total quantity of nitrogen exhibited in the food is 164.84 grams, with a daily average of 16.48 grams; 8.04 per cent of nitrogen is excreted in the feces and 95.78 per cent in the urine. The nitrogen balance is age in a negative quantity, equivalent to -0.64 gram per day. The data in the case of No. 2 , as in the case of No. 1 , show the influence of the preservative in increasing the excretion of nitrogen, if we exclude from consideration 
the first fore subperiod, in which some abnormality is shown to exist, and this influence, as in the first instance, is continued during the after period.

No. 3.

Owing to illness No. 3 had only a partial fore period, and this was taken at the beginning of the preservative period for the other men, extending from October 29 to November 2, inclusive. During this period the total quantity of nitrogen exhibited in the food is 72.58 grams, with an average of 14.52 grams per day; 7.38 per cent of the nitrogen exhibited is excreted in the feces and 87.06 per cent in the urine. The daily nitrogen balance is 0.81 grams. During the preservative period, extending from November 3 to 27, inclusive, five days less than in the other cases, the total quantity of nitrogen exhibited in the food is 369.08 grams, an equivalent of 14.76 grams per day. The percentage of nitrogen excreted in the feces is 7.62 and in the urine 76.20. The excretion of nitrogen in this case is strongly inhibited and the nitrogen balance becomes very largely positive, reaching the very large amount of 2.39 grams per day. The after period in the case of No. 3 was again interrupted by illness, and covers only five days, therefore it is not comparable. It is evident that the data in the case of No. 3 are wholly useless for comparison by reason of interruption of the observations by illness both at the beginning and close of the investigations. -

\section{No. 4 .}

During the fore period the quantity of nitrogen exhibited in the food of No. 4 is 157.10 grams, equivalent to $15.71 \mathrm{grams}$ per day. Of this quantity, 6.86 per cent is excreted in the feces and 92.76 per cent in the urine. The nitrogen balance is positive, but of very small magnitude, amounting to 0.06 gram per day. During the preservative period the total quantity of nitrogen exhibited in the food is 468.11 grams, amounting to 15.60 grams per day. Of this quantity, 6.75 per cent is excreted in the feces and 90.42 per cent in the urine. The nitrogen balance has been considerably increased in magnitude and remains positive, amounting to 0.44 gram per day. During the after period the amount of nitrogen exhibited in the food is 157.79 grams, equivalent to 15.78 grams per day. Of this quantity, 6.43 per cent is excreted in the feces and 92.83 per cent in the urine. The nitrogen balance remains positive, but is diminished in magnitude, amounting to only 0.12 gram per day. The data in this case are in general contrary to those of cases 1 and 2 . The indications here are that the action of the preservative serves to inhibit to a certain extent the excretion of the nitrogen, thus increasing the magnitude of the positive balance.

$7656-$ No. 84 , pt $2-06-8$ 
No. 5 .

During the fore period the quantity of nitrogen exhibited in the food of No. 5 is 158.70 grams, amounting to 15.87 grams per day. Of this quantity, 8.90 per cent is excreted in the feces and 82.79 per cent in the urine. The nitrogen balance is strongly positive, amounting to 1.32 grams per day. During the preservative period the total quantity of nitrogen exhibited in the food is $475.86 \mathrm{grams}$, amounting to 15.86 grams per day. The percentage excreted in the feces is 7.32 and in the urine 87.48. The nitrogen balance remains positive, but is diminished in magnitude. The effect of the preservative in this case is to diminish the percentage of nonmetabolized nitrogen and increase very considerably that of the netabolized. In the after period the amount of nitrogen exhibited in the food is $159.30 \mathrm{grams}$, equivalent to $15.93 \mathrm{grams}$ per day. The percentage excreted in the feces is 6.35 and in the urine 87.87. The nitrogen balance is positive and its average magnitude is 0.92 gram per day. The excretion of the nitrogenin the feces is diminished and slightly increased in the urine. The balance indicates a partial return in the after period to the conditions obtaining in the fore period, but a larger percentage of the nitrogen is metabolized, thus continuing the tendency shown in the preservative period.

No. 6.

The quantity of nitrogen in the food of No. 6 in the fore period is 142.30 grams, equivalent to 14.23 grams per day, of which 10.96 per cent is excreted in the feces and 82.30 in the urine. The nitrogen balance is positive, and has an average daily magnitude of $0.96 \mathrm{gram}$. During the preservative period the total quantity of nitrogen exhibited in the food is 432.39 grams, equivalent to a daily amount of 14.41 grams; 10.73 per cent of this nitrogen is excreted in the feces, and 93.94 in the urine. The nitrogen balance is diminished and becomes a negative quantity equivalent to -0.68 gram per day. The total quantity of nitrogen exhibited in the after period is 143.96 grams, showing an average daily quantity of 14.40 grams; 10.43 per cent is excreted in the feces and 84.07 per cent in the urine. The average balance now returns to a positive quantity with an average value of $0.80 \mathrm{gram}$.

These data show a most marked effect of the preservative in increasing the excretion of metabolized nitrogen, although the nonmetabolized nitrogen in the feces decreased only very slightly. There is also shown a distinct tendency to return to normal conditions in the after period. In connection with this case it is to be observed that the subject suffered a very marked loss in weight, amounting to more than a kilogram, both in the preservative and after period. 
No. $\%$.

The quantity of nitrogen exhibited in the food during the fore period in the case of No. 7 is 139.42 grams, equivalent to 13.94 grams per day; 6.80 per cent is excreted in the feces and 85.76 per cent in the urine. The nitrogen balance is strongly positive, 1.03 grams per day. The quantity of nitrogen in the food during the preservative period is 405.53 grams, equivalent to 13.52 grams daily. Of this quantity, 5.02 per cent is excreted in the feces and 80.19 per cent in the urine. In this case the nitrogen balance is very large, having been increased to 2 grams per day. The total quantity of nitrogen exhibited in the food in the after period is 136.04 grams, equivalent to 13.60 grams per day; of this amount, 8 per cent is excreted in the feces and 80.04 per cent in the urine. The nitrogen balance remains strongly positive, but is decreased to 1.62 grams per day. These data are exactly contrary to those obtained in the former case (No. 6) in that they show a distinct effect of the preservative in this instance in inhibiting the excretion of the metabolized-nitrogen; the nonmetabolized nitrogen excreted is also decreased, while in the case of No. 6 it remained practically constant.

No. 8 .

The quantity of nitrogen exhibited in the food of the fore period in the case of No. 8 is 129.07 grams, representing a daily quantity of 12.91 grams. Of this 9.04 per cent is excreted in the feces and 83.99 per cent in the urine. The nitrogen balance is positive and has an average magnitude of +0.90 gram per day. The quantity of nitrogen in the food during the preservative period is 398.71 grams, with an average daily magnitude of 13.29 grams. Of this quantity 9.88 per cent is excreted in the feces and 80.87 per cent in the urine. The balance is strongly positive and has increased to 1.23 grams per day. These figures indicate that the preservative has decreased the excretion of metabolized nitrogen about 3 per cent and increased the excretion of nonmetabolized nitrogen 0.84 per cent.

During the after period the nitrogen administered in the food amounts to 135.19 grams, equivalent to 13.52 grams per day; 11.53 per cent of this nitrogen is excreted in the feces and 88.42 per cent ir the urine. The balance remains positive, but is very small, amounting only to $0.01 \mathrm{gram}$. It is thus seen that in the after period the excretion of the metabolized nitrogen increased greatly, almost 8 per cent, exceeding that of the fore period, and the excretion of nonmetabolized nitrogen continued to increase also.

Nos. 9 and 10.

The balances in these two cases of 3 and 4 grams show plainly that some radical error is present. The subjects evidently either did not collect and deliver for analysis the whole of the excreta, or ate food 
in addition to that weighed out at the experimental table. The data in these cases are therefore discredited, and the balances are printed merely to show the reason for their exclusion from the summaries and the conclusions.

No. 11.

The quantity of nitrogen in the food during the fore period extending from October 19 to October 28 is 177.42 grams, equivalent to 17.74 grams per day. Of this amount 9.01 per cent is excreted in the feces and 89.35 per cent in the urine. This shows a slightly positive nitrogen balance equivalent to 0.29 gram per day.

'The quantity of nitrogen in the food administered to No. 11 during the preservative period is 528.34 grams, equivalent to 17.61 grams per day. Of this quantity 8.74 per cent is excreted in the feces and 85.15 per cent in the urine. The nitrogen balance is positive for the preservative period and amounts to 1.07 grams per day. During the after period the quantity of nitrogen in the food of No. 11 is 177.67 grams, equivalent to 17.77 grams per day. Of this quantity 7.66 per cent is excreted in the feces and 76.64 per cent in the urine. The balance is positive and very high, amounting to 2.79 grams per day. The excretion of both metabolized and nonmetabolized nitrogen is decreased in the preservative period, and this decrease is still greater in the after period.

No. 12.

The quantity of nitrogen in the food of No. 12 during the fore period is 175.18 grams, equivalent to 17.52 grams daily. Of this amount 8.25 per cent is excreted in the feces and 84.89 per cent in the urine. The nitrogen balance is positive and amounts to 1.20 grams per day. During the preservative period the quantity of nitrogen in the food of No. 12 is 514.30 grams, equivalent to 17.14 grams per day. Of this quantity 5.88 per cent is excreted in the feces and 84.53 per cent in the urine. The nitrogen balance is again positive in the preservative period and has increased to 1.64 grams per day. The quantity of nitrogen in the food of No. 12 during the after period is 170.46 grams, equivalent to 17.05 grams per day. Of this quantity 6.48 per cent is excreted in the feces and 88.27 per cent in the urine. The nitrogen balance is still positive, but has decreased to 0.90 gram per day.

The principal effect of the preservative in this case is to slightly inhibit the excretion of nitrogen. The inhibition is found almost exclusively in the nonmetabolized material, the percentage of nitrogen in the food which was excreted in the feces falling from 8.25 in the fore period to 5.88 in the preservative period and rising again to 6.48 in the after period. 


\section{GENERAL DISCUSSION OF INDIVIDUAL DATA.}

Some of the noticeable variations in the individual data in regard to the excretion of nitrogen are of interest, Nos. 3, 9, and 10 being excluded as usual. The lowest percentage excreted in the feces in the fore period is found in the case of No. 1, namely, 4.66 per cent, and the highest in the case of No. 6 , namely, 10.96 per cent. In regard to the quantity excreted in the urine the largest percentage is found in the case of No. 2, namely, 95.76 per cent, and the lowest in the case of No. 6, namely, 82.30 per cent. No. 2 was decidedly abnormal during the fore period, since the quantity of nitrogen excreted was greater than that ingested in the food.

During the preservative period the following notable variations in individuals from the average may be cited: The smallest percentage of nitrogen excreted in the feces is again found in the case of No. 1, namely, 4.22 per cent, and the largest, 10.73 per cent, again occurs in the case of No. 6. In the after period the smallest percentage excreted in the feces is again in the case of No. 1, namely, 6.31, and the largest, 11.53, No. 8. No. 6, however, maintained a high average, 10.43. These data show a consistent idiosyncrasy in the individuals which is practically indicated during all three of the periods. It is evident that No. 1 was able to utilize the nitrogen in the food more completely than were Nos. 6 and 8 .

In regard to the percentage of nitrogen excreted in the urine, the largest quantity in the preservative period is excreted by No. 1, namely, 96.06 per cent, and the smallest quantity by No. 7, namely, 80.19 per cent. Of the total number three show an excretion of nitrogen during the preservative period in excess of the quantity exhibited in the food, namely, Nos. 1, 2, and 6.

During the after period the largest quantity of nitrogen in the urine is again excreted by No. 1, namely, 96:16 per cent, and the smallest by No. 11, namely, 76.64 per cent. This is a remarkably low number, but no source of error can be detected from a study of the detailed data and of the character of the man. In the after period only two of the men showed an excess of nitrogen excreted over that given in the food, namely, Nos. 1 and 2.

It is evident that in the case of a loss of weight a negative nitrogen balance would not be regarded as abnormal. Likewise, in the case of a gain in weight by growth or otherwise, a large positive nitrogen balance would not be regarded as abnormal. Where, however, the weight remains practically constant, any very large positive balances or negative balances require most careful study in order to determine exactly the sources which have caused the variation. The most plausible explanation of an abnormally large positive balance, the energy and food consumed remaining constant, is an increase in the anabolic 
activities. The most plausible cause of a large negative balance, when the normal quantity of food is consumed and the energy is constant, is an increase of the katabolic activities of the body.

SUMMARY.

In all of the summaries the totals and averages for the nine subjects completing the series satisfactorily are combined in one expression for each subperiod and for the fore, preservative, and after periods as a whole. Thus in the fore period the data are totaled and averaged for all subjects for each of the two subperiods and then for the entire period. In the following discussion only the data for the entire periods will be considered, and these are here inserted in tabular form for convenience in reference:

TABLE XII.-Nitrogen summary, by periods, for nine men, Series TI.

\begin{tabular}{|c|c|c|c|c|c|c|}
\hline Period. & $\begin{array}{l}\text { Nitrogen } \\
\text { in food. }\end{array}$ & $\begin{array}{c}\text { Nitrogen } \\
\text { in feces. }\end{array}$ & $\begin{array}{l}\text { Nitrogen } \\
\text { in urine. }\end{array}$ & $\begin{array}{l}\text { Nitrogen } \\
\text { in feces. }\end{array}$ & $\begin{array}{l}\text { Nitrogen } \\
\text { in urine. }\end{array}$ & Balance. \\
\hline $\begin{array}{l}\text { Fore period } \\
\text { Preservative period } . \ldots \ldots \\
\text { After period } . . . .\end{array}$ & $\begin{array}{r}\text { Grams. } \\
15.46 \\
15.37 \\
15.46\end{array}$ & $\begin{array}{r}\text { Grams. } \\
1.29 \\
1.15 \\
1.21\end{array}$ & $\begin{array}{r}\text { Grams. } \\
13.50 \\
13.51 \\
13.56\end{array}$ & \begin{tabular}{|r|} 
Per cent. \\
8.33 \\
7.50 \\
7.83
\end{tabular} & $\begin{array}{r}\text { Per cent. } \\
87.32 \\
87.89 \\
87.75\end{array}$ & $\begin{array}{r}\text {. Grams. } \\
0.67 \\
.71 \\
.69\end{array}$ \\
\hline
\end{tabular}

The average quantity of nitrogen consumed by each of the nine men daily during the fore period is $15.46 \mathrm{grams}$, of which $1.29 \mathrm{grams}$ are excreted in the feces and 13.50 in the urine. Expressed as percentages, 8.33 per cent of the total nitrogen is excreted in the feces and 87.32 per cent in the urine. The average daily balance is $0.67 \mathrm{gram}$.

For the whole preservative period the average daily quantity of nitrogen ingested is 15.37 grams, of which 1.15 grams is excreted in the feces and 13.51 grams in the urine. Expressed in percentages, 7.50 per cent of the total nitrogen is excreted in the feces and 87.89 per cent in the urine. The average daily balance of the nitrogen is 0.71 gram. For the entire after period the average quantity of nitrogen exhibited in foods is 15.46 grams, of which 1.21 grams is excreted in the feces and 13.56 grams in the urine. Expressed in percentages, 7.83 per cent is found in the feces and 87.75 per cent in the urine. The average daily balance of the nitrogen is $0.69 \mathrm{gram}$.

A comparison of these data by periods shows that the average daily amount of nitrogen in the food during the preservative period is slightly less than in the fore and after periods, where they are the same. The quantity execreted in the feces is considerably diminished during the preservative period, in fact by a larger quantity than could be accounted for by the slight diminution of the amount ingested. The quantity excreted in the urine is a trifle greater than in the fore period, although the total amount in the food is less. That is, the percentage figures show a diminished quantity of nonmetabolized 
nitrogen and a slightly increased quantity of metabolized nitrogen excreted. In the after period a general tendency is shown to return to the conditions of the fore period, but this return is only partial. The percentage of nonmetabolized nitrogen eliminated in the after period is greater than in the preservative period but less than in the fore period, while the amount of metabolized nitrogen excreted is less than in the preservative period but not so small as in the fore period.

The balance shows a slightly decreased total excretion of nitrogen, and this decrease is wholly in the nonmetabolized nitrogen. These data indicate that the exhibition of the preservative tended slightly to increase the digestibility and absorption of the nitrogen ingested.

Had these phenomena been accompanied by an increase in weight, the data would have all pointed in one direction, namely, to a stimulation of the metabolic processes; but, in view of the considerable loss in body weight, the question suggests itself, Can any part of the increased excretion of metabolized nitrogen be due rather to increased katabolic activity, i. e., destruction of tissue? It must be remembered, however, that the excretion of nonmetabolized nitrogen decreased under the influence of the preservative even to a greater degree than the excretion of the metabolized nitrogen increased, and the balance indicates clearly increased digestion and absorption during the preservative period, as already stated, although there appears to have been a decrease in tissue formation. Unless, therefore, the decrease in weight be ascribed to some cause beyond control-such as the advance of the winter season and effect of temperature, psychological influences resulting from restraint, fear, etc.-it would appear that in the case of nitrogen the katabolic processes were stimulated to a greater degree than the anabolic activities. It would not be expected that a marked increase in appetite, as reported in the medical notes, would, under the existing conditions, be accompanied by a loss of weight. In Series XI, page 706, a special study of the nitrogenous bodies in the urine is made which bears directly upon this point, an effort being made to discover the significance of the appearance of these bodies in the urine in various forms. 
Table XIII.-Nitrogen balances for Series VI.

[Averages are per day.]

No. 1 .

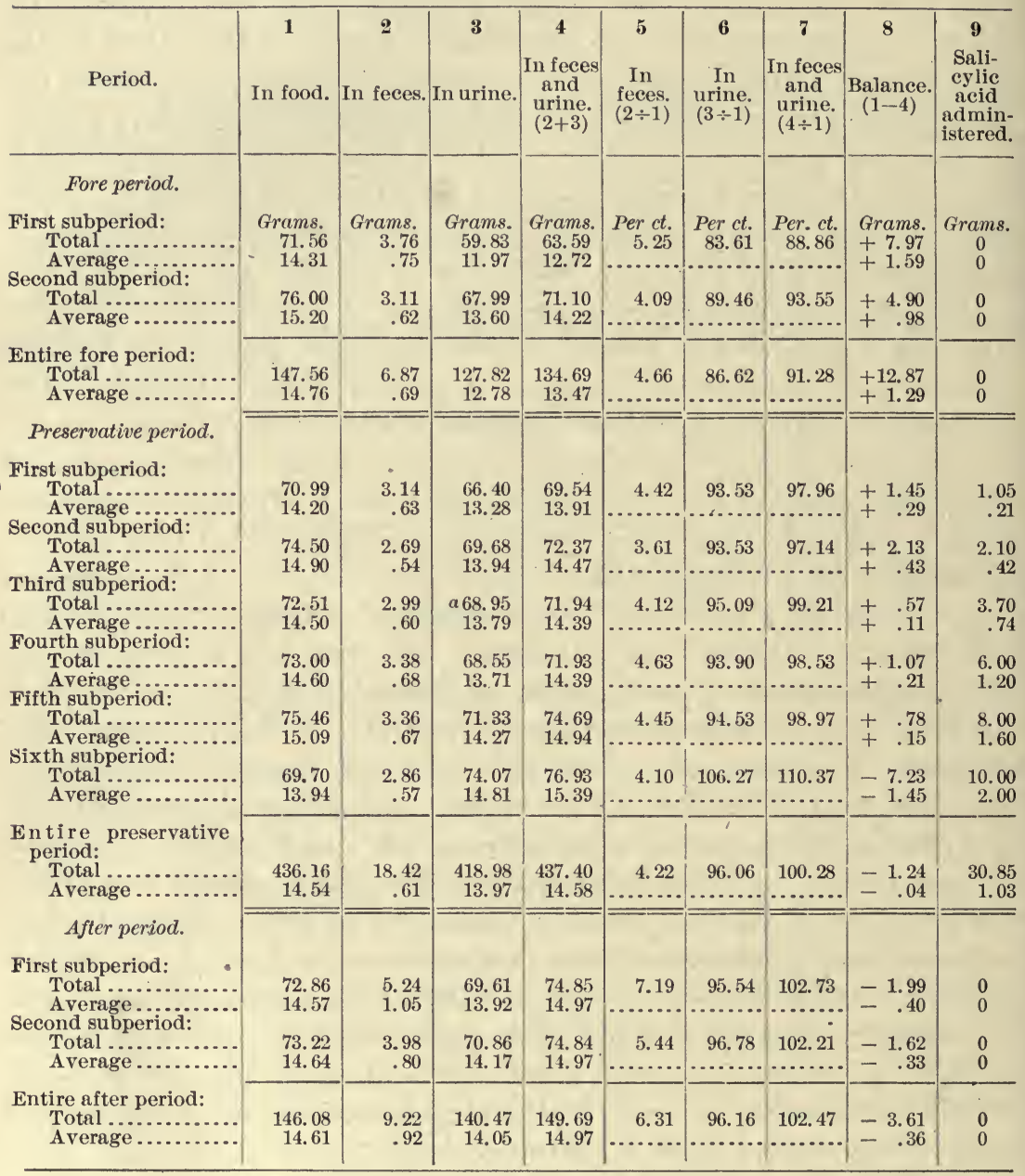

$a$ Daily average added in ord $\approx r$ to complete record. 
TABLE XIII.-Nitrogen balances for Series VI-Continued.

[Averages are per day.]

No. 2.

\begin{tabular}{|c|c|c|c|c|c|c|c|c|c|}
\hline Period. & In food. & $\begin{array}{c}2 \\
\text { In feces. }\end{array}$ & \begin{tabular}{c|}
3 \\
In urine.
\end{tabular} & $\begin{array}{c}4 \\
\text { In feces } \\
\text { and } \\
\text { urine. } \\
(2+3)\end{array}$ & $\begin{array}{c}5 \\
\text { In } \\
\text { feces. } \\
(2 \div 1)\end{array}$ & $\begin{array}{c}6 \\
\text { In } \\
\text { urine. } \\
(3 \div 1)\end{array}$ & \begin{tabular}{|c|}
7 \\
In feces \\
and \\
urine. \\
$(4 \div 1)$
\end{tabular} & $\begin{array}{c}8 \\
\text { Bal- } \\
\text { ance. } \\
(1-4)\end{array}$ & $\begin{array}{l}9 \\
\text { Sali- } \\
\text { cylic } \\
\text { acid } \\
\text { admin- } \\
\text { istered. }\end{array}$ \\
\hline Fore period. & & & & & & & & & \\
\hline 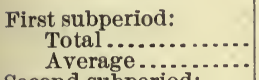 & $\begin{array}{r}\text { Grams. } \\
81.82 \\
16.36\end{array}$ & $\begin{array}{r}\text { Grams. } \\
7.80 \\
\quad 1.56\end{array}$ & $\begin{array}{r}\text { Grams. } \\
a 85.34 \\
17.07\end{array}$ & $\begin{array}{r}\text { Grams. } \\
93.14 \\
18.63\end{array}$ & $\begin{array}{r}\text { Per ct. } \\
9.53\end{array}$ & $\begin{array}{l}\text { Per ct. } \\
104.30\end{array}$ & $\begin{array}{l}\text { Per ct. } \\
113.84\end{array}$ & $\begin{array}{l}\text { Grams. } \\
-11.32 \\
-2.27\end{array}$ & $\begin{array}{c}\text { Grams. } \\
0 \\
0\end{array}$ \\
\hline $\begin{array}{l}\text { Second subperiod: } \\
\text { Total ................ } \\
\text { Average ............ }\end{array}$ & $\begin{array}{l}82.88 \\
16.58\end{array}$ & $\begin{array}{l}9.15 \\
1.83\end{array}$ & $\begin{array}{l}72.37 \\
14.47\end{array}$ & $\begin{array}{l}81.52 \\
16.30\end{array}$ & 11.04 & 87.32 & 98.36 & $\begin{array}{r}+1.36 \\
+\quad .28\end{array}$ & $\begin{array}{l}0 \\
0\end{array}$ \\
\hline $\begin{array}{l}\text { Entire fore period: } \\
\quad \text { Total ................................. } \\
\text { Average }\end{array}$ & $\begin{array}{r}164.70 \\
16.47\end{array}$ & $\begin{array}{r}16.95 \\
1.70\end{array}$ & $\begin{array}{r}157.71 \\
15.77 \\
\end{array}$ & $\begin{array}{r}174.66 \\
17.47 \\
\end{array}$ & 10.29 & 95.76 & 106.05 & $\begin{array}{r}-9.96 \\
-1.00\end{array}$ & $\begin{array}{l}0 \\
0\end{array}$ \\
\hline Preservative period. & & & & & & & & & \\
\hline $\begin{array}{l}\text { First subperiod: } \\
\text { Total .......... } \\
\text { Average ..... }\end{array}$ & $\begin{array}{l}79.78 \\
15,96\end{array}$ & $\begin{array}{l}6.98 \\
1.40\end{array}$ & $\begin{array}{l}71.87 \\
14.37\end{array}$ & $\begin{array}{l}78.85 \\
15.77\end{array}$ & 8.75 & 90.09 & 98.83 & $\begin{array}{r}+\quad \therefore 93 \\
+\quad .19\end{array}$ & $\begin{array}{r}1.05 \\
.21\end{array}$ \\
\hline $\begin{array}{r}\text { Second s } \\
\text { Tota } \\
\text { Aver }\end{array}$ & $\begin{array}{l}83.73 \\
16.75\end{array}$ & $\begin{array}{l}7.83 \\
1.57\end{array}$ & $\begin{array}{r}72.49 \\
14.50\end{array}$ & $\begin{array}{l}80.32 \\
16.06\end{array}$ & 9.35 & 86.58 & 95.93 & $\begin{array}{r}+3.41 \\
+\quad .69\end{array}$ & $\begin{array}{l}2.10 \\
42\end{array}$ \\
\hline $\begin{array}{l}\text { Third su } \\
\text { Tota }\end{array}$ & & & & 84.94 & 9.53 & 94.03 & $103.56^{\circ}$ & $\begin{array}{r}-2.92 \\
-29\end{array}$ & \\
\hline $\begin{array}{c}\text { Ave } \\
\text { Fourth }\end{array}$ & 16. & 1.5 & & 16. 99 & & & & $-\quad .59$ & $\begin{array}{r}3.70 \\
.74\end{array}$ \\
\hline $\begin{array}{l}\text { Tot } \\
\text { Ave }\end{array}$ & $\begin{array}{l}82.59 \\
16.52\end{array}$ & $\begin{array}{l}8.87 \\
1.77\end{array}$ & $\begin{array}{l}71.30 \\
14.26\end{array}$ & $\begin{array}{l}80.17 \\
16.03\end{array}$ & 10.74 & 86.33 & 97.07 & $\begin{array}{r}+\quad 2.42 \\
+\quad .49\end{array}$ & 6.00 \\
\hline $\begin{array}{l}\text { Fifth st } \\
\text { Tot } \\
\text { Ave }\end{array}$ & 85.63 & $\begin{array}{l}4.50 \\
.90\end{array}$ & $\begin{array}{l}82 . \\
16 .\end{array}$ & $\begin{array}{l}86.60 \\
17.32\end{array}$ & 5.26 & 95.88 & 101.13 & - .97 & 8.00 \\
\hline 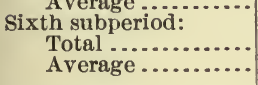 & $\begin{array}{l}79.10 \\
15.82\end{array}$ & $\begin{array}{l}7.97 \\
1.59\end{array}$ & $\begin{array}{l}76.80 \\
15.36\end{array}$ & $\begin{array}{l}84.77 \\
16.95\end{array}$ & $\begin{array}{r}10.08 \\
\end{array}$ & $\begin{array}{c}97.09 \\
\ldots \ldots . .\end{array}$ & 107.17 & $\begin{array}{l}-5.67 \\
-1.13\end{array}$ & $\begin{array}{r}10.00 \\
2.00\end{array}$ \\
\hline $\begin{array}{r}\text { Entire preservative } \\
\text { period: } \\
\text { Total ................. } \\
\text { Average ............. }\end{array}$ & $\begin{array}{r}492.85 \\
16.43\end{array}$ & $\begin{array}{r}43.97 \\
1.47\end{array}$ & $\begin{array}{r}451.68 \\
15.06\end{array}$ & $\begin{array}{r}495.65 \\
16.52\end{array}$ & 8.92 & $\begin{array}{c}91.65 \\
\ldots \ldots \ldots\end{array}$ & $\begin{array}{l}100.57 \\
\cdots \cdots\end{array}$ & $\begin{array}{r}-2.80 \\
-\quad .09\end{array}$ & $\begin{array}{r}30.85 \\
1.03 \\
\end{array}$ \\
\hline After period. & & & & & & & & & \\
\hline $\begin{array}{l}\text { First subperiod: } \\
\text { Total ............ } \\
\text { Average....... } \\
\text { Second subperiod: }\end{array}$ & $\begin{array}{l}82.39 \\
16.48\end{array}$ & $\begin{array}{l}6.05 \\
1.21\end{array}$ & $\begin{array}{l}77.92 \\
15.58\end{array}$ & $\begin{array}{l}83.97 \\
16.79\end{array}$ & 7.34 & 94.57 & 101.92 & $\begin{array}{r}-1.58 \\
-\quad .31\end{array}$ & $\begin{array}{l}0 \\
0\end{array}$ \\
\hline 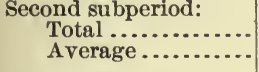 & $\begin{array}{l}82.45 \\
16.49\end{array}$ & $\begin{array}{l}7.21 \\
1.44\end{array}$ & $\begin{array}{l}79.97 \\
15.99\end{array}$ & $\begin{array}{l}87.18 \\
17.44\end{array}$ & 8.74 & 96.99 & 105.74 & $\begin{array}{l}-4.73 \\
-\quad .95\end{array}$ & $\begin{array}{l}0 \\
0\end{array}$ \\
\hline $\begin{array}{l}\text { Entire after period: } \\
\text { Total .................. } \\
\text { Average ............. }\end{array}$ & $\begin{array}{r}164.84 \\
16.48\end{array}$ & $\begin{array}{r}13.26 \\
1.33\end{array}$ & $\begin{array}{r}157.89 \\
15.79\end{array}$ & $\begin{array}{r}171.15 \\
17.12\end{array}$ & 8.04 & 95.78 & 103.83 & $\begin{array}{l}-6.31 \\
-\quad .64\end{array}$ & $\begin{array}{l}0 \\
0\end{array}$ \\
\hline
\end{tabular}

$a$ Daily average added in order to complete record.

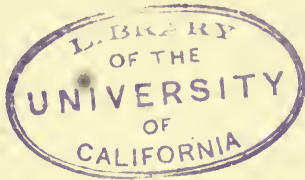


TABLE XIII.-Nitrogen balances for Series VI-Continued.

[Averages are per day.]

No. 3.

\begin{tabular}{|c|c|c|c|c|c|c|c|c|c|}
\hline Period. & In food. & In feces. & In urine. & $\begin{array}{c}4 \\
\text { In feces } \\
\text { and } \\
\text { urine. } \\
(2+3)\end{array}$ & $\begin{array}{c}5 \\
\text { In } \\
\text { feces. } \\
(2 \div 1)\end{array}$ & $\begin{array}{c}6 \\
\text { In } \\
\text { urine. } \\
(3 \div 1)\end{array}$ & $\begin{array}{c}7 \\
\text { In feces } \\
\text { and } \\
\text { urine. } \\
(4 \div 1)\end{array}$ & $\begin{array}{c}8 \\
\text { Bal- } \\
\text { ance. } \\
(1-4)\end{array}$ & $\begin{array}{c}9 \\
\text { Sali- } \\
\text { cylic } \\
\text { acid } \\
\text { admin- } \\
\text { istered. }\end{array}$ \\
\hline Fore period. & & & & & & & & & \\
\hline 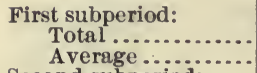 & Grams. & Grame. & Grams. & \multicolumn{6}{|c|}{ Broken by illness. } \\
\hline $\begin{array}{l}\text { Second subperiod: } \\
\quad \text { Total ................... } \\
\text { Average ......... }\end{array}$ & $\begin{array}{l}72.58 \\
14.52\end{array}$ & $\begin{array}{l}5.36 \\
1.07\end{array}$ & $\begin{array}{l}63.19 \\
12.64\end{array}$ & $\begin{array}{l}68.55 \\
13.71\end{array}$ & $\begin{array}{r}7.38 \\
\ldots .\end{array}$ & $\begin{array}{r}87.06 \\
\ldots .\end{array}$ & $\begin{array}{c}94.45 \\
. . . .\end{array}$ & $\begin{array}{l}+4.03 \\
+\quad .81\end{array}$ & $\begin{array}{l}0 \\
0\end{array}$ \\
\hline $\begin{array}{l}\text { Entire fore period: } \\
\text { Total .................. } \\
\text { Average ......... }\end{array}$ & $\begin{array}{l}72.58 \\
14.52\end{array}$ & $\begin{array}{l}5.36 \\
1.07\end{array}$ & $\begin{array}{l}63.19 \\
12.64\end{array}$ & $\begin{array}{l}68.55 \\
13.71\end{array}$ & $\begin{array}{r}7.38 \\
\cdots \cdots \\
\end{array}$ & $\begin{array}{r}87.06 \\
\cdots \cdots \\
\ldots \ldots . .\end{array}$ & $\begin{array}{r}94.45 \\
\cdots \cdots \\
\end{array}$ & $\begin{array}{r}+4.03 \\
+\quad .81\end{array}$ & $\begin{array}{l}0 \\
0\end{array}$ \\
\hline Preservative period. & & & & & & & & & \\
\hline 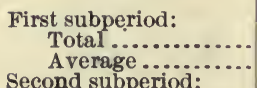 & $\begin{array}{l}75.20 \\
15.04\end{array}$ & $\begin{array}{r}4.17 \\
.83\end{array}$ & $\begin{array}{l}56.15 \\
11.23\end{array}$ & $\begin{array}{l}60.32 \\
12.06\end{array}$ & $\begin{array}{r}5.55 \\
\ldots \ldots\end{array}$ & $\begin{array}{r}74.67 \\
\cdots . . .\end{array}$ & $\begin{array}{r}80.21 \\
\cdots\end{array}$ & $\begin{array}{l}+14.88 \\
+2.98\end{array}$ & $\begin{array}{r}1.05 \\
.21\end{array}$ \\
\hline 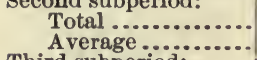 & $\begin{array}{l}71.51 \\
14.30\end{array}$ & $\begin{array}{l}8.72 \\
1.74\end{array}$ & $\begin{array}{r}a 57.33 \\
11.47\end{array}$ & $\begin{array}{l}66.05 \\
13.21\end{array}$ & 12.19 & 80.17 & $\begin{array}{c}92.36 \\
\cdots\end{array}$ & $\begin{array}{l}+5.46 \\
+1.09\end{array}$ & $\begin{array}{r}2.10 \\
.42\end{array}$ \\
\hline $\begin{array}{l}\text { Third subperiod: } \\
\quad \text { Total } \ldots . . . . . . . . . \\
\text { Average }\end{array}$ & $\begin{array}{l}73.03 \\
14.61\end{array}$ & $\begin{array}{r}3.75 \\
.75\end{array}$ & $\begin{array}{l}54.57 \\
10.91\end{array}$ & $\begin{array}{l}58.32 \\
11.66\end{array}$ & 5.13 & 74.72 & 79.86 & $\begin{array}{r}+14.71 \\
+\quad 2.95\end{array}$ & $\begin{array}{l}4.60 \\
80\end{array}$ \\
\hline Fourth subperiod: & 14.01 & & & & & •........ & $\cdots$ & $+2.9)$ & \\
\hline 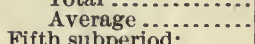 & 15.48 & $\begin{array}{l}7.54 \\
1.51\end{array}$ & $\begin{array}{l}57.67 \\
11.53\end{array}$ & $\begin{array}{l}65.21 \\
13.04\end{array}$ & $\begin{array}{r}9.74 \\
\cdots\end{array}$ & $\begin{array}{l}74.52 \\
\cdots . . .\end{array}$ & $\begin{array}{c}84.26 \\
\ldots . . .\end{array}$ & $\begin{array}{l}+12.18 \\
+\quad 2.44\end{array}$ & $\begin{array}{l}6.00 \\
1.20\end{array}$ \\
\hline $\begin{array}{l}\text { Fifth subperiod: } \\
\quad \text { Total } \ldots . . . . . . . . . \\
\text { Average } . . . . . .\end{array}$ & $\begin{array}{l}71.95 \\
14.39\end{array}$ & $\begin{array}{r}3.93 \\
.79\end{array}$ & $\begin{array}{l}55.51 \\
11.10\end{array}$ & $\begin{array}{l}59.44 \\
11.89\end{array}$ & $\begin{array}{r}5.46 \\
\ldots . . .\end{array}$ & $\begin{array}{r}77.15 \\
\ldots \ldots\end{array}$ & $\begin{array}{r}82.61 \\
\ldots \ldots \ldots\end{array}$ & $\begin{array}{l}+12.51 \\
+2.50\end{array}$ & $\begin{array}{l}8.00 \\
1.60\end{array}$ \\
\hline $\begin{array}{l}\text { Five preservative sub- } \\
\text { periods: } \\
\text { Total } \ldots \ldots \ldots \ldots \ldots \\
\text { Average } \ldots \ldots \ldots \ldots\end{array}$ & $\begin{array}{r}b 369.08 \\
14.76\end{array}$ & $\begin{array}{r}28.11 \\
1.12\end{array}$ & $\begin{array}{r}281.23 \\
11.25\end{array}$ & $\begin{array}{r}309.34 \\
12.37\end{array}$ & $\begin{array}{r}7.62 \\
\cdots \cdots \\
\end{array}$ & $\begin{array}{r}76.20 \\
\cdots \cdots\end{array}$ & $\begin{array}{r}83.81 \\
\cdots\end{array}$ & $\begin{array}{l}+59.74 \\
+\quad 2.39\end{array}$ & $\begin{array}{r}21.15 \\
.85\end{array}$ \\
\hline After period. & & & & & & & & & \\
\hline $\begin{array}{l}\text { First subperiod: } \\
\quad \text { Total .............. } \\
\text { Average .......... }\end{array}$ & $\begin{array}{l}73.16 \\
14.63\end{array}$ & & $\begin{array}{l}53.56 \\
10.71\end{array}$ & & & $\begin{array}{l}73.21 \\
-\ldots .\end{array}$ & & & $\begin{array}{l}0 \\
0\end{array}$ \\
\hline $\begin{array}{l}\text { Second subperiod: } \\
\text { Total } \\
\text { Average................. }\end{array}$ & $\begin{array}{l}74.40 \\
14.88\end{array}$ & $\begin{array}{l}5.30 \\
1.06\end{array}$ & $\begin{array}{l}55.08 \\
11.02\end{array}$ & $\begin{array}{l}60.33 \\
12.08\end{array}$ & $\begin{array}{r}7.12 \\
\cdots \cdots\end{array}$ & $\begin{array}{r}74.03 \\
\ldots . . .\end{array}$ & $\begin{array}{r}81.16 \\
\ldots \ldots\end{array}$ & $\begin{array}{r}+14.02 \\
+2.80\end{array}$ & $\begin{array}{l}0 \\
0\end{array}$ \\
\hline $\begin{array}{l}\text { Entire after period: } \\
\text { Total ........... } \\
\text { Average ........ }\end{array}$ & & & & & & & & & $\begin{array}{l}0 \\
0\end{array}$ \\
\hline
\end{tabular}

a Daily average added in order to complete record. $\quad b$ No. 3 had only five preservative subperiods. 
SALICYLIC ACID AND SALICYLATES.

TABLE XIII.-Nitrogen balances for Series VI-Continued.

[Averages are per day.]

No. 4.

\begin{tabular}{|c|c|c|c|c|c|c|c|c|c|}
\hline Period. & In food. & $\begin{array}{c}2 \\
\text { In feces. }\end{array}$ & $\begin{array}{c}\text { In } \\
\text { urine. }\end{array}$ & $\begin{array}{c}.4 \\
\text { In feces } \\
\text { and } \\
\text { urine. } \\
(2+3)\end{array}$ & $\begin{array}{c}5 \\
\text { In } \\
\text { feces } \\
(2 \div 1)\end{array}$ & $\begin{array}{c}6 \\
\text { In } \\
\text { urine. } \\
(3 \div 1)\end{array}$ & $\begin{array}{c}7 \\
\text { In feces } \\
\text { and } \\
\text { urine. } \\
(4 \div 1)\end{array}$ & $\begin{array}{c}8 \\
\text { Balance. } \\
(1-4)\end{array}$ & $\begin{array}{c}9 \\
\text { Sali- } \\
\text { cylic } \\
\text { acid } \\
\text { admin- } \\
\text { istered. }\end{array}$ \\
\hline Fore period. & & & & & & & & & \\
\hline $\begin{array}{l}\text { First subperiod: } \\
\quad \text { Total } \\
\text { A verage } . . .\end{array}$ & $\begin{array}{r}\text { Grams. } \\
\quad 78.30 \\
15.66\end{array}$ & $\begin{array}{r}\text { Grams. } \\
5.00 \\
1.00\end{array}$ & $\begin{array}{r}\text { Grams. } \\
73.78 \\
14.76\end{array}$ & $\begin{array}{r}\text { Grams } \\
78.78 \\
15.76\end{array}$ & $\begin{array}{r}\text { Per ct. } \\
6.39\end{array}$ & $\begin{array}{r}\text { Per ct. } \\
94.23\end{array}$ & $\begin{array}{l}\text { Per ct. } \\
100.61\end{array}$ & $\begin{array}{l}\text { Gram8. } \\
=\quad .48 \\
-10\end{array}$ & $\underset{0}{\operatorname{Grams} .}$ \\
\hline 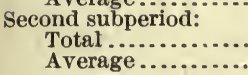 & $\begin{array}{l}78.80 \\
15.76\end{array}$ & $\begin{array}{l}5.78 \\
1.16\end{array}$ & $\begin{array}{l}71.94 \\
14.39\end{array}$ & $\begin{array}{l}77.72 \\
15.54\end{array}$ & 7.34 & 91.29 & $\begin{array}{l}98.63 . \\
\cdots . . .\end{array}$ & $\begin{array}{l}+1.08 \\
+\quad .22\end{array}$ & $\begin{array}{l}0 \\
0\end{array}$ \\
\hline 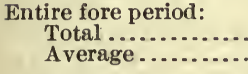 & $\begin{array}{r}157.10 \\
15.71\end{array}$ & $\begin{array}{r}10.78 \\
1.08\end{array}$ & $\begin{array}{r}145.72 \\
14.57\end{array}$ & $\begin{array}{r}156.50 \\
15.65\end{array}$ & $\begin{array}{r}6.86 \\
\ldots . . .\end{array}$ & $\begin{array}{r}92.76 \\
\cdots \cdots . . .\end{array}$ & $\begin{array}{c}99.62 \\
\cdots \cdots \ldots\end{array}$ & $\begin{array}{l}+.60 \\
+\quad .06\end{array}$ & $\begin{array}{l}0 \\
0\end{array}$ \\
\hline Preservative period. & & & & & & & & & \\
\hline $\begin{array}{l}\text { First subperiod: } \\
\text { Total ......... } \\
\text { Average .... }\end{array}$ & $\begin{array}{l}76.45 \\
15.29\end{array}$ & $\begin{array}{r}4.51 \\
.90\end{array}$ & $\begin{array}{l}\text { 70.07 } \\
14.01\end{array}$ & $\begin{array}{l}74.58 \\
14.92\end{array}$ & 5.90 & 91.65 & 97.55 & $\begin{array}{l}+1.87 \\
+\quad .37\end{array}$ & $\begin{array}{r}1.05 \\
.21\end{array}$ \\
\hline Second suk & & & & & & & & & \\
\hline $\begin{array}{r}\text { Tot: } \\
\text { Ave } \\
\text { hird sl }\end{array}$ & $\begin{array}{l}80.18 \\
16.04\end{array}$ & $\begin{array}{l}5.28 \\
1.06\end{array}$ & $\begin{array}{l}74.59 \\
14.92\end{array}$ & $\begin{array}{l}79.87 \\
15.97\end{array}$ & 6.59 & 93.03 & 99.61 & $\begin{array}{l}+.31 \\
+.07\end{array}$ & $\begin{array}{r}2.10 \\
.42\end{array}$ \\
\hline hird st & 78.37 & 5.19 & & 73. & 6.62 & 87.41 & 94.03. & +4.68 & 3.7 \\
\hline $\begin{array}{r}\text { A } \\
\text { Fourtl }\end{array}$ & & & & 14. & & & & & : \\
\hline Total & 77.50 & a 4.71 & 72.07 & 76. 78 & 6.08 & 92.99 & $\$ 9.08$ & +.72 & 6.00 \\
\hline Fifth & & & & 15.8 & & & & & \\
\hline & 81.16 & 6.57 & 66.08 & 72.65 & 8.10 & 81.42 & 89.51 & +8.51 & 8.00 \\
\hline sixth subpe & & & & & & & & & \\
\hline $\begin{array}{l}\text { Total } \ldots \ldots \ldots \\
\text { Average } . . .\end{array}$ & $\begin{array}{l}74.45 \\
14.89\end{array}$ & $\begin{array}{rl}a & 5.35 \\
1.07\end{array}$ & $\begin{array}{l}71.94 \\
14.39\end{array}$ & $\begin{array}{l}77.29 \\
15.46\end{array}$ & 7.19 & 96.63 & 103.81 & -2.84 & $\begin{array}{r}10.00 \\
2.00\end{array}$ \\
\hline 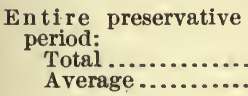 & $\begin{array}{r}468.11 \\
15.60\end{array}$ & $\begin{array}{r}31.61 \\
1.05\end{array}$ & $\begin{array}{r}423.25 \\
14.11\end{array}$ & $\begin{array}{r}454.86 \\
15.16\end{array}$ & 6.75 & 90.42 & 97.17 & $\begin{array}{l}+13.25 \\
+\quad .44\end{array}$ & $\begin{array}{r}30.85 \\
1.03\end{array}$ \\
\hline After period. & & & & & & & & & \\
\hline $\begin{array}{l}\text { First subperiod: } \\
\text { Total ......... } \\
\text { Average..... }\end{array}$ & $\begin{array}{l}78.89 \\
15.78\end{array}$ & $\begin{array}{r}4.24 \\
.85\end{array}$ & $\begin{array}{l}72.04 \\
14.41\end{array}$ & $\begin{array}{l}76.28 \\
15.26\end{array}$ & 5.37 & 91.32 & 96.69 & $\begin{array}{r}+2.61 \\
+\quad .52\end{array}$ & $\begin{array}{l}0 \\
0\end{array}$ \\
\hline $\begin{array}{l}\text { Second subpe } \\
\text { Total .... } \\
\text { Average }\end{array}$ & $\begin{array}{l}78.90 \\
15.78\end{array}$ & & $\begin{array}{l}74.44 \\
14.89\end{array}$ & $\begin{array}{l}80.34 \\
16.07\end{array}$ & 7.48 & 94.35 & 101.83 & $\begin{array}{l}-1.44 \\
-\quad .29\end{array}$ & $\begin{array}{l}0 \\
0\end{array}$ \\
\hline $\begin{array}{l}\text { Entire after period: } \\
\text { Total ................... } \\
\text { Average ......... }\end{array}$ & $\begin{array}{r}157.79 \\
15.78\end{array}$ & $\begin{array}{r}10.14 \\
1.01\end{array}$ & $\begin{array}{r}146.48 \\
14.65\end{array}$ & $\begin{array}{r}156.62 \\
15.66\end{array}$ & 6.43 & 92.83 & 99.26 & $\begin{array}{l}+1.17 \\
+\quad .12\end{array}$ & $\begin{array}{l}0 \\
0\end{array}$ \\
\hline
\end{tabular}

$a$ Daily average added in order to complete record. 
TABLE XIII. - Nitrogen balances for Series VI-Continued.

[Averages are per day.]

No.5.

\begin{tabular}{|c|c|c|c|c|c|c|c|c|c|}
\hline Period. & In food. & In feces. & $\begin{array}{c}3 \\
\text { In } \\
\text { urine. }\end{array}$ & $\begin{array}{c}4 \\
\text { In feces } \\
\text { and } \\
\text { urine. } \\
(2+3)\end{array}$ & $\begin{array}{c}\text { In } \\
\text { feces. } \\
(2 \div 1)\end{array}$ & $\begin{array}{c}6 \\
\text { In } \\
\text { urine. } \\
(3 \div 1)\end{array}$ & $\begin{array}{c}7 \\
\text { In feces } \\
\text { and } \\
\text { urine. } \\
(4 \div 1)\end{array}$ & Balance. & $\begin{array}{c}9 \\
\text { Sali- } \\
\text { cylic } \\
\text { acid } \\
\text { admin- } \\
\text { istered. }\end{array}$ \\
\hline \multicolumn{10}{|l|}{ Fore period. } \\
\hline $\begin{array}{l}\text { First subperiod: } \\
\quad \text { Total ............... } \\
\text { A verage ........... }\end{array}$ & $\begin{array}{r}\text { Grams. } \\
78.77 \\
15.75\end{array}$ & $\begin{array}{r}\text { Grams. } \\
7.41 \\
1.48\end{array}$ & $\begin{array}{r}\text { Grams. } \\
69.53 \\
13.91\end{array}$ & $\begin{array}{c}\text { Grams. } \\
76.94 \\
15.39\end{array}$ & $\begin{array}{r}\text { Per ct. } \\
9.41\end{array}$ & $\begin{array}{l}\text { Per ct. } \\
88.27\end{array}$ & $\begin{array}{c}\text { Per ct. } \\
97.68 \\
\ldots\end{array}$ & $\begin{array}{l}\text { Grams. } \\
+1.83 \\
+\quad .36\end{array}$ & $\begin{array}{c}\text { Grams. } \\
0 \\
0\end{array}$ \\
\hline $\begin{array}{l}\text { Second subperiod: } \\
\text { Total }\end{array}$ & & & & & & & & & \\
\hline $\begin{array}{l}\text { Total ................. } \\
\text { Average.......... }\end{array}$ & $\begin{array}{l}79.93 \\
15.99\end{array}$ & $\begin{array}{l}6.71 \\
1.34\end{array}$ & $\begin{array}{l}61.86 \\
12.37\end{array}$ & $\begin{array}{l}68.57 \\
13.71\end{array}$ & $\begin{array}{r}8.39 \\
\cdots\end{array}$ & $\begin{array}{r}77.39 \\
\ldots \ldots .\end{array}$ & $\begin{array}{r}85.79 \\
\cdots \cdots\end{array}$ & $\begin{array}{l}+11.36 \\
+2.28\end{array}$ & $\begin{array}{l}0 \\
0\end{array}$ \\
\hline $\begin{array}{l}\text { Entire fore period: } \\
\text { Total ................ } \\
\text { Average .......... }\end{array}$ & $\begin{array}{r}158.70 \\
15.87\end{array}$ & $\begin{array}{r}14.12 \\
1.41\end{array}$ & $\begin{array}{r}131.39 \\
13.14 \\
\end{array}$ & $\begin{array}{r}145.51 \\
14.55\end{array}$ & $\begin{array}{r}8.90 \\
\cdots \\
\end{array}$ & $\begin{array}{r}82.79 \\
\cdots \cdots \\
\end{array}$ & $\begin{array}{r}91.69 \\
\cdots \ldots \ldots \\
\end{array}$ & $\begin{array}{l}+13.19 \\
+1.32 \\
\end{array}$ & $\begin{array}{l}0 \\
0\end{array}$ \\
\hline \multicolumn{10}{|l|}{ Preservative period. } \\
\hline $\begin{array}{l}\text { First subperiod: } \\
\text { Total............ } \\
\text { Average....... }\end{array}$ & $\begin{array}{l}77.85 \\
15.57\end{array}$ & $\begin{array}{l}7.15 \\
1.43\end{array}$ & $\begin{array}{l}68.49 \\
13.70\end{array}$ & $\begin{array}{l}75.64 \\
15.13\end{array}$ & 9.18 & 87.98 & $\begin{array}{l}97.16 \\
\cdots \ldots . .\end{array}$ & $\begin{array}{l}+2.21 \\
+\quad .44\end{array}$ & $\begin{array}{r}1.05 \\
.21\end{array}$ \\
\hline $\begin{array}{l}\text { econd subperiod: } \\
\text { Total .......... }\end{array}$ & & & & & & & . & & \\
\hline 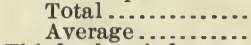 & $\begin{array}{l}80.83 \\
16.17\end{array}$ & $\begin{array}{r}4.69 \\
.94\end{array}$ & $\begin{array}{l}69.02 \\
13.80\end{array}$ & $\begin{array}{l}73.71 \\
14.74\end{array}$ & $\begin{array}{c}5.80 \\
\cdots \cdots\end{array}$ & 85.39 & 91.19 & $\begin{array}{l}+7.12 \\
+1.43\end{array}$ & 2.10 \\
\hline & & & & & & & & & \\
\hline 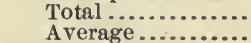 & 80.22 & 6.35 . & $\begin{array}{l}67.90 \\
78\end{array}$ & 74.25 & 7.92 & 84.64 & 92.56 & $\begin{array}{l}+5.97 \\
\end{array}$ & 3.70 \\
\hline \multicolumn{10}{|l|}{ Fourth subperiod: } \\
\hline 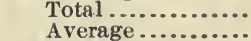 & $\begin{array}{l}79.11 \\
15.82\end{array}$ & $\begin{array}{r}4.47 \\
.89\end{array}$ & $\begin{array}{l}69.38 \\
13.88\end{array}$ & $\begin{array}{l}73.85 \\
14.77\end{array}$ & 5.65 & 87.70 & 93.35 & $\begin{array}{r}+5.26 \\
+1.05\end{array}$ & $\begin{array}{l}6.00 \\
1.20\end{array}$ \\
\hline Fifth subperiod: & & & 10.00 & & & & & & \\
\hline $\begin{array}{l}\text { Total } \\
\text { Average............... }\end{array}$ & 81.99 & 6.00 & 72.81 & 78.81 & 7. 32 & 88.80 & 96.12 & +3.18 & 8.00 \\
\hline \multirow{2}{*}{\multicolumn{10}{|c|}{$\begin{array}{l}\text { Sixth subperiod: } \\
\text { Total ................... }\end{array}$}} \\
\hline & $\begin{array}{l}75.86 \\
15.17\end{array}$ & $\begin{array}{l}6.18 \\
1.24\end{array}$ & 68. 66 & $\begin{array}{l}74.84 \\
14.97\end{array}$ & 8.15 & 90.51 & 98.66 & +1.02 & 10.00 \\
\hline \multirow{2}{*}{\multicolumn{10}{|c|}{$\begin{array}{l}\text { Entire preservative } \\
\text { period: }\end{array}$}} \\
\hline & & & & & & & & & \\
\hline Total ............... & 475.86 & 34.84 & 416.26 & 451.10 & 7.32 & 87.48 & 94.80 & +24.76 & 30.85 \\
\hline Average ....... & 15.86 & 1.16 & 13.88 & 15.04 & & & $\cdots \ldots \ldots$ & +.82 & 1.03 \\
\hline After period. & & & & & & & & & \\
\hline $\begin{array}{l}\text { First subperiod: } \\
\text { Total ................ } \\
\text { Average ........... }\end{array}$ & $\begin{array}{l}79.62 \\
15.92\end{array}$ & $\begin{array}{l}5.91 \\
1.18\end{array}$ & $\begin{array}{r}a 69.30 \\
13.86\end{array}$ & $\begin{array}{l}75.21 \\
15.04\end{array}$ & 7.42 . & 87.04 & 94.46 & $\begin{array}{r}+4.41 \\
+\quad .88\end{array}$ & $\begin{array}{l}0 \\
0\end{array}$ \\
\hline \multirow{2}{*}{\multicolumn{10}{|c|}{ 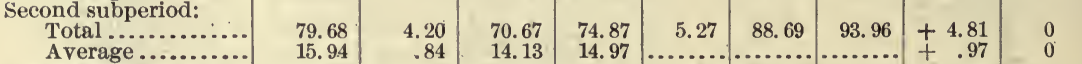 }} \\
\hline & & 4.20 & 70.67 & 74.87 & 5.27 & 88.69 & 93.96 & +4.81 & 0 \\
\hline Arerase............... & & & & & & & & & 0 \\
\hline \multirow{2}{*}{ 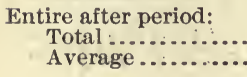 } & 159.30 & 10.11 & 139.97 & 150.08 & 6.35 & 87.87 & 94.21 & +9.22 & 0 \\
\hline & 15.93 & 1.01 & 14.00 & 15.01 & & & & +.92 & 0 \\
\hline
\end{tabular}

$a$ Daily average added in order to complete record. 
TABLE XIII.-Nitrogen balances for Series VI-Continued.

[Averages are per day.]

No. 6.

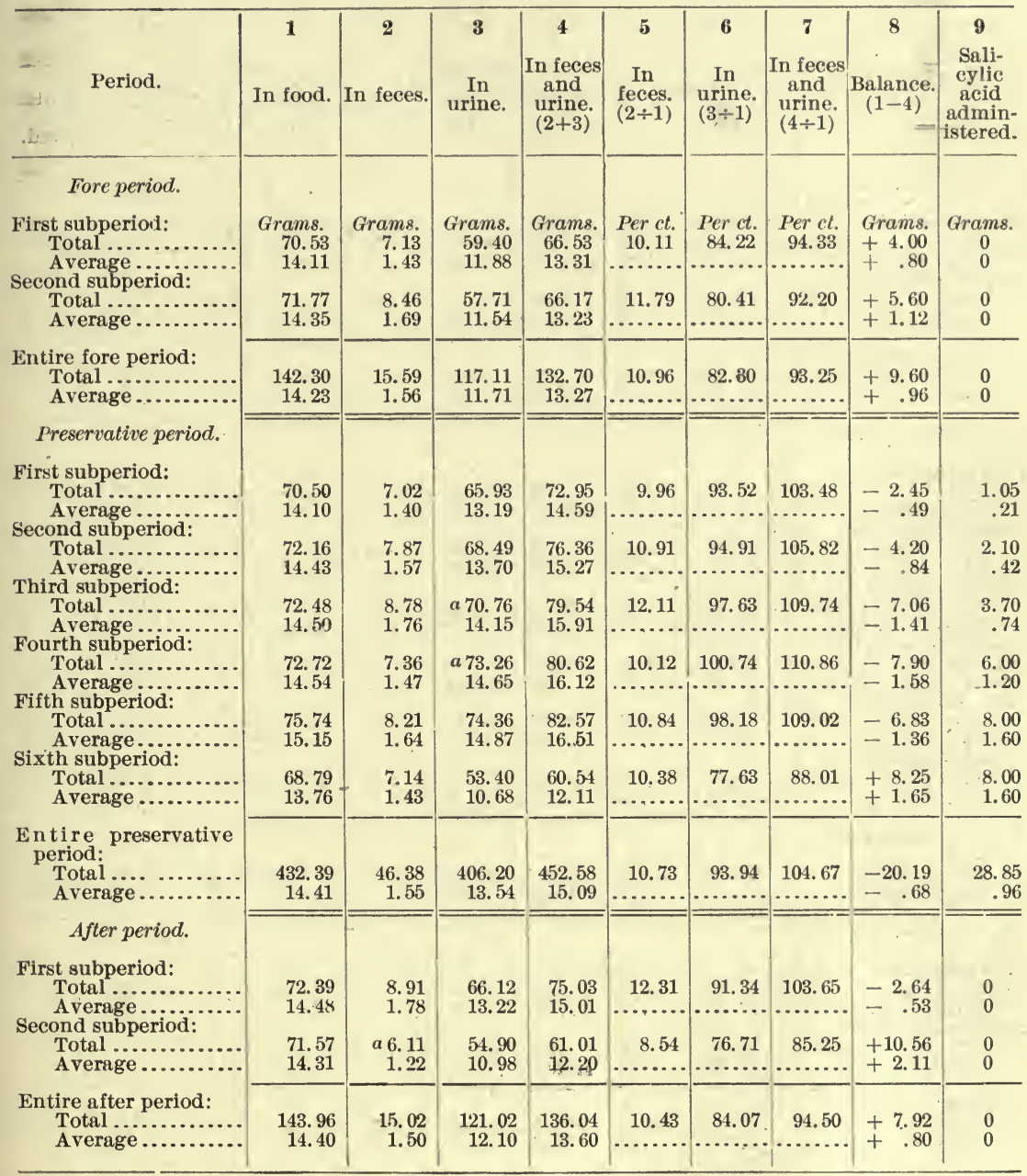

$a$ Daily average added in order to complete record. 
TaBLe XIII.-Nitrogen balances for Series VI-Continued.

[Averages are per day.]

No. 7 .

\begin{tabular}{|c|c|c|c|c|c|c|c|c|c|}
\hline Period. & $\begin{array}{c}1 \\
\text { In food. }\end{array}$ & In feces. & In urine. & $\mid \begin{array}{c}4 \\
\text { In fcces } \\
\text { and } \\
\text { arine. } \\
(2+3)\end{array}$ & $\begin{array}{c}5 \\
\text { In } \\
\text { feces } \\
(2 \div 1)\end{array}$ & $\begin{array}{c}6 \\
\text { In } \\
\text { urine. } \\
(3 \div 1)\end{array}$ & \begin{tabular}{|c}
7 \\
In feces \\
and \\
urine. \\
$(4 \div 1)$
\end{tabular} & $\begin{array}{c}8 \\
\text { Balance. } \\
(1-4)\end{array}$ & $\begin{array}{c}9 \\
\text { Sali- } \\
\text { cylic } \\
\text { acid } \\
\text { admin- } \\
\text { istered. }\end{array}$ \\
\hline Fore period. & & & & & & & & & \\
\hline 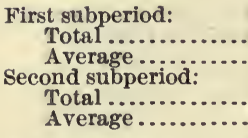 & $\begin{array}{r}\text { Grams. } \\
\quad 5.52 \\
13.10 \\
73.90 \\
-14.78\end{array}$ & $\begin{array}{r}\text { Grams. } \\
6.24 \\
1.25 \\
3.24 \\
.65\end{array}$ & $\begin{array}{r}\text { Grams. } \\
56.57 \\
11.31 \\
63.00 \\
12.60\end{array}$ & $\begin{array}{r}\text { Grams. } \\
62.81 \\
12.56\end{array}$ & $\begin{array}{l}\text { Per ct. } \\
9.52\end{array}$ & $\begin{array}{l}\text { Per ct. } \\
86.34\end{array}$ & $\begin{array}{c}\text { Per ct. } \\
95.86 \\
\\
89.63 \\
\\
.6 .\end{array}$ & $\begin{array}{l}\text { Grams. } \\
+2.71 \\
+\quad .54 \\
+7.66 \\
+1.53\end{array}$ & $\begin{array}{c}\text { Grams. } \\
0 \\
0 \\
0 \\
0\end{array}$ \\
\hline 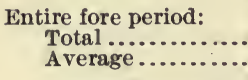 & $\begin{array}{r}139.42 \\
13.94 \\
\end{array}$ & $\begin{array}{r}9.48 \\
.95\end{array}$ & $\begin{array}{r}119.57 \\
11.96 \\
\end{array}$ & $\begin{array}{r}129.05 \\
12.91 \\
\end{array}$ & 6.80 & $\begin{array}{r}85.76 \\
\ldots \ldots \ldots \\
\end{array}$ & $\begin{array}{l}92.56 \\
\cdots \cdots\end{array}$ & $\begin{array}{r}+10.37 \\
+1.03 \\
\end{array}$ & $\begin{array}{l}0 \\
0\end{array}$ \\
\hline Preservative period. & & & & & & & & & \\
\hline $\begin{array}{l}\text { First subperiod: } \\
\text { Total .................. } \\
\text { Average .......... }\end{array}$ & $\begin{array}{l}65.68 \\
13.14\end{array}$ & $\begin{array}{r}4.04 \\
.81\end{array}$ & $\begin{array}{l}50.72 \\
10.14\end{array}$ & $\begin{array}{l}54.76 \\
10.95\end{array}$ & 6.15 & 77.22 & 83.37 & $\begin{array}{l}+10.92 \\
+2.19\end{array}$ & $\begin{array}{r}1.05 \\
.21\end{array}$ \\
\hline $\begin{array}{l}\text { Second subperiod: } \\
\text { Total ................. } \\
\text { Average }\end{array}$ & $\begin{array}{l}71.80 \\
14.36\end{array}$ & $\begin{array}{r}3.08 \\
.62\end{array}$ & $\begin{array}{l}56.98 \\
11.40\end{array}$ & $\begin{array}{l}60.06 \\
12.01\end{array}$ & 4.29 & 79.36 & 83.65 & $\begin{array}{l}+11.74 \\
+2.35\end{array}$ & $\begin{array}{r}2.10 \\
.42\end{array}$ \\
\hline 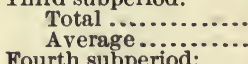 & $\begin{array}{l}68.01 \\
13.60\end{array}$ & $\begin{array}{r}4.09 \\
.82\end{array}$ & $\begin{array}{l}51.24 \\
10.25\end{array}$ & $\begin{array}{l}55.33 \\
11.07\end{array}$ & 6.01 & 75.34 & 81.36 & $\begin{array}{l}+12.68 \\
+2.53\end{array}$ & $\begin{array}{r}3.70 \\
.74\end{array}$ \\
\hline $\begin{array}{l}\text { Fourth subperiod: } \\
\text { Total .................... } \\
\text { Average } \\
\text { Fifth subperiod: }\end{array}$ & $\begin{array}{l}67.02 \\
13.40\end{array}$ & $\begin{array}{r}2.92 \\
.58\end{array}$ & $\begin{array}{r}49.15 \\
9.83\end{array}$ & $\begin{array}{l}52.07 \\
10.41\end{array}$ & 4.36 & 73.34 & 77.69 & $\begin{array}{l}+14.95 \\
+2.99\end{array}$ & $\begin{array}{l}6.00 \\
1.20\end{array}$ \\
\hline $\begin{array}{l}\text { Total ..................... } \\
\text { Average ........... } \\
\text { Sixth subperiod: }\end{array}$ & $\begin{array}{l}68.55 \\
13.71\end{array}$ & $\begin{array}{r}4.01 \\
.80\end{array}$ & $\begin{array}{l}52.92 \\
10.58\end{array}$ & $\begin{array}{l}56.93 \\
11.39\end{array}$ & 5.85 & 77.20 & 83.05 & $\begin{array}{l}+11.62 \\
+2.32\end{array}$ & $\begin{array}{l}8.00 \\
1.60\end{array}$ \\
\hline $\begin{array}{l}\text { Total } \\
\text { Average...................... }\end{array}$ & $\begin{array}{l}64.47 \\
12.89\end{array}$ & $\begin{array}{r}2.23 \\
.45\end{array}$ & $\begin{array}{l}64.17 \\
12.83\end{array}$ & $\begin{array}{l}66.40 \\
13.28\end{array}$ & $\begin{array}{r}3.46 \\
\cdots \cdots \\
\end{array}$ & $\begin{array}{l}99.53 \\
\ldots \ldots \ldots \\
\end{array}$ & $\begin{array}{r}102.99 \\
\cdots \cdots \cdots \\
\end{array}$ & $\begin{array}{l}-1.93 \\
-\quad .39\end{array}$ & $\begin{array}{r}10.00 \\
2.00\end{array}$ \\
\hline $\begin{array}{c}\text { Entire preservative } \\
\text { period: } \\
\text { Total .................. } \\
\text { Average........... }\end{array}$ & $\begin{array}{r}405.53 \\
13.52 \\
\end{array}$ & $\begin{array}{r}20.37 \\
.68 \\
\end{array}$ & $\begin{array}{r}325.18 \\
10.84 \\
\end{array}$ & $\begin{array}{r}345.55 \\
11.52 \\
\end{array}$ & $\begin{array}{r}5.02 \\
\cdots \ldots . . \\
\end{array}$ & $\begin{array}{l}80.19 \\
\cdots \cdots\end{array}$ & $\begin{array}{l}85.21 \\
\cdots \cdots\end{array}$ & $\begin{array}{r}+59.98 \\
+2.00 \\
\end{array}$ & $\begin{array}{r}30.85 \\
1.03 \\
\end{array}$ \\
\hline After period. & & & & & & & & & \\
\hline $\begin{array}{l}\text { First subperiod: } \\
\text { Total ................ } \\
\text { Average....... } \\
\text { Second subperiod: }\end{array}$ & $\begin{array}{l}67.76 \\
13.55\end{array}$ & $\begin{array}{r}4.57 \\
.91\end{array}$ & $\begin{array}{l}52.80 \\
10.56\end{array}$ & $\begin{array}{l}57.37 \\
11.47\end{array}$ & 6.74 & 77.92 & 84.67 & $\begin{array}{r}+10.39 \\
+2.08\end{array}$ & $\begin{array}{l}0 \\
0\end{array}$ \\
\hline $\begin{array}{l}\text { Second subperiod: } \\
\text { Total ..................................... } \\
\text { Average }\end{array}$ & $\begin{array}{l}68.28 \\
13.66\end{array}$ & $\begin{array}{l}6.32 \\
1.26\end{array}$ & $\begin{array}{l}56.09 \\
11.22\end{array}$ & $\begin{array}{l}62.41 \\
12.48\end{array}$ & 9.26 & 82.15 & 91.40 & $\begin{array}{l}+5.87 \\
+1.18\end{array}$ & $\begin{array}{l}0 \\
0\end{array}$ \\
\hline 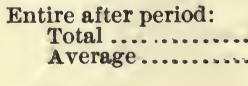 & $\begin{array}{r}136.04 \\
13.60\end{array}$ & $\begin{array}{r}10.89 \\
1.09\end{array}$ & $\begin{array}{r}108.89 \\
10.89\end{array}$ & $\begin{array}{r}119.78 \\
11.98\end{array}$ & 8.00 & 80.04 & 88.05 & $\begin{array}{l}+16.26 \\
+1.62\end{array}$ & $\begin{array}{l}0 \\
0\end{array}$ \\
\hline
\end{tabular}


TABLE XIII.-Nitrogen balances for Series VI-Continued.

[Averages are per day.]

No.8.

\begin{tabular}{|c|c|c|c|c|c|c|c|c|c|}
\hline Period. & In food. & In feces. & In urine. & $\begin{array}{c}4 \\
\text { In feces } \\
\text { and } \\
\text { urine. } \\
(2+3)\end{array}$ & $\begin{array}{c}\text { ๘ } \\
\text { In } \\
\text { feces. } \\
(2 \div 1)\end{array}$ & $\begin{array}{c}\text { In } \\
\text { urine. } \\
(3 \div-1)\end{array}$ & $\begin{array}{c}7 \\
\text { In feces } \\
\text { and } \\
\text { urine. } \\
(4 \div 1)\end{array}$ & $\begin{array}{c}8 \\
\text { Balance. } \\
(1-4)\end{array}$ & $\begin{array}{c}9 \\
\text { Sali- } \\
\text { cylic } \\
\text { acid } \\
\text { admin- } \\
\text { istered. }\end{array}$ \\
\hline \multicolumn{10}{|l|}{ Fore period. } \\
\hline 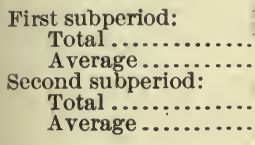 & $\begin{array}{r}\text { Grams. } \\
63.80 \\
12.76 \\
\\
65.27 \\
13.05\end{array}$ & $\begin{array}{r}\text { Grams. } \\
4.76 \\
.95 \\
\\
6.91 \\
1.38\end{array}$ & $\begin{array}{r}\text { Grams. } \\
52.91 \\
10.58\end{array}$ & $\begin{array}{r}\text { Grams. } \\
57.67 \\
11.53 \\
\\
62.41 \\
12.48\end{array}$ & $\begin{array}{r}\text { Per ct. } \\
7.46 \\
\ldots \\
10.59 \\
\ldots \ldots\end{array}$ & $\begin{array}{r}\text { Per ct. } \\
82.93 \\
\ldots \ldots\end{array}$ & $\begin{array}{r}\text { Per ct. } \\
90.39 \\
\cdots\end{array}$ & $\begin{array}{l}\text { Grams. } \\
+6.13 \\
+1.23 \\
+\quad 2.86 \\
+\quad .57\end{array}$ & $\begin{array}{c}\text { Grams. } \\
0 \\
0 \\
\\
0 \\
0\end{array}$ \\
\hline $\begin{array}{l}\text { Entire fore period: } \\
\text { Total ............ } \\
\text { Average....... }\end{array}$ & $\begin{array}{r}129.07 \\
12.91\end{array}$ & $\begin{array}{r}11.67 \\
1.17\end{array}$ & $\begin{array}{r}108.41 \\
10.84\end{array}$ & $\begin{array}{r}120.08 \\
12.01\end{array}$ & $\begin{array}{r}9.04 \\
\cdots \cdots \\
\end{array}$ & $\begin{array}{r}83.99 \\
\cdots\end{array}$ & $\begin{array}{r}93.03 \\
\cdots \cdots \\
\end{array}$ & $\begin{array}{r}+8.99 \\
+\quad .90\end{array}$ & $\begin{array}{l}0 \\
0\end{array}$ \\
\hline \multicolumn{10}{|l|}{ Preservative period. } \\
\hline $\begin{array}{l}\text { First subperiod: } \\
\text { Total ................. } \\
\text { Average.......... } \\
\text { Second subperiod: }\end{array}$ & $\begin{array}{l}64.56 \\
12.91\end{array}$ & $\begin{array}{l}7.72 \\
1.54\end{array}$ & $\begin{array}{l}52.73 \\
10.55\end{array}$ & $\begin{array}{l}60.45 \\
12.09\end{array}$ & $\begin{array}{r}11.96 \\
\ldots\end{array}$ & $\begin{array}{r}81.68 \\
\ldots \ldots\end{array}$ & $\begin{array}{r}93.63 \\
\ldots\end{array}$ & $\begin{array}{l}+4.11 \\
+\quad .82\end{array}$ & $\begin{array}{r}1.05 \\
.21\end{array}$ \\
\hline $\begin{array}{l}\text { Total.... } \\
\text { Average. }\end{array}$ & $\begin{array}{l}69.04 \\
13.81\end{array}$ & $\begin{array}{l}8.40 \\
1.68\end{array}$ & $\begin{array}{l}54.44 \\
10.89\end{array}$ & $\begin{array}{l}62.84 \\
12.57\end{array}$ & $\begin{array}{r}12.17 \\
\cdots\end{array}$ & $\begin{array}{r}78.85 \\
\cdots\end{array}$ & $\begin{array}{r}91.02 \\
\cdots \cdots\end{array}$ & $\begin{array}{r}+6.20 \\
+1.24\end{array}$ & $\begin{array}{r}2.10 \\
.42\end{array}$ \\
\hline 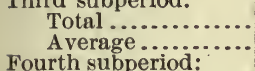 & $\begin{array}{l}65.30^{\circ} \\
13.06\end{array}$ & $\begin{array}{l}5.28 \\
1.06\end{array}$ & $\begin{array}{l}51.74 \\
10.35\end{array}$ & $\begin{array}{l}57.02 \\
11.40\end{array}$ & $\begin{array}{r}8.09 \\
.\end{array}$ & $\begin{array}{r}79.23 \\
\cdots\end{array}$ & $\begin{array}{r}87.32 \\
\ldots \ldots\end{array}$ & $\begin{array}{l}+8.28 \\
+1.66\end{array}$ & $\begin{array}{r}3.70 \\
.74\end{array}$ \\
\hline $\begin{array}{l}\text { Fourth } \\
\text { Tota } \\
\text { Aver }\end{array}$ & $\begin{array}{l}65.80 \\
13.16\end{array}$ & $\begin{array}{l}7.00 \\
1.40\end{array}$ & $\begin{array}{r}a .50 .16 \\
10.03\end{array}$ & $\begin{array}{l}57.16 \\
11.43\end{array}$ & $\begin{array}{l}10.64 \\
\ldots \ldots\end{array}$ & 76.23 & $\begin{array}{r}86.87 \\
\ldots .\end{array}$ & $\begin{array}{l}+8.64 \\
+1.73\end{array}$ & $\begin{array}{l}6.00 \\
1.20\end{array}$ \\
\hline $\begin{array}{l}\text { Fifth sul } \\
\text { Tota } \\
\text { Aver }\end{array}$ & $\begin{array}{l}70.42 \\
14.08\end{array}$ & $\begin{array}{r}4.38 \\
.88\end{array}$ & $\begin{array}{l}57.62 \\
11.52\end{array}$ & $\begin{array}{l}62.00 \\
12.40\end{array}$ & 6.22 & 81.82 & $\begin{array}{l}88.04 \\
\ldots \ldots \ldots\end{array}$ & $\begin{array}{l}+8.42 \\
+1.68\end{array}$ & $\begin{array}{l}8.00 \\
1.60\end{array}$ \\
\hline 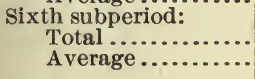 & $\begin{array}{l}63.59 \\
12.72\end{array}$ & $\begin{array}{l}6.60 \\
1.32\end{array}$ & $\begin{array}{l}55.75 \\
11.15\end{array}$ & $\begin{array}{l}62.35 \\
12.47\end{array}$ & $\begin{array}{r}10.38 \\
\cdots\end{array}$ & $\begin{array}{r}87.67 \\
\cdots \cdots\end{array}$ & $\begin{array}{r}98.05 \\
\cdots \cdots\end{array}$ & $\begin{array}{l}+1.24 \\
+\quad .25\end{array}$ & $\begin{array}{r}10.00 \\
2.00\end{array}$ \\
\hline $\begin{array}{l}\text { Entire preservative } \\
\text { period: } \\
\text { Total } \\
\text { Average................ }\end{array}$ & $\begin{array}{r}398.71 \\
13.29\end{array}$ & $\begin{array}{r}39.38 \\
1.31\end{array}$ & $\begin{array}{r}322.44 \\
10.75\end{array}$ & $\begin{array}{r}361.82 \\
12.06\end{array}$ & $\begin{array}{r}9.88 \\
\cdots \\
\end{array}$ & $\begin{array}{r}80.87 \\
\cdots\end{array}$ & $\begin{array}{r}90.75 \\
\ldots \ldots \\
\end{array}$ & $\begin{array}{l}+36.89 \\
+\quad 1.23\end{array}$ & $\begin{array}{r}30.85 \\
1.03\end{array}$ \\
\hline After period. & & & & & & & & & \\
\hline $\begin{array}{l}\text { First subperiod: } \\
\text { Total ............... } \\
\text { Average.......... } \\
\text { Second subperiod: }\end{array}$ & $\begin{array}{l}66.81 \\
13.36\end{array}$ & $\begin{array}{l}8.07 \\
1.61\end{array}$ & $\begin{array}{l}60.36 \\
12.07\end{array}$ & $\begin{array}{l}68.43 \\
13.69\end{array}$ & $\begin{array}{l}12.08 \\
\ldots \ldots\end{array}$ & 90.35 & $\begin{array}{l}102.42 \\
\cdots \ldots\end{array}$ & $\begin{array}{r}-1.62 \\
-\quad .33\end{array}$ & $\begin{array}{l}0 \\
0\end{array}$ \\
\hline 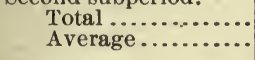 & $\begin{array}{l}68.38 \\
13.68\end{array}$ & $\begin{array}{l}7.52 \\
1.50\end{array}$ & $\begin{array}{l}59.18 \\
11.84\end{array}$ & $\begin{array}{l}66.70 \\
13.34\end{array}$ & $\begin{array}{l}11.00 \\
\cdots \cdots\end{array}$ & $\begin{array}{r}86.55 \\
\ldots . .\end{array}$ & $\begin{array}{r}97.54 \\
. .2 \ldots\end{array}$ & $\begin{array}{r}+1.68 \\
+\quad .34\end{array}$ & $\begin{array}{l}0 \\
0\end{array}$ \\
\hline $\begin{array}{l}\text { Entire after period: } \\
\text { Total ........... } \\
\text { Average ........ }\end{array}$ & $\begin{array}{r}135.19 \\
13.52\end{array}$ & $\begin{array}{r}15.59 \\
1.56\end{array}$ & $\begin{array}{r}119.54 \\
11.95\end{array}$ & $\begin{array}{r}135.13 \\
13.51\end{array}$ & $\begin{array}{c}11.53 \\
\ldots \ldots\end{array}$ & 88.42 & $\begin{array}{r}99.96 \\
\ldots \ldots\end{array}$ & $\begin{array}{l}+.06 \\
+.01\end{array}$ & $\begin{array}{l}0 \\
0\end{array}$ \\
\hline
\end{tabular}

$a$ Daily average added in order to complete record. 
TABLE XIII.-Nitrogen balances for Series VI-Continued.

[Averages are per day.]

No.9.

\begin{tabular}{|c|c|c|c|c|c|c|c|c|c|}
\hline Period. & In food. & In feces. & In urine. & $\begin{array}{c}4 \\
\text { In feces } \\
\text { and } \\
\text { urine. } \\
(2+3)\end{array}$ & $\begin{array}{c}5 \\
\text { In } \\
\text { feces. } \\
(2 \div 1)\end{array}$ & $\begin{array}{c}\mathbf{6} \\
\underset{(3 \div-1)}{\text { In }} \\
\left(3+1 e^{-}\right.\end{array}$ & $\begin{array}{c}7 \\
\text { In feces } \\
\text { and } \\
\text { urine. } \\
(4 \div 1)\end{array}$ & $\begin{array}{c}8 \\
\begin{array}{c}\text { Balance. } \\
(1-4)\end{array}\end{array}$ & $\begin{array}{c}9 \\
\text { Sali- } \\
\text { cylic } \\
\text { acid } \\
\text { admin. } \\
\text { istered. }\end{array}$ \\
\hline Fore period. & & & & & & & & & \\
\hline 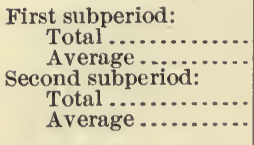 & $\begin{array}{r}\text { Grams. } \\
85.69 \\
17.14 \\
\\
88.92 \\
17.78\end{array}$ & $\begin{array}{r}\text { Grams. } \\
2.30 \\
.46\end{array}$ & $\begin{array}{r}\text { Grams. } \\
70.26 \\
14.05\end{array}$ & $\begin{array}{c}\text { Grams. } \\
72.56 \\
14.51\end{array}$ & $\begin{array}{r}\text { Per ct. } \\
2.68 \\
\times \\
4.99 \\
.\end{array}$ & $\begin{array}{r}\text { Per } \text { ct. } \\
81.99 \\
75.52 \\
\cdots\end{array}$ & $\begin{array}{r}\text { Per ct. } \\
84.68 \\
\cdots\end{array}$ & $\begin{array}{l}\text { Grams. } \\
+13.13 \\
+2.63 \\
+17.33 \\
+3.46 \\
\end{array}$ & $\begin{array}{c}\text { Grams. } \\
0 \\
0 \\
\\
0 \\
0\end{array}$ \\
\hline $\begin{array}{l}\text { Entire forc period: } \\
\text { Total ............... } \\
\text { Average ......... }\end{array}$ & $\begin{array}{r}174.61 \\
17.46 \\
\end{array}$ & $\begin{array}{r}6.74 \\
.67 \\
\end{array}$ & $\begin{array}{r}137.41 \\
13.74 \\
\end{array}$ & $\begin{array}{r}144.15 \\
14.42 \\
\end{array}$ & $\begin{array}{r}3.86 \\
\cdots \cdots\end{array}$ & $\begin{array}{r}78.70 \\
\cdots \cdots\end{array}$ & $\begin{array}{r}82.56 \\
\cdots \cdots \\
\end{array}$ & $\begin{array}{l}+30.46 \\
+3.04\end{array}$ & $\begin{array}{l}0 \\
0\end{array}$ \\
\hline Preservative period. & & & & & & & & & \\
\hline $\begin{array}{l}\text { First subperiod: } \\
\quad \text { Total ............... } \\
\text { Average .......... } \\
\text { Sccond subperiod: }\end{array}$ & $\begin{array}{l}86.60 \\
17.32\end{array}$ & $\begin{array}{l}6.99 \\
1.40\end{array}$ & $\begin{array}{l}72.12 \\
14.42\end{array}$ & $\begin{array}{l}79.11 \\
15.82\end{array}$ & $\begin{array}{c}8.07 \\
\ldots .\end{array}$ & $\begin{array}{r}83.28 \\
\cdots \cdots\end{array}$ & $\begin{array}{r}91.35 \\
\cdots \cdots\end{array}$ & $\begin{array}{l}+7.49 \\
+1.50\end{array}$ & $\begin{array}{r}1.05 \\
.21\end{array}$ \\
\hline $\begin{array}{l}\text { Total ....................... } \\
\text { Average } \\
\text { Third subperiod: }\end{array}$ & $\begin{array}{l}88.77 \\
17.75\end{array}$ & $\begin{array}{l}5.01 \\
1.00\end{array}$ & $\begin{array}{l}66.12 \\
13.22\end{array}$ & $\begin{array}{l}71.13 \\
14.23\end{array}$ & $\begin{array}{r}5.64 \\
\cdots \ldots .\end{array}$ & $\begin{array}{r}74.48 \\
\cdots \cdots\end{array}$ & 80.13 & $\begin{array}{l}+17.64 \\
+3.52\end{array}$ & $\begin{array}{r}2.10 \\
.42\end{array}$ \\
\hline Total ...................... & $\begin{array}{l}88.34 \\
17.67\end{array}$ & $\begin{array}{l}4.98 \\
1.00\end{array}$ & $\begin{array}{l}69.10 \\
13.82\end{array}$ & $\begin{array}{l}74.08 \\
14.82\end{array}$ & $\begin{array}{r}5.64 \\
. . . .\end{array}$ & $\begin{array}{r}78.22 \\
\ldots \ldots \ldots\end{array}$ & $\begin{array}{r}83.86 \\
\cdots \cdots\end{array}$ & $\begin{array}{l}+14.26 \\
+2.85\end{array}$ & $\begin{array}{r}3.70 \\
.74\end{array}$ \\
\hline $\begin{array}{l}\text { Fourth subperiod: } \\
\quad \text { Total } \ldots . . . . . . . . . . \\
\text { Average }\end{array}$ & $\begin{array}{l}88.92 \\
17.78\end{array}$ & $\begin{array}{r}3.90 \\
.78\end{array}$ & $\begin{array}{l}67.84 \\
13.57\end{array}$ & $\begin{array}{l}71.74 \\
14.35\end{array}$ & 4. 39 & 76.29 & 80.68 & $\begin{array}{r}+17.18 \\
+\quad 3.43\end{array}$ & 6.00 \\
\hline $\begin{array}{l}\text { Fifth subperiod: } \\
\text { Total } . . . \ldots \ldots \ldots\end{array}$ & & & & & & & & +3.43 & \\
\hline $\begin{array}{l}\text { Average.................. } \\
\text { Sixth subperiod: }\end{array}$ & $\begin{array}{l}90.94 \\
18.19\end{array}$ & $\begin{array}{l}6.42 \\
1.28\end{array}$ & $\begin{array}{l}63.35 \\
12.67\end{array}$ & $\begin{array}{l}69.77 \\
13.95\end{array}$ & $\begin{array}{r}7.06 \\
\ldots \ldots\end{array}$ & $\begin{array}{r}69.66 \\
\cdots \cdots\end{array}$ & $\begin{array}{r}76.72 \\
\cdots\end{array}$ & $\begin{array}{l}+21.17 \\
+4.24\end{array}$ & $\begin{array}{l}8.00 \\
1.60\end{array}$ \\
\hline Total ....................... & $\begin{array}{l}83.84 \\
16.77\end{array}$ & $\begin{array}{l}5.15 \\
1.03\end{array}$ & $\begin{array}{l}73.44 \\
14.69\end{array}$ & $\begin{array}{l}78.59 \\
15.72\end{array}$ & $\begin{array}{r}6.14 \\
\cdots \cdots\end{array}$ & $\begin{array}{r}87.60 \\
\cdots \cdots\end{array}$ & $\begin{array}{r}93.74 \\
\cdots \cdots\end{array}$ & $\begin{array}{l}+5.25 \\
+1.05\end{array}$ & $\begin{array}{r}10.00 \\
2.00\end{array}$ \\
\hline 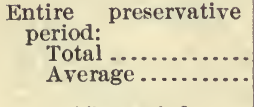 & $\begin{array}{r}527.41 \\
17.58 \\
\end{array}$ & $\begin{array}{r}32.45 \\
1.08 \\
\end{array}$ & $\begin{array}{r}411.97 \\
13.73\end{array}$ & $\begin{array}{r}444.42 \\
14.81 \\
\end{array}$ & $\begin{array}{r}6.15 \\
\cdots \cdots \\
\end{array}$ & $\begin{array}{r}78.11 \\
\cdots \\
\end{array}$ & $\begin{array}{r}84.26 \\
\cdots \cdots \\
\end{array}$ & $\begin{array}{r}+82.99 \\
+2.77 \\
\end{array}$ & $\begin{array}{r}30.85 \\
1.03 \\
\end{array}$ \\
\hline After period. & & & & & & & & & \\
\hline 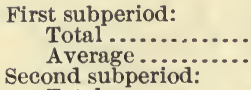 & $\begin{array}{l}89.71 \\
17.94\end{array}$ & $\begin{array}{r}2.63 \\
.53\end{array}$ & $\begin{array}{l}64.45 \\
12.89\end{array}$ & $\begin{array}{l}67.08 \\
13.42\end{array}$ & $\begin{array}{r}2.93 \\
\cdots . . .\end{array}$ & $\begin{array}{r}71.84 \\
\cdots \cdots \cdots\end{array}$ & $\begin{array}{r}74.77 \\
\cdots \ldots\end{array}$ & $\begin{array}{l}+22.63 \\
+4.52\end{array}$ & $\begin{array}{l}0 \\
0\end{array}$ \\
\hline $\begin{array}{l}\text { Total ....................... } \\
\text { Average ...... }\end{array}$ & $\begin{array}{l}88.39 \\
17.68\end{array}$ & $\begin{array}{l}5.80 \\
1.16\end{array}$ & $\begin{array}{l}70.20 \\
14.04\end{array}$ & $\begin{array}{l}76.00 \\
15.20\end{array}$ & $\begin{array}{r}6.56 \\
\cdots \cdots\end{array}$ & $\begin{array}{r}79.42 \\
\ldots \ldots\end{array}$ & $\begin{array}{r}85.98 \\
-\cdots\end{array}$ & $\begin{array}{r}+12.39 \\
+2.48\end{array}$ & $\begin{array}{l}0 \\
0\end{array}$ \\
\hline 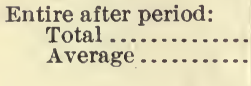 & $\begin{array}{r}178.10 \\
17.81\end{array}$ & $\begin{array}{r}8.43 \\
.84\end{array}$ & $\begin{array}{r}134.65 \\
13.47\end{array}$ & $\begin{array}{r}143.08 \\
14.31\end{array}$ & $\begin{array}{r}4.73 \\
\cdots\end{array}$ & $\begin{array}{r}75.60 \\
7.6\end{array}$ & $\begin{array}{r}80.34 \\
\ldots\end{array}$ & $\begin{array}{l}+35.02 \\
+3.50\end{array}$ & $\begin{array}{l}0 \\
0\end{array}$ \\
\hline
\end{tabular}


TABLE. XIII.-Nitrogen balances for Series VI-Continued.

[Averages are per day.]

No. 10.

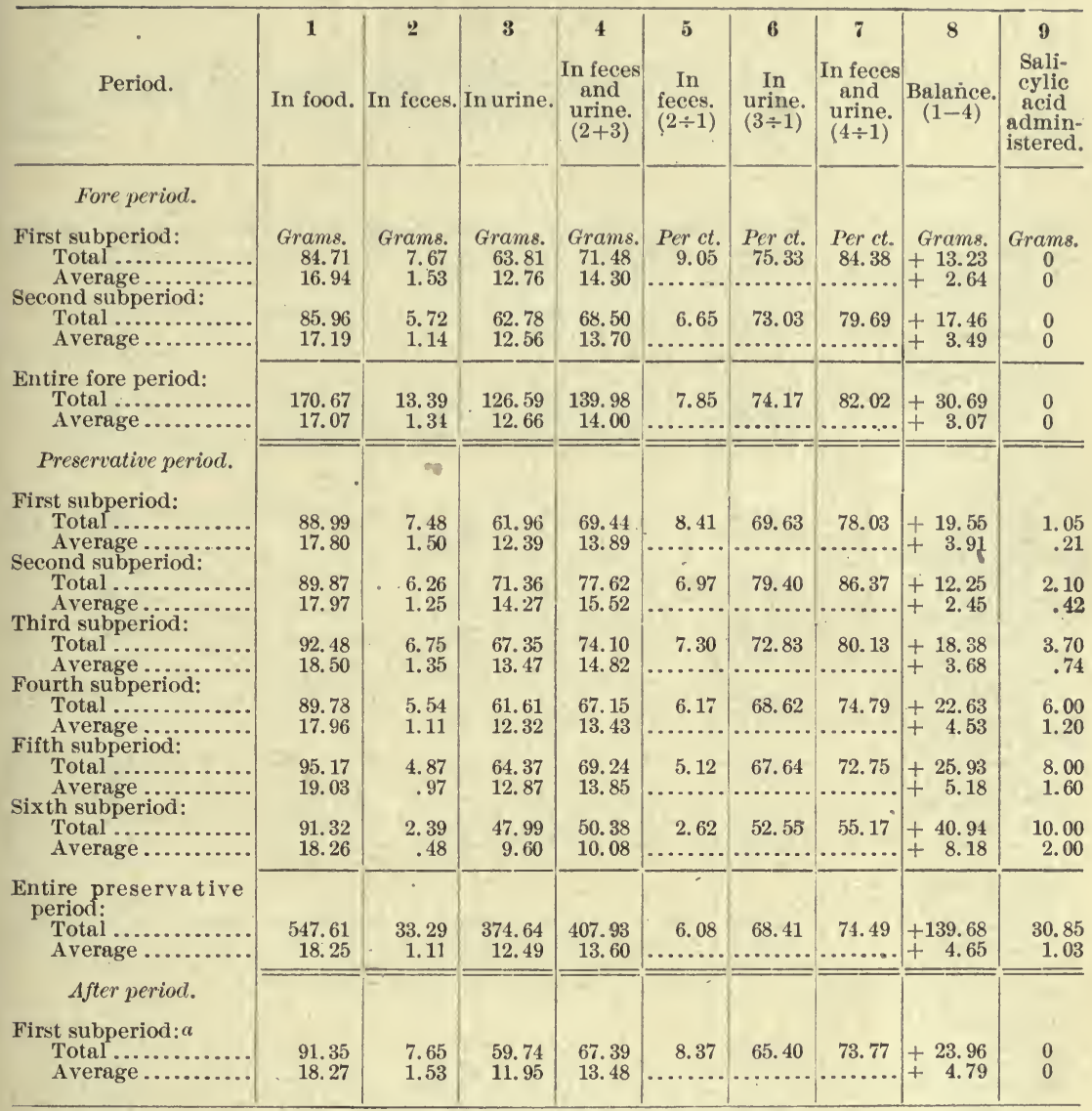

$a$ No second after subperiod; subject ill.

7656-No, 84, pt 2-06-9 
TABLE XIII.-Nitrogen balances for Series VI-Continued.

[Averages are per day.]

No. 11.

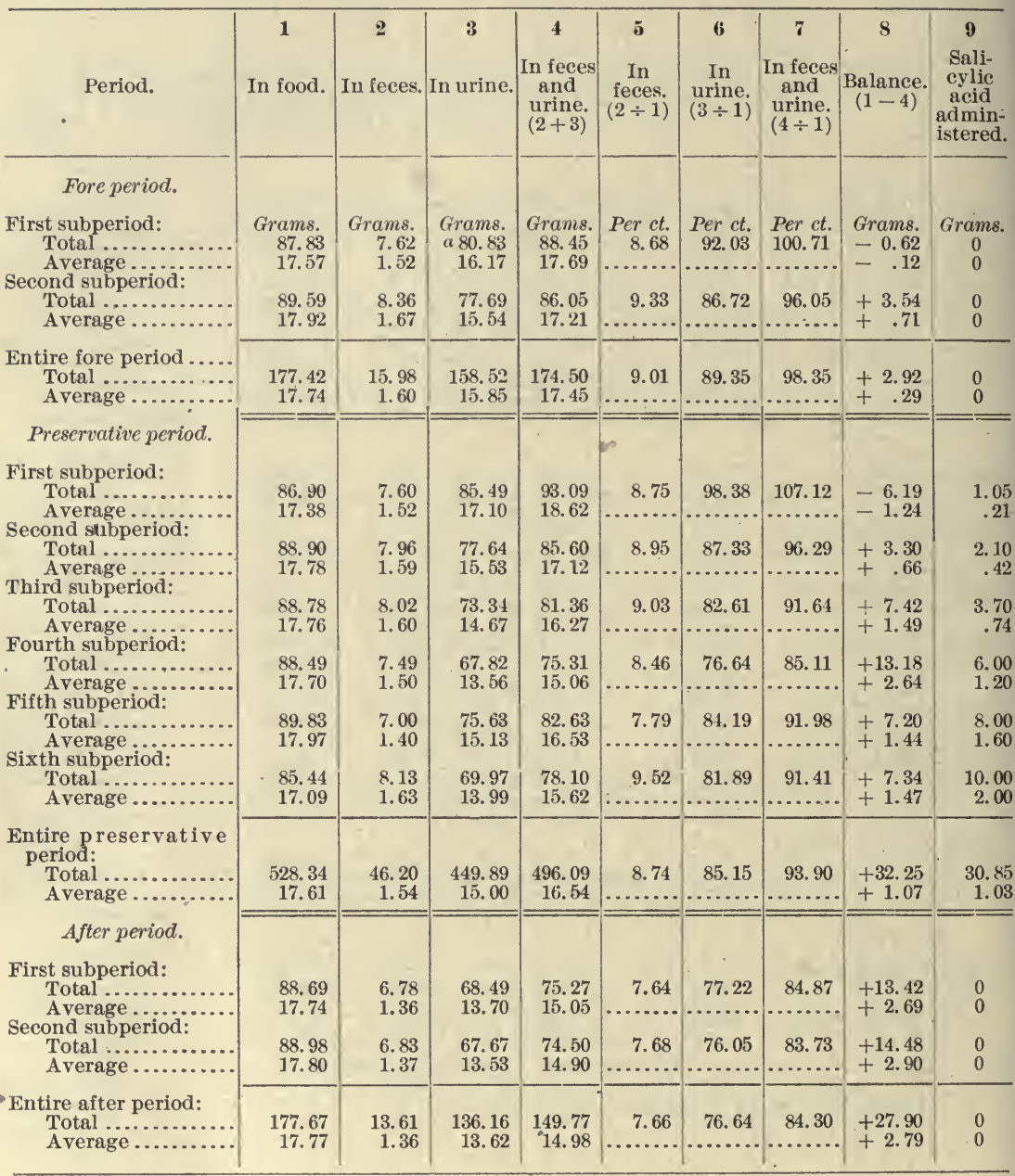

$a$ Daily average added in order to complete record. 
Table XIII.-Nitrogen balances for Series VI-Continued.

[Averages are per day.]

No. 12.

\begin{tabular}{|c|c|c|c|c|c|c|c|c|c|}
\hline Period. & In food. & In feces. & $\begin{array}{c}3 \\
\text { In urine. }\end{array}$ & $\begin{array}{c}4 \\
\text { In feces } \\
\text { and } \\
\text { urine. } \\
(2+3)\end{array}$ & $\begin{array}{c}5 \\
\text { In } \\
\text { feces. } \\
(2 \div 1)\end{array}$ & $\underset{(3 \div 1)}{\operatorname{In}}$ & $\begin{array}{c}7 \\
\text { In feces } \\
\text { and } \\
\text { urine. } \\
(4 \div 1)\end{array}$ & Balance. & \begin{tabular}{c}
\multicolumn{1}{c}{} \\
Sali- \\
cylic \\
acid \\
admin- \\
istered.
\end{tabular} \\
\hline Fore period. & & & & & & & & & \\
\hline $\begin{array}{l}\text { First subperiod: } \\
\text { Total............. } \\
\text { Average }\end{array}$ & $\begin{array}{r}\text { Grams. } \\
85.72 \\
17.14\end{array}$ & $\begin{array}{r}\text { Grams. } \\
6.44 \\
1.29\end{array}$ & $\begin{array}{r}\text { Grams. } \\
76.69 \\
15.34\end{array}$ & $\begin{array}{c}\text { Grams. } \\
83.13 \\
16.63\end{array}$ & $\begin{array}{r}\text { Per } \text { ct. } \\
7.51 \\
\ldots\end{array}$ & $\begin{array}{r}\text { Per ct. } \\
89.47 \\
\ldots\end{array}$ & $\begin{array}{r}\text { Per ct. } \\
96.98 \\
\end{array}$ & $\begin{array}{l}\text { Grams. } \\
+\quad 2.59 \\
+\quad .51\end{array}$ & $\begin{array}{c}\text { Grams. } \\
0 \\
0\end{array}$ \\
\hline 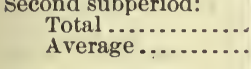 & $\begin{array}{l}89.46 \\
17.89\end{array}$ & $\begin{array}{l}8.01 \\
1.60\end{array}$ & $\begin{array}{l}72.02 \\
14.40\end{array}$ & $\begin{array}{l}80.03 \\
16.01\end{array}$ & 8.95 & 80.51 & $\begin{array}{r}89.46 \\
\ldots . . . .\end{array}$ & $\begin{array}{r}+9.43 \\
+1.88\end{array}$ & $\begin{array}{l}0 \\
0\end{array}$ \\
\hline $\begin{array}{l}\text { Entire fore period: } \\
\text { Total ............ } \\
\text { Average....... }\end{array}$ & $\begin{array}{r}175.18 \\
17.52\end{array}$ & $\begin{array}{r}14.45 \\
1.45\end{array}$ & $\begin{array}{r}148.71 \\
14.87\end{array}$ & $\begin{array}{r}163.16 \\
16.32\end{array}$ & $\begin{array}{r}8.25 \\
\ldots \ldots . . \\
\end{array}$ & $\begin{array}{l}84: 89 \\
\ldots .\end{array}$ & $\begin{array}{r}\bullet \\
93.14 \\
\ldots \ldots \ldots \\
\end{array}$ & $\begin{array}{r}+12.02 \\
+1.20\end{array}$ & $\begin{array}{l}0 \\
0\end{array}$ \\
\hline Preservative period. & & & & , & & & & & \\
\hline $\begin{array}{l}\text { First subperiod: } \\
\text { Total } \ldots . . . . . . \\
\text { Average } . . .\end{array}$ & $\begin{array}{l}84.87 \\
16.97\end{array}$ & $\begin{array}{r}4.04 \\
.81\end{array}$ & $\begin{array}{l}65.06 \\
13.01\end{array}$ & $\begin{array}{l}69.10 \\
13.82\end{array}$ & $\begin{array}{l}\text { 4. } 76 \\
\text {... }\end{array}$ & 76.66 & $\begin{array}{c}81.42 \\
\ldots \ldots . .\end{array}$ & $\begin{array}{l}+15.77 \\
+3.15\end{array}$ & $\begin{array}{r}1.05 \\
.21\end{array}$ \\
\hline $\begin{array}{l}\text { erond s } \\
\text { Tota }\end{array}$ & & 4.10 & & & 4.69 & 85.99 & 90.68 & & \\
\hline $\begin{array}{l}\text { Ave } \\
\text { Third s }\end{array}$ & & .82 & & & & (3) & ........ & +1.63 & $\begin{array}{r}2.10 \\
.42\end{array}$ \\
\hline Tot & & & & & 7. 76 & 87.30 & 95.06 & $\begin{array}{r}+4.23 \\
+\quad .84\end{array}$ & $\begin{array}{r}3.70 \\
.74\end{array}$ \\
\hline Fourth s & & & & & & & & & \\
\hline $\begin{array}{l}\text { Total } \\
\text { Aver }\end{array}$ & $\begin{array}{l}85.49 \\
17.10\end{array}$ & $\begin{array}{r}4.42 \\
.88\end{array}$ & $\begin{array}{l}70.01 \\
14.00\end{array}$ & $\begin{array}{l}74.43 \\
14.89\end{array}$ & 5.17 & $\begin{array}{l}81.89 \\
\ldots . . .\end{array}$ & $\begin{array}{r}87.06 \\
\ldots . . . .\end{array}$ & $\begin{array}{l}+11.06 \\
+2.21\end{array}$ & $\begin{array}{l}6.00 \\
1.20\end{array}$ \\
\hline $\begin{array}{l}\text { Tifth } \\
\text { Tc }\end{array}$ & & & & & & & & & \\
\hline $\begin{array}{l}\text { Tote } \\
\text { Ave }\end{array}$ & $\begin{array}{l}88.6 \\
17.7\end{array}$ & & & & 7.23 & $\begin{array}{r}84.02 \\
\ldots . . .\end{array}$ & 91.26 & $\begin{array}{l}+7.75 \\
+1.55\end{array}$ & $\begin{array}{l}8.00 \\
1.60\end{array}$ \\
\hline $\begin{array}{l}\text { Sixth su } \\
\text { Totg }\end{array}$ & & & & & & & & & \\
\hline Average ................ & & $\begin{array}{r}4.63 \\
.93\end{array}$ & & $\begin{array}{l}79.91 \\
15.98\end{array}$ & $\begin{array}{r}5.63 \\
\cdots \cdots\end{array}$ & $\begin{array}{r}91.50 \\
\cdots \cdots\end{array}$ & $\begin{array}{r}97.13 \\
\ldots \ldots \\
\ldots \ldots\end{array}$ & $\begin{array}{l}+2.36 \\
+\quad .47\end{array}$ & $\begin{array}{r}10.00 \\
2.00\end{array}$ \\
\hline $\begin{array}{l}\text { Entire preservative } \\
\text { period: } \\
\text { Total ................. } \\
\text { Average........... }\end{array}$ & $\begin{array}{r}514.30 \\
17.14\end{array}$ & $\begin{array}{r}30.24 \\
1.01 \\
\end{array}$ & $\begin{array}{r}434.74 \\
14.49\end{array}$ & $\begin{array}{r}464.98 \\
15.50 \\
\end{array}$ & $\begin{array}{r}5.88 \\
\ldots . . .\end{array}$ & $\begin{array}{r}84.53 \\
\ldots . . .\end{array}$ & $\begin{array}{r}90.41 \\
\ldots \ldots\end{array}$ & $\begin{array}{l}+49.32 \\
+1.64\end{array}$ & $\begin{array}{r}30.85 \\
1.03\end{array}$ \\
\hline After period. & & & & & & & & & \\
\hline $\begin{array}{l}\text { First subperiod: } \\
\text { Total .......... } \\
\text { Average ...... }\end{array}$ & $\begin{array}{l}85.76 \\
17.15\end{array}$ & $\begin{array}{l}5.45 \\
1.09\end{array}$ & & $\begin{array}{l}79.12 \\
15.82\end{array}$ & 6.35 & 85.90 & $\begin{array}{l}92.26 \\
\ldots \ldots \ldots\end{array}$ & $\begin{array}{r}+6.64 \\
+1.33\end{array}$ & $\begin{array}{l}0 \\
0\end{array}$ \\
\hline $\begin{array}{l}\text { econd subperiod: } \\
\text { Total ............... } \\
\text { Average........ }\end{array}$ & $\begin{array}{l}84.70 \\
16.94\end{array}$ & $\begin{array}{r}a 5.60 \\
1.12\end{array}$ & $\begin{array}{l}76.80 \\
15.36\end{array}$ & $\begin{array}{l}82.40 \\
16.48\end{array}$ & $\begin{array}{c}6.61 \\
\cdots . .\end{array}$ & $\begin{array}{r}90.67 \\
\ldots \ldots \ldots .\end{array}$ & $\begin{array}{r}97.28 \\
\ldots . . .\end{array}$ & $\begin{array}{l}+2.30 \\
+\quad .46\end{array}$ & $\begin{array}{l}0 \\
0\end{array}$ \\
\hline $\begin{array}{c}\text { Entire after period: } \\
\text { Total ............... } \\
\text { Average ........ }\end{array}$ & $\begin{array}{r}170.46 . \\
17.05\end{array}$ & $\begin{array}{r}11.05 \\
1.11\end{array}$ & $\begin{array}{r}150.47 \\
15.05\end{array}$ & $\begin{array}{r}161.52 \\
16.15\end{array}$ & 6.48 & 88.27 & $\begin{array}{r}94.76 \\
\ldots . \ldots .\end{array}$ & $\begin{array}{r}+8.94 \\
+\quad .90\end{array}$ & $\begin{array}{l}0 \\
0\end{array}$ \\
\hline
\end{tabular}

$a$ Daily average added in order to complete record. 
TABLE XIII.-Nitrogen balances for Series VI-Continued.

[Averages are per man per day.]

Summary for nine men (Nos. 3, 9, and 10 excluded).

\begin{tabular}{|c|c|c|c|c|c|c|c|c|c|}
\hline Period. & In food. & In feces. & In urine. & $\begin{array}{c}4 \\
\text { In feces } \\
\text { and } \\
\text { urine. } \\
(2+3)\end{array}$ & $\begin{array}{c}5 \\
\text { In } \\
\text { feces. } \\
(2 \div-1)\end{array}$ & $\begin{array}{c}6 \\
\text { In } \\
\text { urine. } \\
(3 \div 1)\end{array}$ & $\begin{array}{c}7 \\
\text { In feces } \\
\text { and } \\
\text { urine. } \\
(4 \div 1)\end{array}$ & $\begin{array}{c}\mathbf{8} \\
\begin{array}{c}\text { Balance } \\
(1-4)\end{array}\end{array}$ & $\begin{array}{c}9 \\
\text { Sali- } \\
\text { cylic } \\
\text { acidad- } \\
\text { minis- } \\
\text { tered. }\end{array}$ \\
\hline Fore period. & & & & & & & & & \\
\hline $\begin{array}{l}\text { First subperiod: } \\
\text { Total ........... } \\
\text { Average...... }\end{array}$ & $\begin{array}{r}\text { Grams. } \\
683.85 \\
15.20\end{array}$ & $\begin{array}{r}\text { Grams. } \\
56.16 \\
1.25\end{array}$ & $\begin{array}{r}\text { Grams. } \\
614.88 \\
13.66\end{array}$ & $\begin{array}{c}\text { Grams. } \\
671.04 \\
14.91\end{array}$ & $\begin{array}{l}\text { Perct. } \\
8.21\end{array}$ & $\begin{array}{l}\text { Per ct. } \\
89.91 \\
\end{array}$ & $\begin{array}{c}\text { Per ct. } \\
98.13 \\
\end{array}$ & $\begin{array}{r}\text { Grams. } \\
+\quad 12.81 \\
+\quad .29\end{array}$ & $\begin{array}{c}\text { Grams. } \\
0 \\
0\end{array}$ \\
\hline 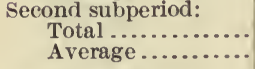 & $\begin{array}{r}707.60 \\
15.72\end{array}$ & $\begin{array}{r}59.73 \\
1.33\end{array}$ & $\begin{array}{r}600.08 \\
13.33\end{array}$ & $\begin{array}{r}659.81 \\
14.66\end{array}$ & $\begin{array}{r}8.44 \\
\ldots . . .\end{array}$ & $\begin{array}{l}84.79 \\
\cdots . . .\end{array}$ & $\begin{array}{r}93.10 \\
\ldots \ldots\end{array}$ & $\begin{array}{l}+47.79 \\
+\quad 1.06\end{array}$ & $\begin{array}{l}0 \\
0\end{array}$ \\
\hline 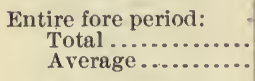 & $\begin{array}{r}1,391.45 \\
15.46\end{array}$ & $\begin{array}{r}115.89 \\
1.29\end{array}$ & $\begin{array}{r}1,214.96 \\
13.50\end{array}$ & $\begin{array}{r}1,330.85 \\
14.79\end{array}$ & $\begin{array}{r}8.33 \\
\cdots . . .\end{array}$ & $\begin{array}{r}87.32 \\
\cdots \cdots \\
\end{array}$ & $\begin{array}{r}95.64 \\
\cdots \cdots \cdots \\
\end{array}$ & $\begin{array}{r}+60.60 \\
+\quad .67 \\
\end{array}$ & $\begin{array}{l}0 \\
0\end{array}$ \\
\hline Preservative period. & & & , & & & & & & \\
\hline $\begin{array}{l}\text { First subperiod: } \\
\quad \text { Total ............... } \\
\text { Average } . . . \ldots \ldots \ldots\end{array}$ & $\begin{array}{r}677.58 \\
15.06\end{array}$ & $\begin{array}{r}52.20 \\
1.16\end{array}$ & $\begin{array}{r}596.76 \\
13.26\end{array}$ & $\begin{array}{r}648.96 \\
14.42\end{array}$ & 7.70 & 88.07 & $\begin{array}{r}95.78 \\
\ldots \ldots \ldots\end{array}$ & $\begin{array}{r}+28.62 \\
+\quad .64\end{array}$ & $\begin{array}{r}9.45 \\
.21\end{array}$ \\
\hline Second subperiod: & & & & & & & (6) & & \\
\hline Total ..................... & $\begin{array}{r}708.56 \\
15.74\end{array}$ & $\begin{array}{r}51.90 \\
1.15\end{array}$ & $\begin{array}{r}618.50 \\
13.74\end{array}$ & $\begin{array}{r}670.40 \\
14.89\end{array}$ & 7.32 & 87.29 & $\begin{array}{r}94.61 \\
\cdots \cdots\end{array}$ & $\begin{array}{l}+38.16 \\
+\quad .85\end{array}$ & $\begin{array}{r}18.90 \\
.42\end{array}$ \\
\hline $\begin{array}{l}\text { Third subperiod: } \\
\text { Total ........... } \\
\text { Average....... }\end{array}$ & $\begin{array}{r}693.31 \\
15.41\end{array}$ & $\begin{array}{r}55.16 \\
1.23\end{array}$ & $\begin{array}{r}604.30 \\
13.43\end{array}$ & $\begin{array}{r}659.46 \\
14.66\end{array}$ & 7.96 & 87.16 & 95.12 & $\begin{array}{l}+33.85 \\
+\quad .75\end{array}$ & $\begin{array}{r}33.30 \\
.74\end{array}$ \\
\hline Fourth subperiod: & f01 70 & & 10.40 & & & & & & \\
\hline $\begin{array}{l}\text { Total ...................... } \\
\text { Average }\end{array}$ & $\begin{array}{r}691.72 \\
15.37\end{array}$ & $\begin{array}{r}50.62 \\
1.13\end{array}$ & $\begin{array}{r}591.70 \\
13.15\end{array}$ & $\begin{array}{r}642.32 \\
14.27\end{array}$ & 7.32 & $\begin{array}{l}85.54 \\
\cdots\end{array}$ & $\begin{array}{r}92.86 \\
\ldots \ldots\end{array}$ & $\begin{array}{r}+\quad 49.40 \\
+\quad 1.10\end{array}$ & $\begin{array}{r}54.00 \\
1.20\end{array}$ \\
\hline $\begin{array}{r}\text { Fifth subper } \\
\text { Total ... }\end{array}$ & 717.41 & 50.44 & 627.32 & 677.76 & 7.03 & 87.45 & 94.48 & $\begin{array}{r}+39.65 \\
\end{array}$ & 72.00 \\
\hline Average & 15.94 & 1.12 & 13.94 & 15.06 & & & & +.88 & 1.60 \\
\hline $\begin{array}{l}\text { Sixth subperiod: } \\
\text { Total .................. } \\
\text { Average .......... }\end{array}$ & $\begin{array}{r}663.67 \\
14.75\end{array}$ & $\begin{array}{r}51.09 \\
1.14\end{array}$ & $\begin{array}{r}610.04 \\
13.56\end{array}$ & $\begin{array}{r}661.13 \\
14.69\end{array}$ & 7.70 & $\begin{array}{r}91.92 \\
\cdots\end{array}$ & $\begin{array}{r}99.62 \\
-. .\end{array}$ & $\begin{array}{r}+\quad 2.54 \\
+\quad .06\end{array}$ & $\begin{array}{r}88.00 \\
1.96\end{array}$ \\
\hline $\begin{array}{l}\text { Entire preservative } \\
\text { period: } \\
\text { Total ................ } \\
\text { Average ........... }\end{array}$ & $\begin{array}{r}4,152.25 \\
15.37\end{array}$ & $\begin{array}{r}311.41 \\
1.15\end{array}$ & $\begin{array}{r}3,648.62 \\
13.51\end{array}$ & $\begin{array}{c}3,960.03 \\
14.66\end{array}$ & $\begin{array}{r}7.50 \\
\cdots \cdots\end{array}$ & $\begin{array}{r}87.89 \\
\ldots \ldots \\
\end{array}$ & $\begin{array}{c}95.39 \\
\cdots \cdots\end{array}$ & $\begin{array}{r}+192.22 \\
+\quad .71 \\
\end{array}$ & $\begin{array}{r}275.65 \\
1.02\end{array}$ \\
\hline After period. & & & & & & & & & \\
\hline $\begin{array}{l}\text { First subperiod: } \\
\quad \text { Total .............. } \\
\text { Average .......... }\end{array}$ & $\begin{array}{r}695.17 \\
15.44\end{array}$ & $\begin{array}{r}55.22 \\
1.23\end{array}$ & $\begin{array}{r}610.31 \\
13.56\end{array}$ & $\begin{array}{r}665.53 \\
14.79\end{array}$ & 7.94 & 87.79 & 95.74 & $\begin{array}{r}+29.64 \\
+\quad .65\end{array}$ & $\begin{array}{l}0 \\
0\end{array}$ \\
\hline $\begin{array}{l}\text { Second subperiod: } \\
\text { Total ................ } \\
\text { Average........ }\end{array}$ & $\begin{array}{r}696.16 \\
15.47\end{array}$ & $\begin{array}{r}53.67 \\
1.19\end{array}$ & $\begin{array}{r}6.0 .58 \\
13.57\end{array}$ & $\begin{array}{r}664.25 \\
14.76\end{array}$ & $\begin{array}{c}7.71 \\
\cdots . .\end{array}$ & $\begin{array}{r}87.71 \\
\cdots \cdots\end{array}$ & $\begin{array}{r}95.42 \\
\cdots \ldots .\end{array}$ & $\begin{array}{r}+31.91 \\
+\quad .71\end{array}$ & 0 \\
\hline $\begin{array}{l}\text { Entire after period: } \\
\quad \text { Total ................. } \\
\text { Average .......... }\end{array}$ & $\begin{array}{r}1,391.33 \\
15.46\end{array}$ & $\begin{array}{r}108.89 \\
1.21\end{array}$ & $\begin{array}{r}1,220.89 \\
13.56\end{array}$ & $\begin{array}{c}1,329.78 \\
14.77\end{array}$ & 7.83 & 87.75 & $\begin{array}{r}95.58 \\
\ldots \ldots \ldots\end{array}$ & $\begin{array}{r}+61.55 \\
+\quad .69\end{array}$ & $\begin{array}{l}0 \\
0\end{array}$ \\
\hline
\end{tabular}

PHOSPHORIC ACID BALANCE.

INDIVIDUAL DATA.

No. 1.

The total quantity of phosphoric acid administered in the food of No. 1 during the fore period is 37.037 grams. The average daily quantity consumed is 3.704 grams. The quantities of phosphoric acid in the two subperiods are not greatly different, amounting to an average of 3.809 and 3.598 grams, respectively. Of the total quantity administered in the food 11.424 grams, equivalent to 1.142 grams per 
day, are found in the feces, and 21.686 grams, equivalent to 2.169 grams per day, -in the urine. 'The percentage of phosphoric acid excreted in the feces is 30.85 and in the urine 58.55. The phosphoric acid balance is positive, amounting to 0.393 gram per day.

The total quantity of phosphoric acid exhibited in the food during the preservative period is 115.833 grams, equivalent to 3.861 grams per day. The quantities in the various subperiods are quite constant, as will be seen by inspection of the table. Of the total quantity exhibited 30.921 grams are excreted in the feces, equivalent to 1.031 grams per day, and 70.508 grams in the urine, equivalent to 2.35 grams per day. Of the phosphoric acid exhibited in the food 26.69 per cent is excreted in the feces and 60.87 per cent in the urine. The phosphoric acid balance remains positive, and amounts to 0.48 gram per day. During the after period the quantity of phosphoric acid exhibited in the food is 37.551 grams, equivalent to 3.755 grams per day. Of this amount there are excreted in the feces 18.39 grams, equivalent to 1.839 grams per day, ${ }^{a}$ and in the urine 21.342 grams, equivalent to 2.134 grams per day. Of the total phosphoric acid in the food 48.97 per cent is excreted in the feces and 56.83 per cent in the urine. This produces a negative phosphoric acid balance for the after period, amounting to -0.218 gram per day.

The data in the case of No. 1 show a slight increase in the magnitude of the balance during the preservative period and a marked change, resulting in a negative balance, in the after period. This remarkable increase in the excretion of phosporic acid in the after period is entirely nonmetabolized phosphoric acid, the percentage excreted being almost twice as great as in the preservative period, while the quantity of metabolized phosphoric acid excreted is slightly decreased in the after period. In this instance, therefore, it appears that the withdrawal of the salicylic acid caused a very decided interruption of the digestion and absorption of the phosphoric acid in the intestinal canal.

No. 2.

The total quantity of phosphoric acid consumed by No. 2 during the fore period is 43.005 grams, equivalent to 4.30 grams per day. Of this quantity 14.101 grams appear in the feces, equivalent to 1.41 grams per day, and 30.292 grams appear in the urine, equivalent to 3.029 grams per day. The percentage of phosphoric acid eliminated in the feces is 32.79 and in the urine 70.44 , causing a negative balance the magnitude of which is -0.139 gram per day. During the fore period the quantity of phosphoric acid excreted was slightly greater than that consumed.

a The weight of the dry feces is 52 grams in the after period, while in the fore period it is 41 grams and in the preservative period 35 grams. The increase in phosphoric acid excreted thus appears to be due to the increased fecal excretion. 
During the preservative period the total quantity of phosphoric acid administered to No. 2 is 132.427 grams, equivalent to 4.414 grams per day. Of this amount 37.553 grams appear in the feces, equivalent to 1.252 grams per day, and 91.662 grams appear in the urine, equivalent to 3.055 grams per day. The percentage of phosphoric acid eliminated in the feces is 28.36 and in the urine 69.22. This shows a slightly positive phosphoric acid balance, the magnitude of which is 0.107 gram.

During the after period 43.577 grams of phosphoric acid were consumed by No. 2, equivalent to 4.358 grams per day. Of this quantity 12.264 grams appear in the feces, equivalent to 1.226 grams per day, and 29.061 grams in the urine, equivalent to 2.906 grams per day. The percentage of phosphoric acid excreted in the feces is 28.14 and in the urine 66.69. This indicates a positive phosphoric-acid balance of an average daily magnitude of 0.225 grams. In this case we have practically a reversal of the conditions which obtained in the case of No. 1. The excess of phosphoric acid which was excreted during the fore period disappeared and the total amount excreted decreased considerably, both in the preservative and after periods.

Considering the nonmetabolized phosphoric acid, it is seen that the amount excreted during the preservative period decreased very decidedly (4.43 per cent) and during the after period very slightly (0.22 per cent), while in the case of the metabolized phosphoric acid the reverse is true; that is, the smaller decrease took place in the preservative period (1.22 per cent) and a very decided decrease in the after period (2.53 per cent). This results, as already stated, in a marked decrease in the total quantity of phosphoric acid excreted during the preservative period, although the amount given in the food was slightly greater in the preservative period than in the fore period. In this instance the exhibition of the salicylic acid seems to exert a marked inhibitive influence upon the excretion of phosphoric acid (5.66 per cent) and this effect continues after the withdrawal of the preservative, the after period showing a decrease as compared with the preservative period of 2.74 per cent.

No. 3.

The average daily quantity of phosphoric acid in the food of No. 3 during the fore period (only five days) is 3.773 grams, of which 0.746 gram was excreted in the feces and 2.14 grams in the urine. The percentage of phosphoric acid excreted in the feces is 19.79 and in the urine 56.73. The average daily quantity of phosphoric acid in the food for the preservative period (five subperiods) is 3.827 grams, of which 0.783 gram appeared in the feces and 2.038 grams in the urine. The percentage appearing in the feces is 20.47 and in the urine 53.26. The balance is strongly positive, amounting to 1.006 grams per day in magnitude. During the after period the average daily quantity of 
phosphoric acid in the food for the second subperiod is 3.834 grams, of which 0.906 gram appears in the feces and 1.993 grams in the urine, equivalent to 23.62 per cent in the feces and 51.99 per cent in the urine. The balance is again strongly positive, amounting to 0.935 gram daily.

These large balances are explained by reason of the fact that No. 3 commenced the observations after a period of illness and constantly gained weight during the progress of the experiment. The phosphoric acid therefore was doubtless utilized to some extent in building the growing tissues. The after period was again interrupted by sickness due to a severe cold.

\section{No.4.}

The quantity of phosphoric acid exhibited in the food of No. 4 during the fore period is 4.133 grams daily, of which 1.068 grams appear in the feces and 2.729 grams in the urine; 25.84 per cent of the total phosphoric acid ingested in the food appears in the feces and 66.03 per cent in the urine. The balance is positive, and its daily magnitude is 0.336 gram. During the preservative period the quantity of phosphoric acid in the food is greater, namely, 4.285 grams per day, of which 1.079 grams appeared in the feces and 2.83 grams in the urine, equivalent to 25.17 and 66.03 per cent, respectively. The balance is positive and amounts to 0.377 gram per day. In the after period No. 4 consumed in his food 4.202 grams of phosphoric acid daily, of which 1.07 grams appeared in the feces and 2.7 grams $^{-}$in the urine, equivalent to 25.47 and 64.25 per cent, respectively. The balance is again positive and is slightly greater than in the two previous periods, namely, 0.432 gram per day.

An inspection of these data shows that the exhibition of the preservative had scarcely any influence upon the excretions of phosphoric acid. What influence was exerted was of an inhibitory character, the quantity of phosphoric acid excreted being slightly less in the preservative period than in the fore period, which tendency became somewhat more marked during the after period.

No. 5 .

The summary of the data for No. 5 shows that during the preservative period the quantity of phosphoric acid ingested in the food daily is 4.177 grams. Of this quantity there appeared in the feces 1.433 grams and in the urine 2.479 grams, equivalent to 34.30 and 59.34 per. cent, respectively. The balance is positive, and its daily average magnitude is 0.266 gram. During the preservative period the quantity of phosphoric acid consumed in the food is slightly greater than in the fore period, averaging $4.326 \mathrm{grams}$ daily. Of this quantity 1.216 grams appear in the feces and 2.62 .3 grams in the urine, equivalent to 
28.11 and 60.63 per cent, respectively. The balance is positive and of an average daily magnitude of $0.488 \mathrm{gram}$. During the after period No. 5 consumed in his food 4.225 grams of phosphoric acid, of which 1.156 grams appear in the feces and 2.462 grams in the urine, equivalent to 27.35 and 58.28 per cent, respectively. The balance is positive and has attained during the after period an average daily magnitude of 0.607 .

A comparison of the average data by periods shows the effect of the preservative in increasing the digestion and the absorption of phosphoric acid from the intestinal canal and in producing a very slight additional excretion of metabolized phosphoric acid. This effect is continued in the after period in so far as the continued absorption of phosphoric acid from the intestinal canal is concerned, but the metabolized phosphoric acid is diminished, thus considerably increasing the daily balance.

No. 6 .

The total quantity of phosphoric acid contained in the food consumed by No. 6 during the fore period represents an average daily quantity of 3.599 grams. Of this amount 1.316 grams appear in the feces and 1.982 grams in the urine, corresponding to 36.56 and 55.09 per cent, respectively. The balance is positive and has an average daily magnitude of 0.301 gram. During the preservative period the average quantity of phosphoric acid exhibited in the food daily is 3.808 grams. The quantity appearing in the feces is 1.334 grams and in the urine 2.252 grams, equivalent to 35.03 and 59.14 per cent, respectively. The balance is still positive, though the daily magnitude thereof is not so great as during the fore period, having been reduced to 0.222 gram. In the after period the average quantity of phosphoric acid consumed daily by No. 6 is 3.713 grams, of which 1.422 grams appear in the feces and 1.989 grams in the urine, corresponding to 38.30 and 53.56 per cent, respectively. The balance is again positive and is almost exactly the same in magnitude as in the fore period, namely, $0.302 \mathrm{gram}$ daily. There is a tendency shown by these data on the part of the preservative to increase the quantity of metabolized phosphoric acid excreted and at the'sarne time to diminish the quantity of the nonmetabolized phosphoric acid. By reason of the greater excretion of phosphoric acid in the urine during the preservative period the total magnitude of the positive balance is reduced by about one-third.

Upon the withdrawal of the preservative the percentage of nonmetabolized phosphoric acid excreted increases 3.27 per cent, exceeding that of the fore period, while the quantity of metabolized phosphoric acid falls 5.58 per cent, reaching a figure considerably below that of the fore period. The data therefore in this case indicate that the pres- 
ence of the salicylic acid tends to increase the digestion and the absorption of the phosphoric acid from the intestinal canal and to increase the quantity of phosphoric acid excreted by the kidneys.

No. 7.

In the case of No. 7 the quantity of phosphoric acid ingested in the food during the fore period averages daily 2.899 grams. Of this quantity $0.771 \mathrm{gram}$ appears in the feces and $1.703 \mathrm{grams}$ in the urine, corresponding to 26.60 and 58.74 per cent, respectively, of the total phosphoric acid in the food. The balance is positive and amounts to 0.425 gram per day. During the preservative period the quantity of phosphoric acid in the food is slightly increased, amounting to a daily average of 2.962 grams. Of this quantity $0.636 \mathrm{gram}$ appears in the feces and 1.456 grams in the urine, corresponding to 21.48 and 49.14 per cent, respectively, of the total phosphoric acid in the food. The phosphoric acid balance is thus phenomenally large, amounting to 0.87 gram per day.

During the after period No. 7 consumed a smaller quantity of phosphoric acid than during either the preservative or fore period, namely, 2.763 grams per day. Of this quantity 0.966 gram appears in the feces and 1.24 grams in the urine, corresponding to 34.95 and 44.97 per cent, respectively, of the total quantity of phosphoric acid consumed. The balance is again very large, though smaller than that of the preservative period, amounting to 0.557 gram per'day. Thus it is seen that the quantity of phosphoric acid excreted in the feces greatly increased in the after period and the quantity in the urine showed a marked decrease.

In the case of No. 7 we again see a tendency on the part of the salicylic acid to increase the digestion of phosphoric acid and its absorption from the intestinal canal. In this case, however, there is no evidence of any increased katabolio activity, in fact the quantity of phosphoric acid excreted in the urine is diminished. The balances are larger than would be expected in a case of this kind, but careful revision of the data for No. 7 failed to reveal any cause of suspicion that the excreta had not been properly collected and examined.

No. 8.

The quantity of phosphoric acid in the food of No. 8 amounts to 2.728 grams daily, of which 0.972 gram appears in the feces and 1.756 grams in the urine, representing 35.63 and 64.36 per cent, respectively, of the total quantity of the phosphoric acid in the food. The balance is 0 , as all except one one-hundredth of 1 per cent of the phosphoric acid is accounted for. During the preservative period the quantity of phosphoric acid is slightly increased, amounting to 2.962 grams 
daily, of which 0.942 gram appears in the feces and 1.744 grams in the urine, equivalent to 31.81 and 58.87 per cent, respectively, of the total quantity of phosphoric acid ingested. The balance now becomes positive and its magnitude is equivalent to $0.276 \mathrm{gram}$ of phosphoric acid per day. During the after period the quantity of phosphoric acid consumed daily by No. 8 is 2.9 grams, of which 1.131 grams appear in the feces and 1.533 grams in the urine, corresponding to 39 and 52.84 per cent, respectively, of the total quantity of phosphoric acid in the food. The balance is again positive, amounting to $0.236 \mathrm{gram}$ per day. These data again show a tendency on the part of the preservative to increase the absorption of the phosphoric acid from the alimentary canal, but there is no evidence of any increased katabolic activity given by the urinalysis. On the contrary, the quantity so excreted is slightly smaller in amount and considerably smaller in percentage than during the fore period.

In the after period the quantity of nonmetabolized phosphoric acid excreted becomes considerably greater, but the quantity of metabolized phosphoric acid is decidedly less and the balance is slightly decreased.

\section{No. 9.}

The daily quantity of phosphoric acid consumed in the food by No. 9 during the fore period amounts to 4.832 grams, of which 1.014 grams appear in the feces and 2.227 grams in the urine. These data correspond to 20.99 and 46.08 per cent, respectively, of the total phosphoric acid in the food. The balance for the fore period is positive, amounting to 1.591 grams per day.

During the preservative period the quantity of phosphoric acid daily consumed is increased, amounting to 5.012 grams per day, of which 1.635 grams oceur in the feces and 2.457 grams in the urine. These data correspond to 32.62 and 49.01 per cent, respectively, of the amount of phosphoric acid in the food. The balance for the preservative period is again positive, though not so great in amount as that of the fore period, amounting to only 0.920 gram per day.

During the after period No. 9 consumed 5.111 grams of phosphoric acid daily, of which 1.429 grams appear in the feces and 2.295 grams in the urine, corresponding to 27.96 and 44.91 per cent, respectively, of the phosphoric acid in the food. The balance is again strongly positive, amounting to 1.387 grams per day.

According to these data the amount of both metabolized and nonmetabolized phosphoric acid eliminated by this subject during the preservative period was greater than in the fore period. The increase of the nonmetabolized phosphoric acid is especially marked. During the after period the amount of metabolized phosphoric acid eliminated is not greatly different from that of the fore period, although it is relatively less considering the fact that the amount of phosphoric acid 
ingested is greater in the after period than in the fore period. The elimination of nonmetabolized phosphoric acid in the after period was distinctly greater than in the fore period, but not so great as in the preservative period.

For reasons given elsewhere (p. 587) the results obtained with this subject are believed to be untrustworthy and are not included in the summaries.

\section{No. 10.}

The quantity of phosphoric acid consumed in the food by No. 10 during the fore period amounts to 4.324 grams daily, of which 1.087 grams appear in the feces and 2.233 grams in the urine. These data correspond to 25.15 and 51.64 per cent, respectively, of the total phosphoric acid in the food. The balance during the fore period was strongly positive, amounting to 1.044 grams per day.

During the preservative period the quantity of phosphoric acid consumed is somewhat greater than in the fore period, amounting to 4.584 grams per day, of which 0.907 gram appears in the feces and 2.244 grams in the urine. This is equivalent to 19.79 and 48.95 per cent, respectively, of the total amount of phosphoric acid in the food. The balance, therefore, is even more strongly positive than in the fore period, amounting to 1.433 grams per day.

Owing to illness, No. 10 was not continued in the experiment during the after period. For reasons given elsewhere (p. 587) this subject is omitted from the summaries. The results are given here, however, as a matter of record.

\section{No. 11}

The quantity of phosphoric acid consumed in the food by No. 11 during the fore period amounts to 4.373 grams per day, of which 1.568 grams appear in the feces and 2.863 grams in the urine. These data correspond to 35.85 and 65.46 per cent, respectively, of the total phosphoric acid in the food. It is seen that this causes a negative balance of small magnitude, equivalent to -0.057 gram per day. During the fore period, therefore, No. 11 was excreting a slightly greater quantity of phosphoric acid than he was ingesting. During the preservative period the quantity of phosphoric acid ingested is slightly increased, amounting to 4.573 grams per day, of which 1.541 grams appear in the feces and 2.481 grams in the urine, corresponding to 33.69 and 54.25 per cent, respectively, of the total phosphoric acid in the food. These data indicate a strongly positive balance the magnitude of which is equivalent to $0.551 \mathrm{gram}$ per day. During the after period No. 11 consumed 4.572 grams of phosphoric acid daily, of which 1.697 appear in the feces and 2.209 grams in the urine, corresponding to 37.12 ard 48.31 per cent, respectively, of the total quantity of phosphoric acid contained in the food. This indicates a still 
more strongly positive balance, the magnitude of which is $0.666 \mathrm{gram}$ per day. Again, we see in this instance a tendency on the part of the preservative to increase the absorption of the phosphoric acid from the alimentary canal. The quantity of phosphoric acid metabolized which was excreted through the kidneys is, however, very greatly reduced, the percentage falling from 65.46 in the fore period to 54.25 in the preservative period. It is this great reduction in the metabolized phosphoric acid which has caused the balance to be so large. Again, in the after period the marked diminution of the metabolic activity as manifested through the kidneys has caused the balance to become even more strongly positive than during the preservative period, amounting to 0.666 gram per day, although the excretion of nonmetabolized phosphoric acid increased.

No. 12 .

No. 12 consumed in his food during the fore period 4.434 grams per day, of which 1.549 grams appear in the feces and 2.569 grams in the urine. These data correspond to 34.93 and 57.93 per cent, respectively, of the total quantity of phosphoric acid in the food. During the preservative period No. 12 consumed 4.517 grams of phosphoric acid, of which 1.279 grams appear in the feces and 2.576 grams in the urine, corresponding to 28.31 and 57.05 per cent, respectively, of the total phosphoric acid contained in the food. This causes a very large positive balance, the magnitude of which is 0.662 gram daily. In the after period No. 12 consumes 4.366 grams of phosphoric acid per day, of which 1.507 grams appear in the feces and 2.456 grams in the urine, corresponding to 34.51 and 56.27 per cent, respectively, of the total quantity of phosphoric acid in the food. The balance is positive and amounts to 0.403 gram per day.

Again, we see in this case a tendency on the part of the preservative to increase the absorption of phosphoric acid from the intestinal canal. There is a very slight increase during the preservative period in the quantity of phosphoric acid excreted by the kidneys, but a decrease in the percentage amount and a decided decrease in the nonmetabolized phosphoric acid excreted. During the after period there is an increase in the nonmetabolized phosphoric acid excreted in the feces and a slight falling off of the quantity of phosphoric acid excreted in the urine. In this case the large positive balance of the preservative period is due chiefly to the diminution of the quantity of nonmetabolized phosphoric acid excreted in the feces.

SUMMARY.

In the general summary of Table XV (p. 626), the figures for Nos. 3,9 , and 10 are omitted for the reasons already given (pp. 585, 587), although presented in detail among the individual data. The follow- 
ing summary of the results obtained by periods for the nine men is repeated for convenience in the discussion:

Table XIV.-Phosphoric acid summary, by periods, for nine men, Series VI.

\begin{tabular}{|c|c|c|c|c|c|c|}
\hline Period. & $\begin{array}{l}\text { Phosphoric } \\
\text { acid in } \\
\text { food. }\end{array}$ & $\begin{array}{l}\text { Phosphoric } \\
\text { acid in } \\
\text { feces. }\end{array}$ & $\begin{array}{l}\text { Phosphoric } \\
\text { acid in } \\
\text { urine. }\end{array}$ & $\begin{array}{l}\text { Phosphoric } \\
\text { acid in } \\
\text { feces. }\end{array}$ & $\begin{array}{l}\text { Phosphoric } \\
\text { acid in } \\
\text { urine. }\end{array}$ & Balance. \\
\hline 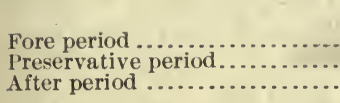 & $\begin{array}{r}\text { Grams. } \\
3.816 \\
3.967 \\
3.873\end{array}$ & $\begin{array}{r}\text { Grams. } \\
1.247 \\
1.145 \\
1.335\end{array}$ & $\begin{array}{r}\text { Grams. } \\
2.364 \\
2.374 \\
2.181\end{array}$ & $\begin{array}{r}\text { Per cent. } \\
32.69 \\
28.87 \\
34.47\end{array}$ & $\begin{array}{r}\text { Per cent. } \\
61.95 \\
59.81 \\
56.32\end{array}$ & $\begin{array}{l}\text { Grams. } \\
+0.205 \\
+0.448 \\
+0.357\end{array}$ \\
\hline
\end{tabular}

It is seen that the average quantities of phosphoric acid ingested daily in the different periods varied but little. There is, however, a slightly larger amount found in the food during the preservative period than in the fore period, while the food of the after period contains an intermediate amount.

The quantity of phosphoric acid contained in the feces is decidedly smaller during the preservative period than in either of the other periods, amounting to 1.145 grams daily as compared with 1.247 grams in the fore period and 1.335 grams in the after period. These average figures, agreeing as they do with the individual figures in every case, show a very distinct effect of the salicylic acid in increasing the absorption of the phosphcric acid from the alimentary canal into the circulation. In respect of the phosphoric acid in the urine it is seen that the amounts during the fore and preservative periods are almost exactly the same, being 2.364 and 2.374 , respectively. In the after period, however, there is a very decided indication that upon the withdrawal of the salicylic acid after the preservative period of six weeks the quantity of phosphoric acid in the feces was increased to a marked degree, while the amount in the urine was diminished. The relative percentages of phosphoric acid excreted in the feces and urine show these relations in a still more pronounced manner.

It thus appears that the effect of the salicylic acid is first to increase the absorption of the phosphoric acid from the intestinal canal, but upon the withdrawal of the preservative much less of the phosphoric acid is absorbed than before the exhibition of the drug. Considering the after period, therefore, the evident conclusion is that the final effect of the salicylic acid has been to diminish the metabolization of the phosphoric acid, and the same conclusion is reached from a study of the percentage data for the amounts excreted in the urine, which decrease steadily from 61.95 per cent in the fore period to 59.84 in the preservative period and 56.32 per cent in the after period.

The most prominent fact brought out by the summary is that during the administration of this preservative the feces are more free from phosphoric acid than during the fore and after periods and there is practically no increase in the amount excreted in the urine, while the 
percentage amount decreases, and in the after period both amount and percentage decrease. There is therefore a well-developed tendency to increase the store of phosphoric acid in the body, as is shown by the balances, which increase in the preservative period from 0.205 gram to 0.448 gram and decrease again in the after period to 0.357 gram. It is fair to presume, therefore, that these results are caused by the action of the preservative. In other words, salicylic acid creates a tendency toward the accumulation of phosphoric acid in the body.

TABLE XV.-Phosphoric acid balances for Series VI.

[Averages are per day.]

No. 1 .

\begin{tabular}{|c|c|c|c|c|c|c|c|c|c|}
\hline Period. & In food. & In feces. & In urine. & $\begin{array}{c}4 \\
\text { In feces } \\
\text { and } \\
\text { urine. } \\
(2+3)\end{array}$ & $\begin{array}{c}\overline{5} \\
\text { In } \\
\text { feces } \\
(2 \div 1)\end{array}$ & $\begin{array}{c}6 \\
\underset{\text { In }}{\text { Inine. }} \\
(3 \div 1)\end{array}$ & $\begin{array}{c}7 \\
\text { In feces } \\
\text { and } \\
\text { urine. } \\
(4 \div 1)\end{array}$ & $\begin{array}{c}\text { Balance. } \\
(1-4)\end{array}$ & $\begin{array}{c}9 \\
\text { Sali- } \\
\text { cylic } \\
\text { acid } \\
\text { admin- } \\
\text { istered. }\end{array}$ \\
\hline \multicolumn{10}{|l|}{ Fore period. } \\
\hline $\begin{array}{l}\text { First subperiod: } \\
\quad \text { Total } \ldots \ldots \ldots \ldots \\
\quad \text { Average.............. }\end{array}$ & $\begin{array}{r}\text { Grams. } \\
19.046 \\
3.809\end{array}$ & $\begin{array}{r}\text { Grams. } \\
6.597 \\
1.319\end{array}$ & $\begin{array}{r}\text { Grams. } \\
10.723 \\
2.145\end{array}$ & $\begin{array}{r}\text { Grams. } \\
17.320 \\
3.464\end{array}$ & $\begin{array}{l}\text { Per ct. } \\
34.64 \\
3 . .\end{array}$ & $\begin{array}{l}\text { Per } \text { et. } \\
56.30 \\
.6 .\end{array}$ & $\begin{array}{c}\text { Per ct. } \\
\text { so. } 94 \\
.\end{array}$ & $\begin{array}{r}\text { Grams } \\
+\quad 1.726 \\
+\quad .345\end{array}$ & $\begin{array}{c}\text { Grams } \\
0 \\
0\end{array}$ \\
\hline $\begin{array}{l}\text { Second subperiod: } \\
\text { Total ................ } \\
\text { Average.......... }\end{array}$ & $\begin{array}{r}17.991 \\
3.598\end{array}$ & $\begin{array}{r}4.827 \\
.965\end{array}$ & $\begin{array}{r}10.963 \\
2.193\end{array}$ & $\begin{array}{r}15.790 \\
3.158\end{array}$ & $\begin{array}{r}26.83 \\
\ldots \ldots\end{array}$ & $\begin{array}{r}60.94 \\
\cdots \cdots\end{array}$ & $\begin{array}{l}87.77 \\
\cdots\end{array}$ & $\begin{array}{r}+2.201 \\
+\quad .440\end{array}$ & $\begin{array}{l}0 \\
0\end{array}$ \\
\hline $\begin{array}{l}\text { Entire fore period: } \\
\quad \text { Total ................... } \\
\text { Average ......... }\end{array}$ & $\begin{array}{r}37.037 \\
3.704\end{array}$ & $\begin{array}{r}11.424 \\
1.14^{\prime 2}\end{array}$ & $\begin{array}{r}-21.686 \\
2.169\end{array}$ & $\begin{array}{r}33.110 \\
3.311\end{array}$ & $\begin{array}{r}30.85 \\
\cdots \cdots\end{array}$ & $\begin{array}{r}58.55 \\
\ldots \ldots \ldots\end{array}$ & $\begin{array}{r}89.40 \\
\ldots\end{array}$ & $\begin{array}{r}+3.927 \\
+\quad .393\end{array}$ & $\begin{array}{l}0 \\
0\end{array}$ \\
\hline Preservative period. & & & & & & & & & \\
\hline $\begin{array}{l}\text { First subperiod: } \\
\text { Total } \ldots . . . . . . \\
\text { Average } . . .\end{array}$ & $\begin{array}{r}18.902 \\
3.780\end{array}$ & $\begin{array}{r}2.982 \\
.596\end{array}$ & $\begin{array}{r}11.271 \\
2.254\end{array}$ & $\begin{array}{r}14.253 \\
2.851\end{array}$ & 15.78 & 59.63 & 75.40 & $\begin{array}{r}+4.649 \\
+\quad .929\end{array}$ & $\begin{array}{r}1.05 \\
.21\end{array}$ \\
\hline $\begin{array}{l}\text { cond su } \\
\text { Total }\end{array}$ & & & & & & & & & \\
\hline $\begin{array}{l}\text { Total } \\
\text { Avera }\end{array}$ & 18.978 & 5.003 & 12.343 & 17.346 & 26.36 & 65.04 & 91.40 & +1.632 & 2.10 \\
\hline $\begin{array}{l}\text { Average } \\
\text { Third subperiod: }\end{array}$ & 796 & & 2.469 & $\begin{array}{l}-3.469 \\
-\end{array}$ & $\cdots \cdots$ & $\ldots \ldots$ & & +.327 & .042 \\
\hline $\begin{array}{l}\text { Total } \\
\text { Average } . . . .\end{array}$ & $\begin{array}{r}19.798 \\
3.960\end{array}$ & $\begin{array}{l}5.345 \\
1.069\end{array}$ & $\begin{array}{r}a \\
11.596 \\
.2319\end{array}$ & $\begin{array}{r}16.941 \\
3.388\end{array}$ & 27.00 & 58.57 & 85.57 & +2.857 & 3.70 \\
\hline $\begin{array}{l}\text { Average ............... } \\
\text { Fourth subperiod: }\end{array}$ & & & & 3.3 & & & & +.012 & .14 \\
\hline $\begin{array}{l}\text { Total } \ldots \ldots \ldots \\
\text { Average } . . .\end{array}$ & $\begin{array}{r}19.211 \\
3.842\end{array}$ & $\begin{array}{l}5.561 \\
1.112\end{array}$ & $\begin{array}{r}12.216 \\
2.443\end{array}$ & $\begin{array}{r}17.777 \\
3.555\end{array}$ & 28.95 & $\begin{array}{r}63.59 \\
\ldots .6 .\end{array}$ & 92.54 & $\begin{array}{r}+1.434 \\
+\quad .287\end{array}$ & $\begin{array}{l}6.00 \\
1.20\end{array}$ \\
\hline Fifth subperiod: & & & & & & & & & \\
\hline $\begin{array}{l}\text { Total } \\
\text { Average.................. }\end{array}$ & $\begin{array}{r}19.894 \\
3.979\end{array}$ & $\begin{array}{l}6.508 \\
1.302\end{array}$ & $\begin{array}{r}11.737 \\
2.347\end{array}$ & $\begin{array}{r}18.245 \\
3.649\end{array}$ & 32.71 & 59.00 & 91.71 & +1.649 & 8.00 \\
\hline Sixth subper & & & & & & & & & \\
\hline Total $\ldots . . . . . . . . .$. & $\begin{array}{r}19.050 \\
3.810\end{array}$ & $\begin{array}{l}5.522 \\
1.104\end{array}$ & $\begin{array}{r}11.345 \\
2.269\end{array}$ & $\begin{array}{r}16.867 \\
3.373\end{array}$ & $\begin{array}{r}28.99 \\
\ldots \ldots\end{array}$ & $\begin{array}{r}59.55 \\
\cdots \cdots\end{array}$ & $\begin{array}{r}88.54 \\
\ldots \ldots\end{array}$ & $\begin{array}{l}+2.183 \\
+\quad .437\end{array}$ & $\begin{array}{r}10.00 \\
2.00\end{array}$ \\
\hline $\begin{array}{l}\text { Entire preservative } \\
\text { period: }\end{array}$ & & & & & & & & & \\
\hline $\begin{array}{l}\text { Total } \\
\text { Average } . . . .\end{array}$ & $\begin{array}{r}115.833 \\
3.861\end{array}$ & $\begin{array}{r}30.921 \\
1.031\end{array}$ & $\begin{array}{r}70.508 \\
2.350\end{array}$ & $\begin{array}{r}101.429 \\
3.381\end{array}$ & $\begin{array}{r}26.69 \\
\ldots \ldots\end{array}$ & $\begin{array}{r}60.87 \\
\ldots \ldots\end{array}$ & 87.56 & $\begin{array}{l}+14.404 \\
+\quad .480\end{array}$ & 30.85 \\
\hline & & & & & & & & & \\
\hline-9 & & & & & & & & & \\
\hline $\begin{array}{l}\text { First subperiod: } \\
\text { Total .......... } \\
\text { Average ...... }\end{array}$ & $\begin{array}{r}18.383 \\
3.677\end{array}$ & $\begin{array}{r}10.438 \\
2.088\end{array}$ & $\begin{array}{r}10.418 \\
2.084\end{array}$ & $\begin{array}{r}20.856 \\
4.171\end{array}$ & 56.78 & 56.67 & 113.45 & $\begin{array}{r}2.473 \\
-\quad .494\end{array}$ & $\begin{array}{l}0 \\
0\end{array}$ \\
\hline $\begin{array}{l}\text { Second subperiod: } \\
\text { Total .................. } \\
\text { Average ......... }\end{array}$ & $\begin{array}{r}19.168 \\
3.834\end{array}$ & $\begin{array}{l}7.952 \\
1.590\end{array}$ & $\begin{array}{r}10.924 \\
2.185\end{array}$ & $\begin{array}{r}18.876 \\
3.775\end{array}$ & $\begin{array}{r}41.49 \\
\cdots \cdots\end{array}$ & $\begin{array}{r}56.99 \\
\ldots . . .\end{array}$ & $\begin{array}{r}98.48 \\
\ldots .\end{array}$ & $\begin{array}{r}+\quad .292 \\
+\quad .059\end{array}$ & $\begin{array}{l}0 \\
0\end{array}$ \\
\hline $\begin{array}{l}\text { Entire after period: } \\
\text { Total } . . . . . . \\
\text { Average } . . . .\end{array}$ & $\begin{array}{r}37.551 \\
3.755\end{array}$ & $\begin{array}{r}18.390 \\
1.839\end{array}$ & $\begin{array}{r}21.342 \\
2.134\end{array}$ & $\begin{array}{r}39.732 \\
3.973\end{array}$ & $\begin{array}{r}48.97 \\
\hdashline \cdots\end{array}$ & $\begin{array}{r}56.83 \\
\ldots . . .\end{array}$ & $\begin{array}{l}105.81 \\
\ldots \ldots\end{array}$ & $\begin{array}{r}2.181 \\
-\quad .218\end{array}$ & $\begin{array}{l}0 \\
0\end{array}$ \\
\hline
\end{tabular}

$a$ Daily average added in order to complete record. 
SALICYLIC ACID AND SALICYLATES.

Table XV.-Phosphoric acid balances for Series VI-Continued.

[Averages are per day.]

No. 2 .

\begin{tabular}{|c|c|c|c|c|c|c|c|c|c|}
\hline Period. . & In food. & In feces. & In urine. & $\begin{array}{c}4 \\
\text { In feces } \\
\text { and } \\
\text { urine. } \\
(2+3)\end{array}$ & $\begin{array}{c}5 \\
\text { In } \\
\text { feces. } \\
(2 \div 1)\end{array}$ & $\underset{\substack{\text { In } \\
(3 \div 1)}}{{ }^{*}}$ & $\begin{array}{c}7 \\
\text { In feces } \\
\text { and } \\
\text { urine. } \\
(4 \div-1)\end{array}$ & $\begin{array}{c}\text { Balance. } \\
(1-4)\end{array}$ & $\begin{array}{c}9 \\
\text { Sali- } \\
\text { cylic } \\
\text { acid } \\
\text { admin. } \\
\text { istered. }\end{array}$ \\
\hline \multicolumn{10}{|l|}{ Fore period. } \\
\hline $\begin{array}{l}\text { First subperiod: } \\
\text { Total ........... } \\
\text { Average ........ } \\
\text { Second subperiod: } \\
\text { Total ............ } \\
\text { Average........ }\end{array}$ & $\begin{array}{r}\text { Grams. } \\
22.060 \\
4.412\end{array}$ & $\begin{array}{r}\text { Grams. } \\
6.816 \\
1.363\end{array}$ & $\begin{array}{r}\text { Grams. } \\
\text { a } 16.025 \\
3.205\end{array}$ & $\begin{array}{r}\text { Grams. } \\
22.841 \\
4.568\end{array}$ & $\begin{array}{c}\text { Per ct. } \\
30.90 \\
\cdots \\
34.78 \\
\cdots\end{array}$ & $\begin{array}{l}\text { Per ct. } \\
72.64 \\
\cdots\end{array}$ & $\begin{array}{c}\text { Per ct. } \\
103.54 \\
\cdots \cdots\end{array}$ & $\begin{array}{l}\text { Grams. } \\
-0.781 \\
-.156 \\
-.607 \\
-.121\end{array}$ & $\begin{array}{c}\text { Grams. } \\
0 \\
0\end{array}$ \\
\hline $\begin{array}{l}\text { Entire fore period: } \\
\text { Total ............. } \\
\text { Average ....... }\end{array}$ & $\begin{array}{r}43.005 \\
4.300\end{array}$ & $\begin{array}{r}14.101 \\
1.410\end{array}$ & $\begin{array}{r}30.292 \\
3.029\end{array}$ & $\begin{array}{r}44.393 \\
4.439\end{array}$ & $\begin{array}{r}32.79 \\
\ldots \ldots \ldots \\
\end{array}$ & $\begin{array}{r}70.44 \\
\ldots \ldots \ldots \\
\end{array}$ & $\begin{array}{c}103.23 \\
\cdots \ldots \ldots\end{array}$ & $\begin{array}{r}-1.388 \\
-.139 \\
\end{array}$ & $\begin{array}{l}0 \\
0\end{array}$ \\
\hline \multicolumn{10}{|l|}{ Preservative period. } \\
\hline 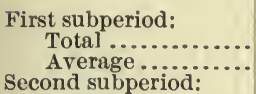 & $\begin{array}{r}21.871 \\
4.374\end{array}$ & $\begin{array}{l}5.151 \\
1.030\end{array}$ & $\begin{array}{r}14.714 \\
2.943\end{array}$ & $\begin{array}{r}19.865 \\
3.973\end{array}$ & $\begin{array}{c}23.55 \\
. \cdots . . .\end{array}$ & $\begin{array}{r}67.28 \\
\ldots \ldots \ldots\end{array}$ & $\begin{array}{r}90.83 \\
\ldots . . .\end{array}$ & $\begin{array}{l}+2,006 \\
+.401\end{array}$ & $\begin{array}{r}1.05 \\
.21\end{array}$ \\
\hline $\begin{array}{r}\text { To } \\
\text { Avird }\end{array}$ & $\begin{array}{r}21.652 \\
4.330\end{array}$ & & $\begin{array}{r}16.061 \\
3.212\end{array}$ & $\begin{array}{r}22.497 \\
4.499\end{array}$ & $\begin{array}{r}29.72 \\
\ldots \ldots . .\end{array}$ & $\begin{array}{r}74.18 \\
\cdots \cdots \cdots\end{array}$ & $\begin{array}{c}103.90 \\
\cdots \ldots\end{array}$ & $\begin{array}{l}-.845 \\
-.169\end{array}$ & $\begin{array}{r}2.10 \\
.42\end{array}$ \\
\hline .................... & $\begin{array}{r}21.844 \\
4.369\end{array}$ & $\begin{array}{l}7.364 \\
1.473\end{array}$ & $\begin{array}{r}15.734 \\
3.147\end{array}$ & $\begin{array}{r}23.098 \\
4.620\end{array}$ & $\begin{array}{c}33.71 \\
\ldots \ldots . \cdots\end{array}$ & $\begin{array}{r}72.03 \\
\cdots \cdots\end{array}$ & $\begin{array}{c}105.74 \\
\cdots \ldots \ldots\end{array}$ & $\begin{array}{l}-1.254 \\
-.251\end{array}$ & $\begin{array}{r}3.70 \\
.7\end{array}$ \\
\hline $\begin{array}{r}\text { Fourth su } \\
\text { Total } \\
\text { Avera }\end{array}$ & $\begin{array}{r}22.092 \\
4.418\end{array}$ & & $\begin{array}{r}14.903^{\circ} \\
2.981\end{array}$ & $\begin{array}{r}22.005 \\
4.401\end{array}$ & $\begin{array}{r}32.15 \\
\cdots \cdots\end{array}$ & $\begin{array}{r}67.46 \\
\cdots \cdots\end{array}$ & $\begin{array}{r}99.61 \\
\ldots \ldots\end{array}$ & $\begin{array}{r}.087 \\
+.087 \\
+.017\end{array}$ & $\begin{array}{l}6.00 \\
1.20\end{array}$ \\
\hline To & $\begin{array}{r}22.879 \\
4.576\end{array}$ & $\begin{array}{r}4.133 \\
.827\end{array}$ & $\begin{array}{r}15.979 \\
3.196\end{array}$ & $\begin{array}{r}20.112 \\
4.022\end{array}$ & $\begin{array}{r}18.06 \\
\ldots \ldots\end{array}$ & $\begin{array}{r}69.84 \\
\cdots \\
\cdots\end{array}$ & $\begin{array}{l}87.91 \\
\ldots . \ldots .\end{array}$ & $\begin{array}{l}+2.767 \\
+.554\end{array}$ & $\begin{array}{l}8.00 \\
1.60\end{array}$ \\
\hline $\begin{array}{l}\text { Sixth subpe } \\
\text { Total. } \\
\text { Averag }\end{array}$ & $\begin{array}{r}22.089 \\
4.418\end{array}$ & $\begin{array}{l}7.367 \\
1.473\end{array}$ & $\begin{array}{r}14.271 \\
2.854\end{array}$ & $\begin{array}{r}21.638 \\
4.328\end{array}$ & $\begin{array}{r}33.35 \\
\ldots . . .\end{array}$ & $\begin{array}{r}64.61 \\
\ldots \ldots\end{array}$ & $\begin{array}{r}97.96 \\
.\end{array}$ & $\begin{array}{r}+.451 \\
+.090\end{array}$ & $\begin{array}{r}10.00 \\
2.00\end{array}$ \\
\hline $\begin{array}{l}\text { Entire preservative } \\
\text { period: } \\
\text { Total } \ldots \ldots \ldots \ldots \ldots \\
\text { Average } \ldots \ldots \ldots \ldots\end{array}$ & $\begin{array}{r}132.427 \\
4.414\end{array}$ & $\begin{array}{r}37.553 \\
1.252 \\
\end{array}$ & $\begin{array}{r}91.662 \\
3.055 \\
\end{array}$ & $\begin{array}{r}129.215 \\
4.307 \\
\end{array}$ & $\begin{array}{r}28.36 \\
\cdots \cdots \\
\end{array}$ & $\begin{array}{r}69.22 \\
\ldots \\
\end{array}$ & $\begin{array}{r}97.57 \\
\cdots \cdots \cdots \\
\end{array}$ & $\begin{array}{r}+3.212 \\
+\quad .107 \\
\end{array}$ & $\begin{array}{r}30.85 \\
1.03\end{array}$ \\
\hline After period. & & & & & & & & & \\
\hline $\begin{array}{l}\text { First subp } \\
\text { Total } \\
\text { Avera }\end{array}$ & $\begin{array}{r}21.409 \\
4,252\end{array}$ & $\begin{array}{l}5.519 \\
1.104\end{array}$ & $\begin{array}{r}14.075 \\
2.815\end{array}$ & $\begin{array}{r}19.594 \\
3.919\end{array}$ & $\begin{array}{r}25.78 \\
\cdots \cdots . . .\end{array}$ & 65.74 & 91.52 & $\begin{array}{l}+1.815 \\
+\cdot .333\end{array}$ & $\begin{array}{l}0 \\
0\end{array}$ \\
\hline $\begin{array}{l}\text { Second subperiod; } \\
\text { Total ................................ } \\
\text { Average }\end{array}$ & $\begin{array}{r}22.168 \\
4.434\end{array}$ & $\begin{array}{l}6.745 \\
1.349\end{array}$ & $\begin{array}{r}14.986 \\
2.997\end{array}$ & $\begin{array}{r}21.731 \\
4.346\end{array}$ & 30.43 & $\begin{array}{r}67.60 \\
\cdots \cdots \\
\end{array}$ & $\begin{array}{r}98.03 \\
\cdots \cdots \\
\end{array}$ & $\begin{array}{r}+.437 \\
+.188 \\
\end{array}$ & $\begin{array}{l}0 \\
0\end{array}$ \\
\hline $\begin{array}{l}\text { Entire after period: } \\
\text { Total ........... } \\
\text { Average........ }\end{array}$ & $\begin{array}{r}43.577 \\
4.358\end{array}$ & $\begin{array}{r}12.264 \\
1.226\end{array}$ & $\begin{array}{r}29.061 \\
2.906\end{array}$ & $\begin{array}{r}41.325 \\
4.133\end{array}$ & 28.14 & 66.69 & $\begin{array}{r}94.83 \\
\cdots \cdots \cdots\end{array}$ & $\begin{array}{l}+2.252 \\
+\quad .225\end{array}$ & $\begin{array}{l}0 \\
0\end{array}$ \\
\hline
\end{tabular}

$a$ Daily average added in order to complete record, 
TABLe XV.-Phosphoric acid balances for Series VI-Continued.

[Averages are per day.]

No. 3.

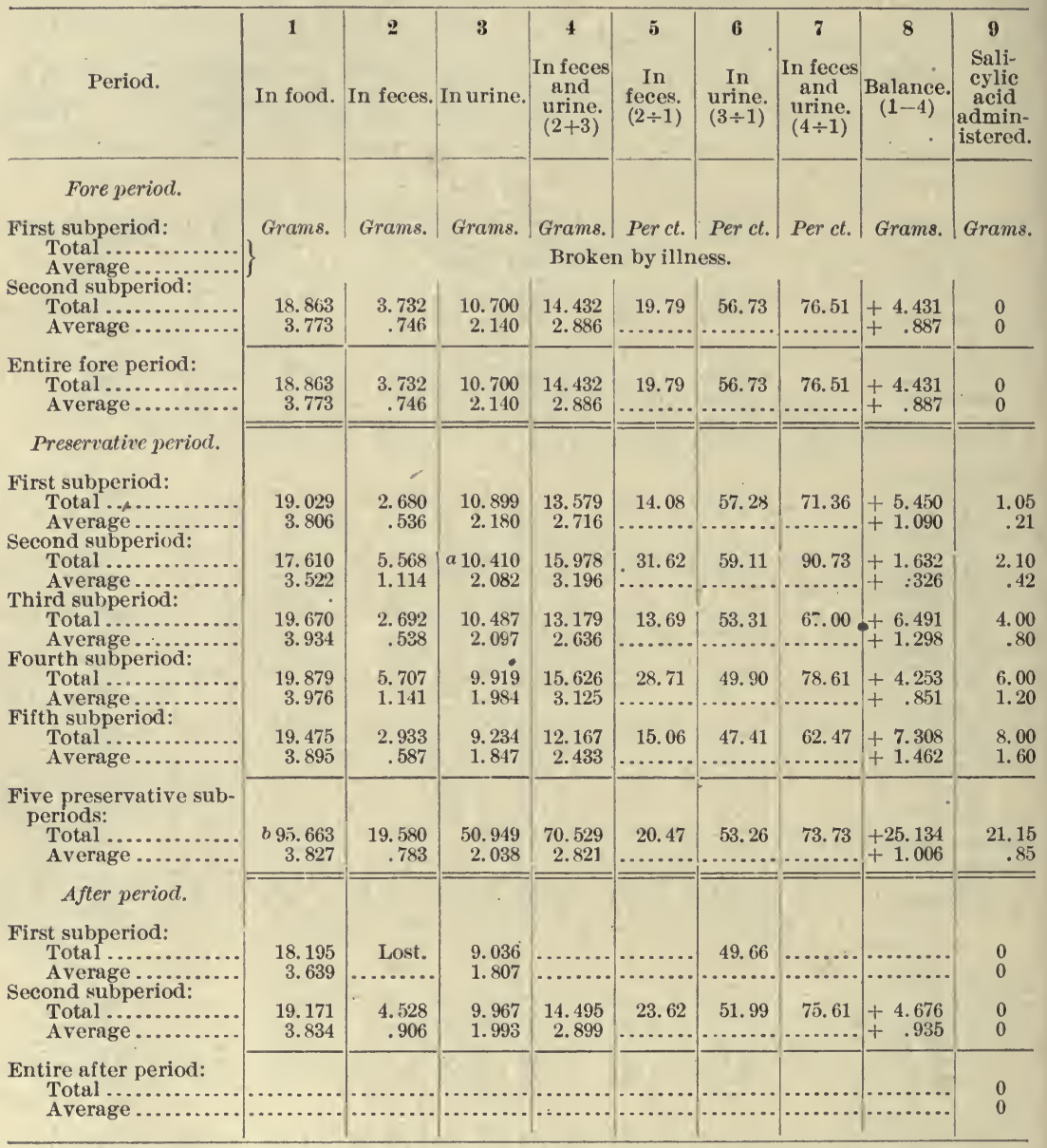

$a$ Daily average added in order to complete record. $b$ No. 3 had only five preservative subperiods. 
Table XV.-Phosphoric acid balances for Series VI-Continued.

[Averages are per day.]

No. 4.

\begin{tabular}{|c|c|c|c|c|c|c|c|c|c|}
\hline Period. & In food. & In feces. & In urine. & $\begin{array}{c}4 \\
\text { In feces } \\
\text { and } \\
\text { urine. } \\
(2+3)\end{array}$ & $\begin{array}{c}5 \\
\text { In } \\
\text { feces. } \\
(2 \div 1)\end{array}$ & $\begin{array}{c}\mathbf{6} \\
\text { In } \\
\text { urine. } \\
(3 \div 1)^{-}\end{array}$ & $\begin{array}{c}\mathbf{7} \\
\text { In feces } \\
\text { and } \\
\text { urine. } \\
(4 \div 1)\end{array}$ & $\begin{array}{c}\text { Balance. } \\
(1-4)\end{array}$ & $\begin{array}{c}9 \\
\text { Sali- } \\
\text { cylic } \\
\text { acid } \\
\text { admin- } \\
\text { istered. }\end{array}$ \\
\hline \multicolumn{10}{|l|}{ Fore period: } \\
\hline 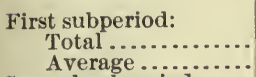 & $\begin{array}{r}\text { Grams. } \\
21.208 \\
4.242\end{array}$ & $\begin{array}{r}\text { Grams. } \\
4.947 \\
.989\end{array}$ & $\begin{array}{r}\text { Grams. } \\
12.787 \\
2.557\end{array}$ & $\begin{array}{r}\text { Grams. } \\
17.734 \\
3.547\end{array}$ & $\begin{array}{r}\text { Per ct. } \\
23.33 \\
2\end{array}$ & $\begin{array}{l}\text { Per ct. } \\
60.29 \\
0 .\end{array}$ & $\begin{array}{l}\text { Per ct. } \\
83.62 \\
\end{array}$ & $\begin{array}{r}\text { Grams } \\
+\quad 3.474 \\
+\quad .695\end{array}$ & $\begin{array}{c}\text { Grams. } \\
0 \\
0\end{array}$ \\
\hline $\begin{array}{l}\text { Second subperiod: } \\
\text { Total ................ } \\
\text { Average .......... }\end{array}$ & $\begin{array}{r}20.125 \\
4.025\end{array}$ & $\begin{array}{l}5.732 \\
1.146\end{array}$ & $\begin{array}{r}14.505 \\
2.901\end{array}$ & $\begin{array}{r}20.237 \\
4.047\end{array}$ & 28.48 & 72.07 & 100.56 & $\begin{array}{l}-.112 \\
-\quad 022\end{array}$ & $\begin{array}{l}0 \\
0\end{array}$ \\
\hline $\begin{array}{l}\text { Entire fore period: } \\
\text { Total ............. } \\
\text { Average } . . . \ldots \ldots\end{array}$ & $\begin{array}{r}41.333 \\
4.133\end{array}$ & $\begin{array}{r}10.679 \\
1.068\end{array}$ & $\begin{array}{r}27.292 \\
2.729\end{array}$ & $\begin{array}{r}37.971 \\
3.797\end{array}$ & $\begin{array}{l}25.84 \\
2 \ldots\end{array}$ & 66.03 & $\begin{array}{l}91.87 \\
\cdots \cdots\end{array}$ & $\begin{array}{l}+3.362 \\
+\quad .336\end{array}$ & $\begin{array}{l}0 \\
0\end{array}$ \\
\hline \multicolumn{10}{|l|}{ - Preservative period. } \\
\hline $\begin{array}{l}\text { First subperiod: } \\
\text { Total ........ } \\
\text { Average..... }\end{array}$ & $\begin{array}{r}21.124 \\
4.225\end{array}$ & $\begin{array}{r}4.929 \\
.986\end{array}$ & $\begin{array}{r}13.843 \\
2.769\end{array}$ & $\begin{array}{r}18.772 \\
3.754\end{array}$ & 23.33 & 65.53 & 88.87 & +2.352 & 1.05 \\
\hline Second subperiod: & & & & & & .......... & $\cdots$ & +.471 & \\
\hline $\begin{array}{l}\text { Total } \ldots . . . . . \\
\text { A verage } . . .\end{array}$ & $\begin{array}{r}21.027 \\
4.205\end{array}$ & 5.679 & 15. 277 & 20.956 & 27.01 & .72 .65 & 99.66 & +.071 & 2.10 \\
\hline Third subperiod: & 4.200 & & & 4. 191 & & & & +.014 & \\
\hline Total ........... & 1.154 & 5. 691 & 13.640 & 19. 331 & 26.90 & 64.48 & 91.38 & +1.823 & 3.70 \\
\hline $\begin{array}{l}\text { Average ........ } \\
\text { Fourth subperiod: }\end{array}$ & & & 728 & & $\cdots$ & & .............. & +.365 & .74 \\
\hline Total .................. & 21.806 & a 4.731 & 14. 891 & 19. 622 & 21.70 & 68.29 & 89.98 & +2.184 & 6.00 \\
\hline $\begin{array}{l}\text { Average ....... } \\
\text { Fifth subperiod: }\end{array}$ & 361 & 946 & & & (......... & & ......... & +.437 & 1.20 \\
\hline Total ........... & 22.177 & 6.128 & 13.402 & 19. 530 & 27.63 & 60.43 & 88.06 & +2.647 & 8.00 \\
\hline $\begin{array}{l}\text { Average } \\
\text { Sixth subperiod: }\end{array}$ & 4.435 & 1.226 & 2.680 & 3.906 & .......... & & & +.529 & 1.60 \\
\hline n......... & 21.273 & a 5.204 & 13. 839 & 19.043 & 24.46 & 65.06 & 89.52 & +2.230 & 10.00 \\
\hline Average & 4.255 & 1. 041 & 2.768 & 3.809 & ........ & ......... & $\ldots \ldots$ & +.446 & 2.00 \\
\hline $\begin{array}{l}\text { Entire preservative } \\
\text { period: }\end{array}$ & & & & & & & & & \\
\hline Total ... & 128.561 & 32.362 & 84.892 & 117.254 & 25.17 & 66.03 & 91.21 & +11.307 & 30.85 \\
\hline Average ............ & 4.285 & -1.079 & 2.830 & 3.908 & .... & $\cdots$ & $\cdots$ & +.377 & 1.03 \\
\hline After period. & & & & & & & & $\cdot$ & \\
\hline $\begin{array}{l}\text { First subperiod: } \\
\text { Total....... }\end{array}$ & & & & & & & & & \\
\hline Averag & 4.112 & .843 & 2.592 & 3.435 & & & & $\begin{array}{r}+ \\
+\quad .677\end{array}$ & 0 \\
\hline $\begin{array}{l}\text { Cond sub } \\
\text { Total .. }\end{array}$ & 21.459 & 6.484 & 14.038 & 20.522 & 30.22 & 65.42 & 95.63 & +.937 & 0 \\
\hline Average......... & 4. 292 & 1.297 & 2.808 & 4.104 & & ........ & ......... & +.188 & 0 \\
\hline $\begin{array}{l}\text { Entire after period: } \\
\text { Total ............... } \\
\text { Average ........ }\end{array}$ & $\begin{array}{r}42.017 \\
4.202\end{array}$ & $\begin{array}{r}10.701 \\
1.070\end{array}$ & $\begin{array}{r}26.997 \\
2.700\end{array}$ & $\begin{array}{r}37.698 \\
3.770\end{array}$ & $\begin{array}{r}25.47 \\
\cdots\end{array}$ & $\begin{array}{r}64.25 \\
\cdots\end{array}$ & $\begin{array}{r}89.72 \\
\ldots .\end{array}$ & $\begin{array}{l}+4.319 \\
+\quad 432\end{array}$ & $\begin{array}{l}0 \\
0\end{array}$ \\
\hline
\end{tabular}

$\alpha$ Daily average added in order to complete record.

7656-No. 84, pt $2-06-10$ 
TABLE XV.-Phosphoric acid balances for Series VI-Continued.

[Averages are per day.]

No. 5.

\begin{tabular}{|c|c|c|c|c|c|c|c|c|c|}
\hline Period. & In food. & In feces. & In urine. & $\begin{array}{c}4 \\
\text { In feces } \\
\text { and } \\
\text { urine. } \\
(2+3)\end{array}$ & $\begin{array}{c}5 \\
\text { In } \\
\text { feces. } \\
(2 \div 1)\end{array}$ & $\begin{array}{c}6 \\
\underset{\substack{\text { In } \\
(3 \div 1)}}{ }\end{array}$ & $\begin{array}{c}7 \\
\text { In feces } \\
\text { and } \\
\text { urine. } \\
(4 \div 1)\end{array}$ & $\begin{array}{c}\text { Balance. } \\
(1-4)\end{array}$ & $\begin{array}{c}9 \\
\text { Sali- } \\
\text { cylic } \\
\text { acid } \\
\text { admin- } \\
\text { istered. }\end{array}$ \\
\hline \multicolumn{10}{|l|}{ Fore period. } \\
\hline $\begin{array}{l}\text { First subperiod: } \\
\text { Total ........... } \\
\text { Average ........ }\end{array}$ & $\begin{array}{r}\text { Grams. } \\
21.244 \\
4.249\end{array}$ & $\begin{array}{r}\text { Grams. } \\
7.249 \\
1.450\end{array}$ & $\begin{array}{r}\text { Grams. } \\
12.890 \\
2.578\end{array}$ & $\begin{array}{r}\text { Grams. } \\
20.139 \\
4.028\end{array}$ & $\begin{array}{l}\text { Per ct. } \\
34.12 \\
\ldots\end{array}$ & $\begin{array}{l}\text { Per ct. } \\
60.68\end{array}$ & $\begin{array}{r}\text { Per ct. } \\
94.80 \\
\\
0 .\end{array}$ & $\begin{array}{r}\text { Grams. } \\
+\quad 1.105 \\
+\quad .221\end{array}$ & $\begin{array}{c}\text { Grams. } \\
0 \\
0\end{array}$ \\
\hline $\begin{array}{l}\text { Second subperiod: } \\
\quad \text { Total .................. } \\
\text { Average .......... }\end{array}$ & $\begin{array}{r}20.526 \\
4.105\end{array}$ & $\begin{array}{l}7.079 \\
1.416\end{array}$ & $\begin{array}{r}11.896 \\
2.379\end{array}$ & $\begin{array}{r}18.975 \\
3.795\end{array}$ & $\begin{array}{r}34.49 \\
\cdots\end{array}$ & $\begin{array}{l}57.96 \\
\cdots \ldots .\end{array}$ & $\begin{array}{c}92.44 \\
\cdots\end{array}$ & $\begin{array}{r}+1.551 \\
+\quad .310\end{array}$ & $\begin{array}{l}0 \\
0\end{array}$ \\
\hline $\begin{array}{l}\text { Entire fore period: } \\
\text { Total } \ldots . . . . \\
\text { Average ......... }\end{array}$ & $\begin{array}{r}41.770 . \\
4.177\end{array}$ & $\begin{array}{r}14.328 \\
1.433\end{array}$ & $\begin{array}{r}24.786 \\
2.479\end{array}$ & $\begin{array}{r}39.114 \\
3.911\end{array}$ & $\begin{array}{r}34.30 \\
\cdots \cdots\end{array}$ & $\begin{array}{r}59.34 \\
\cdots \cdots\end{array}$ & $\begin{array}{r}93.64 \\
\cdots \cdots \cdots \\
\end{array}$ & $\begin{array}{r}+2.656 \\
+\quad .266 \\
\end{array}$ & $\begin{array}{l}0 \\
0\end{array}$ \\
\hline \multicolumn{10}{|l|}{ Preservative period. } \\
\hline $\begin{array}{l}\text { First subperiod: } \\
\text { Total ........... } \\
\text { Average ........ }\end{array}$ & $\begin{array}{r}21.423 \\
4.285\end{array}$ & $\begin{array}{l}7.271 \\
1.454\end{array}$ & $\begin{array}{r}12.791 \\
2.558\end{array}$ & $\begin{array}{r}20.062 \\
4.012\end{array}$ & 33.94 & 59.71 & 93.65 & $\begin{array}{r}+1.361 \\
+\quad .273\end{array}$ & $\begin{array}{r}1.05 \\
.21\end{array}$ \\
\hline $\begin{array}{l}\text { Second subperiod: } \\
\text { Total ............ } \\
\text { Average ....... }\end{array}$ & $\begin{array}{r}21.118 \\
4.224\end{array}$ & $\begin{array}{l}5.110 \\
1.022\end{array}$ & $\begin{array}{r}13.595 \\
2.719\end{array}$ & $\begin{array}{r}18.705 \\
3.741\end{array}$ & $\begin{array}{r}24.20 \\
\ldots \ldots\end{array}$ & 64.38 & $\begin{array}{r}88.57 \\
\ldots \ldots\end{array}$ & $\begin{array}{r}+2.413 \\
+\quad .483\end{array}$ & $\begin{array}{r}2.10 \\
.42\end{array}$ \\
\hline Third subperiod: & & & & & & & $\cdots \cdot$ & & \\
\hline 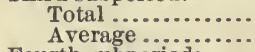 & $\begin{array}{r}21.207 \\
4.241\end{array}$ & $\begin{array}{l}6.012 \\
1.202\end{array}$ & $\begin{array}{r}13.579 \\
2.716\end{array}$ & $\begin{array}{r}19.591 \\
3.918\end{array}$ & $\begin{array}{l}28.35 \\
\ldots \ldots . .\end{array}$ & $\begin{array}{r}64.03 \\
\ldots \ldots \ldots\end{array}$ & $\begin{array}{r}92.38 \\
\ldots . . .\end{array}$ & $\begin{array}{r}1.616 \\
+\quad .323\end{array}$ & $\begin{array}{r}3.70 \\
.74\end{array}$ \\
\hline $\begin{array}{l}\text { Fourth subperiod: } \\
\text { Total }\end{array}$ & & & & & & & & & \\
\hline 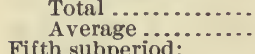 & $\begin{array}{r}22.261 \\
4.452\end{array}$ & $\begin{array}{r}4.777 \\
.955\end{array}$ & $\begin{array}{r}13.216 \\
2.643\end{array}$ & $\begin{array}{r}17.993 \\
3.599\end{array}$ & $\begin{array}{l}21.46 \\
\ldots \ldots \ldots\end{array}$ & $\begin{array}{l}59.37 \\
-\ldots . .\end{array}$ & $\begin{array}{l}80.83 \\
\ldots . . .\end{array}$ & $\begin{array}{r}+\quad 4.268 \\
+\quad .853\end{array}$ & $\begin{array}{l}6.00 \\
1.20\end{array}$ \\
\hline $\begin{array}{l}\text { Fif th subperiod: } \\
\text { Total .......... }\end{array}$ & & & & & & & & ( & \\
\hline $\begin{array}{l}\text { Averag } \\
\text { th subp }\end{array}$ & 4.467 & $\begin{array}{l}0.256 \\
1.256\end{array}$ & 2.617 & 3.873 & 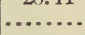 & כ0. 09 & ........... & $+\quad .594$ & $\begin{array}{l}0.00 \\
1.60\end{array}$ \\
\hline $\begin{array}{l}\text { Total ............. } \\
\text { Average ....... }\end{array}$ & $\begin{array}{r}21.428 \\
4.286\end{array}$ & 7.030 & $\begin{array}{r}12.408 \\
2.482\end{array}$ & $\begin{array}{r}19.438 \\
3.888\end{array}$ & 32.81 & 57.91 & 90.71 & +1.990 & 10.00 \\
\hline \\
\hline 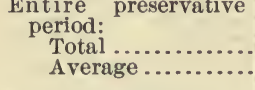 & $\begin{array}{r}129.774 \\
4.326\end{array}$ & $\begin{array}{r}36.478 \\
1.216\end{array}$ & $\begin{array}{r}78.676 \\
2.623\end{array}$ & $\begin{array}{r}115.154 \\
3.838\end{array}$ & $\begin{array}{l}\text { 28.11 } \\
\ldots \ldots \ldots\end{array}$ & $\begin{array}{r}60.63 \\
\cdots\end{array}$ & $\begin{array}{r}88.73 \\
\cdots \cdots\end{array}$ & $\begin{array}{r}+14.620 \\
+\quad .488 \\
\end{array}$ & $\begin{array}{r}30.85 \\
1.03\end{array}$ \\
\hline \multicolumn{10}{|l|}{ After period. } \\
\hline $\begin{array}{l}\text { First subperiod: } \\
\text { Total .......... } \\
\text { Avernge ....... }\end{array}$ & $\begin{array}{r}20.810 \\
4.162\end{array}$ & $\begin{array}{l}6.393 \\
1.279\end{array}$ & $\begin{array}{r}a 11.883 \\
2.377\end{array}$ & $\begin{array}{r}18.276 \\
3.655\end{array}$ & 30.72 & 57.10 & 87.82 & $\begin{array}{r}+2.534 \\
+\quad .507\end{array}$ & $\begin{array}{l}0 \\
0\end{array}$ \\
\hline $\begin{array}{l}\text { Second subperiod: } \\
\text { Total .............. } \\
\text { Average ....... }\end{array}$ & $\begin{array}{r}21.439 \\
4.288\end{array}$ & $\begin{array}{l}5.164 \\
1.033\end{array}$ & $\begin{array}{r}12.738 \\
2.548\end{array}$ & $\begin{array}{r}17.902 \\
3.580\end{array}$ & $\begin{array}{r}24.09 \\
\cdots \cdots \cdots\end{array}$ & $\begin{array}{r}59.42 \\
\cdots \cdots \cdots \\
\end{array}$ & $\begin{array}{r}83.50 \\
\cdots \cdots\end{array}$ & $\begin{array}{l}+3.537 \\
+\quad .708\end{array}$ & $\begin{array}{l}0 \\
0\end{array}$ \\
\hline $\begin{array}{l}\text { Entire after period: } \\
\text { Total .............. } \\
\text { Average ....... }\end{array}$ & $\begin{array}{r}42.249 \\
4.225\end{array}$ & $\begin{array}{r}11.557 \\
1.156\end{array}$ & $\begin{array}{r}24.621 \\
2.462\end{array}$ & $\begin{array}{r}36.178 \\
3.618\end{array}$ & 27.35 & 58.28 & 85.63 & $\begin{array}{l}+6.071 \\
+\quad .607\end{array}$ & $\begin{array}{l}0 \\
0\end{array}$ \\
\hline
\end{tabular}

$a$ Daily average added in order to complete record. 
TABLE XV.-Phosphoric acid balances for Series VI-Continued.

[Averages are per day.]

No. 6.

\begin{tabular}{|c|c|c|c|c|c|c|c|c|c|}
\hline Period. & $\begin{array}{c}1 \\
\text { In food. }\end{array}$ & $\begin{array}{c}2 \\
\text { In feces. }\end{array}$ & $\begin{array}{c}3 \\
\text { In urine. }\end{array}$ & $\begin{array}{c}4 \\
\text { In feces } \\
\text { and } \\
\text { urine. } \\
(2+3)\end{array}$ & $\begin{array}{c}5 \\
\text { In } \\
\text { feces. } \\
(2 \div 1)\end{array}$ & $\begin{array}{c}6 \\
\text { In } \\
\text { urine. } \\
(3 \div-1)\end{array}$ & $\begin{array}{c}7 \\
\text { In feces } \\
\text { and } \\
\text { urine. } \\
(4 \div 1)\end{array}$ & $\begin{array}{c}8 \\
\text { Balance. } \\
(1-4)\end{array}$ & $\begin{array}{c}9 \\
\text { Sali- } \\
\text { cylic } \\
\text { acid } \\
\text { admin- } \\
\text { istercd. }\end{array}$ \\
\hline Fore period. & & & & & & & & & \\
\hline 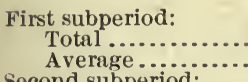 & $\begin{array}{r}\text { Grams } \\
18.365 \\
3.673\end{array}$ & $\begin{array}{r}\text { Grams. } \\
6.658 \\
1.332\end{array}$ & $\begin{array}{r}\text { Grams. } \\
9.814 \\
1.963\end{array}$ & $\begin{array}{l}\text { Grams. } \\
16.472 \\
3.294\end{array}$ & $\begin{array}{r}\text { Per ct. } \\
36.25 \\
\ldots \ldots .\end{array}$ & $\begin{array}{r}\text { Per ct. } \\
53.44 \\
\ldots \ldots\end{array}$ & $\begin{array}{r}\text { Per ct. } \\
89.69\end{array}$ & $\begin{array}{l}\text { Grams. } \\
+1.893 \\
+\quad .379\end{array}$ & $\begin{array}{c}\text { Grams. } \\
0 \\
0\end{array}$ \\
\hline 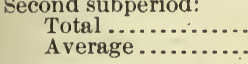 & $\begin{array}{r}17.621 \\
3.524\end{array}$ & $\begin{array}{l}6.498 \\
1.300\end{array}$ & $\begin{array}{r}10.010 \\
2.002\end{array}$ & $\begin{array}{r}16.508 \\
3.302\end{array}$ & 36.58 & 56.81 & 93.68 & $\begin{array}{r}+1.113 \\
+.222\end{array}$ & $\begin{array}{l}0 \\
0\end{array}$ \\
\hline 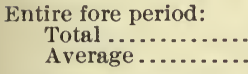 & $\begin{array}{r}35.986 \\
3.599\end{array}$ & $\begin{array}{r}13.156 \\
1.316\end{array}$ & $\begin{array}{r}19.824 \\
1.982\end{array}$ & $\begin{array}{r}32.980 \\
3.298\end{array}$ & 36.56 & 55.09 & 91.65 & $\begin{array}{l}+3.006 \\
+\quad .301\end{array}$ & $\begin{array}{l}0 \\
0\end{array}$ \\
\hline Preservative period. & & & & & & & & & \\
\hline $\begin{array}{l}\text { First subperiod: } \\
\text { Total ............ } \\
\text { Average _...... } \\
\text { Second subperiod: }\end{array}$ & $\begin{array}{r}18.736 \\
3.747\end{array}$ & $\begin{array}{l}6.117 \\
1.223\end{array}$ & $\begin{array}{r}11.315 \\
2.263\end{array}$ & $\begin{array}{r}17.432 \\
3.486\end{array}$ & 32.65 & 60.39 & 93.04 & $\begin{array}{l}+1.304 \\
+.261\end{array}$ & $\begin{array}{r}1.05 \\
.21\end{array}$ \\
\hline $\begin{array}{l}\text { To } \\
\text { A }\end{array}$ & $\begin{array}{r}18.059 \\
3.612\end{array}$ & $\begin{array}{l}7.097 \\
1.419\end{array}$ & $\begin{array}{r}12.020 \\
2.404\end{array}$ & $\begin{array}{r}19.117 \\
3.823\end{array}$ & 39.30 & 66.56 & 105.86 & $\begin{array}{l}-1.058 \\
-.211\end{array}$ & $\begin{array}{r}2.10 \\
.42\end{array}$ \\
\hline $\begin{array}{l}\text { Third subper } \\
\text { Total ... } \\
\text { Average }\end{array}$ & $\begin{array}{r}18.795 \\
3.759\end{array}$ & $\begin{array}{l}7.147 \\
1.429\end{array}$ & $\begin{array}{r}a 11.275 \\
2.255\end{array}$ & $\begin{array}{r}18.422 \\
3.684\end{array}$ & 38.03 & 59.99 & 98.02 & $\begin{array}{l}+.373 \\
+.075\end{array}$ & $\begin{array}{r}3.70 \\
.74\end{array}$ \\
\hline $\begin{array}{l}\text { Fourth s } \\
\text { Total } \\
\text { Aver }\end{array}$ & $\begin{array}{r}19.977 \\
3.995\end{array}$ & $\begin{array}{l}5.956 \\
1.191\end{array}$ & $\begin{array}{r}a 11.928 \\
2.386\end{array}$ & $\begin{array}{r}17.884 \\
3.577\end{array}$ & 29.81 & 59.71 & 89.52 & $\begin{array}{r}+2.093 \\
+\quad .418\end{array}$ & $\begin{array}{l}6.00 \\
1.20\end{array}$ \\
\hline $\begin{array}{l}\text { Fifth sub } \\
\text { Total } \\
\text { A vera }\end{array}$ & $\begin{array}{r}\text { 19. } 960 \\
\text { 3. } 992\end{array}$ & $\begin{array}{l}7.219 \\
1.444\end{array}$ & $\begin{array}{r}11.044 \\
2.209\end{array}$ & \begin{tabular}{|r}
18.263 \\
3.653
\end{tabular} & 36.17 & 55.33 & 91.50 & $\begin{array}{r}+1.697 \\
+\quad .339\end{array}$ & $\begin{array}{l}8.00 \\
1.60\end{array}$ \\
\hline $\begin{array}{l}\text { Sixth subperiod: } \\
\quad \text { Total } \\
\text { Average .................... }\end{array}$ & $\begin{array}{r}18.728 \\
3.746\end{array}$ & $\begin{array}{l}6.482 \\
1.296\end{array}$ & $\begin{array}{l}9.990 \\
1.998\end{array}$ & $\begin{array}{r}16.472 \\
3.294\end{array}$ & 34.61 & 53.34 & $\begin{array}{c}87.95 \\
\quad \cdots \ldots . .\end{array}$ & $\begin{array}{l}+2.256 \\
+.452\end{array}$ & $\begin{array}{l}8.00 \\
1.60\end{array}$ \\
\hline $\begin{array}{l}\text { Entire preservative } \\
\text { period: } \\
\text { Total .................... } \\
\text { Average............. }\end{array}$ & $\begin{array}{r}114.255 \\
3.808\end{array}$ & $\begin{array}{r}40.018 \\
1.334\end{array}$ & $\begin{array}{r}67.572 \\
2.252\end{array}$ & $\begin{array}{r}107.590 \\
3.586\end{array}$ & 35.03 & 59.14 & 94.17 & $\begin{array}{l}+6.665 \\
+\quad .222\end{array}$ & $\begin{array}{r}28.85 \\
.96\end{array}$ \\
\hline After period. & & & & & & & & & \\
\hline $\begin{array}{l}\text { First subperiod: } \\
\text { Total ......... } \\
\text { Average..... }\end{array}$ & $\begin{array}{r}18.354 \\
3.671\end{array}$ & $\begin{array}{l}8.616 \\
1.723\end{array}$ & $\begin{array}{l}9.180 \\
\text { 1. } 836\end{array}$ & $\begin{array}{r}17.796 \\
3.559\end{array}$ & 46.94 & 50.02 & 96.96 & $\begin{array}{l}+.558 \\
+.112\end{array}$ & $\begin{array}{l}0 \\
0\end{array}$ \\
\hline 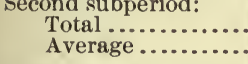 & $\begin{array}{r}18.779 \\
3.756\end{array}$ & $\begin{array}{r}a 5.605 \\
1.121\end{array}$ & $\begin{array}{r}10.707 \\
2.141\end{array}$ & $\begin{array}{r}16.312 \\
3.262\end{array}$ & 29.85 & 57.02 & 86.86 & $\begin{array}{l}+2.467 \\
+\quad .494\end{array}$ & $\begin{array}{l}0 \\
0\end{array}$ \\
\hline $\begin{array}{l}\text { Entire after period: } \\
\quad \text { Total } \ldots \ldots \ldots \ldots . . . \\
\text { Average ................. }\end{array}$ & $\begin{array}{r}37.133 \\
3.713\end{array}$ & $\begin{array}{r}14.221 \\
1.422\end{array}$ & $\begin{array}{r}19.887 \\
1.989\end{array}$ & $\begin{array}{r}34.108 \\
3.411\end{array}$ & 38.30 & 53.56 & 91.85 & $\begin{array}{l}+3.025 \\
+\quad .302\end{array}$ & $\begin{array}{l}0 \\
0\end{array}$ \\
\hline
\end{tabular}

$a$ Daily average added in order to complete record. 
Table XV.-Phosphoric acid balances for Series VI-Continued.

[Averages are per day.]

No. 7.

\begin{tabular}{|c|c|c|c|c|c|c|c|c|c|}
\hline Period. & In food. & In feces. & In urine. & $\begin{array}{c}t \\
\text { In feces } \\
\text { and } \\
\text { urine. } \\
(2+3)\end{array}$ & $\begin{array}{c}5 \\
\text { In } \\
\text { feces. } \\
(2 \div 1)\end{array}$ & $\begin{array}{c}6 \\
\text { In } \\
\text { urine. } \\
(3 \div 1)\end{array}$ & $\begin{array}{c}7 \\
\text { In feces } \\
\text { and } \\
\text { urine. } \\
(4 \div 1)\end{array}$ & $\underset{(1-4)}{\text { Balance. }}$ & $\begin{array}{c}\mathbf{9} \\
\text { Sali- } \\
\text { cylic } \\
\text { acid } \\
\text { admin- } \\
\text { istered. }\end{array}$ \\
\hline \multicolumn{10}{|l|}{ Fore period. } \\
\hline $\begin{array}{l}\text { First subperiod: } \\
\text { Total ............ } \\
\text { Average....... }\end{array}$ & $\begin{array}{r}\text { Grams. } \\
14.252 \\
2.850\end{array}$ & $\begin{array}{r}\text { Grams. } \\
4.781 \\
.956\end{array}$ & $\begin{array}{r}\text { Grams. } \\
8.547 \\
1.709\end{array}$ & $\begin{array}{c}\text { Grams. } \\
13.328 \\
2.666\end{array}$ & $\begin{array}{c}\text { Per ct. } \\
33.55 \\
\end{array}$ & $\begin{array}{l}\text { Per ct. } \\
59.97 \\
.\end{array}$ & $\begin{array}{r}\text { Per ct. } \\
93.52 \\
\ldots \ldots\end{array}$ & $\begin{array}{r}\text { Grams. } \\
+\quad 0.924 \\
+\quad .184\end{array}$ & $\begin{array}{c}\text { Grams. } \\
0 \\
0\end{array}$ \\
\hline Second subperiod: & & & & & & & & & \\
\hline $\begin{array}{l}\text { Total } \ldots . . . \\
\text { Average } . . . .\end{array}$ & $\begin{array}{r}14.733 \\
2.947 .\end{array}$ & $\begin{array}{r}2.930 \\
.586\end{array}$ & $\begin{array}{l}8.480 \\
1.696\end{array}$ & $\begin{array}{r}11.410 \\
2.282\end{array}$ & $\begin{array}{r}19.89 \\
\cdots \ldots .\end{array}$ & $\begin{array}{r}57.56 \\
\cdots \ldots \ldots\end{array}$ & $\begin{array}{r}77.45 \\
\cdots \ldots .\end{array}$ & $\begin{array}{l}+3.323 \\
+\quad .665\end{array}$ & $\begin{array}{l}0 \\
0\end{array}$ \\
\hline $\begin{array}{l}\text { Entire fore period: } \\
\text { Total ................ } \\
\text { Average .......... }\end{array}$ & $\begin{array}{r}28.985 \\
2.899\end{array}$ & $\begin{array}{r}7.711 \\
.771 \\
\end{array}$ & $\begin{array}{r}17.027 \\
1.703 \\
\end{array}$ & $\begin{array}{r}24.738 \\
2.474 \\
\end{array}$ & $\begin{array}{r}26.60 \\
\cdots \cdots \\
\cdots \cdots\end{array}$ & $\begin{array}{r}58.74 \\
\ldots \ldots\end{array}$ & $\begin{array}{r}85.35 \\
\cdots \ldots . .\end{array}$ & $\begin{array}{r}+4.247 \\
+\quad .425 \\
\end{array}$ & $\begin{array}{l}0 \\
0\end{array}$ \\
\hline \multicolumn{10}{|l|}{ Preservative period. } \\
\hline $\begin{array}{l}\text { I irst subperiod: } \\
\text { Total......... } \\
\text { Average..... }\end{array}$ & $\begin{array}{r}14.865 \\
2.973\end{array}$ & $\begin{array}{r}3.839 \\
.768\end{array}$ & $\begin{array}{l}7.354 \\
1.471\end{array}$ & $\begin{array}{r}11.193 \\
2.239\end{array}$ & 25.83 & $\begin{array}{l}49.47 \\
\cdots \ldots . .\end{array}$ & 75.30 & $\begin{array}{r}+3.672 \\
+\quad .734\end{array}$ & $\begin{array}{r}1.05 \\
.21\end{array}$ \\
\hline Second subperiod: & & & & & & & & & \\
\hline $\begin{array}{l}\text { Total } \ldots . . . . \\
\text { Average } . . .\end{array}$ & $\begin{array}{r}15.167 \\
3.033\end{array}$ & $\begin{array}{r}2.650 \\
.530\end{array}$ & $\begin{array}{l}7.903 \\
1.581\end{array}$ & $\begin{array}{r}10.553 \\
2.111\end{array}$ & 17.47 & 52.11 & 69.58 & +4.614 & 2. 10 \\
\hline \multicolumn{10}{|l|}{ Third subperiod: } \\
\hline $\begin{array}{l}\text { Total .............. } \\
\text { Average ...... }\end{array}$ & $\begin{array}{r}15.010 \\
3.002\end{array}$ & $\begin{array}{r}3.870 \\
774\end{array}$ & 6.597 & 10. 467 & 25.78 & 43.95 & 69.73 & +4.543 & 3.70 \\
\hline Fourth subperiod: & & .774 & & & & & & + & \\
\hline $\begin{array}{l}\text { Total } \ldots . . . . \\
\text { Average }\end{array}$ & $\begin{array}{r}14.722 \\
2.944\end{array}$ & $\begin{array}{r}2.757 \\
.551\end{array}$ & $\begin{array}{l}6.450 \\
1.290\end{array}$ & $\begin{array}{l}9.207 \\
1.811\end{array}$ & $\begin{array}{l}18.73 \\
\cdots \cdots . . .\end{array}$ & 43.81 & 62.54 & $\begin{array}{l}+5.515 \\
+1.103\end{array}$ & $\begin{array}{l}6.00 \\
1.20\end{array}$ \\
\hline \multicolumn{10}{|l|}{ Fifth subperiod: } \\
\hline $\begin{array}{l}\text { Total } . . . . . . \\
\text { Average } . . .\end{array}$ & $\begin{array}{r}14.749 \\
2.950\end{array}$ & $\begin{array}{r}4.281 \\
.856\end{array}$ & $\begin{array}{l}6.989 \\
1.398\end{array}$ & $\begin{array}{r}11.270 \\
2.254\end{array}$ & $\begin{array}{c}29.03 \\
\ldots \ldots\end{array}$ & $\begin{array}{l}47.39 \\
\ldots . . .\end{array}$ & 76.41 & $\begin{array}{l}+3.479 \\
+\quad .696\end{array}$ & $\begin{array}{r}8.00 \\
1.60\end{array}$ \\
\hline $\begin{array}{l}\text { Sixth subperiod: } \\
\text { Total }\end{array}$ & & & & & & & & 1000 & \\
\hline $\begin{array}{l}\text { Total ....................... } \\
\text { Average }\end{array}$ & $\begin{array}{r}14.355 \\
2.871\end{array}$ & $\begin{array}{r}1.690 \\
.338\end{array}$ & $\begin{array}{l}8.379 \\
1.676\end{array}$ & $\begin{array}{r}10.069 \\
2.014\end{array}$ & 11.77 & 58.37 & $\begin{array}{l}70.14 \\
\cdots\end{array}$ & $\begin{array}{l}+4.286 \\
+\quad .857\end{array}$ & $\begin{array}{r}10.00 \\
2.00\end{array}$ \\
\hline \multicolumn{10}{|l|}{$\begin{array}{l}\text { Entire preservative } \\
\text { period: }\end{array}$} \\
\hline $\begin{array}{l}\text { Total } \ldots . . . \\
\text { Average } . . .\end{array}$ & $\begin{array}{r}88.868 \\
2.962\end{array}$ & $\begin{array}{r}19.087 \\
.636\end{array}$ & $\begin{array}{r}43.672 \\
1.456\end{array}$ & $\begin{array}{r}62.759 \\
2.092\end{array}$ & 21.48 & 49.14 & 70.61 & $\begin{array}{l}+26.109 \\
+\quad 870\end{array}$ & 30.85 \\
\hline \multirow{2}{*}{\multicolumn{10}{|c|}{ After period. }} \\
\hline & & & & & & & & & \\
\hline $\begin{array}{l}\text { First subperiod: } \\
\text { Total ........... }\end{array}$ & 13. 565 & 4.153 & 5.802 & 9.955 & 30.62 & 42.77 & 73.39 & +3.610 & 0 \\
\hline $\begin{array}{l}\text { Averag } \\
\text { Second sul }\end{array}$ & 2.713 & .831 & 1. 160 & 1. 991 & & & & +.722 & 0 \\
\hline $\begin{array}{l}\text { Total . } \\
\text { Total }\end{array}$ & 14. 066 & 5. 504 & 6.5 & 12.100 & 39.13 & 46.89 & 86.02 & +1.966 & 0 \\
\hline Average......... & 2.813 & 1.101 & 1. 319 & 2.420 & & ......... & ......... & +.393 & 0 \\
\hline $\begin{array}{l}\text { Entire after period: } \\
\text { Total } . . . . . . . . \\
\text { Average ........ }\end{array}$ & $\begin{array}{r}27.631 \\
2.763\end{array}$ & $\begin{array}{r}9.657 \\
.966\end{array}$ & $\begin{array}{r}12.398 \\
1.240\end{array}$ & $\begin{array}{r}22.055 \\
2.206\end{array}$ & 34.95 & 44.87 & $\begin{array}{r}79.82 \\
\cdots\end{array}$ & $\begin{array}{l}+5.576 \\
+\quad .557\end{array}$ & $\begin{array}{l}0 \\
0\end{array}$ \\
\hline
\end{tabular}


TABLE XV.-Phosphoric acid balances for Series VI-Continued.

[Averages are per day.]

No. 8.

\begin{tabular}{|c|c|c|c|c|c|c|c|c|c|}
\hline Period. & In food. & In feces. & In urine. & $\begin{array}{l}4 \\
\text { In feces } \\
\text { and } \\
\text { urine. } \\
(2+3)\end{array}$ & 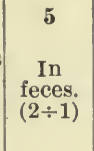 & $\underset{\substack{\text { In } \\
(3 \div 1)}}{6}$ & \begin{tabular}{|c|}
7 \\
In feces \\
and \\
urine. \\
$(4 \div 1)$
\end{tabular} & $\begin{array}{c}8 \\
\text { Balance. } \\
(1-4)\end{array}$ & $\begin{array}{l}\mathbf{9} \\
\text { Sali- } \\
\text { cylic } \\
\text { acid } \\
\text { admin- } \\
\text { istered. }\end{array}$ \\
\hline Fore period. & & & & & & & & & \\
\hline 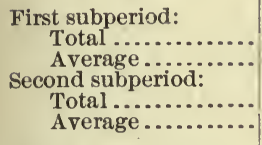 & $\begin{array}{r}\text { Grams. } \\
14.214 \\
2.843 \\
13.065 \\
2.613\end{array}$ & $\begin{array}{r}\text { Grams. } \\
4.014 \\
.803\end{array}$ & $\begin{array}{r}\text { Grams. } \\
8.818 \\
1.764\end{array}$ & $\begin{array}{r}\text { Grams. } \\
12.832 \\
2.566 \\
14.446 \\
2.889\end{array}$ & $\begin{array}{c}\text { Per ct. } \\
28.24 \\
\ldots \\
43.67 \\
\cdots\end{array}$ & $\begin{array}{c}\text { Per ct. } \\
62.04 \\
\cdots \\
66.90 \\
\cdots\end{array}$ & $\begin{array}{c}\text { Per ct. } \\
90.28 \\
110.57 \\
\cdots\end{array}$ & $\begin{array}{l}\text { Grams. } \\
+1.382 \\
+.277 \\
-1.381 \\
-.277\end{array}$ & $\begin{array}{c}\text { Grams. } \\
0 \\
0 \\
0 \\
0\end{array}$ \\
\hline $\begin{array}{l}\text { Entire fore period: } \\
\quad \text { Total } \ldots . . . \ldots \ldots \\
\text { Average } . . . \ldots \ldots\end{array}$ & $\begin{array}{r}27.279 \\
2.728\end{array}$ & $\begin{array}{r}9.720 \\
.972\end{array}$ & $\begin{array}{r}17.558 \\
1.756\end{array}$ & $\begin{array}{r}27.278 \\
2.728\end{array}$ & $\begin{array}{r}35.63 \\
\ldots . . \ldots\end{array}$ & $\begin{array}{r}61.36 \\
\ldots \ldots \ldots\end{array}$ & $\begin{array}{c}100.00 \\
\cdots \ldots\end{array}$ & $\begin{array}{l}+.001 \\
\pm .000\end{array}$ & $\begin{array}{l}0 \\
0\end{array}$ \\
\hline Preservative period. & & & & & & & & & \\
\hline $\begin{array}{l}\text { First subperiod: } \\
\text { Total ........... } \\
\text { Average....... }\end{array}$ & $\begin{array}{r}14.739 \\
2.948\end{array}$ & $\begin{array}{l}5.119 \\
1.024\end{array}$ & $\begin{array}{l}8.882 \\
1.776\end{array}$ & $\begin{array}{r}14.001 \\
2.800\end{array}$ & 34.73 & 60.26 & $\begin{array}{r}94.99 \\
\ldots\end{array}$ & $\begin{array}{r}+.738 \\
+.148\end{array}$ & $\begin{array}{r}1.05 \\
.21\end{array}$ \\
\hline $\begin{array}{l}\text { Second subperiod: } \\
\text { Total ............... } \\
\text { Average ........... }\end{array}$ & $\begin{array}{r}14.596 \\
2.919\end{array}$ & $\begin{array}{l}6.091 \\
1.218\end{array}$ & $\begin{array}{l}9.500 \\
1.900\end{array}$ & $\begin{array}{r}15.591 \\
3.118\end{array}$ & $\begin{array}{r}41.73 \\
\cdots . . . . . .\end{array}$ & $\begin{array}{c}65.09 \\
\ldots . .\end{array}$ & $\begin{array}{c}106.82 \\
\ldots \ldots \ldots\end{array}$ & $\begin{array}{r}-.995 \\
-.199\end{array}$ & $\begin{array}{r}2.10 \\
.42\end{array}$ \\
\hline $\begin{array}{l}\text { Third subperiod: } \\
\text { Total ................. } \\
\text { Average } . . .\end{array}$ & $\begin{array}{r}14.510 \\
2.902\end{array}$ & $\begin{array}{r}3.681 \\
.736\end{array}$ & $\begin{array}{l}8.820 \\
1.764\end{array}$ & $\begin{array}{r}12.501 \\
2.500\end{array}$ & $\begin{array}{r}25.37 \\
\ldots . . . . .\end{array}$ & $\begin{array}{r}60.79 \\
\ldots . . .\end{array}$ & $\begin{array}{r}86.15 \\
\ldots . . .\end{array}$ & $\begin{array}{r}+2.009 \\
+\quad .402\end{array}$ & $\begin{array}{r}3.70 \\
.74\end{array}$ \\
\hline 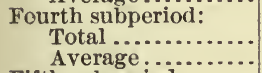 & $\begin{array}{r}14.787 \\
2.957\end{array}$ & $\begin{array}{l}5.474 \\
1.095\end{array}$ & $\begin{array}{r}a 8.888 \\
1.778\end{array}$ & $\begin{array}{r}14.362 \\
2.872\end{array}$ & $\begin{array}{r}37.02 \\
\ldots \ldots . . .\end{array}$ & $\begin{array}{r}60.11 \\
\ldots \ldots \ldots .\end{array}$ & $\begin{array}{r}97.13 \\
\ldots . . . .\end{array}$ & $\begin{array}{r}+.425 \\
+.085\end{array}$ & $\begin{array}{l}6.00 \\
1.20\end{array}$ \\
\hline $\begin{array}{l}\text { Fifth subperiod: } \\
\text { Total ................... } \\
\text { Average }\end{array}$ & $\begin{array}{r}15.671 \\
3.134\end{array}$ & $\begin{array}{r}3.377 \\
.675\end{array}$ & $\begin{array}{l}8.432 \\
1.686\end{array}$ & $\begin{array}{r}11.809 \\
2.362\end{array}$ & $\begin{array}{r}21.55 \\
\ldots \ldots \ldots\end{array}$ & $\begin{array}{r}53.81 \\
\ldots .\end{array}$ & $\begin{array}{r}75.36 \\
\ldots .\end{array}$ & $\begin{array}{r}+3.862 \\
+\quad .772\end{array}$ & $\begin{array}{l}8.00 \\
1.60\end{array}$ \\
\hline $\begin{array}{l}\text { Sixth subperiod: } \\
\text { Total ................. } \\
\text { Average } . . . \ldots \ldots \ldots\end{array}$ & $\begin{array}{r}14.546 \\
2.909\end{array}$ & $\begin{array}{r}4.521 \\
.904\end{array}$ & $\begin{array}{l}7.783 \\
1.557\end{array}$ & $\begin{array}{r}12.304 \\
2.461\end{array}$ & $\begin{array}{r}31.08 \\
\cdots \cdots\end{array}$ & $\begin{array}{r}53.51 \\
\ldots . . .\end{array}$ & $\begin{array}{r}84.59 \\
\ldots \ldots \ldots \\
\ldots \ldots\end{array}$ & $\begin{array}{r}+2.242 \\
+.448\end{array}$ & $\begin{array}{r}10.00 \\
2.00\end{array}$ \\
\hline $\begin{array}{l}\text { Entire preservative } \\
\text { period: } \\
\text { Total ................. } \\
\text { Average .......... }\end{array}$ & $\begin{array}{r}88.849 \\
2.962\end{array}$ & $\begin{array}{r}28.263 \\
.942 \\
\end{array}$ & $\begin{array}{r}52.305 \\
1.744\end{array}$ & $\begin{array}{r}80.568 \\
2.686 \\
\end{array}$ & $\begin{array}{r}31.81 \\
\cdots \cdots \cdots \\
\end{array}$ & $\begin{array}{r}58.87 \\
\ldots \ldots \ldots \\
\end{array}$ & $\begin{array}{r}90.68 \\
\ldots \ldots\end{array}$ & $\begin{array}{r}+8.281 \\
+\quad .276 \\
\end{array}$ & $\begin{array}{r}30.85 \\
1.03\end{array}$ \\
\hline $\begin{array}{c}\text { After period. } \\
\text { First subperiod: } \\
\text { Total ................ } \\
\text { Average .......... }\end{array}$ & $\begin{array}{r}14.010 \\
2.802\end{array}$ & $\begin{array}{l}5.878 \\
1.176\end{array}$ & $\begin{array}{l}7.387 \\
1.477\end{array}$ & $\begin{array}{r}13.265 \\
2.653\end{array}$ & $\begin{array}{r}41.96 \\
4 . . .\end{array}$ & $\begin{array}{r}52.73 \\
\ldots .\end{array}$ & $\begin{array}{r}94.68 \\
\ldots . . .\end{array}$ & $\begin{array}{r}+.745 \\
+.149\end{array}$ & $\begin{array}{l}0 \\
0\end{array}$ \\
\hline 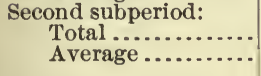 & $\begin{array}{r}14.994 \\
2.999\end{array}$ & $\begin{array}{l}5.435 \\
1.087\end{array}$ & $\begin{array}{l}7.938 \\
1.588\end{array}$ & $\begin{array}{r}13.373 \\
2.675\end{array}$ & $\begin{array}{r}36.25 \\
\cdots \cdots\end{array}$ & $\begin{array}{r}52.94 \\
\ldots \ldots\end{array}$ & $\begin{array}{r}89.19 \\
\ldots . .\end{array}$ & $\begin{array}{r}+1.621 \\
+.324\end{array}$ & $\begin{array}{l}0 \\
0\end{array}$ \\
\hline $\begin{array}{l}\text { Entire after period: } \\
\text { Total ................ } \\
\text { Average .......... }\end{array}$ & $\begin{array}{r}29.004 \\
2.900\end{array}$ & $\begin{array}{r}11.313 \\
1.131\end{array}$ & $\begin{array}{r}15.325 \\
1.533\end{array}$ & $\begin{array}{r}26.638 \\
2.664\end{array}$ & $\begin{array}{r}39.00 \\
\ldots . . .\end{array}$ & 52.84 & 91.84 & $\begin{array}{l}+2.366 \\
+.236\end{array}$ & $\begin{array}{l}0 \\
0\end{array}$ \\
\hline
\end{tabular}

$a$ Daily average added in order to complete record. 
TABLE XV.-Phosphoric acid balances for Series VI-Continued.

[Averages are per day.]

No. 9.

\begin{tabular}{|c|c|c|c|c|c|c|c|c|c|}
\hline Period. & In food. & In feces. & In urine. & $\begin{array}{c}\mathbf{4} \\
\text { In feces } \\
\text { and } \\
\text { urine. } \\
(2+3)\end{array}$ & $\begin{array}{c}5 \\
\text { In } \\
\text { feces. } \\
(2 \div 1)\end{array}$ & $\begin{array}{c}\mathbf{6} \\
\underset{\text { In }}{\text { urine. }} \\
(3 \div 1)\end{array}$ & $\begin{array}{c}7 \\
\text { In feces } \\
\text { and } \\
\text { urine. } \\
(4 \div 1)\end{array}$ & $\begin{array}{c}8 \\
\begin{array}{c}\text { Balance. } \\
(1-4)\end{array}\end{array}$ & $\begin{array}{c}\mathbf{9} \\
\text { Sali- } \\
\text { cylic } \\
\text { acid } \\
\text { admin- } \\
\text { istered. }\end{array}$ \\
\hline \multicolumn{10}{|l|}{ Fore period. } \\
\hline $\begin{array}{l}\text { First subperiod: } \\
\text { Total .............. } \\
\text { Average ......... }\end{array}$ & $\begin{array}{r}\text { Grams. } \\
23.981 \\
4.796\end{array}$ & $\begin{array}{r}\text { Grams. } \\
4.020 \\
.804\end{array}$ & $\begin{array}{r}\text { Grams. } \\
11.857 \\
2.371\end{array}$ & $\begin{array}{c}\text { Grams. } \\
15.877 \\
3.175\end{array}$ & $\begin{array}{c}\text { Per } \text { ct. } \\
16.76 \\
\ldots\end{array}$ & $\begin{array}{l}\text { Perct. } \\
49.44 \\
4.4 .\end{array}$ & $\begin{array}{l}\text { Per ct. } \\
66.21 \\
.\end{array}$ & $\begin{array}{r}\text { Grams. } \\
+8.104 \\
+1.621\end{array}$ & $\begin{array}{c}\text { Grams. } \\
0 \\
0\end{array}$ \\
\hline Second subperiod: & & & & & & & & & \\
\hline $\begin{array}{l}\text { Total ................... } \\
\text { Average }\end{array}$ & $\begin{array}{r}24.313 \\
4.869\end{array}$ & $\begin{array}{l}6.122 \\
1.224\end{array}$ & $\begin{array}{r}10.410 \\
2.082\end{array}$ & $\begin{array}{r}16.532 \\
3.306\end{array}$ & $\begin{array}{r}25.15 \\
\cdots\end{array}$ & $\begin{array}{r}42.76 \\
\cdots \ldots \ldots\end{array}$ & $\begin{array}{r}67.91 \\
\ldots . . .\end{array}$ & $\begin{array}{r}+7.811 \\
+1.563\end{array}$ & $\begin{array}{l}0 \\
0\end{array}$ \\
\hline $\begin{array}{l}\text { Entire fore period: } \\
\text { Total ............... } \\
\text { Average....... }\end{array}$ & $\begin{array}{r}48.324 \\
4.832\end{array}$ & $\begin{array}{r}10.142 \\
1.014\end{array}$ & $\begin{array}{r}22.267 \\
2.227\end{array}$ & $\begin{array}{r}32.409 \\
3.211\end{array}$ & $\begin{array}{r}20.99 \\
\cdots \cdots \\
\end{array}$ & $\begin{array}{l}46.08 \\
\ldots \ldots\end{array}$ & $\begin{array}{r}67.07 \\
\ldots \ldots \ldots \\
\end{array}$ & $\begin{array}{r}+15.915 \\
+1.591 \\
\end{array}$ & $\begin{array}{l}0 \\
0\end{array}$ \\
\hline \multicolumn{10}{|l|}{ Preservative period. } \\
\hline $\begin{array}{l}\text { First subperiod: } \\
\text { Total......... } \\
\text { Average..... }\end{array}$ & $\begin{array}{r}25.052 \\
5.010\end{array}$ & $\begin{array}{r}11.610 \\
2.322\end{array}$ & $\begin{array}{r}11.750 \\
2.350\end{array}$ & $\begin{array}{r}23.360 \\
4.672\end{array}$ & $\begin{array}{l}46.34 \\
\ldots . . .\end{array}$ & 46.90 & 93.25 & $\begin{array}{r}+1.692 \\
+\quad .338\end{array}$ & $\begin{array}{r}1.05 \\
.21\end{array}$ \\
\hline $\begin{array}{l}\text { Second subperiod: } \\
\text { Total } \ldots \ldots \ldots \ldots\end{array}$ & & & & & & & & & \\
\hline $\begin{array}{l}\text { Total } \\
\text { Avera }\end{array}$ & $\begin{array}{r}24.095 \\
4.819\end{array}$ & $\begin{array}{l}7.838 \\
1.568\end{array}$ & $\begin{array}{r}11.997 \\
2.399\end{array}$ & $\begin{array}{r}19.835 \\
3.967\end{array}$ & $\begin{array}{l}32.53 \\
\ldots . . .\end{array}$ & $\begin{array}{r}49.79 \\
\cdots\end{array}$ & $\begin{array}{l}82.32 \\
\ldots .\end{array}$ & $\begin{array}{r}+4.260 \\
+\quad .852\end{array}$ & $\begin{array}{r}2.10 \\
.42\end{array}$ \\
\hline $\begin{array}{l}\text { Third subperiod: } \\
\text { Total }\end{array}$ & & & & & & & & & \\
\hline $\begin{array}{l}\text { Total ............... } \\
\text { Average ...... }\end{array}$ & $\begin{array}{r}24.818 \\
4.964\end{array}$ & $\begin{array}{l}7.195 \\
1.439\end{array}$ & $\begin{array}{r}12.249 \\
2.450\end{array}$ & $\begin{array}{r}19.444 \\
3.889\end{array}$ & 28.99 & 49.36 & 78.35 & $\begin{array}{r}+5.374 \\
+1.075\end{array}$ & $\begin{array}{r}3.70 \\
.74\end{array}$ \\
\hline Fourth subperiod: & & & & & & & & & \\
\hline $\begin{array}{l}\text { Total .............. } \\
\text { Average..... }\end{array}$ & $\begin{array}{r}25.591 \\
5.118\end{array}$ & $\begin{array}{r}4.812 \\
.962\end{array}$ & $\begin{array}{r}13.981 \\
2.796\end{array}$ & $\begin{array}{r}18.793 \\
3.759\end{array}$ & $\begin{array}{r}18.80 \\
\ldots \ldots \ldots\end{array}$ & 54.63 & 73.44 & $\begin{array}{r}+6.798 \\
+1.359\end{array}$ & $\begin{array}{l}6.00 \\
1.20\end{array}$ \\
\hline Fifth subperiod: & & & & & & & & & \\
\hline $\begin{array}{l}\text { Total. } \\
\text { Avera }\end{array}$ & $\begin{array}{r}25.776 \\
5.155\end{array}$ & $\begin{array}{l}9.892 \\
1.978\end{array}$ & $\begin{array}{r}10.919 \\
2.184\end{array}$ & $\begin{array}{r}20.811 \\
4.162\end{array}$ & $\begin{array}{r}38.38 \\
\ldots .6 .\end{array}$ & $\begin{array}{r}42.36 \\
\cdots\end{array}$ & $\begin{array}{r}80.74 \\
\ldots . . .\end{array}$ & $\begin{array}{r}+4.965 \\
+\quad .993\end{array}$ & $\begin{array}{l}8.00 \\
1.60\end{array}$ \\
\hline $\begin{array}{l}\text { Sixth sub } \\
\text { Total }\end{array}$ & & & & 20.508 & 30.76 & 51.14 & 81.91 & +4.530 & 10.00 \\
\hline Average & 008 & 1.541 & & 4.102 & .... & & ......... & +.906 & 2.00 \\
\hline \multicolumn{10}{|l|}{$\begin{array}{l}\text { Entire preservative } \\
\text { period: }\end{array}$} \\
\hline 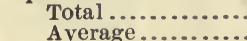 & $\begin{array}{r}150.370 \\
5.012\end{array}$ & $\begin{array}{r}49.050 \\
1.635\end{array}$ & $\begin{array}{r}73.701 \\
2.457\end{array}$ & $\begin{array}{r}122.751 \\
4.09^{\circ}\end{array}$ & 32.62 & 49.01 & 81.63 & $\begin{array}{r}+27.619 \\
+\quad .920\end{array}$ & $\begin{array}{r}30.85 \\
1.03\end{array}$ \\
\hline & & & & & & & & & \\
\hline After period. & & 1. & & & & & & & \\
\hline $\begin{array}{l}\text { First subperiod: } \\
\quad \text { Total ................... } \\
\text { Average......... }\end{array}$ & $\begin{array}{r}25.380 \\
5.076\end{array}$ & $\begin{array}{r}3.271 \\
.654\end{array}$ & $\begin{array}{r}10.423 \\
2.085\end{array}$ & $\begin{array}{r}13.694 \\
2.739\end{array}$ & $\begin{array}{r}12.89 \\
\cdots \ldots . .\end{array}$ & 41.07 & $\begin{array}{r}53.96 \\
\ldots \ldots\end{array}$ & $\begin{array}{r}+11: 686 \\
+2.337\end{array}$ & $\begin{array}{l}0 \\
0\end{array}$ \\
\hline $\begin{array}{l}\text { Second subperiod: } \\
\text { Total } \ldots \ldots \ldots \ldots\end{array}$ & & & & & & & & & \\
\hline 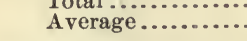 & 5.146 & $\begin{array}{r}2.018 \\
2.204\end{array}$ & $\begin{array}{r}12.531 \\
2.506\end{array}$ & $\begin{array}{r}20.749 \\
4.710\end{array}$ & $\begin{array}{r}42.02 \\
\cdots \ldots \ldots\end{array}$ & (10.11 & ........ & $+\quad .436$ & 0 \\
\hline $\begin{array}{r}\text { Entire after period: } \\
\text { Total ............ } \\
\text { Average ....... }\end{array}$ & $\begin{array}{r}51.108 \\
5.111\end{array}$ & $\begin{array}{r}14.289 \\
1.429\end{array}$ & $\begin{array}{r}22.954 \\
2.295\end{array}$ & $\begin{array}{r}37.243 \\
3.724\end{array}$ & 27.96 & 44.91 & 72.87 & $\begin{array}{r}+13.865 \\
+1.387\end{array}$ & $\begin{array}{l}0 \\
0\end{array}$ \\
\hline
\end{tabular}


SALICYLIC ACID AND SALICYLATES.

TABLe XV.-Phosphoric acid balances for Series VI-Continued.

[Avcrages are per day.]

Nò. 10 .

\begin{tabular}{|c|c|c|c|c|c|c|c|c|c|}
\hline Period. & $\begin{array}{c}1 \\
\text { In food. }\end{array}$ & $\begin{array}{c}2 \\
\text { In feces. }\end{array}$ & $\begin{array}{c}3 \\
\text { In urine. }\end{array}$ & $\begin{array}{l}\quad 4 \\
\text { In feces } \\
\text { and } \\
\text { urine. } \\
(2+3)\end{array}$ & $\begin{array}{c}5 \\
\text { In } \\
\text { feces. } \\
(2 \div 1)\end{array}$ & $\begin{array}{c}\mathbf{6} \\
\text { In } \\
\text { urine. } \\
(3 \div 1)\end{array}$ & $\begin{array}{c}17 \\
\text { In feces } \\
\text { and } \\
\text { urine. } \\
(4 \div 1)\end{array}$ & $\mid \begin{array}{c}8 \\
\text { Balance. } \\
(1-4)\end{array}$ & $\begin{array}{c}\mathbf{9} \\
\text { Sali- } \\
\text { cylic } \\
\text { acid } \\
\text { admin- } \\
\text { istered. }\end{array}$ \\
\hline Fore period. & & & & & & & & & \\
\hline 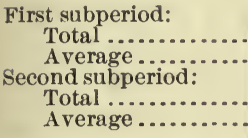 & $\begin{array}{r}\text { Grams. } \\
22.277 \\
4.455\end{array}$ & $\begin{array}{r}\text { Grams. } \\
6.672 \\
1.334 \\
4.200 \\
.840\end{array}$ & $\begin{array}{r}\text { Grams. } \\
10.727 \\
2.145 \\
11.600 \\
2.320\end{array}$ & $\begin{array}{r}\text { Grams. } \\
17.399 \\
3.480 \\
15.800 \\
3.160\end{array}$ & $\begin{array}{c}\text { Per ct. } \\
29.95 \\
\cdots \\
20.04 \\
2 . . .\end{array}$ & $\begin{array}{r}\text { Per ct. } \\
48.15 \\
\ldots \\
55.35\end{array}$ & $\begin{array}{c}\text { Per ct. } \\
78.10 \\
.\end{array}$ & $\begin{array}{r}\text { Grams } \\
+\quad 4.878 \\
+\quad .975 \\
+\quad 5.158 \\
+1.032\end{array}$ & $\begin{array}{c}\text { Grams. } \\
0 \\
0 \\
0 \\
0\end{array}$ \\
\hline 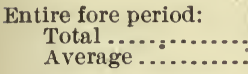 & $\begin{array}{r}43.235 \\
4.324\end{array}$ & $\begin{array}{r}10.872 \\
1.087\end{array}$ & $\begin{array}{r}22.327 \\
2.233\end{array}$ & $\begin{array}{r}33.199 \\
3.320\end{array}$ & 25.15 & 51.64 & 76.79 & $\begin{array}{r}+10.036 \\
+1.004\end{array}$ & $\begin{array}{l}0 \\
0\end{array}$ \\
\hline Preservative period. & & & & & & & & & \\
\hline 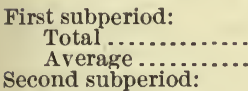 & $\begin{array}{r}22.883 \\
4.577\end{array}$ & $\begin{array}{l}5.843 \\
1.169\end{array}$ & $\begin{array}{r}10.851 \\
2.170\end{array}$ & $\begin{array}{r}16.694 \\
3.339\end{array}$ & 25.53 & 47.42 & 72.95 & $\begin{array}{r}+\quad 6.189 \\
+\quad 1.238\end{array}$ & $\begin{array}{r}1.05 \\
.21\end{array}$ \\
\hline 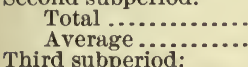 & $\begin{array}{r}22.599 \\
4.520\end{array}$ & $\begin{array}{l}5.222 \\
1.044\end{array}$ & $\begin{array}{r}12.768 \\
2.554\end{array}$ & $\begin{array}{r}17.990 \\
3.598\end{array}$ & $\begin{array}{l}23.11 \\
\ldots . . .\end{array}$ & 56.50 & 79.61 & $\begin{array}{r}+\quad 4.609 \\
+\quad .922\end{array}$ & $\begin{array}{r}2.10 \\
.42\end{array}$ \\
\hline od: & $\begin{array}{r}22.319 \\
4.464\end{array}$ & $\begin{array}{l}6.068 \\
1.214\end{array}$ & $\begin{array}{r}11.612 \\
2.322\end{array}$ & $\begin{array}{r}17.680 \\
3.536\end{array}$ & $\begin{array}{r}27.18 \\
\ldots . . .\end{array}$ & 52.03 & 79.22 & $\begin{array}{r}+4.639 \\
+\quad .928\end{array}$ & $\begin{array}{r}3.70 \\
.74\end{array}$ \\
\hline $\begin{array}{l}\text { Fourth subperiod: } \\
\text { Total ....................... } \\
\text { Average }\end{array}$ & $\begin{array}{r}22.680 \\
4.536\end{array}$ & $\begin{array}{r}4.434 \\
.887\end{array}$ & $\begin{array}{r}11.513 \\
2.303\end{array}$ & $\begin{array}{r}15.947 \\
3.189\end{array}$ & $\begin{array}{l}19.55 \\
\cdots \cdots . . .\end{array}$ & 50.76 & 70.31 & $\begin{array}{l}+6.733 \\
+1.347\end{array}$ & $\begin{array}{l}6.00 \\
1.20\end{array}$ \\
\hline $\begin{array}{l}\text { Fifth subperiod: } \\
\text { Total ............ } \\
\text { Average }\end{array}$ & $\begin{array}{r}23.925 \\
4.785\end{array}$ & $\begin{array}{r}3.936 \\
.787\end{array}$ & $\begin{array}{r}10.823 \\
2.165\end{array}$ & $\begin{array}{r}14.759 \\
2.952\end{array}$ & $\begin{array}{r}16.45 \\
\cdots \cdots . . .\end{array}$ & 45.24 & 61.69 & $\begin{array}{r}+9.166 \\
+1.833\end{array}$ & $\begin{array}{l}8.00 \\
1.60\end{array}$ \\
\hline 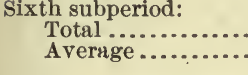 & $\begin{array}{r}23.103 \\
4.621\end{array}$ & $\begin{array}{r}1.704 \\
.341\end{array}$ & $\begin{array}{l}9.744 \\
1.949\end{array}$ & $\begin{array}{r}11.448 \\
2.290\end{array}$ & 7.38 & 42.18 & 49.55 & $\begin{array}{r}+11.655 \\
+2.331 \\
\end{array}$ & $\begin{array}{r}10.00 \\
2.00\end{array}$ \\
\hline 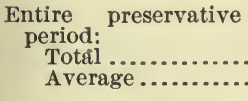 & $\begin{array}{r}137.509 \\
4.584 \\
\end{array}$ & $\begin{array}{r}27.207 \\
.907 \\
\end{array}$ & $\begin{array}{r}67.311 \\
2.244\end{array}$ & $\begin{array}{r}94.518 \\
3.151\end{array}$ & 19.79 & 48.95 & 68.74 & $\begin{array}{r}+42.991 \\
+1.433 \\
\end{array}$ & $\begin{array}{r}30.85 \\
1.03 \\
\end{array}$ \\
\hline $\begin{array}{l}\text { First subperiod: } a \\
\quad \text { Total } \ldots . . . . . . \\
\text { Average ........ }\end{array}$ & $\begin{array}{r}22.591 \\
4.518\end{array}$ & $\begin{array}{l}5.422 \\
1.084\end{array}$ & $\begin{array}{l}9.498 \\
1.900\end{array}$ & $\begin{array}{r}14.920 \\
2.984\end{array}$ & 24.00 & 42.04 & 66.04 & $\begin{array}{r}7.671 \\
+1.534\end{array}$ & $\begin{array}{l}0 \\
0\end{array}$ \\
\hline
\end{tabular}

$a$ No second after subperiod; subject ill. 
TABle XV.-Phosphoric acid balances for Series. VI-Continued

[Averages are per day.]

No. 11 .

\begin{tabular}{|c|c|c|c|c|c|c|c|c|c|}
\hline Period. & In food. & In feces. & In urine. & $\begin{array}{c}4 \\
\text { In feces } \\
\text { and } \\
\text { urine. } \\
(2+3)\end{array}$ & $\begin{array}{c}5 \\
\text { In } \\
\text { feces. } \\
(2 \div 1)\end{array}$ & $\begin{array}{c}6 \\
\text { In } \\
\text { urine. } \\
(3 \div 1)\end{array}$ & $\begin{array}{c}7 \\
\text { In feces } \\
\text { and } \\
\text { urine. } \\
(4 \div 1)\end{array}$ & $\begin{array}{c}\text { Balance. } \\
(1-4)\end{array}$ & $\begin{array}{c}9 \\
\text { Sali- } \\
\text { cylic } \\
\text { acid } \\
\text { admin- } \\
\text { istered. }\end{array}$ \\
\hline \multicolumn{10}{|l|}{ Fore period. } \\
\hline 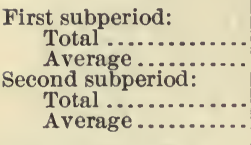 & $\begin{array}{r}\text { Grams. } \\
21.970 \\
4.394 \\
\\
21.762 \\
4.352\end{array}$ & $\begin{array}{r}\text { Grams. } \\
7.691 \\
1.538\end{array}$ & $\begin{array}{r}\text { Grams. } \\
\text { a 14. } 139 \\
2.828 \\
14.489 \\
2.898\end{array}$ & $\begin{array}{r}\text { Grams. } \\
21.830 \\
4.366\end{array}$ & $\begin{array}{r}\text { Per ct. } \\
35.01 \\
\cdots \cdots \\
36.69 \\
\ldots \ldots\end{array}$ & $\begin{array}{r}\text { Per ct. } \\
64.36 \\
\cdots\end{array}$ & $\begin{array}{r}\text { Per ct. } \\
99.36 \\
\cdots\end{array}$ & $\begin{array}{r}\text { Grams. } \\
+\quad 0.140 \\
+\quad .028 \\
-\quad .712 \\
-\quad .143 \\
\end{array}$ & $\begin{array}{c}\text { Grams. } \\
0 \\
0\end{array}$ \\
\hline $\begin{array}{l}\text { Entire fore period: } \\
\quad \text { Total ................ } \\
\text { Average .......... }\end{array}$ & $\begin{array}{r}43.732 \\
4.373\end{array}$ & $\begin{array}{r}15.676 \\
1.568\end{array}$ & $\begin{array}{r}28.628 \\
2.863 \\
\end{array}$ & $\begin{array}{r}44.304 \\
4.430 \\
\end{array}$ & $\begin{array}{r}35.85 \\
\cdots \cdots . . \\
\end{array}$ & $\begin{array}{r}65.46 \\
\ldots \ldots \ldots \\
\end{array}$ & $\begin{array}{r}101.31 \\
\ldots \ldots \ldots\end{array}$ & $\begin{array}{r}-\quad .572 \\
-\quad .057 \\
\end{array}$ & $\begin{array}{l}0 \\
0\end{array}$ \\
\hline \multicolumn{10}{|l|}{ Preservative period. } \\
\hline $\begin{array}{l}\text { First subperiod: } \\
\text { Total ............... } \\
\text { Average .......... } \\
\text { Second subperiod: }\end{array}$ & $\begin{array}{r}22.991 \\
4.598\end{array}$ & $\begin{array}{l}7.781 \\
1.556\end{array}$ & $\begin{array}{r}13.572 \\
2.714\end{array}$ & $\begin{array}{r}21.353 \\
4.271\end{array}$ & $\begin{array}{r}33.84 \\
\cdots \ldots\end{array}$ & $\begin{array}{r}59.03 \\
\cdots \cdots . . .\end{array}$ & $\begin{array}{r}92.88 \\
\ldots \ldots \ldots \\
\ldots \ldots . .\end{array}$ & $\begin{array}{r}+1.638 \\
+\quad .327\end{array}$ & $\begin{array}{r}1.05 \\
.21\end{array}$ \\
\hline $\begin{array}{l}\text { Total ..................... } \\
\text { Average } \\
\text { Third subperiod: }\end{array}$ & $\begin{array}{r}22.046 \\
4.409\end{array}$ & $\begin{array}{l}8.012 \\
1.602\end{array}$ & $\begin{array}{r}12.829 \\
2.566\end{array}$ & $\begin{array}{r}20.841 \\
4.168\end{array}$ & $\begin{array}{r}36.34 \\
\cdots \cdots\end{array}$ & $\begin{array}{r}58.19 \\
\ldots . . . . .\end{array}$ & $\begin{array}{r}94.53 \\
\ldots \ldots . .\end{array}$ & $\begin{array}{r}+1.205 \\
+\quad .241\end{array}$ & $\begin{array}{r}2.10 \\
.42\end{array}$ \\
\hline 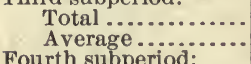 & $\begin{array}{r}22.757 \\
4.551\end{array}$ & $\begin{array}{l}7.378 \\
1.476\end{array}$ & $\begin{array}{r}12.616 \\
2.523\end{array}$ & $\begin{array}{r}19.994 \\
3.999\end{array}$ & $\begin{array}{r}32.42 \\
\ldots \ldots\end{array}$ & $\begin{array}{r}55.44 \\
\cdots . . . . .\end{array}$ & $\begin{array}{r}87.86 \\
\ldots \ldots \ldots\end{array}$ & $\begin{array}{r}+2.763 \\
+\quad .552\end{array}$ & $\begin{array}{r}3.70 \\
.74\end{array}$ \\
\hline 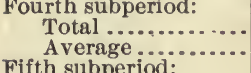 & $\begin{array}{r}23.065 \\
4.613\end{array}$ & $\begin{array}{l}7.433 \\
1.487\end{array}$ & $\begin{array}{r}11.968 \\
2.394\end{array}$ & $\begin{array}{r}19.401 \\
3.880\end{array}$ & $\begin{array}{r}32.23 \\
\cdots \cdots\end{array}$ & $\begin{array}{r}51.89 \\
\ldots \ldots\end{array}$ & $\begin{array}{c}84.11 \\
\ldots \ldots\end{array}$ & $\begin{array}{r}+3.664 \\
+\quad .733\end{array}$ & $\begin{array}{l}600 \\
1.20\end{array}$ \\
\hline $\begin{array}{r}\text { Total } \\
\text { Average............................ } \\
\text { Sixth subperiod: }\end{array}$ & $\begin{array}{r}23.394 \\
4.679\end{array}$ & $\begin{array}{l}7.253 \\
1.451\end{array}$ & $\begin{array}{r}12.792 \\
2.558\end{array}$ & $\begin{array}{r}20.045 \\
4.009\end{array}$ & $\begin{array}{r}31.00 \\
\cdots\end{array}$ & $\begin{array}{r}54.68 \\
\ldots \ldots \ldots\end{array}$ & $\begin{array}{r}85.68 \\
\ldots \ldots \ldots\end{array}$ & $\begin{array}{r}+3.349 \\
+\quad .670\end{array}$ & $\begin{array}{l}8.00 \\
1.60\end{array}$ \\
\hline $\begin{array}{l}\text { Sixth subperiod: } \\
\text { Total ................ } \\
\text { Average ........... }\end{array}$ & $\begin{array}{r}22.935 \\
4.587\end{array}$ & $\begin{array}{l}8.366 \\
1.673\end{array}$ & $\begin{array}{r}10.648 \\
2.130\end{array}$ & $\begin{array}{r}19.014 \\
3.803 \\
\end{array}$ & $\begin{array}{r}36.48 \\
\ldots . . .\end{array}$ & $\begin{array}{r}46.43 \\
\ldots \ldots \ldots\end{array}$ & $\begin{array}{r}82.90 \\
\ldots\end{array}$ & $\begin{array}{r}+3.921 \\
+\quad .781 \\
\end{array}$ & $\begin{array}{r}10.00 \\
2.00\end{array}$ \\
\hline 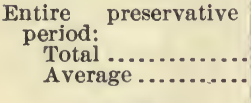 & $\begin{array}{r}137.188 \\
4.573 \\
\end{array}$ & $\begin{array}{r}46.223 . \\
1.541\end{array}$ & $\begin{array}{r}74.425 \\
2.481 \\
\end{array}$ & $\begin{array}{r}120.648 \\
4.022 \\
\end{array}$ & $\begin{array}{r}33.69 \\
\ldots \ldots . .\end{array}$ & $\begin{array}{r}54.25 \\
\ldots \ldots \ldots \\
\end{array}$ & $\begin{array}{r}87.94 \\
\ldots . . \ldots \\
\end{array}$ & $\begin{array}{r}+16.540 \\
+\quad .551 \\
\end{array}$ & $\begin{array}{r}30.85 \\
1.03 \\
\end{array}$ \\
\hline After period. & & & & & & & & & \\
\hline $\begin{array}{l}\text { First subperiod: } \\
\text { Total .............. } \\
\text { Average .......... } \\
\text { Second subperiod: }\end{array}$ & $\begin{array}{r}22.447 \\
4.489\end{array}$ & $\begin{array}{l}8.606 \\
1.721\end{array}$ & $\begin{array}{r}10.377 \\
2.075\end{array}$ & $\begin{array}{r}18.983 \\
3.797\end{array}$ & $\begin{array}{r}38.34 \\
\ldots . . .\end{array}$ & $\begin{array}{r}46.23 \\
\cdots \cdot . . .\end{array}$ & $\begin{array}{r}84.57 \\
\ldots . . .\end{array}$ & $\begin{array}{r}+3.464 \\
+\quad .692\end{array}$ & $\begin{array}{l}0 \\
0\end{array}$ \\
\hline 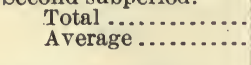 & $\begin{array}{r}23.273 \\
4.655\end{array}$ & $\begin{array}{l}8.367 \\
1.673\end{array}$ & $\begin{array}{r}11.710 \\
2.342\end{array}$ & $\begin{array}{r}20.977 \\
4.015 \\
\end{array}$ & $\begin{array}{r}35.95 \\
\ldots \ldots . . .\end{array}$ & $\begin{array}{r}50.32 \\
\ldots . . .\end{array}$ & $\begin{array}{r}86.27 \\
\ldots \ldots \\
\end{array}$ & $\begin{array}{r}3.196 \\
+\quad .640\end{array}$ & $\begin{array}{l}0 \\
0\end{array}$ \\
\hline $\begin{array}{l}\text { Entire after period: } \\
\text { Total .............. } \\
\text { Average ........ }\end{array}$ & $\begin{array}{r}45.720 \\
4.572\end{array}$ & $\begin{array}{r}16.973 \\
1.697\end{array}$ & $\begin{array}{r}22.087 \\
2.209\end{array}$ & $\begin{array}{r}39.060 \\
3.906\end{array}$ & $\begin{array}{r}37.12 \\
\ldots \ldots\end{array}$ & $\begin{array}{c}48.31 \\
\ldots . . .\end{array}$ & $\begin{array}{r}85.43 \\
\cdots \cdots\end{array}$ & $\begin{array}{r}+6.660 \\
+\quad .666\end{array}$ & $\begin{array}{l}0 \\
0 .\end{array}$ \\
\hline
\end{tabular}

$\alpha$ Daily a verage added in order to complete record. 
TABLe XV.-Phosphoric acid balances for Series VI-Continued.

[Averages are per day.]

No. 12 .

\begin{tabular}{|c|c|c|c|c|c|c|c|c|c|}
\hline Period. & In food. & In feces. & In urine. & $\begin{array}{c}4 \\
\text { In feces } \\
\text { and } \\
\text { urine. } \\
(2+3)\end{array}$ & $\begin{array}{c}5 \\
\text { In } \\
\text { feces. } \\
(2 \div 1)\end{array}$ & $\begin{array}{c}6 \\
\text { In } \\
\text { urine. } \\
(3 \div 1)\end{array}$ & $\begin{array}{c}7 \\
\text { In feces } \\
\text { and } \\
\text { urine. } \\
(4 \div 1)\end{array}$ & $\begin{array}{c}\text { Balance. } \\
(1-4)\end{array}$ & $\begin{array}{c}9 \\
\text { Sali- } \\
\text { cylic } \\
\text { acid } \\
\text { admin } \\
\text { istered. }\end{array}$ \\
\hline Fore period. & & & & & & & & & \\
\hline $\begin{array}{l}\text { First subperiod: } \\
\text { Total ........... } \\
\text { Average....... }\end{array}$ & $\begin{array}{r}\text { Grams. } \\
22.259 \\
\mathbf{4 . 4 5 2}\end{array}$ & $\begin{array}{r}\text { Grams. } \\
7.643 \\
1.529\end{array}$ & $\begin{array}{r}\text { Grams. } \\
12.731 \\
2.546\end{array}$ & $\begin{array}{c}\text { Grams. } \\
20.374 \\
4.075\end{array}$ & $\begin{array}{r}\text { Per ct. } \\
34.34\end{array}$ & $\begin{array}{r}\text { Per ct. } \\
57.19\end{array}$ & $\begin{array}{r}\text { Per ct. } \\
91.53 \\
\end{array}$ & $\begin{array}{r}\text { Grams } \\
+\quad 1.885 \\
+\quad .377\end{array}$ & $\begin{array}{c}\text { Grams. } \\
0 \\
0\end{array}$ \\
\hline $\begin{array}{l}\text { Second subperiod: } \\
\text { Total ............. } \\
\text { Average....... }\end{array}$ & $\begin{array}{r}22.085 \\
4.417\end{array}$ & $\begin{array}{l}7.846 \\
1.569\end{array}$ & $\begin{array}{r}12.955 \\
2.591\end{array}$ & $\begin{array}{r}20.801 \\
4.160\end{array}$ & $\begin{array}{l}35.53 \\
\cdots\end{array}$ & $\begin{array}{r}58.66 \\
\ldots \ldots \ldots\end{array}$ & $\begin{array}{l}94.19 \\
\cdots \cdots\end{array}$ & $\begin{array}{r}+1.284 \\
+\quad .257\end{array}$ & $\begin{array}{l}0 \\
0\end{array}$ \\
\hline $\begin{array}{l}\text { Entire fore period: } \\
\quad \text { Total ............... } \\
\text { Average .......... }\end{array}$ & $\begin{array}{r}44.344 \\
4.434\end{array}$ & $\begin{array}{r}15.489 \\
1.549\end{array}$ & $\begin{array}{r}25.686 \\
2.569\end{array}$ & $\begin{array}{r}41.175 \\
4.118\end{array}$ & $\begin{array}{l}34.93 \\
3.3\end{array}$ & $\begin{array}{r}57.93 \\
\ldots \ldots \ldots\end{array}$ & $\begin{array}{l}92.85 \\
\cdots\end{array}$ & $\begin{array}{r}+3.169 \\
+\quad .316\end{array}$ & $\begin{array}{l}0 \\
0\end{array}$ \\
\hline \multicolumn{10}{|l|}{ Preservative period. } \\
\hline $\begin{array}{l}\text { First subperiod: } \\
\text { Total .............. } \\
\text { Average........ }\end{array}$ & $\begin{array}{r}22.566 \\
4.513\end{array}$ & $\begin{array}{l}5.167 \\
1.033\end{array}$ & $\begin{array}{r}11.591 \\
2.318\end{array}$ & $\begin{array}{r}16.758 \\
3.352\end{array}$ & $\begin{array}{r}22.90 \\
2 .\end{array}$ & 51.36 & 74.26 & $\begin{array}{l}+5.808 \\
+1.161\end{array}$ & $\begin{array}{r}1.05 \\
.21\end{array}$ \\
\hline $\begin{array}{l}\text { Second } \\
\text { Tots } \\
\text { Ave }\end{array}$ & $\begin{array}{r}22.450 \\
4.490\end{array}$ & $\begin{array}{l}5.345 \\
1.069\end{array}$ & $\begin{array}{r}13.235 \\
2.647\end{array}$ & $\begin{array}{r}18.580 \\
3.716\end{array}$ & 23.81 & 58.95 & 82.76 & $\begin{array}{l}+3.870 \\
+\quad 774\end{array}$ & 2.10 \\
\hline od: & & & & & & & & 154 & \\
\hline (......... & $\begin{array}{r}22.233 \\
4.447\end{array}$ & & $\begin{array}{r}14.425 \\
2.885\end{array}$ & $\begin{array}{r}22.079 \\
4.416\end{array}$ & $\begin{array}{l}34.42 \\
\ldots \ldots . .\end{array}$ & $\begin{array}{r}64.88 \\
\ldots \ldots \ldots\end{array}$ & $\begin{array}{l}99.31 \\
\ldots . . .\end{array}$ & $\begin{array}{l}+\quad .154 \\
+\quad .031\end{array}$ & $\begin{array}{r}3.70 \\
.74\end{array}$ \\
\hline $\begin{array}{r}\text { Fourtl } \\
\text { To }\end{array}$ & & & & & 22.50 & 57.40 & 79.91 & +4.5 & \\
\hline $\begin{array}{l}\text { Av } \\
\text { fth s }\end{array}$ & 4.505 & & & 3.600 & & & & +.905 & 1. \\
\hline $\begin{array}{l}\text { Fifth s } \\
\text { To } \\
\text { Av }\end{array}$ & $\begin{array}{r}23.425 \\
4.685\end{array}$ & & & $\begin{array}{r}20.882 \\
4.176\end{array}$ & 37.54 & 51.60 & 89.14 & $\begin{array}{r}+2.543 \\
+\quad 509\end{array}$ & 8.0 \\
\hline $\begin{array}{l}\text { Sixth su } \\
\text { Tota }\end{array}$ & & & & & & & & & \\
\hline 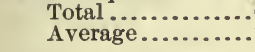 & $\begin{array}{r}22.305 \\
4.461\end{array}$ & & $\begin{array}{r}13.015 \\
2.603\end{array}$ & $\begin{array}{r}19.347 \\
3.869\end{array}$ & $\begin{array}{r}28.39 \\
\cdots\end{array}$ & $\begin{array}{r}58.35 \\
\ldots \ldots \ldots\end{array}$ & $\begin{array}{r}86.74 \\
\cdots \ldots \ldots\end{array}$ & $\begin{array}{r}+\quad 2.958 \\
+\quad .592\end{array}$ & $\begin{array}{r}10.00 \\
2.00\end{array}$ \\
\hline 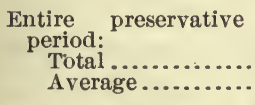 & $\begin{array}{r}135.504 \\
4.517\end{array}$ & $\begin{array}{r}38.361 \\
1.279\end{array}$ & $\begin{array}{r}77.284 \\
2.576\end{array}$ & $\begin{array}{r}115.645 \\
3.855\end{array}$ & $\begin{array}{r}28.31 \\
\ldots\end{array}$ & $\begin{array}{r}57.05 \\
\ldots \ldots\end{array}$ & $\begin{array}{r}85.34 \\
\cdots\end{array}$ & $\begin{array}{r}+19.859 \\
+\quad .662\end{array}$ & $\begin{array}{r}30.85 \\
1.08\end{array}$ \\
\hline After period. & & & & & & & & & \\
\hline $\begin{array}{l}\text { First subperi } \\
\text { Total... } \\
\text { Average }\end{array}$ & $\begin{array}{r}21.483 \\
4.297\end{array}$ & $\begin{array}{l}7.067 \\
1.413\end{array}$ & $\begin{array}{r}11.870 \\
2.374\end{array}$ & $\begin{array}{r}18.937 \\
3.787\end{array}$ & 32.89 & 55.25 & 88.15 & $\begin{array}{l}+2.546 \\
+\quad .510\end{array}$ & $\begin{array}{l}0 \\
0\end{array}$ \\
\hline $\begin{array}{l}\text { Second subperiod: } \\
\text { Total } \\
\text { Average............ }\end{array}$ & $\begin{array}{r}22.174 \\
4.435\end{array}$ & $\begin{array}{r}a 7.998 \\
1.600\end{array}$ & $\begin{array}{r}12.694 \\
2.539\end{array}$ & $\begin{array}{r}20.692 \\
4.138\end{array}$ & 36.07 & $\begin{array}{r}57.25 \\
\cdots\end{array}$ & $\begin{array}{r}93.32 \\
\ldots \ldots . .\end{array}$ & $\begin{array}{r}+1.482 \\
+\quad .297\end{array}$ & $\begin{array}{l}0 \\
0\end{array}$ \\
\hline $\begin{array}{r}\text { Entire after period: } \\
\text { Total ............ } \\
\text { Average ........ }\end{array}$ & $\begin{array}{r}43.657 \\
4.366\end{array}$ & $\begin{array}{r}15.065 \\
1.507\end{array}$ & $\begin{array}{r}24.564 \\
2.456\end{array}$ & $\begin{array}{r}39.629 \\
3.963\end{array}$ & $\begin{array}{r}34.51 \\
\ldots . . .\end{array}$ & 56.27 & $\begin{array}{r}90.77 \\
\ldots . . .\end{array}$ & $\begin{array}{r}+4.028 \\
+\quad .403\end{array}$ & $\begin{array}{l}0 \\
0\end{array}$ \\
\hline
\end{tabular}

a Daily average added in order to complete record. 
Table XV.-Phosphoric acid balances for Series VI-Continued.

[Averages are per man per day.]

Summary for nine men (Nos. 3, 9, and 10 excluded).

\begin{tabular}{|c|c|c|c|c|c|c|c|c|c|}
\hline Period. & $\begin{array}{c}1 \\
\text { In food. }\end{array}$ & In feees. & In urine. & $\begin{array}{c}4 \\
\text { In feces } \\
\text { and } \\
\text { urine. } \\
(2+3)\end{array}$ & $\begin{array}{c}5 \\
\text { In } \\
\text { feces. } \\
(2 \div-1)\end{array}$ & $\begin{array}{c}\mathbf{6} \\
\underset{\text { In }}{\text { Inine. }}(3 \div 1)\end{array}$ & $\begin{array}{c}7 \\
\text { In feces } \\
\text { and } \\
\text { urine. } \\
(4 \div 1)\end{array}$ & $\begin{array}{c}8 \\
\text { Balance. } \\
(1-4)\end{array}$ & $\begin{array}{c}9 \\
\text { Sali- } \\
\text { cylic } \\
\text { acid } \\
\text { admin- } \\
\text { istered. }\end{array}$ \\
\hline - Fore period. & & & & & & & & & \\
\hline $\begin{array}{l}\text { First subperiod: } \\
\text { Total ........... } \\
\text { Average...... }\end{array}$ & $\begin{array}{r}\text { Grams. } \\
174.618 \\
3.880\end{array}$ & $\begin{array}{r}\text { Grams. } \\
56.396 \\
1.253\end{array}$ & $\begin{array}{r}\text { Grams. } \\
106.474 \\
2.366\end{array}$ & $\begin{array}{r}\text { Grams. } \\
162.870 \\
3.619\end{array}$ & $\begin{array}{r}\text { Per ct. } \\
32.30 \\
\ldots\end{array}$ & $\begin{array}{r}\text { Per } c \text { t. } \\
60.98 \\
\end{array}$ & $\begin{array}{r}\text { Per ct. } \\
93.27 \\
.\end{array}$ & $\begin{array}{r}\text { Grams. } \\
+11.748 \\
+\quad .261\end{array}$ & $\begin{array}{c}\text { Grams. } \\
0 \\
0\end{array}$ \\
\hline 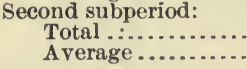 & $\begin{array}{r}168.853 \\
3.753\end{array}$ & $\begin{array}{r}55.888 \\
1.242\end{array}$ & $\begin{array}{r}106.305 \\
2.362\end{array}$ & $\begin{array}{r}162.193 \\
3.604\end{array}$ & $\begin{array}{c}33.10 \\
\ldots \ldots\end{array}$ & $\begin{array}{r}62.96 \\
\therefore \ldots .\end{array}$ & $\begin{array}{r}96.05 \\
\ldots \ldots . .\end{array}$ & $\begin{array}{r}+6.660 \\
+\quad .148\end{array}$ & $\begin{array}{l}0 \\
0\end{array}$ \\
\hline $\begin{array}{l}\text { Entire fore period: } \\
\text { Total ............... } \\
\text { Average ........ }\end{array}$ & $\begin{array}{r}343.471 \\
3.816\end{array}$ & $\begin{array}{r}112.284 \\
1.247\end{array}$ & $\begin{array}{r}212.779 \\
2.364\end{array}$ & $\begin{array}{r}325.063 \\
3.611\end{array}$ & $\begin{array}{r}32.69 \\
\cdots\end{array}$ & $\begin{array}{l}61.95 \\
\cdots \ldots . .\end{array}$ & $\begin{array}{r}94.64 \\
\cdots \cdots\end{array}$ & $\begin{array}{r}+18.408 \\
+\quad .205\end{array}$ & $\begin{array}{l}0 \\
0\end{array}$ \\
\hline Preservative period. & & & & & & & & & \\
\hline $\begin{array}{l}\text { First subperiod: } \\
\text { Total ........... } \\
\text { Average } . . . .\end{array}$ & $\begin{array}{r}177.217 \\
3.938\end{array}$ & $\begin{array}{r}48.356 \\
1.074\end{array}$ & $\begin{array}{r}105.333 \\
2.341\end{array}$ & $\begin{array}{r}153.689 \\
3.415\end{array}$ & 27.29 & 59.44 & $\begin{array}{c}86.72 \\
\cdots\end{array}$ & $\begin{array}{l}+23.528 \\
+\quad .523\end{array}$ & $\begin{array}{r}9.45 \\
.21\end{array}$ \\
\hline $\begin{array}{l}\text { Second subperiod: } \\
\text { Total ............. } \\
\text { Average....... }\end{array}$ & $\begin{array}{r}175.093 \\
3.891\end{array}$ & $\begin{array}{r}51.423 \\
1.143\end{array}$ & $\begin{array}{r}112.763 \\
2.506\end{array}$ & $\begin{array}{r}164.186 \\
3.648\end{array}$ & $\begin{array}{l}29.37 \\
\cdots\end{array}$ & $\begin{array}{l}64.40 \\
\ldots \ldots . .\end{array}$ & $\begin{array}{r}93.77 \\
\cdots\end{array}$ & $\begin{array}{l}+10.907 \\
+\quad .243\end{array}$ & $\begin{array}{r}18.90 \\
.42\end{array}$ \\
\hline Third subperiod: & & & & & & & & & \\
\hline Total .... & $\begin{array}{r}177.308 \\
3.940\end{array}$ & $\begin{array}{r}54.142 \\
1.203\end{array}$ & $\begin{array}{r}108.282 \\
2.407\end{array}$ & $\begin{array}{r}162.424 \\
3.609\end{array}$ & $\begin{array}{l}30.54 \\
\ldots . . .\end{array}$ & $\begin{array}{l}61.07 \\
\ldots \ldots .\end{array}$ & $\begin{array}{l}91.61 \\
\cdots \cdots\end{array}$ & $\begin{array}{l}+14.884 \\
+\quad .331\end{array}$ & $\begin{array}{r}33.30 \\
.74\end{array}$ \\
\hline Fourth subperiod: & & & & & & & & & \\
\hline Total ................ & $\begin{array}{r}180.446 \\
4.010\end{array}$ & $\begin{array}{r}48.860 \\
1.086\end{array}$ & $\begin{array}{r}107.390 \\
2.386\end{array}$ & $\begin{array}{r}156.250 \\
3.472\end{array}$ & 27.08 & 59.51 & 86.59 & $\begin{array}{l}+24.196 \\
+\quad .538\end{array}$ & $\begin{array}{r}54.00 \\
1.20\end{array}$ \\
\hline $\begin{array}{l}\text { Fifth subl } \\
\text { Total }\end{array}$ & & & & & & & & & \\
\hline $\begin{array}{l}\text { Total } \\
\text { Avera }\end{array}$ & $\begin{array}{r}184.486 \\
4.100\end{array}$ & $\begin{array}{r}53.971 \\
1.199\end{array}$ & 105.550 & 159.521 & 29.25 & 57.21 & 86.47 & +24.965 & 72.00 \\
\hline Sixth subperiod: & T. 100 & & & 0.010 & & & & T $\quad$ T & 1.00 \\
\hline Total $\ldots . . . . \ldots \ldots \ldots$ & $\begin{array}{r}176.708 \\
3.927\end{array}$ & $\begin{array}{r}52.514 \\
1.167\end{array}$ & $\begin{array}{r}101.678 \\
2.260\end{array}$ & $\begin{array}{r}154.192 \\
3.426\end{array}$ & $\begin{array}{c}29.72 \\
\ldots \ldots\end{array}$ & $\begin{array}{r}57.54 \\
\cdots \cdots\end{array}$ & $\begin{array}{r}87.26 \\
\cdots \ldots . .\end{array}$ & $\begin{array}{r}+22.510 \\
+\quad .501\end{array}$ & $\begin{array}{r}88.00 \\
1.96\end{array}$ \\
\hline $\begin{array}{l}\text { Entire preservative } \\
\text { period: }\end{array}$ & & & & & & & & & \\
\hline 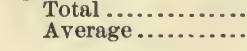 & $\begin{array}{r}1,071.258 \\
3.967\end{array}$ & $\begin{array}{r}309.266 \\
1.145\end{array}$ & $\begin{array}{r}640.996 \\
2.374\end{array}$ & $\begin{array}{r}950.262 \\
3.519\end{array}$ & $\begin{array}{r}28.87 \\
\ldots \ldots \ldots \\
\end{array}$ & 59.84 & $\begin{array}{r}88.71 \\
\cdots \cdots\end{array}$ & $\begin{array}{r}+120.996 \\
+\quad .448\end{array}$ & $\begin{array}{r}275.65 \\
1.02\end{array}$ \\
\hline After period. & & & & & & & & & \\
\hline $\begin{array}{l}\text { First subperiod: } \\
\text { Total } \ldots \ldots \ldots \\
\text { Average } \ldots \ldots\end{array}$ & $\begin{array}{r}171.019 \\
3.800\end{array}$ & $\begin{array}{r}60.887 \\
1.353\end{array}$ & $\begin{array}{r}93.951 \\
2.088\end{array}$ & $\begin{array}{r}154.838 \\
3.441\end{array}$ & $\begin{array}{c}35.60 \\
\ldots . . . .\end{array}$ & 54.94 & 90.54 & $\begin{array}{l}+16.181 \\
+\quad .359\end{array}$ & $\begin{array}{l}0 \\
0\end{array}$ \\
\hline $\begin{array}{l}\text { Second subperiod: } \\
\text { Total ................ } \\
\text { Average........ }\end{array}$ & $\begin{array}{r}177.520 \\
3.945\end{array}$ & $\begin{array}{r}59.254 \\
1.317\end{array}$ & $\begin{array}{r}102.331 \\
2.274\end{array}$ & $\begin{array}{r}161.585 \\
3.591\end{array}$ & $\begin{array}{r}39.01 \\
\cdots . . .\end{array}$ & $\begin{array}{r}63.28 \\
\cdots . . .\end{array}$ & $\begin{array}{r}91.02 \\
\ldots . . . \\
\end{array}$ & $\begin{array}{r}+15.935 \\
+\quad .354\end{array}$ & $\begin{array}{l}0 \\
0\end{array}$ \\
\hline $\begin{array}{l}\text { Entire after period: } \\
\text { Total ............... } \\
\text { Average ........ }\end{array}$ & $\begin{array}{r}348.539 \\
3.873\end{array}$ & $\begin{array}{r}120.141 \\
1.335\end{array}$ & $\begin{array}{r}196.282 \\
2.181\end{array}$ & $\begin{array}{r}316.423 \\
3.516\end{array}$ & $\begin{array}{r}34.47 \\
\ldots . . . . .\end{array}$ & 56.32 & 90.79 & $\begin{array}{l}+32.116 \\
+\quad .357\end{array}$ & $\begin{array}{l}0 \\
0\end{array}$ \\
\hline
\end{tabular}

\section{SULPHUR BALANCE.}

The sulphur which enters the body in the food exists in at least two states, namely, organic sulphur, as a constituent of the protein matter, and inorganic sulphur, as found in sulphuric and sulphurous acids and their salts. In the discussion of the balance these two kinds of sulphur are considered together, whereas in the further investigation of the metabolic products a separation is secured.

The principal change which sulphur undergoes in the metabolic process is its oxidation from the organic form to the highly oxidized form of sulphuric acid, or sulphates. In other words, the greater 
part of the sulphur which enters the body in the food is organic, and the greater part which is excreted with the feces is inorganic. The data discussed below are to be found in Table XVII, page 633 .

INDIVIDUAL DATA.

No. 1 .

The quantity of sulphur in the food of No. 1 is almost the same during the three periods. The daily amount is $0.904 \mathrm{gram}$ in the fore period, $0.952 \mathrm{gram}$ in the preservative period, and $0.933 \mathrm{gram}$ in the after period. Of this quantity 0.083 gram appears in the feces in the fore period, 0.077 gram in the preservative period, and 0.111 gram in the after period. In the urine is found $0.825 \mathrm{gram}$ in the fore period, 0.907 gram in the preservative period, and $0.935 \mathrm{gram}$ in the after period. Expressed as percentages it is found that of the total sulphur exhibited in the food 9.23 per cent appears in the feces in the fore period, 8.12 per cent in the preservative period, and 11.88 per cent in the after period, while in the urine 91.33 per cent appears in the fore period, 95.27 per cent in the preservative period, and 100.20 per cent in the after period. The balance is negative in all cases, amounting to only 0.005 gram in the fore period, rising to 0.032 gram in the preservative period, and amounting to the comparatively very large quantity of $0.113 \mathrm{gram}$ in the after period. In this case it is seen that there is a marked tendency in the case of No. 1 while unc'er observation to excrete a larger quantity of sulphur than he is eating in his food. During the fore period this excess is extremely minute, but it is increased in the preservative and after periods. The principal excess of excretion is found in the urine both in the preservative and after periods-that is, the general effect of the preservative appears to be to increase the excretion of metabolized sulphur, and this is done even at the expense of the tissues of the body during the preservative period and to a greater extent in the after period.

\section{No. 2.}

The quantities of sulphur administered in the food of No. 2 for the three periods are 1.047 grams, 1.079 grams, and 1.054 grams, respectively. Of this quantity $0.153 \mathrm{gram}, 0.147 \mathrm{gram}$, and 0.131 gram appears in the feces, respectively; and 1.009 grams, 0.985 gram, and 1.039 grams appear in the urine, respectively. Based upon the percentages of sulphur in the food it is seen that 14.65 per cent, 13.65 per cent, and 12.40 per cent, respectively, are excreted in the feces during the three periods, while in the urine 96.38 per cent, 91.28 per cent, and 98.55 per cent are excreted, respectively, in the three periods. The balance in all cases is strongly negative but its magnitude during the preservative period is less than half of that of the 
fore and after periods. In this case also we find a greater amount of sulphur excreted than is found in the food, but the effect of the preservative seems to have been to diminish this excessive amount.

No. 3.

The average daily quantities of sulphur administered in the food of No. 3 for the three periods are $0.916 \mathrm{gram}, 0.968 \mathrm{gram}$, and 0.954 gram, respectively. Only the second subperiod of the after period is considered, the first subperiod being broken by illness of the subject. Of this quantity there appear daily in the feces for the three periods 0.104 gram, 0.122 gram, and 0.103 gram, respectively, and in the urine 0.825 gram, 0.734 gram, and $0.803 \mathrm{gram}$, respectively. Expressed in percentage of the total quantity of sulphur in the food there appear in the feces for the three periods 11.38, 12.61, and 10.84 per cent, respectively, and in the urine 90.13 per cent, 75.88 per cent, and 84.13 per cent, respectively. The balance is slightly negative in the fore period, strongly positive in the preservative period, and very slightly positive in the after period. The effect of the preservative in this case seems to have been to decrease the excretion of metabolized sulphur. More nonmetabolized sulphur is excreted during the preservative period than in either of the other periods, but the quantity of metabolized sulphur excreted is very much less in the preservative period than in either of the other periods. The fact that the preservative period was a period of convalescence for this subject probably partly explains the variations in balances, and on account of the illness of No. 3 the data have no comparative value.

\section{No. 4 .}

The quantities of sulphur contained in the food of No. 4 for the three periods of observation are 0.989 gram, 1.020 grams, and 0.998 gram, respectively. Of this quantity there appears in the feces for the three periods 0.113 gram, 0.117 gram, and 0.117 gram, respectively, and in the urine 0.948 gram, 0.911 gram, and 0.959 gram, respectively. Expressed as percentages, of the total quantity of sulphur in the food, it appears that for the three periods 11.37 per cent, 11.47 per cent, and 11.70 per cent, respectively, are excreted in the feces, and 95.82 per cent, 89.35 per cent, and 96.03 per cent, respectively, in the urine. The balance is negative throughout, but is very small, especially in the preservative period, being represented by the quantity $0.071,0.008$, and 0.077 gram, respectively, for the three periods. We have in this instance a slight tendency on the part of the preservative to increase the excretion of nonmetabolized sulphur and to diminish the excretion of metabolized sulphur. 
No. 5.

The total quantities of sulphur ingested by No. 5 in the three periods are 1.008 grams, 1.033 grams, and 1.015 grams, respectively. Of this quantity there appear in the feces 0.146 gram, 0.126 gram, and 0.106 gram, respectively, and in the urine $0.828 \mathrm{gram}, 0.908$ gram, and 0.919 gram, respectively. Expressed in percentages, of the sulphur in the food, it is seen that there appears in the feces for the three periods 14.46 per cent, 12.20 per cent, and 10.41 per cent, respectively, and in the urine 82.14 per cent, 87.91 per cent, and 90.54 per cent, respectively.

The balance is slightly positive in the fore period, very slightly negative in the preservative period, and slightly negative in the after period. These data show that the general effect of the preservative in this case is to decrease the quantity of nonmetabolized sulphur excreted and to increase the quantity of metabolized sulphur.

\section{No. 6 .}

The quantities of sulphur in the food of No. 6 for the three periods are 0.912 gram, 0.961 gram, and 0.929 gram, respectively. Of this quantity there appear in the feces for the three periods 0.145 gram, $0.159 \mathrm{gram}$, and $0.147 \mathrm{gram}$, respectively, and in the urine $0.783 \mathrm{gram}$, 0.920 gram, and 0.896 gram, respectively. Expressed in percentages, of the total sulphur in the food, it is seen that there appear in the feces 15.92 per cent, 16.50 per cent, and 15.80 per cent, respectively, for the three periods, and in the urine 85.95 per cent, 95.80 per cent, and 96.42 per cent, respectively, for the three periods. The balarice is slightly negative in the fore period and very decidedly negative in both the preservative and after periods. The data show a slight increase in the nonmetabolized sulphur excreted during the preservative period and a very marked increase in the excretion of the metabolized sulphur in the preservative period.

No $\%$.

The quantities of sulphur in the food of No. 7 for the three periods are 0.826 gram, 0.875 gram, and $0.869 \mathrm{gram}$, respectively. Of this quantity there appear in the feces 0.094 gram, 0.074 gram, and 0.114 gram, respectively, for the three periods, and in the urine 0.804 gram, 0.827 gram, and 0.754 gram, respectively. Expressed in percentages, of the total sulphur in the food there appear in the feces for the three periods, respectively, 11.35 per cent, 8.42 per cent, and 13.14 per cent, and in the urine 97.25 per cent, 94.62 per cent, and 86.74 per cent, respectively. The balance is negative in the fore period, slightly negative in the preservative period, and very slightly positive in the 
after period. The general effect of the administration of the preservative appears to be a decrease in the excretion of both nonmetabolized and metabolized sulphur in the preservative period. In the after period the quantity of nonmetabolized sulphur is considerably increased, while the quantity of metabolized sulphur excreted, is again very decidedly diminished.

No. 8.

The quantities of sulphur contained in the food of No. 8 during the three periods are 0.821 gram, 0.882 gram, and 0.879 gram, respectively. Of this quantity there appear in the feces $0.111,0.130$, and 0.145 gram, respectively, for the three periods, and in the urine $0.768,0.741$, and 0.838 gram, respectively. Expressed in percentages, of the total sulphur in the food there appear in the feces 13.56, 14.79, and 16.52 per cent, respectively, for the three periods, and in the urine $93.53,83.99$, and 95.27 per cent, respectively, for the three periods. The balance is negative in the fore period, slightly positive in the preservative period, and strongly negative in the after period. The general effect of the preservative appears to have been to slightly increase the percentage of the nonmetabolized sulphur excreted in the feces in the preservative period and to decidedly increase it in the after period, and to greatly diminish the quantity of metabolized sulphur excreted during the preservative period, while a very marked increase occurs in the after period.

No. 9 .

The sulphur daily consumed in the food by No. 9 for the three periods was $1.097,1.204$, and 1.154 grams, respectively. Of this there appear in the feces $0.079,0.119$, and 0.089 grams, respectively, and in the urine $0.922,0.927$, and 0.892 grams, respectively, for the three periods. Expressed in percentages, of the total amount of sulphur in the food there appear for the three periods in the feces 7.23, 9.92 , and 7.71 per cent, respectively, and in the urine 84.04, 76.98, and 77.34 per cent, respectively.

The balance is positive in all cases. Its magnitude is considerably increased during the preservative period and still further increased during the after period. This increase of balance, however, is not sufficient to compensate for the increase in the amount of sulphur in the food.

The amount of nonmetabolized sulphur excreted appears to be considerably increased during the preservative period, but returns during the after period to approximately the same amount as in the fore period. The amount of metabolized sulphur excreted during the preservative, period is almost the same as that of the fore period, but the percentage amount is greatly decreased. 
For reasons given elsewhere (p. 587) the results obtained with this subject are not included in the summaries. The $y$ are stated here, however, as a matter of record.

No. 10.

On account of illness this subject did not complete the experiment, but left the table during the after period. For reasons given elsewhere (p. 587) this subject is omitted from the summaries. The results are given here as a matter of record.

The amount of sulphur consumed in the food by No. 10 is 1.086 and 1.186 grams, daily, for the fore and preservative periods, respectively. Of this amount 0.125 and 0.118 gram, respectively, appear in the feces, and 0.852 and 0.840 gram, respectively, in the urine. Expressed in percentages, of the total sulphur contained in the food there appear for the fore period and preservative period in the feces 11.53 and 9.93 per cent, and in the urine 78.41 and 70.77 per cent, respectively.

The balance is positive in both periods, its magnitude being increased in the preservative period.

No. 11.

The quantities of sulphur in the food of No. 11 for the three periods are 1.217 grams, 1.168 grams, and 1.153 grams, respectively. Of this there appear in the feces $0.152 \mathrm{gram}, 0.154 \mathrm{gram}$, and $0.133 \mathrm{gram}$, respectively, for the three periods, and in the urine 1.000 gram, 0.985 gram, and 0.963 gram, respectively, for the three periods. Expressed in percentages, of the total amount of sulphur contained in the food there appear for the three periods in the feces 12.48 per cent, 13.21 per cent, and 11.56 per cent, respectively, and in the urine 82.13 per cent, 84.34 per cent, and 83.54 per cent, respectively. The balance is slightly positive in all cases, but its magnitude is considerably diminished during the preservative period. The preservative in this case appears to have slightly increased the percentage both of nonmetabolized and metabolized sulphur excreted.

No. 12 .

The quantity of sulphur which is contained in the food of No. 12 for the three periods is $1.114,1.146$, and 1.098 grams, respectively. Of this amount there appear in the feces $0.139,0.115$, and 0.122 gram, respectively, for the three periods, and in the urine 1.008, 0.972, and 1.016 grams, respectively. Expressed in percentages of the total amount of sulphur in the food there appear in the feces 12.51, 10.03, and 11.15 per cent respectively, for the three periods, and in the urine $90.53,84.76$, and 92.54 per cent, respectively, for the three periods. The balance is slightly negative in the fore period and after period 
and positive in the preservative period. The general effect of the preservative in this case is to distinctly decrease the quantity both of nonmetabolized and metabolized sulphur excreted.

SUMMARY.

Combining the data for the nine men in one expression for each period the following general results are obtained:

TABle XVI.-Sulphur summary, by periods, for nine men, Series VI.

\begin{tabular}{|c|c|c|c|c|c|c|}
\hline Period. & $\begin{array}{l}\text { Sulphur } \\
\text { in food. }\end{array}$ & $\begin{array}{l}\text { Sulphur } \\
\text { in feces. }\end{array}$ & $\begin{array}{l}\text { Sulphur } \\
\text { in urine. }\end{array}$ & $\begin{array}{l}\text { Sulphur } \\
\text { in feces. }\end{array}$ & $\begin{array}{l}\text { Sulphur } \\
\text { in urine. }\end{array}$ & Balance. \\
\hline 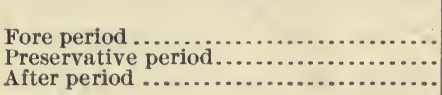 & $\begin{array}{r}\text { Grams. } \\
0.982 \\
1.013 \\
.992\end{array}$ & $\begin{array}{r}\text { Grams. } \\
0.126 \\
.122 \\
.125\end{array}$ & $\begin{array}{r}\text { Grams. } \\
0.886 \\
.906 \\
.924\end{array}$ & $\begin{array}{r}\text { Per cent. } \\
12.86 \\
12.06 \\
12.61\end{array}$ & $\begin{array}{r}\text { Per cent. } \\
90.22 \\
89.48 \\
93.16\end{array}$ & $\begin{array}{l}\text { Grams. } \\
-0.030 \\
-.015 \\
--.057\end{array}$ \\
\hline
\end{tabular}

It is seen that the balance is negative in all cases, being smallest in the preservative period and largest in the after period. The general tendency of the preservative seems to be to diminish very slightly the excretion of nonmetabolized sulphur-that is, the quantity appearing in the feces-and to increase the quantity but decrease the percentage of metabolized sulphur, that is the sulphur appearing in the urine. These changes are so slight that it may be said that sulphur metabolism is practically unaffected by salicylic acid, as far as these data show.

In regard to the apparent irregularity of a negative balance in the fore period especially, it may be stated that the methods which are in common use by analysts for determining organic sulphur-that is, sulphur existing principally in foods-are not so complete as the methods for determining inorganic sulphur. All the modern methods and precautions for securing the whole of the organic sulphur were observed during the analytical operations, but there is probably still sufficient difficulty in the methods of sampling and analysis to account for the slightly irregular results indicated by the above data. 
SALICYLIC ACID AND SALICYLATES.

TABLE XVII.-Sulphur balances for Series VI.

[Averages are per day.]

No. 1.

\begin{tabular}{|c|c|c|c|c|c|c|c|c|c|}
\hline Period. & In food. & $\begin{array}{c}2 \\
\text { In feces. }\end{array}$ & $\begin{array}{c}3 \\
\text { In } \\
\text { urine. }\end{array}$ & \begin{tabular}{|c|}
$\mathbf{4}$ \\
In feces \\
and \\
urine. \\
$(2+3)$
\end{tabular} & $\begin{array}{c}5 \\
\text { In } \\
\text { feces. } \\
(2 \div 1)\end{array}$ & $\begin{array}{c}6 \\
\text { In } \\
\text { urine. } \\
(3 \div 1)\end{array}$ & \begin{tabular}{|c|}
7 \\
In feces \\
and \\
urine. \\
$(4 \div 1)$
\end{tabular} & $\left|\begin{array}{c}8 \\
\text { Balance. } \\
(1-4)\end{array}\right|$ & \begin{tabular}{|c}
9 \\
Sali- \\
cylic \\
acid \\
admin- \\
istered.
\end{tabular} \\
\hline Fore period. & & & & & & & & & \\
\hline 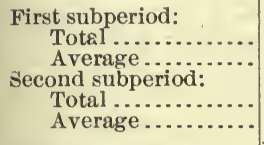 & $\begin{array}{r}\text { Grams. } \\
4.815 \\
.963 \\
-\quad 4.223 \\
.845 \\
\end{array}$ & $\begin{array}{r}\text { Grams. } \\
0.440 \\
.088 \\
\quad .394 \\
.079 \\
\end{array}$ & $\begin{array}{r}\text { Grams. } \\
4.002 \\
.800 \\
4.252 \\
.850 \\
\end{array}$ & $\begin{array}{r}\text { Grams. } \\
4.442 \\
.888 \\
4.646 \\
.929 \\
\end{array}$ & $\begin{array}{r}\text { Per ct. } \\
9.14 \\
\ldots . . .\end{array}$ & $\begin{array}{l}\text { Per ct. } \\
83.12\end{array}$ & $\begin{array}{r}\text { Per ct. } \\
92.25 \\
\ldots \ldots\end{array}$ & $\begin{array}{r}\text { Grams. } \\
+0.373 \\
+.075 \\
-.423 \\
-.084 \\
\end{array}$ & $\begin{array}{c}\text { Grams. } \\
0 \\
0 \\
0 \\
0 \\
0\end{array}$ \\
\hline $\begin{array}{l}\text { Entire fore period: } \\
\text { Total ............... } \\
\text { Average............ }\end{array}$ & $\begin{array}{r}9.038 \\
.904 \\
\end{array}$ & $\begin{array}{l}.834 \\
.083 \\
\end{array}$ & $\begin{array}{r}8.254 \\
.825 \\
\end{array}$ & $\begin{array}{r}9.088 \\
.909 \\
\end{array}$ & $\begin{array}{r}9.23 \\
\ldots .2 .\end{array}$ & 91.33 & 100.55 & $\begin{array}{r}-.050 \\
-.005 \\
\end{array}$ & $\begin{array}{l}0 \\
0\end{array}$ \\
\hline Preservative period. & & & & & & & & & \\
\hline 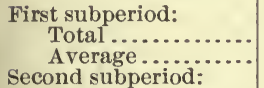 & $\begin{array}{r}4.406 \\
.881\end{array}$ & $\begin{array}{l}.360 \\
.072\end{array}$ & $\begin{array}{r}4.415 \\
.883\end{array}$ & $\begin{array}{r}4.775 \\
.955\end{array}$ & 8.17 & 100.20 & 108.37 & $\begin{array}{r}-.369 \\
-.074\end{array}$ & $\begin{array}{r}1.05 \\
.21\end{array}$ \\
\hline a: & $\begin{array}{r}4.735 \\
.947\end{array}$ & $\begin{array}{l}.333 \\
.067\end{array}$ & $\begin{array}{r}4.654 \\
.931\end{array}$ & $\begin{array}{r}4.987 \\
.998\end{array}$ & 7.03 & 98.29 & 105.32 & $\begin{array}{l}-.252 \\
-.051\end{array}$ & $\begin{array}{r}2.10 \\
.42\end{array}$ \\
\hline $\begin{aligned} \text { Third } & \text { T } \\
\text { A } & \end{aligned}$ & $\begin{array}{r}4.683 \\
.937\end{array}$ & $\begin{array}{l}.386 \\
.077\end{array}$ & $\begin{array}{r}a 4.376 \\
.875\end{array}$ & $\begin{array}{r}4.762 \\
.952\end{array}$ & 8.24 & 93.44 & 101.69 & $=.079$ & $\begin{array}{r}3.70 \\
.74\end{array}$ \\
\hline $\begin{array}{r}\text { Fourtl } \\
\text { To } \\
\text { A } \\
\text { Fifth }\end{array}$ & $\begin{array}{r}4.759 \\
.952\end{array}$ & $\begin{array}{l}.406 \\
.081\end{array}$ & $\begin{array}{r}4.720 \\
.944\end{array}$ & $\begin{array}{l}5.126 \\
1.025\end{array}$ & 8.53 & 99.18 & 107.71 & $\begin{array}{l}-.367 \\
-.073\end{array}$ & $\begin{array}{l}6.00 \\
1.20\end{array}$ \\
\hline $\begin{array}{r}\text { Fifth s } \\
\text { To } \\
\text { Av }\end{array}$ & $\begin{array}{l}5.082 \\
1.016\end{array}$ & $\begin{array}{l}.454 \\
.091\end{array}$ & $\begin{array}{r}4.429 \\
.886\end{array}$ & $\begin{array}{r}4.883 \\
.977\end{array}$ & 8.93 & 87.15 & 96.08 & $\begin{array}{r}+.199 \\
+.039\end{array}$ & $\begin{array}{l}8.00 \\
1.60\end{array}$ \\
\hline $\begin{array}{l}\text { Sixth subperiod: } \\
\text { Total .......... } \\
\text { Average ........ }\end{array}$ & $\begin{array}{r}4.904 \\
.981\end{array}$ & $\begin{array}{r}.380 \\
.076 \\
\end{array}$ & $\begin{array}{r}4.621 \\
.924 \\
\end{array}$ & $\begin{array}{l}5.001 \\
1.000\end{array}$ & 7.75 & .94 .23 & 101.98 & $\begin{array}{l}-.097 \\
-.019 \\
\end{array}$ & $\begin{array}{r}10.00 \\
2.00\end{array}$ \\
\hline $\begin{array}{l}\text { Entire preservative pe- } \\
\text { riod: } \\
\text { Total ................ } \\
\text { Average.............. }\end{array}$ & $\begin{array}{r}28.569 \\
.952 \\
\end{array}$ & $\begin{array}{r}2.319 \\
.077\end{array}$ & $\begin{array}{r}27.215 \\
.907 \\
\end{array}$ & $\begin{array}{r}29.534 \\
.984 \\
\end{array}$ & 8.12 & $\bullet 95.27$ & 103.38 & $=.965$ & $\begin{array}{r}30.85 \\
1.03 \\
\end{array}$ \\
\hline After period. & & & & & & & & & \\
\hline $\begin{array}{l}\text { First subpe } \\
\text { Total. } \\
\text { Averag } \\
\text { Second sub }\end{array}$ & $\begin{array}{r}4.639 \\
.928\end{array}$ & $\begin{array}{l}.652 \\
.130\end{array}$ & $\begin{array}{r}4.580 \\
.916\end{array}$ & $\begin{array}{l}5.232 \\
1.046\end{array}$ & 14.05 & 98.73 & 112.78 & $\begin{array}{l}-.593 \\
-.118\end{array}$ & $\begin{array}{l}0 \\
0\end{array}$ \\
\hline $\begin{array}{l}\text { Second subperiod: } \\
\text { Total ............ } \\
\text { Average........ }\end{array}$ & $\begin{array}{r}4.693 \\
.939\end{array}$ & $\begin{array}{l}.457 \\
.091\end{array}$ & $\begin{array}{r}4,771 \\
.954\end{array}$ & $\begin{array}{l}5.228 \\
1.046\end{array}$ & 9.74 & 101.66 & 111.40 & $\begin{array}{r}-.535 \\
-.107 \\
\end{array}$ & $\begin{array}{l}0 \\
0\end{array}$ \\
\hline $\begin{array}{l}\text { Entire after period: } \\
\quad \text { Total } \\
\text { Average............... }\end{array}$ & $\begin{array}{r}9.332 \\
.933\end{array}$ & $\begin{array}{r}1.109 \\
.111\end{array}$ & $\begin{array}{r}9.351 \\
.935\end{array}$ & $\begin{array}{r}10.460 \\
1.046\end{array}$ & 11.88 & 100.20 & 112.09 & $\begin{array}{l}-1.128 \\
-.113\end{array}$ & $\begin{array}{l}0 \\
0\end{array}$ \\
\hline
\end{tabular}

$a$ Daily average added in order to complete record.

7656-No. 84, pt 2-06-11 
TABle XVII.-Sulphur balances for Series VI-Continued.

[Averages are per day.]

No. 2 .

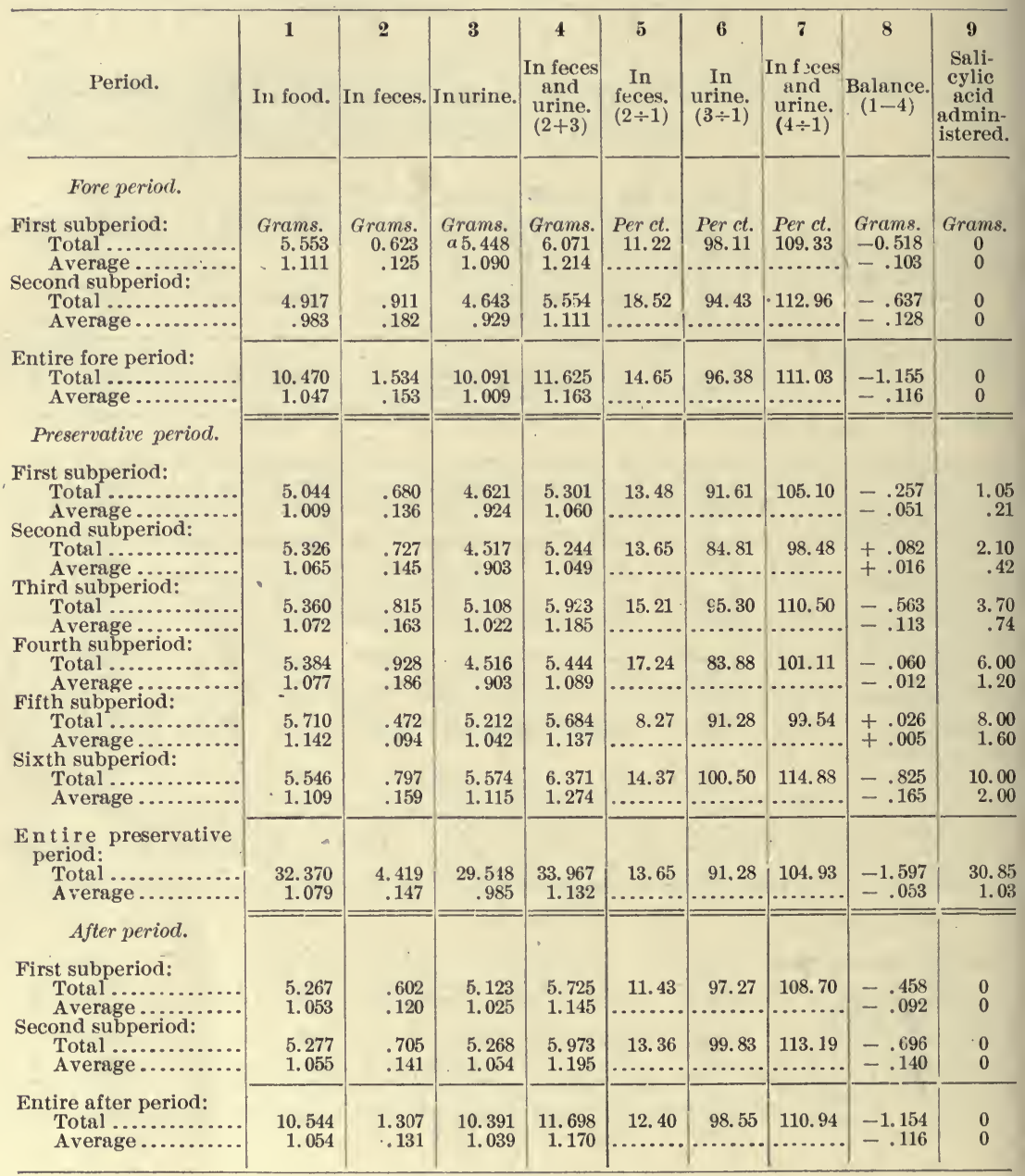

a Daily average added in order to complete record. 
SALICYLIC ACID AND SALICYLATES.

TABLE XVII.-Sulphur balances for Series VI-Continued.

[Averages are per day.]

No. 3.

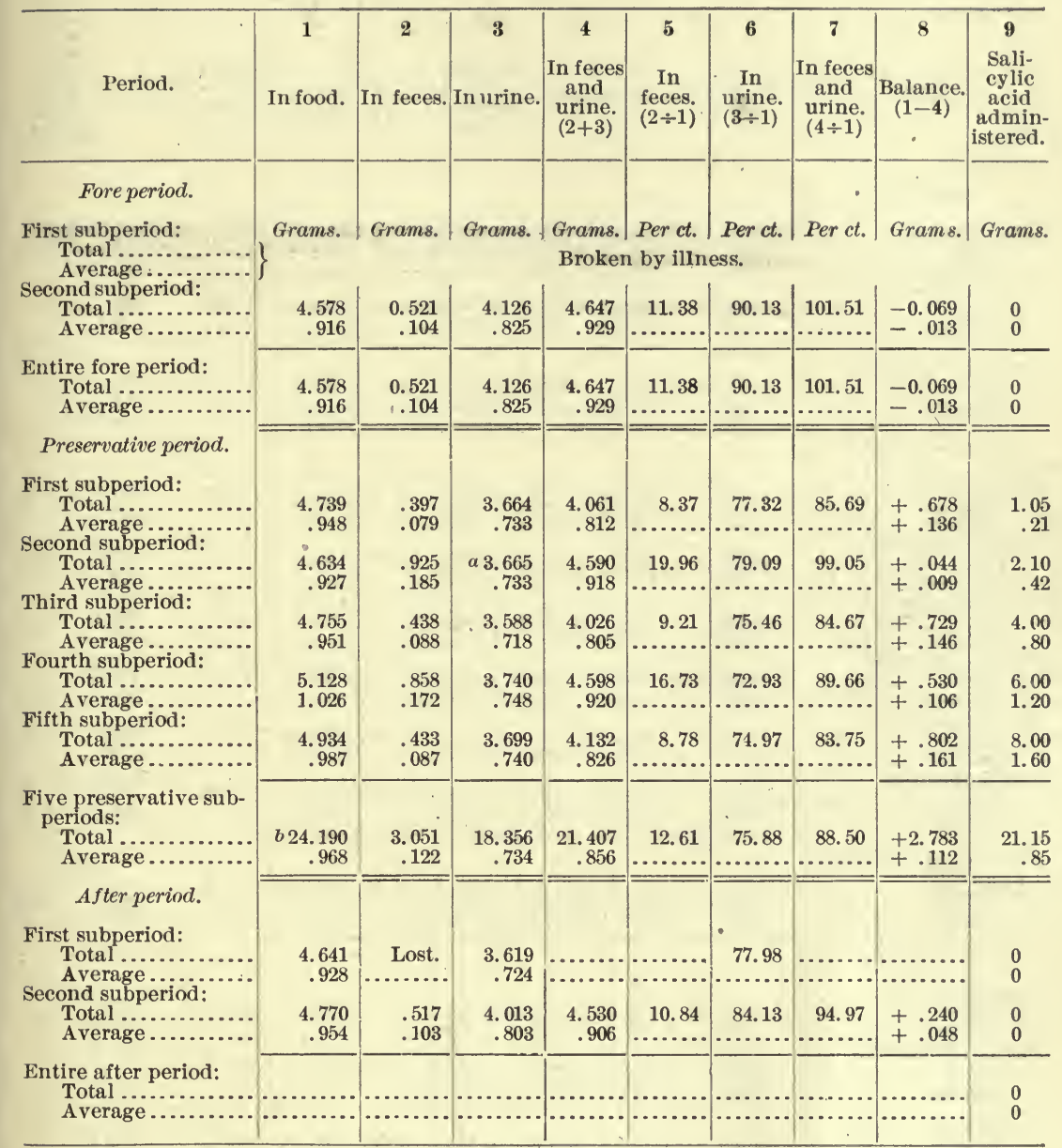

$a$ Daily average added in order to complete record.

$b$ No. 3 had only five preservative subperiods. 
TABLE XVII.-Sulphur balances for Series VI-Continued.

[Averages are per day.]

No. 4.

\begin{tabular}{|c|c|c|c|c|c|c|c|c|c|}
\hline Period. & In food. & In feces. & In urine. & $\begin{array}{c}4 \\
\text { In feces } \\
\text { and } \\
\text { urine. } \\
(2+3)\end{array}$ & $\begin{array}{c}5 \\
\text { In } \\
\text { feces. } \\
(2 \div 1)\end{array}$ & $\begin{array}{c}6 \\
\underset{(3 \div 1)}{\text { In }} \\
\text { urine. }\end{array}$ & $\begin{array}{c}7 \\
\text { In feces } \\
\text { and } \\
\text { urine. } \\
(4 \div 1)\end{array}$ & $\begin{array}{c}\text { Balance. } \\
(1-4)\end{array}$ & $\begin{array}{c}9 \\
\text { Sali- } \\
\text { cylic } \\
\text { acid } \\
\text { admin- } \\
\text { istered. }\end{array}$ \\
\hline \multicolumn{10}{|l|}{ Fore periud. . } \\
\hline $\begin{array}{l}\text { First subpcriod: } \\
\quad \text { Total ............... } \\
\text { Average } . . .6 . . .\end{array}$ & $\begin{array}{r}\text { Grams. } \\
5.283 \\
1.057\end{array}$ & $\begin{array}{r}\text { Grams. } \\
0.469 \\
.094\end{array}$ & $\begin{array}{r}\text { Grams. } \\
4.905 \\
.981\end{array}$ & $\begin{array}{r}\text { Grams. } \\
5.374 \\
1.075\end{array}$ & $\begin{array}{r}\text { Per } c t . \\
8.88 \\
\quad .1 .\end{array}$ & $\begin{array}{r}\text { Per ct. } \\
92.84 \\
. \ldots \ldots\end{array}$ & $\begin{array}{l}\text { Per ct. } \\
101.72 \\
\end{array}$ & $\begin{array}{l}\text { Grams. } \\
-0.091 \\
-\quad .018\end{array}$ & $\begin{array}{c}\text { Grams. } \\
0 \\
0\end{array}$ \\
\hline Sccond subperiod: & & & & & & & $\cdots \cdots$ & -.018 & \\
\hline Total ................. & 4. 610 & .656 & 4.574 & 5. 230 & 14.23 & 99.22 & 113.43 & -.620 & 0 \\
\hline Average ........... & .922 & .131 & .915 & 1.016 & ......... & .......... & ......... & -.124 & 0 \\
\hline $\begin{array}{l}\text { Entire fore period: } \\
\text { Total ........... }\end{array}$ & 9.893 & 1.125 & 9.479 & 10.604 & 11.37 & 95.82 & 107.19 & -.711 & 0 \\
\hline Average............. & .989 & .113 & .948 & 1.060 & & ........ & ........ & -.071 & 0 \\
\hline \multicolumn{10}{|l|}{ Preservative period. } \\
\hline First subperiod: & & & & & & & & & \\
\hline $\begin{array}{l}\text { Total. } \\
\text { Averag }\end{array}$ & $\begin{array}{r}4.767 \\
.953\end{array}$ & $\begin{array}{r}.464 \\
.093\end{array}$ & $\begin{array}{r}4.444 \\
.889\end{array}$ & $\begin{array}{r}4.908 \\
.982\end{array}$ & 9.73 & 93.22 & 102.96 & $\begin{array}{l}-.141 \\
-\quad 029\end{array}$ & 1. 05 \\
\hline Second subperiod: & & & & & & & & & \\
\hline Total ............. & 5.084 & .557 & 4. 724 & 5.281 & 10.96 & 92.92 & 103.87 & -.197 & 2.10 \\
\hline $\begin{array}{l}\text { Average } \ldots . . . . . \\
\text { Third subperiod: }\end{array}$ & 1.017 & .111 & .945 & 1.056 & ......... & $\because \cdot \cdots \cdot$ & $\cdots \ldots \ldots$ & -.039 & .42 \\
\hline Total ........... & 5.077 & .618 & 4.548 & 5.166 & 12.17 & 89.58 & 101.75 & -.089 & 3.70 \\
\hline \multirow{2}{*}{\multicolumn{10}{|c|}{ Fourth subperiod: }} \\
\hline & & & & & & & & & \\
\hline $\begin{array}{l}\text { Total .......... } \\
\text { Average ..... }\end{array}$ & $\begin{array}{l}5.038 \\
1.008\end{array}$ & a. 555 & $\begin{array}{r}4.559 \\
912\end{array}$ & 5.114 & 11.01 & 90.49 & 101.50 & -.076 & 6.00 \\
\hline \multicolumn{10}{|l|}{ Fifth subperiod: } \\
\hline Total ............ & 5.441 & .753 & 4. 299 & 5.052 & 13.83 & 79.01 & 92.85 & +.389 & 8.00 \\
\hline $\mathrm{d}:$ & 1.088 & .151 & .860 & 1.010 & (......... & … & . $\ldots \ldots$ & +.078 & 1.60 \\
\hline Total.. & 5. 191 & $a .564$ & 4. 766 & 5.330 & 10.86 & 91.81 & 102.68 & -.139 & 10.00 \\
\hline Average & 1.038 & .113 & .953 & 1.066 & .......... & .......... & .......... & -.028 & 2.00 \\
\hline \multicolumn{10}{|l|}{$\begin{array}{l}\text { Entire preservative } \\
\text { period: }\end{array}$} \\
\hline $\begin{array}{l}\text { perod: } \\
\text { Total .............. }\end{array}$ & 30.598 & 3.511 & 27.340 & 30.851 & 11.47 & 89.35 & 100.82 & -.253 & 30.85 \\
\hline Average ............. & 1.020 & .117 & - $\quad .911$ & 1.028 & & & .......... & -.008 & 1.03 \\
\hline After period. & & & & & & & & & \\
\hline First subperiod: & 4.961 & .542 & 4.737 & 5,279 & 10,93 & 95,48 & 106.41 & & 0 \\
\hline Average .......... & .992 & .108 & .947 & 1. 056 & & & & -.064 & 0 \\
\hline \multicolumn{10}{|l|}{$\begin{array}{l}\text { Second subperiod: } \\
\text { Total }\end{array}$} \\
\hline $\begin{array}{l}\text { Total .............. } \\
\text { Average........ }\end{array}$ & 5. 020 & .626 & 4.848 & 5.474 & 12.47 & 96.57 & 109.04 & -.454 & 0 \\
\hline Entire after period: & 9.981 & 1,168 & 9.585 & 10.7 & 11.70 & 96.03 & 107.73 & & 0 \\
\hline Average ................ & .998 & .117 & .959 & 1.075 & & & & -.077 & 0 \\
\hline
\end{tabular}

$a$ Daily average added in order to complete record. 
SALICYLIC ACID AND SALICYLATES.

Table XVII.-Sulphur balances for Series VI-Continued.

[Averages are per day.]

No. 5.

\begin{tabular}{|c|c|c|c|c|c|c|c|c|c|}
\hline Period. & In food. & In feces. & In urine. & $\begin{array}{c}4 \\
\text { In feces } \\
\text { and } \\
\text { urine. } \\
(2+3)\end{array}$ & $\begin{array}{c}5 \\
\text { In } \\
\text { feces. } \\
(2 \div 1)\end{array}$ & $\underset{\substack{\text { In } \\
\text { urine. } \\
(3 \div 1)}}{6}$ & $\begin{array}{c}7 \\
\text { In feces } \\
\text { and } \\
\text { urine. } \\
(4 \div 1)\end{array}$ & $\begin{array}{c}\text { Balance. } \\
(1-4)\end{array}$ & $\begin{array}{l}\mathbf{9} \\
\text { Sali- } \\
\text { cylic } \\
\text { acid } \\
\text { admin- } \\
\text { istered. }\end{array}$ \\
\hline \multicolumn{10}{|l|}{ Fore period. } \\
\hline 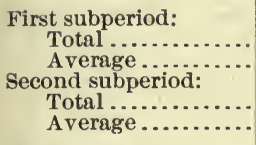 & $\begin{array}{r}\text { Grams. } \\
5.351 \\
1.070 \\
4.725 \\
.945\end{array}$ & $\begin{array}{r}\text { Grams. } \\
0.765 \\
.153 \\
\\
.692 \\
.138\end{array}$ & $\begin{array}{r}\text { Grams. } \\
4.565 \\
\quad .913\end{array}$ & $\begin{array}{c}\text { Grams. } \\
5.330 \\
1.066 \\
\\
4.403 \\
.881\end{array}$ & $\begin{array}{c}\text { Per ct. } \\
14.30 \\
\cdots \\
14.65 \\
\cdots\end{array}$ & $\begin{array}{l}\text { Perct. } \\
85.31 \\
\text {. }\end{array}$ & $\begin{array}{c}\text { Per ct. } \\
99.61 \\
\cdots \\
93.19 \\
\cdots\end{array}$ & $\begin{array}{l}\text { Grams. } \\
+0.021 \\
+.004 \\
+.322 \\
+.064\end{array}$ & $\begin{array}{c}\text { Grams. } \\
0 \\
0 \\
0 \\
0 \\
0\end{array}$ \\
\hline $\begin{array}{l}\text { Entire fore period: } \\
\text { Total ................. } \\
\text { Average ........... }\end{array}$ & $\begin{array}{r}10.076 \\
1.008\end{array}$ & $\begin{array}{r}1.457 \\
.146\end{array}$ & $\begin{array}{r}8.276 \\
.828\end{array}$ & $\begin{array}{r}9.733 \\
.973\end{array}$ & $\begin{array}{r}14.46 \\
\ldots \ldots \ldots \\
\end{array}$ & $\begin{array}{r}82.14 \\
\ldots \ldots \ldots\end{array}$ & $\begin{array}{r}96.60 \\
\ldots \ldots \ldots \\
\end{array}$ & $\begin{array}{r}+.343 \\
+.035\end{array}$ & $\begin{array}{l}0 \\
0\end{array}$ \\
\hline \multicolumn{10}{|l|}{ Preservative period. } \\
\hline $\begin{array}{l}\text { First subperiod: } \\
\text { Total ............. } \\
\text { Average......... }\end{array}$ & $\begin{array}{r}4.894 \\
.979\end{array}$ & $\begin{array}{l}.727 \\
.145\end{array}$ & $\begin{array}{r}4.342 \\
.868\end{array}$ & $\begin{array}{l}5.069 \\
1.014\end{array}$ & $\begin{array}{r}14.85 \\
\ldots \ldots\end{array}$ & $\begin{array}{r}88.72 \\
\ldots \ldots \ldots\end{array}$ & $\begin{array}{c}103.58 \\
\ldots \ldots \ldots\end{array}$ & $\begin{array}{r}-.175 \\
-.035\end{array}$ & $\begin{array}{r}1.05 \\
.21\end{array}$ \\
\hline $\begin{array}{l}\text { Tota } \\
\text { Aver }\end{array}$ & $\begin{array}{l}5.143 \\
1.029\end{array}$ & $\begin{array}{l}.531 \\
.106\end{array}$ & $\begin{array}{r}4.495 \\
.899\end{array}$ & $\begin{array}{l}5.026 \\
1.005\end{array}$ & $\begin{array}{l}10.32 \\
\ldots \ldots . .\end{array}$ & $\begin{array}{r}87.40 \\
\ldots . . . .\end{array}$ & $\begin{array}{r}97.73 \\
\ldots \ldots \ldots\end{array}$ & $\begin{array}{r}+.117 \\
+.024\end{array}$ & $\begin{array}{r}2.10 \\
.42\end{array}$ \\
\hline $\begin{array}{l}\text { Third sul } \\
\text { Total }\end{array}$ & & .662 & 4. 566 & 5.228 & 12.84 & 88.54 & 101.38 & +.024 & 3. 70 \\
\hline $\begin{array}{l}\text { Aver } \\
\text { Fourth s }\end{array}$ & & .132 & .913 & 1. 046 & & & .......... & -.015 & \\
\hline $\begin{array}{l}\text { Total } \\
\text { Aver: }\end{array}$ & $\begin{array}{l}5.175 \\
1.035\end{array}$ & $\begin{array}{l}.506 \\
.101\end{array}$ & $\begin{array}{r}4.632 \\
.926\end{array}$ & $\begin{array}{l}5.138 \\
1.028\end{array}$ & $\begin{array}{r}9.78 \\
\ldots . .6\end{array}$ & 89.51 & $\begin{array}{r}99.29 \\
\ldots \ldots \ldots\end{array}$ & $\begin{array}{r}+.037 \\
+.007\end{array}$ & $\begin{array}{l}6.00 \\
1.20\end{array}$ \\
\hline Fifth sub & & & & 1.020 & & & & +.001 & \\
\hline $\begin{array}{l}\text { Tot } \\
\text { Ave }\end{array}$ & $\begin{array}{l}5.316 \\
1.063\end{array}$ & $\begin{array}{l}.659 \\
.132\end{array}$ & $\begin{array}{r}4.670 \\
.934\end{array}$ & $\begin{array}{l}5.329 \\
1.066\end{array}$ & $\begin{array}{r}12.40 \\
\ldots . . .\end{array}$ & 87.85 & $\begin{array}{l}100.24 \\
\ldots \ldots \ldots\end{array}$ & $\begin{array}{l}-.013 \\
-.003\end{array}$ & $\begin{array}{l}8.00 \\
1.60\end{array}$ \\
\hline $\begin{array}{l}\text { Sixth subperic d: } \\
\text { Total ................ } \\
\text { Average ............ }\end{array}$ & $\begin{array}{l}5.300 \\
1.060\end{array}$ & $\begin{array}{l}.696 \\
.139\end{array}$ & $\begin{array}{r}4.534 \\
.907\end{array}$ & $\begin{array}{l}5.230 \\
1.046\end{array}$ & $\begin{array}{r}13.13 \\
\ldots \ldots .\end{array}$ & $\begin{array}{r}85.55 \\
\therefore \ldots \ldots\end{array}$ & $\begin{array}{r}98.68 \\
\ldots \ldots\end{array}$ & $\begin{array}{r}+.070 \\
+.014\end{array}$ & $\begin{array}{r}10.00 \\
2.00\end{array}$ \\
\hline $\begin{array}{l}\text { Entire preservative } \\
\text { period: } \\
\text { Total .................. } \\
\text { Average........... }\end{array}$ & $\begin{array}{r}30.985 \\
1.033\end{array}$ & $\begin{array}{r}3.781 \\
.126\end{array}$ & $\begin{array}{r}27.239 \\
.908\end{array}$ & $\begin{array}{r}31.020 \\
1.034\end{array}$ & $\begin{array}{r}12.20 \\
\cdots \cdots\end{array}$ & $\begin{array}{c}87.91 \\
\ldots .6 .\end{array}$ & $\begin{array}{c}100.11 \\
\ldots \ldots\end{array}$ & $\begin{array}{l}-.035 \\
-.001\end{array}$ & $\begin{array}{r}30.85 \\
1.03\end{array}$ \\
\hline After period. & & & & & & & & & \\
\hline $\begin{array}{l}\text { First subperiod: } \\
\quad \text { Total ............ } \\
\text { Average....... }\end{array}$ & $\begin{array}{l}5.075 \\
1.015\end{array}$ & $\begin{array}{l}.637 \\
.127\end{array}$ & $\begin{array}{r}a 4.516 \\
.903\end{array}$ & $\begin{array}{l}5.153 \\
1.031\end{array}$ & $\begin{array}{c}12.55 \\
\ldots . .2 .\end{array}$ & 88.99 & $\begin{array}{c}101.51 \\
\ldots\end{array}$ & $\begin{array}{l}-.078 \\
-.016\end{array}$ & $\begin{array}{l}0 \\
0\end{array}$ \\
\hline $\begin{array}{l}\text { Second subperiod: } \\
\text { Total ................. } \\
\text { Average ......... }\end{array}$ & $\begin{array}{l}5.079 \\
1.016\end{array}$ & $\begin{array}{l}.420 \\
.084\end{array}$ & $\begin{array}{r}4.677 \\
.935\end{array}$ & $\begin{array}{l}5.097 \\
1.019\end{array}$ & $\begin{array}{r}8.27 \\
\ldots . . .\end{array}$ & $\begin{array}{r}92.09 \\
\ldots \ldots\end{array}$ & $\begin{array}{c}100.35 \\
\cdots \ldots \ldots\end{array}$ & $\begin{array}{r}-.018 \\
-.003\end{array}$ & $\begin{array}{l}0 \\
0\end{array}$ \\
\hline $\begin{array}{l}\text { Entire after period: } \\
\text { Total .............. } \\
\text { Average....... }\end{array}$ & $\begin{array}{r}10.154 \\
1.015\end{array}$ & $\begin{array}{r}1.057 \\
.106\end{array}$ & $\begin{array}{r}9.193 \\
.919\end{array}$ & $\begin{array}{r}10.250 \\
1.025\end{array}$ & $\begin{array}{r}10.41 \\
\ldots \ldots\end{array}$ & $\begin{array}{r}90.54 \\
\ldots . . .\end{array}$ & $\begin{array}{c}100.95 \\
\cdots \ldots\end{array}$ & $\begin{array}{r}-.096 \\
-.010\end{array}$ & $\begin{array}{l}0 \\
0\end{array}$ \\
\hline
\end{tabular}

$a$ Daily average added in order to complete record. 
TABLE XVII.-Sulphur balances for Series VI-Continued.

[Averages are per day.]

No. 6.

\begin{tabular}{|c|c|c|c|c|c|c|c|c|c|}
\hline Period. & In food. & In feces. & $\underset{\text { In }}{\mathbf{3}}$ & $\begin{array}{c}4 \\
\text { In feces } \\
\text { and } \\
\text { urine. } \\
(2+3)\end{array}$ & $\begin{array}{c}5 \\
\text { In } \\
\text { feces. } \\
(2 \div 1)\end{array}$ & $\underset{\substack{\text { In } \\
\text { urine. } \\
(3 \div 1)}}{6}$ & $\begin{array}{c}7 \\
\text { In feces } \\
\text { and } \\
\text { urine. } \\
(4 \div 1)\end{array}$ & $\begin{array}{c}\text { Balance. } \\
(1-4)\end{array}$ & $\begin{array}{c}9 \\
\text { Sali- } \\
\text { cylic } \\
\text { acid } \\
\text { admin- } \\
\text { istered. }\end{array}$ \\
\hline Fore period. & & & & a. & & & & & \\
\hline 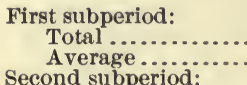 & $\begin{array}{r}\text { Grams. } \\
\quad 4.824 \\
\quad .965\end{array}$ & $\begin{array}{r}\text { Grams. } \\
0.680 \\
.136\end{array}$ & $\begin{array}{r}\text { Grams. } \\
4.038 \\
.808\end{array}$ & $\begin{array}{r}\text { Grams. } \\
4.718 \\
.944\end{array}$ & $\begin{array}{l}\text { Per ct. } \\
14.10 \\
1.1 .\end{array}$ & $\begin{array}{l}\text { Per ct. } \\
83.71 \\
.\end{array}$ & $\begin{array}{r}\text { Per ct. } \\
97.81 \\
\\
\end{array}$ & $\begin{array}{l}\text { Grams. } \\
+0.106 \\
+.021\end{array}$ & $\begin{array}{l}\text { Grams. } \\
0 \\
0\end{array}$ \\
\hline $\begin{array}{l}\text { Total ................ } \\
\text { Average ........ }\end{array}$ & $\begin{array}{r}4.291 \\
.858\end{array}$ & $\begin{array}{l}.771 \\
.154\end{array}$ & $\begin{array}{r}3.796 \\
.759\end{array}$ & $\begin{array}{r}4.567 \\
.913\end{array}$ & $\begin{array}{r}17.97 \\
1.2 .\end{array}$ & $\begin{array}{r}88.46 \\
\ldots\end{array}$ & $\begin{array}{l}106.43 \\
\ldots \ldots\end{array}$ & $\begin{array}{l}-.276 \\
-.055\end{array}$ & $\begin{array}{l}0 \\
0\end{array}$ \\
\hline $\begin{array}{l}\text { Entire fore period: } \\
\text { Total ................. } \\
\text { Average ........ }\end{array}$ & $\begin{array}{r}9.115 \\
.912\end{array}$ & $\begin{array}{r}1.451 \\
.145\end{array}$ & $\begin{array}{r}7.834 \\
.783\end{array}$ & $\begin{array}{r}9.285 \\
.929\end{array}$ & $\begin{array}{r}15.92 \\
\cdots \cdots\end{array}$ & $\begin{array}{r}85.95 \\
\cdots\end{array}$ & $\begin{array}{l}101.87 \\
\cdots\end{array}$ & $\begin{array}{l}-.170 \\
-.017\end{array}$ & $\begin{array}{l}0 \\
0\end{array}$ \\
\hline Preservative period. & & & & & & & & & \\
\hline 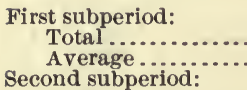 & $\begin{array}{r}4.493 \\
.899\end{array}$ & $\begin{array}{l}.722 \\
.144\end{array}$ & $\begin{array}{r}4.090 \\
.818\end{array}$ & $\begin{array}{r}4.812 \\
.962\end{array}$ & $\begin{array}{r}16.07 \\
\cdots\end{array}$ & 91.03 & $\begin{array}{l}107.10 \\
\cdots \cdots\end{array}$ & $\begin{array}{l}-.319 \\
-.063\end{array}$ & $\begin{array}{r}1.05 \\
.21\end{array}$ \\
\hline $\begin{array}{r}\text { Total ......................... } \\
\text { Average }\end{array}$ & $\begin{array}{r}4.655 \\
.931\end{array}$ & $\begin{array}{l}.733 \\
.147\end{array}$ & $\begin{array}{r}4.521 \\
.904\end{array}$ & $\begin{array}{l}5.254 \\
1.051\end{array}$ & $\begin{array}{r}15.75 \\
\cdots \cdots \cdots\end{array}$ & $\begin{array}{r}97.12 \\
\ldots . \cdots\end{array}$ & $\begin{array}{l}112.87 \\
\cdots\end{array}$ & $\begin{array}{l}-.599 \\
-.120\end{array}$ & $\begin{array}{r}2.10 \\
.42\end{array}$ \\
\hline 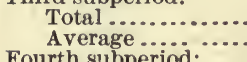 & $\begin{array}{r}4.789 \\
.958\end{array}$ & $\begin{array}{l}.926 \\
.185\end{array}$ & $\begin{array}{r}a 4.748 \\
\quad .950\end{array}$ & $\begin{array}{l}5.674 \\
1.135\end{array}$ & $\begin{array}{r}19.34 \\
\cdots\end{array}$ & $\begin{array}{r}99.14 \\
\cdots\end{array}$ & $\begin{array}{l}118.48 \\
\ldots \ldots .\end{array}$ & $\begin{array}{l}-.885 \\
-.177\end{array}$ & $\begin{array}{r}3.70 \\
.74\end{array}$ \\
\hline $\begin{array}{l}\text { Fourth subperiod: } \\
\text { Total ............................. } \\
\text { Average }\end{array}$ & $\begin{array}{r}4.867 \\
.973\end{array}$ & $\begin{array}{l}.784 \\
.157\end{array}$ & $\begin{array}{r}a 5.065 \\
1.013\end{array}$ & $\begin{array}{l}5.849 \\
1.170\end{array}$ & $\begin{array}{r}16.11 \\
\ldots \ldots \ldots\end{array}$ & $\begin{array}{c}104.07 \\
\ldots \ldots \ldots\end{array}$ & $\begin{array}{c}120.18 \\
. \ldots \ldots\end{array}$ & $\begin{array}{l}-.982 \\
-.197\end{array}$ & $\begin{array}{l}6.00 \\
1.20\end{array}$ \\
\hline $\begin{array}{l}\text { Fifth subperiod: } \\
\text { Total } \ldots . . . . . . \\
\text { Average }\end{array}$ & $\begin{array}{l}5.123 \\
1.025\end{array}$ & $\begin{array}{r}.873 \\
.175\end{array}$ & $\begin{array}{r}4.800 \\
.960\end{array}$ & $\begin{array}{l}5.673 \\
1.135\end{array}$ & 17.04 & 93.70 & 110.74 & $\begin{array}{l}-.550 \\
-.110\end{array}$ & $\begin{array}{l}8.00 \\
1.60\end{array}$ \\
\hline 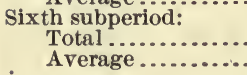 & $\begin{array}{r}4.898 \\
.980\end{array}$ & $\begin{array}{l}.719 \\
.144\end{array}$ & $\begin{array}{r}4.390 \\
.878\end{array}$ & $\begin{array}{l}5.109 \\
1.022\end{array}$ & $\begin{array}{r}14.68 \\
\cdots\end{array}$ & 89.63 & $\begin{array}{l}104.31 \\
\ldots .\end{array}$ & $\begin{array}{l}-.211 \\
-.042\end{array}$ & $\begin{array}{l}8.00 \\
1.60\end{array}$ \\
\hline 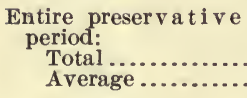 & $\begin{array}{r}28.825 \\
.961\end{array}$ & $\begin{array}{r}4.757 \\
.159\end{array}$ & $\begin{array}{r}27.614 \\
.920\end{array}$ & $\begin{array}{r}32.371 \\
1.079\end{array}$ & $\begin{array}{l}16.50 \\
\cdots . . .\end{array}$ & $\begin{array}{c}95.80 \\
\ldots \ldots \ldots\end{array}$ & $\begin{array}{c}112.30 \\
. \ldots .\end{array}$ & $\begin{array}{r}-3.546 \\
-.118\end{array}$ & $\begin{array}{r}28.85 \\
.96\end{array}$ \\
\hline After period. & & & & & & & & & \\
\hline 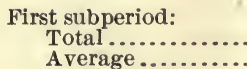 & $\begin{array}{r}4.645 \\
.929\end{array}$ & $\begin{array}{l}.878 \\
.176\end{array}$ & $\begin{array}{r}4.346 \\
.869\end{array}$ & $\begin{array}{l}5.224 \\
1.045\end{array}$ & 18.90 & 93.56 & 112.46 & $\begin{array}{r}-.579 \\
-.116\end{array}$ & $\begin{array}{l}0 \\
0\end{array}$ \\
\hline $\begin{array}{l}\text { Second subperiod: } \\
\quad \text { Total ...................... } \\
\text { Average ........ }\end{array}$ & $\begin{array}{r}4.645 \\
.929\end{array}$ & $\begin{array}{r}a .590 \\
.118\end{array}$ & $\begin{array}{r}4.611 \\
.922\end{array}$ & $\begin{array}{l}5.201 \\
1.040\end{array}$ & $\begin{array}{r}12.70 \\
\ldots \ldots\end{array}$ & $\begin{array}{r}99.27 \\
\ldots \ldots . .\end{array}$ & $\begin{array}{r}111.97 \\
\ldots . . .\end{array}$ & $\begin{array}{l}-.556 \\
-.111\end{array}$ & $\begin{array}{l}0 \\
0\end{array}$ \\
\hline $\begin{array}{c}\text { Entire after period: } \\
\text { Total } \ldots \ldots \ldots \\
\text { Average } \ldots \ldots\end{array}$ & $\begin{array}{r}9.290 \\
.929\end{array}$ & $\begin{array}{r}1.468 \\
.147\end{array}$ & $\begin{array}{r}8.957 \\
.896\end{array}$ & $\begin{array}{r}10.425 \\
1.043\end{array}$ & $\begin{array}{r}15.80 \\
\cdots . . . .\end{array}$ & $\begin{array}{r}96.42 \\
\ldots \ldots \ldots .\end{array}$ & $\begin{array}{c}112.22 \\
\cdots \ldots . . .\end{array}$ & $\begin{array}{r}-1.135 \\
-.114\end{array}$ & $\begin{array}{l}0 \\
0\end{array}$ \\
\hline
\end{tabular}

$a$ Daily average added in order to complete record. 
Table XVII.-Sulphur balances for Series VI-Continued.

[A verages are per day.]

No. 7.

\begin{tabular}{|c|c|c|c|c|c|c|c|c|c|}
\hline Period. & In food. & $\begin{array}{c}2 \\
\text { In feces. }\end{array}$ & $\underset{\substack{\text { In } \\
\text { urine. }}}{3}$ & $\begin{array}{l}\mathbf{4} \\
\text { In feces } \\
\text { and } \\
\text { urine. } \\
(2+3)\end{array}$ & $\begin{array}{c}5 \\
\text { In } \\
\text { feces. } \\
(2 \div 1)\end{array}$ & $\underset{\substack{\text { In } \\
(3 \div 1)}}{6}$ & $\begin{array}{l}7 \\
\text { In feces } \\
\text { and } \\
\text { urine. } \\
(4 \div 1)\end{array}$ & $\begin{array}{c}8 \\
\begin{array}{c}\text { Balance. } \\
(1-4)\end{array}\end{array}$ & $\begin{array}{l}\mathbf{9} \\
\text { Sali- } \\
\text { cylic } \\
\text { acid } \\
\text { admin- } \\
\text { istered. }\end{array}$ \\
\hline Fore period. & & & & & & & & & \\
\hline 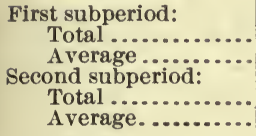 & $\begin{array}{r}\text { Grams. } \\
4.328 \\
.866 \\
3.935 \\
.787\end{array}$ & $\begin{array}{r}\text { Grams. } \\
0.607 \\
.121 \\
.331 \\
.066\end{array}$ & $\begin{array}{r}\text { Grams. } \\
3.756 \\
.751 \\
4.280 \\
.856\end{array}$ & $\begin{array}{r}\text { Grams. } \\
4.363 \\
.873 \\
4.611 \\
.922\end{array}$ & $\begin{array}{r}\text { Per ct. } \\
14.02 \\
8.41 \\
8 .\end{array}$ & $\begin{array}{c}\text { Per ct. } \\
86.78 \\
103.77 \\
\ldots\end{array}$ & $\begin{array}{l}\text { Per ct. } \\
100.81 \\
\ldots \ldots \\
117.18 \\
\cdots\end{array}$ & $\begin{array}{l}\text { Grams. } \\
-0.035 \\
-.007 \\
-.676 \\
-.135\end{array}$ & $\begin{array}{c}\text { Grams. } \\
0 \\
0 \\
0 \\
0\end{array}$ \\
\hline $\begin{array}{l}\text { Fintire fore period: } \\
\text { Total ................ } \\
\text { Average .......... }\end{array}$ & $\begin{array}{r}8.263 \\
.826\end{array}$ & $\begin{array}{l}.938 \\
.094\end{array}$ & $\begin{array}{r}8.036 \\
.804\end{array}$ & $\begin{array}{r}8.974 \\
.897\end{array}$ & $\begin{array}{r}11.35 \\
\ldots \ldots . .\end{array}$ & $\begin{array}{r}97.25 \\
\ldots \ldots\end{array}$ & $\begin{array}{r}108.60 \\
\cdots \ldots\end{array}$ & $\begin{array}{l}-.711 \\
-.071\end{array}$ & $\begin{array}{l}0 \\
0\end{array}$ \\
\hline Preservative period. & & & & & & & & & \\
\hline $\begin{array}{l}\text { First subperiod: } \\
\text { Total } \ldots . . . . . . . . \\
\text { Average.......... }\end{array}$ & $\begin{array}{r}3.980 \\
.796\end{array}$ & $\begin{array}{l}.428 \\
.086\end{array}$ & $\begin{array}{r}3.513 \\
.703\end{array}$ & $\begin{array}{r}3.941 \\
.788\end{array}$ & 10.75 & 88.27 & 99.02 & $\begin{array}{r}+.039 \\
+.008\end{array}$ & $\begin{array}{r}1.05 \\
.21\end{array}$ \\
\hline $\begin{array}{l}\text { Second subperiod: } \\
\text { Total } \ldots . . . . . . . . . . \\
\text { Average }\end{array}$ & $\begin{array}{r}4.448 \\
.890\end{array}$ & $\begin{array}{r}.287 \\
.057\end{array}$ & $\begin{array}{r}4.085 \\
.817\end{array}$ & $\begin{array}{r}4.372 \\
.874\end{array}$ & 6.45 & 91.84 & 98.29 & +.076 & $\begin{array}{r}2.10 \\
42\end{array}$ \\
\hline $\begin{array}{l}\text { Third subperiod: } \\
\text { Total ........... } \\
\text { Average ....... } \\
\text { Fourth subperiod: }\end{array}$ & $\begin{array}{r}4.280 \\
.856\end{array}$ & $\begin{array}{r}.448 \\
.090\end{array}$ & $\begin{array}{r}3.368 \\
.674\end{array}$ & $\begin{array}{r}3.816 \\
.763\end{array}$ & $\begin{array}{r}10.47 \\
\ldots \ldots \ldots\end{array}$ & $\begin{array}{r}78.69 \\
\ldots \ldots \ldots\end{array}$ & $\begin{array}{r}89.16 \\
\cdots\end{array}$ & $\begin{array}{r}+.464 \\
+.093\end{array}$ & $\begin{array}{r}3.70 \\
.74\end{array}$ \\
\hline 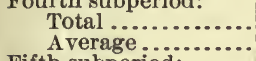 & $\begin{array}{l}4.346 \\
. .869\end{array}$ & $\begin{array}{l}.337 \\
.067\end{array}$ & $\begin{array}{r}3.564 \\
.713\end{array}$ & $\begin{array}{r}3.901 \\
.780\end{array}$ & 7.75 & $\begin{array}{r}82.01 \\
\ldots \ldots \ldots\end{array}$ & $\begin{array}{r}89.76 \\
\ldots . . . . .\end{array}$ & $\begin{array}{r}+.445 \\
+.089\end{array}$ & $\begin{array}{l}6.00 \\
1.20\end{array}$ \\
\hline $\begin{array}{l}\text { Fifth subperiod: } \\
\text { Total........... } \\
\text { Average....... }\end{array}$ & $\begin{array}{r}4.642 \\
.928\end{array}$ & $\begin{array}{l}.471 \\
.094\end{array}$ & $\begin{array}{r}3.870 \\
.774\end{array}$ & $\begin{array}{r}4.341 \\
.868\end{array}$ & $\begin{aligned} 10.15 \\
.\end{aligned}$ & 83.37 & $\begin{array}{r}93.52 \\
\ldots . . .\end{array}$ & $\begin{array}{r}+.301 \\
+.060\end{array}$ & $\begin{array}{l}8.00 \\
1.60\end{array}$ \\
\hline $\begin{array}{l}\text { Sixth subperiod: } \\
\text { Total ................ } \\
\text { Average .......... }\end{array}$ & $\begin{array}{r}4.540 \\
.908\end{array}$ & $\begin{array}{l}.239 \\
.048\end{array}$ & $\begin{array}{l}6.424 \\
1.285\end{array}$ & $\begin{array}{l}6.663 \\
1.333\end{array}$ & $\begin{array}{r}5.26 \\
\ldots \ldots\end{array}$ & $\begin{array}{r}141.50 \\
\ldots .\end{array}$ & $\begin{array}{c}146.76 \\
\ldots \ldots\end{array}$ & $\begin{array}{r}-2.123 \\
-.425\end{array}$ & $\begin{array}{r}10.00 \\
2.00\end{array}$ \\
\hline $\begin{array}{l}\text { Entire preservative } \\
\text { period: } \\
\text { Total ................. } \\
\text { Average.......... }\end{array}$ & $\begin{array}{r}26.236 \\
.875\end{array}$ & $\begin{array}{r}2.210 \\
.074\end{array}$ & $\begin{array}{r}24.824 \\
.827\end{array}$ & $\begin{array}{r}27.034 \\
.901\end{array}$ & $\begin{array}{r}8.42 \\
\ldots . . .\end{array}$ & $\begin{array}{r}94.62 \\
\ldots \ldots \ldots\end{array}$ & $\begin{array}{c}103.04 \\
\ldots \ldots \ldots\end{array}$ & $\begin{array}{r}-.798 \\
-.026\end{array}$ & $\begin{array}{r}30.85 \\
1.03\end{array}$ \\
\hline - After period. & & & & & & & & & \\
\hline $\begin{array}{l}\text { First subperiod: } \\
\text { Total............. } \\
\text { Average } . . . . . .\end{array}$ & $\begin{array}{r}4.282 \\
.856\end{array}$ & $\begin{array}{l}.504 \\
.101\end{array}$ & $\begin{array}{r}3.650 \\
.730\end{array}$ & $\begin{array}{r}4.154 \\
.831\end{array}$ & $\begin{array}{r}11.77 \\
\ldots . . .\end{array}$ & $\begin{array}{r}85.24 \\
\ldots . . .\end{array}$ & 97.01 & $\begin{array}{r}+.128 \\
+.025\end{array}$ & $\begin{array}{l}0 \\
0\end{array}$ \\
\hline $\begin{array}{l}\text { Second subperiod: } \\
\text { Total ................... } \\
\text { Average ........ }\end{array}$ & $\begin{array}{r}4.412 \\
.882\end{array}$ & $\begin{array}{l}.638 \\
.128\end{array}$ & $\begin{array}{r}3.891 \\
6778\end{array}$ & $\begin{array}{r}4.529 \\
.906\end{array}$ & $\begin{array}{r}14.46 \\
\ldots . . .\end{array}$ & $\begin{array}{r}88.19 \\
\ldots \ldots\end{array}$ & $\begin{array}{r}102.65 \\
\cdots\end{array}$ & $\begin{array}{l}-.117 \\
-.024\end{array}$ & $\begin{array}{l}0 \\
0\end{array}$ \\
\hline $\begin{array}{l}\text { Entire after period: } \\
\text { Total ................ } \\
\text { Average }\end{array}$ & $\begin{array}{r}8.694 \\
.869\end{array}$ & $\begin{array}{r}1.142 \\
.114\end{array}$ & $\begin{array}{r}7.541 \\
.754\end{array}$ & $\begin{array}{r}8.683 \\
.868\end{array}$ & 13.14 & 86.74 & 99.87 & $\begin{array}{r}+.011 \\
+.001\end{array}$ & $\begin{array}{l}0 \\
0\end{array}$ \\
\hline
\end{tabular}


TABLE XVII.-Sulphur balances for Series VI-Continued.

[Averages are per day.]

No. 8.

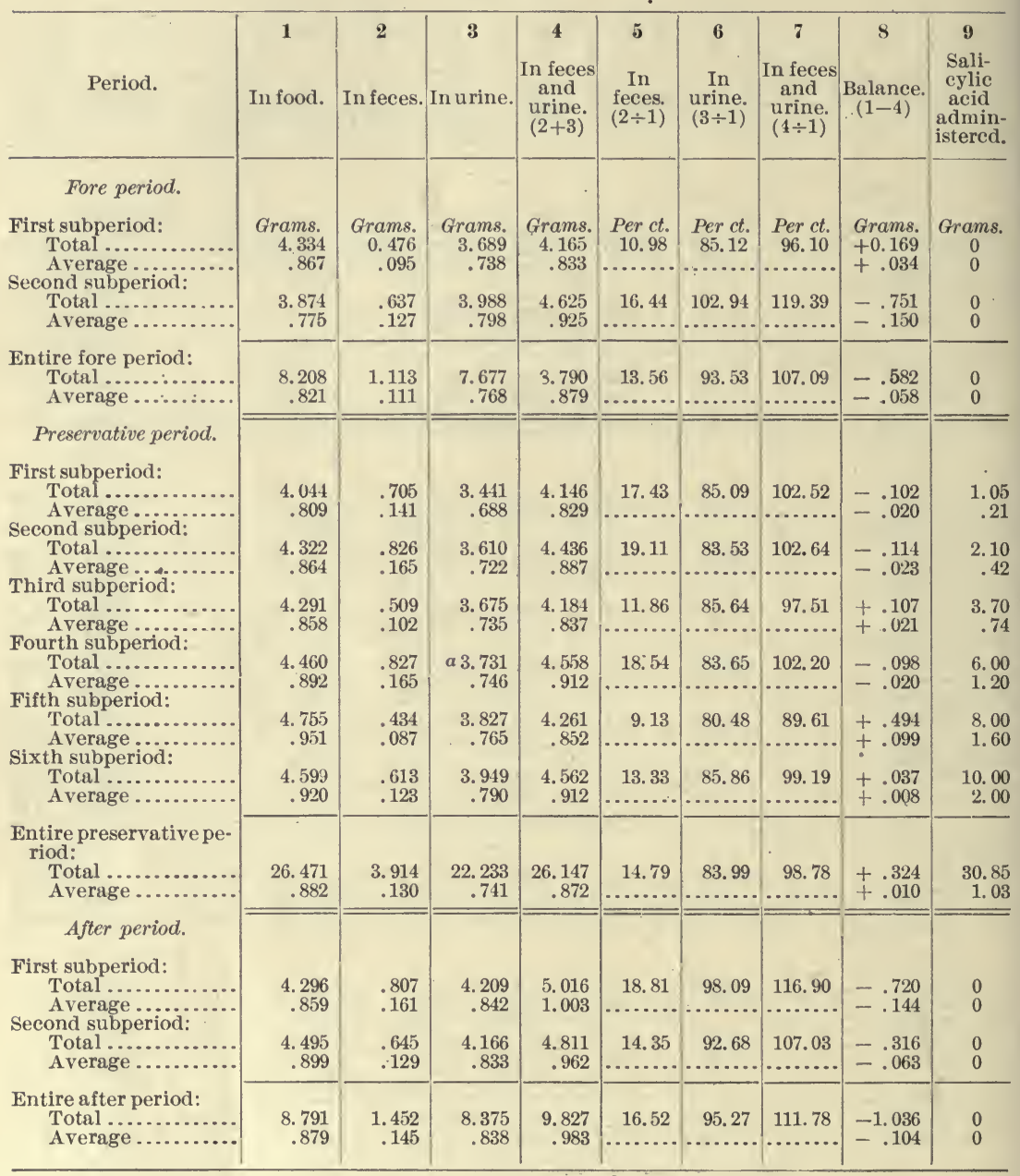

$a$ Daily average added in order to complete record. 
SALICYLIC ACID AND SALICYLATES.

TABLE XVII.-Sulphur balances for Series VI-Continued.

[Averages are per day.]

No. 9.

\begin{tabular}{|c|c|c|c|c|c|c|c|c|c|}
\hline Period. & $\begin{array}{c}1 \\
\text { In food. }\end{array}$ & $\begin{array}{c}2 \\
\text { In feces. }\end{array}$ & In urine. & \begin{tabular}{|c}
4 \\
In feces \\
and \\
urine. \\
$(2+3)$
\end{tabular} & $\begin{array}{c}5 \\
\text { In } \\
\text { feces. } \\
(2 \div 1)\end{array}$ & $\begin{array}{c}6 \\
\text { In } \\
\text { urine. } \\
(3 \div 1)\end{array}$ & \begin{tabular}{|c|}
7 \\
In feces \\
and \\
urine. \\
$(4 \div 1)$
\end{tabular} & $\left|\begin{array}{c}8 \\
\text { Balance. } \\
(1-4)\end{array}\right|$ & $\begin{array}{c}9 \\
\text { Sali- } \\
\text { cylic } \\
\text { acid } \\
\text { admin- } \\
\text { istered. }\end{array}$ \\
\hline Fore period. & & & & & & & & & \\
\hline $\begin{array}{l}\text { First subperiod: } \\
\text { Total.................. } \\
\text { Average ........ } \\
\text { Second subperiod: } \\
\text { Total ............. } \\
\text { Average........ }\end{array}$ & $\begin{array}{r}\text { Grams. } \\
5.683 \\
1.137 . \\
5.285 \\
1.057\end{array}$ & $\begin{array}{r}\text { Grams. } \\
0.310 \\
.062 \\
.483 \\
.097 \\
\end{array}$ & $\begin{array}{r}\text { Grams. } \\
4.671 \\
.934 \\
4.547 \\
.909\end{array}$ & $\begin{array}{r}\text { Grams. } \\
4.981 \\
.996 \\
\\
5.030 \\
1.006 \\
\end{array}$ & $\begin{array}{r}\text { Per ct. } \\
5.45 \\
\times \\
9.14\end{array}$ & $\begin{array}{r}\text { Per ct. } \\
82.19\end{array}$ & $\begin{array}{r}\text { Per ct. } \\
87.65\end{array}$ & $\begin{array}{l}\text { Grams. } \\
+0.702 \\
+. .141 \\
+.255 \\
+.051\end{array}$ & $\begin{array}{c}\text { Grams. } \\
0 \\
0 \\
0 \\
0\end{array}$ \\
\hline $\begin{array}{l}\text { Entire fore period: } \\
\text { Total .............. } \\
\text { Average ....... }\end{array}$ & $\begin{array}{r}10.968 \\
1.097 \\
\end{array}$ & $\begin{array}{r}.793 \\
.079 \\
\end{array}$ & $\begin{array}{r}9.218 \\
.922 \\
\end{array}$ & $\begin{array}{r}10.011 \\
1.001 \\
\end{array}$ & $\begin{array}{r}-7.23 \\
\cdots . . .\end{array}$ & 84.04 & $\begin{array}{c}91.27 \\
\ldots \ldots \ldots .\end{array}$ & $\begin{array}{r}+.957 \\
+.096 \\
\end{array}$ & $\begin{array}{l}0 \\
0\end{array}$ \\
\hline Preservative period. & & & & & & & & & \\
\hline $\begin{array}{l}\text { First subperiod: } \\
\text { Total ........... } \\
\text { Average..... } \\
\text { Second subperiod }\end{array}$ & $\begin{array}{l}5.585 \\
1.117\end{array}$ & $\begin{array}{l}.716 \\
.143\end{array}$ & $\begin{array}{r}4.948 \\
.990\end{array}$ & $\begin{array}{l}5.664 \\
1.133\end{array}$ & 12. 82 & 88.59 & 101.41 & $\begin{array}{l}-.079 \\
-.016\end{array}$ & $\begin{array}{r}1.05 \\
.21\end{array}$ \\
\hline $\begin{array}{l}\text { Secono } \\
\text { Tot } \\
\text { Av } \\
\text { Third s }\end{array}$ & $\begin{array}{l}5.759 \\
1.152\end{array}$ & $\begin{array}{l}.540 \\
.108\end{array}$ & $\begin{array}{r}4.570 \\
.914\end{array}$ & $\begin{array}{l}5.110 \\
1.022\end{array}$ & 9.38 & 79.35 & 88.73 & $\begin{array}{r}+.649 \\
+.130\end{array}$ & $\begin{array}{r}2.10 \\
.42\end{array}$ \\
\hline $\begin{array}{l}\text { Third s } \\
\text { Tot } \\
\text { Av }\end{array}$ & $\begin{array}{l}5.830 \\
1.166\end{array}$ & $\begin{array}{l}.528 \\
.106\end{array}$ & $\begin{array}{r}4.780 \\
.956\end{array}$ & $\begin{array}{l}5.308 \\
1.062\end{array}$ & 9.06 & 81.99 & 91.05 & $\begin{array}{r}+.522 \\
+.104\end{array}$ & $\begin{array}{r}3.70 \\
.74\end{array}$ \\
\hline $\begin{array}{r}\text { Fourth su } \\
\text { Total } \\
\text { Avera } \\
\text { Fifth subr }\end{array}$ & $\begin{array}{l}7.164 \\
1.433\end{array}$ & $\begin{array}{l}.445 \\
.089\end{array}$ & $\begin{array}{r}4.567 \\
.913\end{array}$ & $\begin{array}{l}5.012 \\
1.002\end{array}$ & 6.21 & 63.74 & 69.96 & $\begin{array}{l}+2.152 \\
+.431\end{array}$ & $\begin{array}{l}6.00 \\
1.20\end{array}$ \\
\hline $\begin{aligned} \text { Fifth } \\
\text { To } \\
\text { AV }\end{aligned}$ & $\begin{array}{l}5.864 \\
1.173\end{array}$ & $\begin{array}{r}.762 \\
.152\end{array}$ & $\begin{array}{r}4.072 \\
.814\end{array}$ & $\begin{array}{r}4.834 \\
.967\end{array}$ & 12.99 & 69.44 & 82.44 & $\begin{array}{l}+1.030 \\
+.206\end{array}$ & $\begin{array}{l}8.00 \\
1.60\end{array}$ \\
\hline $\begin{array}{l}\text { Sixth subperiod: } \\
\quad \text { Total ................................... } \\
\text { Average }\end{array}$ & $\begin{array}{l}5.913 \\
1.183 \\
\end{array}$ & $\begin{array}{r}.592 \\
.118 \\
\end{array}$ & $\begin{array}{r}4.864 \\
.973 \\
\end{array}$ & $\begin{array}{l}5.456 \\
1.091 \\
\end{array}$ & $\begin{array}{r}10.01 \\
\cdots \cdots \cdots \\
\cdots\end{array}$ & 82.26 & $\begin{array}{c}92.27 \\
\ldots \ldots \ldots\end{array}$ & $\begin{array}{r}+.457 \\
+.092 \\
\end{array}$ & $\begin{array}{r}10.00 \\
2.00\end{array}$ \\
\hline $\begin{array}{l}\text { Entire preservative } \\
\text { period: } \\
\text { Total ................ } \\
\text { Average ............ }\end{array}$ & $\begin{array}{r}36.115 \\
1.204 \\
\end{array}$ & $\begin{array}{r}3.583 \\
.119 \\
\end{array}$ & $\begin{array}{r}27.801 \\
.927\end{array}$ & $\begin{array}{r}31.384 \\
1.046 \\
\end{array}$ & $\begin{array}{r}9.92 \\
\cdots \cdots \cdots \\
\end{array}$ & 76.98 & $\begin{array}{c}86.90 \\
\ldots \ldots . .\end{array}$ & $\begin{array}{r}+4.731 \\
+\quad .158 \\
\end{array}$ & $\begin{array}{r}30.85 \\
1.03 \\
\end{array}$ \\
\hline After period. & & & & & & & & & \\
\hline $\begin{array}{l}\text { First subperiod: } \\
\text { Total ......... } \\
\text { Average ..... } \\
\text { Second subperiod }\end{array}$ & $\begin{array}{l}\text { 5.798 } \\
1.160\end{array}$ & $\begin{array}{l}.263 \\
.053\end{array}$ & $\begin{array}{r}4.345 \\
.869\end{array}$ & $\begin{array}{r}4.608 \\
.922\end{array}$ & 4.54 & 74.94 & 79.48 & $\begin{array}{r}+1.190 \\
+\quad .238\end{array}$ & $\begin{array}{l}0 \\
0\end{array}$ \\
\hline $\begin{array}{l}\text { Second subperiod: } \\
\text { Total .................. } \\
\text { Average ............. }\end{array}$ & $\begin{array}{l}5.739 \\
1.148\end{array}$ & $\begin{array}{l}.626 \\
.125\end{array}$ & $\begin{array}{r}4.578 \\
.916\end{array}$ & $\begin{array}{l}5.204 \\
1.041\end{array}$ & 10.91 & 79.77 & 90.68 & $\begin{array}{r}+.535 \\
+.107 \\
\end{array}$ & $\begin{array}{l}0 \\
0\end{array}$ \\
\hline $\begin{array}{l}\text { Entire after period: } \\
\quad \text { Total ............... } \\
\text { Average ........ }\end{array}$ & $\begin{array}{r}11.537 \\
1.154\end{array}$ & $\begin{array}{r}.889 \\
: 089\end{array}$ & $\begin{array}{r}8.923 \\
.892\end{array}$ & $\begin{array}{r}9.812 \\
.981\end{array}$ & 7. 71 & 77.34 & 85.05 & $\begin{array}{r}+1.725 \\
+.173\end{array}$ & $\begin{array}{l}0 \\
0\end{array}$ \\
\hline
\end{tabular}


TABLE XVII.-Sulphur balances for Series VI-Continued.

[Averages are per day.]

No. 10.

\begin{tabular}{|c|c|c|c|c|c|c|c|c|c|}
\hline - & In food. & In feces. & $\begin{array}{c}3 \\
\text { In urine. }\end{array}$ & $\begin{array}{c}4 \\
\text { In feces } \\
\text { and } \\
\text { urine. } \\
(2+3)\end{array}$ & $\begin{array}{c}5 \\
\text { In } \\
\text { feces. } \\
(2 \div 1)\end{array}$ & $\begin{array}{c}6 \\
\text { In } \\
\text { urine. } \\
(3 \div 1)\end{array}$ & \begin{tabular}{|c|} 
\\
In feces \\
and \\
urine. \\
$(4 \div 1)$
\end{tabular} & $\begin{array}{c}8 \\
\begin{array}{c}\text { Balance. } \\
(1-4)\end{array}\end{array}$ & \begin{tabular}{|c}
$\mathbf{9}$ \\
Sali- \\
cylic \\
acid \\
admin- \\
istered.
\end{tabular} \\
\hline Fore period. & & & & & & & & & \\
\hline 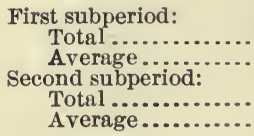 & $\begin{array}{r}\text { Grams. } \\
5.678 \\
1.136 \\
\\
5.183 \\
1.037\end{array}$ & $\begin{array}{r}\text { Grams. } \\
0.702 \\
.140 \\
\\
.550 \\
.110\end{array}$ & $\begin{array}{r}\text { Grams. } \\
4.215 \\
.843\end{array}$ & $\begin{array}{r}\text { Grams. } \\
4.917 \\
.983 \\
4.851 \\
.970\end{array}$ & $\begin{array}{r}\text { Per ct. } \\
12.36 \\
10.61 \\
\ldots\end{array}$ & $\begin{array}{r}\text { Per ct. } \\
74.23 \\
. \\
82.98 \\
\cdots\end{array}$ & $\begin{array}{c}\text { Per ct. } \\
86.60 \\
\\
93.59 \\
\ldots\end{array}$ & $\begin{array}{l}\text { Grams. } \\
+0.761 \\
+.153 \\
+.332 \\
+.067\end{array}$ & $\begin{array}{c}\text { Grams. } \\
0 \\
0 \\
\\
0 \\
0\end{array}$ \\
\hline $\begin{array}{l}\text { Entire fore period: } \\
\quad \text { Total ................ } \\
\text { Average ......... }\end{array}$ & $\begin{array}{r}10.861 \\
1.086\end{array}$ & $\begin{array}{r}1.252 \\
.125\end{array}$ & $\begin{array}{r}8.516 \\
.852\end{array}$ & $\begin{array}{r}9.768 \\
.977\end{array}$ & $\begin{array}{r}11.53 \\
\ldots \ldots . . .\end{array}$ & $\begin{array}{r}78.41 \\
\ldots \ldots\end{array}$ & $\begin{array}{r}89.94 \\
\ldots .\end{array}$ & $\begin{array}{r}+1.093 \\
+\quad .109\end{array}$ & $\begin{array}{l}0 \\
0\end{array}$ \\
\hline Preservative period. & & & & & & & & & \\
\hline $\begin{array}{l}\text { First subperiod: } \\
\quad \text { Total ............... } \\
\text { Average } . . . . . . .\end{array}$ & $\begin{array}{l}5.546 \\
1.109\end{array}$ & $\begin{array}{l}.768 \\
.154\end{array}$ & $\begin{array}{r}3.997 \\
.799\end{array}$ & $\begin{array}{r}4.765 \\
.953\end{array}$ & 13.85 & $\begin{array}{r}72.07 \\
\ldots \ldots \ldots\end{array}$ & $\begin{array}{l}85.92 \\
\ldots \ldots\end{array}$ & $\begin{array}{l}+.781 \\
+.156\end{array}$ & $\begin{array}{r}1.05 \\
.21\end{array}$ \\
\hline 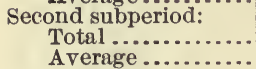 & $\begin{array}{l}5.721 \\
1.144\end{array}$ & $\begin{array}{l}.671 \\
.134\end{array}$ & $\begin{array}{r}4.786 \\
.957\end{array}$ & $\begin{array}{l}5.457 \\
1.091\end{array}$ & 11.73 & 83.66 & 95.39 & $\begin{array}{r}+.264 \\
+.053\end{array}$ & $\begin{array}{r}2.10 \\
.42\end{array}$ \\
\hline $\begin{array}{l}\text { Third subperiod: } \\
\text { Total } \ldots . . . . . . . . \\
\text { Average }\end{array}$ & $\begin{array}{l}5.887 \\
1.177\end{array}$ & $\begin{array}{r}.692 \\
.138\end{array}$ & $\begin{array}{r}4.073 \\
.815\end{array}$ & $\begin{array}{r}4.765 \\
.953\end{array}$ & 11.75 & 69.19 & 80.94 & $\begin{array}{r}+1.122 \\
+\quad 224\end{array}$ & 3.70 \\
\hline 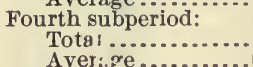 & $\begin{array}{l}5.926 \\
1.185\end{array}$ & - .630 & $\begin{array}{r}4.264 \\
.853\end{array}$ & $\begin{array}{r}4.894 \\
979\end{array}$ & 10.63 & 71.95 & 82.59 & $\begin{array}{r}+1.032 \\
+\quad 206\end{array}$ & 6.00 \\
\hline $\begin{array}{l}\text { Fifth sub, eriod: } \\
\text { Total ....................... } \\
\text { Average }\end{array}$ & $\begin{array}{l}6.357 \\
1.271\end{array}$ & $\begin{array}{l}.521 \\
.104\end{array}$ & $\begin{array}{r}4.135 \\
.827\end{array}$ & $\begin{array}{r}4.656 \\
.931\end{array}$ & 8.20 & 65.05 & 73.24 & $\begin{array}{l}+1.701 \\
+\quad .340\end{array}$ & $\begin{array}{l}8.00 \\
1.60\end{array}$ \\
\hline 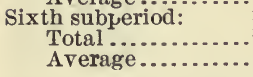 & $\begin{array}{l}6.156 \\
1.231\end{array}$ & $\begin{array}{l}.251 \\
.050\end{array}$ & $\begin{array}{r}3.934 \\
.787\end{array}$ & $\begin{array}{r}4.185 \\
.837\end{array}$ & $\begin{array}{r}4.08 \\
\ldots \ldots . .\end{array}$ & $\begin{array}{r}63.91 \\
\ldots . . .\end{array}$ & $\begin{array}{r}67.98 \\
\ldots \ldots\end{array}$ & $\begin{array}{r}+1.971 \\
+\quad .394\end{array}$ & $\begin{array}{r}10.00 \\
2.00\end{array}$ \\
\hline $\begin{array}{l}\text { Entire prese rvative } \\
\text { period: } \\
\text { Total ................ } \\
\text { Average .......... }\end{array}$ & $\begin{array}{r}35.593 \\
1.186\end{array}$ & $\begin{array}{r}3.533 \\
.118\end{array}$ & $\begin{array}{r}25.189 \\
.840\end{array}$ & $\begin{array}{r}28.722 \\
.957\end{array}$ & $\begin{array}{r}9.93 \\
\ldots . . .\end{array}$ & $\begin{array}{r} \\
70.77 \\
-\ldots . .\end{array}$ & $\begin{array}{r}80.70 \\
\hdashline\end{array}$ & $\begin{array}{r}+6.871 \\
+\quad .229\end{array}$ & $\begin{array}{r}30.85 \\
1.03\end{array}$ \\
\hline $\begin{array}{l}\text { After period. } \\
\text { First subperiod: } a \\
\text { Total............ } \\
\text { Average...... }\end{array}$ & $\begin{array}{l}5.833 \\
1.167\end{array}$ & $\begin{array}{l}.775 \\
.155\end{array}$ & $\begin{array}{r}4.033 \\
.807\end{array}$ & $\begin{array}{r}4.808 \\
.962\end{array}$ & 13. 29 & 69.14 & $\begin{array}{r}82.43 \\
\ldots .\end{array}$ & $\begin{array}{l}+1.025 \\
+\quad .205\end{array}$ & $\begin{array}{l}0 \\
0\end{array}$ \\
\hline
\end{tabular}

$a$ Left out in second after subperiod-sick. 
SALICYLIC ACID AND SALICYLATES.

TÁble XVII.-Sulphur balances for Series VI-Continued.

[Averages are per day.]

No. 11.

\begin{tabular}{|c|c|c|c|c|c|c|c|c|c|}
\hline Period. & $\begin{array}{c}1 \\
\text { In food. }\end{array}$ & In feces. & In urine. & \begin{tabular}{|c|}
4 \\
In feces \\
and \\
urine. \\
$(2+3)$
\end{tabular} & $\begin{array}{c}\text { o } \\
\text { In } \\
\text { feces. } \\
(2 \div 1)\end{array}$ & $\begin{array}{c}\mathbf{6} \\
\underset{(3 \div 1)}{\text { In }} \\
\text { urine. }\end{array}$ & \begin{tabular}{|c|}
7 \\
In feces \\
and \\
urine. \\
$(4 \div 1)$
\end{tabular} & $\begin{array}{c}8 \\
\text { Balance. } \\
(1-4)\end{array}$ & \begin{tabular}{|c}
$\mathbf{9}$ \\
Sali- \\
cylic \\
acid \\
admin- \\
istered.
\end{tabular} \\
\hline Fore period. & & & & & & & & & \\
\hline 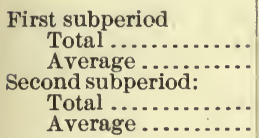 & $\begin{array}{r}\text { Grams. } \\
6.886 \\
1.377 \\
5.288 \\
1.058\end{array}$ & $\begin{array}{r}\text { Grams. } \\
0.708 \\
.142 \\
.811 \\
.162\end{array}$ & $\begin{array}{r}\text { Grams. } \\
\text { a } 4.673 \\
.935\end{array}$ & $\begin{array}{r}\text { Grams. } \\
5.381 \\
1.076 \\
6.136 \\
1.227\end{array}$ & $\begin{array}{r}\text { Per ct. } \\
10.28 \\
\ldots \ldots\end{array}$ & $\begin{array}{r}\text { Per ct. } \\
67.86\end{array}$ & $\begin{array}{l}\text { Per ct. } \\
78.14\end{array}$ & $\begin{array}{l}\text { Grams. } \\
+1.505 \\
+.301 \\
-.848 \\
-.169\end{array}$ & $\begin{array}{c}\text { Grams. } \\
0 \\
0\end{array}$ \\
\hline $\begin{array}{l}\text { Entire fore period: } \\
\quad \text { Total ................ } \\
\text { A verage ........... }\end{array}$ & $\begin{array}{r}12.174 \\
1.217\end{array}$ & $\begin{array}{r}1.519 \\
.152\end{array}$ & $\begin{array}{l}9.998 \\
1.000\end{array}$ & $\begin{array}{r}11.517 \\
1.152\end{array}$ & 12.48 & 82.13 & 94.60 & $\begin{array}{r}+.657 \\
+.065 \\
\end{array}$ & $\begin{array}{l}0 \\
0\end{array}$ \\
\hline Preservative period. & & & & & & & & & \\
\hline $\begin{array}{l}\text { First subperiod: } \\
\text { Total ............ } \\
\text { Average...... } \\
\text { Second subperiod: }\end{array}$ & $\begin{array}{l}5.410 \\
1.082\end{array}$ & $\begin{array}{l}.750 \\
.150\end{array}$ & $\begin{array}{l}5.469 \\
1.094\end{array}$ & $\begin{array}{l}6.219 \\
1.244\end{array}$ & 13.86 & 101.09 & 114. 95 & $\begin{array}{l}-.809 \\
-.162\end{array}$ & $\begin{array}{r}1.05 \\
.21\end{array}$ \\
\hline $\begin{array}{l}\text { Second s } \\
\text { Total } \\
\text { Aver } \\
\text { Third sul }\end{array}$ & $\begin{array}{l}5.688 \\
1.138\end{array}$ & $\begin{array}{r}.796 \\
.159\end{array}$ & $\begin{array}{l}5.338 \\
1.068\end{array}$ & $\begin{array}{l}6.134 \\
1.227\end{array}$ & 13. 99 & 93.85 & 107.84 & $\begin{array}{l}-.446 \\
-.089\end{array}$ & $\begin{array}{r}2.10 \\
.42\end{array}$ \\
\hline $\begin{array}{l}\text { Total } \\
\text { Aver }\end{array}$ & $\begin{array}{l}5.842 \\
1.168\end{array}$ & $\begin{array}{l}.813 \\
.163\end{array}$ & $\begin{array}{r}4.455 \\
.891\end{array}$ & $\begin{array}{l}5.268 \\
1.054\end{array}$ & 13. 92 & 76.26 & 90.17 & $\begin{array}{r}+.574 \\
+.114\end{array}$ & $\begin{array}{r}3.70 \\
.74\end{array}$ \\
\hline $\begin{array}{l}\text { Fourth su } \\
\text { Total } \\
\text { Avera }\end{array}$ & $\begin{array}{l}5.908 \\
1.182\end{array}$ & $\begin{array}{l}.776 \\
.155\end{array}$ & $\begin{array}{r}4.602 \\
.920\end{array}$ & $\begin{array}{l}1.004 \\
5.378 \\
1.076\end{array}$ & 13.13 & 77.89 & 91.03 & $\begin{array}{r}+.114 \\
+.530 \\
+.106\end{array}$ & $\begin{array}{l}.14 \\
6.00 \\
1.20\end{array}$ \\
\hline $\begin{array}{l}\text { Fifth sul } \\
\text { Tota } \\
\text { Ave }\end{array}$ & $\begin{array}{l}1.102 \\
6.154 \\
1.231\end{array}$ & $\begin{array}{l}.100 \\
.681 \\
.136\end{array}$ & $\begin{array}{l}5.021 \\
1.004\end{array}$ & $\begin{array}{l}1.060 \\
5.702 \\
1.140\end{array}$ & 11.07 & 81.59 & 92.66 & $\begin{array}{r}+.100 \\
+.452 \\
+.091\end{array}$ & $\begin{array}{l}8.00 \\
1.60\end{array}$ \\
\hline 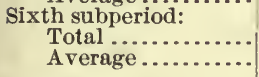 & $\begin{array}{l}6.031 \\
1.206\end{array}$ & .813 & $\begin{array}{r}4.661 \\
.932\end{array}$ & $\begin{array}{l}5.474 \\
1.095\end{array}$ & 13.48 & 77.28 & 90.76 & $\begin{array}{l}+.091 \\
+.557 \\
+.111\end{array}$ & $\begin{array}{r}10.00 \\
2.00\end{array}$ \\
\hline $\begin{array}{l}\text { Entire preservative } \\
\text { period: } \\
\text { Total } \ldots \ldots \ldots \ldots \ldots . . . . . . \\
\text { Average } . . . . . . . . . .\end{array}$ & $\begin{array}{r}35.033 \\
1.168\end{array}$ & $\begin{array}{r}4.629 \\
.154\end{array}$ & $\begin{array}{r}29.546 \\
.985\end{array}$ & $\begin{array}{r}34.175 \\
1.139\end{array}$ & 13. 21 & 84.34 & 97.55 & $\begin{array}{r}+\quad 858 \\
+\quad .029\end{array}$ & $\begin{array}{r}30.85 \\
1.03\end{array}$ \\
\hline After period. & & & & & & & & & \\
\hline $\begin{array}{r}\text { First subperio } \\
\text { Total..... } \\
\text { Average... }\end{array}$ & $\begin{array}{l}5.722 \\
1.144\end{array}$ & $\begin{array}{l}.697 \\
.139\end{array}$ & $\begin{array}{r}4.854 \\
.971\end{array}$ & $\begin{array}{l}5.551 \\
1.110\end{array}$ & 12.18 & 84.83 & 97.01 & $\begin{array}{r}+.171 \\
+.034\end{array}$ & $\begin{array}{l}0 \\
0\end{array}$ \\
\hline 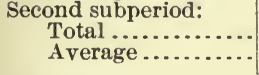 & & $\begin{array}{l}.636 \\
.127\end{array}$ & $\begin{array}{r}4.780 \\
.956\end{array}$ & $\begin{array}{l}5.416 \\
1.083\end{array}$ & 10.96 & 82.27 & 93.22 & $\begin{array}{l}+.394 \\
+.079\end{array}$ & $\begin{array}{l}0 \\
0\end{array}$ \\
\hline $\begin{array}{l}\text { Entire after period: } \\
\text { Total ............. } \\
\text { Average....... }\end{array}$ & $\begin{array}{r}11.532 \\
1.153\end{array}$ & $\begin{array}{r}1.333 \\
.133\end{array}$ & $\begin{array}{r}9.634 \\
.963\end{array}$ & $\begin{array}{r}10.967 \\
1.097\end{array}$ & 11.56 & 83.54 & 95.10 & $\begin{array}{l}+.565 \\
+.056\end{array}$ & $\begin{array}{l}0 \\
0\end{array}$ \\
\hline
\end{tabular}

$a$ Daily average added in order to complete record. 
TABLE XVII.-Sulphur balances for Series VI-Continued.

[Averages are per day.]

No. 12 .

\begin{tabular}{|c|c|c|c|c|c|c|c|c|c|}
\hline Period. & In food. & In feces. & In urine, & $\begin{array}{c}4 \\
\text { In feces } \\
\text { and } \\
\text { urine. } \\
(2+3)\end{array}$ & $\begin{array}{c}5 \\
\text { In } \\
\text { feces. } \\
(2 \div 1)\end{array}$ & $\begin{array}{c}6 \\
\text { In } \\
\text { urine. } \\
(3 \div 1)\end{array}$ & $\begin{array}{c}7 \\
\text { In feces } \\
\text { and } \\
\text { urine. } \\
(4 \div 1)\end{array}$ & Balance. & $\begin{array}{c}9 \\
\text { Sali- } \\
\text { cylic } \\
\text { acid } \\
\text { admin- } \\
\text { istered. }\end{array}$ \\
\hline \multicolumn{10}{|l|}{ Fore period. } \\
\hline $\begin{array}{l}\text { First, subperiod: } \\
\text { Total ............ } \\
\text { Average ....... }\end{array}$ & $\begin{array}{r}\text { Grams. } \\
5.805 \\
1.161\end{array}$ & $\begin{array}{r}\text { Grams. } \\
0.726 \\
.145\end{array}$ & $\begin{array}{r}\text { Grams. } \\
5.166 \\
1.033\end{array}$ & $\begin{array}{r}\text { Grams. } \\
5.892 \\
1.178\end{array}$ & $\begin{array}{l}\text { Per ct. } \\
12.51 \\
\ldots\end{array}$ & $\begin{array}{r}\text { Per ct. } \\
88.99 \\
\quad . .\end{array}$ & $\begin{array}{l}\text { Perct. } \\
101.50 \\
\end{array}$ & $\begin{array}{l}\text { Grams. } \\
-0.087 \\
-\quad 017\end{array}$ & $\begin{array}{c}\text { Grams. } \\
0 \\
0\end{array}$ \\
\hline Second subperiod: & & & & & & & & -.016 & \\
\hline $\begin{array}{l}\text { Total ........................ } \\
\text { Average }\end{array}$ & $\begin{array}{l}5.334 \\
1.067\end{array}$ & $\begin{array}{l}.667 \\
.133\end{array}$ & $\begin{array}{r}4.918 \\
.984\end{array}$ & $\begin{array}{l}5.585 \\
1.117\end{array}$ & $\begin{array}{r}12.50 \\
\cdots \cdots\end{array}$ & $\begin{array}{r}92.20 \\
\cdots \cdots\end{array}$ & $\begin{array}{l}104.71 \\
\ldots \ldots \ldots\end{array}$ & $\begin{array}{l}-.251 \\
-.050\end{array}$ & $\begin{array}{l}0 \\
0\end{array}$ \\
\hline $\begin{array}{l}\text { Entire fore period: } \\
\quad \text { Total ............... } \\
\text { Average.......... }\end{array}$ & $\begin{array}{r}11.139 \\
1.114\end{array}$ & $\begin{array}{r}1.393 \\
.139\end{array}$ & $\begin{array}{r}10.084 \\
1.008\end{array}$ & $\begin{array}{r}11.477 \\
1.148\end{array}$ & $\begin{array}{r}12.51 \\
\cdots \cdots\end{array}$ & $\begin{array}{r}90.53 \\
\ldots \ldots \ldots \\
\end{array}$ & $\begin{array}{l}103 .(3 \\
\cdots \cdots\end{array}$ & $\begin{array}{l}-.338 \\
-.034\end{array}$ & $\begin{array}{l}0 \\
0\end{array}$ \\
\hline Preservative period. & - & & & & & & & & \\
\hline $\begin{array}{l}\text { First subperiod: } \\
\text { Total ............... } \\
\text { Average .......... }\end{array}$ & $\begin{array}{l}5.425 \\
1.085\end{array}$ & $\begin{array}{l}.443 \\
.089\end{array}$ & $\begin{array}{r}4.236 \\
.847\end{array}$ & $\begin{array}{r}4.679 \\
.936\end{array}$ & 8.17 & 78.08 & $\begin{array}{r}86.25 \\
\ldots \ldots \ldots\end{array}$ & $\begin{array}{r}+.746 \\
+.149\end{array}$ & $\begin{array}{r}1.05 \\
.21\end{array}$ \\
\hline Second su & & & & & & & & & \\
\hline Total & 5. 611 & .455 & 5.129 & 5.584 & 8.11 & 91.41 & 99.52 & +.027 & 2.10 \\
\hline $\begin{array}{l}\text { Average ............. } \\
\text { Third subperiod: }\end{array}$ & 122 & .091 & 1. 026 & 1.117 & & & & +.005 & .42 \\
\hline $\begin{array}{l}\text { Total .................. } \\
\text { Average }\end{array}$ & 5. 707 & .820 & 4. 906 & 5.726 & 14.37 & 85.96 & 100.33 & -.019 & 3.70 \\
\hline Fourth subperiod: & 141 & .164 & .981 & $1.14 b$ & & & & -.004 & .74 \\
\hline $\begin{array}{l}\text { Total } \ldots . . . . . . \\
\text { Average } . . .\end{array}$ & $\begin{array}{l}5.746 \\
1.149\end{array}$ & $\begin{array}{l}.517 \\
.103\end{array}$ & $\begin{array}{r}4.734 \\
.947\end{array}$ & $\begin{array}{l}5.251 \\
1.050\end{array}$ & 9.00 & 82.39 & 91.39 & $\begin{array}{l}+.495 \\
+.099\end{array}$ & $\begin{array}{l}6.00 \\
1.20\end{array}$ \\
\hline Fifth subperiod: & & & & & & & & & \\
\hline $\begin{array}{l}\text { Total ............ } \\
\text { Average....... }\end{array}$ & $\begin{array}{l}6.075 \\
1.215\end{array}$ & $\begin{array}{r}.720 \\
144\end{array}$ & $\begin{array}{l}5.173 \\
1.035\end{array}$ & $\begin{array}{l}5.893 \\
1.179\end{array}$ & 11.85 & 85.15 & 97.00 & +.182 & 8.00 \\
\hline $\begin{array}{l}\text { Sixth subperiod: } \\
\text { Total }\end{array}$ & 5827 & & & & & & & & \\
\hline Average........... & 1.165 & $\begin{array}{l}.493 \\
.099\end{array}$ & $\begin{array}{r}4.973 \\
.995\end{array}$ & $\begin{array}{l}5.466 \\
1.093\end{array}$ & $\begin{array}{r}8.46 \\
\ldots . .\end{array}$ & $\begin{array}{r}85.34 \\
\ldots . . . .\end{array}$ & $\begin{array}{r}98.80 \\
\ldots \ldots . .\end{array}$ & $\begin{array}{l}+.361 \\
+.072\end{array}$ & $\begin{array}{r}10.00 \\
2.00\end{array}$ \\
\hline $\begin{array}{l}\text { Entire preservative } \\
\text { period: }\end{array}$ & & & & & & & & & \\
\hline $\begin{array}{l}\text { Total } \ldots . . . . . . . \\
\text { Average } . . . .\end{array}$ & $\begin{array}{r}34.391 \\
1.146\end{array}$ & $\begin{array}{r}3.448 \\
.115\end{array}$ & $\begin{array}{r}29.151 \\
.972\end{array}$ & $\begin{array}{r}32.599 \\
1.087\end{array}$ & $\begin{array}{r}10.03 \\
\ldots \ldots \ldots\end{array}$ & $\begin{array}{r}\$ 4.76 \\
\ldots . . . .\end{array}$ & $\begin{array}{r}94.79 \\
\ldots . \ldots\end{array}$ & $\begin{array}{l}+1.792 \\
+\quad .059\end{array}$ & $\begin{array}{r}30.85 \\
1.03\end{array}$ \\
\hline & & & & & & & & & \\
\hline & & & & & & & & & \\
\hline $\begin{array}{l}\text { First subperiod: } \\
\text { Total ........... } \\
\text { Average...... }\end{array}$ & $\begin{array}{l}5.463 \\
1.093\end{array}$ & $\begin{array}{l}.644 \\
.129\end{array}$ & $\begin{array}{l}4.999 \\
1.000\end{array}$ & $\begin{array}{l}5.643 \\
1.129\end{array}$ & 11.79 & 91.51 & 103.29 & $\begin{array}{l}-.180 \\
-.036\end{array}$ & $\begin{array}{l}0 \\
0\end{array}$ \\
\hline $\begin{array}{l}\text { Second subperiod: } \\
\text { Total .................. } \\
\text { Average .......... }\end{array}$ & $\begin{array}{l}5.519 \\
1.104\end{array}$ & $\begin{array}{r}a .580 \\
.116\end{array}$ & $\begin{array}{l}5.164 \\
1.033\end{array}$ & $\begin{array}{l}5.744 \\
1.149\end{array}$ & $\begin{array}{r}10.51 \\
\ldots \ldots \ldots\end{array}$ & $\begin{array}{r}93.57 \\
\ldots \ldots\end{array}$ & \begin{tabular}{|l}
104.08 \\
$\cdots \ldots .$.
\end{tabular} & $\begin{array}{l}-.225 \\
-.045\end{array}$ & $\begin{array}{l}0 \\
0\end{array}$ \\
\hline $\begin{array}{c}\text { Entire after period: } \\
\text { Total ............... } \\
\text { Average } . . . . .\end{array}$ & $\begin{array}{r}10.982 \\
1.098\end{array}$ & $\begin{array}{r}1.224 \\
.122\end{array}$ & $\begin{array}{r}10 \dot{ } .163 \\
1.016\end{array}$ & $\begin{array}{r}11.387 \\
1.139\end{array}$ & $\begin{array}{r}11.15 \\
\cdots \cdots\end{array}$ & $\begin{array}{r}92.54 \\
\cdots \cdots\end{array}$ & $\begin{array}{l}103.67 \\
\cdots \ldots . .\end{array}$ & $\begin{array}{l}-.405 \\
-.041\end{array}$ & $\begin{array}{l}0 \\
0\end{array}$ \\
\hline
\end{tabular}

$a$ Daily average added in order to complete record. 
TABLE XVII.-Sulphur balances for Series VI-Continued.

[Averages are per man per day.]

Summary for nine men.

\begin{tabular}{|c|c|c|c|c|c|c|c|c|c|}
\hline Period. & In food. & In feces. & In urine. & \begin{tabular}{|}
4 \\
In feces \\
and \\
urine. \\
$(2+3)$
\end{tabular} & $\underset{\substack{\text { In } \\
\text { feces. } \\
(2 \div 1)}}{5}$ & $\underset{\substack{\text { In } \\
(3 \div 1)}}{6}$ & $\begin{array}{c}7 \\
\text { In feces } \\
\text { and } \\
\text { urine. } \\
(4 \div 1)\end{array}$ & $\begin{array}{c}\text { Balance. } \\
(1-4)\end{array}$ & $\begin{array}{l}9 \\
\text { Sali- } \\
\text { cylic } \\
\text { acid } \\
\text { admin- } \\
\text { istered. }\end{array}$ \\
\hline \multicolumn{10}{|l|}{ Fore period. } \\
\hline 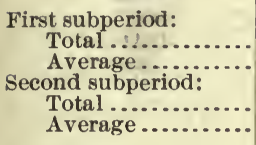 & $\begin{array}{r}\text { Grams. } \\
47.179 \\
1.048\end{array}$ & $\begin{array}{r}\text { Grams. } \\
5.494 \\
.122\end{array}$ & $\begin{array}{r}\text { Grams. } \\
40.242 \\
.894\end{array}$ & $\begin{array}{c}\text { Grams. } \\
45.736 \\
1.016\end{array}$ & $\begin{array}{c}\text { Per ct. } \\
11.65 \\
\cdots\end{array}$ & $\begin{array}{r}\text { Per ct. } \\
85.30 \\
\cdots\end{array}$ & $\begin{array}{c}\text { Per ct. } \\
96.94 \\
\cdots \\
110.10 \\
\cdots\end{array}$ & $\begin{array}{r}\text { Grams. } \\
+1.443 \\
+.032 \\
-4.160 \\
-.093\end{array}$ & $\begin{array}{c}\text { Grams. } \\
0 \\
0 \\
\\
0 \\
0\end{array}$ \\
\hline $\begin{array}{l}\text { Entire fore period: } \\
\text { Total ............... } \\
\text { Average ......... }\end{array}$ & $\begin{array}{r}88.376 \\
.982 \\
\end{array}$ & $\begin{array}{r}11.364 \\
.126 \\
\end{array}$ & $\begin{array}{r}79.729 \\
.886 \\
\end{array}$ & $\begin{array}{r}91.093 \\
1.012\end{array}$ & $\begin{array}{r}12.86 \\
\ldots \ldots \\
\end{array}$ & $\begin{array}{r}90.22 \\
\cdots \cdots \\
\end{array}$ & $\begin{array}{c}103.07 \\
\ldots \ldots\end{array}$ & $\begin{array}{r}-2.717 \\
-.030 \\
\end{array}$ & $\begin{array}{l}0 \\
0\end{array}$ \\
\hline Preservative period. & & & & & . & & & & \\
\hline 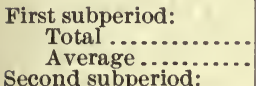 & $\begin{array}{r}42.463 \\
.943\end{array}$ & $\begin{array}{r}5.279 \\
.117\end{array}$ & $\begin{array}{r}38.571 \\
.857\end{array}$ & $\begin{array}{r}43.850 \\
.974\end{array}$ & $\begin{array}{r}12.43 \\
\ldots\end{array}$ & $\begin{array}{r}90.83 \\
\cdots \ldots . . .\end{array}$ & $\begin{array}{r}103.27 \\
\cdots \ldots \ldots\end{array}$ & $\begin{array}{l}-1.387 \\
-.031\end{array}$ & $\begin{array}{r}9.45 \\
.21\end{array}$ \\
\hline T & $\begin{array}{r}45.012 \\
1.000\end{array}$ & $\begin{array}{r}5.245 \\
.116\end{array}$ & $\begin{array}{r}41.073 \\
.913\end{array}$ & $\begin{array}{r}46.318 \\
1.029\end{array}$ & $\begin{array}{c}11.65 \\
\text {......... }\end{array}$ & 91.25 & $\begin{array}{c}102.90 \\
\ldots \ldots \ldots\end{array}$ & $\begin{array}{l}-1.306 \\
-.029\end{array}$ & $\begin{array}{r}18.90 \\
.42\end{array}$ \\
\hline $\begin{array}{r}\text { Third } \\
\text { Tr } \\
\text { A }\end{array}$ & $\begin{array}{r}45.186 \\
1.004\end{array}$ & $\begin{array}{r}5.997 \\
.133\end{array}$ & $\begin{array}{r}39.750 \\
.883\end{array}$ & $\begin{array}{r}45.747 \\
1.016\end{array}$ & $\begin{array}{c}13.27 \\
\ldots . . .\end{array}$ & $\begin{array}{r}87.97 \\
\hdashline \ldots . . .\end{array}$ & $\begin{array}{r}101.24 \\
\ldots \ldots \ldots\end{array}$ & $\begin{array}{l}-.561 \\
-.012\end{array}$ & $\begin{array}{r}33.30 \\
.74\end{array}$ \\
\hline & & 5. 636 & 40.123 & & 12.34 & 87.83 & 100.17 & -.076 & 54.00 \\
\hline Fifth & & & 80.120 & & & & ........... & & 1.20 \\
\hline & $\begin{array}{r}48.298 \\
1.073\end{array}$ & $\begin{array}{r}5.517 \\
.122\end{array}$ & $\begin{array}{r}41.301 \\
.918\end{array}$ & $\begin{array}{r}46.818 \\
1.040\end{array}$ & $\begin{array}{r}11.42 \\
\ldots \ldots \ldots\end{array}$ & $\begin{array}{r}85.51 \\
\cdots . . . .\end{array}$ & $\begin{array}{r}96.94 \\
\ldots . .2\end{array}$ & $\begin{array}{r}+1.480 \\
+\quad .033\end{array}$ & $\begin{array}{r}72.00 \\
1.60\end{array}$ \\
\hline $\begin{array}{l}\text { Sixth subperio } \\
\text { Total ..... } \\
\text { Average... }\end{array}$ & $\begin{array}{r}46.836 \\
1.041\end{array}$ & $\begin{array}{r}5.314 \\
.118\end{array}$ & $\begin{array}{r}43.892 \\
.975\end{array}$ & $\begin{array}{r}49.206 \\
1.093\end{array}$ & $\begin{array}{r}11.35 \\
\ldots . . . \cdots\end{array}$ & $\begin{array}{r}93.71 \\
\ldots\end{array}$ & $\begin{array}{c}105.06 \\
\ldots \ldots\end{array}$ & $\begin{array}{r}-2.370 \\
-.052\end{array}$ & $\begin{array}{r}88.00 \\
1.96\end{array}$ \\
\hline $\begin{array}{l}\text { Entire preservative } \\
\text { period: } \\
\text { Total .................. } \\
\text { Average........... }\end{array}$ & $\begin{array}{r}273.478 \\
1.013\end{array}$ & $\begin{array}{r}32.988 \\
.122 \\
\end{array}$ & $\begin{array}{r}244.710 \\
.906 \\
\end{array}$ & $\begin{array}{r}277.698 \\
1.028 \\
\end{array}$ & $\begin{array}{r}12.06 \\
\cdots \cdots \\
\end{array}$ & $\begin{array}{r}89.48 \\
\cdots \cdots \\
\end{array}$ & $\begin{array}{r}101.54 \\
\cdots \cdots \\
\end{array}$ & $\begin{array}{r}-4.220 \\
-.015 \\
\end{array}$ & $\begin{array}{r}275.65 \\
1.02 \\
\end{array}$ \\
\hline After period. & & & & & & & & & \\
\hline $\begin{array}{r}\text { First s } \\
\text { To } \\
\text { Av } \\
\text { Second }\end{array}$ & $\begin{array}{r}44.350 \\
.985\end{array}$ & $\begin{array}{r}5.963 \\
.133\end{array}$ & $\begin{array}{r}41.014 \\
.911\end{array}$ & $\begin{array}{r}46.977 \\
1.044\end{array}$ & $\begin{array}{c}13.44 \\
\cdots . . . .\end{array}$ & $\begin{array}{r}92.49 \\
\ldots \ldots .\end{array}$ & $\begin{array}{c}105.99 \\
\cdots \ldots . . .\end{array}$ & $\begin{array}{l}-2.627 \\
-.059\end{array}$ & $\begin{array}{l}0 \\
0\end{array}$ \\
\hline 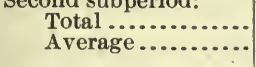 & $\begin{array}{r}44.950 \\
.998\end{array}$ & $\begin{array}{r}5.297 \\
.117\end{array}$ & $\begin{array}{r}42.176 \\
.937\end{array}$ & $\begin{array}{r}47.473 \\
1.055\end{array}$ & $\begin{array}{r}11.80 \\
\ldots \ldots \ldots\end{array}$ & $\begin{array}{l}93.93 \\
\ldots \ldots \ldots\end{array}$ & $\begin{array}{r}105.73 \\
\ldots \ldots\end{array}$ & $\begin{array}{r}-2.523 \\
-.057\end{array}$ & $\begin{array}{l}0 \\
0\end{array}$ \\
\hline $\begin{array}{l}\text { Entire after period: } \\
\text { Total ............... } \\
\text { Average........ }\end{array}$ & $\begin{array}{r}89.300 \\
.992\end{array}$ & $\begin{array}{r}11.260 \\
. .125\end{array}$ & $\begin{array}{r}83.190 \\
.924\end{array}$ & $\begin{array}{r}94.450 \\
1.049\end{array}$ & $\begin{array}{r}12.61 \\
\ldots . . . .\end{array}$ & $\begin{array}{l}93.16 \\
\ldots \ldots \ldots\end{array}$ & $\begin{array}{c}105.77 \\
\ldots \ldots \ldots\end{array}$ & $\begin{array}{r}-5.150 \\
-.057\end{array}$ & $\begin{array}{l}0 \\
0\end{array}$ \\
\hline
\end{tabular}




\section{FAT BALANCE.}

INDIVIDUAL DATA.

An interesting problem is presented in connection with this work in respect of the relative absorption and digestibility of the food in the various periods of the experiment, inasmuch as in the fat balance the total amount excreted is found in the feces. (See Table XIX, page 651$)$.

No. 1 .

During the fore period the quantity of fat in the food of No. 1 amounted daily to 87.16 grams, during the preservative period 85.15 grams, and during the after period to 83.64 grams. This shows a progressive decrease in the quantity of fat in the food. There appears in the feces for the fore period 1.88 grams daily of fat, in the preservative period 1.70 grams, and in the after period 2.74 grams. Expressed in percentages, there were excreted of fat in the feces in the fore period 2.15 per cent, in the preservative period 1.99 per cent, and in the after period 3.27 per cent.

These data show a very slight tendency on the part of the preservative to decrease the quantity of fat in the feces; in other words, to increase the absorption of one of the principal heat-forming constituents. The remarkable fact in connection with these data is that on the withdrawal of the preservative the quantity of unabsorbed fat in the feces is very largely increased and the balance is correspondingly low.

No. 2.

In the case of No. 2 the average quantity of fat consumed was largest in the fore period, namely, 97.57, smaller in the preservative period, namely, 94.16, and smallest in the after period, namely, 91.77 grams. Of this quantity there appear in the feces in the fore period 4.73 grams, in the preservative period $3.35 \mathrm{grams}$, and in the after period 3.49 grams. - The percentage of fat in the food appearing in the feces daily is 4.84 per cent in the fore period, 3.56 per cent in the preservative period, and 3.80 per cent in the after period. By reason of the varying quantity of fat ingested the percentages of fat excreted in the feces afforded a better means of comparison than the total quantities. These percentages show that the greatest percentage of fat was excreted during the fore period, namely, 4.84 per cent, the smallest in the preservative period, namely, 3.56 per cent, while in the after period nature appeared to make an effort to reestablish the normal condition existing in the fore period, the quantity of fat excreted rising to 3.80 per cent.

These data show a very marked tendency on the part of the preservative to increase the absorption of the heat-forming elements of the fatty food from the alimentary canal. 
No. 3.

The data for No. 3 are exhibited without comment by reason of the conditions heretofore stated in connection with this member of the table.

No. 4.

The average daily quantity of fat in the food of No. 4 in the fore period is 96.51 grams, in the preservative period 92.77 grams, and in the after period 91.42 grams. The relative amounts appearing in the feces for the three periods daily are as follows:

For the fore period 2.96 grams, for the preservative period 2.81 grams, and for the after period 2.58 grams. Inasmuch as there is a slightly diminished quantity of fat in the food in the after period, as in the previous case, a more comprehensive idea of the amount of fat absorbed from the alimentary canal is found by an inspection of the percentage column. This shows that 3.06 per cent of the fat was excreted in the fore period in the feces, 3.03 per cent in the preservative period, and 2.82 per cent in the after period. These data show practically no influence of the preservative as affecting the absorption of the fat from the alimentary canal, but a considerable increase in this absorption is noticed during the after period.

No. 5 .

The average daily quantity of fat consumed by No. 5 in the fors period is 96.82 grams, in the preservative period 94.03 grams, and in the after period 91.37 grams. Here again we find a slightly diminishing quantity of fat throughout the three periods. The amounts appearing in the feces during these three periods are, respectively, 3 grams, 2.80 grams, and 2.93 grams. Expressed in percentages, it is seen that of the total fat in the food 3.10 per cent is excreted daily in the feces of the fore period, 2.98 per cent in the preservative period, and 3.21 per cent in the after period.

These data again show the influence of the preservative in increasing the absorption of the fatty substances from the alimentary canal.

No. 6 .

The average daily quantity of fat in the food of No. 6 in the fore period is 96.91 grams, in the preservative period 94.49 grams, and in the after period 92.63 grams. The quantity appearing in the feces for the fore period is 3.24 grans, for the preservative period 3.92 grams, and for the after period $3.39 \mathrm{grams}$, which, expressed in percentages, represents an excretion of fat in the feces in the fore period of 3.34 per cent, in the preservative period 4.14 per cent, and in the after period 3.66 per cent. In this case the data are exactly the opposite in significance from those of the preceding cases, the administration of the 
preservative having decidedly increased the quantity of fat in the feces, thus indicating a decrease in the quantity absorbed from the alimentary canal.

No. $\%$.

In the case of No. 7 the average daily quantity of fat in the food for the fore period is 67 grams, for the preservative period 66.51 grams, and for the after period 64.94 grams. Of this quantity there appear in the feces of the fore period 2.17 grams, in the preservative period 1.75 grams, and in the after period 2.55 grams. Expressed in percentages of the total quantity of fat in the food there is found to be excreted in the feces in the fore period 3.24 per cent, in the preservative period 2.64 per cent, and in the after period 3.93 per. cent. In this case the data again indicate the marked effect of the preserv ative in increasing the quantity of fatty substances absorbed from the alimentary canal, and thus decreasing the quantity appearing in the feces.

No. 8 .

The average daily quantity of fat in the food of No. 8 in the fore period is 63.14 grams, in the preservative period 63.34 grams, and in the after period 62.58 grams. Of this quantity there appear in the feces of the fore period 2.25 grams, of the preservative period 2.60 grams, and of the after period 2.40 grams. Expressed as percentages, the total amount of fat in the food of No. 8 excreted in the feces of the fore period is 3.56 per cent, of the preservative period 4.10 per cent, and of the after period 3.83 per cent. These data agree with those of No. 6, and show the effect of the administration of the preservative in decreasing the absorption of the fatty substances from the alimentary canal and the increase of these substances in the feces.

No. 9 .

The average daily quantity of fat in the food of No. 9 in the fore period was 136.79 grams, in the preservative period 133.95 grams, and in the after period 128.91 grams. Of this quantity there appeared in the feces of the fore period 2.49 grams, in the preservative period 3.45 grams, and in the after period 2.57 grams. Expressed as percentage of the total amount of fat in the food, No. 9 excreted 1.82 per cent in the fore period, 2.57 per cent in the preservative period, and 1.99 per cent in the after period.

For reasons given elsewhere (p. 587) the results obtained with this subject are not included in the summaries. They are stated here, however, as a matter of record.

No. 10.

The average daily quantity of fat in the food of No. 10 in the fore period is 97.51 grams and in the preservative period 91.49 grams. Of 
this quantity there appear in the feces in the fore period 3.82 grams and in the preservative period 2.74 grams. Expressed as percentage of the total amount of fat in the food, No. 10 excreted 3.92 per cent during the fore period and 2.99 per cent during the preservative period.

Owing to illness, No. 10 was absent from the table during the after period. For reasons given elsewhere (p. 587) this subject is omitted from the summaries. The results are given here, however, as a matter of record.

$$
\text { No. } 11 .
$$

In the case of No. 11 the average daily quantity of fat in the food in the fore period is 97.67 grams, in the preservative period 95.03 . grams, and in the after period 93.26 grams. Of this quantity there appear in the feces of the fore period $3.49 \mathrm{grams}$, in the preservative period 3.04 grams, and in the after period 2.64 grams. Expressed in percentages of the total quantity of fat in the food there was excreted in the feces of the fore period 3.58 per cent, of the preservative period 3.20 per cent, and of the after period 2.83 per cent. These data show a tendency on the part of the preservative to increase the absorption of the fatty substances from the alimentary canal and diminish the quantity excreted in the feces. This tendency is continued in the after period.

No. 12.

The average daily quantity of fat in the food of No. 12 in the fore period is 117.22 grams, in the preservative period 119.10 grams, and in the after period 113.74-grams. Of this quantity there appear in the feces of the fore period 5.03 grams, of the preservative period 2.55 grams, and of the after period 3.11 grams. Expressed as percentages of the total quantity of fat in the food there appear in the feces of the fore period 4.29 per cent, of the preservative period 2.14 per cent, and of the after period 2.73 per cent. These data show a very marked tendency on the part of the preservative to increase the absorption of the fatty substances from the alimentary canal and to decrease the quantity appearing in the feces. This tendency is only partially corrected during the after period.

SUMMARY.

The average figures for the nine men, by periods, taken from Table XIX on the fat balances are here inserted for convenience:

TABLE XVIII.-Fat summary, by periods, for nine men, Series VI.

\begin{tabular}{|c|c|c|c|c|}
\hline Period. & Fat & Fat in & feces. & Balance. \\
\hline 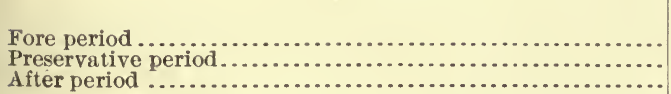 & $\begin{array}{r}\text { Grams. } \\
91.11 \\
89.40 \\
87.26\end{array}$ & $\begin{array}{r}\text { Grams. } \\
3.19 \\
2.72 \\
2.87\end{array}$ & $\begin{array}{r}\text { Per cent. } \\
3.50 \\
3.05 \\
3.29\end{array}$ & $\begin{array}{r}\text { Grams. } \\
87.92 \\
86.68 \\
84.39\end{array}$ \\
\hline
\end{tabular}


The daily average quantity of fat in the food of the nine men included in the general average is 91.11 grams for the fore period, 89.40 grams for the preservative period, and 87.26 grams for the after period. Of this quantity there appear in the feces of the fore period 3.19 grams, of the preservative period 2.72 grams, and of the after period 2.87 grams. Expressed in percentages of the total quantity of fat in the food, the amount excreted in the feces of the fore period is 3.50 per cent, of the preservative period 3.05 per cent, and of the after period 3.29 per cent.

These data show a tendency on the part of the preservative to increase the absorption of the fatty substances in the alimentary canal and to decrease the quantity appearing in the feces. This tendency is only partly overcome in the after period, during which the quantity of fat excreted is greater than in the preservative period, but less than in the fore period.

In the consideration of the fat balances it should be remembered that the experimental work continued from October to December. The colder weather might have been expected to cause a more complete oxidation of the fat ingested in the food, and the data show such a condition in the preservative period; therefore the effect produced can not be ascribed entirely to the preservative. In the after period, however, the amount of fat excreted increases slightly, which would seem to indicate that the increasing cold weather had no marked effect. 
TABLE XIX. - Fat balances for Series VI.

[Averages are per day.]

No. 1.

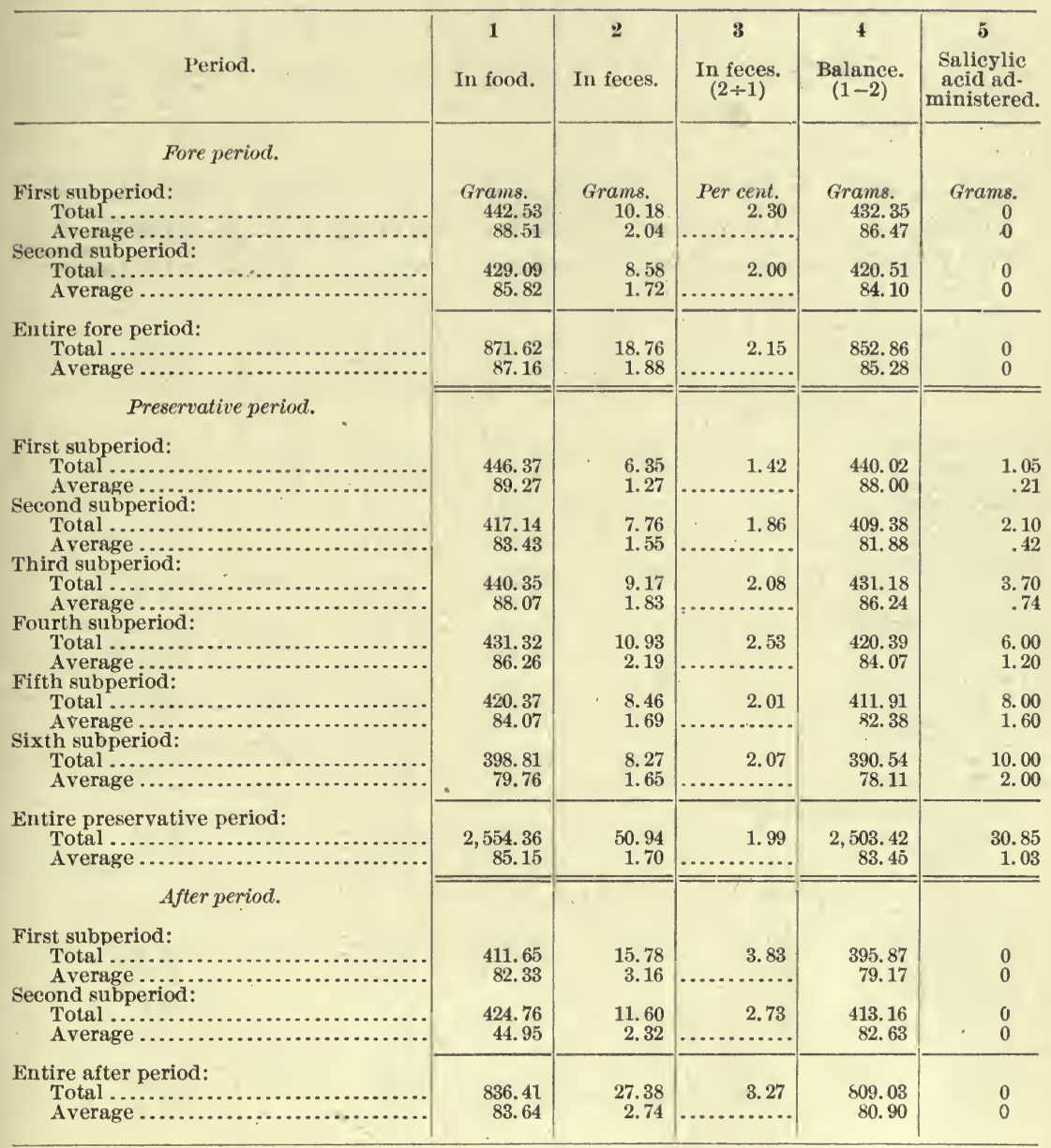


TABLE XIX.-Fat balances for Series VI-Continued.

[Averages are per day.]

No.2.

\begin{tabular}{|c|c|c|c|c|c|}
\hline Period. & In food. & $\begin{array}{c}2 \\
\text { In feces. }\end{array}$ & $\begin{array}{l}3 \\
\text { In feces. } \\
(2 \div 1)\end{array}$ & $\begin{array}{c}t \\
\text { Balance. } \\
(1-2)\end{array}$ & $\begin{array}{l}\tilde{\mathbf{o}} \\
\text { Salicylic } \\
\text { acid ad- } \\
\text { ministered. }\end{array}$ \\
\hline \multicolumn{6}{|l|}{ Fore period. } \\
\hline $\begin{array}{l}\text { First subperiod: } \\
\quad \text { Total } \\
\text { Average } \ldots \ldots \ldots \\
\text { Second subperiod: } \\
\quad \text { Total } \\
\text { Average }\end{array}$ & $\begin{array}{r}\text { Grams. } \\
494.38 \\
98.88 \\
481.36 \\
96.27\end{array}$ & $\begin{array}{r}\text { Grams. } \\
23.19 \\
4.64 \\
24.07 \\
4.81\end{array}$ & 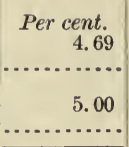 & $\begin{array}{r}\text { Grams. } \\
471.19 \\
94.24 \\
457.29 \\
91.46\end{array}$ & $\begin{array}{r}\text { Grams. } \\
0 \\
0 \\
0 \\
0\end{array}$ \\
\hline 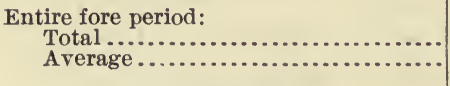 & $\begin{array}{r}975.74 \\
97.57 \\
\end{array}$ & $\begin{array}{r}47.26 \\
4.73 \\
\end{array}$ & $\begin{array}{r}4.84 \\
\cdots \cdots \\
\end{array}$ & $\begin{array}{r}928.48 \\
92.84 \\
\end{array}$ & $\begin{array}{l}0 \\
0\end{array}$ \\
\hline \multicolumn{6}{|l|}{ Preservative period. } \\
\hline $\begin{array}{l}\text { First subperiod: } \\
\quad \text { Total } \ldots \ldots \ldots \ldots \ldots \ldots \ldots \ldots \ldots \ldots \\
\text { Average } \ldots \ldots \ldots \ldots \ldots \\
\text { Second subperiod: }\end{array}$ & $\begin{array}{r}492.56 \\
98.51\end{array}$ & $\begin{array}{r}13.84 \\
2.77\end{array}$ & $\begin{array}{l}2.81 \\
2 . . .\end{array}$ & $\begin{array}{r}478.72 \\
95.74\end{array}$ & $\begin{array}{r}1.05 \\
.21\end{array}$ \\
\hline $\begin{array}{r}\text { Total } \\
\text { Average } \\
\text { Third subperiod: }\end{array}$ & $\begin{array}{r}460.44 \\
92.09\end{array}$ & $\begin{array}{r}16.58 \\
3.32\end{array}$ & $\begin{array}{l}3.60 \\
\cdots \ldots\end{array}$ & $\begin{array}{r}443.86 \\
88.77\end{array}$ & $\begin{array}{r}2.10 \\
.42\end{array}$ \\
\hline 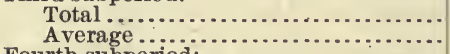 & $\begin{array}{r}487.78 \\
97.56\end{array}$ & $\begin{array}{r}21.69 \\
4.34\end{array}$ & $\begin{array}{l}4.45 \\
\ldots . .\end{array}$ & $\begin{array}{r}466.09 \\
93.22\end{array}$ & $\begin{array}{r}3.70 \\
.74\end{array}$ \\
\hline $\begin{array}{l}\text { Fourth subperiod: } \\
\quad \text { Total } \ldots \ldots \ldots \\
\text { Average }\end{array}$ & $\begin{array}{r}474.01 \\
94.80\end{array}$ & $\begin{array}{r}18.24 \\
3.65\end{array}$ & $\begin{array}{l}3.85 \\
\cdots \cdots\end{array}$ & $\begin{array}{r}45 \hbar .77 \\
91.15\end{array}$ & $\begin{array}{l}6.00 \\
1.20\end{array}$ \\
\hline $\begin{array}{l}\text { Fifth subperiod: } \\
\quad \text { Total } \ldots \ldots \ldots \ldots \ldots \ldots \ldots \ldots \ldots \ldots \\
\text { Average } \ldots \ldots \ldots \ldots \ldots \ldots \ldots\end{array}$ & $\begin{array}{r}457.39 \\
91.48\end{array}$ & $\begin{array}{r}11.64 \\
2.33\end{array}$ & $\begin{array}{l}2.54 \\
\cdots \ldots\end{array}$ & $\begin{array}{r}445.75 \\
89.15\end{array}$ & $\begin{array}{l}8.00 \\
1.60\end{array}$ \\
\hline 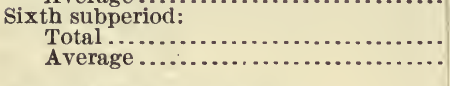 & $\begin{array}{r}452.72 \\
90.54\end{array}$ & $\begin{array}{r}18.52 \\
3.70\end{array}$ & $\begin{array}{r}4.09 \\
\cdots \cdots \\
\end{array}$ & $\begin{array}{r}434.20 \\
86.84\end{array}$ & $\begin{array}{r}10.00 \\
2.00 \\
\end{array}$ \\
\hline 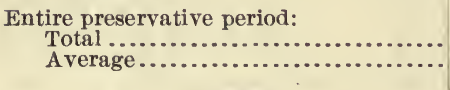 & $\begin{array}{r}2,824.90 \\
94.16 \\
\end{array}$ & $\begin{array}{r}100.51 \\
3.35\end{array}$ & $\begin{array}{r}3.56 \\
\cdots \cdots \\
\end{array}$ & $\begin{array}{r}2,724.39 \\
90.81\end{array}$ & $\begin{array}{r}30.85 \\
1.03\end{array}$ \\
\hline After period. & & & & & \\
\hline 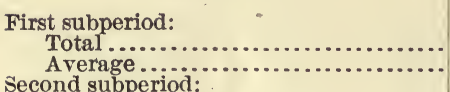 & $\begin{array}{r}452.46 \\
90.49\end{array}$ & $\begin{array}{r}15.56 \\
3.11\end{array}$ & $\begin{array}{c}3.44 \\
\cdots \ldots . .\end{array}$ & $\begin{array}{r}436.90 \\
87.38\end{array}$ & $\begin{array}{l}0 \\
0\end{array}$ \\
\hline 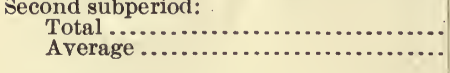 & $\begin{array}{r}465.27 \\
93.05\end{array}$ & $\begin{array}{r}19.33 \\
3.87\end{array}$ & $\begin{array}{r}4.15 \\
\cdots \\
\end{array}$ & $\begin{array}{r}445.94 \\
89.18\end{array}$ & $\begin{array}{l}0 \\
0\end{array}$ \\
\hline $\begin{array}{l}\text { Entire after period: } \\
\quad \text { Total } \ldots \ldots \ldots \ldots \ldots \ldots \ldots \ldots \\
\quad \text { Average } \ldots \ldots \ldots\end{array}$ & $\begin{array}{r}917.73 \\
91.77\end{array}$ & $\begin{array}{r}34.89 \\
3.49\end{array}$ & $\begin{array}{r}3.80 \\
\cdots \cdots . . .\end{array}$ & $\begin{array}{r}882.84 \\
88.28\end{array}$ & $\begin{array}{l}0 \\
0\end{array}$ \\
\hline
\end{tabular}




\section{Table XIX. - Fat balances for Series VI-Continued.}

[Averages are per day.]

No. 3.

\begin{tabular}{|c|c|c|c|c|c|}
\hline Period. & $\begin{array}{c}1 \\
\text { In food. }\end{array}$ & $\begin{array}{c}2 \\
\text { In feces. }\end{array}$ & $\begin{array}{l}3 \\
\text { In feces. } \\
(2 \div 1)\end{array}$ & $\begin{array}{c}4 \\
\text { Balance. } \\
(1-2)\end{array}$ & $\begin{array}{l}\mathbf{5} \\
\text { Salicylic } \\
\text { acid ad- } \\
\text { ministered. }\end{array}$ \\
\hline Fore period. & & & & & \\
\hline $\begin{array}{l}\text { First subperiod: } \\
\quad \text { Total } \ldots \ldots \ldots \ldots \ldots \ldots \ldots \\
\text { Average } \ldots \ldots \ldots \ldots \ldots \ldots\end{array}$ & 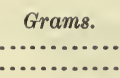 & $\begin{array}{l}\text { Grams. } \\
\text { G.......... }\end{array}$ & $\begin{array}{l}\text { Per cent. } \\
\cdots \\
\cdots\end{array}$ & $\begin{array}{c}\text { Grams. } \\
\cdots \cdots \\
\cdots \cdots\end{array}$ & $\begin{array}{r}\text { Grams. } \\
0 \\
0\end{array}$ \\
\hline $\begin{array}{l}\text { Second subperiod: } \\
\quad \text { Total } \ldots \ldots \ldots \ldots \ldots \ldots \ldots \ldots \ldots \ldots \ldots \ldots \ldots \\
\text { Average } \ldots \ldots \ldots \ldots \ldots \ldots \ldots \ldots\end{array}$ & $\begin{array}{r}362.43 \\
72.49\end{array}$ & $\begin{array}{l}9.81 \\
1.96\end{array}$ & 2.71 & $\begin{array}{r}352.62 \\
70.53\end{array}$ & . \\
\hline $\begin{array}{l}\text { Entire fore period: } \\
\quad \text { Total } \ldots \ldots \ldots \ldots \\
\text { Average } \ldots \ldots \ldots \\
\end{array}$ & & & $\cdots \cdots$ & & $\begin{array}{l}0 \\
0\end{array}$ \\
\hline Preservative period. & . & & & & \\
\hline $\begin{array}{l}\text { First subperiod: } \\
\quad \text { Total } \ldots \ldots \ldots \ldots \ldots \ldots \ldots \ldots \\
\text { Average } \ldots \ldots \ldots \ldots \ldots\end{array}$ & $\begin{array}{r}321.50 \\
64.30\end{array}$ & $\begin{array}{l}9.23 \\
1.85\end{array}$ & $\begin{array}{l}2.87 \\
\cdots\end{array}$ & $\begin{array}{r}312.27 \\
62.45\end{array}$ & $\begin{array}{r}1.05 \\
-\quad .21\end{array}$ \\
\hline $\begin{array}{l}\text { Second subperiod: } \\
\text { Total } \ldots \ldots \ldots \ldots \ldots \ldots \ldots\end{array}$ & $\begin{array}{r}334.39 \\
66.88\end{array}$ & $\begin{array}{r}14.77 \\
2.95\end{array}$ & 4.42 & $\begin{array}{r}319.62 \\
63.93\end{array}$ & $\begin{array}{r}2.10 \\
42\end{array}$ \\
\hline Third subperiod: & 00.80 & & & 05.95 & \\
\hline 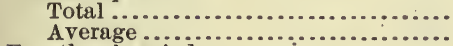 & $\begin{array}{r}331.51 \\
66.30\end{array}$ & $\begin{array}{l}9.56 \\
1.91\end{array}$ & 2.88 & $\begin{array}{r}321.95 \\
64.39\end{array}$ & $\begin{array}{r}4.00 \\
.80\end{array}$ \\
\hline Fourth subperiod: & & & & & \\
\hline Total ${ }^{4}, \ldots \ldots$ & 331.93 & 13. 33 & 4.02 & 318.60 & 6.00 \\
\hline Fifth subperiod: & 66.39 & 2.67 & ....... & 63.72 & 1.20 \\
\hline 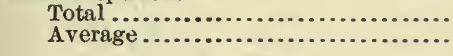 & $\begin{array}{r}308.01 \\
61.60\end{array}$ & $\begin{array}{r}10.27 \\
2.05\end{array}$ & $\begin{aligned} & 3.33 \\
\ldots \ldots \ldots & \end{aligned}$ & $\begin{array}{r}297.74 \\
59.55\end{array}$ & $\begin{array}{l}8.00 \\
1.60\end{array}$ \\
\hline 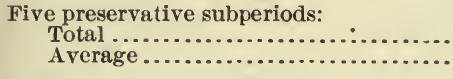 & $\begin{array}{r}a 1,627.34 \\
65.09\end{array}$ & $\begin{array}{r}57.16 \\
2.29\end{array}$ & $\begin{array}{c}3.51 \\
\cdots \ldots . .\end{array}$ & $\begin{array}{r}1,570.18 \\
62.80\end{array}$ & $\begin{array}{r}21.15 \\
.85\end{array}$ \\
\hline After period. & & & & & \\
\hline $\begin{array}{l}\text { First subperiod: } \\
\quad \text { Total } \ldots \ldots \ldots \ldots \ldots \ldots \ldots \ldots \ldots \ldots \ldots \ldots \\
\quad \text { Average } \ldots \ldots \ldots \ldots \ldots \ldots \ldots \ldots \ldots \\
\text { Second subperiod: }\end{array}$ & $\begin{array}{r}311.28 \\
62.26\end{array}$ & $\begin{array}{l}\text { Lost. } \\
\text { L.... }\end{array}$ & & & $\begin{array}{l}0 \\
0\end{array}$ \\
\hline 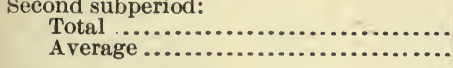 & $\begin{array}{r}330.03 \\
66.01\end{array}$ & $\begin{array}{r}10.35 \\
2.07\end{array}$ & 3.14 & $\begin{array}{r}319.68 \\
63.94\end{array}$ & $\begin{array}{l}0 \\
0\end{array}$ \\
\hline $\begin{array}{l}\text { Entire after period: } \\
\text { Total } \ldots . . . . . . . . . \\
\text { Average } . . .\end{array}$ & & & & & $\begin{array}{l}0 \\
0\end{array}$ \\
\hline
\end{tabular}

$a$ No. 3 had only five preservative subperiods. 
Table XIX.-Fat balances for Series VI-Continued.

[Averages are per day.]

No. 4.

\begin{tabular}{|c|c|c|c|c|c|}
\hline 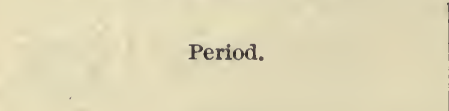 & $\begin{array}{c}1 \\
\text { In food. }\end{array}$ & $\begin{array}{c}2 \\
\text { In feces. }\end{array}$ & $\begin{array}{c}3 \\
\text { In feces. } \\
(2 \div 1)\end{array}$ & $\begin{array}{c}4 \\
\text { Balance. } \\
(1-2)\end{array}$ & $\begin{array}{c}\mathbf{5} \\
\text { Salicylic } \\
\text { acid ad- } \\
\text { ministered. }\end{array}$ \\
\hline Fore period. & & - & & & \\
\hline $\begin{array}{l}\text { First subperiod: } \\
\quad \text { Total } \ldots \ldots \ldots \ldots \ldots \ldots \ldots \\
\quad \text { Average } \ldots \ldots \ldots \ldots \ldots \ldots\end{array}$ & $\begin{array}{r}\text { Grams. } \\
488.58 \\
97.72\end{array}$ & $\begin{array}{r}\text { Grams. } \\
16.70 \\
3.34\end{array}$ & $\begin{array}{l}\text { Per cent. } \\
3.42 \\
3 .\end{array}$ & $\begin{array}{r}\text { Grams. } \\
471.88 \\
94.38\end{array}$ & $\begin{array}{r}\text { Grams. } \\
0 \\
0\end{array}$ \\
\hline Second subperiod: & & & & & \\
\hline $\begin{array}{l}\text { Total } \ldots . . . . \ldots \ldots \\
\text { Average } \ldots \ldots \ldots\end{array}$ & $\begin{array}{r}476.49 \\
95.30\end{array}$ & $\begin{array}{r}12.86 \\
2.57\end{array}$ & $\begin{array}{r}2.70 \\
2 .\end{array}$ & $\begin{array}{r}463.63 \\
92.73\end{array}$ & $\begin{array}{l}0 \\
0\end{array}$ \\
\hline 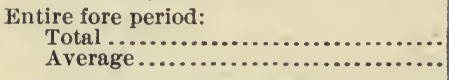 & $\begin{array}{r}965.07 \\
96.51\end{array}$ & $\begin{array}{r}29.56 \\
2.96\end{array}$ & $\begin{array}{l}3.06 \\
\cdots\end{array}$ & $\begin{array}{r}935.51 \\
93.55\end{array}$ & $\begin{array}{l}0 \\
0\end{array}$ \\
\hline Preservative period. & & & & & \\
\hline $\begin{array}{l}\text { First subperiod: } \\
\quad \text { Total } \ldots \ldots \ldots \ldots \ldots \\
\quad \text { Average } \ldots \ldots \ldots \\
\end{array}$ & $\begin{array}{r}490.87 \\
98.17\end{array}$ & $\begin{array}{r}10.17 \\
2.03\end{array}$ & 2.07 & $\begin{array}{r}480.70 \\
96.14\end{array}$ & $\begin{array}{r}1.05 \\
.21\end{array}$ \\
\hline $\begin{array}{l}\text { Second subperiod: } \\
\quad \text { Total } \ldots \ldots \ldots \ldots \ldots \ldots \ldots \\
\quad \text { Average } \ldots \ldots \ldots \ldots\end{array}$ & $\begin{array}{r}429.08 \\
85.82\end{array}$ & $\begin{array}{r}13.08 \\
2.62\end{array}$ & 3. 05 & $\begin{array}{r}416.00 \\
83.20\end{array}$ & $\begin{array}{r}2.10 \\
.42\end{array}$ \\
\hline $\begin{array}{l}\text { Third subperiod: } \\
\text { Total ........... }\end{array}$ & & & & & \\
\hline 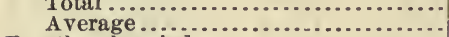 & $\begin{array}{r}484.45 \\
96.89\end{array}$ & $\begin{array}{r}12.54 \\
2.51\end{array}$ & 2.59 & $\begin{array}{r}471.91 \\
94.38\end{array}$ & 3. 70 \\
\hline Fourth subperiod: & & & & & \\
\hline 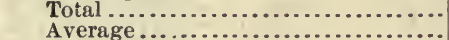 & $\begin{array}{r}460.38 \\
92.08\end{array}$ & a 12.76 & 2. 77 & 447.62 & 6.00 \\
\hline Fifth subperiod: & & 2.00 & & 89.53 & 1. \\
\hline 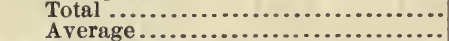 & $\begin{array}{r}468.92 \\
03.78\end{array}$ & 20.48 & 4.37 & 448.44 & 8.00 \\
\hline Sixth subperiod: & 70.10 & 4.10 & & 89.08 & 1.60 \\
\hline 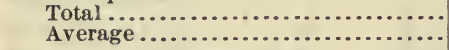 & $\begin{array}{r}449.32 \\
89.86\end{array}$ & $\begin{array}{r}a 15.41 \\
3.08\end{array}$ & $\begin{array}{l}3.43 \\
\ldots \ldots\end{array}$ & $\begin{array}{r}433.91 \\
86.78\end{array}$ & $\begin{array}{r}10.00 \\
2.00\end{array}$ \\
\hline Entire preservative period: & & & & & \\
\hline 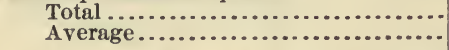 & $\begin{array}{r}2,783.02 \\
92.77\end{array}$ & $\begin{array}{r}84.44 \\
2.81\end{array}$ & $\begin{array}{c}3.03 \\
\cdots . . .\end{array}$ & $\begin{array}{r}2,698.58 \\
89.96\end{array}$ & $\begin{array}{r}30.85 \\
1.03\end{array}$ \\
\hline After period. & & & & & \\
\hline 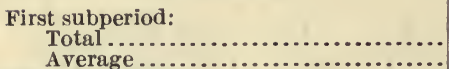 & $\begin{array}{r}448.48 \\
.89 .70\end{array}$ & $\begin{array}{r}11.35 \\
2.27\end{array}$ & $\begin{array}{c}2.53 \\
\cdots . . .\end{array}$ & $\begin{array}{r}437.13 \\
87.43\end{array}$ & $\begin{array}{l}0 \\
0\end{array}$ \\
\hline $\begin{array}{l}\text { Second subperiod: } \\
\text { Total }\end{array}$ & & & & & \\
\hline 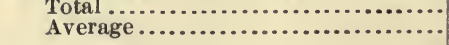 & $\begin{array}{r}465.73 \\
93.15\end{array}$ & $\begin{array}{r}14.47 \\
2.89\end{array}$ & $\begin{array}{l}3.11 \\
\ldots . . .\end{array}$ & $\begin{array}{r}451.26 \\
90.26\end{array}$ & $\begin{array}{l}0 \\
0\end{array}$ \\
\hline $\begin{array}{l}\text { Entire after period: } \\
\quad \text { Total } \ldots \ldots \ldots \ldots \\
\text { Average } \ldots \ldots \\
\end{array}$ & $\begin{array}{r}914.21 \\
91.42\end{array}$ & $\begin{array}{r}25.82 \\
2.58\end{array}$ & $\begin{array}{r}2.82 \\
\ldots . . .\end{array}$ & $\begin{array}{r}888.39 \\
88.84\end{array}$ & $\begin{array}{l}0 \\
0\end{array}$ \\
\hline
\end{tabular}

$a$ Daily average added in order to complete record. 
TABle XIX.-Fat balances for Series VI-Continued.

[Averages are per day.]

No: 5.

\begin{tabular}{|c|c|c|c|c|c|}
\hline Period. & In food. & $\begin{array}{c}2 \\
\text { In feces. }\end{array}$ & $\begin{array}{l}\mathbf{3} \\
\text { In feces. } \\
\quad(2 \div 1)\end{array}$ & $\begin{array}{c}\mathbf{4} \\
\text { Balance. } \\
(1-2)\end{array}$ & $\begin{array}{l}\mathbf{5} \\
\text { Salicylic } \\
\text { acid ad- } \\
\text { ministered. }\end{array}$ \\
\hline \multicolumn{6}{|l|}{ Fore period. } \\
\hline 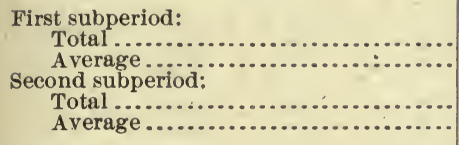 & $\begin{array}{r}\text { Grams. } \\
487.58 \\
97.52 \\
\\
480.64 \\
96.13\end{array}$ & $\begin{array}{r}\text { Grams. } \\
16.08 \\
3.22 \\
13.90 \\
2.78\end{array}$ & $\begin{array}{r}\text { Per cent. } \\
3.30 \\
\\
2.89 \\
\cdots\end{array}$ & $\begin{array}{r}\text { Grams. } \\
471.50 \\
94.30 \\
\\
466.74 \\
93.35\end{array}$ & $\begin{array}{r}\text { Grams. } \\
0 \\
0 \\
0 \\
0\end{array}$ \\
\hline $\begin{array}{l}\text { Entire fore period: } \\
\text { Total } \ldots \ldots \ldots \ldots \ldots \ldots \ldots \\
\text { Average } . \ldots \ldots \ldots \ldots \ldots\end{array}$ & $\begin{array}{r}968.22 \\
96.82\end{array}$ & $\begin{array}{r}29.98 \\
3.00\end{array}$ & $\begin{array}{r}3.10 \\
\cdots\end{array}$ & $\begin{array}{r}938.24 \\
93.82\end{array}$ & $\begin{array}{l}0 \\
0\end{array}$ \\
\hline $\begin{array}{l}\text { Preservative period. } \\
\text { Pirst }\end{array}$ & & & & & \\
\hline $\begin{array}{l}\text { First subperiod: } \\
\quad \text { Total } \ldots \ldots \ldots \\
\text { Average } \ldots \ldots \ldots \\
\text { Second subperiod: }\end{array}$ & $\begin{array}{r}491.79 \\
98.36\end{array}$ & $\begin{array}{r}14.53 \\
2.91\end{array}$ & $\begin{array}{l}2.95 \\
2 . .\end{array}$ & $\begin{array}{r}477.26 \\
95.45\end{array}$ & $\begin{array}{r}1.05 \\
.21\end{array}$ \\
\hline $\begin{array}{r}\text { Total } \\
\text { Average } \ldots \ldots \\
\text { Third subperiod: }\end{array}$ & $\begin{array}{r}457.62 \\
91.52\end{array}$ & $\begin{array}{r}12.20 \\
2.44\end{array}$ & $\begin{array}{r}2.67 \\
2 .\end{array}$ & $\begin{array}{r}445.42 \\
89.08\end{array}$ & $\begin{array}{r}2.10 \\
.42\end{array}$ \\
\hline $\begin{array}{l}\text { Total } \\
\text { Average }{ }_{1} \ldots \ldots \ldots \\
\end{array}$ & $\begin{array}{r}485.12 \\
97.02\end{array}$ & $\begin{array}{r}15.16 \\
3.03\end{array}$ & $\begin{array}{r}3.12 \\
\ldots \ldots\end{array}$ & $\begin{array}{r}469.96 \\
93.99\end{array}$ & $\begin{array}{r}3.70 \\
.74\end{array}$ \\
\hline $\begin{array}{l}\text { Fourth subperiod: } \\
\text { Total } \\
\text { Average } \\
\text { Fif }\end{array}$ & $\begin{array}{r}467.39 \\
93.48\end{array}$ & $\begin{array}{r}12.26 \\
2.45\end{array}$ & $\begin{array}{r}2.62 \\
\cdots\end{array}$ & $\begin{array}{r}455.13 \\
91.03\end{array}$ & $\begin{array}{l}6.00 \\
1.20\end{array}$ \\
\hline 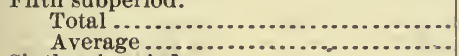 & $\begin{array}{r}469.73 \\
93.95\end{array}$ & $\begin{array}{r}16.66 \\
3.33\end{array}$ & $\begin{array}{l}3.55 \\
\cdots\end{array}$ & $\begin{array}{r}453.07 \\
90.62\end{array}$ & $\begin{array}{l}8.00 \\
1.60\end{array}$ \\
\hline $\begin{array}{l}\text { Sixth subperiod: } \\
\quad \text { Total } \ldots \ldots \ldots \ldots \\
\text { Average } \ldots \ldots \ldots \\
\end{array}$ & $\begin{array}{r}449.19 \\
89.84\end{array}$ & $\begin{array}{r}13.26 \\
2.65\end{array}$ & $\begin{array}{r}2.95 \\
\ldots \ldots .\end{array}$ & $\begin{array}{r}435.93 \\
87.19\end{array}$ & $\begin{array}{r}10.00 \\
2.00\end{array}$ \\
\hline $\begin{array}{l}\text { Entire preservative period: } \\
\quad \text { Total } \ldots \ldots \ldots \\
\text { Average } . \ldots \ldots \\
\end{array}$ & $\begin{array}{r}2,820.84 \\
94.03\end{array}$ & $\begin{array}{r}84.07 \\
2.80\end{array}$ & $\begin{array}{r}2.98 \\
\ldots \ldots . .6 \\
\end{array}$ & $\begin{array}{r}2,736.77 \\
91.23\end{array}$ & $\begin{array}{r}30.85 \\
1.03\end{array}$ \\
\hline After period. & & & & & \\
\hline $\begin{array}{l}\text { First subperiod: } \\
\quad \text { Total } \ldots \ldots \ldots \ldots \ldots \ldots \\
\text { A verage................... } \\
\text { Second subperiod: }\end{array}$ & $\begin{array}{r}450.18 \\
90.04\end{array}$ & $\begin{array}{r}18.48 \\
3.70\end{array}$ & $\begin{array}{l}4.11 \\
\cdots . . .\end{array}$ & $\begin{array}{r}431.70 \\
86.34\end{array}$ & $\begin{array}{l}0 \\
0\end{array}$ \\
\hline 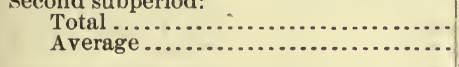 & $\begin{array}{r}463.48 \\
92.70\end{array}$ & $\begin{array}{r}10.82 \\
2.16\end{array}$ & $\begin{array}{c}2.33 \\
\cdots . . .\end{array}$ & $\begin{array}{r}452.66 \\
90.54\end{array}$ & $\begin{array}{l}0 \\
0\end{array}$ \\
\hline 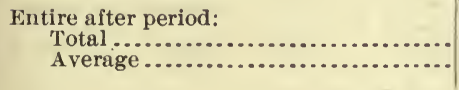 & $\begin{array}{r}913.66 \\
91.37\end{array}$ & $\begin{array}{r}29.30 \\
2.93\end{array}$ & $\begin{array}{c}3.21 \\
\cdots\end{array}$ & $\begin{array}{r}884.36 \\
88.44\end{array}$ & $\begin{array}{l}0 \\
0\end{array}$ \\
\hline
\end{tabular}




\section{TABLe XIX.-Fat balances for Series VI-Continued.}

[Averages are per day.]

No. 6.

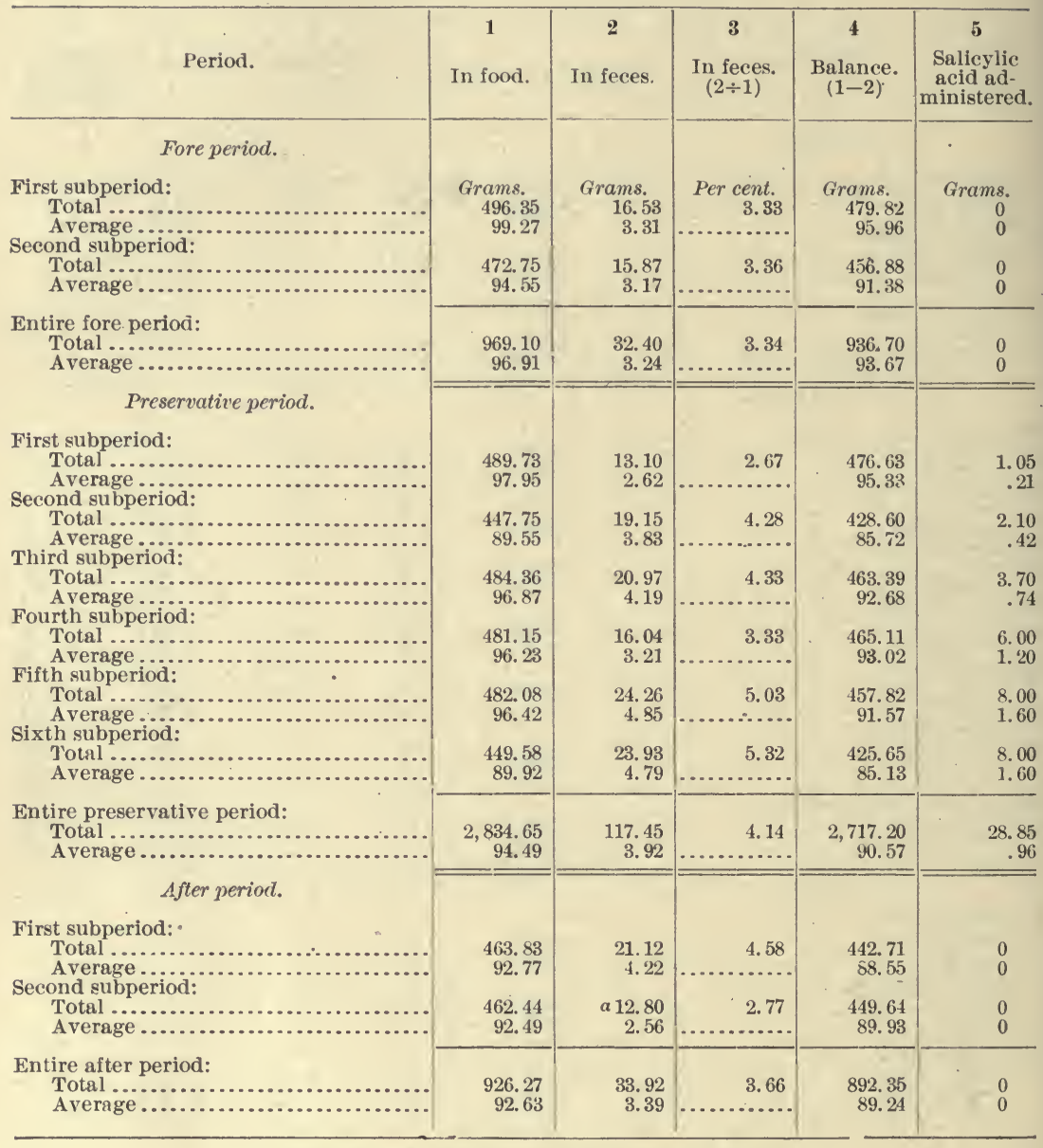

$a$ Daily average added in order to complete record. 
Table XIX._Fat balances for Series VI-Continued.

[A verages are per day.]

No. 7.

\begin{tabular}{|c|c|c|c|c|c|}
\hline Period. & In food. & $\begin{array}{c}2 \\
\text { In feces. }\end{array}$ & $\begin{array}{l}\mathbf{3} \\
\text { In feces. } \\
(2 \div 1)\end{array}$ & $\begin{array}{c}\mathbf{4} \\
\text { Balance. } \\
(1-2)\end{array}$ & $\begin{array}{l}\mathbf{5} \\
\text { Salicylic } \\
\text { acid ad- } \\
\text { ministered. }\end{array}$ \\
\hline \multicolumn{6}{|l|}{ Fore period. } \\
\hline $\begin{array}{l}\text { First subperiod: } \\
\quad \text { Total } \ldots \ldots \ldots \ldots \ldots \ldots \\
\text { Average } \ldots \ldots \ldots \ldots \\
\text { Second subneriod: }\end{array}$ & $\begin{array}{r}\text { Grams. } \\
321.37 \\
64.27\end{array}$ & $\begin{array}{r}\text { Grams. } \\
13.58 \\
2.72\end{array}$ & $\begin{array}{r}\text { Per cent. } \\
4.23 \\
4\end{array}$ & $\begin{array}{r}\text { Grams. } \\
307.79 \\
61.55\end{array}$ & $\begin{array}{r}\text { Grams. } \\
0 \\
0\end{array}$ \\
\hline 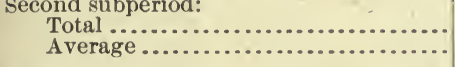 & $\begin{array}{r}348.63 \\
69.73\end{array}$ & $\begin{array}{l}8.14 \\
1.63\end{array}$ & - 2.33 & $\begin{array}{r}340.49 \\
68.10\end{array}$ & $\begin{array}{l}0 \\
0\end{array}$ \\
\hline $\begin{array}{l}\text { Entire fore period: } \\
\text { Total } \ldots \ldots \ldots \ldots \ldots \ldots \\
\text { Average } \ldots \ldots \ldots \ldots \ldots\end{array}$ & $\begin{array}{r}670.00 \\
67.00\end{array}$ & $\begin{array}{r}21.72 \\
2.17\end{array}$ & $\begin{array}{r}3.24 \\
-2 .\end{array}$ & $\begin{array}{r}648.28 \\
64.83\end{array}$ & $\begin{array}{l}0 \\
0\end{array}$ \\
\hline \multicolumn{6}{|l|}{ Preservative period. } \\
\hline 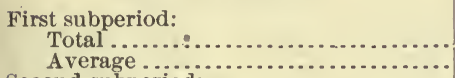 & $\begin{array}{r}350.78 \\
70.16\end{array}$ & $\begin{array}{r}10.92 \\
2.18\end{array}$ & 3.11 & $\begin{array}{r}339.86 \\
67.98\end{array}$ & $\begin{array}{r}1.05 \\
.21\end{array}$ \\
\hline Second subperiod: & 319.18 & & & & \\
\hline $\begin{array}{l}\text { Total } \\
\text { Average } \\
\text { Third subperiod: }\end{array}$ & $\begin{array}{r}319.18 \\
63.84\end{array}$ & $\begin{array}{l}8.00 \\
1.60\end{array}$ & $\begin{aligned} 2.51 \\
-\ldots . .\end{aligned}$ & $\begin{array}{r}311.18 \\
62.24\end{array}$ & $\begin{array}{r}2.10 \\
.42\end{array}$ \\
\hline 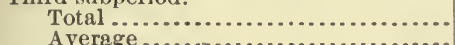 & $\begin{array}{r}339.72 \\
67.94\end{array}$ & 11.06 & 3.26 & 328.66 & 3. 70 \\
\hline Average .............................. & 67.94 & 2. 21 . & & 65.73 & .74 \\
\hline Total $\ldots \ldots \ldots \ldots$ & 337.70 & 7.71 & 2.28 & 329.99 & 6.00 \\
\hline $\begin{array}{l}\text { Average } \\
\text { Fifth subperiod: }\end{array}$ & 67.54 & 1.54 & $\cdots \cdots$ & 66.00 & 1.20 \\
\hline $\begin{array}{l}\text { Total } \\
\text { Average } . . .6\end{array}$ & $\begin{array}{r}341.48 \\
68.30\end{array}$ & $\begin{array}{l}9.73 \\
1.95\end{array}$ & 2.85 & $\begin{array}{r}331.75 \\
66.35\end{array}$ & $\begin{array}{l}8.00 \\
1.00\end{array}$ \\
\hline Sixth subperiod: & & & & 00.00 & 1.00 \\
\hline $\begin{array}{l}\text { Total } \\
\text { Average } \ldots\end{array}$ & $\begin{array}{r}306.58 \\
61.32\end{array}$ & $\begin{array}{l}5.20 \\
1.04\end{array}$ & $\begin{array}{l}1.70 \\
2 . \cdots\end{array}$ & $\begin{array}{r}301.38 \\
60.28\end{array}$ & $\begin{array}{r}10.00 \\
2.00\end{array}$ \\
\hline Entire preservative period: & & & & & \\
\hline 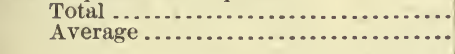 & $\begin{array}{r}1,995.44 \\
66.51\end{array}$ & $\begin{array}{r}52.62 \\
1.75\end{array}$ & $\begin{array}{l}2.64 \\
. . . .\end{array}$ & $\begin{array}{r}1,942.82 \\
64.76\end{array}$ & $\begin{array}{r}30.85 \\
1.03\end{array}$ \\
\hline After period. & & & & & \\
\hline $\begin{array}{l}\text { First subperiod: } \\
\text { Total }\end{array}$ & & & & & \\
\hline Average............... & $\begin{array}{r}323.35 \\
64.67\end{array}$ & $\begin{array}{r}11.79 \\
2.36\end{array}$ & $\begin{array}{l}3.65 \\
\ldots . . .\end{array}$ & $\begin{array}{r}311.56 \\
62.31\end{array}$ & $\begin{array}{l}0 \\
0\end{array}$ \\
\hline $\begin{array}{l}\text { Second subperiod: } \\
\text { Total .............. }\end{array}$ & 326.04 & 13,71 & 4. 21 & 20 & 0 \\
\hline Average............... & $\begin{array}{r}520.04 \\
65.21\end{array}$ & $\begin{array}{r}15.71 \\
2.74\end{array}$ & 4.21 & 62.47 & 0 \\
\hline $\begin{array}{l}\text { Entire after period: } \\
\quad \text { Total } \ldots \ldots \ldots \ldots \ldots \ldots \ldots \\
\text { Average } \ldots \ldots \ldots \ldots\end{array}$ & $\begin{array}{r}649.39 \\
64.94\end{array}$ & $\begin{array}{r}25.50 \\
2.55\end{array}$ & $\begin{array}{c}3.93 \\
\ldots . .6\end{array}$ & $\begin{array}{r}623.89 \\
62.39\end{array}$ & $\begin{array}{l}0 \\
0\end{array}$ \\
\hline
\end{tabular}


TABLE XLX._Fat balances for Series VI-Continued.

[Averages are per day.]

No.8.

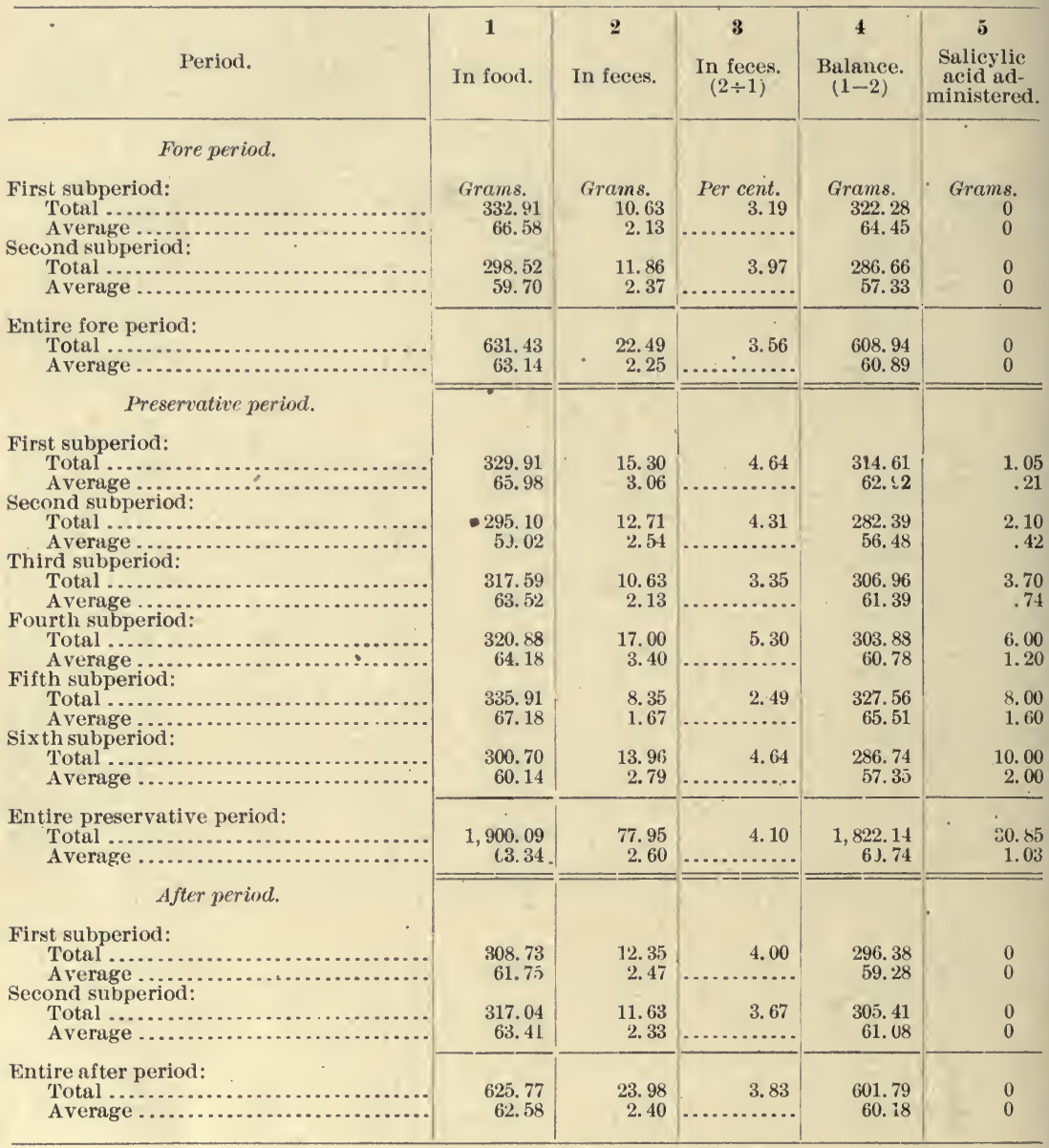


TABle XIX.-Fat balances for Series VI-Continued

[Averages are per day.]

No. 9.

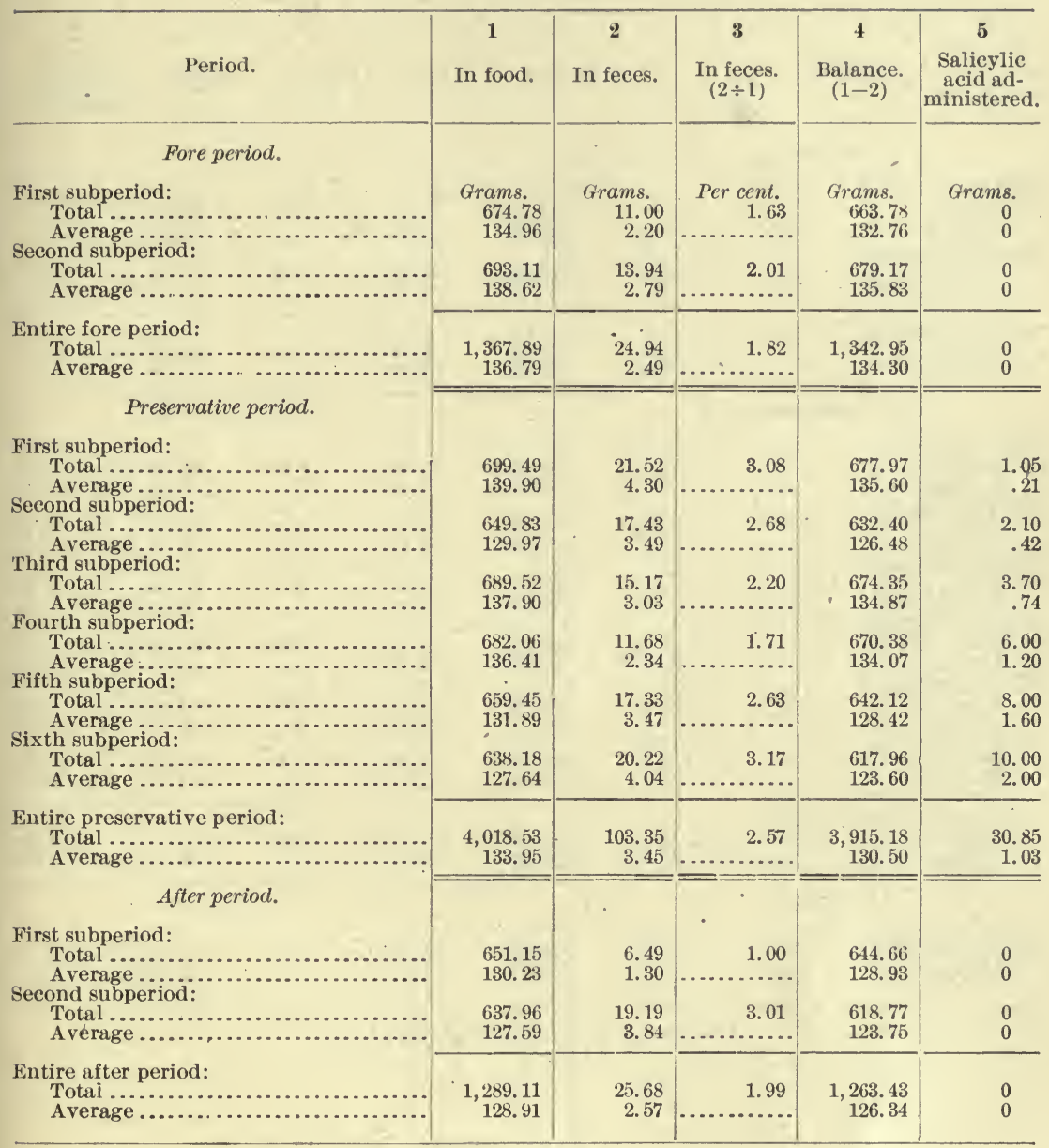


TaBLE XIX. - Fat balances for Series VI-Continued.

[Averages are per day.]

No. 10.

\begin{tabular}{|c|c|c|c|c|c|}
\hline Period. & $\begin{array}{c}1 \\
\text { In food. }\end{array}$ & $\begin{array}{l}2 \\
\text { In feces. }\end{array}$ & $\begin{array}{l}3 \\
\text { In feces. } \\
(2 \div 1)\end{array}$ & $\begin{array}{c}4 \\
\text { Balance. } \\
(1-2)\end{array}$ & $\begin{array}{l}\text { s } \\
\text { Balicylic } \\
\text { acid ad- } \\
\text { ministered. }\end{array}$ \\
\hline \multicolumn{6}{|l|}{ Fore period. } \\
\hline 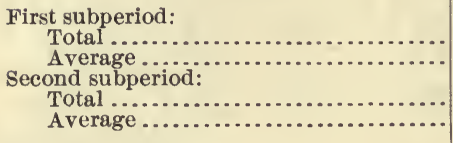 & $\begin{array}{r}\text { Grams. } \\
502.65 \\
100.53 \\
472.44 \\
94.49\end{array}$ & $\begin{array}{r}\text { Grams. } \\
22.31 \\
4.46 \\
\\
15.89 \\
3.18\end{array}$ & $\begin{array}{r}\text { Per cent. } \\
4.44 \\
3.36 \\
3 .\end{array}$ & $\begin{array}{r}\text { Grams. } \\
480.34 \\
96.07 \\
456.55 \\
91.31\end{array}$ & $\begin{array}{r}\text { Grams. } \\
0 \\
0 \\
0 \\
0\end{array}$ \\
\hline $\begin{array}{l}\text { Entire fore period: } \\
\quad \text { Total } \ldots \ldots \ldots \\
\text { Average } \ldots \ldots \\
\end{array}$ & $\begin{array}{r}975.09 \\
97.51 \\
\end{array}$ & $\begin{array}{r}38.20 \\
3.82 \\
\end{array}$ & $\begin{array}{r}3.92 \\
\cdots \cdots . \\
\end{array}$ & $\begin{array}{r}936.89 \\
93.69\end{array}$ & $\begin{array}{l}0 \\
0 \\
\end{array}$ \\
\hline \multicolumn{6}{|l|}{ Preservative period. } \\
\hline $\begin{array}{l}\text { First subperiod: } \\
\quad \text { Total } \ldots \ldots \ldots \ldots \ldots \ldots \ldots \ldots \ldots \ldots \ldots \ldots \\
\quad \text { Average } \ldots \ldots \ldots \ldots \ldots \ldots \ldots \ldots \ldots \\
\text { Second subperiod: }\end{array}$ & $\begin{array}{r}480.34 \\
96.07\end{array}$ & $\begin{array}{r}17.89 \\
3.58\end{array}$ & $\begin{array}{l}3.72 \\
\ldots \ldots \ldots\end{array}$ & $\begin{array}{r}462.45 \\
92.49\end{array}$ & $\begin{array}{r}1.05 \\
.21\end{array}$ \\
\hline 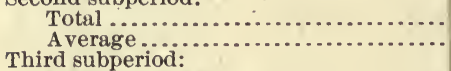 & $\begin{array}{r}455.80 \\
91.16\end{array}$ & $\begin{array}{r}13.41 \\
2.68\end{array}$ & $\begin{array}{c}2.94 \\
\cdots \ldots .\end{array}$ & $\begin{array}{r}442.39 \\
88.48\end{array}$ & $\begin{array}{r}2.10 \\
.42\end{array}$ \\
\hline 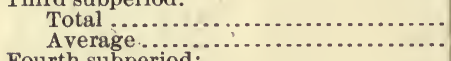 & $\begin{array}{r}453.31 \\
90.66\end{array}$ & $\begin{array}{r}15.71 \\
3.14\end{array}$ & 3.47 & $\begin{array}{r}437.60 \\
87.52\end{array}$ & $\begin{array}{r}3.70 \\
.74\end{array}$ \\
\hline $\begin{array}{l}\text { Fourth subperiod: } \\
\quad \text { Total } \ldots \ldots \ldots \\
\text { Average }\end{array}$ & $\begin{array}{r}461.06 \\
92.21\end{array}$ & $\begin{array}{r}15.47 \\
3.09\end{array}$ & $\begin{array}{r}3.36 \\
\ldots \ldots . .\end{array}$ & $\begin{array}{r}445.59 \\
89.12\end{array}$ & $\begin{array}{l}6.00 \\
1.20\end{array}$ \\
\hline $\begin{array}{l}\text { Fifth subperiod: } \\
\quad \text { Total } \ldots \ldots \ldots \ldots \\
\text { Average } \ldots \ldots \\
\end{array}$ & $\begin{array}{r}465.91 \\
93.18\end{array}$ & $\begin{array}{r}14.24 \\
2.85\end{array}$ & $\begin{array}{c}3.06 \\
\ldots \ldots\end{array}$ & $\begin{array}{r}451.67 \\
90.33\end{array}$ & $\begin{array}{l}8.00 \\
1.60\end{array}$ \\
\hline $\begin{array}{l}\text { Six th subperiod: } \\
\quad \text { Total }{ }^{\prime} \ldots \ldots \ldots \\
\quad \text { Average } \ldots \ldots \\
\end{array}$ & $\begin{array}{r}428.18 \\
85.64\end{array}$ & $\begin{array}{l}5.38 \\
1.08\end{array}$ & $\begin{array}{c}1.26 \\
\cdots \cdots . . \\
\end{array}$ & $\begin{array}{r}422.80 \\
84.56\end{array}$ & $\begin{array}{r}10.00 \\
2.00\end{array}$ \\
\hline 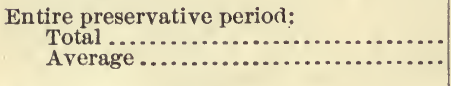 & $\begin{array}{r}2,744.60 \\
91.49 \\
\end{array}$ & $\begin{array}{r}82.10 \\
2.74 \\
\end{array}$ & $\begin{array}{r}2.99 \\
\cdots \cdots \\
\end{array}$ & $\begin{array}{r}2,662.50 \\
88.75\end{array}$ & $\begin{array}{r}30.85 \\
1.03\end{array}$ \\
\hline After period. & . & & & $x^{2}$ & \\
\hline $\begin{array}{l}\text { First subperiod: } a \\
\quad \text { Total } \ldots \ldots \ldots \ldots \ldots \\
\quad \text { Average } \ldots \ldots \ldots \ldots\end{array}$ & $\begin{array}{r}451.44 \\
90.29\end{array}$ & $\begin{array}{r}16.77 \\
3.35\end{array}$ & $\begin{array}{c}3.71 \\
\cdots . . .\end{array}$ & $\begin{array}{r}434.67 \\
86.94\end{array}$ & $\begin{array}{l}0 \\
0\end{array}$ \\
\hline
\end{tabular}

$a$ No second after subperiod; subject $i l l$. 
TABLE XIX._Fat balances for Series VI-Continued.

[Averages are per day.]

No. 11.

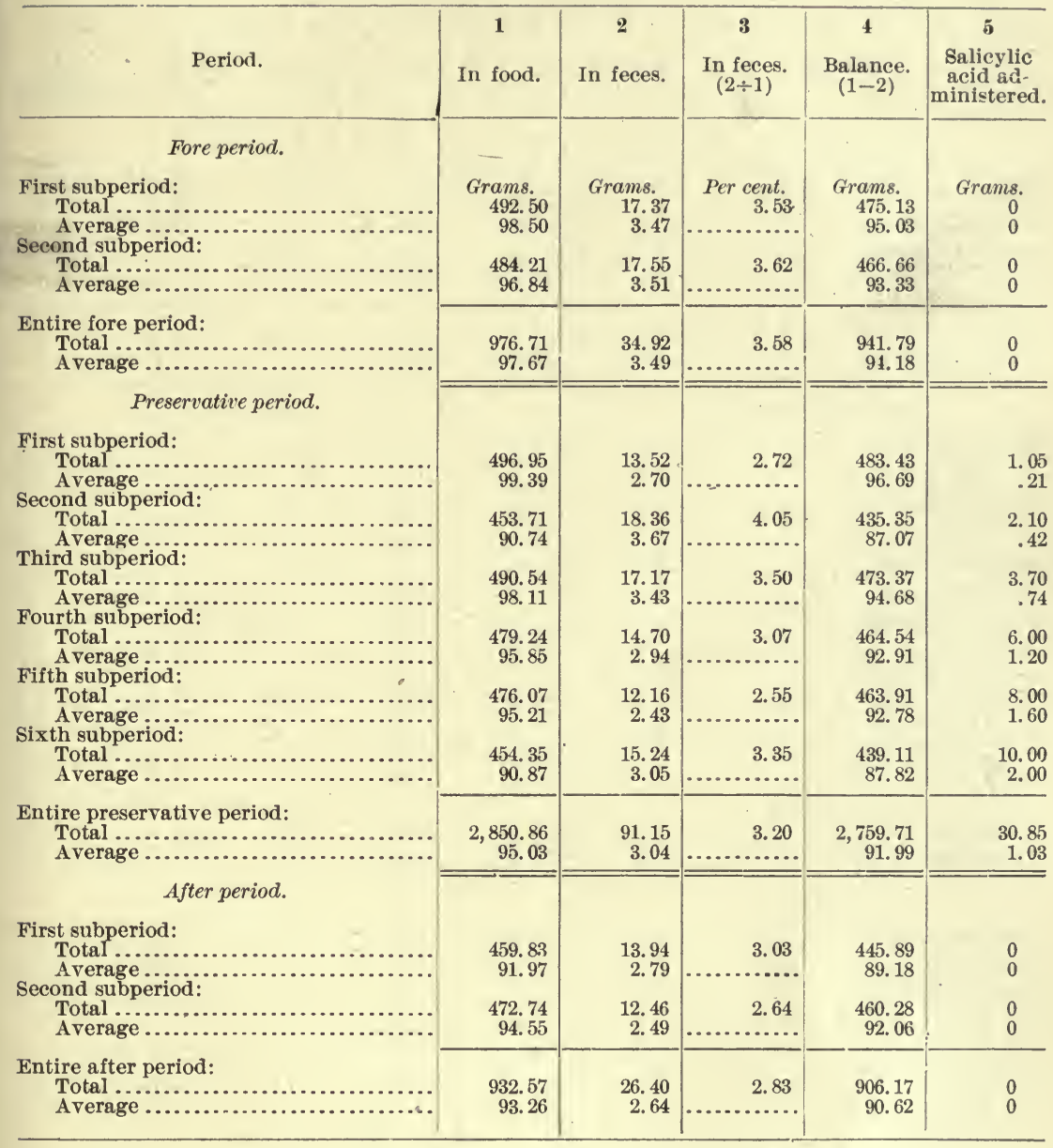


Table XIX._Fat balances for Series VI-Continued.

[Averages are per day.]

No. 12.

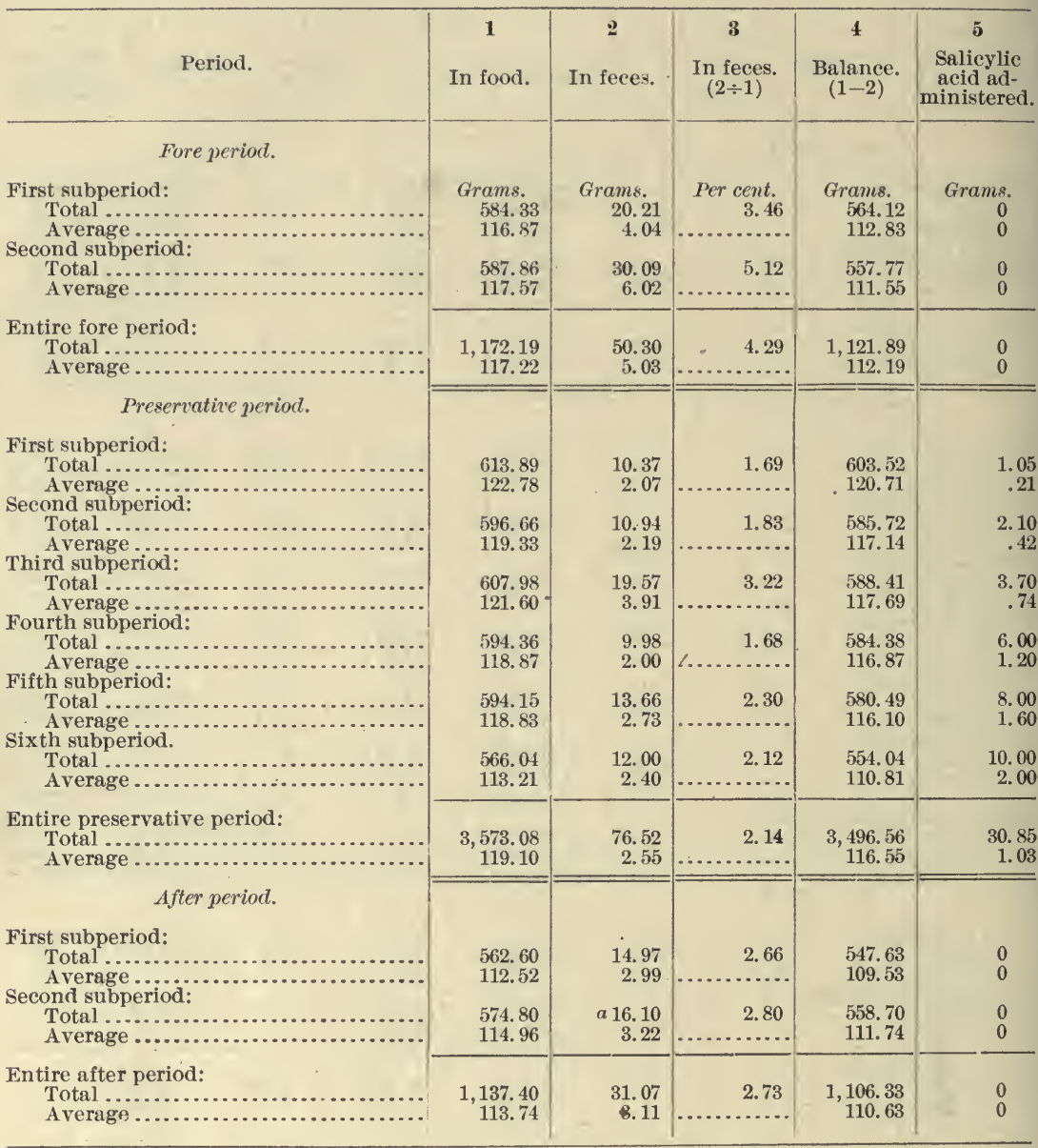

$a$ Daily average added in order to complete record. 
TABle XIX.-Fat balances for Series VI-Continued.

[Averages are per man per day.]

Summary for nine men.

\begin{tabular}{|c|c|c|c|c|c|}
\hline Period. & $\begin{array}{c}1 \\
\text { In food. }\end{array}$ & $\begin{array}{c}2 \\
\text { In feces. }\end{array}$ & $\begin{array}{c}3 \\
\text { In feces. } \\
(2 \div 1)\end{array}$ & $\begin{array}{c}\mathbf{4} \\
\text { Balance. } \\
(1-2)\end{array}$ & $\begin{array}{c}\mathbf{5} \\
\text { Salicylic } \\
\text { acid ad- } \\
\text { ministered. }\end{array}$ \\
\hline Fore period. & & & & & \\
\hline 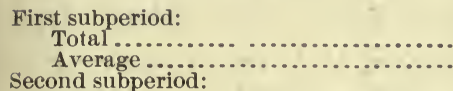 & $\begin{array}{r}\text { Grams. } \\
4,140.53 \\
89.79\end{array}$ & $\begin{array}{l}\text { Grams. } \\
\quad 144.47 \\
\quad 3.21\end{array}$ & $\begin{array}{r}\text { Per cent. } \\
3.58\end{array}$ & $\begin{array}{l}\text { Grams. } \\
3,896.06 \\
86.58\end{array}$ & $\begin{array}{r}\text { Grams. } \\
0 \\
0\end{array}$ \\
\hline 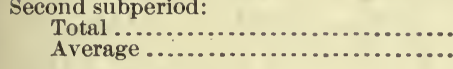 & $\begin{array}{r}4,059.55 \\
90.21\end{array}$ & $\begin{array}{r}142.92 \\
3.18\end{array}$ & 3.52 & $\begin{array}{r}3,916.63 \\
87.03\end{array}$ & $\begin{array}{l}0 \\
0\end{array}$ \\
\hline 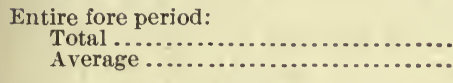 & $\begin{array}{r}8,200.08 \\
91.11\end{array}$ & $\begin{array}{r}287.39 \\
3.19\end{array}$ & $\begin{array}{l}3.50 \\
\cdots \cdots\end{array}$ & $\begin{array}{r}7,912.69 \\
87.92\end{array}$ & $\begin{array}{l}0 \\
0\end{array}$ \\
\hline Preservative period. & & s & & & \\
\hline $\begin{array}{l}\text { First subperiod: } \\
\text { Total ............. } \\
\text { Average...... } \\
\text { Second subperiod: }\end{array}$ & $\begin{array}{r}4,202.85 \\
93.40\end{array}$ & $\begin{array}{r}108.10 \\
2.40\end{array}$ & 2.57 & $\begin{array}{r}4,094.75 \\
91.00\end{array}$ & $\begin{array}{r}9.45 \\
.21\end{array}$ \\
\hline $\begin{array}{l}\text { Second subperiod: } \\
\text { Total ........... } \\
\text { Average ........ }\end{array}$ & $\begin{array}{r}3,876.68 \\
86.15\end{array}$ & $\begin{array}{r}118.78 \\
2.64\end{array}$ & 3.06 & $\begin{array}{r}3,757.90 \\
83.51\end{array}$ & 18.90 \\
\hline $\begin{array}{c}\text { Third subperiod: } \\
\text { Total .............. }\end{array}$ & $4,137.89$ & 137.96 & 3,33 & 05.01 & \\
\hline $\begin{array}{l}\text { Average....... } \\
\text { Fourth subperiod: }\end{array}$ & 0910 & 3.07 & & $\begin{array}{r}3,999.93 \\
88.88\end{array}$ & $\begin{array}{r}33.30 \\
.74\end{array}$ \\
\hline $\begin{array}{l}\text { Total ............. } \\
\text { Average....... }\end{array}$ & $4,046.43$ & 119. 62 & 2.96 & $3,926.81$ & 54. \\
\hline $\begin{array}{l}\text { Average } \\
\text { Fifth subperiod: } \\
\text { Total } . . . . . . .\end{array}$ & & 2.66 & & 87.26 & 1. \\
\hline $\begin{array}{l}\text { Total............ } \\
\text { Average ...... } \\
\text { Sixth subperiod: }\end{array}$ & $\begin{array}{r}4,046.10 \\
89.91\end{array}$ & $\begin{array}{r}125.40 \\
2.79\end{array}$ & 3.10 & $\begin{array}{r}3,920.70 \\
87.12\end{array}$ & $\begin{array}{r}72.00 \\
1.60\end{array}$ \\
\hline 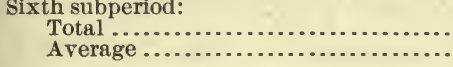 & $\begin{array}{r}3,827.29 \\
85.05\end{array}$ & $\begin{array}{r}125.79 \\
2.80\end{array}$ & 3.29 & $\begin{array}{r}3,701.50 \\
82.25\end{array}$ & $\begin{array}{r}88.00 \\
1.96\end{array}$ \\
\hline 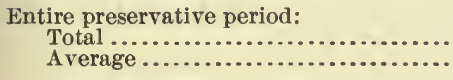 & $\begin{array}{r}24,137.24 \\
89.40\end{array}$ & $\begin{array}{r}735.65 \\
2.72\end{array}$ & 3.05 & $\begin{array}{r}23,401.59 \\
86.68\end{array}$ & $\begin{array}{r}275.65 \\
1.02\end{array}$ \\
\hline After period. & & & & & \\
\hline $\begin{array}{l}\text { First subperiod: } \\
\quad \text { Total } \ldots . . . . . . . . \\
\text { Average } . . . . . .\end{array}$ & $\begin{array}{r}3,881.11 \\
86.25\end{array}$ & $\begin{array}{r}135.34 \\
3.01\end{array}$ & 3.49 & $\begin{array}{r}3,745.77 \\
83.24\end{array}$ & $\begin{array}{l}0 \\
0\end{array}$ \\
\hline 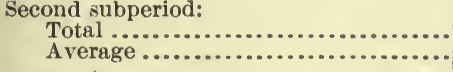 & $\begin{array}{r}3,972.30 \\
88.27\end{array}$ & $\begin{array}{r}122.92 \\
2.73\end{array}$ & 3.09 & $\begin{array}{r}3,849.38 \\
85.54\end{array}$ & $\begin{array}{l}0 \\
0\end{array}$ \\
\hline 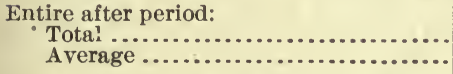 & $\begin{array}{r}7,853.41 \\
87.26\end{array}$ & $\begin{array}{r}258.26 \\
2.87\end{array}$ & 3.29 & $\begin{array}{r}7,595.15 \\
84.39\end{array}$ & $\begin{array}{l}0 \\
0\end{array}$ \\
\hline
\end{tabular}

\section{CALORIES BALANCE.}

A comparison of the total heat value of the food with the heat value of the residual matter in the feces and urine will give an indication of the activity of the organism in respect of its relations to the heat and energy supplied by the food. The calories were determined by calculation, a comparison of such results with those obtained by combustion in an atmosphere of oxidation, in the borax experiment, having shown that the two methods give comparable results on a large number of samples. The figures used in the following discussion are found in Table XXI, page 669. 
INDIVIDUAL DATA.

No. 1 .

The average daily number of calories in the food of No. 1 for the fore period is 2,442 , for the preservative period 2,453 , and for the after period 2,454 . The data show a very close agreement in the daily quantity of heat-forming material ingested with the food. The residual calories in the feces for the fore period are 62 , for the preservative period 60 , and for the after period 96. The residual calories in the urine for the fore period are 72 , for the preservative period 79, and for the after period 79. Expressed in percentages, the calories in the fore period in the feces are 2.55 per cent, in the preservative period $2.43 \mathrm{per}$ cent, and in the after period 3.92 per cent; in the urine, 2.95 per cent, $3.2 \mathrm{l}$ per cent, and 3.23 per cent, respectively. The balance for the fore period is 2,308 , for the preservative period 2,315 , and for the after period 2,279. These figures represent the actual quantity of heat furnished by the food during the progress of the experiment. The data show a slight tendency on the part of the preservative to decrease the calories in the feces. On the withdrawal of the preservative, however, the calories in the feces increase in a very marked degree. There was but little change in the calories occurring in the urine, there being a slight increase during the preservative period, which was maintained without change in the after period. The most notable fact in connection with the data is the increase in the calories in the feces upon the withdrawal of the preservative.

\section{No. 2.}

The average number of calories in the food of No. 2 for the three periods is $3,015,3,008$, and 3,011 , respectively, showing a very close agreement in the heat value for the three periods. There appear in the feces for the fore period 137 calories daily, in the preservative period 114, and in the after period 106. There appear in the urine in the fore period 89 calories, in the preservative period 85 calories, and in the after period 89 calories. The largest percentage of calories appears in the feces in the fore period and the smallest in the after period. The largest percentage of calories in the urine is in the after period, but it is almost identical with the percentage of the fore period. There is a slight diminution in the percentage of calories in the urine during the preservative period. The largest balance, namely, 2,816, occurs in the after period and the smallest in the fore period. The most noted change indicated by the data is in the diminution of the calories in the feces upon the addition of the preservative, and this diminution is continued, but to a less extent, in the after period. 
No. 3.

The sheet for No. 3 is offered without comment on account of the illness and consequent interruption of the normal functions during a part of the period of observation.

No. 4.

In the case of No. 4 it is seen that the average daily calories in the food number 2,769 in the fore period, 2,833 in the preservative period, and 2,904 in the after period, showing a progressive increase in the calories of the food. There is a corresponding decrease in the average daily calories in the feces, falling from 94 in the fore period to 91 in the preservative period and 85 in the after period. In the urine the quantity of calories in the preservative period is slightly less than during the fore period and the after period, being practically identical for these two periods. In this connection, however, the increase in the calories in the food in both the preservative and after periods must be remembered. The largest balance is found in the after period and the smallest in the fore period. The decrease in the calories in the feces and their almost constant value in the urine do not correspond to the increase of the calories in the food. The data therefore show an increased consumption of the heat values of the foods during the administration of the preservative and in the after period.

No. 5 .

The quantity of calories in the food of No. 5 is almost the same for all three periods, being identical for the fore and preservative periods and only 8 calories less for the after period. The calories in the feces are greatest in the fore period, namely, 116, and decrease throughout, being least in the after period. There is but little change in the calories in the urine, a slightly larger number appearing in the preservative period and the after period than in the fore period. The balances are almost the same for all the periods, increasing very slightly in the preservative and after periods. The data show again a tendency to diminish the calories in the feces by the administration of the preservative, although a corresponding decrease is not observed in the urine. This indicates an increased consumption of the heat values of the food during the preservative period, and this tendency is continued in the after period.

No. 6 .

The calories in the food for No. 6 are somewhat greater in the preservative period and in the after period than in the fore period. The quantity appearing in the feces is almost the same for the fore and

$7656-$ No. 84 , pt $2-06-13$ 
preservative periods and slightly less in the after period. The quantity appearing in the urine is greater in the preservative period than at any other time. These data show but little effect of the preservative in decreasing the calories in the feces, but a somewhat marked effect in increasing the calories in the urine. The general tendency therefore is to excrete more organic matter in the urine during the administration of the preservative.

No. $\%$.

The calories in the food of No. 7 for the fore and preservative periods are almost the same, but they were slightly increased in the after period. The data show a very notable decrease in the calories of the feces during the preservative period and a slight decrease in the calories of the urine. These data show a very marked tendency on the part of the preservative to increase the heat consumption of the food during the metabolic processes.

\section{No. 8 .}

The calories in the food of No. 8 are very markedly less in the fore period than in the preservative and after periods. The quantity appearing in the feces is correspondingly increased with the increase of the amount in the food. This is not true, however, of the calories in the urine during the preservative period, but is partially true in the after period. In this case there seems to be no notable effect as respects the calories resulting from the administration of the preservative. Considering the percentage figures, however, it is seen that there is an increase in the amount excreted in the feces and a decrease in the amount in the urine, resulting in a very slight total increase in the total percentage excreted in the preservative period. In the after period this slight total increase continues, due to the increase in the amount excreted in the urine, the amount in the feces remaining the same as in the preservative period.

\section{No. 9 .}

The calories in the food of No. 9 are somewhat greater in the fore period than in either the preservative or after period. Notwithstanding this, however, the calories of the feces are greater in the preservative period than in the fore period, and are somewhat greater in the after period than in the fore period. The calories of the urine are practically the same for the three periods.

For reasons given elsewhere (p. 587) the results obtained with this subject are not included in the summaries. They are stated here, however, as a matter of record. 
No. 10.

Owing to illness, No. 10 was not under observation during the last half of the after period.

The calories in the food were greater in the preservative period than in the fore period. Notwithstanding this, however, the calories appearing in the feces were somewhat greater in the fore period than in the preservative period. The calories appearing in the urine were practically the same for the two periods.

For reasons given elsewhere (p. 587) this subject is omitted from the summaries. The results are given here, however, as a matter of record.

\section{No. 11.}

In the case of No. 11 the calories in the food are slightly diminished in the preservative period and still further decreased in the after period. There is also a diminution in the calories in the feces during the same periods, which, as shown by the percentage data, is relatively a larger decrease than the diminution of the calories in the food. It is fair, therefore, to attribute a part of this decrease to the influence of the preservative. There is a slightly smaller number of calories in the urine during the preservative period, corresponding very closely to the diminution of the number in the food, and the same is true of the after period, as is shown by the percentage of elimination. Thus in this case the diminution is somewhat greater than the smaller quantity in the food would account for, and the data show a tendency on the part of the preservative to diminish the calories in the feces and also in the urine, and this tendency is more marked in the urine in the after period.

\section{No. 12.}

In the case of No. 12 there is a rapid diminution in the calories in the food from the fore to the after period. There is a very marked decrease in the calories in the feces in the preservative period, greater than can be accounted for by the diminished calories in the food. In the after period there is a larger quantity of calories in the feces than in the preservative period, although the amount in the food is smaller. The number of ealories excreted in the urine during the three periods remains almost the same. The data show a marked effect of the preservative in diminishing the calories in the feces during the preservative period with practically no effect upon the calories in the urine. Inasmuch, however, as the calories in the food are diminished, there is an increase in the percentage eliminated in the urine in the after period. 
SUMMARY.

In the following table are given the averages by periods for the nine men satisfactorily completing the series:

TABLE XX.-Calories summary, by periods, for nine men, Series VI.

\begin{tabular}{|c|c|c|c|c|c|c|}
\hline Period. & $\begin{array}{l}\text { Calories } \\
\text { in food. }\end{array}$ & $\begin{array}{l}\text { Calories } \\
\text { in feces. }\end{array}$ & $\begin{array}{l}\text { Calories } \\
\text { in urine. }\end{array}$ & $\begin{array}{l}\text { Calories } \\
\text { in feces. }\end{array}$ & $\begin{array}{l}\text { Calories } \\
\text { in urine. }\end{array}$ & Balance. \\
\hline 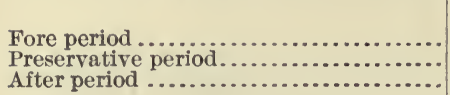 & $\begin{array}{l}2,818 \\
2,819 \\
2,818\end{array}$ & $\begin{array}{r}104 \\
92 \\
95\end{array}$ & $\begin{array}{l}76 \\
76 \\
77\end{array}$ & $\begin{array}{r}\text { Per cent. } \\
3.70 \\
3.24 \\
3.34\end{array}$ & $\begin{array}{r}\text { Per cent. } \\
2.70 \\
2.68 \\
2.69\end{array}$ & $\begin{array}{l}2,638 \\
2,680 \\
2,676\end{array}$ \\
\hline
\end{tabular}

There is seen to be an average increase in the calories of the food of the pressrvative period of 31 , and in the after period of 30 over the quantity in the fore period. Although the number of calories in the food was smallest in the fore period, the number excreted in the feces is the largest, amounting to 104 calories daily. The calories excreted in the preservative period in the feces is 92 daily, and in the after period 95 .

These data show the marked tendency on the part of the preservative to diminish the calories in the feces; in other words, to increase the combustion of the heat-forming constituents of the food during the period of the administration of the preservative. This effect continues to some extent in the after period, although there is a marked tendency shown to return to the condition of the fore period.

The actual quantity of calories in the urine is almost the same for the three periods. When, however, it is remembered that there is a smaller number of calories in the food during the fore period, it is evident that there is very slight tendency on the part of the preservative to decrease the excretion of calories in the urine. This decrease, however, is very slight, and there is a slight increase in the after period. The largest percentage of calories appears in the feces in the fore period and the smallest in the preservative period. The percentage occurring in the urine is almost the same for all the periods, but there is a slightly larger percentage found in the urine of the fore period.

These data show a tendency to increase the combustion of the food in the metabolic process, showing a total greater heat consumption induced by the administration of the preservative. The balances only corroborate in a different form of expression the conclusions already drawn. 
TABLE XXI.-Calories balances for Series VI.

[Averages are per day.]

No. 1.

\begin{tabular}{|c|c|c|c|c|c|c|c|c|c|}
\hline Period. & $\begin{array}{c}1 \\
\text { In food. }\end{array}$ & $\begin{array}{c}2 \\
\text { In } \\
\text { feces. }\end{array}$ & $\begin{array}{c}\mathbf{3} \\
\text { In } \\
\text { urine. }\end{array}$ & $\begin{array}{c}4 \\
\text { In } \\
\text { feces } \\
\text { and } \\
\text { urine. } \\
(2+3)\end{array}$ & $\begin{array}{c}5 \\
\text { In } \\
\text { feces. } \\
(2 \div 1)\end{array}$ & $\begin{array}{c}6 \\
\text { In } \\
\text { urine. } \\
(3 \div 1)\end{array}$ & $\begin{array}{c}7 \\
\text { In } \\
\text { feces } \\
\text { and } \\
\text { urine. } \\
(4 \div 1)\end{array}$ & $\begin{array}{c}8 \\
\text { Balance. } \\
(1-4)\end{array}$ & $\begin{array}{c}9 \\
\text { Sali- } \\
\text { cylic } \\
\text { acid } \\
\text { admin- } \\
\text { istered. }\end{array}$ \\
\hline \multicolumn{10}{|l|}{ Fore period. } \\
\hline 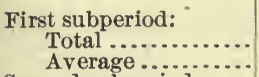 & $\begin{array}{r}\text { Calories. } \\
12,453 \\
2,491\end{array}$ & $\begin{array}{c}\text { Calories } \\
345 \\
69\end{array}$ & $\begin{array}{c}\text { Calories } \\
337 \\
67\end{array}$ & $\begin{array}{c}\text { Calories } \\
682 \\
136\end{array}$ & $\begin{array}{r}\text { Per ct. } \\
2.77 \\
\end{array}$ & $\begin{array}{c}\text { Per ct. } \\
2.71 \\
\end{array}$ & $\begin{array}{r}\text { Per ct. } \\
5.48 \\
\end{array}$ & $\begin{array}{r}\text { Calories. } \\
11,771 \\
2,355\end{array}$ & $\begin{array}{c}\text { Grams. } \\
0 \\
0\end{array}$ \\
\hline 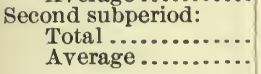 & $\begin{array}{r}11,964 \\
2,393\end{array}$ & $\begin{array}{r}278 \\
56\end{array}$ & $\begin{array}{r}383 \\
77\end{array}$ & $\begin{array}{l}661 \\
132\end{array}$ & $\begin{array}{l}2.32 \\
\text { (..... }\end{array}$ & $\begin{array}{r}3.20 \\
\cdots\end{array}$ & $\begin{array}{c}5.52 \\
\cdots\end{array}$ & $\begin{array}{r}11,303 \\
2,261\end{array}$ & $\begin{array}{l}0 \\
0\end{array}$ \\
\hline $\begin{array}{l}\text { Entire fore period: } \\
\text { Total ................ } \\
\text { Average ......... }\end{array}$ & $\begin{array}{r}24,417 \\
2,442\end{array}$ & $\begin{array}{r}623 \\
62\end{array}$ & $\begin{array}{r}720 \\
72\end{array}$ & $\begin{array}{r}1,343 \\
134\end{array}$ & 2.55 & $\begin{array}{l}2.95 \\
-\ldots\end{array}$ & $\begin{array}{l}5.50 \\
\cdots\end{array}$ & $\begin{array}{r}23,074 \\
2,308\end{array}$ & $\begin{array}{l}0 \\
0\end{array}$ \\
\hline \multicolumn{10}{|l|}{ Preservative period. } \\
\hline $\begin{array}{l}\text { First subperiod: } \\
\text { Total......... } \\
\text { Average..... }\end{array}$ & $\begin{array}{r}12,325 \\
2,465\end{array}$ & $\begin{array}{r}263 \\
53\end{array}$ & $\begin{array}{r}374 \\
75\end{array}$ & $\begin{array}{l}637 \\
127\end{array}$ & $\begin{array}{l}2.13 \\
\ldots \ldots . .\end{array}$ & 3.03 & 5.77 & & \\
\hline Second sul & & & & & & & & & \\
\hline $\begin{array}{l}\text { Total } \\
\text { Avera: }\end{array}$ & 12,110 & 271 & 393 & 664 & 2.24 & 3.25 & 5.49 & 11,446 & 2.10 \\
\hline Third sul & & & 79 & 133 & ........... & …....... & .... & & \\
\hline $\begin{array}{l}\text { Total } \\
\text { Aver }\end{array}$ & $\begin{array}{r}12,218 \\
2,444\end{array}$ & $\begin{array}{r}304 \\
61\end{array}$ & $\begin{array}{r}\text { a } 389 \\
78\end{array}$ & $\begin{array}{l}693 \\
139\end{array}$ & 2.49 & 3.18 & 5.67 & $\begin{array}{r}11,525 \\
2,305\end{array}$ & $\begin{array}{r}3.70 \\
.74\end{array}$ \\
\hline Fourth subperiod: & & & & & & & & & \\
\hline $\begin{array}{l}\text { Tot } \\
\text { Av }\end{array}$ & $\begin{array}{r}12,031 \\
2,406\end{array}$ & $\begin{array}{r}333 \\
67\end{array}$ & $\begin{array}{r}387 \\
77\end{array}$ & $\begin{array}{l}720 \\
144\end{array}$ & 2.77 & 3.22 & 5.98 & $\begin{array}{r}11,311 \\
2,262\end{array}$ & $\begin{array}{l}6.00 \\
1.20\end{array}$ \\
\hline Fifth subpe & & & & & & & & & \\
\hline $\begin{array}{l}\text { Total } \\
\text { Averag }\end{array}$ & 13,047 & 343 & 402 & 745 & 2.63 & 3.08 & 5.71 & 12,302 & $\begin{array}{l}8.0 \\
1.6\end{array}$ \\
\hline Sixth subperi & & 69 & 80 & 149 & & & & & \\
\hline Total ... & $\begin{array}{r}11,859 \\
2,372\end{array}$ & 271 & 418 & 689 & 2.29 & 3.52 & 5.81 & & 10.00 \\
\hline \multirow{2}{*}{\multicolumn{10}{|c|}{$\begin{array}{l}\text { Entire preservative } \\
\text { period: }\end{array}$}} \\
\hline & & & & & & & & & \\
\hline 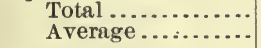 & $\begin{array}{r}73,590 \\
2,453\end{array}$ & $\begin{array}{r}1,785 \\
60\end{array}$ & $\begin{array}{r}2,363 \\
79\end{array}$ & $\begin{array}{r}4,148 \\
138\end{array}$ & 2.43 & 3.21 & 5.64 & $\begin{array}{r}69,442 \\
2,315\end{array}$ & $\begin{array}{r}30.8 \\
1.0\end{array}$ \\
\hline & & & & & & & & & \\
\hline \multicolumn{10}{|l|}{ First subperiod: } \\
\hline Total ......... & 12,016 & 550 & 393 & 943 & 4.58 & 3.27 & 7.85 & 11,073 & 0 \\
\hline $\begin{array}{l}\text { Average } \\
\text { Second subperiod: }\end{array}$ & 2,403 & 110 & 79 & 189 & & ........ & ....... & 2,214 & 0 \\
\hline Total ............... & 12,520 & 411 & 400 & 811 & 3.28 & 3.19 & Second subperiod: & 11 , & 0 \\
\hline Average........ & 2,504 & 82 & 80 & 162 & & & & & 0 \\
\hline 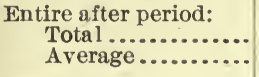 & $\begin{array}{r}24,536 \\
2,454\end{array}$ & $\begin{array}{r}961 \\
96\end{array}$ & $\begin{array}{r}793 \\
79\end{array}$ & $\begin{array}{r}1,754 \\
175\end{array}$ & $\begin{array}{r}3.92 \\
-\ldots .\end{array}$ & 3.23 & 7.15 & $\begin{array}{r}22,782 \\
2,279\end{array}$ & $\begin{array}{l}0 \\
0\end{array}$ \\
\hline
\end{tabular}

$a$ Daily average added in order to complete record. 
TABLE XXI.-Calories balances for Series VI-Continued.

[Averages are per day.]

No. 2.

\begin{tabular}{|c|c|c|c|c|c|c|c|c|c|}
\hline Period. & $\begin{array}{c}1 \\
\text { In food. }\end{array}$ & $\begin{array}{c}2 \\
\text { In } \\
\text { feces. }\end{array}$ & $\begin{array}{c}3 \\
\text { In } \\
\text { urine. }\end{array}$ & $\begin{array}{c}4 \\
\text { In } \\
\text { feces } \\
\text { and } \\
\text { urine. } \\
(2+3)\end{array}$ & $\begin{array}{c}5 \\
\text { In } \\
\text { feces. } \\
(2 \div-1)\end{array}$ & $\begin{array}{c}6 \\
\text { In } \\
\text { urine. } \\
(3 \div 1)\end{array}$ & $\begin{array}{c}7 \\
\text { In } \\
\text { feces } \\
\text { and } \\
\text { urine. } \\
(4 \div 1)\end{array}$ & $\begin{array}{c}8 \\
\begin{array}{c}\text { Balance. } \\
(1-4)\end{array}\end{array}$ & $\begin{array}{c}\mathbf{9} \\
\text { Sali- } \\
\text { cylic } \\
\text { acid } \\
\text { admin- } \\
\text { istered. }\end{array}$ \\
\hline Fore period. & & & & & & & & & \\
\hline $\begin{array}{l}\text { First subperiod: } \\
\quad \text { Total } \ldots \ldots \ldots \\
\text { Average................. }\end{array}$ & $\begin{array}{r}\text { Calories. } \\
15,213 \\
3,013\end{array}$ & $\begin{array}{c}\text { Calories } \\
650 \\
130\end{array}$ & $\begin{array}{c}\text { Calories } \\
\text { a } 481 \\
96\end{array}$ & $\begin{array}{c}\text { Calories } \\
1,131 \\
226\end{array}$ & $\begin{array}{r}\text { Per ct. } \\
4.27 \\
\ldots \ldots \ldots\end{array}$ & $\begin{array}{r}\text { Per ct. } \\
3.16\end{array}$ & $\begin{array}{r}\text { Per ct. } \\
7.43\end{array}$ & $\begin{array}{r}\text { Calories. } \\
14,082 \\
2,817\end{array}$ & $\begin{array}{c}\text { Grams. } \\
0 \\
0\end{array}$ \\
\hline $\begin{array}{l}\text { Second subperiod: } \\
\text { Total } \\
\text { Average............................. }\end{array}$ & $\begin{array}{r}14,933 \\
2,987\end{array}$ & $\begin{array}{l}718 \\
144\end{array}$ & $\begin{array}{r}408 \\
82\end{array}$ & $\begin{array}{r}1,126 \\
225\end{array}$ & $\begin{array}{r}4.81 \\
\cdots \cdots\end{array}$ & 2.73 & 7.54 & $\begin{array}{r}13,807 \\
2,762\end{array}$ & $\begin{array}{l}0 \\
0\end{array}$ \\
\hline $\begin{array}{l}\text { Entire fore period: } \\
\quad \text { Total ................ } \\
\text { Average .............. }\end{array}$ & $\begin{array}{r}30,146 \\
3,015\end{array}$ & $\begin{array}{r}1,368 \\
137\end{array}$ & $\begin{array}{r}889 \\
89\end{array}$ & $\begin{array}{r}2,257 \\
226\end{array}$ & 4.54 & 2.95 & 7.49 & $\begin{array}{r}27,889 \\
2,789\end{array}$ & $\begin{array}{l}0 \\
0\end{array}$ \\
\hline Preservative period. & & & & & & & & & \\
\hline $\begin{array}{l}\text { First subperiod: } \\
\text { Total ............ } \\
\text { Average...... }\end{array}$ & $\begin{array}{r}14,890 \\
2,978\end{array}$ & $\begin{array}{l}520 \\
104\end{array}$ & $\begin{array}{r}405 \\
81\end{array}$ & $\begin{array}{l}925 \\
185\end{array}$ & 3.49 & 2.72 & $\begin{array}{c}6.21 \\
\cdots \cdots\end{array}$ & $\begin{array}{r}13,965 \\
2,793\end{array}$ & $\begin{array}{r}1.05 \\
.21\end{array}$ \\
\hline $\begin{array}{r}\text { Total ............................. } \\
\text { Average }\end{array}$ & $\begin{array}{r}14,803 \\
2,961\end{array}$ & $\begin{array}{l}598 \\
120\end{array}$ & $\begin{array}{r}409 \\
82\end{array}$ & $\begin{array}{r}1,007 \\
201\end{array}$ & 4.04 & 2.76 & .80 & $\begin{array}{r}13,796 \\
2,760\end{array}$ & $\begin{array}{r}2.10 \\
.42\end{array}$ \\
\hline $\begin{array}{l}\text { Third subperiod: } \\
\text { Total ................. } \\
\text { Average....... }\end{array}$ & $\begin{array}{r}15,162 \\
3,032\end{array}$ & $\begin{array}{l}643 \\
129\end{array}$ & $\begin{array}{r}435 \\
87\end{array}$ & $\begin{array}{r}1,078 \\
216\end{array}$ & 4.24 & 2.87 & 7.11 & $\begin{array}{r}14,084 \\
2,816\end{array}$ & $\begin{array}{l}3.70 \\
74\end{array}$ \\
\hline Fourth subperiod: & 0,002 & 120 & & 1058 & 4.33 & 265 & 699 & & .74 \\
\hline $\begin{array}{l}\text { Average } \\
\text { Fifth subperiod.............. }\end{array}$ & $\begin{array}{r}15,142 \\
3,028\end{array}$ & $\begin{array}{l}600 \\
131\end{array}$ & 80 & $\begin{array}{r}1,008 \\
212\end{array}$ & 4.33 & 2.65 & 6.99 & $\begin{array}{r}14,084 \\
2,816\end{array}$ & $\begin{array}{l}6.00 \\
1.20\end{array}$ \\
\hline $\begin{array}{r}\text { Total ................ } \\
\text { Average ...... }\end{array}$ & $\begin{array}{r}15,443 \\
3,089\end{array}$ & $\begin{array}{r}370 \\
74\end{array}$ & $\begin{array}{r}463 \\
93\end{array}$ & $\begin{array}{l}833 \\
167\end{array}$ & 2.40 & 3.00 & 5. 39 & $\begin{array}{r}14,610 \\
2,922\end{array}$ & $\begin{array}{l}8.00 \\
1.60\end{array}$ \\
\hline $\begin{array}{l}\text { Sixth subperiod: } \\
\quad \text { Total } \\
\text { Average .......................... }\end{array}$ & $\begin{array}{r}14,798 \\
2,960\end{array}$ & $\begin{array}{l}635 \\
127\end{array}$ & $\begin{array}{r}433 \\
87\end{array}$ & $\begin{array}{r}1,068 \\
-214\end{array}$ & $\begin{array}{r}4.29 \\
\ldots . . .\end{array}$ & 2.93 & 7.22 & $\begin{array}{r}13,730 \\
2,746\end{array}$ & $\begin{array}{r}10.00 \\
2.00\end{array}$ \\
\hline 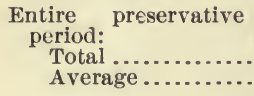 & $\begin{array}{r}90,238 \\
3,008\end{array}$ & $\begin{array}{r}3,422 \\
114\end{array}$ & $\begin{array}{r}2,547 \\
85\end{array}$ & $\begin{array}{r}5,969 \\
199\end{array}$ & 3.79 & 2.82 & 6.61 & $\begin{array}{r}84,269 \\
2,809\end{array}$ & $\begin{array}{r}30.85 \\
1.03\end{array}$ \\
\hline After period. & & & & & & & & & \\
\hline $\begin{array}{l}\text { First subperiod: } \\
\text { Total ............. }\end{array}$ & $\begin{array}{r}14,681 \\
2,936\end{array}$ & $\begin{array}{r}467 \\
93\end{array}$ & $\begin{array}{r}439 \\
88\end{array}$ & $\begin{array}{l}906 \\
181\end{array}$ & 3.18 & 2. 99 & 6.17 & $\begin{array}{r}13,775 \\
2,755\end{array}$ & $\begin{array}{l}0 \\
0\end{array}$ \\
\hline 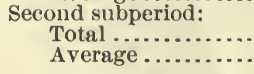 & $\begin{array}{r}15,432 \\
3,086\end{array}$ & $\begin{array}{l}592 \\
118\end{array}$ & $\begin{array}{r}451 \\
90\end{array}$ & $\begin{array}{r}1,043 \\
209\end{array}$ & 3.84 & 2.92 & 6.76 & $\begin{array}{r}14,389 \\
2,877\end{array}$ & $\begin{array}{l}0 \\
0\end{array}$ \\
\hline $\begin{array}{c}\text { Entire after period: } \\
\text { Total } \ldots \ldots \ldots \ldots \ldots . . \\
\text { Average ................ }\end{array}$ & $\begin{array}{r}30,113 \\
3,011\end{array}$ & $\begin{array}{r}1,059 \\
106\end{array}$ & $\begin{array}{r}890 \\
89\end{array}$ & $\begin{array}{r}1,949 \\
195\end{array}$ & 3.52 & 2.96 & 6.47 & $\begin{array}{r}28,164 \\
2,816\end{array}$ & $\begin{array}{l}0 \\
0\end{array}$ \\
\hline
\end{tabular}

$a$ Daily average added in order to complete record. 
Table XXI.-Calories balances for Series VI-Continued.

[Averages are per day.]

No. 3.

\begin{tabular}{|c|c|c|c|c|c|c|c|c|c|}
\hline Period. & $\begin{array}{c}1 \\
\text { In food. }\end{array}$ & $\begin{array}{c}2 \\
\text { In } \\
\text { feces. }\end{array}$ & $\begin{array}{c}3 \\
\text { In } \\
\text { urine. }\end{array}$ & $\begin{array}{c}4 \\
\text { In } \\
\text { feces } \\
\text { and } \\
\text { urine. } \\
(2+3)\end{array}$ & 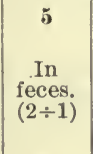 & $\begin{array}{c}\mathbf{6} \\
\underset{\text { In }}{\text { urine. }} \\
(3 \div-1)\end{array}$ & $\begin{array}{c}7 \\
\text { In } \\
\text { feces } \\
\text { and } \\
\text { urine. } \\
(4 \div 1)\end{array}$ & $\begin{array}{c}8 \\
\begin{array}{c}\text { Balance. } \\
(1-4)\end{array}\end{array}$ & $\begin{array}{l}9 \\
\text { Sali- } \\
\text { cylic } \\
\text { acid } \\
\text { admin- } \\
\text { istered. }\end{array}$ \\
\hline $\begin{array}{l}\text { Fore period. } \\
\text { First subperiod: } \\
\text { Total .............. } \\
\text { Average....... }\end{array}$ & Calories. & |Calories & Calories & $\begin{array}{r}\text { Calories } \\
\text { Brok }\end{array}$ & $\begin{array}{l}\text { Per ct. } \\
\text { ken by il }\end{array}$ & $\begin{array}{l}\text { Per ct. } \\
\text { llness. }\end{array}$ & Per ct. & Calories. & Grams. \\
\hline 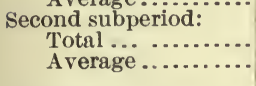 & $\begin{array}{r}13,068 \\
2,614\end{array}$ & $\begin{array}{r}388 \\
78\end{array}$ & $\begin{array}{r}356 \\
71\end{array}$ & $\begin{array}{l}744 \\
149\end{array}$ & $\begin{array}{r}2.97 \\
\ldots \ldots . .\end{array}$ & $\begin{array}{c}2.72 \\
\cdots \cdots\end{array}$ & 5.69 & $\begin{array}{r}12,324 \\
2,465\end{array}$ & $\begin{array}{l}0 \\
0\end{array}$ \\
\hline $\begin{array}{l}\text { Entire fore period: } \\
\quad \text { Total .................... } \\
\text { Average ............ }\end{array}$ & $\begin{array}{r}13,068 \\
2,614\end{array}$ & $\begin{array}{r}388 \\
78\end{array}$ & $\begin{array}{r}356 \\
71\end{array}$ & $\begin{array}{l}744 \\
149\end{array}$ & 2.97 & 2.72 & 5.69 & $\begin{array}{r}12,324 \\
2,465\end{array}$ & $\begin{array}{l}0 \\
0\end{array}$ \\
\hline Preservative period. & & & & & & & & & \\
\hline $\begin{array}{l}\text { First subperiod: } \\
\text { Total ............ } \\
\text { Average...... } \\
\text { Second subperiod: }\end{array}$ & $\begin{array}{r}13,580 \\
2,716\end{array}$ & $\begin{array}{r}316 \\
63\end{array}$ & $\begin{array}{r}317 \\
63\end{array}$ & $\begin{array}{l}633 \\
127\end{array}$ & 2.33 & 2.33 & 4.66 & $\begin{array}{r}12,947 \\
2,589\end{array}$ & $\begin{array}{r}1.05 \\
.21\end{array}$ \\
\hline 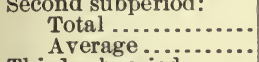 & $\begin{array}{r}13,797 \\
2,759\end{array}$ & $\begin{array}{l}635 \\
127\end{array}$ & $\begin{array}{r}a 324 \\
65\end{array}$ & $\begin{array}{l}959 \\
192\end{array}$ & 4.60 & 2.35 & $6.9 \overline{0}$ & $\begin{array}{r}12,838 \\
2,567\end{array}$ & $\begin{array}{r}2.10 \\
.42\end{array}$ \\
\hline 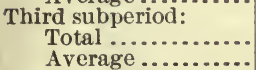 & $\begin{array}{r}13,860 \\
2,772\end{array}$ & $\begin{array}{r}304 \\
61\end{array}$ & $\begin{array}{r}308 \\
62\end{array}$ & $\begin{array}{l}612 \\
122\end{array}$ & 2.19 & 2.22 & 4.42 & $\begin{array}{r}13,248 \\
2,650\end{array}$ & $\begin{array}{l}4.00 \\
.80\end{array}$ \\
\hline $\begin{array}{l}\text { Fourth subperiod: } \\
\text { Total } \\
\text { Average }\end{array}$ & $\begin{array}{r}14,603 \\
2,921\end{array}$ & $\begin{array}{l}01 \\
571 \\
114\end{array}$ & $\begin{array}{r}325 \\
65\end{array}$ & $\begin{array}{l}896 \\
179\end{array}$ & 3.91 & 2.23 & 6.14 & $\begin{array}{r}13,707 \\
2,742\end{array}$ & $\begin{array}{l}6.00 \\
1.20\end{array}$ \\
\hline $\begin{array}{r}\text { Fifth subperiod: } \\
\text { Total ................ } \\
\text { Average................ }\end{array}$ & $\begin{array}{r}2,921 \\
14,711 \\
2,942\end{array}$ & $\begin{array}{r}114 \\
326 \\
65\end{array}$ & $\begin{array}{r}00 \\
313 \\
63\end{array}$ & $\begin{array}{l}639 \\
128\end{array}$ & 2.22 & 2.13 & 4.34 & $\begin{array}{r}14,072 \\
2,814\end{array}$ & $\begin{array}{l}3.00 \\
1.60\end{array}$ \\
\hline $\begin{array}{l}\text { Five preservative sub- } \\
\text { periods: } \\
\text { Total } . . . . . . . . . . . . . \\
\text { Average ............ }\end{array}$ & $\begin{array}{r}b 70,551 \\
2,822 \\
\end{array}$ & $\begin{array}{r}2,152 \\
86\end{array}$ & $\begin{array}{r}1,587 \\
63\end{array}$ & $\begin{array}{r}3,739 \\
\quad 150 \\
\end{array}$ & 3.05 & $\begin{array}{r}2.25 \\
\ldots \ldots . \\
\end{array}$ & 5.30 & $\begin{array}{r}66,812 \\
2,672 \\
\end{array}$ & $\begin{array}{r}21.15 \\
.85\end{array}$ \\
\hline After period. & & & & & & & & & \\
\hline $\begin{array}{l}\text { First subperiod: } \\
\text { Total ................ } \\
\text { Average...................... }\end{array}$ & $\begin{array}{r}14,397 \\
2,879\end{array}$ & & $\begin{array}{r}302 \\
60\end{array}$ & & & 2.10 & & & $\begin{array}{l}0 \\
0\end{array}$ \\
\hline 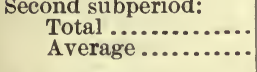 & $\begin{array}{r}14,964 \\
2,993\end{array}$ & $\begin{array}{r}399 \\
80\end{array}$ & $\begin{array}{r}311 \\
62\end{array}$ & $\begin{array}{l}710 \\
142\end{array}$ & $\begin{array}{r}2.67 \\
\ldots \ldots \ldots \\
\end{array}$ & 2.08 & 4.74 & $\begin{array}{r}14,254 \\
2,851\end{array}$ & $\begin{array}{l}0 \\
0\end{array}$ \\
\hline 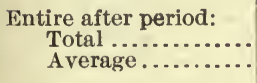 & & & & & & & & & $\begin{array}{l}0 \\
0\end{array}$ \\
\hline
\end{tabular}

$a$ Daily average added in order to complete record.

$b$ No. 3 had only five preservative subperiods. 
TABLE XXI.-Calories balances for Series VI-Continued.

[Averages are per day.]

No. 4.

\begin{tabular}{|c|c|c|c|c|c|c|c|c|c|}
\hline Period. & In food. & $\begin{array}{c}2 \\
\text { In } \\
\text { feces. }\end{array}$ & $\underset{\text { In }}{\mathbf{3}}$ & $\begin{array}{l}4 \\
\text { In } \\
\text { feces } \\
\text { and } \\
\text { urine. } \\
(2+3)\end{array}$ & $\begin{array}{c}5 \\
\text { In } \\
\text { feces. } \\
(2 \div 1)\end{array}$ & $\begin{array}{c}\mathbf{6} \\
\text { In } \\
\text { urine. } \\
(3 \div 1)\end{array}$ & $\begin{array}{c}7 \\
\text { In } \\
\text { feces } \\
\text { and } \\
\text { urine. } \\
(4 \div 1)\end{array}$ & $\begin{array}{c}8 \\
\underset{(1-4)}{\text { Balance. }}\end{array}$ & $\begin{array}{l}9 \\
\text { Sali- } \\
\text { cylic } \\
\text { acid } \\
\text { admin- } \\
\text { istered. }\end{array}$ \\
\hline Fore period. & & & & & & & & & \\
\hline 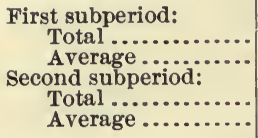 & $\begin{array}{r}\text { Calories. } \\
13,890 \\
2,778 \\
13,802 \\
2,760\end{array}$ & \begin{tabular}{|c|} 
Calories \\
455 \\
91
\end{tabular} & \begin{tabular}{|} 
Calories \\
416 \\
83
\end{tabular} & $\begin{array}{c}\text { Calories } \\
871 \\
174\end{array}$ & $\begin{array}{r}\text { Per ct. } \\
3.28 \\
\cdots \\
3.53 \\
\cdots\end{array}$ & $\begin{array}{r}\text { Per ct. } \\
2.99 \\
2.94 \\
\ldots\end{array}$ & $\begin{array}{r}\text { Per ct. } \\
6.27 \\
\ldots \\
6.47 \\
\ldots\end{array}$ & $\begin{array}{r}\text { Calories. } \\
13,019 \\
2,604 \\
12,909 \\
2,581\end{array}$ & $\begin{array}{c}\text { Grams. } \\
0 \\
0 \\
0 \\
0\end{array}$ \\
\hline $\begin{array}{l}\text { Entire fore period: } \\
\text { Total } \ldots . . . \ldots \ldots \ldots \\
\text { Average } \ldots \ldots \ldots \ldots \\
\end{array}$ & $\begin{array}{r}27,692 \\
2,769\end{array}$ & $\begin{array}{r}942 \\
94\end{array}$ & $\begin{array}{r}822 \\
82\end{array}$ & $\begin{array}{r}1,764 \\
176\end{array}$ & $\begin{array}{r}3.40 \\
\cdots \cdots \\
\end{array}$ & $\begin{array}{r}2.97 \\
\cdots . . . \\
\end{array}$ & $\begin{array}{c}6.37 \\
\cdots \cdots\end{array}$ & $\begin{array}{r}25,928 \\
2,593 \\
\end{array}$ & $\begin{array}{l}0 \\
0\end{array}$ \\
\hline Preservative period. & & & & & & & & & \\
\hline $\begin{array}{l}\text { First subperiod: } \\
\text { Total ........................ } \\
\text { Average ........... } \\
\text { Second subperiod: }\end{array}$ & $\begin{array}{r}14,162 \\
2,832\end{array}$ & $\begin{array}{r}381 \\
76\end{array}$ & $\begin{array}{r}395 \\
79\end{array}$ & $\begin{array}{l}776 \\
155\end{array}$ & 2.69 & $\begin{array}{r}2.79 \\
\cdots \cdots\end{array}$ & $\begin{array}{l}5.48 \\
\cdots \cdots\end{array}$ & $\begin{array}{r}13,386 \\
2,677\end{array}$ & $\begin{array}{r}1.05 \\
.21\end{array}$ \\
\hline $\begin{array}{l}\text { Total } \\
\text { Average } \\
\text { Third subperiod: }\end{array}$ & $\begin{array}{r}13,9 \overline{7} 7 \\
2,791\end{array}$ & $\begin{array}{r}463 \\
93\end{array}$ & $\begin{array}{r}421 \\
84\end{array}$ & $\begin{array}{l}884 \\
177\end{array}$ & $\begin{array}{r}3.32 \\
\cdots \cdots\end{array}$ & 3.02 & 6.33 & $\begin{array}{r}13,073 \\
2,614\end{array}$ & $\begin{array}{r}2.10 \\
.42\end{array}$ \\
\hline 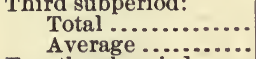 & $\begin{array}{r}14,286 \\
2,857\end{array}$ & $\begin{array}{r}442 \\
88\end{array}$ & $\begin{array}{r}386 \\
77\end{array}$ & $\begin{array}{l}828 \\
166\end{array}$ & 3.09 & $\begin{array}{l}2.70 \\
\cdots . .\end{array}$ & 5.80 & $\begin{array}{r}13,458 \\
2,691\end{array}$ & $\begin{array}{r}3.70 \\
.74\end{array}$ \\
\hline 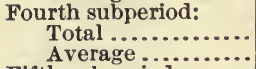 & $\begin{array}{r}14,056 \\
2,811\end{array}$ & $\begin{array}{r}a 400 \\
80\end{array}$ & $\begin{array}{r}406 \\
81\end{array}$ & $\begin{array}{l}806 \\
161\end{array}$ & 2.84 & 2.89 & 5. 73 & $\begin{array}{r}13,250 \\
2,650\end{array}$ & $\begin{array}{l}6.00 \\
1.20\end{array}$ \\
\hline $\begin{array}{l}\text { Fifth subperiod: } \\
\text { Total ................. } \\
\text { Average ......... }\end{array}$ & $\begin{array}{r}14,518 \\
2,904\end{array}$ & $\begin{array}{l}570 \\
114\end{array}$ & $\begin{array}{r}373 \\
75\end{array}$ & $\begin{array}{l}943 \\
189\end{array}$ & 3.93 & 2.57 & 6.50 & $\begin{array}{r}13,575 \\
2,715\end{array}$ & $\begin{array}{l}8.00 \\
1.60\end{array}$ \\
\hline 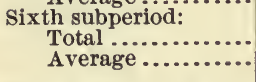 & $\begin{array}{r}14,018 \\
2,804\end{array}$ & $\begin{array}{r}a 461 \\
92\end{array}$ & $\begin{array}{r}406 \\
81\end{array}$ & $\begin{array}{l}867 \\
173\end{array}$ & $\begin{array}{r}3.29 \\
\cdots \cdots\end{array}$ & $\begin{array}{r}2.90 \\
\cdots\end{array}$ & $\begin{array}{r}6.18 \\
\cdots \cdots\end{array}$ & $\begin{array}{r}13,151 \\
2,631\end{array}$ & $\begin{array}{r}10.00 \\
2.00\end{array}$ \\
\hline 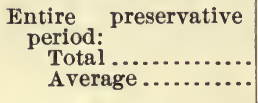 & $\begin{array}{r}84,997 \\
2,833 \\
\end{array}$ & $\begin{array}{r}2,717 \\
91\end{array}$ & $\begin{array}{r}2,387 \\
80\end{array}$ & $\begin{array}{r}5,104 \\
170\end{array}$ & $\begin{array}{r}3.20 \\
\cdots \cdots \\
\end{array}$ & $\begin{array}{r}2.81 \\
\cdots\end{array}$ & $\begin{array}{r}6.00 \\
\cdots \cdots \cdots \\
\end{array}$ & $\begin{array}{r}79,893 \\
2,663\end{array}$ & $\begin{array}{r}30.85 \\
1.03\end{array}$ \\
\hline After period. & & & & & & & & & \\
\hline $\begin{array}{l}\text { First subperiod: } \\
\quad \text { Total } \ldots . . . \ldots \ldots \ldots \\
\text { A verage } \ldots \ldots \ldots \ldots\end{array}$ & $\begin{array}{r}14,020 \\
2,804\end{array}$ & $\begin{array}{r}362 \\
72\end{array}$ & $\begin{array}{r}406 \\
81\end{array}$ & $\begin{array}{l}768 \\
154\end{array}$ & $\begin{array}{l}2.58 \\
\cdots\end{array}$ & $\begin{array}{r}2.90 \\
\ldots . . .\end{array}$ & $\begin{array}{r}5.48 \\
\ldots \ldots\end{array}$ & $\begin{array}{r}13,252 \\
2,650\end{array}$ & $\begin{array}{l}0 \\
0\end{array}$ \\
\hline $\begin{array}{l}\text { Second subperiod: } \\
\text { Total ................ } \\
\text { Average........... }\end{array}$ & $\begin{array}{r}15,023 \\
3,005\end{array}$ & $\begin{array}{r}487 \\
97\end{array}$ & $\begin{array}{r}420 \\
84\end{array}$ & $\begin{array}{l}907 \\
181\end{array}$ & $\begin{array}{r}3.24 \\
\cdots \cdots \\
\end{array}$ & $\begin{array}{r}2.80 \\
\cdots \cdots \\
\end{array}$ & $\begin{array}{r}6.04 \\
\cdots \\
\cdots\end{array}$ & $\begin{array}{r}14,116 \\
2,824\end{array}$ & $\begin{array}{l}0 \\
0\end{array}$ \\
\hline $\begin{array}{l}\text { Entire after period: } \\
\text { Total ............. } \\
\text { Average ......... }\end{array}$ & $\begin{array}{r}29,043 \\
2,904\end{array}$ & $\begin{array}{r}849 \\
85\end{array}$ & $\begin{array}{r}826 \\
83\end{array}$ & $\begin{array}{r}1,675 \\
168\end{array}$ & 2.92 & 2.84 & $\begin{array}{c}5.77 \\
\ldots \ldots .\end{array}$ & $\begin{array}{r}27,368 \\
2,736\end{array}$ & $\begin{array}{l}0 \\
0\end{array}$ \\
\hline
\end{tabular}

$a$ Daily average added in order to complete record. 
SALICYLIC ACID AND SALICYLATES.

TABLe XXI.-Calories balances for Series VI-Continued.

[Averages are per day.]

No. 5 .

\begin{tabular}{|c|c|c|c|c|c|c|c|c|c|}
\hline Period. & $\begin{array}{c}1 \\
\text { In food. }\end{array}$ & $\begin{array}{l}2 \\
\text { In } \\
\text { feces. }\end{array}$ & $\begin{array}{c}3 \\
\text { In } \\
\text { urine. }\end{array}$ & $\begin{array}{c}t \\
\text { In } \\
\text { feces } \\
\text { and } \\
\text { urine. } \\
(2+3)\end{array}$ & $\begin{array}{c}5 \\
\text { In } \\
\text { feces. } \\
(2 \div 1)\end{array}$ & $\begin{array}{c}6 \\
\text { In } \\
\text { urine. } \\
(3 \div 1)\end{array}$ & $\begin{array}{c}7 \\
\text { In } \\
\text { feces } \\
\text { and } \\
\text { urine. } \\
(4 \div-1)\end{array}$ & $\begin{array}{c}8 \\
\substack{\text { Balanec. } \\
(1-4)}\end{array}$ & $\begin{array}{c}9 \\
\text { Sali- } \\
\text { cylic } \\
\text { acid } \\
\text { admin- } \\
\text { istered. }\end{array}$ \\
\hline Fore period. & & & & & & & & & \\
\hline $\begin{array}{l}\text { First subperiod: } \\
\text { Total ................. } \\
\text { Average ............ }\end{array}$ & $\begin{array}{r}\text { Calories. } \\
14,467 \\
2,893\end{array}$ & $\begin{array}{c}\text { Calories } \\
611 \\
122\end{array}$ & $\begin{array}{c}\text { Calories } \\
392 \\
78\end{array}$ & $\begin{array}{c}\text { Calories } \\
1,003 \\
201\end{array}$ & $\begin{array}{r}\text { Per ct. } \\
4.22 \\
\ldots \ldots \ldots . .\end{array}$ & $\begin{array}{r}\text { Per ct. } \\
2.71\end{array}$ & $\begin{array}{r}\text { Per ct. } \\
6.93 \\
\ldots \ldots\end{array}$ & $\begin{array}{r}\text { Calories. } \\
13,464 \\
2,692\end{array}$ & $\begin{array}{c}\text { Grams. } \\
0 \\
0\end{array}$ \\
\hline 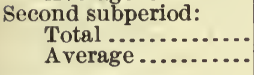 & $\begin{array}{r}14,191 \\
2,838\end{array}$ & $\begin{array}{l}550 \\
110\end{array}$ & $\begin{array}{r}349 \\
70\end{array}$ & $\begin{array}{l}899 \\
180\end{array}$ & $\begin{array}{r}3.88 \\
\ldots .\end{array}$ & 2.46 & 6.34 & $\begin{array}{r}13,292 \\
2,658\end{array}$ & $\begin{array}{l}0 \\
0\end{array}$ \\
\hline $\begin{array}{l}\text { Entire fore period: } \\
\quad \text { Total ................ } \\
\text { Average ........... }\end{array}$ & $\begin{array}{r}28,658 \\
2,866\end{array}$ & $\begin{array}{r}1,161 \\
116\end{array}$ & $\begin{array}{r}741 \\
74\end{array}$ & $\begin{array}{r}1,902 \\
190 \\
\end{array}$ & 4.06 & 2.59 & 6.64 & $\begin{array}{r}26,756 \\
2,676\end{array}$ & $\begin{array}{l}0 \\
0\end{array}$ \\
\hline Preservative period. & & & & & & & & & \\
\hline 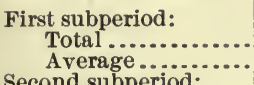 & $\begin{array}{r}14,652 \\
2,930\end{array}$ & $\begin{array}{l}579 \\
116\end{array}$ & $\begin{array}{r}386 \\
77\end{array}$ & $\begin{array}{l}965 \\
193\end{array}$ & 3.95 & 2.63 & 6.59 & $\begin{array}{r}13,687 \\
2,737\end{array}$ & $\begin{array}{r}1.05 \\
.21\end{array}$ \\
\hline 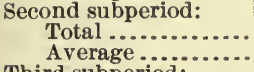 & $\begin{array}{r}14,041 \\
2,808\end{array}$ & $\begin{array}{r}408 \\
82\end{array}$ & $\begin{array}{r}389 \\
78\end{array}$ & $\begin{array}{l}797 \\
159\end{array}$ & 2.91 & 2.77 & 5.68 & $\begin{array}{r}13,244 \\
2,649\end{array}$ & $\begin{array}{r}2.10 \\
.42\end{array}$ \\
\hline 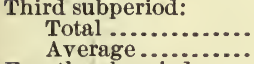 & $\begin{array}{r}14,315 \\
2,863\end{array}$ & $\begin{array}{l}526 \\
105\end{array}$ & $\begin{array}{r}383 \\
77\end{array}$ & $\begin{array}{l}909 \\
182\end{array}$ & 3.67 & 2.68 & 6.35 & $\begin{array}{r}13,406 \\
2,681\end{array}$ & $\begin{array}{r}3.70 \\
.74\end{array}$ \\
\hline $\begin{array}{l}\text { Fourth subperiod: } \\
\quad \text { Total } \ldots . . . . . . . . .\end{array}$ & $\begin{array}{r}14,267 \\
2,853\end{array}$ & $\begin{array}{r}388 \\
78\end{array}$ & $\begin{array}{r}391 \\
78\end{array}$ & $\begin{array}{l}779 \\
156\end{array}$ & 2.72 & 2.74 & 5.46 & $\begin{array}{r}13,488 \\
2,697\end{array}$ & $\begin{array}{l}6.00 \\
1.20\end{array}$ \\
\hline $\begin{array}{l}\text { Fifth subperiod: } \\
\text { Total } \\
\text { Avera................... }\end{array}$ & $\begin{array}{r}14,536 \\
2,907\end{array}$ & $\begin{array}{l}509 \\
102\end{array}$ & $\begin{array}{r}411 \\
82\end{array}$ & $\begin{array}{l}920 \\
184\end{array}$ & 3.50 & 2.83 & 6.33 & $\begin{array}{r}13,616 \\
2,723\end{array}$ & $\begin{array}{l}8.00 \\
1.60\end{array}$ \\
\hline $\begin{array}{l}\text { Sixth subperiod: } \\
\quad \text { Total } \\
\text { Average............................ }\end{array}$ & $\begin{array}{r}14,169 \\
2,834\end{array}$ & $\begin{array}{l}516 \\
103\end{array}$ & $\begin{array}{r}387 \\
77\end{array}$ & $\begin{array}{l}903 \\
181\end{array}$ & 3.64 & 2.73 & 6.37 & $\begin{array}{r}13,266 \\
2,653\end{array}$ & $\begin{array}{r}10.00 \\
2.00\end{array}$ \\
\hline $\begin{array}{l}\text { Entire preservative } \\
\text { peiiod: } \\
\text { Total } \\
\text { Average } \\
\end{array}$ & $\begin{array}{r}85,980 \\
2,866\end{array}$ & $\begin{array}{r}2,926 \\
98 \\
\end{array}$ & $\begin{array}{r}2,347 \\
78\end{array}$ & $\begin{array}{r}5,273 \\
176\end{array}$ & 3.40 & 2.73 & 6.13 & $\begin{array}{r}80,707 \\
2,690\end{array}$ & $\begin{array}{r}30.85 \\
1.03\end{array}$ \\
\hline After period. & & & & & & & & & \\
\hline $\begin{array}{l}\text { First subperiod: } \\
\text { Total } \ldots \ldots \ldots \ldots . . \\
\text { Average }\end{array}$ & $\begin{array}{r}14,141 \\
2,828\end{array}$ & $\begin{array}{r}491 \\
98\end{array}$ & $\begin{array}{r}a 391 \\
78\end{array}$ & $\begin{array}{l}882 \\
176\end{array}$ & 3.47 & 2.77 & 6.24 & $\begin{array}{r}13,259 \\
2,652\end{array}$ & $\begin{array}{l}0 \\
0\end{array}$ \\
\hline $\begin{array}{l}\text { Second subperiod: } \\
\text { Total } \\
\text { Average........................... }\end{array}$ & $\begin{array}{r}14,437 \\
2,887\end{array}$ & $\begin{array}{r}359 \\
72\end{array}$ & $\begin{array}{r}399 \\
80\end{array}$ & $\begin{array}{l}758 \\
152\end{array}$ & 2.49 & 2.76 & 5.25 & $\begin{array}{r}13,679 \\
2,735\end{array}$ & $\begin{array}{l}0 \\
0\end{array}$ \\
\hline $\begin{array}{l}\text { Entire after period: } \\
\text { Total ............. } \\
\text { Average ......... }\end{array}$ & $\begin{array}{r}28,578 \\
2,858\end{array}$ & $\begin{array}{r}850 \\
85\end{array}$ & $\begin{array}{r}790 \\
79\end{array}$ & $\begin{array}{r}1,640 \\
164\end{array}$ & 2.97 & 2.76 & 5.74 & $\begin{array}{r}26,938 \\
2,694\end{array}$ & $\begin{array}{l}0 \\
0\end{array}$ \\
\hline
\end{tabular}

$a$ Daily average added in order to complete record. 
TABle XXI.-Calories balances for Series VI-Continued.

[Averages are per day.]

No. 6.

\begin{tabular}{|c|c|c|c|c|c|c|c|c|c|}
\hline Period. & In food. & $\begin{array}{c}2 \\
\text { In } \\
\text { feces. }\end{array}$ & $\underset{\text { urine. }}{3}$ & $\begin{array}{c}4 \\
\text { In } \\
\text { feces } \\
\text { and } \\
\text { urine. } \\
(2+3)\end{array}$ & $\begin{array}{c}5 \\
\text { In } \\
\text { feces. } \\
(2 \div 1)\end{array}$ & $\underset{\substack{\text { In } \\
(3 \div 1)}}{6}$ & $\begin{array}{c}7 \\
\text { In } \\
\text { feces } \\
\text { and } \\
\text { urine. } \\
(4 \div 1)\end{array}$ & $\begin{array}{c}\text { Balance. } \\
(1-4)\end{array}$ & $\begin{array}{c}9 \\
\text { Sali- } \\
\text { cylic } \\
\text { acid } \\
\text { admin- } \\
\text { istered. }\end{array}$ \\
\hline \multicolumn{10}{|l|}{ Fore period. } \\
\hline $\begin{array}{l}\text { First subperiod: } \\
\text { Total ................ } \\
\text { Average .......... }\end{array}$ & $\begin{array}{r}\text { Calories. } \\
13,700 \\
2,740\end{array}$ & \begin{tabular}{|c|} 
Calories \\
574 \\
115
\end{tabular} & $\begin{array}{c}\text { Calories } \\
335 \\
67\end{array}$ & $\begin{array}{c}\text { Calories } \\
909 \\
182\end{array}$ & $\begin{array}{r}\text { Per ct. } \\
4.19 \\
\ldots\end{array}$ & $\begin{array}{l}\text { Per ct. } \\
2.45 \\
\end{array}$ & $\begin{array}{r}\text { Per } c t . \\
6.64 \\
\end{array}$ & $\begin{array}{r}\text { Calories. } \\
12,791 \\
2,558\end{array}$ & $\begin{array}{c}\text { Grams. } \\
0 \\
0\end{array}$ \\
\hline $\begin{array}{l}\text { Second subperiod: } \\
\text { Total .................... } \\
\text { Average ......... }\end{array}$ & $\begin{array}{r}14,017 \\
2,803\end{array}$ & $\begin{array}{l}666 \\
133\end{array}$ & $\begin{array}{r}325 \\
65\end{array}$ & $\begin{array}{l}991 \\
198\end{array}$ & $\begin{array}{r}4.75 \\
\ldots . . .\end{array}$ & $\begin{array}{r}2.32 \\
\ldots . . .\end{array}$ & $\begin{array}{r}7.07 \\
\ldots . . .\end{array}$ & $\begin{array}{r}13,026 \\
2,605\end{array}$ & $\begin{array}{l}0 \\
0\end{array}$ \\
\hline $\begin{array}{l}\text { Entire fore period: } \\
\text { Total ................. } \\
\text { Average .......... }\end{array}$ & $\begin{array}{r}27,717 \\
2,772 \\
\end{array}$ & $\begin{array}{r}1,240 \\
124\end{array}$ & $\begin{array}{r}660 \\
66\end{array}$ & $\begin{array}{r}1,900 \\
190\end{array}$ & $\begin{array}{r}4.47 \\
\ldots . . .\end{array}$ & $\begin{array}{r}2.38 \\
\ldots . . .\end{array}$ & $\begin{array}{r}6.86 \\
\ldots . . . \cdots\end{array}$ & $\begin{array}{r}25,817 \\
2,582\end{array}$ & $\begin{array}{l}0 \\
0\end{array}$ \\
\hline \multicolumn{10}{|l|}{ Preservative period. } \\
\hline 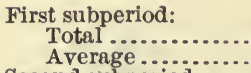 & $\begin{array}{r}13,572 \\
2,714\end{array}$ & $\begin{array}{l}532 \\
106\end{array}$ & $\begin{array}{r}372 \\
74\end{array}$ & $\begin{array}{l}904 \\
181\end{array}$ & 3.92 & 2.74 & 6. 66 & $\begin{array}{r}12,668 \\
2,533\end{array}$ & $\begin{array}{r}1.05 \\
.21\end{array}$ \\
\hline 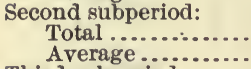 & $\begin{array}{r}13,806 \\
2,761\end{array}$ & $\begin{array}{l}637 \\
127\end{array}$ & $\begin{array}{r}386 \\
77\end{array}$ & $\begin{array}{r}1,023 \\
205\end{array}$ & 4.61 & 2.80 & $\begin{array}{c}7.41 \\
\cdots . . .\end{array}$ & $\begin{array}{r}12,783 \\
2,556\end{array}$ & $\begin{array}{r}2.10 \\
.42\end{array}$ \\
\hline $\begin{array}{l}\text { Third subperiod: } \\
\text { Total .................. } \\
\text { Average ......... }\end{array}$ & $\begin{array}{r}14,131 \\
2,826\end{array}$ & $\begin{array}{l}693 \\
139\end{array}$ & $\begin{array}{r}\alpha 399 \\
80\end{array}$ & $\begin{array}{r}1,092 \\
218\end{array}$ & 4.90 & 2.82 & 7.73 & $\begin{array}{r}13,039 \\
2,608\end{array}$ & $\begin{array}{r}3.70 \\
.74\end{array}$ \\
\hline Fourth subperiod: & 2,020 & & & & & & & & \\
\hline 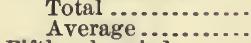 & $\begin{array}{r}14,197 \\
2,839\end{array}$ & $\begin{array}{l}564 \\
113\end{array}$ & $\begin{array}{r}a 414 \\
83\end{array}$ & $\begin{array}{l}978 \\
196\end{array}$ & $\begin{array}{r}3.97 \\
\ldots . . .\end{array}$ & $\begin{array}{r}2.92 \\
\ldots .\end{array}$ & $\begin{array}{r}6.89 \\
\ldots \ldots . .\end{array}$ & $\begin{array}{r}13,219 \\
2,643\end{array}$ & $\begin{array}{l}6.00 \\
1.20\end{array}$ \\
\hline $\begin{array}{l}\text { Fifth subperiod: } \\
\text { Total } \\
\text { Average }\end{array}$ & $\begin{array}{r}14,614 \\
2,923\end{array}$ & $\begin{array}{l}643 \\
129\end{array}$ & $\begin{array}{r}419 \\
84\end{array}$ & $\begin{array}{r}1,062 \\
212\end{array}$ & $\begin{array}{c}4.40 \\
\ldots . .\end{array}$ & 2.87 & $\begin{array}{c}7.27 \\
\ldots .\end{array}$ & $\begin{array}{r}13,552 \\
2,711\end{array}$ & $\begin{array}{l}8.00 \\
1.60\end{array}$ \\
\hline 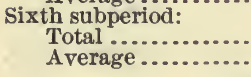 & $\begin{array}{r}14,707 \\
2,941\end{array}$ & $\begin{array}{l}633 \\
127\end{array}$ & $\begin{array}{r}301 \\
60\end{array}$ & $\begin{array}{l}934 \\
187\end{array}$ & $\begin{array}{r}4.30 \\
\ldots . . .\end{array}$ & $\begin{array}{r}2.05 \\
\ldots .2 .\end{array}$ & $\begin{array}{r}6.35 \\
\ldots . . .\end{array}$ & $\begin{array}{r}13,773 \\
2,754\end{array}$ & $\begin{array}{l}8.00 \\
1.60\end{array}$ \\
\hline $\begin{array}{l}\text { Entire preservative } \\
\text { period: } \\
\text { Total ................. } \\
\text { Average ......... }\end{array}$ & $\begin{array}{r}85,027 \\
2,834\end{array}$ & $\begin{array}{r}3,702 \\
123\end{array}$ & $\begin{array}{r}2,291 \\
76\end{array}$ & $\begin{array}{r}5,993 \\
200\end{array}$ & $\begin{array}{r}4.35 \\
\ldots . . .\end{array}$ & 2. 69 & $\begin{array}{r}7.05 \\
\ldots \ldots \ldots \\
\end{array}$ & $\begin{array}{r}79,034 \\
2,634\end{array}$ & $\begin{array}{r}28.85 \\
.96 \\
\end{array}$ \\
\hline \multicolumn{10}{|l|}{ After period. } \\
\hline $\begin{array}{l}\text { First subperiod: } \\
\quad \text { Total ................ } \\
\text { A verage ........... }\end{array}$ & $\begin{array}{r}14,040 \\
2,808\end{array}$ & $\begin{array}{l}698 \\
140\end{array}$ & $\begin{array}{r}373 \\
75\end{array}$ & $\begin{array}{r}1,071 \\
214\end{array}$ & 4.97 & 2.69 & 7.63 & $\begin{array}{r}12,969 \\
2,594\end{array}$ & $\begin{array}{l}0 \\
0\end{array}$ \\
\hline $\begin{array}{l}\text { Second subperiod: } \\
\text { Total ...................... } \\
\text { Average .......... }\end{array}$ & $\begin{array}{r}14,366 \\
2,873\end{array}$ & $\begin{array}{r}a 456 \\
91\end{array}$ & $\begin{array}{r}310 \\
62\end{array}$ & $\begin{array}{l}766 \\
153\end{array}$ & $\begin{array}{r}3.17 \\
\cdots \cdots \cdots\end{array}$ & $\begin{array}{r}2.16 \\
\therefore .2 .\end{array}$ & $\begin{array}{r}5.33 \\
\cdots \cdots \\
\end{array}$ & $\begin{array}{r}13,600 \\
2,720\end{array}$ & $\begin{array}{l}0 \\
0\end{array}$ \\
\hline $\begin{array}{l}\text { Entire after period: } \\
\text { Total ................... } \\
\text { Average ......... }\end{array}$ & $\begin{array}{r}28,406 \\
2,841 .\end{array}$ & $\begin{array}{r}1,154 \\
115\end{array}$ & $\begin{array}{r}683 \\
68\end{array}$ & $\begin{array}{r}1,837 \\
184\end{array}$ & $\begin{array}{r}4.06 \\
\cdots . . . . .\end{array}$ & 2.40 & $\begin{array}{c}6.47 \\
\ldots \ldots . .\end{array}$ & $\begin{array}{r}26,569 \\
2,657\end{array}$ & $\begin{array}{l}0 \\
0\end{array}$ \\
\hline
\end{tabular}

$a$ Daily average added in order to complete record. 
TABLE XXI.-Calories balances for Series VI-Continued.

[Averages are per day.]

No. 7 .

\begin{tabular}{|c|c|c|c|c|c|c|c|c|c|}
\hline Period. & $\begin{array}{c}1 \\
\text { In food. }\end{array}$ & $\begin{array}{c}2 \\
\text { In } \\
\text { feces. }\end{array}$ & $\underset{\text { In }}{3}$ & $\begin{array}{l}\mathbf{4} \\
\text { In } \\
\text { feces } \\
\text { and } \\
\text { urine. } \\
(2+3)\end{array}$ & $\begin{array}{c}5 \\
\text { In } \\
\text { feces. } \\
(2 \div 1)\end{array}$ & $\begin{array}{c}6 \\
\text { In } \\
\text { urine. } \\
(3 \div 1)\end{array}$ & $\begin{array}{l}7 \\
\text { In } \\
\text { feces } \\
\text { and } \\
\text { urine. } \\
(4 \div 1)\end{array}$ & $\begin{array}{c}8 \\
\substack{\text { Balance. } \\
(1-4)}\end{array}$ & $\begin{array}{c}9 \\
\text { Sali- } \\
\text { cylic } \\
\text { acid } \\
\text { admin- } \\
\text { istered. }\end{array}$ \\
\hline Fore period. & & & & & & & & & \\
\hline 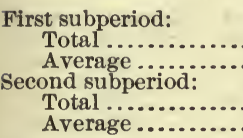 & $\begin{array}{r}\text { Calories. } \\
11,483 \\
2,297\end{array}$ & $\begin{array}{c}\text { Calories } \\
455 \\
91\end{array}$ & $\begin{array}{r}\text { Calories } \\
319 \\
64\end{array}$ & $\begin{array}{c}\text { Calories } \\
774 \\
155\end{array}$ & $\begin{array}{r}\text { Per ct. } \\
3.96 \\
\ldots \ldots \ldots . .\end{array}$ & $\begin{array}{r}\text { Per ct. } \\
2.78 \\
\ldots . . .\end{array}$ & $\begin{array}{r}\text { Per ct. } \\
6.74 \\
\ldots \ldots . . .\end{array}$ & $\begin{array}{r}\text { Calories. } \\
10,709 \\
2,142\end{array}$ & $\begin{array}{c}\text { Grams. } \\
0 \\
0\end{array}$ \\
\hline $\begin{array}{l}\text { Entire fore period: } \\
\quad \text { Total .................................. } \\
\text { Average }\end{array}$ & $\begin{array}{r}23,893 \\
2,389 \\
\end{array}$ & $\begin{array}{r}706 \\
71\end{array}$ & $\begin{array}{r}674 \\
67\end{array}$ & $\begin{array}{r}1,380 \\
138\end{array}$ & 2.95 & 2.82 & 5.78 & $\begin{array}{r}22,513 \\
2,251\end{array}$ & $\begin{array}{l}0 \\
0\end{array}$ \\
\hline Preservative period. & & & & & & & & & \\
\hline 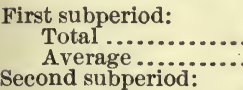 & $\begin{array}{r}11,864 \\
2,373\end{array}$ & $\begin{array}{r}313 \\
63\end{array}$ & $\begin{array}{r}286 \\
57\end{array}$ & $\begin{array}{l}599 \\
120\end{array}$ & 2.64 & 2.41 & 5.05 & $\begin{array}{r}11,265 \\
2,253\end{array}$ & $\begin{array}{r}1.05 \\
.21\end{array}$ \\
\hline $\begin{array}{l}\text { Total ................... } \\
\text { Average ............ } \\
\text { Third subperiod: }\end{array}$ & $\begin{array}{r}11,869 \\
2,374\end{array}$ & $\begin{array}{r}238 \\
48\end{array}$ & $\begin{array}{r}321 \\
64\end{array}$ & $\begin{array}{l}559 \\
112\end{array}$ & 2.01 & 2.70 & 4.71 & $\begin{array}{r}11,310 \\
2,262\end{array}$ & $\begin{array}{r}2.10 \\
.42\end{array}$ \\
\hline $\begin{array}{l}\text { Total ................. } \\
\text { Average }\end{array}$ & $\begin{array}{r}11,908 \\
2,382\end{array}$ & $\begin{array}{r}330 \\
66\end{array}$ & $\begin{array}{r}289 \\
58\end{array}$ & $\begin{array}{l}619 \\
124\end{array}$ & 2.77 & 2.43 & 5.20 & $\begin{array}{r}11,289 \\
2,258\end{array}$ & $\begin{array}{r}3.70 \\
.74\end{array}$ \\
\hline $\begin{array}{l}\text { Fourth subperiod: } \\
\text { Total .......... } \\
\text { Average....... }\end{array}$ & $\begin{array}{r}12,025 \\
2,405\end{array}$ & $\begin{array}{r}229 \\
46\end{array}$ & $\begin{array}{r}277 \\
55\end{array}$ & $\begin{array}{l}506 \\
101\end{array}$ & 1.90 & 2.30 & 4.21 & $\begin{array}{r}11,519 \\
2,304\end{array}$ & $\begin{array}{l}6.00 \\
1.20\end{array}$ \\
\hline 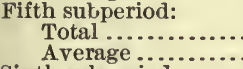 & $\begin{array}{r}12,084 \\
2,417\end{array}$ & $\begin{array}{r}321 \\
64\end{array}$ & $\begin{array}{r}298 \\
60\end{array}$ & $\begin{array}{l}619 \\
124\end{array}$ & 2.66 & 2.47 & 5.12 & $\begin{array}{r}11,465 \\
2,293\end{array}$ & $\begin{array}{l}8.00 \\
1.60\end{array}$ \\
\hline 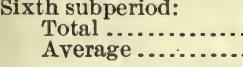 & $\begin{array}{r}11,849 \\
2,370\end{array}$ & $\begin{array}{r}167 \\
33\end{array}$ & $\begin{array}{r}362 \\
72\end{array}$ & $\begin{array}{l}529 \\
106\end{array}$ & 1.41 & 3.06 & 4.46 & $\begin{array}{r}11,320 \\
2,264\end{array}$ & $\begin{array}{r}10.00 \\
2.00\end{array}$ \\
\hline $\begin{array}{l}\text { Entire } \\
\text { period: } \\
\text { Total } \\
\text { Average } \ldots \ldots \ldots \ldots \\
\end{array}$ & $\begin{array}{r}71,599 \\
2,387\end{array}$ & $\begin{array}{r}1,598 \\
53\end{array}$ & $\begin{array}{r}1,833 \\
61\end{array}$ & $\begin{array}{r}3,431 \\
114\end{array}$ & 2.23 & 2.56 & 4.79 & $\begin{array}{r}68,168 \\
2,273\end{array}$ & $\begin{array}{r}30.85 \\
1.03\end{array}$ \\
\hline After period. & & & & & & & & & \\
\hline $\begin{array}{l}\text { First subperiod: } \\
\text { Total........... } \\
\text { Average...... }\end{array}$ & $\begin{array}{r}11,822 \\
2,364\end{array}$ & $\begin{array}{r}351 \\
70\end{array}$ & $\begin{array}{r}298 \\
60\end{array}$ & $\begin{array}{l}649 \\
130\end{array}$ & 2.97 & 2.52 & 5.49 & $\begin{array}{r}11,173 \\
2,234\end{array}$ & $\begin{array}{l}0 \\
0\end{array}$ \\
\hline 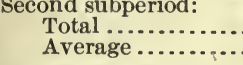 & $\begin{array}{r}12,212 \\
2,442\end{array}$ & $\begin{array}{r}478 \\
96\end{array}$ & $\begin{array}{r}316 \\
63\end{array}$ & $\begin{array}{l}794 \\
159\end{array}$ & 3.91 & 2.59 & 6.50 & $\begin{array}{r}11,418 \\
2,283\end{array}$ & $\begin{array}{l}0 \\
0\end{array}$ \\
\hline 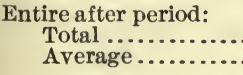 & $\begin{array}{r}24,034 \\
2,403\end{array}$ & $\begin{array}{r}829 \\
83\end{array}$ & $\begin{array}{r}614 \\
61\end{array}$ & $\begin{array}{r}1,443 \\
144\end{array}$ & 3.45 & 2.55 & 6.01 & $\begin{array}{r}22,591 \\
2,259\end{array}$ & $\begin{array}{l}0 \\
0\end{array}$ \\
\hline
\end{tabular}


TABLE XXI.-Calories balances for Series VI-Continued.

[Averages are per day.]

No. 8.

\begin{tabular}{|c|c|c|c|c|c|c|c|c|c|}
\hline Period. & In food. & In & $\begin{array}{l}\mathbf{3} \\
\text { In } \\
\text { urine. }\end{array}$ & $\begin{array}{c}4 \\
\text { In } \\
\text { feces } \\
\text { and } \\
\text { urine. } \\
(2+3)\end{array}$ & $\begin{array}{l}5 \\
\text { In } \\
\text { feces. } \\
(2 \div 1)\end{array}$ & $\begin{array}{c}6 \\
\text { In } \\
(3 \div 1)\end{array}$ & $\begin{array}{c}7 \\
\text { In } \\
\text { feces } \\
\text { and } \\
\text { urine. } \\
(4 \div 1)\end{array}$ & $\begin{array}{c}8 \\
\text { Balance. } \\
(1-4)\end{array}$ & $\begin{array}{c}9 \\
\text { Sali- } \\
\text { cylic } \\
\text { acid } \\
\text { admin- } \\
\text { istered. }\end{array}$ \\
\hline Fore period. & & & & & & & & & \\
\hline $\begin{array}{l}\text { First subperiod: } \\
\text { Total ........... } \\
\text { Average ....... }\end{array}$ & $\begin{array}{r}\text { Calories. } \\
12,913 \\
2,583\end{array}$ & $\begin{array}{c}\text { Calories } \\
364 \\
73\end{array}$ & $\begin{array}{c}\text { Calories } \\
298 \\
60\end{array}$ & $\begin{array}{c}\text { Calories } \\
662 \\
132\end{array}$ & $\begin{array}{r}\text { Per ct. } \\
2.82 \\
2 . . .\end{array}$ & $\begin{array}{r}\text { Per ct. } \\
2.31 \\
\quad \ldots . .\end{array}$ & $\begin{array}{r}\text { Per ct. } \\
5.13 \\
\end{array}$ & $\begin{array}{r}\text { Calories. } \\
12,251 \\
2,451\end{array}$ & $\begin{array}{c}\text { Grams. } \\
0 \\
0\end{array}$ \\
\hline $\begin{array}{l}\text { Second subperiod: } \\
\text { Total ........................ } \\
\text { Average ....... }\end{array}$ & $\begin{array}{r}12,665 \\
2,533\end{array}$ & $\begin{array}{r}466 \\
93\end{array}$ & $\begin{array}{r}313 \\
63\end{array}$ & $\begin{array}{r}779 \\
156\end{array}$ & $\begin{array}{r}3.68 \\
\ldots \ldots . . .\end{array}$ & $\begin{array}{r}2.47 \\
\ldots \ldots \ldots\end{array}$ & $\begin{array}{r}6.15 \\
\ldots . . . .\end{array}$ & $\begin{array}{r}11,886 \\
2,377\end{array}$ & $\begin{array}{l}0 \\
0\end{array}$ \\
\hline $\begin{array}{l}\text { Entire fore period: } \\
\text { Total .................... } \\
\text { Average ........ }\end{array}$ & $\begin{array}{r}25,578 \\
2,558\end{array}$ & $\begin{array}{r}830 \\
83\end{array}$ & $\begin{array}{r}611 \\
61\end{array}$ & $\begin{array}{r}1,441 \\
144\end{array}$ & $\begin{array}{r}3.25 \\
\cdots \cdots . . .\end{array}$ & $\begin{array}{r}2.39 \\
\ldots \ldots \ldots\end{array}$ & $\begin{array}{r}5.63 \\
\cdots . . . . . .\end{array}$ & $\begin{array}{r}24,137 \\
2,414\end{array}$ & $\begin{array}{l}0 \\
0\end{array}$ \\
\hline Preservative period. & & & & & & & & & \\
\hline $\begin{array}{l}\text { First subperiod: } \\
\text { Total .......... } \\
\text { Average...... }\end{array}$ & $\begin{array}{r}13,622 \\
2,724\end{array}$ & $\begin{array}{l}550 \\
110\end{array}$ & $\begin{array}{r}297 \\
59\end{array}$ & $\begin{array}{l}847 \\
169\end{array}$ & 4.04 & $\begin{array}{l}2.18 \\
\cdots \ldots\end{array}$ & $\begin{array}{c}6.22 \\
\ldots \ldots\end{array}$ & $\begin{array}{r}12,775 \\
2,555\end{array}$ & $\begin{array}{r}1.05 \\
.21\end{array}$ \\
\hline Second sul & & & & & & & & & \\
\hline $\begin{array}{l}\text { Total } \\
\text { Avera }\end{array}$ & 14,033 & 573 & $\begin{array}{r}307 \\
61\end{array}$ & 880 & 4.08 & 2.19 & 6.27 & 13,153 & 2.10 \\
\hline Third subperiod: & $.2,001$ & & 01 & & 0 & & & 2,031 & \\
\hline $\begin{array}{l}\text { Tota] } \\
\text { Aver }\end{array}$ & $\begin{array}{r}13,202 \\
2,640\end{array}$ & $\begin{array}{r}374 \\
75\end{array}$ & $\begin{array}{r}292 \\
58\end{array}$ & $\begin{array}{l}666 \\
133\end{array}$ & $\begin{array}{r}2.83 \\
\ldots \ldots\end{array}$ & $\begin{array}{r}2.21 \\
\ldots \ldots\end{array}$ & 5.04 & $\begin{array}{r}12,536 \\
2,507\end{array}$ & $\begin{array}{r}3.70 \\
74\end{array}$ \\
\hline Fourth si & & & & & & & & & \\
\hline a......... & $\begin{array}{r}13,485 \\
, 697\end{array}$ & 575 & a 283 & 858 & 4.26 & 2.10 & 6.36 & 12,627 & 6.00 \\
\hline $\begin{array}{l}\text { Ave } \\
\text { Fifth su }\end{array}$ & 2,697 & 115 & 57 & 172 & $\cdots \cdots \cdot$ & ....... & $\cdots \cdots \cdots$ & 525 & 1.2 \\
\hline $\begin{array}{l}\text { Total } \\
\text { Avera }\end{array}$ & $\begin{array}{r}14,335 \\
2,867\end{array}$ & $\begin{array}{r}301 \\
60\end{array}$ & $\begin{array}{r}325 \\
65\end{array}$ & $\begin{array}{l}626 \\
125\end{array}$ & 2.10 & $\begin{array}{r}2.27 \\
\ldots . . .\end{array}$ & 4.37 & $\begin{array}{r}13,709 \\
2,742\end{array}$ & $\begin{array}{l}8.00 \\
1.60\end{array}$ \\
\hline Sixth subperiod: & & & & & & & & & \\
\hline 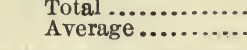 & $\begin{array}{r}14,264 \\
-\quad 2,853 \\
\end{array}$ & $\begin{array}{r}634 \\
+\quad 127\end{array}$ & $\begin{array}{r}314 \\
63\end{array}$ & $\begin{array}{l}948 \\
190\end{array}$ & $\begin{array}{r}4.44 \\
\ldots . . .\end{array}$ & $\begin{array}{r}2.20 \\
\ldots \ldots \ldots\end{array}$ & $\begin{array}{r}6.65 \\
\ldots \ldots \ldots \\
\ldots \ldots\end{array}$ & $\begin{array}{r}13,316 \\
2,663\end{array}$ & $\begin{array}{r}10.00 \\
2.00\end{array}$ \\
\hline 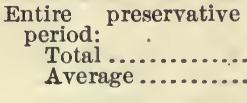 & $\begin{array}{r}82,941 \\
2,765\end{array}$ & $\begin{array}{r}3,007 \\
100\end{array}$ & $\begin{array}{r}1,818 \\
61\end{array}$ & $\begin{array}{r}4,825 \\
161\end{array}$ & $\begin{array}{r}3.63 \\
\cdots \cdots . . . \\
\end{array}$ & $\begin{array}{r}2.19 \\
\ldots \ldots . .\end{array}$ & $\begin{array}{r}5.82 \\
\ldots . . . \\
\end{array}$ & $\begin{array}{r}78,116 \\
2,604 \\
\end{array}$ & $\begin{array}{r}30.85 \\
1.08\end{array}$ \\
\hline After period. & & & & & & & & & \\
\hline $\begin{array}{l}\text { First subperiod: } \\
\quad \text { Total .................. } \\
\text { Average ........... }\end{array}$ & $\begin{array}{r}13,356 \\
2,671\end{array}$ & $\begin{array}{l}504 \\
101\end{array}$ & $\begin{array}{r}340 \\
68\end{array}$ & $\begin{array}{l}844 \\
169\end{array}$ & $\begin{array}{r}3.77 \\
\ldots . . .\end{array}$ & $\begin{array}{r}2.55 \\
\cdots\end{array}$ & $\begin{array}{c}6.32 \\
\ldots \ldots .\end{array}$ & $\begin{array}{r}12,512 \\
2,502\end{array}$ & $\begin{array}{l}0 \\
0\end{array}$ \\
\hline $\begin{array}{l}\text { Second subperiod: } \\
\text { Total } \ldots \ldots \ldots \ldots \ldots \\
\text { Average................ }\end{array}$ & $\begin{array}{r}14,469 \\
2,893\end{array}$ & $\begin{array}{l}507 \\
101\end{array}$ & $\begin{array}{r}334 \\
67\end{array}$ & $\begin{array}{l}841 \\
168\end{array}$ & $\begin{array}{r}3.50 \\
\ldots . . . .\end{array}$ & $\begin{array}{r}2.31 \\
\ldots . . . .\end{array}$ & $\begin{array}{r}5.81 \\
\ldots . . .\end{array}$ & $\begin{array}{r}13,628 \\
2,726\end{array}$ & $\begin{array}{l}0 \\
0\end{array}$ \\
\hline $\begin{array}{l}\text { Entire after period: } \\
\text { Total ............... } \\
\text { Average ....... }\end{array}$ & $\begin{array}{r}27,825 \\
2,783\end{array}$ & $\begin{array}{r}1,011 \\
101\end{array}$ & $\begin{array}{r}674 \\
67\end{array}$ & $\begin{array}{r}1,685 \\
169\end{array}$ & $\begin{array}{r}3.63 \\
\ldots . . .\end{array}$ & $\begin{array}{r}2.42 \\
\ldots \ldots .\end{array}$ & $\begin{array}{r}6.06 \\
\cdots \ldots . . .\end{array}$ & $\begin{array}{r}26,140 \\
2,614\end{array}$ & $\begin{array}{l}0 \\
0\end{array}$ \\
\hline
\end{tabular}

$a$ Daily average added in order to complete record. 
TABLE XXI.-Calories balances for Series VI-Continued.

[Averages are per day.]

No. 9.

\begin{tabular}{|c|c|c|c|c|c|c|c|c|c|}
\hline Period. & $\begin{array}{c}1 \\
\text { In food. }\end{array}$ & $\begin{array}{c}2 \\
\text { In } \\
\text { feces. }\end{array}$ & $\underset{\text { In }}{3}$ & $\begin{array}{c}4 \\
\text { In } \\
\text { feces } \\
\text { and } \\
\text { urine. } \\
(2+3)\end{array}$ & $\begin{array}{c}5 \\
\text { In } \\
\text { feces. } \\
(2 \div 1)\end{array}$ & $\begin{array}{c}\text { 6 } \\
\text { In } \\
\text { urine. } \\
(3 \div 1)\end{array}$ & $\begin{array}{l}7 \\
\text { In } \\
\text { feees } \\
\text { and } \\
\text { urine. } \\
(4 \div 1)\end{array}$ & $\begin{array}{c}8 \\
\substack{\text { Balance. } \\
(1-4)}\end{array}$ & $\begin{array}{l}\mathbf{9} \\
\text { Sali- } \\
\text { cylic } \\
\text { acid } \\
\text { admin- } \\
\text { istered. }\end{array}$ \\
\hline Fore period. & & & & & & & & & \\
\hline 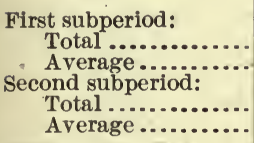 & $\begin{array}{r}\text { Calories. } \\
16,793 \\
3,359\end{array}$ & $\begin{array}{r}\text { Calories } \\
270 \\
54 \\
437 \\
87\end{array}$ & $\begin{array}{c}\text { Calories } \\
396 \\
79\end{array}$ & $\begin{array}{c}\text { Calories } \\
666 \\
133\end{array}$ & $\begin{array}{r}\text { Per ct. } \\
1.61 \\
\ldots \\
2.56\end{array}$ & $\begin{array}{r}\text { Per ct. } \\
2.36 \\
\cdots \\
2.22\end{array}$ & $\begin{array}{l}\text { Per ct. } \\
3.97 \\
\ldots . . .\end{array}$ & $\begin{array}{r}\text { Calories. } \\
16,127 \\
3,226 \\
16,250 \\
3,250\end{array}$ & $\begin{array}{c}\text { Grams. } \\
0 \\
0\end{array}$ \\
\hline $\begin{array}{l}\text { Entire fore period: } \\
\text { Total ............... } \\
\text { Average } . . . . . .\end{array}$ & $\begin{array}{r}33,859 \\
3,386\end{array}$ & $\begin{array}{r}707 \\
71\end{array}$ & $\begin{array}{r}775 \\
78\end{array}$ & $\begin{array}{r}1,482 \\
148\end{array}$ & 2.09 & 2.29 & 4.38 & $\begin{array}{r}32,377 \\
3,238\end{array}$ & $\begin{array}{l}0 \\
0\end{array}$ \\
\hline Preservative period. & & & & & & & & & \\
\hline 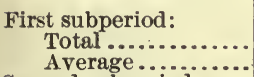 & $\begin{array}{r}16,793 \\
3,359\end{array}$ & $\begin{array}{l}692 \\
138\end{array}$ & $\begin{array}{r}407 \\
81\end{array}$ & $\begin{array}{r}1,099 \\
220\end{array}$ & 4.12 & 2.42 & 6.54 & $\begin{array}{r}15,694 \\
3,139\end{array}$ & $\begin{array}{l}1.05 \\
.21\end{array}$ \\
\hline $\begin{array}{l}\text { Second s } \\
\text { Total } \\
\text { Aver }\end{array}$ & $\begin{array}{r}15,829 \\
3,166\end{array}$ & $\begin{array}{r}485 \\
97\end{array}$ & $\begin{array}{l}373 \\
75\end{array}$ & $\begin{array}{l}858 \\
172\end{array}$ & 3.06 & 2.36 & 5.42 & $\begin{array}{r}14,971 \\
2,994\end{array}$ & 2.10 \\
\hline $\begin{array}{l}\text { Third st } \\
\text { Tota } \\
\text { Ave }\end{array}$ & $\begin{array}{r}16,812 \\
3,362\end{array}$ & $\begin{array}{r}484 \\
97\end{array}$ & $\begin{array}{r}390 \\
78\end{array}$ & $\begin{array}{l}874 \\
175\end{array}$ & 2.88 & 2.32 & 5.20 & $\begin{array}{r}15 ; 938 \\
3,187\end{array}$ & $\begin{array}{l}3.70 \\
74\end{array}$ \\
\hline $\begin{aligned} \text { Fourtl } \\
\text { To } \\
\text { Av }\end{aligned}$ & $\begin{array}{r}16,835 \\
3,367\end{array}$ & 254 & $\begin{array}{r}383 \\
77\end{array}$ & $\begin{array}{l}637 \\
127\end{array}$ & 1.51 & 2,28 & 3.78 & $\begin{array}{r}16,198 \\
3,240\end{array}$ & $\begin{array}{l}6.00 \\
1.20\end{array}$ \\
\hline $\begin{array}{l}\text { Fifth su } \\
\text { Tot: } \\
\text { Ave }\end{array}$ & $\begin{array}{r}0,000 \\
16,712 \\
3,342\end{array}$ & $\begin{array}{r}01 \\
599 \\
120\end{array}$ & $\begin{array}{r}357 \\
71\end{array}$ & $\begin{array}{l}956 \\
191\end{array}$ & $\begin{array}{l}3.58 \\
\therefore \ldots .\end{array}$ & 2.14 & 5.72 & $\begin{array}{r}15,756 \\
3,151\end{array}$ & $\begin{array}{l}8.00 \\
1.60\end{array}$ \\
\hline $\begin{array}{l}\text { Sixth subperiod: } \\
\quad \text { Total ................. } \\
\text { Average ................ }\end{array}$ & $\begin{array}{r}16,195 \\
3,239\end{array}$ & $\begin{array}{l}538 \\
108\end{array}$ & $\begin{array}{r}414 \\
83\end{array}$ & $\begin{array}{l}952 \\
190\end{array}$ & 3.32 & 2.56 & 5. 88 & $\begin{array}{r}15,243 \\
3,049\end{array}$ & $\begin{array}{r}10.00 \\
2.00\end{array}$ \\
\hline $\begin{array}{l}\text { Entire preservative } \\
\text { period: } \\
\text { Total ................. } \\
\text { Average ........... }\end{array}$ & $\begin{array}{r}99,176 \\
3,306\end{array}$ & $\begin{array}{r}3,052 \\
102\end{array}$ & $\begin{array}{r}2,324 \\
77\end{array}$ & $\begin{array}{r}5,376 \\
179\end{array}$ & $\begin{array}{r}3.08 \\
\ldots .0 .\end{array}$ & 2.34 & $\begin{array}{r}5.42 \\
\ldots . . .\end{array}$ & $\begin{array}{r}93,800 \\
3,127\end{array}$ & $\begin{array}{r}30.85 \\
1.03\end{array}$ \\
\hline After period. & & & & & & & & 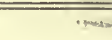 & \\
\hline $\begin{array}{l}\text { First subperiod: } \\
\text { Total .......... } \\
\text { Average..... }\end{array}$ & $\begin{array}{r}16,551 \\
3,310\end{array}$ & $\begin{array}{r}230 \\
46\end{array}$ & $\begin{array}{r}363 \\
73\end{array}$ & $\begin{array}{l}593 \\
119\end{array}$ & 1.39 & 2.19 & 3.58 & $\begin{array}{r}15 ; 958 \\
3,192\end{array}$ & $\begin{array}{l}0 \\
0\end{array}$ \\
\hline 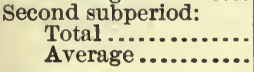 & $\begin{array}{r}16,622 \\
3,324\end{array}$ & $\begin{array}{l}581 \\
116\end{array}$ & $\begin{array}{r}396 \\
79\end{array}$ & $\begin{array}{l}977 \\
195\end{array}$ & 3.50 & 2.38 & 5.88 & $\begin{array}{r}15,645 \\
3,129\end{array}$ & $\begin{array}{l}0 \\
0\end{array}$ \\
\hline 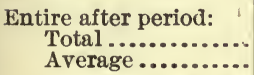 & $\begin{array}{r}33,173 \\
3,317\end{array}$ & $\begin{array}{r}811 \\
81\end{array}$ & $\begin{array}{r}759 \\
76\end{array}$ & $\begin{array}{r}1,570 \\
157\end{array}$ & 2.44 & 2.29 & 4.73 & $\begin{array}{r}31,603 \\
3,160\end{array}$ & $\begin{array}{l}0 \\
0\end{array}$ \\
\hline
\end{tabular}


TABLE XXI.-Calories balances for Series VI-Continued.

[Averages are per day.]

No. 10.

\begin{tabular}{|c|c|c|c|c|c|c|c|c|c|}
\hline Period. & In food. & $\begin{array}{c}2 \\
\text { In } \\
\text { feces. }\end{array}$ & $\begin{array}{l}3 \\
\text { In } \\
\text { urine. }\end{array}$ & $\begin{array}{l}4 \\
\text { In } \\
\text { feces } \\
\text { and } \\
\text { urine. } \\
(2+3)\end{array}$ & $\begin{array}{c}\text { In } \\
\text { feces. } \\
(2 \div 1)\end{array}$ & $\underset{\substack{\text { In } \\
\text { urine. } \\
(3 \div 1)}}{6}$ & $\begin{array}{c}7 \\
\text { In } \\
\text { feces } \\
\text { and } \\
\text { urine. } \\
(4 \div 1)\end{array}$ & $\begin{array}{c}8 \\
\text { Balance. } \\
(1-4)\end{array}$ & $\begin{array}{l}\mathbf{9} \\
\text { Sali- } \\
\text { cylic } \\
\text { acid } \\
\text { admin- } \\
\text { istered. }\end{array}$ \\
\hline Fore period. & & & & & & & & & \\
\hline $\begin{array}{l}\text { First subperiod: } \\
\text { Total ............... } \\
\text { Average ........... } \\
\text { Second subperiod: } \\
\text { Total .................. } \\
\text { Average ............ }\end{array}$ & $\begin{array}{r}\text { Calories. } \\
16,400 \\
3,280 \\
16,377 \\
3,275\end{array}$ & $\begin{array}{c}\text { Calories } \\
631 \\
126\end{array}$ & \begin{tabular}{|} 
Calories \\
360 \\
72
\end{tabular} & $\begin{array}{c}\text { Calories } \\
991 \\
198\end{array}$ & $\begin{array}{r}\text { Per } r t . \\
3.85 \\
\ldots \\
2.72 \\
\ldots\end{array}$ & $\begin{aligned} \text { Per } c t . \\
2.20 \\
\end{aligned}$ & $\begin{array}{r}\text { Per } c t . \\
6.04 \\
\ldots \\
4.88 \\
\ldots\end{array}$ & $\begin{array}{r}\text { Calories. } \\
15,409 \\
3,082 \\
\\
15,578 \\
3,115\end{array}$ & $\begin{array}{c}\text { Grams. } \\
0 \\
0 \\
0 \\
0 \\
0\end{array}$ \\
\hline $\begin{array}{l}\text { Entire fore period: } \\
\text { Total ............... } \\
\text { Average .......... }\end{array}$ & $\begin{array}{r}32,777 \\
3,278\end{array}$ & $\begin{array}{r}1,076 \\
108\end{array}$ & $\begin{array}{r}714 \\
71\end{array}$ & $\begin{array}{r}1,790 \\
179\end{array}$ & $\begin{array}{r}3.28 \\
\ldots \ldots \ldots\end{array}$ & $\begin{array}{r}2.18 \\
\ldots . . .\end{array}$ & $\begin{array}{r}5.46 \\
\cdots . . .\end{array}$ & $\begin{array}{r}30,987 \\
3,099\end{array}$ & $\begin{array}{l}0 \\
0\end{array}$ \\
\hline Preservative period. & & & & & & & & & \\
\hline $\begin{array}{l}\text { First subperiod: } \\
\quad \text { Total............... } \\
\text { Average .......... }\end{array}$ & $\begin{array}{r}16,886 \\
3,377\end{array}$ & $\begin{array}{l}591 \\
118\end{array}$ & $\begin{array}{r}349 \\
70\end{array}$ & $\begin{array}{l}940 \\
188\end{array}$ & 3.50 & 2.07 & 5.57 & $\begin{array}{r}15,946 \\
3,189\end{array}$ & $\begin{array}{r}1.05 \\
.21\end{array}$ \\
\hline 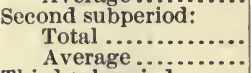 & $\begin{array}{r}16,697 \\
3,339\end{array}$ & $\begin{array}{l}499 \\
100\end{array}$ & $\begin{array}{r}402 \\
80\end{array}$ & $\begin{array}{l}901 \\
180\end{array}$ & 2.99 & 2.41 & 5.40 & $\begin{array}{r}15,796 \\
3,159\end{array}$ & $\begin{array}{r}2.10 \\
.42\end{array}$ \\
\hline 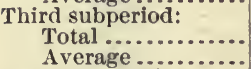 & $\begin{array}{r}16,740 \\
3,348\end{array}$ & $\begin{array}{l}549 \\
110\end{array}$ & $\begin{array}{r}380 \\
76\end{array}$ & $\begin{array}{l}929 \\
186\end{array}$ & 3.28 & 2.27 & $\begin{array}{c}5.55 \\
\ldots . . .\end{array}$ & $\begin{array}{r}15,811 \\
3,162\end{array}$ & $\begin{array}{r}3.70 \\
.74\end{array}$ \\
\hline $\begin{array}{l}\text { Fourth subperiod: } \\
\text { Total .................... } \\
\text { Average ........... }\end{array}$ & $\begin{array}{r}16,485 \\
3,297\end{array}$ & $\begin{array}{r}452 \\
90\end{array}$ & $\begin{array}{r}347 \\
69\end{array}$ & $\begin{array}{l}799 \\
160\end{array}$ & 2.74 & 2.10 & 4.85 & $\begin{array}{r}15,686 \\
3,137\end{array}$ & $\begin{array}{l}6.00 \\
1.20\end{array}$ \\
\hline 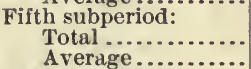 & $\begin{array}{r}17,078 \\
3,416\end{array}$ & $\begin{array}{r}398 \\
80\end{array}$ & $\begin{array}{r}363 \\
73\end{array}$ & $\begin{array}{l}761 \\
152\end{array}$ & 2.33 & 2.13 & 4.46 & $\begin{array}{r}16,317 \\
3,264\end{array}$ & $\begin{array}{l}8.00 \\
1.60\end{array}$ \\
\hline 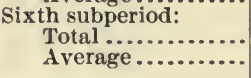 & $\begin{array}{r}16,444 \\
.3,289\end{array}$ & $\begin{array}{r}185 \\
37\end{array}$ & $\begin{array}{r}271 \\
54\end{array}$ & $\begin{array}{r}456 \\
91\end{array}$ & $\begin{array}{r}\cdot 1.13 \\
\ldots \ldots \ldots\end{array}$ & $\begin{array}{r}1.65 \\
\ldots . . .\end{array}$ & $\begin{array}{r}2.77 \\
\ldots . . .\end{array}$ & $\begin{array}{r}15,988 \\
3,198\end{array}$ & $\begin{array}{r}10.00 \\
2.00\end{array}$ \\
\hline $\begin{array}{l}\text { Entire preservative } \\
\text { period: } \\
\quad \text { Total ................. } \\
\text { Average.......... }\end{array}$ & $\begin{array}{r}100,330 \\
3,344\end{array}$ & $\begin{array}{r}2,674 \\
89\end{array}$ & $\begin{array}{r}2,112 \\
70\end{array}$ & $\begin{array}{r}4,786 \\
160\end{array}$ & $\begin{array}{r}2.67 \\
\ldots \ldots\end{array}$ & $\begin{array}{r}2.11 \\
\ldots .2 .\end{array}$ & $\begin{array}{r}4.77 \\
\ldots . . .\end{array}$ & $\begin{array}{r}95,544 \\
3,184\end{array}$ & $\begin{array}{r}30.85 \\
1.03\end{array}$ \\
\hline $\begin{array}{l}\text { After period. } \\
\text { First subperiod:a } \\
\text { Total ........... } \\
\text { Average ....... }\end{array}$ & $\begin{array}{r}16,273 \\
3,255\end{array}$ & $\begin{array}{l}596 \\
119\end{array}$ & $\begin{array}{r}337 \\
67\end{array}$ & $\begin{array}{l}933 \\
187\end{array}$ & $\begin{array}{c}3.66 \\
\cdots . . .\end{array}$ & 2.07 & 5. 73 & $\begin{array}{r}15,340 \\
3,068\end{array}$ & 0 \\
\hline
\end{tabular}

$a$ No second after subperiod; subject ill. 
Table XXI.-Calories balances for Series VI-Continued.

[Averages are per day.]

No. 11.

\begin{tabular}{|c|c|c|c|c|c|c|c|c|c|}
\hline Period. & $\begin{array}{c}1 \\
\text { In food. }\end{array}$ & $\begin{array}{c}2 \\
\text { In } \\
\text { feces. }\end{array}$ & $\begin{array}{c}3 \\
\text { In } \\
\text { urine. }\end{array}$ & $\begin{array}{c}4 \\
\text { In } \\
\text { feces } \\
\text { and } \\
\text { urine. } \\
(2+3)\end{array}$ & $\begin{array}{c}5 \\
\text { In } \\
\text { feces. } \\
(2 \div 1)\end{array}$ & $\begin{array}{c}6 \\
\text { In } \\
\text { urine. } \\
(3 \div 1)\end{array}$ & $\begin{array}{c}7 \\
\text { In } \\
\text { feces } \\
\text { and } \\
\text { urine. } \\
(4 \div 1)\end{array}$ & $\begin{array}{c}8 \\
\underset{(1-4)}{\text { Balance. }}\end{array}$ & $\begin{array}{c}9 \\
\text { Sali- } \\
\text { cylic } \\
\text { acid } \\
\text { admin- } \\
\text { istered. }\end{array}$ \\
\hline Fore period. & & & & & - & & & & \\
\hline 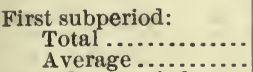 & $\begin{array}{r}\text { Calories. } \\
15,790 \\
3,158\end{array}$ & $\begin{array}{c}\text { Calories } \\
582 \\
116\end{array}$ & $\begin{array}{c}\text { Calories } \\
\text { a } 456 \\
91\end{array}$ & $\begin{array}{c}\text { Calories } \\
1,038 \\
203\end{array}$ & $\begin{array}{r}\text { Per ct. } \\
3.69 \\
\ldots \ldots\end{array}$ & $\begin{array}{r}\text { Per rt. } \\
2.83\end{array}$ & $\begin{array}{r}\text { Per ct. } \\
6.57\end{array}$ & $\begin{array}{r}\text { Calories. } \\
14,752 \\
2,9=0\end{array}$ & $\begin{array}{c}\text { Grams. } \\
0 \\
0\end{array}$ \\
\hline 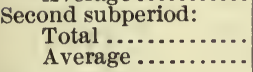 & $\begin{array}{r}15,668 \\
3,134\end{array}$ & $\begin{array}{l}616 \\
123\end{array}$ & $\begin{array}{r}438 \\
89\end{array}$ & $\begin{array}{r}1,0: 4 \\
211\end{array}$ & 3.93 & 2.80 & 6.73 & $\begin{array}{r}14,614 \\
2,923\end{array}$ & $\begin{array}{l}0 \\
0\end{array}$ \\
\hline $\begin{array}{l}\text { Entire fore period: } \\
\text { Total ................ } \\
\text { Average ............... }\end{array}$ & $\begin{array}{r}31,458 \\
3,146\end{array}$ & $\begin{array}{r}1,198 \\
120\end{array}$ & $\begin{array}{r}894 \\
89\end{array}$ & $\begin{array}{r}2,092 \\
209\end{array}$ & 3.81 & 2.84 & 6.65 & $\begin{array}{r}29,366 \\
2,937\end{array}$ & $\begin{array}{l}0 \\
0\end{array}$ \\
\hline Preservative period. & & & & & & & & & \\
\hline $\begin{array}{l}\text { First subperiod: } \\
\text { Total ............ } \\
\text { Average ....... }\end{array}$ & $\begin{array}{r}16,036 \\
3,207\end{array}$ & $\begin{array}{l}544 \\
109\end{array}$ & $\begin{array}{r}482 \\
96\end{array}$ & $\begin{array}{r}1,026 \\
205\end{array}$ & 3.39 & 3. 01 & 6.40 & $\begin{array}{r}15,010 \\
3,002\end{array}$ & $\begin{array}{r}1.05 \\
.21\end{array}$ \\
\hline $\begin{array}{l}\text { riod: } \\
\cdots \ldots . . . . . \\
\cdots \ldots \ldots . . .\end{array}$ & $\begin{array}{r}15,458 \\
3,092\end{array}$ & $\begin{array}{l}596 \\
119\end{array}$ & $\begin{array}{r}438 \\
88\end{array}$ & $\begin{array}{r}1,034 \\
207\end{array}$ & 3.86 & 2.83 & 6.69 & $\begin{array}{r}14,424 \\
2,88.5\end{array}$ & $\begin{array}{r}2.10 \\
.42\end{array}$ \\
\hline $\begin{array}{l}\text { Third st } \\
\text { Tote } \\
\text { Ave }\end{array}$ & $\begin{array}{r}15,657 \\
3,127\end{array}$ & $\begin{array}{l}592 \\
118\end{array}$ & $\begin{array}{r}414 \\
83\end{array}$ & $\begin{array}{r}1,006 \\
201\end{array}$ & 3.79 & 2.65 & 6.43 & $\begin{array}{r}14,631 \\
2,926\end{array}$ & $\begin{array}{r}3.70 \\
.74\end{array}$ \\
\hline $\begin{array}{l}\text { Fourth su } \\
\text { Total } \\
\text { Avera }\end{array}$ & $\begin{array}{r}15,565 \\
3,113\end{array}$ & $\begin{array}{r}212 \\
42\end{array}$ & $\begin{array}{r}383 \\
77\end{array}$ & $\begin{array}{l}595 \\
119\end{array}$ & 1.36 & 2.46 & 3.82 & $\begin{array}{r}14,970 \\
2,994\end{array}$ & $\begin{array}{l}6.00 \\
1.20\end{array}$ \\
\hline $\begin{array}{r}\text { Fifth sub } \\
\text { Total } \\
\text { Aver\& }\end{array}$ & $\begin{array}{r}15,776 \\
3,155\end{array}$ & $\begin{array}{l}499 \\
100\end{array}$ & $\begin{array}{r}427 \\
85\end{array}$ & $\begin{array}{l}926 \\
185\end{array}$ & 3.16 & 2.71 & 5.87 & $\begin{array}{r}14,850 \\
2,970\end{array}$ & $\begin{array}{l}8.00 \\
1.60\end{array}$ \\
\hline $\begin{array}{l}\text { Sixth subperiod: } \\
\quad \text { Total ................ } \\
\text { Average ................ }\end{array}$ & $\begin{array}{r}15,302 \\
3,060\end{array}$ & $\begin{array}{l}574 \\
.115\end{array}$ & $\begin{array}{r}395 \\
79\end{array}$ & $\begin{array}{l}969 \\
194\end{array}$ & 3.75 & 2. 58 & 6. 33 & $\begin{array}{r}14,333 \\
2,866\end{array}$ & $\begin{array}{r}10.00 \\
2.00\end{array}$ \\
\hline $\begin{array}{l}\text { Entire preservative } \\
\text { period: } \\
\text { Total ................ } \\
\text { Average ............... }\end{array}$ & $\begin{array}{r}93,774 \\
3,126\end{array}$ & $\begin{array}{r}3,017 \\
101\end{array}$ & $\begin{array}{r}2,539 \\
85\end{array}$ & $\begin{array}{r}5,556 \\
185\end{array}$ & 3.22 & 2.71 & 5.92 & $\begin{array}{r}88,218 \\
2,941\end{array}$ & $\begin{array}{r}30.85 \\
1.03\end{array}$ \\
\hline After period. & & & & & & & & & \\
\hline $\begin{array}{l}\text { First subperiod: } \\
\text { Total ............. } \\
\text { Average ....... }\end{array}$ & $\begin{array}{r}15,271 \\
3,054\end{array}$ & $\begin{array}{r}436 \\
87\end{array}$ & $\begin{array}{r}386 \\
77\end{array}$ & $\begin{array}{l}822 \\
164\end{array}$ & 2.86 & 2.53 & 5.38 & $\begin{array}{r}14,449 \\
2,890\end{array}$ & $\begin{array}{l}0 \\
0\end{array}$ \\
\hline 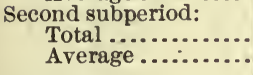 & $\begin{array}{r}15,620 \\
-\quad 3,124\end{array}$ & $\begin{array}{r}431 \\
86\end{array}$ & $\begin{array}{r}382 \\
76\end{array}$ & $\begin{array}{l}813 \\
163\end{array}$ & 2.76 & 2.45 & 5.20 & $\begin{array}{r}14,807 \\
2,961\end{array}$ & $\begin{array}{l}0 \\
0\end{array}$ \\
\hline 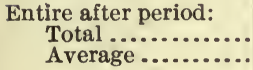 & $\begin{array}{r}30,891 \\
3,089\end{array}$ & $\begin{array}{r}867 \\
87\end{array}$ & $\begin{array}{r}768 \\
77\end{array}$ & $\begin{array}{r}1,635 \\
164\end{array}$ & 2.81 & 2.49 & 5.29 & $\begin{array}{r}29,256 \\
2,925\end{array}$ & $\begin{array}{l}0 \\
0\end{array}$ \\
\hline
\end{tabular}

$a$ Daily average added in order to complete record: 
TABLe XXI.-Calories balances for Series VI-Continued.

[Averages are per day.]

No. 12 .

\begin{tabular}{|c|c|c|c|c|c|c|c|c|c|}
\hline Period. & $\begin{array}{c}1 \\
\text { In food. }\end{array}$ & $\begin{array}{c}2 \\
\text { In } \\
\text { feces. }\end{array}$ & $\begin{array}{c}3 \\
\text { In } \\
\text { urine. }\end{array}$ & $\begin{array}{c}4 \\
\text { In } \\
\text { feces } \\
\text { and } \\
\text { urine. } \\
(2+3)\end{array}$ & $\begin{array}{c}5 \\
\text { In } \\
\text { feces. } \\
(2 \div 1)\end{array}$ & $\begin{array}{c}6 \\
\text { In } \\
\text { urine. } \\
(3 \div 1)\end{array}$ & $\begin{array}{c}7 \\
\text { In } \\
\text { feces } \\
\text { and } \\
\text { urine. } \\
(4 \div 1)\end{array}$ & $\underset{(1-4)}{\text { Balance. }}$ & $\begin{array}{c}9 \\
\text { Sali- } \\
\text { cylic } \\
\text { acid } \\
\text { admin- } \\
\text { istered. }\end{array}$ \\
\hline \multicolumn{10}{|l|}{ Fore period. } \\
\hline $\begin{array}{l}\text { First subperiod: } \\
\quad \text { Total ............... } \\
\text { Average } . . . . . . .\end{array}$ & $\begin{array}{r}\text { Calories. } \\
16,766 \\
3,353\end{array}$ & $\begin{array}{c}\text { Calories } \\
656 \\
131\end{array}$ & $\begin{array}{r}\text { Calories } \\
433 \\
87\end{array}$ & $\begin{array}{r}\text { Calories } \\
-1,089 \\
218\end{array}$ & $\begin{array}{r}\text { Per et. } \\
3.91 \\
.\end{array}$ & $\begin{array}{l}\text { Per ct. } \\
2.58 \\
2 . . .\end{array}$ & $\begin{array}{l}\text { Per ct. } \\
6.50 \\
\ldots .\end{array}$ & $\begin{array}{r}\text { Calories. } \\
15,677 \\
3,135\end{array}$ & $\begin{array}{c}\text { Grams. } \\
0 \\
0\end{array}$ \\
\hline Second subperiod: & & & & & & & & & \\
\hline 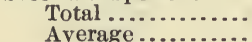 & 17,325 & 655 & 406 & 1,061 & 3. 78 & 2.34 & 6.12 & 16,264 & 0 \\
\hline Average ............ & 3,465 & 131 & 81 & 212 & ........ & (......... & .......... & 3,253 & 0 \\
\hline $\begin{array}{l}\text { Entire fore period: } \\
\quad \text { Total ............... } \\
\text { Average .......... }\end{array}$ & $\begin{array}{r}34,091 \\
3,409\end{array}$ & $\begin{array}{r}1,311 \\
131\end{array}$ & $\begin{array}{r}839 \\
84\end{array}$ & $\begin{array}{r}2,150 \\
215\end{array}$ & $\begin{array}{l}3.85 \\
\cdots \cdots\end{array}$ & $\begin{array}{c}2.46 \\
\cdots \cdots\end{array}$ & $\begin{array}{c}6.31 \\
\cdots . . .\end{array}$ & $\begin{array}{r}31,941 \\
3,194\end{array}$ & $\begin{array}{l}0 \\
0\end{array}$ \\
\hline \multicolumn{10}{|l|}{ Preservative period. } \\
\hline $\begin{array}{l}\text { First subperiod: } \\
\quad \text { Total } \ldots . . . \ldots \ldots \ldots \\
\text { Average } . . . . . . .\end{array}$ & $\begin{array}{r}16,958 \\
3,392\end{array}$ & $\begin{array}{r}387 \\
77\end{array}$ & $\begin{array}{r}367 \\
73\end{array}$ & $\begin{array}{l}754 \\
151\end{array}$ & 2.28 & 2.17 & 4.45 & 16,204 & 1.05 \\
\hline Second subperiod: & & & & & & & & & .21 \\
\hline Total ................ & 16,810 & 387 & 424 & 811 & 2.30 & 2.52 & 4.82 & 15,999 & 2. 10 \\
\hline 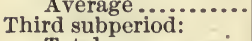 & 3,362 & 77 & 85 & 162 & $\ldots \ldots \ldots$ & (......... & ........ & 3,200 & .42 \\
\hline 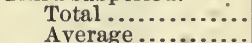 & 16,730 & 642 & 422 & 1,064 & 3. 84 & 2.52 & 6.36 & 15,666 & 3. 70 \\
\hline $\begin{array}{l}\text { Average ............. } \\
\text { Fourth subperiod: }\end{array}$ & 3,346 & 128 & 84 & 213 & & .... & ...... & 3,133 & .74 \\
\hline Total ................... & 16,878 & 380 & 395 & 775 & 2.25 & 2.34 & 4.59 & 16,103 & 6.00 \\
\hline $\begin{array}{l}\text { Average } \\
\text { Fifth subperiod: }\end{array}$ & 3,376 & 76 & 79 & 155 & ........ & $\ldots \ldots$ & ......... & 3,221 & 1.20 \\
\hline Total................... & 17,114 & 558 & 420 & 978 & 3.26 & 2.45 & 5.71 & 16,136 & 8.00 \\
\hline $\begin{array}{l}\text { Average ........ } \\
\text { Sixth subperiod: }\end{array}$ & 3,423 & 112 & 84 & 196 & 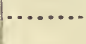 & -..... & (....... & 3,227 & 1.60 \\
\hline Total $\ldots . . . . . .$. & 16,498 & 430 & 425 & 855 & 2.61 & 2.58 & 5.18 & 15,643 & 10.00 \\
\hline Average ........ & 3,300 & 86 & 85 & 171 & ......... & …...... & …..... & 3,129 & 2.00 \\
\hline \multicolumn{10}{|l|}{$\begin{array}{l}\text { Entire preservative } \\
\text { period: }\end{array}$} \\
\hline Total ................ & 100,988 & 2,784 & 2,453 & 5,237 & 2.76 & 2.43 & 5.19 & 95,751 & 30.85 \\
\hline Average....... & 3,366 & 93 & 82 & 175 & …..... & $\ldots \ldots \ldots$ & .......... & & 1.03 \\
\hline \multicolumn{10}{|l|}{ After period. } \\
\hline $\begin{array}{l}\text { First subperiod: } \\
\text { Total ........... }\end{array}$ & 16,376 & 463 & 415 & 878 & 2.83 & 2.53 & 5.36 & 15,498 & 0 \\
\hline $\begin{array}{l}\text { Averag } \\
\text { Second sub }\end{array}$ & & 93 & 83 & 176 & & & & 3,099 & 0 \\
\hline $\begin{array}{l}\text { Total .... } \\
\text { Average }\end{array}$ & 16,498 & a 523 & 433 & 956 & 3.17 & 2.62 & 5. 79 & 15,542 & 0 \\
\hline Average ............ & 3,300 & 105 & 87 & 191 & …....... & …....... & …...... & & 0 \\
\hline $\begin{array}{l}\text { Entire after period: } \\
\text { Total ............. } \\
\text { Average . . . }\end{array}$ & 32,874 & 986 & 848 & 1,834 & 3.00 & 2.58 & 5.58 & 31,040 & 0 \\
\hline Average... & & 99 & 85 & 183 & & & & 3,104 & 0 \\
\hline
\end{tabular}

$a$ Daily average added in order to complete record. 
SALICYLIC ACID AND SALICYLATES.

TABLE XXI._Calories balances for Series VI-Continued.

[Averages are per man per day.]

Summary for nine men.

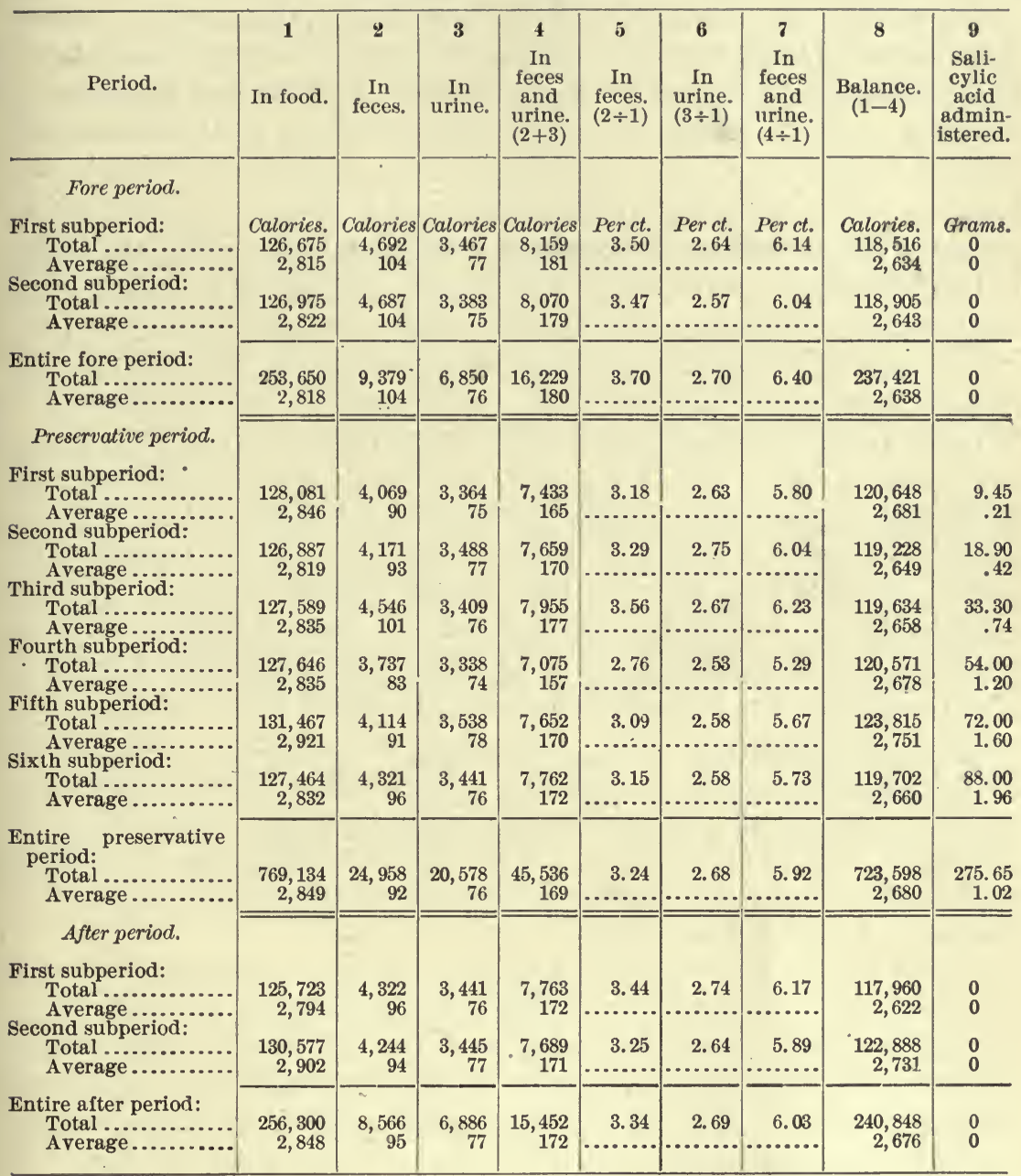

\section{SOLIDS BALANCE.}

A study of the solids balance gives valuable indications respecting the effect of the preservative upon the process of digestion and assimilation. It also furnishes data of important physiological significance in other respects.

\section{INDIVIDUAL DATA.}

No. 1.

The average daily quantity of solids in the food of No. 1 for the fore period is 467 grams, for the preservative period 474 grams, and for the after period 476 grams. It is thus seen that there is very little 
variation in the total quantity of solids, the amounts being as nearly alike as can be secured when a varied ration of uncomminuted food is used. Of this quantity there appear in the feces 14 grams daily in the fore period, 13 grams daily in the preservative period, and 19 grams daily in the after period. In the urine there are found 58 grams daily in the fore period, 62 grams in the preservative period, and 63 grams in the after period. Expressed in percentages, of the total quantity of solids it is found that 3 per cent appear in the feces in the fore period, 2.66 per cent in the preservative period, and 4.08 per cent in the after period. The respective percentages occurring in the urine are 12.32 in the fore period, 13.12 in the preservative period, and 13.28 in the after period. Inasmuch as by far the greater quantity of solid matter in the food is consumed in the production of heat and energy, the balance must be strongly positive. In this case the daily balance for the fore period is 395 grams, for the preservative period 399 grams, and for the after period 393 grams, showing very little difference in respect of the quantity of solids excreted. It is noticed that there is a slightly smaller quantity of solids in the feces in the preservative period than during either the fore or after period; a slightly greater quantity in the urine during the preservative period than in the fore period and a somewhat greater quantity during the after period. The percentage numbers show the same tendency as the weights, the smallest percentage of solids in the feces being in the preservative period and the largest in the after period. The effect of the preservative in this case appears to have been to secure a slightly greater degree of absorption during the preservative period than in the fore period, and on the withdrawal of the preservative the absorption of the food was much less complete.

\section{No. 2.}

A much larger quantity of solid food was consumed by No. 2 than by No. 1 . In the fore period the exact daily quantity of dry food consumed is 604 grams, in the preservative period 598 grams, and in the after period 612 grams. There appear in the feces for the fore period 27 grams of solids, during the preservative period 24 grams, and during the after period 22 grams. In the urine are found 66 grams of solids daily in the fore period, 67 grams in the preservative period, and 69 grams in the after period. Expressed in percentages, the quantity in the feces in the fore period is 4.47 per cent, in the preservative period 3.95 per cent, and in the after period 3.52 per cent. The percentages occurring in the urine during these periods are 10.88 in the fore period, 11.22 in the preservative period, and 11.30 in the after period. The balances are 511 grams, 507 grams, and 521 grams for the three periods, respectively. 
These data show that the quantity of solids in the feces diminished slightly in the preservative period and also in the after period. The solids in the urine increase slightly in the preservative period and to a greater extent in the after period. The percentage of solids in the feces is greatest in the fore period and smallest in the after period. The percentage of solids in the urine is least in the fore period and greatest in the after period.

\section{No. 3.}

The quantity of solids in the food during the fore period is 530 grams, during the preservative period 592 grams, and during the after period (second subperiod only) 633 grams. This shows a very marked increase in the amount of food eaten in the preservative period over the fore period and in the after period over the preservative period, due to the fact that in this case the quantity of food was increased after the recovery of the subject from the illness occurring in the fore period. Expressed in percentages, the largest quantity of solids eliminated in the feces is in the preservative period, namely, 2.98, and the smallest in the after period, namely, 2.56. In the urine the smallest quantity was eliminated in the preservative period, namely, 7.92, and the largest in the fore period, namely, 9.51. The balances are very marked, being highest in the after period and lowest in the fore period.

No. 3 is, of course, an exceptional case by reason of the illness experienced in the early part of the experiment. The data, therefore, are given merely for their individual worth and not because they have any value for comparison.

\section{No.4.}

The total quantity of solids consumed in the food by No. 4 during the fore period is 535 grams, during the preservative period 556 grams, and during the after period 569 grams. In the feces there appear in the fore period 20 grams daily of solids, in the preservative period 19 grams daily, and in the after period 18 grams daily. In the urine during the fore period are found 63 grams of solids, in the preservative period 67 grams, and in the after period 70 grams. The percentage of solids in the fore period recovered in the feces is 3.76 daily, in the preservative period 3.50, and in the after period 3.24. The percentages of solids recovered in the urine daily are 11.72 in the fore period, 12.06 in the preservative period, and 12.27 in the after period. The balances are 452 grams daily in the fore period, 469 in the preservative period, and 481 in the after period. There appears to be a slight tendency in this case for the preservative to diminish the quantity of solids in the feces, and this tendency is continued through the after period. There is a progressive increase of the solids in the urine 
in the preservative and after periods, and this increase is only slightly greater than the increase in the quantity of solids in the food consumed, as shown by the percentages for the three periods, namely, 11.72, 12.06, and 12.27. The largest percentage of solids in the feces is found during the fore period and the smallest in the after period, and this must be considered in connection with the increasing amount of food, from which it might be expected that the solids in the feces would increase proportionately, but this is not the case.

No. 5 .

The total solids consumed daily in the food is 558 grams in the fore period, 563 grams in the preservative period, and 564 grams in the after period, showing a very small variation during the entire period of observation. There appear in the feces during the fore period 25 grams of solids daily, in the preservative period $21 \mathrm{grams}$, and in the after period 18 grams. In the urine there appear daily 57 grams of solids in the fore period, 62 grams in the preservative period, and 62 grams in the after period. The largest percentage of solids in the feces is in the fore period, namely, 4.53 , and the smallest in the after period, namely, 3.17. The largest percentage of solids in the urine is found in the after period, namely, 11.03, and the smallest is found in the fore period, namely, 10.23. The largest balance is in the after period, namely, 484 grams, and the smallest in the fore period, namely, 476 grams. The apparent tendency of the preservative in this case is to diminish the percentage of solids in the feces, and this tendency is continued through the after period. On the other hand, there is a slight tendency to increase the quantity of solids in the urine.

\section{No. 6 .}

In the case of No. 6 the average daily quantity of solids in the food is 537 grams in the fore period, 556 grams in the preservative period, and 561 grams in the after period. Of this quantity there appear in the feces during the fore period 26 grams daily of solids, during the preservative period 25 grams daily, and during the after period 24 grams daily. There also appear in the urine 49 grams daily in the fore period, 57 grams daily in the preservative period, and 56 grams daily in the after period. Expressed in percentages, 4.84 per cent of the solids appear in the feces in the fore period, 4.53 per cent in the preservative period, and 4.28 in the after period. In the urine there are found 9.20 per cent of solids in the fore period, 10.33 per cent in the preservative period, and 9.98 per cent in the after period. There is an apparent tendency in this case to slightly decrease the quantity of solids in the feces, and this is continued through the after period, and to slightly increase the quantity of solids in the urine during the preservative period, and this is only slightly diminished in the after period. 
No. $\%$.

The quantity of solids found in the food of No. 7 during the fore period is 481 grams daily, during the preservative period 482 grams daily, and during the after period 488 grams daily. Of this there appear in the feces 14 grams daily in the fore period, 11 grams daily in the preservative period, and 17 grams daily in the after period. In the urine are found $58 \mathrm{grams}$ daily during the fore period, 54 grams daily during the preservative period, and 49 grams daily during the after period. Expressed in percentages the quantity of solids found in the feces during the fore period is 2.95 per cent daily, in the preservative period 2.21 per cent daily, and in the after period 3.44 per cent daily, while in the urine are found during the fore period 12.02 per cent daily, in the preservative period 11.12 per cent daily, and in the after period 10.12 per cent daily. The largest balance is in the after period, namely, 422 grams daily, and the smallest in. the fore period, namely, 409 grams daily. The principal effect of the preservative in this case is to decrease the amount of solids in the feces during the preservative period, a decrease which is more than regained during the after period. The tendency also appears to be to decrease the quantity of solids in the urine during the preservative period, and this tendency is continued during the after period.

No. 8 .

The quantity of solids in the food of No. 8 in the fore period is 531 grams daily, in the preservative period 575 grams daily, and in the after period 587 grams daily. Of this quantity there are found in the feces 17 grams in the fore period, 19 grams in the preservative period, and $21 \mathrm{grams}$ daily in the after period. In the urine there are found 54 grams daily in the fore period, 56 grams daily in the preservative period, and 60 grams daily in the after period. The largest percentage of solids in the feces occurs in the after period, namely, 3.56 per cent, and the smallest in the fore period, namely, 3.14. The largest per centage of solids in the urine occurs in the after period, namely, 10.25 and the smallest during the preservative period, namely, 9.71 per cent. The largest balance is found in the after period, namely, 506 grams daily, and the smallest in the fore period, namely, 461 grams daily. There is an apparent tendency in this case for the preservative to increase the quantity of solids in the feces, and this tendency is maintained through the after period. There is also manifested a tendency on the part of the preservative to increase the actual amount of solids in the urine in the preservative period, and this is continued in the after period. There is, however, a decrease in the percentage amount excreted in the urine in the preservative period. 
No. 9 .

The daily quantity of solids in the food of No. 9 is 634 grams in the fore period, 624 grams in the preservative period, and 623 grams in after period. Of this quantity there are found in the feces 16 grams in the fore period, 23 grams in the preservative period, and 18 grams in the after period. In the urine there are found $66 \mathrm{grams}$ in the fore period, 71 grams in the preservative period, and 72 grams in the after period.

The largest percentage of solids in the feces occurs in the preservative period, namely, 3.75 per cent, and the smallest in the fore period, namely, 2.44 per cent. The largest percentage of solids in the urine occurs in the after period-11.55 per cent-and the smallest in the fore period-10.48 per cent. The balance was positive in all cases, the magnitude being greatest in the fore period and least in the preservative period.

For reasons given elsewhere (p. 587), the results obtained with this subject are not included in the summaries. They are stated here, however, as a matter of record.

\section{No. 10}

Owing to illness this subject was not connected with the experiment during the second half of the after period. The daily quantity of solids in the food of No. 10 in the fore period was 660 grams and in the preservative period 676 grams. Of this quantity there are found daily in the feces 21 and 18 grams, respectively, and in the urine 54 and 56 grams, respectively.

Expressed in the form of percentage, we have in the feces the elimination of 3.20 and 2.68 per cent, respectively, and in the urine 8.11 and 8.27 per cent, respectively.

The balance is somewhat increased in the preservative period. Notwithstanding the slightly increased consumption of solids in the preservative period, the solids eliminated in the feces are slightly less during the preservative period than in the fore period. The solids eliminated in the urine are approximately the same.

For reasons given elsewhere (p. 587), this subject is omitted from the summaries. The results are given here, however, as a matter of record.

\section{No. 11 .}

The total quantity of solids in the food of No. 11 in the fore period amounts to 621 grams daily, in the preservative period 622 grams daily, and in the after period 615 grams daily. It is thus seen that there is very little variation in the quantity of solids during the whole course of the observation. Of this amount 25 grams daily appear in 
the feces in the fore period, 24 grams in the preservative period, and 22 grams in the after period; 70 grams of solids appear daily in the urine in the fore period, 69 grams in the preservative period, and 66 grams daily in the after period. Expressed in percentages the largest percentage of the solids in the feces is in the fore period, namely, 4.04 per cent, and the smallest in the after period, namely, 3.53 per cent. The largest percentage of solids is found in the urine in the fore period, namely, 11.27 per cent, and the smallest in the after period, namely, 10.73 per cent. The largest balance in this case is in the preservative period, namely, 530 grams daily, and the smallest in the fore period, namely, 526 grams daily. In this case the effect of the preservative upon the solids balance is very slight. There is a tendency, however, to diminish the solids in the feces during the preservative period and this tendency is increased in the after period. The solids in the urine in the fore period and preservative period are almost identical, but there is a small decrease in the after period perhaps due in part to the slightly decreased quantity of solids in the food; the percentage of decrease, however, is slightly greater in the after than in the preservative period.

No. 12.

The total quantity of solids in the food of No. 12 in the fore period is 660 grams daily, in the preservative period 653 grams daily, and in the after period 640 grams daily. The total solids in the food in this case diminished slightly in the preservative period and again in the after period. Of these solids 27 grams were found daily in the feces of the fore period, 21 grams in the preservative period, and 23 grams in the after period. In the urine 63 grams of solids daily are found in the fore period, 65 grams in the preservative period, and 67 grams in the after period. The largest percentage of solids in the feces is in the fore period, namely, 4.12 per cent, and the smallest in the preservative period, namely, 3.20 per cent. In the urine the largest percentage of solids is found in the after period, namely, 10.54 per cent, and the smallest in the fore period, namely, 9.56 per cent. The largest balance is found in the fore period, namely, 570 grams daily, and the smallest in the after period, namely, 550 grams daily. In this case the preservative exerts a marked tendency to diminish the amount of solids in the feces during the preservative period, a tendency which was only partly overcome in the after period. 
SUMMARY.

The summary by periods only for the nine men completing the series is given in the table following, the averages having been taken from Table XXIII (page 689), giving the solids balances in full:

TABLE XXII.-Solids summary, by periods, for nine men, Series VI.

\begin{tabular}{|c|c|c|c|c|c|c|}
\hline Period. & $\begin{array}{c}\text { Solids in } \\
\text { food. }\end{array}$ & $\begin{array}{c}\text { Solids in } \\
\text { feces. }\end{array}$ & $\begin{array}{l}\text { Solids in } \\
\text { urine. }\end{array}$ & $\begin{array}{l}\text { Solidis in } \\
\text { feces. }\end{array}$ & $\begin{array}{l}\text { Solids in } \\
\text { urine. }\end{array}$ & Balance. \\
\hline 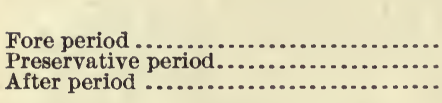 & $\begin{array}{r}\text { Grams. } \\
\mathbf{5 5 5} \\
564 \\
568\end{array}$ & $\begin{array}{r}\text { Grams. } \\
22 \\
19 \\
20\end{array}$ & $\begin{array}{r}\text { Grams. } \\
59 \\
62 \\
63\end{array}$ & $\begin{array}{r}\text { Per cent. } \\
3.92 \\
3.46 \\
3.59\end{array}$ & $\begin{array}{r}\text { Per cent. } \\
10.75 \\
11.01 \\
11.02\end{array}$ & $\begin{array}{r}\text { Grams. } \\
474 \\
483 \\
485\end{array}$ \\
\hline
\end{tabular}

The average daily amount of solids consumed increased slightly from period to period, there being an increase of 9 grams in the preservative period and 4 grams additional increase in the after period. The largest percentage of solids in the feces is found in the fore period, namely, 3.92 per cent, and the smallest in the preservative period, namely, 3.46 per cent, while the quantity in the after period is 3.59 per cent. The smallest percentage of solids in the urine is found in the fore period, namely, 10.75 per cent, while the quantities in the preservative period and the after period are almost identical. The largest balance is found in the after period, namely, 485 grams daily, the smallest in the fore period, namely, 474 grams daily, while the balance for the preservative period is almost the same as that of the after period, namely, 483 grams daily.

These data show a tendency on the part of the preservative to increase the absorption from the alimentary canal of the solids in the food, as shown by the decrease of solids in the feces, and also to increase the katabolic activities of the body as indicated by the increased excretion of solids in the urine. 
TABLE XXIII.-Solids balances for Series VI.

[Averages are per day.]

No. 1 .

\begin{tabular}{|c|c|c|c|c|c|c|c|c|c|}
\hline Period. & $\begin{array}{c}1 \\
\text { In food. }\end{array}$ & In feces. & $\begin{array}{c}3 \\
\text { In urine. }\end{array}$ & $\begin{array}{c}4 \\
\text { In feces } \\
\text { and } \\
\text { urine. } \\
(2+3)\end{array}$ & $\begin{array}{c}5 \\
\text { In } \\
\text { feces. } \\
(2 \div 1)\end{array}$ & $\mid \begin{array}{c}6 \\
\text { In } \\
\text { urine. } \\
(3 \div 1)\end{array}$ & \begin{tabular}{|c|}
7 \\
\\
In feces \\
and \\
urine. \\
$(4 \div 1)$
\end{tabular} & $\begin{array}{c}8 \\
\begin{array}{c}\text { Balance. } \\
(1-4)\end{array}\end{array}$ & $\begin{array}{c}\mathbf{9} \\
\text { Sali- } \\
\text { cylic } \\
\text { acid } \\
\text { ad- } \\
\text { minis- } \\
\text { tered. }\end{array}$ \\
\hline Fore period. & & & & & & & & & \\
\hline $\begin{array}{l}\text { First subperiod: } \\
\quad \text { Total............. } \\
\text { Average } . . . . . .\end{array}$ & $\begin{array}{r}\text { Grams. } \\
2,394 \\
479\end{array}$ & $\begin{array}{r}\text { Grams. } \\
78 \\
16\end{array}$ & $\begin{array}{r}\operatorname{Grams.} \\
269 \\
54\end{array}$ & $\begin{array}{r}\text { Grams. } \\
347 \\
69\end{array}$ & $\begin{array}{r}\text { Per ct. } \\
3.26\end{array}$ & $\begin{array}{l}\text { Per ct. } \\
11.24\end{array}$ & $\begin{array}{l}\text { Per ct. } \\
14.49\end{array}$ & $\begin{array}{r}\underset{2,047}{\operatorname{Grams}} \\
410\end{array}$ & $\begin{array}{c}\text { Grams. } \\
0 \\
0\end{array}$ \\
\hline 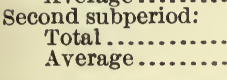 & $\begin{array}{r}2,275 \\
455\end{array}$ & $\begin{array}{l}62 \\
12\end{array}$ & $\begin{array}{r}306 \\
61\end{array}$ & $\begin{array}{r}368 \\
74\end{array}$ & 2.73 & 13.45 & 16.18 & $\begin{array}{r}1,907 \\
381\end{array}$ & $\begin{array}{l}0 \\
0\end{array}$ \\
\hline $\begin{array}{l}\text { Entire fore period: } \\
\text { Total .................. } \\
\text { Average........ }\end{array}$ & $\begin{array}{r}4,669 \\
467 \\
\end{array}$ & $\begin{array}{r}140 \\
14\end{array}$ & $\begin{array}{r}575 \\
58\end{array}$ & $\begin{array}{r}715 \\
72\end{array}$ & 3.00 & $\begin{array}{l}12.32 \\
\ldots . . .\end{array}$ & 15. 31 & $\begin{array}{r}3,954 \\
395\end{array}$ & $\begin{array}{l}0 \\
0\end{array}$ \\
\hline Preservative period. & & & & & & & & & \\
\hline $\begin{array}{l}\text { First subperiod: } \\
\quad \text { Total } \ldots . . . . . . . . . \\
\text { Average........ }\end{array}$ & $\begin{array}{r}2,360 \\
472\end{array}$ & $\begin{array}{l}62 \\
12\end{array}$ & $\begin{array}{r}301 \\
60\end{array}$ & $\begin{array}{r}363 \\
73\end{array}$ & 2.63 & 12.75 & 15.38 & $\begin{array}{r}1,997 \\
399\end{array}$ & $\begin{array}{r}1.05 \\
.21\end{array}$ \\
\hline $\begin{array}{l}\text { Second sub } \\
\text { Total. } \\
\text { Averag }\end{array}$ & $\begin{array}{r}2,338 \\
468\end{array}$ & $\begin{array}{l}56 \\
11\end{array}$ & $\begin{array}{r}314 \\
63\end{array}$ & $\begin{array}{r}370 \\
74\end{array}$ & 2.40 & 13.43 & 15.83 & $\begin{array}{r}1,968 \\
394\end{array}$ & $\begin{array}{r}2.10 \\
.42\end{array}$ \\
\hline $\begin{array}{l}\text { od: } \\
\cdots \cdots . . . \\
\cdots \cdots\end{array}$ & $\begin{array}{r}2,340 \\
468\end{array}$ & $\begin{array}{l}63 \\
13\end{array}$ & $\begin{array}{r}\alpha 308 \\
62\end{array}$ & $\begin{array}{r}371 \\
74\end{array}$ & 2.69 & 13.16 & 15.85 & $\begin{array}{r}1,969 \\
394\end{array}$ & $\begin{array}{r}3.70 \\
.74\end{array}$ \\
\hline $\begin{array}{l}\text { Fourth si } \\
\text { Total } \\
\text { Aver }\end{array}$ & $\begin{array}{r}2,305 \\
461\end{array}$ & $\begin{array}{l}66 \\
13\end{array}$ & $\begin{array}{r}314 \\
63\end{array}$ & $\begin{array}{r}380 \\
76\end{array}$ & 2.86 & 13.62 & 16.49 & $\begin{array}{r}1,925 \\
385\end{array}$ & $\begin{array}{l}6.00 \\
1.20\end{array}$ \\
\hline Fifth subp & $\begin{aligned} 2,568 \\
514\end{aligned}$ & 73 & $\begin{array}{r}311 \\
62\end{array}$ & $\begin{array}{r}384 \\
77\end{array}$ & 2.84 & 12.11 & 14.95 & $\begin{array}{r}2,184 \\
437\end{array}$ & $\begin{array}{l}8.00 \\
1.60\end{array}$ \\
\hline $\begin{array}{l}\text { Sixth subperiod: } \\
\quad \text { Total } \\
\text { Average..................... }\end{array}$ & $\begin{array}{r}2,315 \\
463\end{array}$ & $\begin{array}{l}59 \\
12\end{array}$ & $\begin{array}{r}319 \\
64\end{array}$ & $\begin{array}{r}378 \\
76\end{array}$ & 2.55 & 13.78 & 16.33 & $\begin{array}{r}1,937 \\
387\end{array}$ & $\begin{array}{r}10.00 \\
2.00\end{array}$ \\
\hline $\begin{array}{l}\text { Entire preservative } \\
\text { period: } \\
\text { Total.............. } \\
\text { Average......... }\end{array}$ & $\begin{array}{r}14,226 \\
474\end{array}$ & $\begin{array}{r}379 \\
13\end{array}$ & $\begin{array}{r}1,867 \\
62\end{array}$ & $\begin{array}{r}2,246 \\
75\end{array}$ & 2.66 & 13. 12 & 15.79 & $\begin{array}{r}11,980 \\
399\end{array}$ & $\begin{array}{r}30.85 \\
1.03\end{array}$ \\
\hline After period. & & & & & & & & & \\
\hline $\begin{array}{l}\text { First subperiod: } \\
\text { Total.............. } \\
\text { Average......... }\end{array}$ & $\begin{array}{r}2,325 \\
465\end{array}$ & $\begin{array}{r}110 \\
22\end{array}$ & $\begin{array}{r}317 \\
63\end{array}$ & $\begin{array}{r}427 \\
85\end{array}$ & 4.73 & 13.63 & 18.37 & $\begin{array}{r}1,898 \\
380\end{array}$ & $\begin{array}{l}0 \\
0\end{array}$ \\
\hline 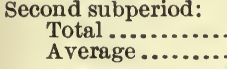 & $\begin{array}{r}2,435 \\
487 .\end{array}$ & $\begin{array}{l}84 \\
17\end{array}$ & $\begin{array}{r}315 \\
63\end{array}$ & $\begin{array}{r}399 \\
80\end{array}$ & 3.45 & 12.94 & 16.39 & $\begin{array}{r}2,036 \\
407\end{array}$ & $\begin{array}{l}0 \\
0\end{array}$ \\
\hline $\begin{array}{l}\text { Entire after period: } \\
\text { Total .............. } \\
\text { Average ........ }\end{array}$ & $\begin{array}{r}4,760 \\
476\end{array}$ & $\begin{array}{r}194 \\
19\end{array}$ & $\begin{array}{r}632 \\
63\end{array}$ & $\begin{array}{r}826 \\
83\end{array}$ & 4.08 & 13.28 & 17.35 & $\begin{array}{r}3,934 \\
393\end{array}$ & $\begin{array}{l}0 \\
0\end{array}$ \\
\hline
\end{tabular}

$a$ Daily average added in order to complete record. 
TABLE XXIII.-Solids balances for Series VI-Continued.

[Averages are per day.]

No. 2 .

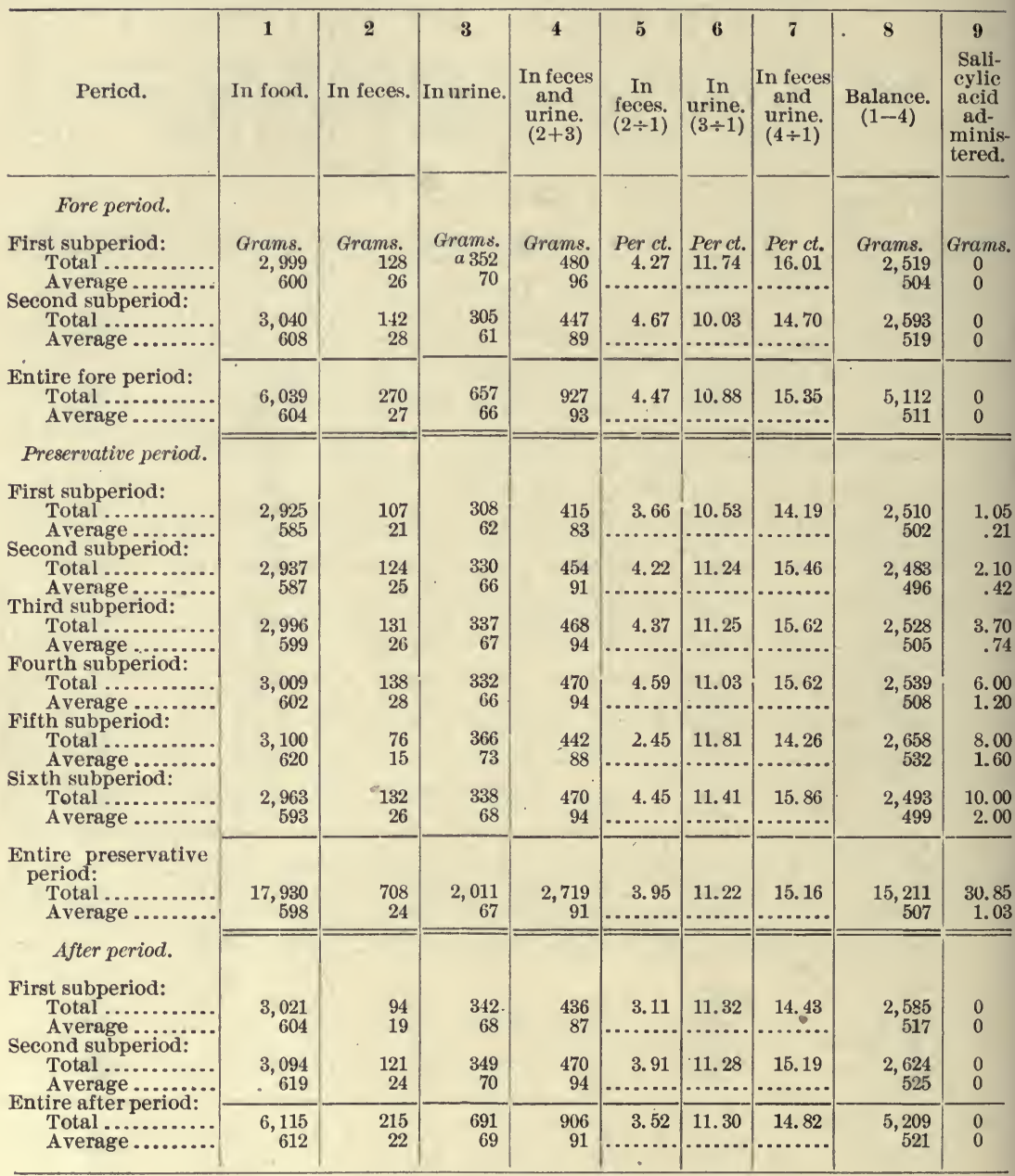

$a$ Daily average added in order to complete record. 
SALICYLIC ACID AND SALICYLATES.

TABLE XXIII.-Solids balances for Series VI-Continued.

[Averages are per day.]

No. 3.

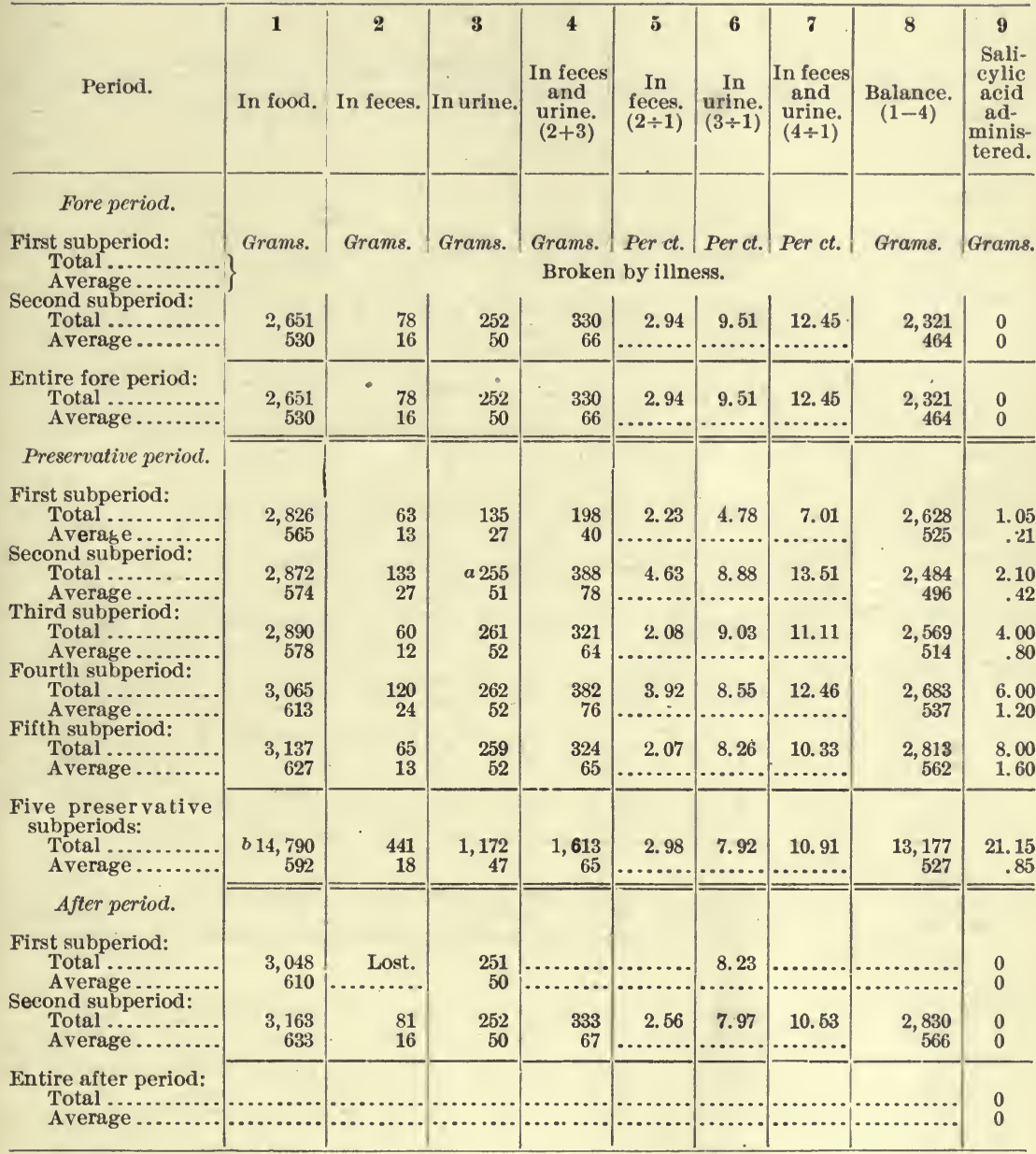

$a$ Daily average added in order to complete record.

$b$ No. 3 had only five preservative subperiods. 
TABle XXIII.-Solids balances for Series VI-Continued.

[Averages are per day.]

No. 4.

\begin{tabular}{|c|c|c|c|c|c|c|c|c|c|}
\hline Period. & In food. & In feces. & In urine. & $\begin{array}{c}4 \\
\text { In feces } \\
\text { and } \\
\text { urine. } \\
(2+3)\end{array}$ & $\begin{array}{c}5 \\
\text { In } \\
\text { feces. } \\
(2 \div 1)\end{array}$ & $\begin{array}{c}\text { In } \\
\text { urine. } \\
(3 \div 1)\end{array}$ & $\begin{array}{c}7 \\
\text { In feces } \\
\text { and } \\
\text { urine. } \\
(4 \div 1)\end{array}$ & $\begin{array}{c}\text { Balance. } \\
(1-4)\end{array}$ & $\begin{array}{c}9 \\
\text { Sali- } \\
\text { cylic } \\
\text { acid } \\
\text { ad- } \\
\text { minis- } \\
\text { tered. }\end{array}$ \\
\hline \multicolumn{10}{|l|}{ Fore period. } \\
\hline $\begin{array}{l}\text { First subperiod: } \\
\text { Total ............ } \\
\text { Average } \ldots \ldots \ldots \ldots\end{array}$ & $\begin{array}{r}\text { Grams. } \\
2,680 \\
536\end{array}$ & $\begin{array}{r}\text { Grams. } \\
93 \\
19\end{array}$ & $\begin{array}{r}\text { Grams. } \\
315 \\
63\end{array}$ & $\begin{array}{r}\text { Grams. } \\
408 \\
82\end{array}$ & $\begin{array}{r}\text { Per ct. } \\
3.47 \\
\ldots\end{array}$ & $\begin{array}{l}\text { Perct. } \\
11.75 \\
\cdots\end{array}$ & $\begin{array}{c}\text { Per ct. } \\
15.22 \\
\cdots \cdots\end{array}$ & $\begin{array}{r}\text { Grams. } \\
2,272 \\
454\end{array}$ & $\begin{array}{c}\text { Grams. } \\
0 \\
0\end{array}$ \\
\hline $\begin{array}{l}\text { Second subperiod: } \\
\text { Total ............... } \\
\text { Average ......... }\end{array}$ & $\begin{array}{r}2,671 \\
534\end{array}$ & $\begin{array}{r}108 \\
22\end{array}$ & $\begin{array}{r}312 \\
62\end{array}$ & $\begin{array}{r}420 \\
84\end{array}$ & $\begin{array}{r}4.04 \\
\ldots . . .\end{array}$ & $\begin{array}{c}11.68 \\
\cdots \cdots\end{array}$ & $\begin{array}{r}15.72 \\
\ldots \ldots . \cdots\end{array}$ & $\begin{array}{r}2,251 \\
450\end{array}$ & $\begin{array}{l}0 \\
0\end{array}$ \\
\hline $\begin{array}{l}\text { Entire fore period: } \\
\text { Total } \ldots . . . \\
\text { Average } \ldots \ldots \\
\end{array}$ & $\begin{array}{r}5,351 \\
535\end{array}$ & $\begin{array}{r}201 \\
20\end{array}$ & $\begin{array}{r}627 \\
63 .\end{array}$ & $\begin{array}{r}828 \\
\quad 83\end{array}$ & $\begin{array}{r}3.76 \\
\cdots \cdots . . .\end{array}$ & $\begin{array}{c}11.72 \\
\cdots \cdots\end{array}$ & $\begin{array}{r}15.47 \\
\cdots \cdots\end{array}$ & $\begin{array}{r}4,523 \\
452\end{array}$ & $\begin{array}{l}0 \\
0\end{array}$ \\
\hline \multicolumn{10}{|l|}{ Preservative period. } \\
\hline $\begin{array}{l}\text { First subperiod: } \\
\text { Total............. } \\
\text { Average......... }\end{array}$ & $\begin{array}{r}2,751 \\
550\end{array}$ & $\begin{array}{l}84 \\
17\end{array}$ & $\begin{array}{r}319 \\
64\end{array}$ & $\begin{array}{r}403 \\
81\end{array}$ & 3.05 & 11. 60 & 14.65 & $\begin{array}{r}2,348 \\
469\end{array}$ & $\begin{array}{r}1.05 \\
.21\end{array}$ \\
\hline $\begin{array}{l}\text { Second subperiod: } \\
\text { Total }\end{array}$ & 2,736 & 102 & 330 & 432 & 3. 73 & 1206 & & & \\
\hline Average............... & $\begin{array}{r}2,736 \\
547\end{array}$ & 20 & $\begin{array}{r}350 \\
66\end{array}$ & $\begin{array}{r}432 \\
86\end{array}$ & 3.73 & 12.06 & $\begin{array}{l}15.79 \\
\ldots \ldots \ldots\end{array}$ & $\begin{array}{r}2,304 \\
461\end{array}$ & $\begin{array}{r}2.10 \\
.42\end{array}$ \\
\hline $\begin{array}{l}\text { Third subperiod: } \\
\text { Total .......... }\end{array}$ & & & & & & & & & \\
\hline Avera & $\begin{array}{r}2,788 \\
558\end{array}$ & $\begin{array}{l}98 \\
20\end{array}$ & $\begin{array}{r}332 \\
66\end{array}$ & $\begin{array}{r}430 \\
86\end{array}$ & 3.52 & 11.91 & 15.42 & $\begin{array}{r}2,358 \\
472\end{array}$ & $\begin{array}{r}3.70 \\
.74\end{array}$ \\
\hline ibperiod: & & & & & & & & & \\
\hline (n....... & 2,765 & a 85 & 346 & 431 & 3.07 & 12.51 & 15.59 & 2,334 & 6.00 \\
\hline Fifth subperiod: & bอ3 & 17. & 69 & 86 & & ….... & (........ & 467 & 1.20 \\
\hline $\begin{array}{l}\text { Total } \ldots \ldots \ldots \\
\text { Average } \ldots\end{array}$ & 2,862 & 117 & 330 & 447 & 4. 09 & 11.53 & 15.62 & 2,415 & 8.00 \\
\hline Sixth subperiod: & & & 66 & 89 & & & & 483 & 1.60 \\
\hline $\begin{array}{l}\text { Total } \\
\text { Average.............. }\end{array}$ & 2,781 & $\begin{array}{r}a 98 \\
20\end{array}$ & 355 & $\begin{array}{r}453 \\
91\end{array}$ & 3.52 & 12.77 & 16.29 & 2,328 & 10.00 \\
\hline \\
\hline & & & & & & & & & \\
\hline 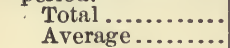 & 16,683 & 584 & 2,012 & 2,596 & 3.50 & 12.06 & 15.56 & 14,087 & 30.85 \\
\hline & & & & & & & & & 1.03 \\
\hline After period. & & & & & & & & & \\
\hline First subperiod: & 2,766 & 78 & 343 & 421 & 2.82 & 12.40 & 15.22 & 2,345 & 0 \\
\hline $\begin{array}{l}\text { Average } \\
\text { cond suberiod:............... }\end{array}$ & 553 & 16 & 69 & 84 & & & & 469 & 0 \\
\hline $\begin{array}{l}\text { Second subperiod: } \\
\text { Total ............. }\end{array}$ & 2,922 & 106 & 355 & 461 & 3.63 & 12,15 & 15. 78 & 2,461 & 0 \\
\hline Average............... & 584 & 21 & 71 & 92 & ……. & & & 492 & 0 \\
\hline $\begin{array}{l}\text { Entire after period: } \\
\text { Total ............ } \\
\text { Average ......... }\end{array}$ & $\begin{array}{r}5,688 \\
569\end{array}$ & $\begin{array}{r}184 \\
18\end{array}$ & $\begin{array}{r}698 \\
70\end{array}$ & $\begin{array}{r}882 \\
88\end{array}$ & 3.24 & 12.27 & 15.51 & $\begin{array}{r}4,806 \\
481\end{array}$ & $\begin{array}{l}0 \\
0\end{array}$ \\
\hline
\end{tabular}

$a$ Daily average added in order to complete record. 
Table XXIII.-Solids balances for Series VI-Continued.

[Averages are per day.]

No. 5.

\begin{tabular}{|c|c|c|c|c|c|c|c|c|c|}
\hline Period. & In food. & In feces. & In urine. & $\begin{array}{l}\mathbf{4} \\
\text { In feces } \\
\text { and } \\
\text { urine. } \\
(2+3)\end{array}$ & $\begin{array}{c}\text { 5 } \\
\text { In } \\
\text { feces. } \\
(2 \div 1)\end{array}$ & $\begin{array}{l}6 \\
\text { In } \\
\text { urine. } \\
(3 \div 1)\end{array}$ & \begin{tabular}{|}
7 \\
In feces \\
and \\
urine. \\
$(4 \div 1)$
\end{tabular} & $\begin{array}{c}\text { Balance. } \\
(1-4)\end{array}$ & $\begin{array}{c}9 \\
\text { Sali- } \\
\text { cylic } \\
\text { acid } \\
\text { ad- } \\
\text { minis- } \\
\text { tered. }\end{array}$ \\
\hline Fore period. & & & & & & & & & \\
\hline 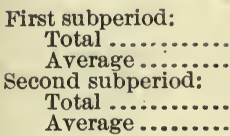 & $\begin{array}{r}\text { Grams. } \\
2,824 \\
565 \\
\\
2,758 \\
552\end{array}$ & $\begin{array}{r}\text { Grams. } \\
132 \\
26\end{array}$ & $\begin{array}{r}\text { Grams. } \\
295 \\
59\end{array}$ & $\begin{array}{r}\text { Grams. } \\
427 \\
85\end{array}$ & $\begin{array}{r}\text { Per ct. } \\
4.67 \\
4.39 \\
4.39\end{array}$ & $\begin{array}{l}\text { Per ct. } \\
10.45 \\
\cdots \\
10.01 \\
\cdots\end{array}$ & $\begin{array}{r}\text { Per ct. } \\
15.12 \\
\cdots\end{array}$ & $\begin{array}{r}\text { Grams. } \\
2,397 \\
480 \\
\\
2,361 \\
473\end{array}$ & $\begin{array}{c}\text { Grams. } \\
0 \\
0\end{array}$ \\
\hline $\begin{array}{l}\text { Entire fore period: } \\
\text { Total .............. } \\
\text { Average ....... }\end{array}$ & $\begin{array}{r}5,582 \\
558\end{array}$ & $\begin{array}{r}253 \\
25\end{array}$ & $\begin{array}{r}571 \\
57\end{array}$ & $\begin{array}{r}824 \\
.82 \\
\end{array}$ & $\begin{array}{r}4.53 \\
\cdots . . .\end{array}$ & $\begin{array}{l}10.23 \\
\ldots \ldots . .\end{array}$ & \begin{tabular}{|c|}
14.76 \\
$\cdots$
\end{tabular} & $\begin{array}{r}4,758 \\
476\end{array}$ & $\begin{array}{l}0 \\
0\end{array}$ \\
\hline Preservative period. & & & & & & & & & \\
\hline $\begin{array}{l}\text { First subperiod: } \\
\text { Total ............ } \\
\text { Average......... }\end{array}$ & $\begin{array}{r}2,867 \\
573\end{array}$ & $\begin{array}{r}126 \\
25\end{array}$ & $\begin{array}{r}297 \\
59\end{array}$ & $\begin{array}{r}423 \\
85\end{array}$ & $\begin{array}{c}4.40 \\
\ldots . . .\end{array}$ & $\begin{array}{l}10.36 \\
\cdots\end{array}$ & $\begin{array}{c}14.75 \\
\ldots \ldots . .\end{array}$ & $\begin{array}{r}2,444 \\
488\end{array}$ & $\begin{array}{r}1.05 \\
.21\end{array}$ \\
\hline $\begin{array}{l}\text { Second subperiod: } \\
\text { Total ............... } \\
\text { Average ......... } \\
\text { Third subperiod: }\end{array}$ & $\begin{array}{r}2,753 \\
551\end{array}$ & $\begin{array}{l}89 \\
18\end{array}$ & $\begin{array}{r}292 \\
58\end{array}$ & $\begin{array}{r}381 \\
76\end{array}$ & $\begin{array}{r}3.23 \\
\cdots \\
\cdots\end{array}$ & $\begin{array}{l}10.61 \\
\ldots \ldots .\end{array}$ & $\begin{array}{r}13.84 \\
\ldots . . .\end{array}$ & $\begin{array}{r}2,372 \\
475\end{array}$ & $\begin{array}{r}2.10 \\
.42\end{array}$ \\
\hline 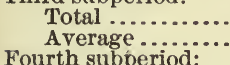 & $\begin{array}{r}2,787 \\
557\end{array}$ & $\begin{array}{r}113 \\
23\end{array}$ & $\begin{array}{r}306 \\
61\end{array}$ & $\begin{array}{r}419 \\
84\end{array}$ & $\begin{array}{r}4.05 \\
\cdots\end{array}$ & $\begin{array}{l}10.98 \\
\ldots . .\end{array}$ & $\begin{array}{r}15.03 \\
\ldots . . .\end{array}$ & $\begin{array}{r}2,368 \\
473\end{array}$ & $\begin{array}{r}3.70 \\
.74\end{array}$ \\
\hline $\begin{array}{r}\text { Total ................... } \\
\text { Average }\end{array}$ & $\begin{array}{r}2,804 \\
561\end{array}$ & $\begin{array}{l}82 \\
16\end{array}$ & $\begin{array}{r}321 \\
64\end{array}$ & $\begin{array}{r}403 \\
81\end{array}$ & $\begin{array}{r}2.92 \\
\ldots .\end{array}$ & $\begin{array}{l}11.45 \\
\cdots\end{array}$ & $\begin{array}{r}14.37 \\
\ldots . . .\end{array}$ & $\begin{array}{r}2,401 \\
480\end{array}$ & $\begin{array}{l}6.00 \\
1.20\end{array}$ \\
\hline 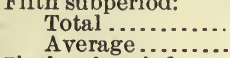 & $\begin{array}{r}2,862 \\
572\end{array}$ & $\begin{array}{r}107 \\
21\end{array}$ & $\begin{array}{r}321 \\
64\end{array}$ & $\begin{array}{r}428 \\
86\end{array}$ & $\begin{array}{r}3.74 \\
\ldots . . .\end{array}$ & $\begin{array}{l}11.22 \\
\cdots . . .\end{array}$ & $\begin{array}{r}14.95 \\
\ldots \ldots .\end{array}$ & $\begin{array}{r}2,434 \\
486\end{array}$ & $\begin{array}{l}8.00 \\
1.60\end{array}$ \\
\hline $\begin{array}{l}\text { Sixth subperiod: } \\
\text { Total .............. } \\
\text { Average........ }\end{array}$ & $\begin{array}{r}2,814 \\
563\end{array}$ & $\begin{array}{r}115 \\
23\end{array}$ & $\begin{array}{r}317 \\
63\end{array}$ & $\begin{array}{r}432 \\
86\end{array}$ & $\begin{array}{r}4.09 \\
\ldots .\end{array}$ & $\begin{array}{l}11.26 \\
\cdots \ldots .\end{array}$ & $\begin{array}{c}15.35 \\
\therefore \ldots .\end{array}$ & $\begin{array}{r}2,382 \\
477\end{array}$ & $\begin{array}{r}10.00 \\
2.00\end{array}$ \\
\hline $\begin{array}{l}\text { Entire preservative } \\
\text { period: } \\
\text { Total .............. } \\
\text { Average .......... }\end{array}$ & $\begin{array}{r}16,887 \\
563\end{array}$ & $\begin{array}{r}632 \\
21\end{array}$ & $\begin{array}{r}1,854 \\
62\end{array}$ & $\begin{array}{r}2,486 \\
83\end{array}$ & $\begin{array}{r}3.74 \\
\ldots . . .\end{array}$ & $\begin{array}{l}10.98 \\
\cdots \ldots . .\end{array}$ & $\begin{array}{r}14.72 \\
\ldots \ldots\end{array}$ & $\begin{array}{r}14,401 \\
480\end{array}$ & $\begin{array}{r}30.85 \\
1.03\end{array}$ \\
\hline After period. & & & & & & & & & \\
\hline $\begin{array}{l}\text { First subperiod: } \\
\text { Total } \ldots . . . . . . . \\
\text { Average......... }\end{array}$ & $\begin{array}{r}2,791 \\
558\end{array}$ & $\begin{array}{r}101 \\
20\end{array}$ & $\begin{array}{r}a 312 \\
62\end{array}$ & $\begin{array}{r}413 \\
83\end{array}$ & 3.62 & 11.18 & 14.80 & $\begin{array}{r}2,378 \\
475\end{array}$ & $\begin{array}{l}0 \\
0\end{array}$ \\
\hline $\begin{array}{l}\text { Second subperiod: } \\
\text { Total ................ } \\
\text { Average ........ }\end{array}$ & $\begin{array}{r}2,850 \\
570\end{array}$ & $\begin{array}{l}78 \\
16\end{array}$ & $\begin{array}{r}310 \\
62\end{array}$ & $\begin{array}{r}388 \\
78\end{array}$ & $\begin{array}{r}2.74 \\
\ldots . .6\end{array}$ & $\begin{array}{c}10.88 \\
\ldots \ldots . .\end{array}$ & $\begin{array}{r}13.61 \\
\ldots . . .\end{array}$ & $\begin{array}{r}2,462 \\
492\end{array}$ & $\begin{array}{l}0 \\
0\end{array}$ \\
\hline $\begin{array}{l}\text { Entire after period: } \\
\text { Total ............. } \\
\text { Average ......... }\end{array}$ & $\begin{array}{r}5,641 \\
564\end{array}$ & $\begin{array}{r}179 \\
18\end{array}$ & $\begin{array}{r}622 \\
62\end{array}$ & $\begin{array}{r}801 \\
80\end{array}$ & 3.17 & 11.03 & $\begin{array}{r}14.20 \\
\ldots . . .\end{array}$ & $\begin{array}{r}4,840 \\
484\end{array}$ & $\begin{array}{l}0 \\
0\end{array}$ \\
\hline
\end{tabular}

$a$ Daily average added in order to complete record. 
Table XXIII.-Solids balances for Series VI-Continued.

[Averages are per day.]

No. 6.

\begin{tabular}{|c|c|c|c|c|c|c|c|c|c|}
\hline Period. & In food. & In feces. & In urine. & $\begin{array}{c}4 \\
\text { In feces } \\
\text { and } \\
\text { urine. } \\
(2+3)\end{array}$ & $\begin{array}{c}\text { 5 } \\
\text { In } \\
\text { feces. } \\
(2 \div 1)\end{array}$ & $\begin{array}{c}6 \\
\text { In } \\
\text { urine. } \\
(3 \div 1)\end{array}$ & $\begin{array}{c}7 \\
\text { In feces } \\
\text { and } \\
\text { urine. } \\
(4 \div 1)\end{array}$ & $\begin{array}{c}\text { Balance. } \\
(1-4)\end{array}$ & $\begin{array}{c}9 \\
\text { Sali- } \\
\text { cylic } \\
\text { acid } \\
\text { ad- } \\
\text { minis- } \\
\text { tered. }\end{array}$ \\
\hline \multicolumn{10}{|l|}{ Fore period. } \\
\hline $\begin{array}{l}\text { First subperiod: } \\
\text { Total ............... }\end{array}$ & $\begin{array}{l}\text { Grams. } \\
2,631\end{array}$ & $\begin{array}{r}\text { Grams. } \\
119\end{array}$ & $\underset{242}{\text { Grams. }}$ & Grams. & $\begin{array}{l}\text { Per ct. } \\
\quad 4.52\end{array}$ & $\begin{array}{c}\text { Per ct. } \\
9.20\end{array}$ & $\begin{array}{r}\text { Per ct. } \\
13.72\end{array}$ & $\begin{array}{l}\text { Grams. } \\
2,270\end{array}$ & $\begin{array}{l}\text { Grams. } \\
\quad 0\end{array}$ \\
\hline $\begin{array}{l}\text { Average ............ } \\
\text { Second suberiod: }\end{array}$ & 526 & 24 & 48 & 72 & & & .......... & 454 & 0 \\
\hline $\begin{array}{l}\text { Second subperiod: } \\
\text { Total .................. }\end{array}$ & 2,736 & 141 & 252 & 393 & 5.15 & 9.21 & 14.36 & 2,343 & 0 \\
\hline Average............ & 547 & 28 & 50 & 79 & ......... & ....... & ........ & 468 & 0 \\
\hline $\begin{array}{c}\text { Entire fore period: } \\
\text { Total ............. }\end{array}$ & 5,367 & $\begin{array}{r}260 \\
26\end{array}$ & $\begin{array}{r}494 \\
49\end{array}$ & 754 & 4.84 & 9.20 & 14.05 & 4,613 & 0 \\
\hline Average....$\ldots \ldots$ & 537 & 26 & 49 & 75 & - $\ldots \ldots$ & $\ldots \ldots$ & ......... & 462 & 0 \\
\hline \multicolumn{10}{|l|}{ Preservative period. } \\
\hline \multicolumn{10}{|l|}{ First subperiod: } \\
\hline & 520 & 22 & 54 & 77 & 1.00 & 10.70 & ....... & 443 & $\begin{array}{r}1.00 \\
.21\end{array}$ \\
\hline \multicolumn{10}{|l|}{$\begin{array}{c}\text { Second subperiod: } \\
\text { Total ...................... }\end{array}$} \\
\hline $\begin{array}{l}\text { Average } \\
\text { Third subperiod: }\end{array}$ & 544 & 26 & $\begin{array}{r}270 \\
55\end{array}$ & $\begin{array}{r}405 \\
81\end{array}$ & $\begin{array}{c}4.78 \\
\ldots \ldots . .\end{array}$ & $\begin{array}{c}10.11 \\
\ldots \ldots\end{array}$ & $\begin{array}{r}14.88 \\
\ldots . . .\end{array}$ & $\begin{array}{r}2,316 \\
463\end{array}$ & $\begin{array}{r}2.10 \\
42\end{array}$ \\
\hline \multicolumn{10}{|l|}{ Third subperiod: } \\
\hline 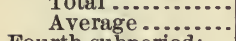 & $\begin{array}{r}2,745 \\
549\end{array}$ & $\begin{array}{r}143 \\
29\end{array}$ & $\begin{array}{r}a 276 \\
55\end{array}$ & - $\begin{array}{r}419 \\
84\end{array}$ & 5.21 & 10.05 & 15.27 & 2,326 & 3. 70 \\
\hline \multicolumn{10}{|l|}{ Fourth subperiod: } \\
\hline Total ........................ & $\begin{array}{l}2,778 \\
556\end{array}$ & $\begin{array}{r}117 \\
23\end{array}$ & $\begin{array}{r}a 307 \\
61\end{array}$ & 424 & 4.21 & 11.05 & 15.26 & 2,354 & 6.00 \\
\hline \multicolumn{10}{|l|}{ Fifth subperiod: } \\
\hline 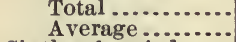 & 2,874 & 132 & 304 & 436 & 4.59 & 10.58 & 15.17 & 2,438 & 8.00 \\
\hline \multicolumn{10}{|l|}{ Sixth subperiod: } \\
\hline Total.......... & $\begin{array}{r}2,960 \\
592\end{array}$ & $\begin{array}{r}121 \\
24\end{array}$ & $\begin{array}{r}289 \\
58\end{array}$ & $\begin{array}{r}410 \\
82\end{array}$ & $\begin{array}{l}4.09 \\
\cdots \cdots\end{array}$ & 9.76 & $\begin{array}{r}13.85 \\
\cdots \cdots\end{array}$ & $\begin{array}{r}2,550 \\
510\end{array}$ & $\begin{array}{l}8.00 \\
1.60\end{array}$ \\
\hline \multicolumn{10}{|l|}{$\begin{array}{l}\text { Entire preservative } \\
\text { period: }\end{array}$} \\
\hline $\begin{array}{l}\text { periof: } \\
\text { Total ............... }\end{array}$ & 16,680 & 755 & 1,723 & 2,478 & 4.53 & 10,33 & 14.86 & & \\
\hline Average...... & 556 & 25 & 57 & 83 & & .......... & .......... & 473 & .96 \\
\hline \multicolumn{10}{|l|}{ After period. } \\
\hline 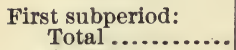 & & & & & & & & & \\
\hline $\begin{array}{l}\text { Average } \\
\text { Second subperiod: }\end{array}$ & 552 & $\begin{array}{r}140 \\
29\end{array}$ & 56 & $\begin{array}{r}425 \\
85\end{array}$ & $\begin{array}{c}5.25 \\
\cdots \cdots\end{array}$ & 10.07 & 15.32 & $\begin{array}{r}2,338 \\
467\end{array}$ & $\begin{array}{l}0 \\
0\end{array}$ \\
\hline $\begin{array}{l}\text { Second subperiod: } \\
\text { Total .................. }\end{array}$ & 2,848 & $a 95$ & 282 & & 3. 34 & 9.90 & 13.24 & 2,471 & 0 \\
\hline Average .......... & 570 & 19 & 56 & 75 & ........ & & .......... & 495 & 0 \\
\hline $\begin{array}{r}\text { Entire after period: } \\
\text { Total } \ldots . . . \\
\text { Average }\end{array}$ & $\begin{array}{r}5,609 \\
561\end{array}$ & $\begin{array}{r}240 \\
24\end{array}$ & $\begin{array}{r}560 \\
56\end{array}$ & $\begin{array}{r}800 \\
80\end{array}$ & $\begin{array}{r}4.28 \\
\ldots . . .\end{array}$ & 9.98 & 14.26 & $\begin{array}{r}4,809 \\
481\end{array}$ & $\begin{array}{l}0 \\
0\end{array}$ \\
\hline
\end{tabular}

$a$ Daily average added in order to complete record. 
SALICYLIC ACID AND SALICYLATES.

TABLE XXIII.-Solids balances for Series VI-Continued.

[Averages are per day.]

No. 7.

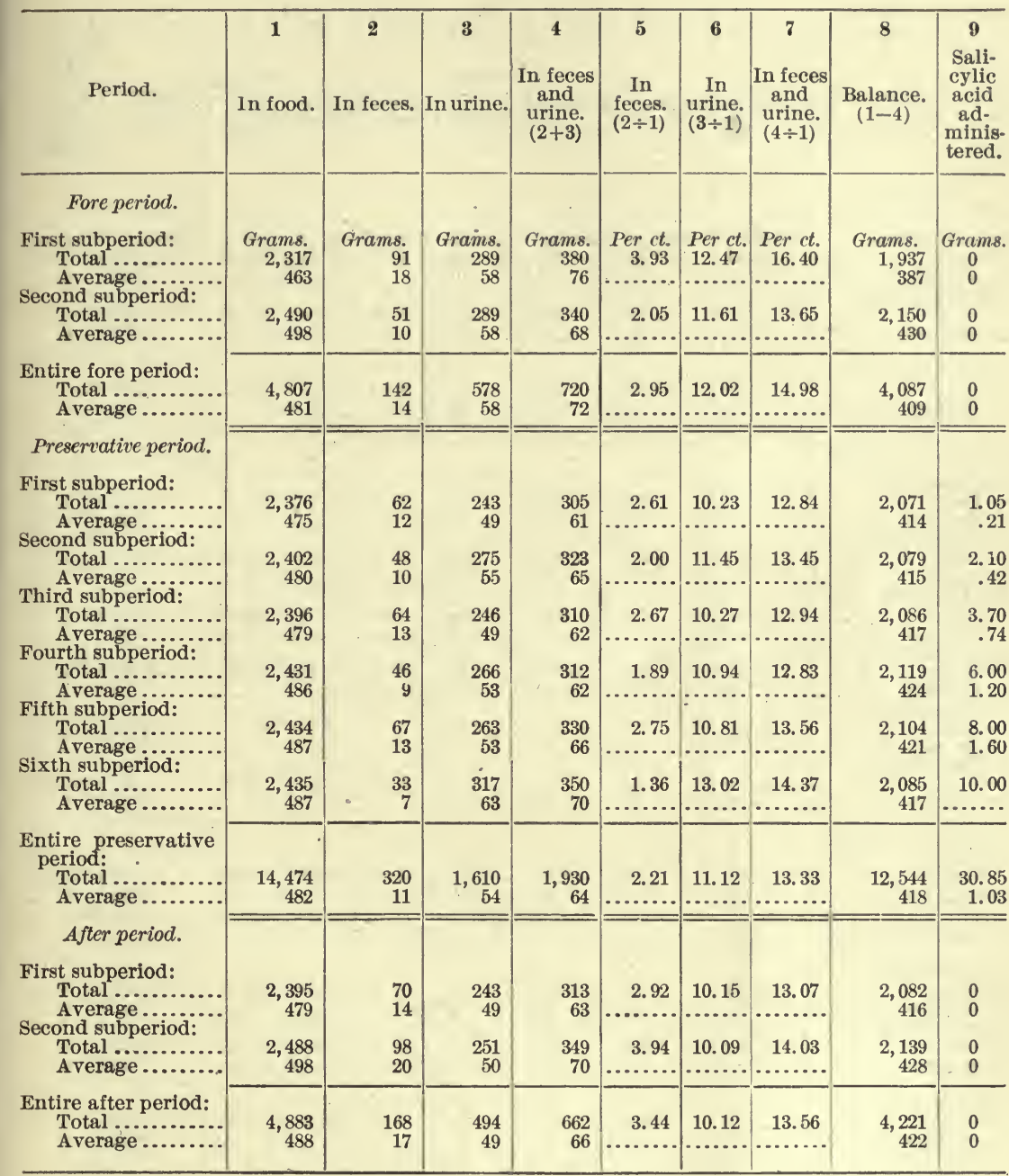


Table XXIII.-Solids balances for Series VI-Continued.

[Averages are per day.]

No. 8.

\begin{tabular}{|c|c|c|c|c|c|c|c|c|c|}
\hline Period. & $\begin{array}{c}1 \\
\text { In food. }\end{array}$ & In feces. & In urine. & $\begin{array}{c}4 \\
\text { In feces } \\
\text { and } \\
\text { urine. } \\
(2+3)\end{array}$ & $\begin{array}{c}5 \\
\text { In } \\
\text { feces. } \\
(2 \div 1)\end{array}$ & $\begin{array}{c}6 \\
\text { In } \\
\text { urine. } \\
(3 \div 1)\end{array}$ & $\begin{array}{c}7 \\
\text { In feces } \\
\text { and } \\
\text { urine. } \\
(4 \div 1)\end{array}$ & $\begin{array}{l}\text { Balance. } \\
(1-4)\end{array}$ & $\begin{array}{c}9 \\
\text { Sali- } \\
\text { cylic } \\
\text { acid } \\
\text { ad- } \\
\text { minis- } \\
\text { tered. }\end{array}$ \\
\hline Fore period. & & & & & & & & & \\
\hline $\begin{array}{l}\text { First subperiod: } \\
\text { Total } \ldots . . . \ldots \ldots \\
\text { Average .......... }\end{array}$ & $\begin{array}{r}\text { Grams. } \\
2,667 \\
533\end{array}$ & $\begin{array}{r}\text { Grams. } \\
73 \\
15\end{array}$ & $\begin{array}{r}\text { Grams. } \\
254 \\
51\end{array}$ & $\begin{array}{r}\text { Arains. } \\
327 \\
65\end{array}$ & $\begin{array}{r}\text { Per ct. } \\
2.74 \\
\ldots . . .\end{array}$ & $\begin{array}{r}\text { Per ct. } \\
9.52\end{array}$ & $\begin{array}{r}\text { Per ct. } \\
12.26 \\
\ldots \ldots\end{array}$ & $\begin{array}{r}\text { Grams. } \\
2,340 \\
468\end{array}$ & $\begin{array}{c}\text { Grams. } \\
0 \\
0\end{array}$ \\
\hline Second subperiod: & & & & & & & & & \\
\hline Total ................ & $\begin{array}{r}2,641 \\
529\end{array}$ & $\begin{array}{l}94 \\
19\end{array}$ & $\begin{array}{r}281 \\
56\end{array}$ & $\begin{array}{r}375 \\
75\end{array}$ & $\begin{array}{r}3.56 \\
\cdots\end{array}$ & $\begin{array}{l}10.63 \\
\cdots \ldots \ldots\end{array}$ & $\begin{array}{r}14.18 \\
\ldots \ldots . .\end{array}$ & $\begin{array}{r}2,269 \\
454\end{array}$ & $\begin{array}{l}0 \\
0\end{array}$ \\
\hline $\begin{array}{l}\text { Entire fore period: } \\
\text { Total .............. } \\
\text { Average ........ }\end{array}$ & $\begin{array}{r}5,311 \\
531\end{array}$ & $\begin{array}{r}167 \\
17\end{array}$ & $\begin{array}{r}535 \\
54\end{array}$ & $\begin{array}{r}702 \\
70\end{array}$ & $\begin{array}{l}3.14 \\
\ldots\end{array}$ & $\begin{array}{l}10.07 \\
\cdots \ldots\end{array}$ & $\begin{array}{r}13.22 \\
\cdots\end{array}$ & $\begin{array}{r}4,609 \\
461\end{array}$ & $\begin{array}{l}0 \\
0\end{array}$ \\
\hline Preservative period. & & & & & & & & & \\
\hline $\begin{array}{l}\text { First subperiod: } \\
\quad \text { Total ............. } \\
\text { Average ......... }\end{array}$ & $\begin{array}{r}2,847 \\
569\end{array}$ & $\begin{array}{r}108 \\
22\end{array}$ & $\begin{array}{r}259 \\
52\end{array}$ & $\begin{array}{r}367 \\
73\end{array}$ & $\begin{array}{r}3.79 \\
\cdots \ldots\end{array}$ & $\begin{array}{c}9.10 \\
\cdots \cdots\end{array}$ & $\begin{array}{r}12.89 \\
\ldots\end{array}$ & $\begin{array}{r}2,480 \\
496\end{array}$ & $\begin{array}{r}1.05 \\
.21\end{array}$ \\
\hline Second subperiod: & & & & & & & & & \\
\hline Total ............... & $\begin{array}{r}2,777 \\
555\end{array}$ & $\begin{array}{r}114 \\
23\end{array}$ & $\begin{array}{r}277 \\
55\end{array}$ & $\begin{array}{r}391 \\
78\end{array}$ & 4.11 & 9.98 & 14.08 & 2,386 & 2.10 \\
\hline Third subperiod: & 050 & & bb & 78 & & $\cdots$ & & 477 & .42 \\
\hline Total .............. & 2,760 & 73 & 271 & 344 & 2.64 & 9.82 & 12.46 & 2,416 & 3. 70 \\
\hline $\begin{array}{l}\text { Average ........... } \\
\text { Fourth subperiod: }\end{array}$ & 552 & 15 & 54 & 69 & $\cdots$ & $\ldots$ & .......... & 483 & .74 \\
\hline $\begin{array}{l}\text { Total ................ } \\
\text { Average }\end{array}$ & $\begin{array}{r}2,827 \\
565\end{array}$ & $\begin{array}{r}114 \\
23\end{array}$ & $a 272$ & 386 & 4.03 & 9.62 & 13.65 & 2,441 & 6.00 \\
\hline Fifth subperiod: & & & 34 & 16 & & & & 400 & 1.20 \\
\hline $\begin{array}{l}\text { Total.......... } \\
\text { Average.... }\end{array}$ & $\begin{array}{r}3,008 \\
602\end{array}$ & $\begin{array}{l}60 \\
12\end{array}$ & $\begin{array}{r}302 \\
60\end{array}$ & $\begin{array}{r}362 \\
72\end{array}$ & 1.99 & $\begin{array}{l}10.04 \\
\ldots \ldots\end{array}$ & 12.03 & 2,646 & 8.00 \\
\hline Sixth subperiod: & & & & & & & & & \\
\hline $\begin{array}{l}\text { Total } . . . . . . . . . . . . . \\
\text { Average } . . . \ldots \ldots\end{array}$ & $\begin{array}{r}3,040 \\
608\end{array}$ & $\begin{array}{l}92 \\
18\end{array}$ & $\begin{array}{r}294 \\
59\end{array}$ & $\begin{array}{r}386 \\
77\end{array}$ & $\begin{array}{r}3.03 \\
\cdots+2 .\end{array}$ & $\begin{array}{r}9.67 \\
\ldots . .\end{array}$ & $\begin{array}{c}12.70 \\
\ldots\end{array}$ & $\begin{array}{r}2,654 \\
531\end{array}$ & $\begin{array}{r}10.00 \\
2.00\end{array}$ \\
\hline $\begin{array}{l}\text { Entire preservative } \\
\text { period: }\end{array}$ & & & & & & & & & \\
\hline 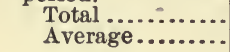 & $\begin{array}{r}17,259 \\
575\end{array}$ & $\begin{array}{r}561 \\
19\end{array}$ & $\begin{array}{r}1,675 \\
56\end{array}$ & $\begin{array}{r}2,236 \\
75\end{array}$ & $\begin{array}{r}3.25 \\
\cdots\end{array}$ & 9.71 & $\begin{array}{r}12.96 \\
\cdots \cdots\end{array}$ & $\begin{array}{r}15,023 \\
500\end{array}$ & $\begin{array}{r}30.85 \\
1.03\end{array}$ \\
\hline After period. & - & & & & & & & & \\
\hline $\begin{array}{l}\text { First subperiod: } \\
\quad \text { Total } \ldots . . . . . . \\
\text { Average } . . . . .\end{array}$ & $\begin{array}{r}2,807 \\
561\end{array}$ & $\begin{array}{r}106 \\
21\end{array}$ & $\begin{array}{r}312 \\
62\end{array}$ & $\begin{array}{r}418 \\
84\end{array}$ & 3.78 & 11.12 & 14.89 & $\begin{array}{r}2,389 \\
477\end{array}$ & $\begin{array}{l}0 \\
0\end{array}$ \\
\hline $\begin{array}{l}\text { Second subperiod: } \\
\text { Total ................ } \\
\text { Average ........ }\end{array}$ & $\begin{array}{r}3,058 \\
612\end{array}$ & $\begin{array}{r}103 \\
21\end{array}$ & $\begin{array}{r}289 \\
58\end{array}$ & $\begin{array}{r}392 \\
78\end{array}$ & $\begin{array}{r}3.37 \\
\cdots . . .\end{array}$ & $\begin{array}{r}9.45 \\
\ldots\end{array}$ & $\begin{array}{r}12.82 \\
\ldots \ldots\end{array}$ & $\begin{array}{r}2,666 \\
534\end{array}$ & $\begin{array}{l}0 \\
0\end{array}$ \\
\hline $\begin{array}{c}\text { Entire after period: } \\
\text { Total ............... } \\
\text { Average ........ }\end{array}$ & $\begin{array}{r}5,865 \\
587\end{array}$ & $\begin{array}{r}209 \\
21\end{array}$ & $\begin{array}{r}601 \\
60\end{array}$ & $\begin{array}{r}810 \\
81\end{array}$ & $\begin{array}{r}3.56 \\
\cdots \cdots\end{array}$ & 10.25 & 13.81 & $\begin{array}{r}5,055 \\
506\end{array}$ & $\begin{array}{l}0 \\
0\end{array}$ \\
\hline
\end{tabular}

$a$ Daily average added in order to complete record. 
Table XXIII.--Solids balances for Series V'I-Continued.

[Averages are per day.]

No. 9.

\begin{tabular}{|c|c|c|c|c|c|c|c|c|c|}
\hline Period. & In food. & In feces. & $\begin{array}{c}3 \\
\text { In urine. }\end{array}$ & $\begin{array}{c}4 \\
\text { In feces } \\
\text { and } \\
\text { urine. } \\
(2+3)\end{array}$ & $\begin{array}{c}5 \\
\text { In } \\
\text { feces. } \\
(2 \div 1)\end{array}$ & $\begin{array}{c}6 \\
\text { In } \\
\text { urine. } \\
(3 \div 1)\end{array}$ & $\begin{array}{c}7 \\
\text { In feces } \\
\text { and } \\
\text { urine. } \\
(4 \div 1)\end{array}$ & $\begin{array}{c}\text { Balance. } \\
(1-4)\end{array}$ & $\begin{array}{c}9 \\
\text { Sali- } \\
\text { cylic } \\
\text { acid } \\
\text { ad- } \\
\text { minis- } \\
\text { tered. }\end{array}$ \\
\hline \multicolumn{10}{|l|}{ Fore period. } \\
\hline $\begin{array}{l}\text { First subperiod: } \\
\text { Total } \ldots . . . . . \\
\text { A verage } . . . . .\end{array}$ & $\begin{array}{r}\text { Grams. } \\
3,157 \\
631\end{array}$ & $\begin{array}{r}\text { Grams. } \\
60 \\
12\end{array}$ & $\begin{array}{r}\text { Grams. } \\
330 \\
66\end{array}$ & $\begin{array}{r}\text { Grams. } \\
390 \\
78\end{array}$ & $\begin{array}{r}\text { Per ct. } \\
1.90 \\
.\end{array}$ & $\begin{array}{l}\text { Perct. } \\
10.45 \\
\cdots\end{array}$ & $\begin{array}{c}\text { Per ct. } \\
12.35 \\
\ldots\end{array}$ & $\begin{array}{r}\text { Grams. } \\
2,767 \\
553\end{array}$ & $\begin{array}{c}\text { Grams. } \\
0 \\
0\end{array}$ \\
\hline $\begin{array}{c}\text { Second subperiod: } \\
\text { Total ............ }\end{array}$ & & & & & & & & & \\
\hline $\begin{array}{l}\text { Total } \ldots . . . . . \\
\text { Average ............. }\end{array}$ & $\begin{array}{r}3,178 \\
636\end{array}$ & $\begin{array}{l}95 \\
19\end{array}$ & $\begin{array}{r}334 \\
67\end{array}$ & $\begin{array}{r}429 \\
86\end{array}$ & $\begin{array}{r}2.99 \\
\ldots .2 .\end{array}$ & $\begin{array}{l}10.51 \\
\ldots \ldots .\end{array}$ & $\begin{array}{r}13.50 \\
\cdots\end{array}$ & $\begin{array}{r}2,749 \\
550\end{array}$ & $\begin{array}{l}0 \\
0\end{array}$ \\
\hline $\begin{array}{l}\text { Entire fore period: } \\
\text { Total ............... } \\
\text { Average } . . .6 .\end{array}$ & $\begin{array}{r}6,335 \\
634\end{array}$ & $\begin{array}{r}155 \\
16\end{array}$ & $\begin{array}{r}664 \\
66\end{array}$ & $\begin{array}{r}819 \\
82\end{array}$ & $\begin{array}{r}2.44 \\
\ldots .\end{array}$ & $\begin{array}{l}10.48 \\
\cdots \cdots\end{array}$ & $\begin{array}{r}12.93 \\
\ldots \ldots\end{array}$ & $\begin{array}{r}5,516 \\
552\end{array}$ & $\begin{array}{l}0 \\
0\end{array}$ \\
\hline \multicolumn{10}{|l|}{ Preservative period. } \\
\hline $\begin{array}{l}\text { First subperiod: } \\
\text { Total............ } \\
\text { Average ......... }\end{array}$ & $\begin{array}{r}3,117 \\
623\end{array}$ & $\begin{array}{r}159 \\
32\end{array}$ & $\begin{array}{r}373 \\
75\end{array}$ & $\begin{array}{l}532 \\
106\end{array}$ & 5.10 & 11.97 & 17.07 & 2,585 & 1. 05 \\
\hline Second subperiod: & & & & & & 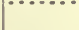 & …... & & .2 \\
\hline ( & $\begin{array}{r}3,130 \\
626\end{array}$ & $\begin{array}{r}106 \\
21\end{array}$ & $\begin{array}{r}334 \\
67\end{array}$ & $\begin{array}{r}440 \\
88\end{array}$ & 3.39 & 10.67 & 14.06 & $\begin{array}{r}2,690 \\
538\end{array}$ & 2. 10 \\
\hline Third subp & & & & & & & & & \\
\hline ( & $\begin{array}{r}3,129 \\
626\end{array}$ & $\begin{array}{r}108 \\
22\end{array}$ & $\begin{array}{r}341 \\
68\end{array}$ & $\begin{array}{r}449 \\
90\end{array}$ & 3.45 & 10.90 & 14.35 & 2,680 & $\begin{array}{l}3.70 \\
74\end{array}$ \\
\hline $\begin{array}{l}\text { Fourth subperiod: } \\
\text { Total }\end{array}$ & & & & & & & & & \\
\hline $\begin{array}{l}\text { Total ................ } \\
\text { Average }\end{array}$ & $\begin{array}{r}3,146 \\
629\end{array}$ & $\begin{array}{l}78 \\
16\end{array}$ & $\begin{array}{r}366 \\
73\end{array}$ & $\begin{array}{r}444 \\
89\end{array}$ & 2.48 & 11.63 & 14.11 & 2,702 & 6.00 \\
\hline $\begin{array}{l}\text { Fifth subperiod: } \\
\text { Total }\end{array}$ & 3141 & & & & & & & & 1.2 \\
\hline Aver & $\begin{array}{r}3,141 \\
628\end{array}$ & $\begin{array}{r}139 \\
28\end{array}$ & $\begin{array}{r}335 \\
67\end{array}$ & $\begin{array}{r}474 \\
95\end{array}$ & 4.43 & 10.67 & 15.09 & $\begin{array}{r}2,667 \\
533\end{array}$ & $\begin{array}{l}8.00 \\
1.60\end{array}$ \\
\hline $\begin{array}{l}\text { Sixth subp } \\
\text { Total. }\end{array}$ & & & & - & & & & & \\
\hline Average ............. & 612 & 23 & $\begin{array}{r}384 \\
77\end{array}$ & $\begin{array}{r}497 \\
99\end{array}$ & ......... & 12.04 & & $\begin{array}{r}2,565 \\
513\end{array}$ & $\begin{array}{r}10.00 \\
2.00\end{array}$ \\
\hline \multicolumn{10}{|l|}{$\begin{array}{l}\text { Entire preservative } \\
\text { period: }\end{array}$} \\
\hline $\begin{array}{l}\text { Total ................. } \\
\text { Average }\end{array}$ & $\begin{array}{r}18,725 \\
624\end{array}$ & $\begin{array}{r}703 \\
23\end{array}$ & $\begin{array}{r}2,133 \\
71\end{array}$ & $\begin{array}{r}2,836 \\
95\end{array}$ & 3.75 & 11.39 & 15.15 & $\begin{array}{r}15,889 \\
529\end{array}$ & 30.85 \\
\hline \multicolumn{8}{|l|}{ After period. } & & \\
\hline $\begin{array}{l}\text { First subperiod: } \\
\text { Total ............ } \\
\text { Average ......... }\end{array}$ & $\begin{array}{r}3,113 \\
623\end{array}$ & $\begin{array}{r}46 \\
9\end{array}$ & $\begin{array}{r}339 \\
68\end{array}$ & $\begin{array}{r}385 \\
77\end{array}$ & $\begin{array}{l}1.48 \\
\cdots\end{array}$ & $\begin{array}{l}10.89 \\
\cdots\end{array}$ & $\begin{array}{l}12.37 \\
\ldots . . .\end{array}$ & $\begin{array}{r}2,728 \\
546\end{array}$ & $\begin{array}{l}0 \\
0\end{array}$ \\
\hline $\begin{array}{l}\text { Second subperiod: } \\
\text { Total ............... } \\
\text { Average ........ }\end{array}$ & $\begin{array}{r}3,119 \\
624\end{array}$ & $\begin{array}{r}133 \\
27\end{array}$ & $\begin{array}{r}381 \\
76\end{array}$ & $\begin{array}{l}514 \\
103\end{array}$ & $\begin{array}{r}4.26 \\
\ldots . . .\end{array}$ & $\begin{array}{l}12.22 \\
\ldots \ldots \ldots\end{array}$ & $\begin{array}{r}16.48 \\
\ldots \ldots . .\end{array}$ & $\begin{array}{r}2,605 \\
521\end{array}$ & $\begin{array}{l}0 \\
0\end{array}$ \\
\hline $\begin{array}{r}\text { Entire after period: } \\
\text { Total .............. } \\
\text { Average ....... }\end{array}$ & $\begin{array}{r}6,232 \\
623\end{array}$ & $\begin{array}{r}179 \\
18\end{array}$ & $\begin{array}{r}720 \\
72\end{array}$ & $\begin{array}{r}899 \\
90\end{array}$ & 2.87 & 11.55 & 14.43 & $\begin{array}{r}5,333 \\
533\end{array}$ & $\begin{array}{l}0 \\
0\end{array}$ \\
\hline
\end{tabular}

7656-No. 84, pt 2-06-15 
TABLE XXIII.-Solids balances for Series $V I$-Continued.

[Averages are per day.]

No. 10 .

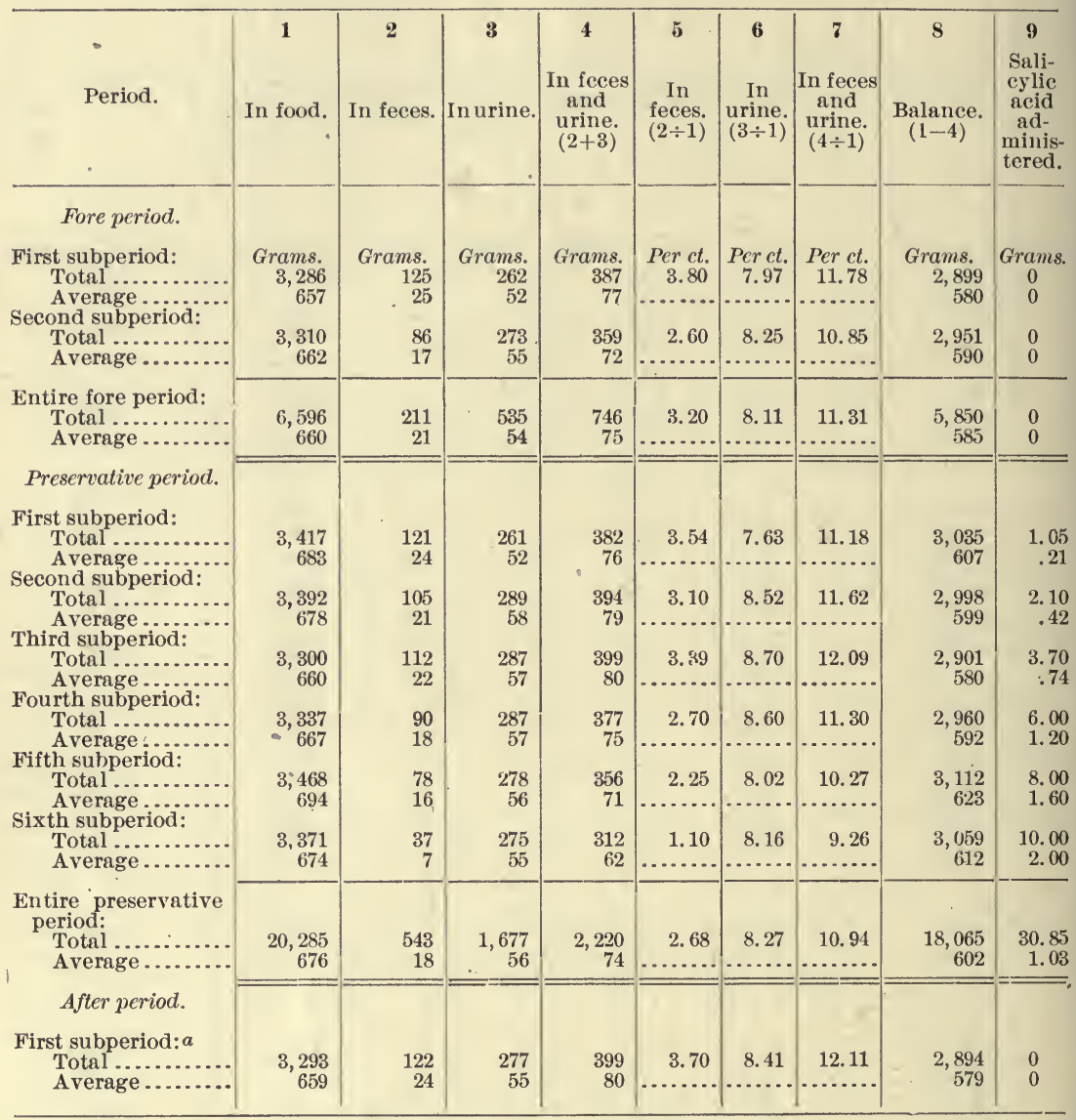

$a$ No second after subperiod; subject ill. 
TABLE XXIII.-Solids balances for Series VI-Continued.

[Averages are per day.]

No. 11 .

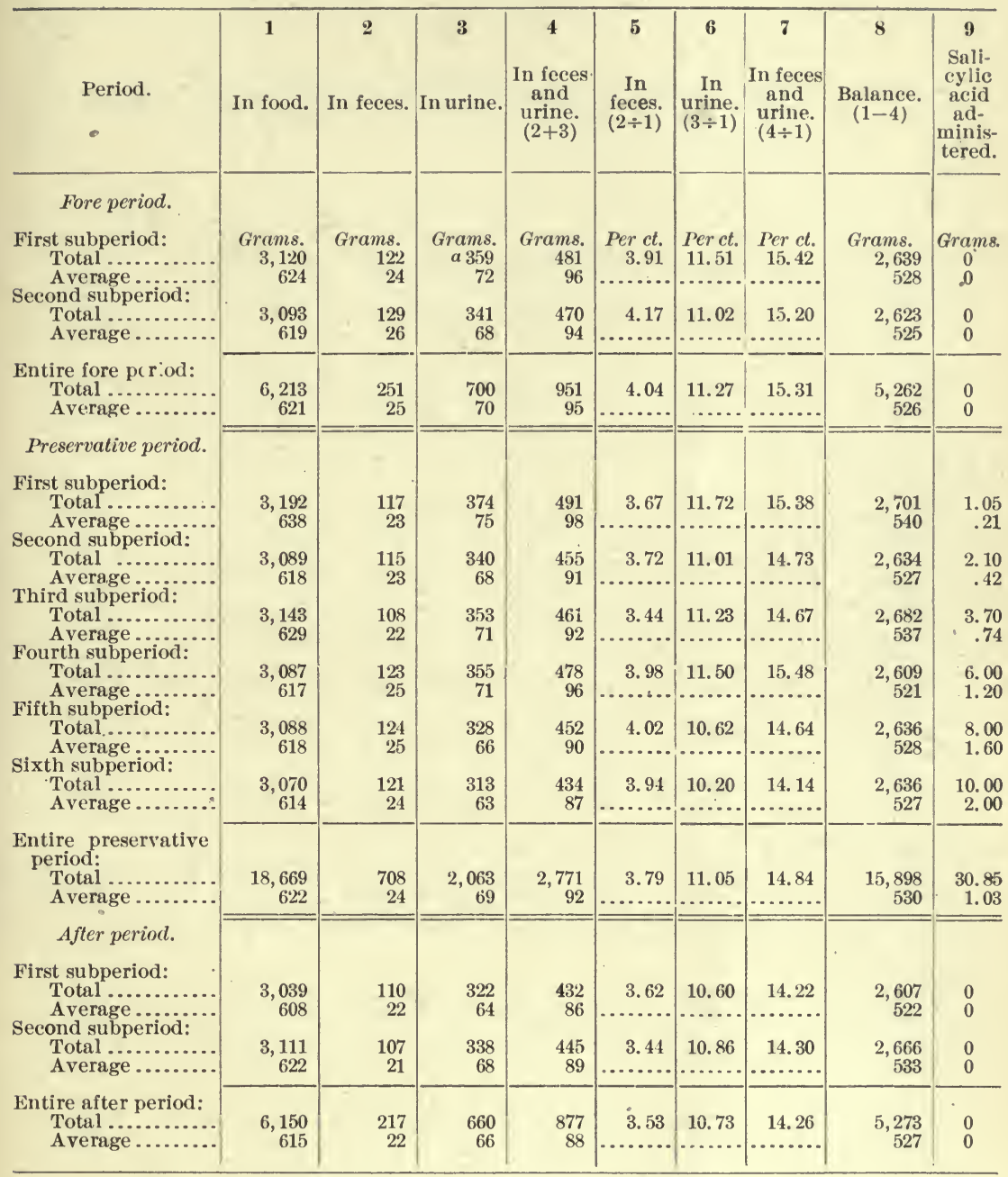

a Daily av rage added in order to complete record. 
TAbLe XXIII.-Solids balances for Series VI-Continued.

[Averages are per day.]

No. 12 .

\begin{tabular}{|c|c|c|c|c|c|c|c|c|c|}
\hline Period. & In food. & In feces. & In urine. & $\begin{array}{c}4 \\
\text { In feces } \\
\text { and } \\
\text { urine. } \\
(2+3)\end{array}$ & $\begin{array}{c}\mathbf{5} \\
\text { In } \\
\text { feces. } \\
(2 \div-1)\end{array}$ & $\begin{array}{c}6 \\
\text { In } \\
\text { urine. } \\
(3 \div-1)\end{array}$ & $\begin{array}{c}\boldsymbol{7} \\
\text { In feces } \\
\text { and } \\
\text { urine. } \\
(4 \div 1)\end{array}$ & $\begin{array}{c}8 \\
\substack{\text { Balance. } \\
(1-4)}\end{array}$ & $\begin{array}{c}9 \\
\text { Sali- } \\
\text { eylic } \\
\text { acid } \\
\text { ad- } \\
\text { minis- } \\
\text { tered. }\end{array}$ \\
\hline Fore period. & & & & & & & & & \\
\hline $\begin{array}{l}\text { First subperiod: } \\
\quad \text { Total ............. } \\
\text { Average......... }\end{array}$ & $\begin{array}{r}\text { Grams. } \\
3,247 \\
649\end{array}$ & $\begin{array}{r}\text { Grams. } \\
147 \\
29\end{array}$ & $\begin{array}{r}\text { Grams. } \\
324 \\
65\end{array}$ & $\begin{array}{r}\text { Grams. } \\
471 \\
94\end{array}$ & $\begin{array}{r}\text { Per ct. } \\
4.53\end{array}$ & $\begin{array}{c}\text { Per ct. } \\
9.98\end{array}$ & $\begin{array}{r}\text { Per et. } \\
14.51\end{array}$ & $\begin{array}{r}\text { Grams. } \\
2,776 \\
555\end{array}$ & $\begin{array}{l}\text { Grams } \\
0 \\
0\end{array}$ \\
\hline $\begin{array}{l}\text { Second subperiod: } \\
\text { Total ............... } \\
\text { Average....... }\end{array}$ & $\begin{array}{r}3,356 \\
671\end{array}$ & $\begin{array}{r}125 \\
25\end{array}$ & $\begin{array}{r}307 \\
61\end{array}$ & $\begin{array}{r}432 \\
86\end{array}$ & $\begin{array}{r}3.72 \\
\ldots .3\end{array}$ & $\begin{array}{r}9.15 \\
\cdots \ldots .\end{array}$ & $\begin{array}{r}12.87 \\
\ldots \ldots \ldots\end{array}$ & $\begin{array}{r}2,924 \\
585\end{array}$ & $\begin{array}{l}0 \\
0\end{array}$ \\
\hline $\begin{array}{l}\text { Entire fore period: } \\
\text { Total ................ } \\
\text { Average ......... }\end{array}$ & $\begin{array}{r}6,603 \\
660\end{array}$ & $\begin{array}{r}272 \\
-\quad 27\end{array}$ & $\begin{array}{r}631 \\
63\end{array}$ & $\begin{array}{r}903 \\
90\end{array}$ & $\begin{array}{r}4.12 \\
\ldots \ldots \ldots\end{array}$ & $\begin{array}{r}9.56 \\
\ldots \ldots\end{array}$ & $\begin{array}{r}13.68 \\
\ldots .\end{array}$ & $\begin{array}{r}5,700 \\
570\end{array}$ & $\begin{array}{l}0 \\
0\end{array}$ \\
\hline Preservative period. & & & & & & & & & \\
\hline $\begin{array}{l}\text { First subperiod: } \\
\quad \text { Total ............ } \\
\text { A verage }\end{array}$ & $\begin{array}{r}3,274 \\
655\end{array}$ & $\begin{array}{l}87 \\
17\end{array}$ & $\begin{array}{r}283 \\
57\end{array}$ & $\begin{array}{r}370 \\
74\end{array}$ & $\begin{array}{l}2.66 \\
\cdots \cdots\end{array}$ & $\begin{array}{r}8.64 \\
\ldots . . .\end{array}$ & 11.30 & $\begin{array}{r}2,904 \\
581\end{array}$ & $\begin{array}{r}1.05 \\
.21\end{array}$ \\
\hline $\begin{array}{l}\text { Second subperiod: } \\
\text { Total ............. } \\
\text { Average........ }\end{array}$ & $\begin{array}{r}3,252 \\
650\end{array}$ & $\begin{array}{l}88 \\
18\end{array}$ & $\begin{array}{r}314 \\
63\end{array}$ & $\begin{array}{r}402 \\
80\end{array}$ & 2.71 & 9.66 & $12.36^{\circ}$ & $\begin{array}{r}2,850 \\
570\end{array}$ & 2.10 \\
\hline Third subperiod: & & & & & & & & & \\
\hline $\begin{array}{l}\text { Total ................. } \\
\text { Average..... }\end{array}$ & $\begin{array}{r}3,237 \\
647\end{array}$ & $\begin{array}{r}142 \\
28\end{array}$ & $\begin{array}{r}338 \\
68\end{array}$ & $\begin{array}{r}480 \\
96\end{array}$ & 4.39 & 10.44 & 14.83 & 2,757 & 3.70 \\
\hline Fourth subperiod: & & & & & & & .......... & & \\
\hline $\begin{array}{l}\text { Total ................... } \\
\text { Average }\end{array}$ & $\begin{array}{r}3,273 \\
655\end{array}$ & $\begin{array}{l}85 \\
17\end{array}$ & $\begin{array}{r}327 \\
65\end{array}$ & $\begin{array}{r}412 \\
82\end{array}$ & 2.60 & 9.99 & 12.59 & $\begin{array}{r}2,861 \\
573\end{array}$ & 6.00 \\
\hline $\begin{array}{l}\text { Fifth subperiod: } \\
\text { Total }\end{array}$ & & & & & & & & & \\
\hline 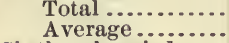 & $\begin{array}{r}3,326 \\
665\end{array}$ & $\begin{array}{r}128 \\
26\end{array}$ & $\begin{array}{r}340 \\
68\end{array}$ & $\begin{array}{r}468 \\
94\end{array}$ & 3.85 & 10.22 & 14.07 & $\begin{array}{r}2,858 \\
571\end{array}$ & $\begin{array}{l}8.00 \\
1.60\end{array}$ \\
\hline $\begin{array}{l}\text { Sixth subperiod: } \\
\text { Total } \ldots \ldots \ldots \\
\text { Average } . . .\end{array}$ & $\begin{array}{r}3,230 \\
646\end{array}$ & $\begin{array}{l}97 \\
19\end{array}$ & $\begin{array}{r}357 \\
71\end{array}$ & $\begin{array}{r}454 \\
91\end{array}$ & 3.00 & $\begin{array}{l}11.05 \\
\cdots . . . .\end{array}$ & $\begin{array}{r}14.06 \\
\ldots . . .\end{array}$ & $\begin{array}{r}2,776 \\
555\end{array}$ & $\begin{array}{r}10.00 \\
2.00\end{array}$ \\
\hline $\begin{array}{l}\text { Entire preservative } \\
\text { period: } \\
\text { Total ............... } \\
\text { A verage } . . . . .\end{array}$ & $\begin{array}{r}19,592 \\
653\end{array}$ & $\begin{array}{r}627 \\
21\end{array}$ & $\begin{array}{r}1,959 \\
65\end{array}$ & $\begin{array}{r}2,586 \\
86\end{array}$ & 3.20 & $\begin{array}{l}10 .(10 \\
\cdots \cdots\end{array}$ & $\begin{array}{r}13.20 \\
\ldots \ldots \ldots\end{array}$ & $\begin{array}{r}17,006 \\
567\end{array}$ & $\begin{array}{r}30.85 \\
1.03\end{array}$ \\
\hline After period. & & & & & & & & & \\
\hline $\begin{array}{l}\text { First subperiod: } \\
\text { Total } \ldots . . . . . . . \\
\text { Average ......... }\end{array}$ & $\begin{array}{r}3,188 \\
638\end{array}$ & $\begin{array}{r}114 \\
23\end{array}$ & $\begin{array}{r}329 \\
66\end{array}$ & $\begin{array}{r}443 \\
89\end{array}$ & 3.58 & 10.32 & 13.90 & $\begin{array}{r}2,745 \\
549\end{array}$ & $\begin{array}{l}0 \\
0\end{array}$ \\
\hline $\begin{array}{l}\text { Second subperiod: } \\
\text { Total .................. } \\
\text { Average .......... }\end{array}$ & $\begin{array}{r}3,208 \\
642\end{array}$ & $\begin{array}{r}a 116 \\
22\end{array}$ & $\begin{array}{r}345 \\
69\end{array}$ & $\begin{array}{r}461 \\
92\end{array}$ & $\begin{array}{c}3.62 \\
\cdots \cdots . .\end{array}$ & 10.75 & $\begin{array}{r}14.37 \\
\ldots . . . . .\end{array}$ & $\begin{array}{r}2,747 \\
550\end{array}$ & $\begin{array}{l}0 \\
0\end{array}$ \\
\hline $\begin{array}{l}\text { Entire after period: } \\
\text { Total ............. } \\
\text { Average....... }\end{array}$ & $\begin{array}{r}6,396 \\
640\end{array}$ & $\begin{array}{r}230 \\
23\end{array}$ & $\begin{array}{r}674 \\
67\end{array}$ & $\begin{array}{r}904 \\
90\end{array}$ & 3.60 & 10.54 & $\begin{array}{r}14.13 \\
\ldots \ldots \ldots\end{array}$ & $\begin{array}{r}5,492 \\
550\end{array}$ & $\begin{array}{l}0 \\
0\end{array}$ \\
\hline
\end{tabular}

$a$ Daily average added in order to complete record. 
TABLE XXIII.-Solids balances for Series VI-Continued.

[Averages are per man per day.]

Summary for mine men.

\begin{tabular}{|c|c|c|c|c|c|c|c|c|c|}
\hline Period. & In food. & In feces. & In urine. & \begin{tabular}{|c|}
$\mathbf{4}$ \\
In feces \\
and \\
urine. \\
$(2+3)$
\end{tabular} & $\begin{array}{c}5 \\
\text { In } \\
\text { feces. } \\
(2 \div 1)\end{array}$ & $\begin{array}{c}\mathbf{6} \\
\text { In } \\
\text { urine. } \\
(3 \div 1]\end{array}$ & $\begin{array}{c}7 \\
\text { In feces } \\
\text { and } \\
\text { urine. } \\
(4 \div 1)\end{array}$ & $\underset{(1-4)}{\text { Balance. }}$ & $\begin{array}{c}\mathbf{9} \\
\text { Sali- } \\
\text { cylic } \\
\text { acid } \\
\text { ad- } \\
\text { minis- } \\
\text { tered. }\end{array}$ \\
\hline Fore period. & & & & & & & & & \\
\hline $\begin{array}{l}\text { First subperiod: } \\
\text { Total } 1 . \ldots \ldots \ldots \\
\text { Average ................ }\end{array}$ & $\begin{array}{r}\operatorname{Grams} 8 \\
24,879 \\
551\end{array}$ & $\begin{array}{r}\text { Grams. } \\
983 \\
22\end{array}$ & $\begin{array}{r}\text { Grams. } \\
2,699 \\
60\end{array}$ & $\begin{array}{r}\text { Grams. } \\
3,682 \\
82\end{array}$ & $\begin{array}{r}\text { Per. ct. } \\
\quad 3.97\end{array}$ & $\begin{array}{l}\text { Per ct. } \\
10.89\end{array}$ & $\begin{array}{l}\text { Per ct. } \\
14.86\end{array}$ & $\begin{array}{r}\text { Grams. } \\
21,197 \\
469\end{array}$ & $\begin{array}{c}\text { Grams. } \\
0 \\
0\end{array}$ \\
\hline $\begin{array}{l}\text { Second subperiod: } \\
\text { Total ................... } \\
\text { Average ......... }\end{array}$ & $\begin{array}{r}25,063 \\
557\end{array}$ & $\begin{array}{r}973 \\
22\end{array}$ & $\begin{array}{r}2,669 \\
59\end{array}$ & $\begin{array}{r}3,642 \\
81\end{array}$ & 3.88 & $\begin{array}{l}11.05 \\
\cdots \ldots . .\end{array}$ & 14. 53 & $\begin{array}{r}21,421 \\
476\end{array}$ & $\begin{array}{l}0 \\
0\end{array}$ \\
\hline $\begin{array}{l}\text { Entire fore period: } \\
\quad \text { Total ............... } \\
\text { Average ......... }\end{array}$ & $\begin{array}{r}49,942 \\
555\end{array}$ & $\begin{array}{r}1,956 \\
22 \\
\end{array}$ & $\begin{array}{r}5,368 \\
59\end{array}$ & $\begin{array}{r}7,324 \\
81 \\
\end{array}$ & 3.92 & 10. 75 & 14.67 & $\begin{array}{r}42,618 \\
474 \\
\end{array}$ & $\begin{array}{l}0 \\
0\end{array}$ \\
\hline Preservative period. & & & & & & & & & \\
\hline $\begin{array}{l}\text { First subperiod: } \\
\text { Total.................... } \\
\text { Average...... } \\
\text { Second subperiod: }\end{array}$ & 25,194 & $\begin{array}{r}865 \\
19\end{array}$ & $\begin{array}{r}2,656 \\
59\end{array}$ & $\begin{array}{r}3,521 \\
78\end{array}$ & 3.43 & 10.54 & 13.98 & $\begin{array}{r}21,673 \\
482\end{array}$ & $\begin{array}{r}9.45 \\
.21\end{array}$ \\
\hline $\begin{array}{l}\text { Total } \\
\text { Aver }\end{array}$ & $\begin{array}{r}25,005 \\
556\end{array}$ & $\begin{array}{r}866 \\
19\end{array}$ & $\begin{array}{r}2,747 \\
61\end{array}$ & $\begin{array}{r}3,613 \\
80\end{array}$ & 3.46 & 10.99 & 14.45 & $\begin{array}{l}21,392 \\
476\end{array}$ & $\begin{array}{r}18.90 \\
. \quad .42\end{array}$ \\
\hline $\begin{array}{l}\text { Third sub } \\
\text { Total }\end{array}$ & 25,192 & 935 & 2,767 & 3,702 & 3,71 & & 14.70 & 21,490 & .72 \\
\hline $\begin{array}{l}\text { Average.................. } \\
\text { Fourth subperiod: }\end{array}$ & 560 & 21 & 61 & 82 & 3. 61 & 10.98 & 14.70 & 478 & $\begin{array}{l}35.30 \\
.74\end{array}$ \\
\hline 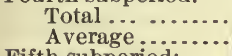 & $\begin{array}{r}25,279 \\
562\end{array}$ & $\begin{array}{r}856 \\
19\end{array}$ & $\begin{array}{r}2,840 \\
63\end{array}$ & $\begin{array}{r}3,696 \\
82\end{array}$ & 3. 39 & 11. 23 & 14.62 & $\begin{array}{r}21,583 \\
480\end{array}$ & $\begin{array}{r}54.00 \\
1.20\end{array}$ \\
\hline $\begin{array}{l}\text { Fifth subperiod: } \\
\text { Total } \\
\text { Average.................. }\end{array}$ & 26,122 & $\begin{array}{r}884 \\
20\end{array}$ & $\begin{array}{r}2,865 \\
64\end{array}$ & $\begin{array}{r}3,749 \\
83\end{array}$ & 3.38 & 10.97 & 14.35 & $\begin{array}{r}22,373 \\
497\end{array}$ & $\begin{array}{r}72.00 \\
1.60\end{array}$ \\
\hline $\begin{array}{l}\text { Sixth subperiod: } \\
\quad \text { Total } \\
\text { Average..................... }\end{array}$ & $\begin{array}{r}25,608 \\
569\end{array}$ & $\begin{array}{r}868 \\
19\end{array}$ & $\begin{array}{r}2,899 \\
64\end{array}$ & $\begin{array}{r}3,767 \\
84\end{array}$ & 3. 39 & 11. 32 & 14.71 & $\begin{array}{r}21,841 \\
485\end{array}$ & $\begin{array}{r}88.00 \\
1.96\end{array}$ \\
\hline $\begin{array}{l}\text { Entire preservative } \\
\text { period: } \\
\text { Total .............. } \\
\text { Average.......... }\end{array}$ & $\begin{array}{r}152,400 \\
564 \\
\end{array}$ & $\begin{array}{r}5,274 \\
19 \\
\end{array}$ & $\begin{array}{r}16,7 ? 4 \\
62 \\
\end{array}$ & $\begin{array}{r}22,048 \\
82 \\
\end{array}$ & 3.46 & $\begin{array}{l}11.01 \\
\ldots \ldots . .\end{array}$ & 14.47 & $\begin{array}{r}130,352 \\
483\end{array}$ & $\begin{array}{r}275.65 \\
1.02 \\
\end{array}$ \\
\hline After period. & & & & & & & & & \\
\hline $\begin{array}{l}\text { First subperiod: } \\
\text { Total ............ } \\
\text { A verage......... } \\
\text { Second subperiod: }\end{array}$ & $\begin{array}{r}25,093 \\
557\end{array}$ & $\begin{array}{r}928 \\
21\end{array}$ & $\begin{array}{r}2,798 \\
62\end{array}$ & $\begin{array}{r}3,726 \\
83\end{array}$ & 3. 70 & 11.15 & 14.85 & $\begin{array}{r}21,367 \\
474\end{array}$ & $\begin{array}{l}0 \\
0\end{array}$ \\
\hline $\begin{array}{l}\text { Second subperiod: } \\
\text { Total ................. } \\
\text { Average ........... }\end{array}$ & $\begin{array}{r}26,014 \\
578\end{array}$ & $\begin{array}{r}908 \\
20\end{array}$ & $\begin{array}{r}2,854 \\
63\end{array}$ & $\begin{array}{r}3,742 \\
83\end{array}$ & 3.49 & 10.89 & 14.38 & $\begin{array}{r}22,272 \\
495\end{array}$ & $\begin{array}{l}0 \\
0\end{array}$ \\
\hline $\begin{array}{l}\text { Entire after period: } \\
\quad \text { Total .............. } \\
\text { A verage ......... }\end{array}$ & $\begin{array}{r}51,107 \\
568\end{array}$ & $\begin{array}{r}1,836 \\
20\end{array}$ & $\begin{array}{r}5,632 \\
63\end{array}$ & $\begin{array}{r}7,468 \\
83\end{array}$ & 3. 59 & 11.02 & 14.61 & $\begin{array}{r}43,639 \\
485\end{array}$ & $\begin{array}{l}0 \\
0\end{array}$ \\
\hline
\end{tabular}

SUMMARY OF RESULTS.

MEDICAL AND CLINICAL .DATA.

A study of the clinical and medical history of the men under observation indicates that the administration of the salicylic acid at first produces a stimulating effect upon the processes of solution and absorption of the food materials from the alimentary canal. There is a smaller proportion of the food products in the feces, both in the individual cases and as a whole, during the preservative period, and part of the after period is subject still to the effect of the administration of the 
preservative. There is also reported in the clinical and medical history an increased appetite in the case of the majority of the subjects. Although the quantity of food which had been found sufficient for the normal functions of the body during the fore period is not diminished, and even to a slight extent in most instances increases, a feeling of hunger develops in almost every case, showing a disturbance of some kind in the metabolic process. The nature of this disturbance is disclosed in the chemical studies, while its observation is a prominent feature of the clinical and medical history. Judged by the development of hunger alone, the administration of the salicylic acid might be considered a stimulant. When, however, all the functions of the body are in a normal state there is no need of a stimulant, and the effect produced by the administration of the acid is evidently therefore an abnormal one. In cases where it is advisable to stimulate temporarily the digestive organs an effect such as that produced would be desirable, if not continued too long. The physiological history of the use of stimulants, however, shows that they are temporary in their effects; that the increased activity induced by them is at the expense of the total vitality of the organs. Hence, stimulants are indicated only for temporary or intermittent use. The truth of this statement is wholly established by the subsequent data gathered from the clinical and medical history of the subjects. The temporary hunger, while accompanied in a number of cases by heaviness and uneasiness in the epigastric region, does not cause any very great discomfort, and in the majority of cases the abnormal desire for food soon decreases. The same quantity or a slightly increased quantity of food is consumed throughout the administration of the preservative.

The loss in weight which is observed in almost all cases indicates that the apparent stimulation of the digestive process is not attended with any corresponding benefit in the building up of the tissues of the body. Assuming, as is done constantly in these studies, that the energy developed by each individual remains practically constant, any increased absorption of food materials ought to have been followed by an increase in body weight. On the contrary, as is shown in the study of the balances, the katabolic activities are increased more strongly than the anabolic. There is a more vigorous tearing down of the tissues of the body than there is a building up thereof, and thus the observations made in the clinical and medical history are thoroughly corroborated by the chemical studies of the foods and the products of metabolism.

The general study of the medical data shows in some instances decidedly unfavorable symptoms attending the use of salicylic acid, while in a minority of cases no unfavorable symptoms of a diagnostic character are developed. After carefully weighing all the dat:t, favorable and unfavorable to the salicylic acid, disclosed in the detailed 
statement of the medical history, the conclusion is inevitable that, taken as a whole, the effects produced by its administration are unfavorable. It is true that there are individual cases which, taken alone, would lead presumably to a contrary opinion, and to these due weight is given in the general conclusion. As a jury eonsidering conflicting testimony gives weight to that which seems most convincing and least open to doubt, so in the decision of this case from the medical history the verdict must follow the weight of testimony and be given against the defendant, namely, salicylic acid.

A summary of the most important indications leading to these conclusions is as follows, dealing with the average results obtained in the body weight, effect on the blood and the urine, and the metabolism of nitrogen and phosphoric acid.

BODY WEIGHT.

If all the variations in weight be taken as a whole for the nine men who completed the periods, it is noticed that there is a gradual diminution in weight, which falls from 62.71 kilograms with an average of 555 grams of dry food per day in the fore period to an average of 62.27 kilograms with an average of 564 grams of dry food per day in the preservative period. This loss of weight is continued in a more marked degree in the after period, where the average weight is 61.61 kilograms with 568 grams of dry food. Thus, although the quantity of food is increased,the weight of the body is diminished. The general conclusion, therefore, is in regard to the effect of the preservative upon the weight of the body, that there is a greater waste than there is a building up of the tissues, assuming, as we may practically do, that the amount of energy and the temperature remain reasonably constant. The general effect, therefore, of the salicylic acid is, under the conditions specified, to diminish the weight of the body; in other words, to interfere with the processes of nutrition by exciting the katabolic activities to a greater degree than the anabolic. The comparison of the weights of each of the subjects, as well as of their average weights, is best shown by consulting the graphic charts in connection with the text. (Figs. 1 and 2.)

\section{MICROSCOPIC BODIES IN THE BLOOD.}

There is an increase in the number of red corpuscles in the blood and also a slight increase in the number of white corpuscles during the period of the administration of the preservative. The intensity of the color of the blood diminishes, however, both in the preservative period and in the after period. There is a marked decrease in the number both of red and white corpuscles in the after period. The apparent increase, therefore, in the preservative period is followed by 
a very considerable decrease in the after period. No conclusion, favorable or unfavorable, can be drawn from this observation regarding the use of the preservative, though, apparently, if there is any effect produced it should be attributed to a favorable influence of the preservative in increasing the number of red corpuscles.

THE URINE.

VOLUME.

There is but little influence noticed due to the salicylic acid on the volume of the urine. The average quantity of urine excreted per day is very slightly larger in the preservative period than in the fore period for the nine men taken together, while in the after period it is slightly less. There is, therefore, a very slight tendency manifested, which is of no particular significance, to increase the volume of the urine. There is also noticed a slight increase in the total solids excreted in the urine, and this increase is maintained in the after period. This observation is in harmony with that indicated by many of the other phenomena which show that the salicylic acid has increased the katabolic activities of the body.

PRESENCE OF ALBUMIN.

In so far as the limited observations show, the administration of the salicylic acid did not produce any notable effect upon the occurrence of albumin in the urine during Series VI. There was, however, a marked tendency shown in the special study, Series XI, to increase the occurrence of albumin in the urine. (See p. 726.)

MICROSCOPIC BODIEs.

The occurrence of microscopic bodies in the urine is a normal condition, and therefore the only point which can be considered here is to determine whether or not the exhibition of the salicylic acid tended to increase or diminish this number. The mass data collected for the nine men indicate that there was a tendency on the part of the salicylic acid to increase the number of microscopic bodies in the urine, the average relative occurrence rising from 68.3 per cent in the fore period to 78.3 per cent in the preservative period, and showing still an additional rise to 79.4 per cent in the after period. Inasmuch as most of the microscopic bodies are considered to be more or less associated with the katabolic products of the body, their increase tends to confirm the supposition already entertained, namely, that the salicylic acid has a greater influence upon the destruction of the tissues of the body than it has upon their restoration. To this extent the increased appearance of microscopic bodies is to be regarded as an unfavorable indication. 
EXCRETION OF THE SALICYLIC ACID.

As in most of the cases when an additional and extraneous substance is added to a food product, the kidneys are called upon to bear the principal effort of excretion. In the case of salicylic acid a large part of it is excreted unchanged in the urine. Other portions undergo changes of a more or less definite nature, and these changed products are also excreted to a large extent by the kidneys, and thus the burden of their work is increased. It is evident, therefore, that the exhibition of the salicylic acid tends to increase the burden which is placed upon the kidneys as the principal excretory organ of the body. Every increase of a burden of this kind must tend to shorten the period of activity of this organ and thus produce a deleterious effect. This is shown, therefore, to be the case in this instance, and for this reason it may be fairly supposed that salicylic acid is a deleterious substance, in that it increases the amount of work demanded of the kidneys.

NITROGEN METABOLISM.

The data collected show that the general effect of the salicylic acid is to slightly increase the quantity of metabolized nitrogen excreted by the kidneys, while the quantity of nonmetabolized nitrogen excreted in the feces is slightly decreased, resulting in a small decrease in the total percentage of nitrogen eliminated. The balance is somewhat greater in the preservative period, although the amount of nitrogen ingested is slightly decreased. These data indicate that the preservative tended to increase slightly the digestibility and absorption of the nitrogen ingested.

\section{PHOSPHORIC ACID METABOLISM.}

While in the case of nitrogen the general tendency of the salicylic acid is to increase the quantity of metabolized nitrogen excreted, the contrary effect is shown in respect of the phosphoric acid. There is a vell-developed tendency during the administration of the salicylic acid to increase the store of phosphoric acid in the body, since the amount absorbed from the alimentary canal is slightly increased and the quantity excreted by the kidneys is decreased. It is evident, therefore, that there is a storing of phosphatic material in the tissues, due to the effect of salicylic acid. It is doubtful if such an increased store would prove of any lasting benefit in its effects, nor would it be just to claim that it would be injurious. The most that can be said in this case is that there is a decided disturbance of phosphoric acid metabolism in the direction of increasing the stores of phosphorus in the body, while in the case of nitrogen there is no marked effect produced on the metabolic process. 


\section{SERIES XI.}

\section{THE EFFECT OF SALICYLIC ACID AND SODIUM SALICYLATE UPON THE NITROGENOUS ELEMENTS OF THE URINE.}

\section{PRELIMINARY STUDY FOR THE DETERMINATION OF THE RATION AND METHODS OF ANALYSIS.}

Before entering upon the special study respecting the influence of salicylic acid and salicylates upon the excretion and composition of the urine, a preliminary experiment was conducted to determine the kind of ration best suited to this particular investigation and also for the purpose of comparing the two most promising methods, namely, the Mörner-Sjöqvist and the Braunstein methods ${ }^{a}$ for the determination of urea. It will also be noted that analyses were made of the daily samples and of a composite sample made by mixing aliquot portions of the daily samples. These samples were composited each day and preserved until the end of the period by the addition of chloroform and thymol, the object in view being to determine whether any material change took place in the samples upon standing.

\section{The Ration.}

For the purposes of this preliminary investigation three men were placed on a definite diet for a limited time, the character of the ration being unchanged throughout the series of observations, as shown in Table I.

TABLE I.-Daily ration, showing amount of food and quantity of nitrogen ingested in the preliminary experiment, Series $X I$.

\begin{tabular}{|c|c|c|c|c|c|c|}
\hline \multirow[b]{2}{*}{ Ration. } & \multicolumn{2}{|c|}{ No. 1.} & \multicolumn{2}{|c|}{ No. 2.} & \multicolumn{2}{|c|}{ No. 3.} \\
\hline & $\begin{array}{l}\text { Weight } \\
\text { of food. }\end{array}$ & Nitrogen. & $\begin{array}{l}\text { Weight } \\
\text { of food. }\end{array}$ & Nitrogen. & $\begin{array}{l}\text { Weight } \\
\text { of food. }\end{array}$ & Nitrogen. \\
\hline $\begin{array}{l}\text { Breakfast: } \\
\quad \text { Bananas ...... }\end{array}$ & $\underset{80}{\operatorname{Grams.}}$ & $\underset{0.15}{\text { Grams. }}$ & $\begin{array}{r}\text { Grams. } \\
100\end{array}$ & $\begin{array}{r}\text { Grams. } \\
0.19\end{array}$ & $\begin{array}{r}\text { Grams. } \\
80\end{array}$ & $\begin{array}{l}\text { Grams. } \\
0.15\end{array}$ \\
\hline Oatmeal $. . . \ldots \ldots \ldots . . . . . . .$. & 70 & & 104 & & 70 & \\
\hline Cream of wheat................. & 100 & .28 & 150 & .42 & 100 & .28 \\
\hline 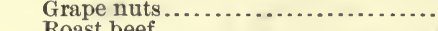 & 16 & & 23 & & 16 & \\
\hline Beefsteak ........... & 80 & 3.58 & 50 & 2.24 & 80 & 3.58 \\
\hline 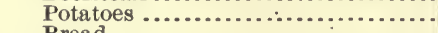 & 100 & 33 & 80 & .27 & 100 & .33 \\
\hline 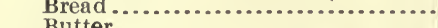 & $\begin{array}{l}70 \\
98\end{array}$ & 1.12 & 50 & .83 & 70 & 1.12 \\
\hline $\begin{array}{l}\text { Butter } \ldots \ldots \ldots \ldots \ldots \ldots \ldots \ldots \ldots \ldots \ldots \\
\text { Coffee }\end{array}$ & $\begin{array}{r}28 \\
150\end{array}$ & .03 & $\begin{array}{r}14 \\
150\end{array}$ & .01 & 28 & .03 \\
\hline $\begin{array}{l}\text { Coffee } \ldots \ldots \ldots \\
\text { Milk } . . .1 .\end{array}$ & $\begin{array}{l}100 \\
206\end{array}$ & 1.06 & $\begin{array}{l}150 \\
206\end{array}$ & 1.06 & $\begin{array}{l}150 \\
206\end{array}$ & 1.06 \\
\hline Total nitrogen for breakfast.. & .... & 6.66 & .... & 5.13 & ..... & 6.66 \\
\hline
\end{tabular}


TABLE I.-Daily ration, showirg amount of food and quantity of nitrogen ingested in the preliminary experiment, Series $X I$-Continued.

\begin{tabular}{|c|c|c|c|c|c|c|}
\hline \multirow[b]{2}{*}{ Ration. } & \multicolumn{2}{|c|}{ No. 1.} & \multicolumn{2}{|c|}{ No. 2.} & \multicolumn{2}{|c|}{ No. 3.} \\
\hline & $\begin{array}{l}\text { Weight } \\
\text { of food. }\end{array}$ & Nitrogen. & $\begin{array}{l}\text { Weight } \\
\text { of food. }\end{array}$ & Nitrogen. & $\begin{array}{l}\text { Weight } \\
\text { of food. }\end{array}$ & Nitrogen. \\
\hline 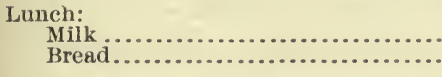 & $\begin{array}{r}\text { Grams. } \\
206 \\
25\end{array}$ & $\begin{array}{r}\text { Grams. } \\
1.11 \\
.41\end{array}$ & $\begin{array}{r}\text { Grams. } \\
203 \\
40\end{array}$ & $\begin{array}{r}\text { Grams. } \\
1.11 \\
.66\end{array}$ & $\begin{array}{r}\text { Grams. } \\
206 \\
25\end{array}$ & $\begin{array}{r}\text { Grams. } \\
1.11 \\
.41\end{array}$ \\
\hline Total nitrogen for lunch...... & ............ & 1.52 & $\ldots \ldots \ldots \ldots$ & 1.77 & ........... & 1.52 \\
\hline $\begin{array}{l}\text { Dinner: } \\
\quad \text { Beefsteak }\end{array}$ & & & & & & \\
\hline Roast beef . & $\begin{array}{r}80 \\
-\quad 150\end{array}$ & 3.58 & 100 & 4.47 & 80 & 3.58 \\
\hline es & $\begin{array}{r}150 \\
33\end{array}$ & .50 & $\begin{array}{r}200 \\
33\end{array}$ & .67 & $\begin{array}{r}150 \\
33\end{array}$ & .50 \\
\hline 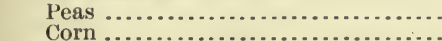 & $\begin{array}{l}45 \\
70\end{array}$ & .29 & $\begin{array}{l}45 \\
70\end{array}$ & .29 & 45 & .29 \\
\hline idding & 150 & & 150 & & 150 & \\
\hline Rice & 172 & .92 & 172 & .92 & 172 & .92 \\
\hline $\begin{array}{l}\text { Bake } \\
\text { Breac }\end{array}$ & $\begin{array}{r}113 \\
60\end{array}$ & .99 & $\begin{array}{r}113 \\
90\end{array}$ & & $\begin{array}{r}113 \\
60\end{array}$ & \\
\hline Butt & 28 & .05 & 28 & .03 & 28 & .03 \\
\hline " & $\begin{array}{l}150 \\
206\end{array}$ & $\begin{array}{r}.06 \\
1.11\end{array}$ & $\begin{array}{l}150 \\
206\end{array}$ & $\begin{array}{r}.06 \\
1.11\end{array}$ & …..... & $\ddot{2} \ddot{2} \ddot{2}$ \\
\hline Total nitrogen for dinner.... & & 7.48 & & 9.04 & & 8.53 \\
\hline Total nitrogen for day ... & $\ldots$ & 15.66 & & 15.94 & & 16. 71 \\
\hline
\end{tabular}

The important point in selecting the ration was to secure a uniform ingestion of nitrogen. Table I shows that No. 1 consumed for breakfast 6.66 grams of nitrogen, for luncheon 1.52 grams of nitrogen, and for dinner 7.48 grams of nitrogen, making a total of 15.66 grams per day. No. 2 had for breakfast 5.13 grams of nitrogen, for luncheon 1.77 grams of nitrogen, and for dinner 9.04 grams of nitrogen, making a total of 15.94 grams of nitrogen per day. No. 3 consumed for breakfast 6.66 grams, for luncheon 1.52 grams, and for dinner 8.53 grams, making a total of 16.71 grams per day. The periods of observation were short in order to avoid any dislike of the food which might have occurred had so uniform a diet been enforced for a long time. The fore period covered only three days, the period of administration of the salicylic acid six days, and the after period three days, making altogether a period of twelve days of observation. During the first preservative subperiod of four days 0.25 gram of salicylic acid and during the second subperiod of two days $0.50 \mathrm{gram}$ of salicylic acid is administered daily. The results obtained in the preliminary study are given in Table II.

\section{Analytical Results. \\ INDIVIDUAL DATA.}

No. 1.

In the case of No. 1 the average volume of the urine excreted daily in the fore period is $967 \mathrm{cc}$. The average daily quantity for the entire preservative period is $1,008 \mathrm{cc}$, and for the after period $1,107 \mathrm{cc}$. 
The administration of the salicylic acid apparently produced an increase in the volume of urine, which tendency continued during the after period.

During the fore period the average daily nitrogen eliminated in the urine is 14.653 grams, during the preservative period 13.286 grams, and during the after period 14.566 grams. These data show a slight tendency on the part of the preservative to decrease the amount of nitrogen eliminated in the urine. A comparison of the nitrogen in the urea by the two methods of observation shows quite concordant results. Unfortunately the determination of the total nitrogen eliminated in the urea for the after period in the composite sample was lost. There is distinctly less nitrogen eliminated as urea in the preservative period than in the fore period. In respect of uric acid nitrogen the average daily quantity eliminated in the fore period is $0.243 \mathrm{gram}$ in the composite sample, in the composite sample of the preservative period $0.210 \mathrm{gram}$, and in the composite sample of the after period $0.258 \mathrm{gram}$. These data also show a distinct diminution in the amount of uric acid nitrogen eliminated during the administration of the salicylic acid.

The study of the total quantities and percentages of urea and uric acid eliminated is a matter of interest. The total quantities of urea eliminated, as determined by the two methods, are practically the same, and the comparison will be based upon the first method alone. It is seen that the average daily weight of urea eliminated in the fore period is 28.346 grams in the composite sample. For the composite sample of the preservative period it is 25.75 grams. There was no composite sample examined for the after period. The average for the daily examinations of the after period shows an elimination of 28.511 grams of urea daily. These data show a markedly depressing effect produced by the preservative upon the quantity of urea eliminated. In respect of uric acid, the average daily quantity eliminated in the fore period, as determined in the composite sample, is $0.727 \mathrm{gram}$, in the preservative period in the composite sample 0.628 gram, and in the composite sample of the after period 0.772 gram. We find here again a marked tendency on the part of the preservative to diminish the average weight of the uric acid eliminated. The ratio of the uric acid nitrogen to the total nitrogen eliminated in the fore period in the composite sample is 60.3 , in the preservative period in the composite sample 63.3, and in the after period in the composite sample 56.5. This ratio shows that the depressing effect of the preservative upon the elimination of nitrogen is exerted more powerfully upon the nitrogen in uric acid than on the other nitrogenous constituents.

In the fore period the average daily percentage of ingested nitrogen which is eliminated in the urine is 93.6 in the composite sample, in the composite sample of the preservative period 84.8 , and in the com- 
posite sample of the after period 93. Thus it is seen that the exhibition of the preservative has diminished in a marked degree the percentage of the total nitrogen of the food eliminated in the urine. Confining the comparison to the first method for the determination of the urea, it is found that 90.4 per cent of the total daily nitrogen eliminated in the urine is eliminated as urea during the fore period, 90.5 per cent during the preservative period, and 91.5 per cent in the after period, showing that the administration of the preservative has not changed the percentage amount of nitrogen eliminated as urea to any great extent.

The percentage of total nitrogen that is eliminated as uric-acid nitrogen in the fore period is 1.7 per cent in the composite sample, in the preservative period 1.6 per cent in the composite sample, and in the after period 1.8 per cent in the composite sample. Again, there is à very slight tendency shown here on the part of the preservative to diminish the percentage of uric-acid nitrogen eliminated.

\section{No. 2.}

In the case of No. 2 the average daily volume of urine in the fore period is $1,037 \mathrm{cc}$, the average daily volume in the preservative period is $1,212 \mathrm{cc}$, and the average for the after period $1,863 \mathrm{cc}$. The average for the after period, however, is only for two days, as the urine for the second day was lost. These data show a slight diuretic effect of the salicylic acid during the 'period' of administration, and this effect is increased enormously in the after period, which can only be attributed to the continued action of the drug.

The average daily quantity of nitrogen eliminated in the urine in

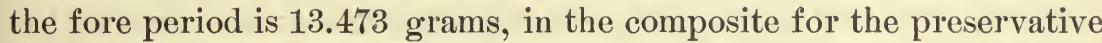
period 13.664 grams, and in the composite for the after period 13.279 grams. These data show a slight increase in the elimination of nitrogen in the urine during the administration of the preservative, both over the fore period and over the after period. The quantities of nitrogen eliminated as urea, determined by the two methods, agree very well. There was no composite, however, examined for the after period. There was a slight increase in the amount of nitrogen excreted as urea during the preservative period. In respect of uric acid, the average daily amount of nitrogen eliminated in the form of uric acid in the fore period in the composite sample is $0.168 \mathrm{gram}$. In the composite sample for the preservative period it is $0.156 \mathrm{gram}$, and for the after period 0.139 gram. Again, we find here a tendency manifested by the preservative to diminish the excretion of nitrogen in the form of uric acid.

The average daily quantity of urea excreted by No. 2 in the fore period is 25.727 grams in the composite sample; in the composite sample for the preservative period 26.669 grams, and for the average 
of daily samples for the after period 25.542 grams. In this case there is a slight tendency on the part of the preservative to increase the average daily amount of urea excreted. The average daily quantity of uric acid excreted by No. 2 in the fore period is 0.503 gram in the composite sample, in the preservative period it is $0.468 \mathrm{gram}$, and in the after period $0.415 \mathrm{gram}$. In this case there seems to be a marked tendency to diminish the average daily quantity of uric acid excreted, due to the influence of the preservative, and this tendeney is continued in the after period. The ratio of the uric-acid nitrogen to the total nitrogen eliminated in the fore period is 80.2 in the composite sample, in the preservative period 87.4, and in the after period in the composite sample 95.5. These data indicate a diminished quantity of uric-acid nitrogen in relation to total nitrogen eliminated under the influence of the preservative, and this tendency is markedly increased in the after period.

Of the total nitrogen ingested in the food 84.5 per cent is eliminated in the urine by No. 2 in the composite sample of the fore period, in the composite sample of the preservative period 85.7, and in the composite sample of the after period 83.3. These data indicate a slight tendency on the part of the preservative to increase the quantity of nitrogen in the urine under the action of the preservative. The percentage of the total nitrogen in the urine excreted as urea during the fore period in the composite sample is 89.2 , in the preservative period 91.2 in the composite sample, and in the average of daily samples of the after period 89.2. These data show a slight tendency on the part of the preservative to increase the percentage of total nitrogen excreted as urea. The percentage of uric-acid nitrogen excreted in the composite sample in the fore period is 1.2 , in the composite sample of the preservative period 1.1, and in the composite sample of the after period 1.0. There is practically no influence, therefore, exerted by the preservative in this case in changing the relative percentage of uric-acid nitrogen excreted.

No. 3.

In the case of No. 3 the average daily volume of urine excreted in the fore period is $675 \mathrm{cc}$; in the preservative period $760 \mathrm{cc}$, and in the after period $1,114 \mathrm{cc}$. These data show again a slight tendency on the part of the preservative to increase the volume of urine, which is greatly accentuated on the withdrawal of the preservative in the after period. This phenomenon having occurred in all three cases would indicate a tendency on the part of the salicylic acid to stimulate the secretory organs connected with the elimination of the urine to extraordinary activity upon the withdrawal of the salicylic acid. This is an apparent tendency which is worthy of further investigation. 
The average daily quantity of nitrogen eliminated by No. 3 in the fore period is 12.941 grams, in the preservative period 13.380 grams, and in the after period 13.939 grams. These data show a slight tendency on the part of the preservative to increase the elimination of nitrogen during the administration of the preservative, and this tendency is maintained in the after period, though not in proportion to the great increase in the volume of the urine. The average daily quantity of urea excreted, as determined by the first method of examination, in the fore period is 11.785 grams in the composite sample, in the preservative period 12.099 grams in the composite sample, and in the after period, in the average daily samples, 12.429 grams. Here there is manifested a slight influence on the part of the preservative to increase the total quantity of nitrogen excreted as urea. In respect of the uric acid the average daily amount excreted by No. 3 in the composite sample is $0.191 \mathrm{gram}$, in the preservative period in the composite sample 0.161 gram, and in the after period in the composite sample 0.202 gram. Here we find again a tendency on the part of the preservative to diminish the quantity of nitrogen eliminated as uric acid.

In regard to the average daily quantity of urea excreted by No. 3, as determined by the first method, it is found to be 25.227 grams in the composite sample, in the composite sample of the preservative period 25.902 grams, and in the average of the daily samples of the after period 26.607 grams. The average daily quantity of uric acid excreted by No. 3 in the composite sample of the fore period is 0.571 gram, in the composite sample of the preservative period 0.483 gram, and in the composite sample of the after period $0.603 \mathrm{gran}$. In this case there is an apparent tendency on the part of the preservative to diminish the average daily quantity of uric acid excreted. If, now, we consider the ratio of the uric acid nitrogen to the total nitrogen eliminated, we find that this ratio in the composite sample of the fore period is 67.7 , in the composite sample of the preservative period 83.1 , and in the composite sample of the after period 69.1. Here there is indicated a very strong tendency on the part of the preservative to increase the ratio of the uric acid excreted to the total nitrogen eliminated, and thus to apparently decrease the relative quantities of uric acid nitrogen excreted, a tendency which has been uniform in all three of the present cases. The total percentage of nitrogen of the food excreted in the urine by No. 3 in the composite sample of the fore period is 77.4 , in the composite sample of the preservative period 80 , and in the composite sample of the after period 83.4. There is here an apparent tendency on the part of the preservative to increase the percentage of the total nitrogen in the food eliminated in the urine. The total nitrogen in the urine eliminated as urea in the composite 
sample of the fore period in the case of No. 3 is 91.1 , in the composite sample of the preservative period 90.4, and in the average of daily samples of the after period 91, indicating a slight tendency on the part of the preservative to decrease the relative amount of nitrogen excreted as urea.

In regard to the percentage of nitrogen excreted as uric acid in the case of No. 3 in the fore period, it is 1.5 , in the preservative period 1.2 , and in the after period 1.4 per cent, in each case determined in the composite sample. These data indicate a tendency on the part of the preservative to diminish the relative proportion of uric acid nitrogen eliminated.

SUMMARY.

We now have to consider the case of Nos. 1, 2, and 3 as a whole. It is seen that the average daily volume of urine excreted in the fore period is $893 \mathrm{cc}$, in the preservative perior $981 \mathrm{cc}$, and in the after period 1,298 cc. These data indicate that the general effect of the preservative is to act as a diuretic, and this effect is enormously increased immediately after the withdrawal of the drug. This would seem to indicate that the maximum excitation produced by the exhibition of salicylic acid is not manifested immediately at the time of its administration, nor within six days, but is shown in a much more marked degree immediately after the administration is withdrawn. In other words, the stimulation of the excretory organs eliminating urine does not reach its maximum until some time, at least six days, after the first administration of the preservative.

The average quantity of nitrogen ingested daily is 16.10 grams. Of this, there is eliminated in the fore period in the urine $13.689 \mathrm{grams}$, in the preservative period 13.429 grams, and in the after period 14.009 grams. These data show a slight general tendency on the part of the preservative to diminish the quantity of nitrogen eliminated in the urine during the administration of the preservative, but the elimination is increased in the after period.

In regard to the nitrogen eliminated as urea, as determined by the first method, it is seen that in the fore period the average daily amount is 12.348 grams, for the preservative period $12.180 \mathrm{grams}$, in the composite samples, respectively, and for the after period 12.638 grams in the average of daily samples. The general indication, therefore, is that the effect of the preservative is to slightly diminish the total quantity of nitrogen eliminated as urea, but the quantity eliminated is increased to above normal on the withdrawal of the preservative.

The average daily quantity of uric-acid nitrogen excreted in the composite sample of the fore period is $0.201 \mathrm{gram}$; in the composite sample of the preservative period $0.177 \mathrm{gram}$, and in the composite 
sample of the after period 0.207 gram. Here there is a manifest tendency on the part of the preservative to diminish the daily quantity of uric-acid nitrogen excreted.

When the total quantity of urea excreted is taken into consideration, it is seen that in the composite sample of the fore period it is 26.433 grams by the first method of determination; in the composite sample of the preservative period it is 26.074 grams, and in the average of daily samples of the after period 27.055 grams. Here is noted a tendency on the part of the preservative to diminish the average daily quantity of urea excreted, and there is an increase in the quantity of urea in the after period.

The average daily quantity of uric acid excreted in the fore period is $0.601 \mathrm{gram}$, in the preservative period $0.529 \mathrm{gram}$, and in the after period 0.620 gram in the composite samples. These data indicate a tendency on the part of the preservative to diminish the total quantity of uric acid excreted.

The ratio of the uric-acid nitrogen excreted to the total nitrogen eliminated in the fore period, in the composite sample, is 68.3 , in the composite sample of the preservative period 75.9, and in the composite sample of the after period 67.6. This increase in the ratio shows a corresponding decrease in the relative amount of uric-acid nitrogen excreted in relation to total nitrogen eliminated.

The average percentage of nitrogen eliminated in the fore period in the composite sample is 85 , in the composite sample of the preservative period 83.4, and in the composite sample of the after period 87. There is a slight tendency, therefore, on the part of the preservative to diminish the average daily percentage of total nitrogen eliminated in the urine.

The total nitrogen in the urine eliminated as urea in the fore period is 90.2 per cent, in the preservative period 91.4 in the composite sample, respectively, and in the average daily samples of the after period 90.7. There is here, therefore, manifested a tendency on the part of the preservative to increase the relative percentage of nitrogen eliminated as urea, the conclusion being based upon the results obtained by the Mörner-Sjöqvist method.

The percentage of uric acid nitrogen excreted per day in the fore period (based on total nitrogen eliminated) is 1.5 , in the preservative period 1.3, and in the after period 1.5, indicating a slight tendency to decrease the relative quantity of nitrogen eliminated as uric acid nitrogen.

$7656-$ No. 84 , pt $2-06-16$ 


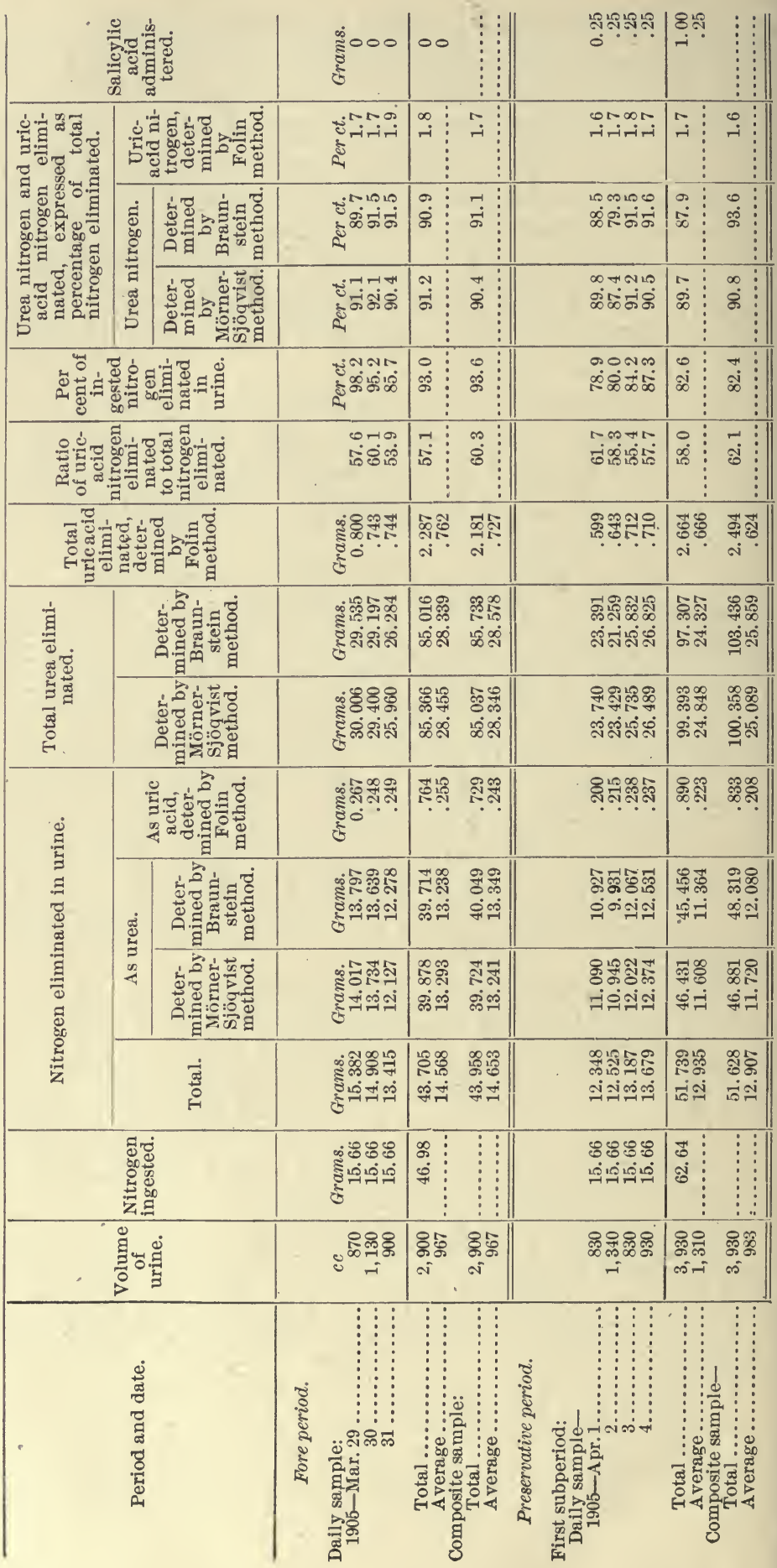




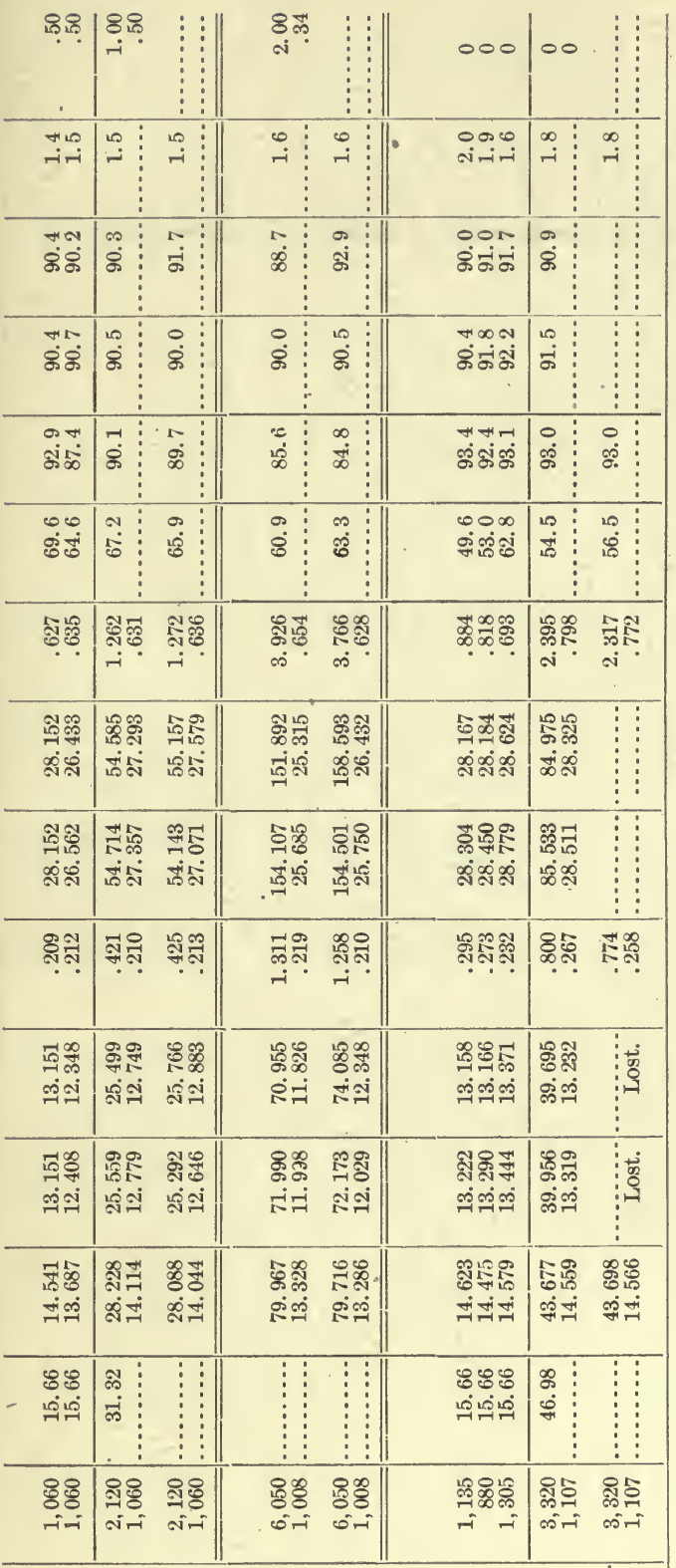

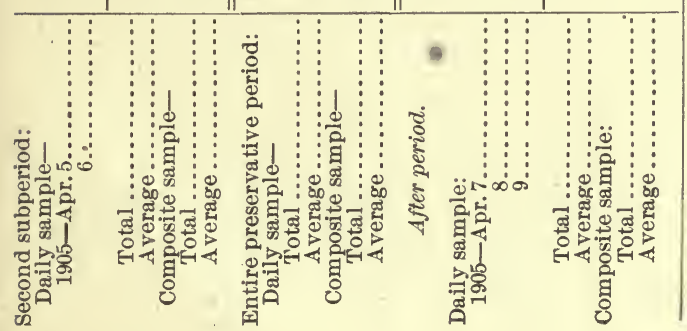




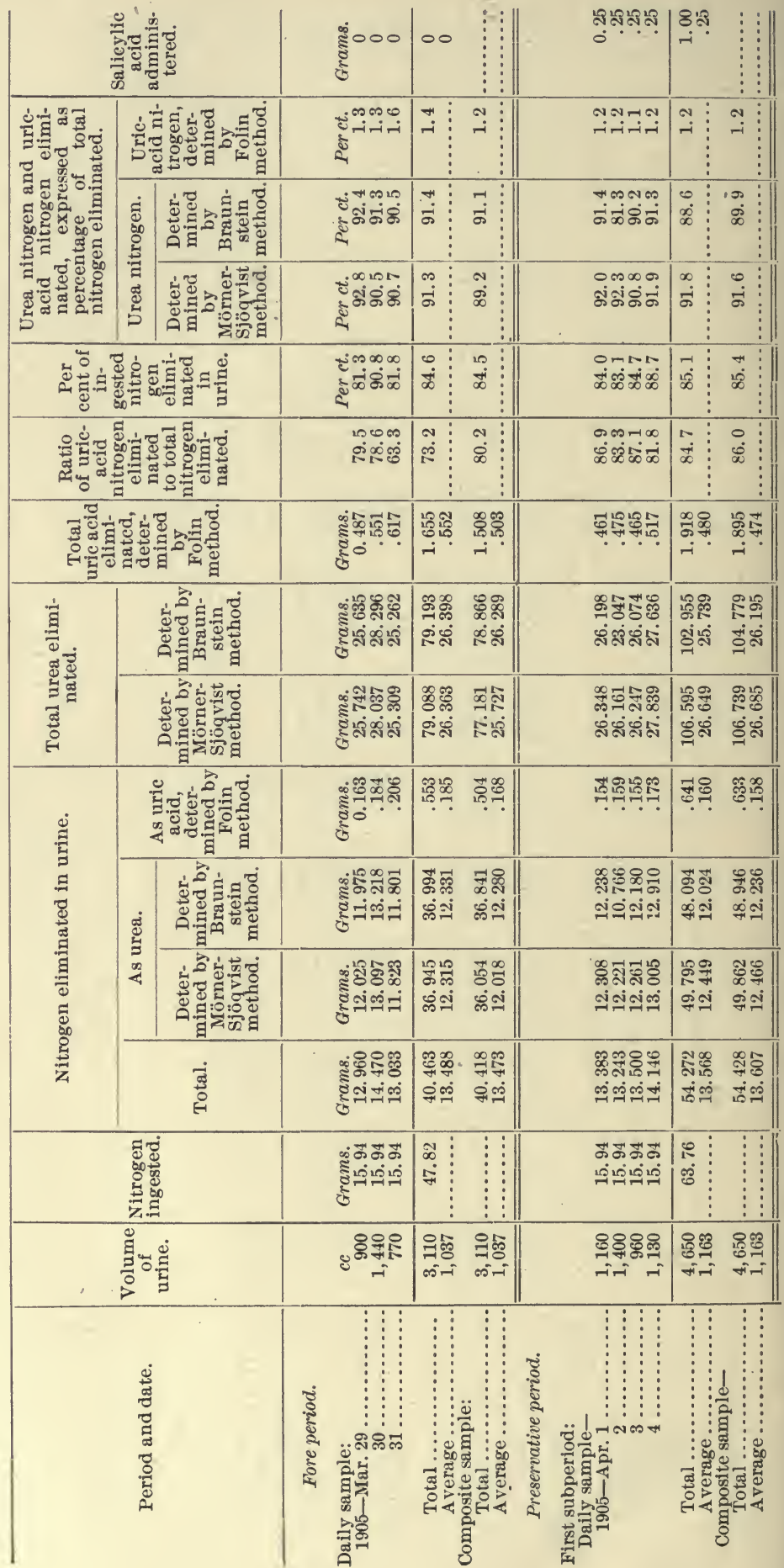




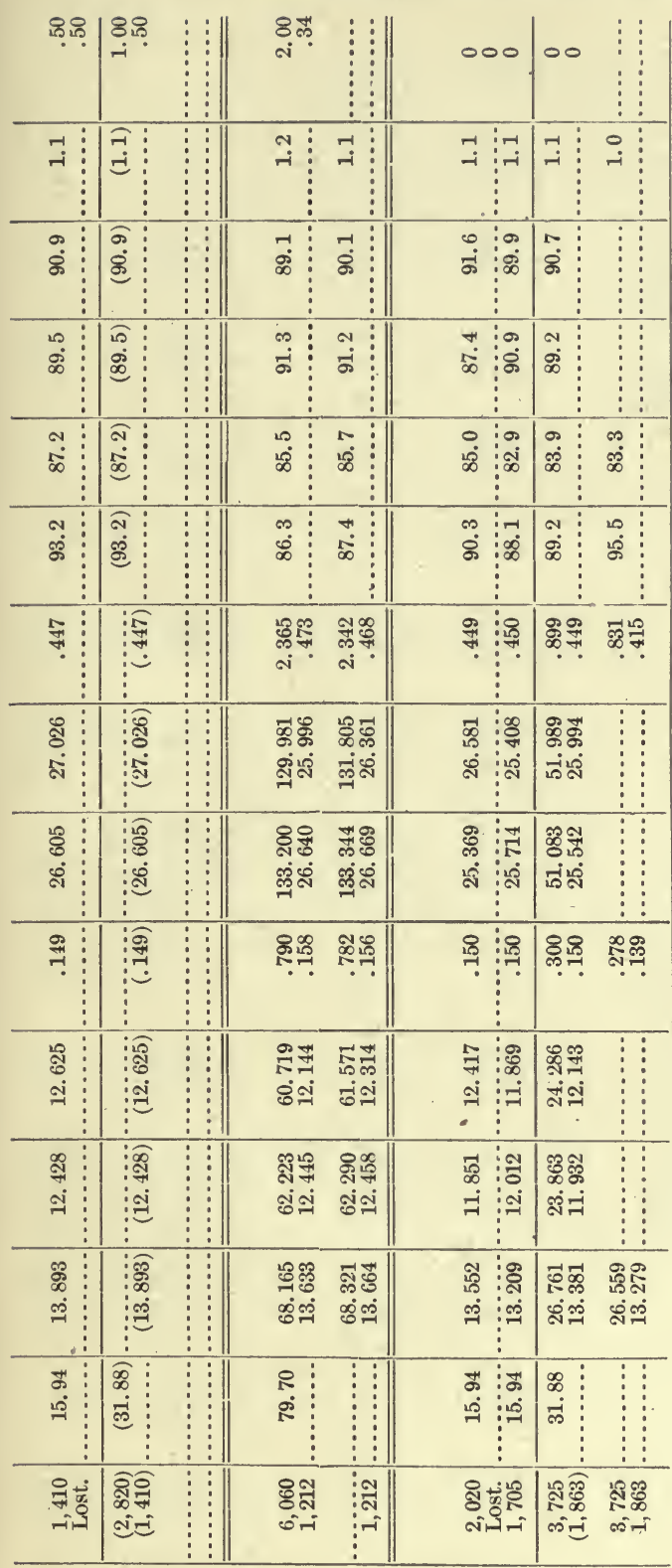

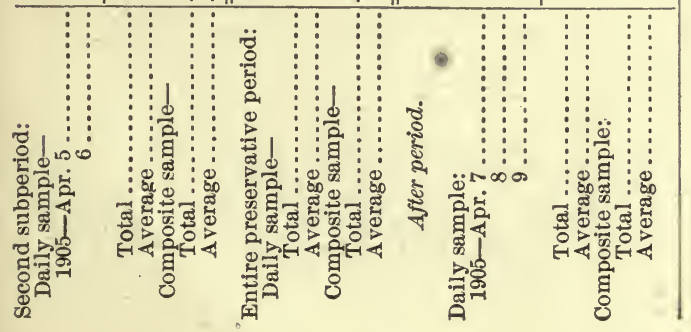




\begin{tabular}{|c|c|c|c|c|c|c|c|c|}
\hline \multicolumn{3}{|c|}{ 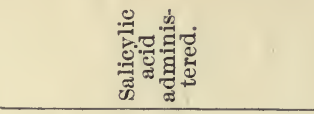 } & $\begin{array}{l}\text { 胥 } 000 \\
\text { के }\end{array}$ & 00 & & ลัง & ․․ & \\
\hline \multirow{3}{*}{ 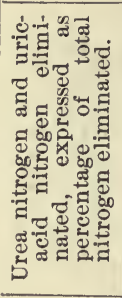 } & \multicolumn{2}{|c|}{ 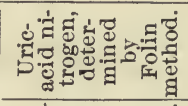 } & 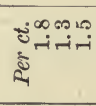 & | & ن- & 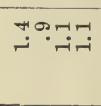 & & \\
\hline & 离 & 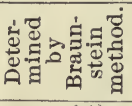 & 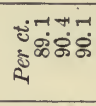 & \begin{tabular}{c|c}
$\infty$ \\
$\dot{\infty}$ \\
$\infty$
\end{tabular} & : & 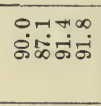 & & \\
\hline & & 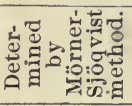 & 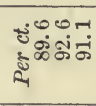 & & 祭 & க்கే் & & \\
\hline \multicolumn{3}{|c|}{ 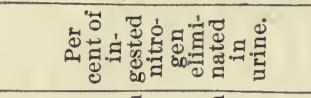 } & 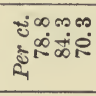 & $\begin{array}{l}\infty \\
\infty \\
\stackrel{N}{*} \vdots\end{array}$ & 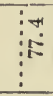 & வுळ & 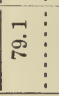 & \\
\hline \multicolumn{3}{|c|}{ 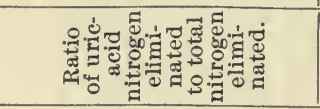 } & 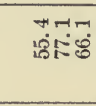 & त्: & 5 & 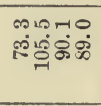 & $\mid \begin{array}{l:}10 \\
8 \\
8 \\
8\end{array}$ & $\stackrel{\dot{\infty}}{\dot{\infty}}$ \\
\hline \multicolumn{3}{|c|}{ 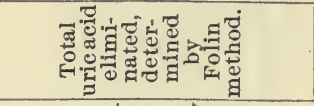 } & 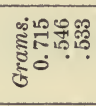 & 㞼足 & 里 & 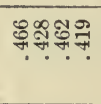 & 路苐 & 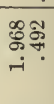 \\
\hline \multirow{2}{*}{ 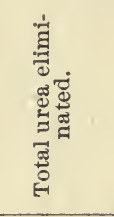 } & \multicolumn{2}{|c|}{ 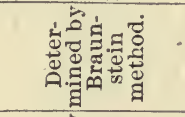 } & 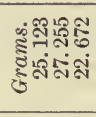 & 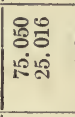 & 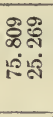 & 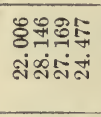 & 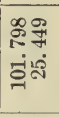 & 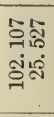 \\
\hline & \multicolumn{2}{|c|}{ 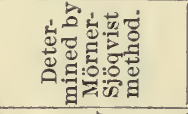 } & 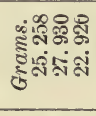 & 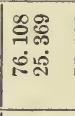 & 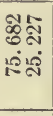 & 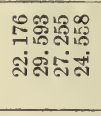 & 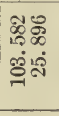 & 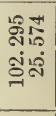 \\
\hline \multirow{4}{*}{ 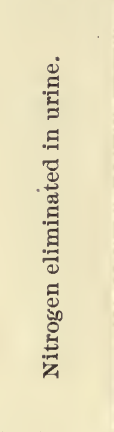 } & \multicolumn{2}{|c|}{ 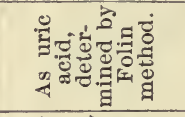 } & 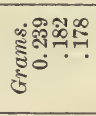 & 㞧局 & สำ & 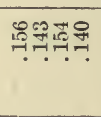 & 骂: & 둥. \\
\hline & \multirow{2}{*}{ 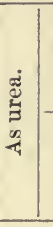 } & 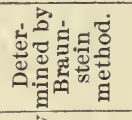 & 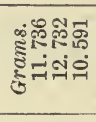 & 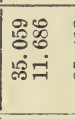 & 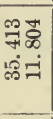 & 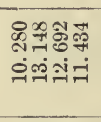 & 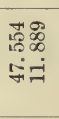 & 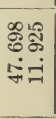 \\
\hline & & 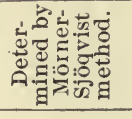 & 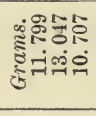 & 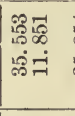 & 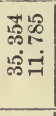 & 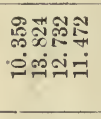 & 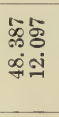 & 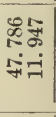 \\
\hline & & हूँّ & 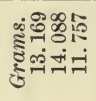 & 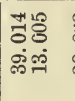 & 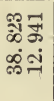 & 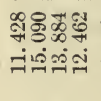 & 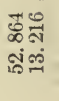 & 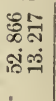 \\
\hline \multicolumn{3}{|c|}{ 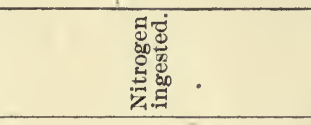 } & 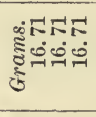 & 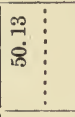 & & 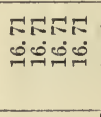 & $\begin{array}{l}\vec{\infty} \\
\dot{8}\end{array}$ & \\
\hline \multicolumn{3}{|c|}{ 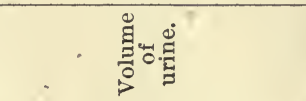 } & 웅유: & $\begin{array}{l}\text { \$2: } \\
\text { कi } \\
\text { oi }\end{array}$ & 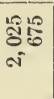 & 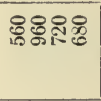 & 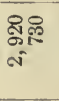 & $\begin{array}{l}\text { హ్ } \\
\text { तi }\end{array}$ \\
\hline
\end{tabular}

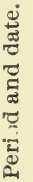

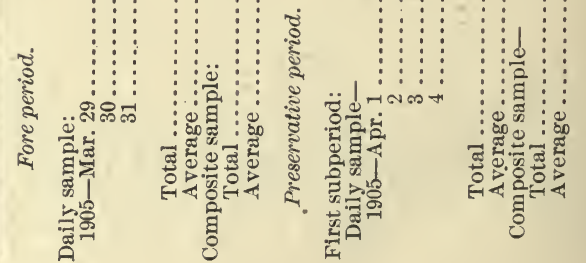




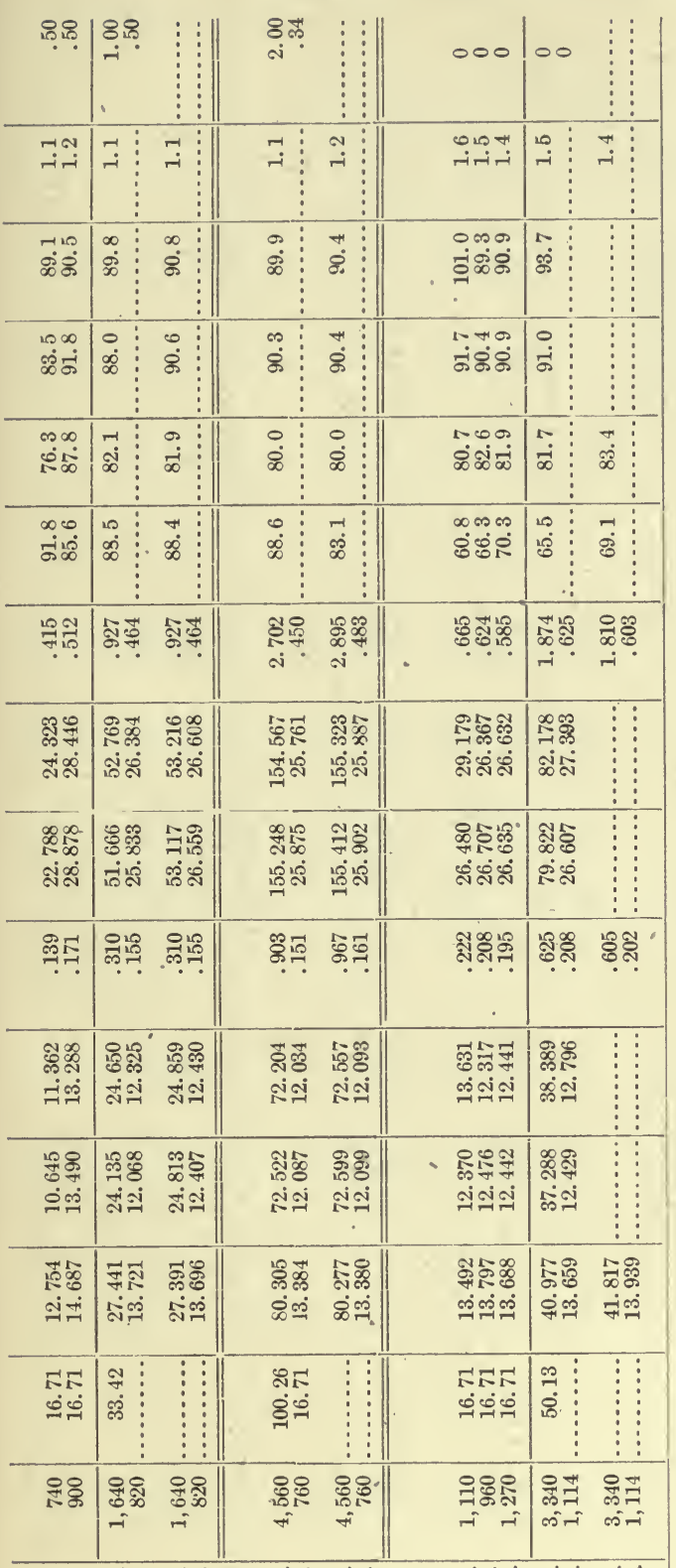

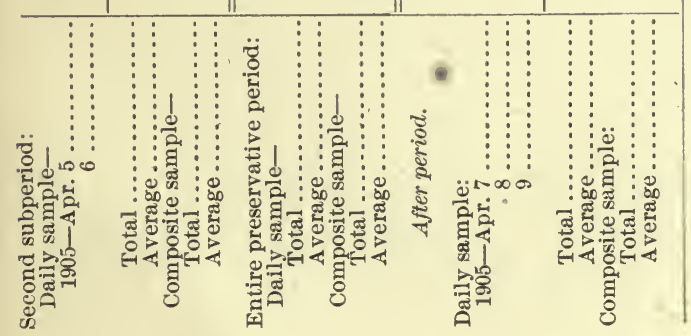




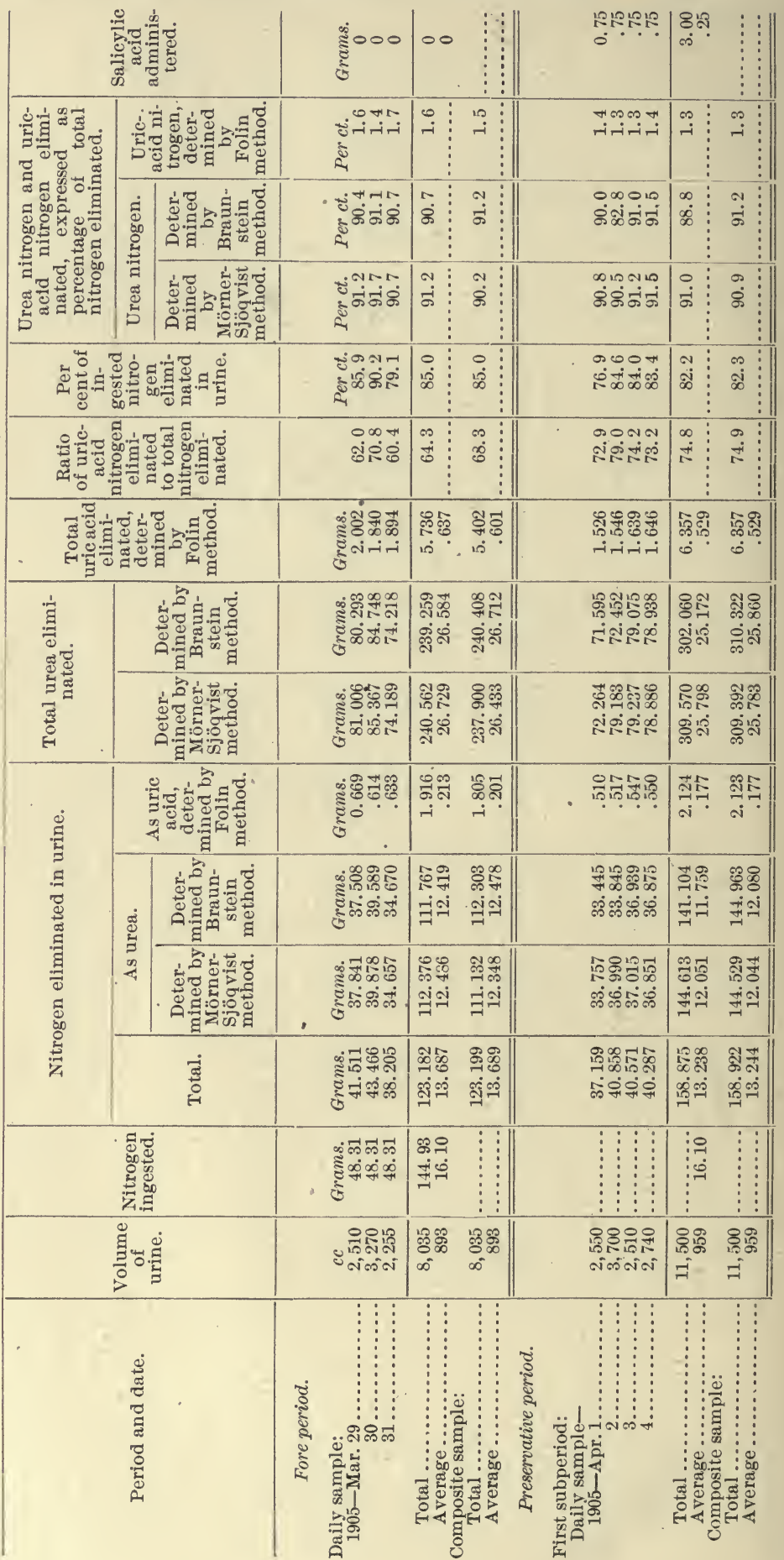




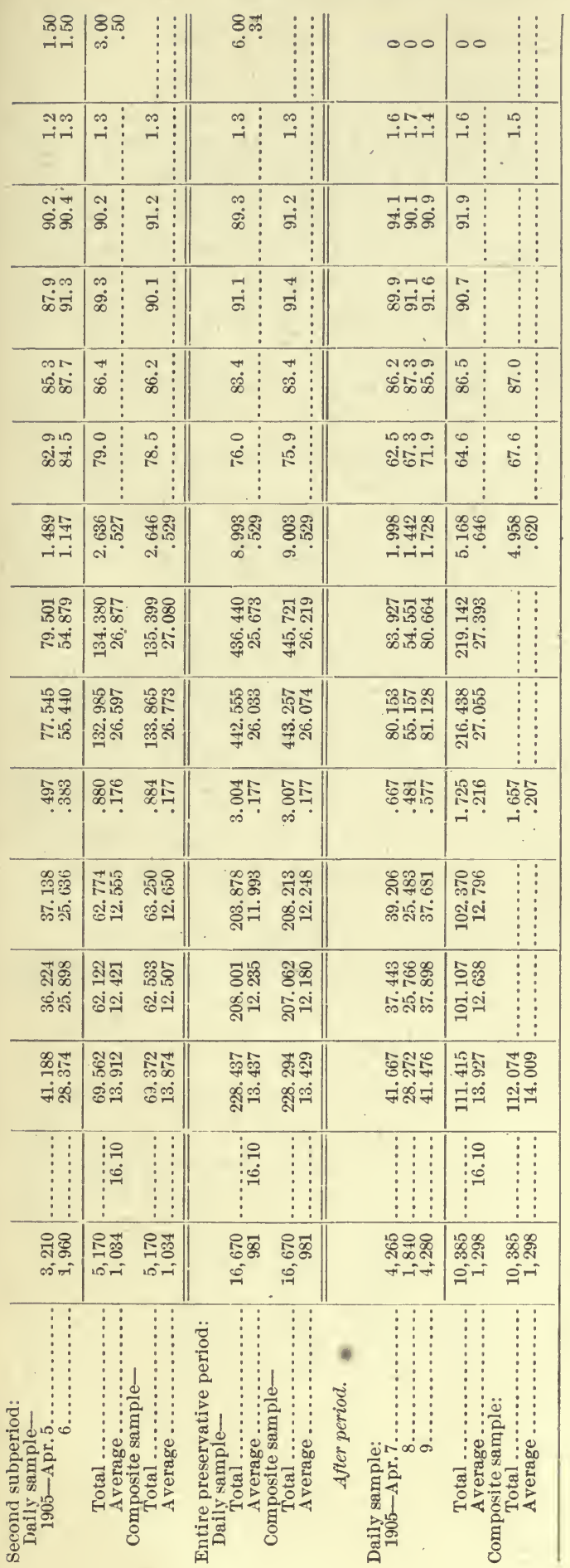


Methods of Analysis Employed and Comparison of Results.

UREA DETERMINATIONS.

Two methods of determining the urea, which are regarded as the best of those in use, were employed, and the data obtained were compared. These were the Mörner-Sjöqvist and the Braunstein method. The principle of the Mörner-Sjöqvist method depends on the fact that the nitrogenous constituents of the urine, with the exception of urea, ammonia, hippuric acid, and kreatinin, are precipitated by means of a solution of barium chlorid and barium hydroxid $\left(50\right.$ grams $\mathrm{Ba}(\mathrm{OH})_{2}$ and 350 grams $\mathrm{BaCl}_{2}$ per liter) and a mixture of alcohol and ether $(2: 1)$; 5 cc of urine, 5 ec of the barium solution, and 100 ce of the alcohol-ether solution are mixed and allowed to stand over night. It is then filtered into a beaker or porcelain dish and the precipitate washed with 50 to 75 cc of the alcohol-ether mixture. The filtrate is then evaporated at a temperature not exceeding $55^{\circ}$ C., and when solvents have disappeared a small quantity of water is added and about 0.5 gram of magnesium oxid. It is then evaporated to dryness or till the fumes are no longer alkaline. The residue is transferred into a Kjeldahl flask and nitrogen determined in the usual manner. The nitrogen found is calculated to urea by multiplying by the factor 2.1407 .

According to Braunstein the above method is inapplicable in the presence of hippuric acid. His modification of the method consists in , taking up the evaporated residue in a small quantity of water and adding 10 grams of crystallized phosphoric acid and heating in an air bath for four and one-half hours at $140^{\circ}-145^{\circ} \mathrm{C}$. The residue is then transferred to a Kjeldahl digestion flask and nitrogen determined.

In the following table are given the comparative data obtained by the two methods on the daily samples and the composite samples:

TABLE III.-Comparison of the two methods for the determination of urea.

\begin{tabular}{|c|c|c|c|c|c|c|}
\hline \multirow{2}{*}{ Subject. } & \multicolumn{2}{|c|}{ Daily sample. } & \multicolumn{2}{|c|}{ Composite sample. } & \multirow{2}{*}{$\begin{array}{l}\text { Daily } \\
\text { sample, } \\
\text { M. \& S. } \\
\text { method. }\end{array}$} & \multirow{2}{*}{$\begin{array}{l}\text { Compo- } \\
\text { site } \\
\text { sample, } \\
\text { Braun- } \\
\text { stein } \\
\text { method. }\end{array}$} \\
\hline & $\begin{array}{l}\text { M. \& S. } \\
\text { method. }\end{array}$ & $\begin{array}{l}\text { Braun- } \\
\text { stein } \\
\text { method. }\end{array}$ & $\begin{array}{l}\text { M. \& S. } \\
\text { method. }\end{array}$ & $\begin{array}{l}\text { Braun- } \\
\text { stein } \\
\text { method. }\end{array}$ & & \\
\hline $\begin{array}{l}\text { No. } 2 . . \\
\text { No. } 3 . .\end{array}$ & $\begin{array}{r}28.455 \\
24.848 \\
27.357 \\
26.363 \\
26.649 \\
25.369 \\
25.896 \\
25.833\end{array}$ & $\begin{array}{l}28.339 \\
24.327 \\
27.293 \\
26.398 \\
25.739 \\
25.016 \\
25.449 \\
26.384\end{array}$ & $\begin{array}{l}28.346 \\
25.089 \\
27.071 \\
25.727 \\
26.685 \\
25.227 \\
25.574 \\
26.559\end{array}$ & $\begin{array}{l}28.578 \\
25.859 \\
27.579 \\
26.289 \\
26.195 \\
25.269 \\
25.527 \\
26.608\end{array}$ & $\begin{array}{l}+ \\
+ \\
+ \\
+ \\
+ \\
+ \\
+\end{array}$ & $\begin{array}{l}+ \\
+ \\
+ \\
+ \\
+ \\
+\end{array}$ \\
\hline
\end{tabular}


URIC ACID DETERMINATIONS (FOLIN'S MODIFICATION OF HOPKINS'S METHOD). $a$

In order to precipitate the uric acid and to remove the small amount of mucoid substance which is always present the following reagent is employed: 500 grams of ammonium sulphate and 5 grams of uranium acetate are dissolved in $650 \mathrm{cc}$ of water, to which $60 \mathrm{cc}$ of a 10 per cent solution of acetic acid are further added.

Seventy-five cubic centimeters of this reagent are added to $300 \mathrm{cc}$ of urine. After standing five minutes the mixture is filtered through two folded filters, the filtrate is divided into two portions of $125 \mathrm{cc}$ each, representing $100 \mathrm{cc}$ of the original sample, and 5 cc of concentrated ammonia added. The solution, after stirring, is set aside until the next day.

The precipitated ammonium urate settles to the bottom of the beaker during this time. The supernatant liquid is poured through a filter (Schleicher and Schüll, No. 575), a hardened filter being found the most serviceable, and the precipitate is collected and washed by a small amount of a 10 per cent solution of ammonium sulphate.

After washing three or four times on the filter with the 10 per cent ammonium sulphate solution the precipitate is washed back into the same beaker in which the precipitation was made, using about $100 \mathrm{cc}$ of water. Fifteen cubic centimeters of concentrated sulphuric acid are then added and the solution titrated immediately with one-twentieth normal potassium permanganate. The first trace of a rose color throughout the entire fluid is taken as the end point. Each cubic centimeter of the standard permanganate $\mathrm{N} / 20$ is equivalent to 0.00375 gram of uric acid. Owing to the solubility of ammonium urate, a final correction of $0.003 \mathrm{gram}$ for each 100 ce of urine employed is necessary.

KREATININ DETERMINATIONS (FOLIN'S METHOD). ${ }^{b}$

In this work kreatin was determined along with and calculated as kreatinin. The method is based on the reaction of kreatinin with alkaline picric acid solution. The red colored solution produced by this reaction has, when in proper dilution, the same shade of color as potassium bichromate solution.

The solutions required are: Half normal potassium bichromate; 10 per cent caustic soda; saturated (1.2 per cent) picric acid solution and normal hydrochloric acid where kreatin and kreatinin are determined together.

The determination is carried out in the following manner: Ten cc of urine are placed in a 500 ec graduated flask, 5 ec of normal hydrochloric acid added and the mixture heated on the water bath for three 
and one-half to four hours, with a proper return condenser attached. At the end of this time the flask is cooled, the acid is neutralized, and 15 ec picric acid solution and $5 \mathrm{ce}$ of the caustic soda solution are added. The contents of the flask are thoroughly mixed and allowed to stand for five minutes. It is now made up to mark and compared with the half normal bichromate solution in a colorimeter. The Duboseq colorimeter was used in this work.

Ten milligrams of pure kreatinin treated in same way gives a depth of color $8.1 \mathrm{~mm}$ of which corresponds to $8 \mathrm{~mm}$ of the bichromate solution. The urine picrate solutions are all compared with $8 \mathrm{~mm}$ of the half normal bichromate solution and adjusted till the depth of color is the same as the standard.

If the reading after adjustment of the urine picrate solution is 6.2 $\mathrm{mm}$, then $10 \mathrm{cc}$ of the urine would contain $10 \times \frac{8.1}{6.2}=13.06 \mathrm{mg}$ of kreatinin.

XANTHIN DETERMINATIONS (KRÜGER-SCHMID METHON). $a$

Free 400 ec of urine from proteids by coagulation and filtration, add 24 grams of sodium acetate and 35 ec of 12 per cent sodium bisulphite, heat to boiling and add 30 ec of 15 per cent copper sulphate. Boil three minutes with careful watching and stirring. After cooling and filtering, the precipitate is washed until colorless. The precipitate is returned to the original beaker and 200 cc of water added. After heating to boiling, $30 \mathrm{cc}$ of sodium sulphid is added, then acetic acid to acidity and the solution warmed on the steam bath until the copper sulphid settles. Filter while warm and wash with warm water, add 10 ec of 10 per cent hydrochloric acid and evaporate in a 300 ec porcelain dish to a volume of $10-15 \mathrm{cc}$. During the process and on standing two hours the uric acid settles out. This is filtered on a small filter paper washed with 3 per cent sulphuric acid until the total volume of filtrate and washings is $75 \mathrm{cc}$. The nitrogen of the precipitate multiplied by 3 gives uric acid, to which add $3.5 \mathrm{mg}$.

The uric acid filtrate is made alkaline with sodium hydroxid and then acidified with acetic acid. After warming to $70^{\circ}, 1$ ce of 10 per cent acetic acid and $10 \mathrm{cc}$ of potassium permanganate are added. Shake one minute, treat with $10 \mathrm{cc}$ of sodium bisulphite and $6 \mathrm{cc}$ of 15 per cent copper sulphate, boil for three minutes, filter the wash with dilute copper sulphate, and determine the nitrogen in the precipitate, which is that of the xanthin bases.

$a$ Hoppe Seyler's Thierfelder, 1903, p. 435. 
SPECIAL STUDY OF THE DISTRIBUTION OF THE NITROGENOUS CONSTITUENTS OF THE URINE AS AFFECTED BY THE PRESERVATIVES.

INTRODUCTION.

It is a very common opinion among medical practitioners and physiologists that salicylic acid when properly administered has some specific effect upon the excretion of certain of the nitrogenous elements of the urine, notably uric acid. One of the principal difficulties attending investigations of this character heretofore has been the uncertainty attending the methods of determination with regard to accuracy as to the quantities of nitrogen present in various forms. Some of these nitrogenous elements are present only in small quantities, and therefore any inherent fault of the method itself or any failure in proper manipulation on the part of the analyst is likely to introduce very serious errors into the results. The data which follow have been obtained in accordance with the methods of investigation which have been described in detail.

The study of the individual data shows often a wide daily variation in the various forms of nitrogenous constituents excreted, and the daily quantity of total nitrogen excreted also shows notable variations.

The total nitrogen of the urine having first been determined, the quantities of nitrogen which were present as urea, uric acid, xanthin, kreatinin, and ammonia were separately determined. The difference between the total nitrogen contained in these bodies and the total nitrogen of the urine represents the nitrogenous elements undetermined. On account of the amount of analytical work in connection with the study of the metabolic processes during Series VI, it was found impossible to properly conduct a study of the distribution of the nitrogen in the urine. To remedy this fault a supplemental study was made of four men subsequent to the investigations described as Series VI. 
SCHEDULE OF ADMINISTRATION OF THE PRESERVATIVE.

The schedule of administration of the preservative and the dates covered by the periods of observation are shown in Table IV.

TABLE IV.-Schedule of administration of salicylic acid and salicylates for special study on four men-Series $X I$.

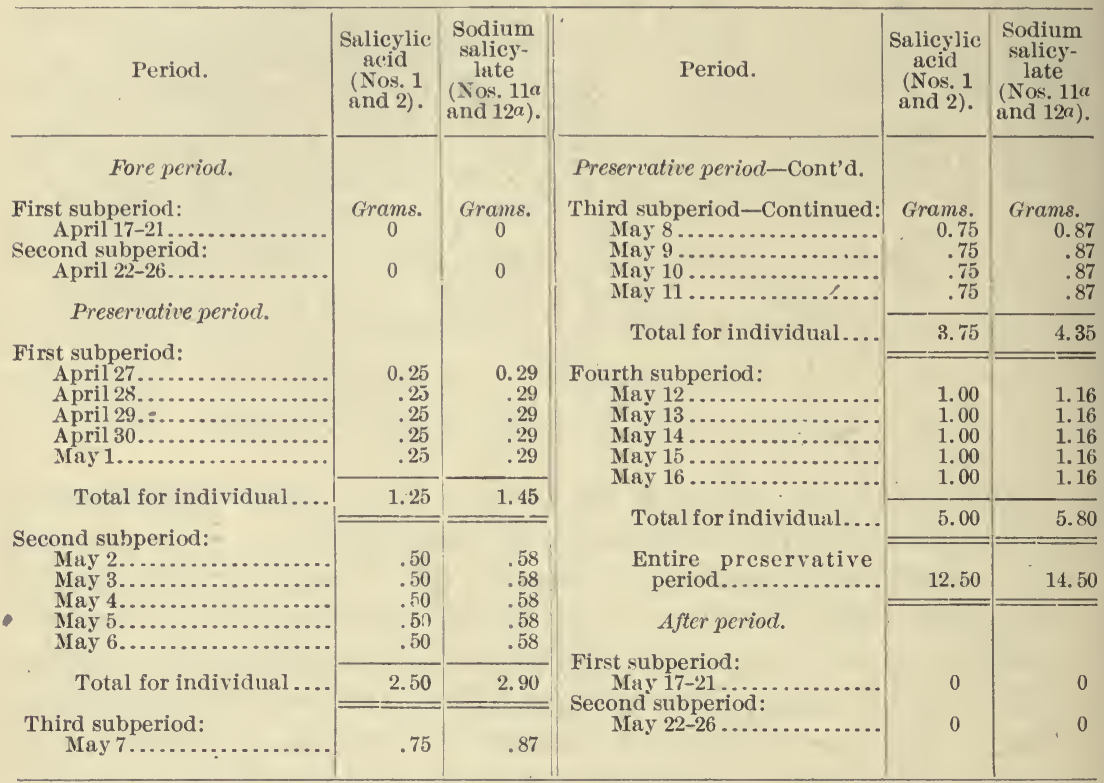

$a$ The amounts of sodium salicylate administered to Nos. 11 and 12 contained amounts of salicylic acid equivalent to the corresponding doses given Nos. 1 and $2-1$. e., $0.25,0.50,0.75$, and 1 gram in the respective subperiods.

SUPPlemental study of the PRESENCe of albumin aNd the ReACtion of the URine.

A further study of the effect of salicylic acid and sodium salicylate on the acidity of the urine and the occurrence of albumin therein was made in the case of the four subjects of the special study. In Table V are given the individual and summarized results of this investigation.

The acidity of the urine is comparatively expressed by the number of cubic centimeters of tenth-normal sodium hydroxid solution required to neutralize $100 \mathrm{cc}$ of the urine, using phenolphthalein as indicator. The average acidity for Nos. 1 and 2, receiving salicylic acid, in the fore period is 38.9 , in the preservative period 41.7 , and in the after period 37.1. These figures indicate a tendency on the part of the salicylic acid to increase the acidity of the urine. 
In the case of Nos. 11 and 12 receiving sodium salicylate the average acidity for the fore period is represented by 34.0 , for the preservative period by 33.6 , and for the after period by 36.2 , showing a tendency to decrease the acidity.

The percentage of cases in which albumin was found in the fore period is 25 , in the preservative period 36 , and in the after period 30 . It appears from these data that the preservative distinctly favored the formation of traces of albumin in the urine, the result being much more marked, however, when the salicylic acid was administered than in the case of the sodium salicylate. 


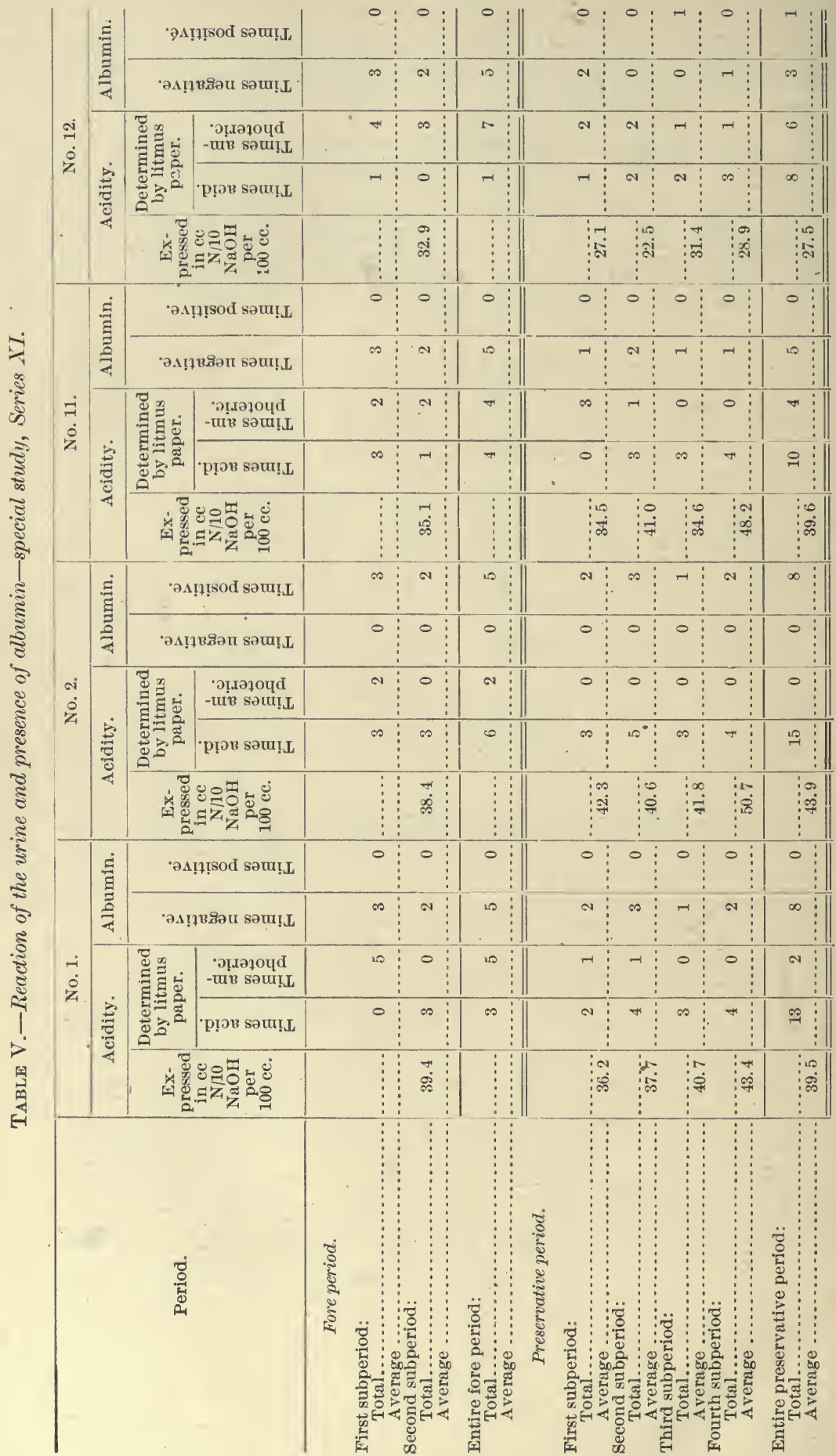


SALICYLIC ACID AND SALICYLATES.

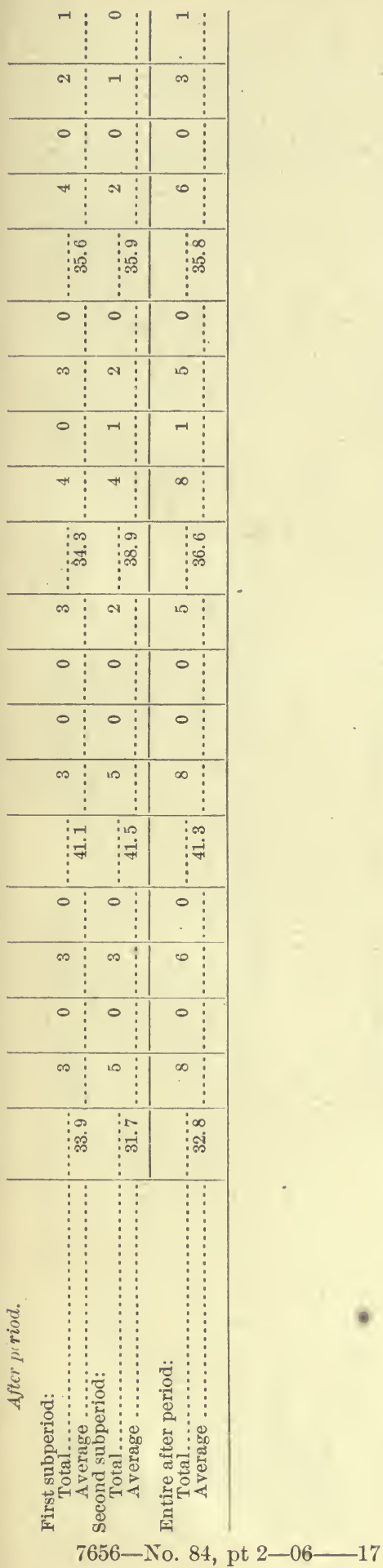




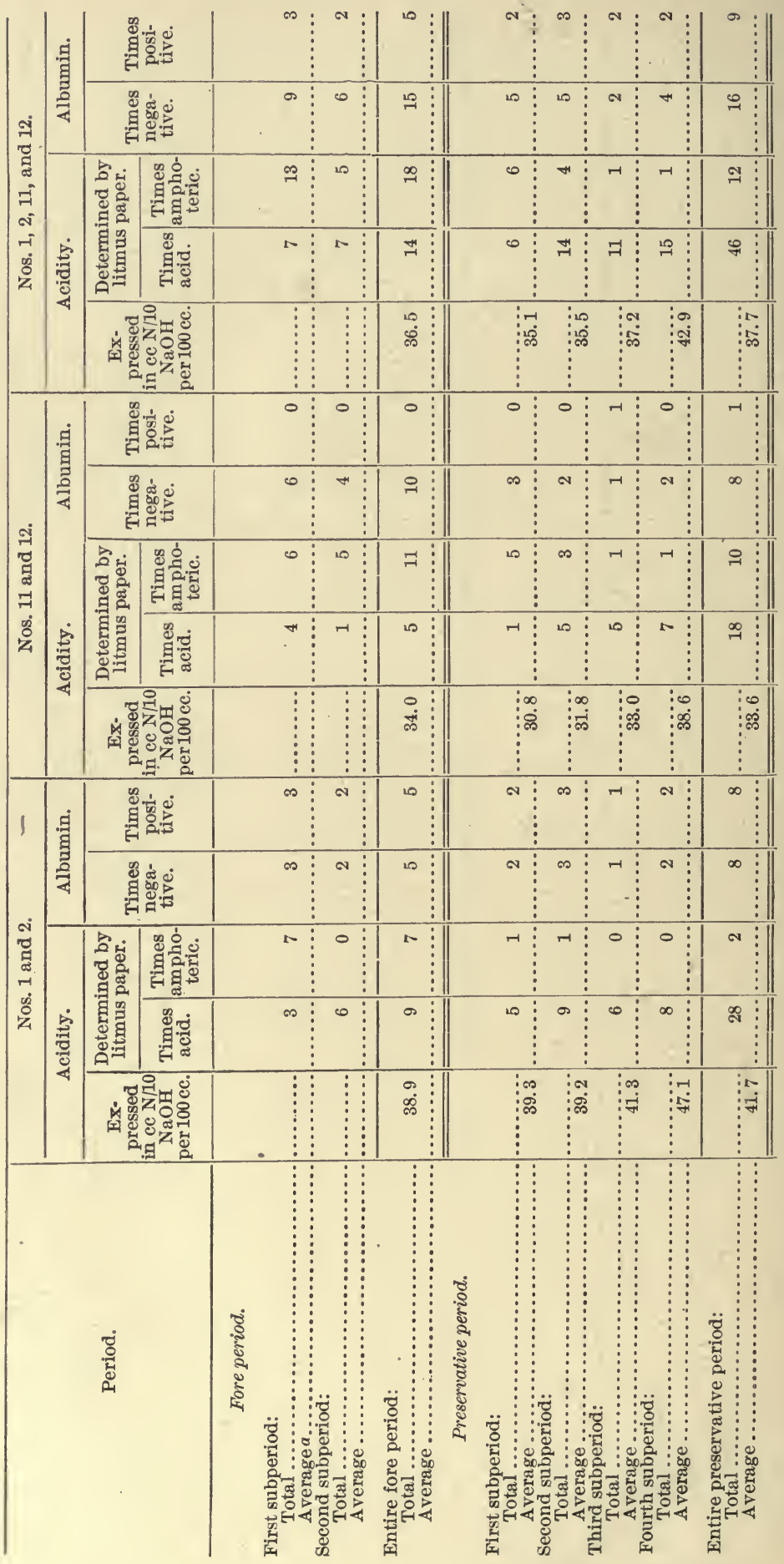




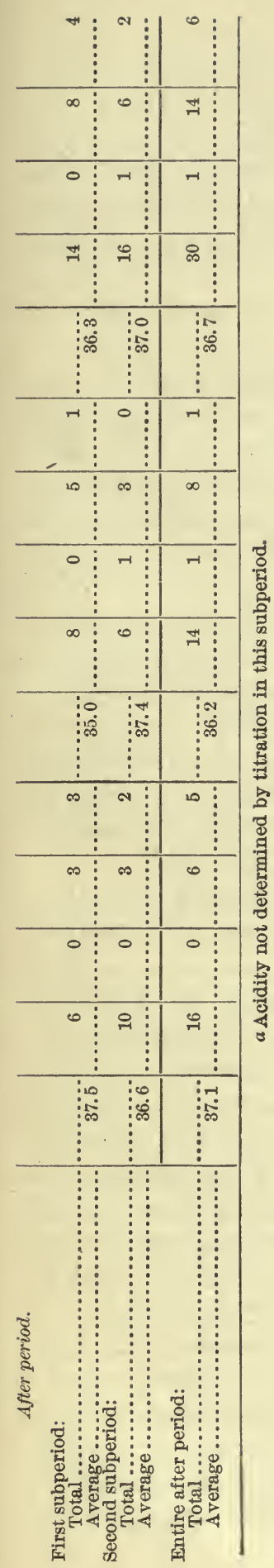


INDIVIDUAL ANALYTICAL DATA.

No. 1.

In the case of No. 1, Table VI, the average daily volume of the urine excreted during the fore period is 921 cc, containing 56.7 grams of solid matter. The total nitrogen ingested daily in the food is 16.46 grams, the total nitrogen excreted in the urine 13.81 grams. Of this quantity 12.77 grams are present as urea, 0.15 as uric acid, 0.04 as xanthin, 0.42 gram as kreatinin, 0.22 gram as ammonia, and 0.22 gram undetermined.

During the preservative period there was a slight increase in the volume of the urine and a considerable increase in the amount of total solids therein. There was an increase in the amount of urea, a slight increase in the amount of kreatinin and ammonia excreted, and a slight decrease in the amount of uric acid found, leaving a total of only 0.18 gram daily of nitrogen unaccounted for by a direct determination of the various nitrogenous constituents.

In the after period there was again an increase in the quantity of the urine, a decrease in the amount of total solids to practically the same figure as in the fore period, and a decrease in the quantity of nitrogen in the urine and the amount of urea excreted.

In respect of the quantity of materials eliminated, it is seen that the average excretion per day of total urea is 27.33 grams in the fore period, for uric acid $0.45 \mathrm{gram}$, for xanthin $0.10 \mathrm{gram}$, for kreatinin 1.13 grams, and for ammonia 0.27 gram for the fore period.

In the preservative period the quantity of urea daily excreted is almost exactly 1 gram greater than in the fore period. The quantity of kreatinin is also somewhat increased.

In the after period the several amounts excreted are almost the same as in the fore period. In the case of No. 1, therefore, it is apparent that the effect of the salicylic acid was to increase the quantity of urea and kreatinin excreted, but not to increase the quantity of uric acid and the other nitrogenous constituents. The percentage of nitrogen excreted in the urine, computed upon the total quantity of nitrogen in the foods in the case of No. 1 in the fore period, is 83.9. The percentages of the various nitrogenous constituents in the urine (based on the total nitrogen excreted) are urea 92.5, uric acid 1.1, xanthin 0.3 , kreatinin 3 , ammonia 1.6 , and undetermined 1.5 .

In the preservative period the percentage of nitrogen in the food excreted in the urine is very markedly increased. The percentage in the urine in the various forms of nitrogenous constituents does not differ greatly from the fore period, except in the cases of kreatinin and ammonia, where there is a considerable increase and the percentage of undetermined is less. 
In the after period the percentages are restored almost to their original value in the fore period.

In general it appears, therefore, that in the case of No. 1 the salicylic acid has a stimulating effect upon the excretion of nitrogen in the urine, but does not increase either in quantity or percentage the amount of uric acid excreted, but does slightly increase the proportions of kreatinin, urea, and ammonia excreted.

No. 2.

In the case of No. 2 the volume of urine was very much greater than in the case of No. 1 and the quantities of total solids are correspondingly increased. There is a progressive decrease in the total nitrogen excreted in the urine, the daily quantity in the fore period being 17.63 grams, in the preservative period 16.04 grams, and in the after period 13.84 grams. This corresponds with the decrease in the volume of the urine during these periods. A similar decrease is found also in the quantity of urea and the other nitrogenous constituents, all except kreatinin and xanthin being more or less diminished in quantity.

The total weight of urea daily excreted in the fore period is 35.02 grams, in the preservative period 31.56 grams, and in the after period 27.22 grams. It would appear that the exhibition of the salicylic acid tends to interfere with the metabolism of the nitrogen as manifested in the urine, and these indications are of an opposite character to those shown in the case of No. 1.

Of the total nitrogen in the food there was excreted in the urine during the fore period of No. 285.6 per cent, during the preservative period 77.9 per cent, and during the after period 67.2 per cent. If the relations of the various nitrogenous constituents in the urine be considered it will be seen that there is but little variation in the excretion of urea nitrogen in relation to the total nitrogen in the urine. During the fore period 92.8 per cent is excreted as urea; during the preservative and after period 91.9 per cent. In the fore period 1.1 per cent of the total nitrogen in the urine is excreted as uric acid, 1 per cent in the preservative period, and 1.2 per cent in the after period. The percentage of nitrogen excreted as kreatinin (based on the total nitrogen in the urine) is 3.4 in the fore period, 4.0 in the preservative period, and 3.6 in the after period. For ammonia the figures are 1.9 per cent in the fore period, 1.4 per cent in the preservative period, and 1.9 per cent in the after period.

These data show that the mass effect in No. 2 was to inhibit in a very striking manner the metabolism of the nitrogen, but that the urine retained its normal composition in respect of the relative amounts of nitrogenous constituents, although the excretion of nitrogen was progressively diminished throughout the preservative and after periods. 
No. 11 .

In the case of No. 11 there was a marked increase in the volume of urine during the preservative period, rising from 748 cc daily in the fore period to-943 in the preservative period, and falling slightly, namely, to 913 , in the after period. The total solids excreted in the urine rise from 44.2 grams in the fore period to 52.9 grams in the preservative period and fall to $50.3 \mathrm{grams}$ in the after period. The total nitrogen in the urine rose from 10.74 grams in the fore period to 12.34 grams in the preservative period, falling only slightly in the after period, namely, to 12.23 grams. There was a corresponding increase in the urea nitrogen, rising from 9.77 grams in the fore period to 11.08 grams in the preservative period and rising still higher, namely, to 11.31 grams, in the after period. There was no increase in the uric acid excretion during the preservative period, but a slight increase is shown in the after period. There was a slight increase in the kreatinin during the preservative period and a decrease in the ammonia.

There was an average daily excretion of 20.9 grams of urea in the fore period, 23.71 grams in the preservatıve period, rising to 24.20 grams in the after period. There was no increase in the excretion of uric acid in the preservative period, but an increase is shown in the after period. There was a marked increase in the excretion of kreatinin during the preservative period. Seventy per cent of the total nitrogen in the food was excreted in the urine in the fore period, 80.4 per cent in the preservative period, and 79.7 per cent in the after period.

In the total percentage of nitrogen excreted as urea there was a decrease in the preservative period, and an increase in the after period exceeding the amount excreted in the fore period. There was markedly less uric acid excreted in the preservative period, but an increase in the after period. No appreciable influence was exerted by the salicylate of soda upon the percentage of nitrogen excreted as kreatinin, but less was excreted as ammonia.

In general it may be said in the case of No. 11 that the exhibition of the salicylate of soda has an apparent tendency to increase the metabolism of nitrogen. It produces no increase in the amount of uric acid eliminated in the preservative period and has very little effect upon the form in which the nitrogen was excreted.

No. 12.

In the case of No. 12 the volume of urine during the preservative period was slightly increased, falling again in the after period to a little below the normal for the fore period. The quantity of total solids excreted in the urine was also slightly increased in the preservative period and fell considerably below the normal of the fore period 
in the after period. In this connection attention is called to the fact that the quantity of nitrogen ingested in the food was somewhat greater in the preservative period and after period than in the fore period. But the total quantity of nitrogen excreted in the urine was considerably less in the preservative period than in the fore period, although the amount ingested is greater. There was some tendency to a larger excretion in the after period, but it did not reach the amount found in the fore period, in spite of the larger quantity of nitrogen in the food. The uric acid in this case is again less in quantity in the preservative period than in the fore period, but is restored to the amount of the fore period in the after period. There appears to be no effect upon the amount of kreatinin excreted, while the ammonia is somewhat less in the preservative period than in the fore period.

In regard to the total quantities of the various nitrogenous constituents in the urine it is seen that the amount of urea excreted is markedly less in the preservative period than in the fore period, and somewhat increased over the preservative period in the after period. The quantity of uric acid excreted during the preservative period is markedly less than in the fore or after period. There was no appreciable effect produced by the sodium salicylate upon the amount of kreatinin excreted. The ammonia is less in the preservative period than in either of the other periods. In the study of the distribution of the nitrogen among the various nitrogenous elements it is seen that an abnormal percentage of nitrogen in the food is excreted in the urine, amounting to 99 per cent in the fore period, 84.4 per cent in the preservative period, and 88.8 per cent in the after period. The administration of the salicylate of soda did not have any effect on the proportions of nitrogen excreted as urea, these being 90 per cent, 90.1 per cent, and 90.2 per cent, respectively, for the three periods.

Again, it is found that the percentage of nitrogen excreted as uric acid is diminished in the preservative period, and is restored in the after period. The percentage of nitrogen excreted as kreatinin is slightly larger in the preservative period than in either of the other periods, while the percentage of nitrogen excreted as ammonia is less.

In this case it is noticed that the administration of the preservative tends to restrict the activity of nitrogen metabolism, that it has a distinct tendency to diminish the amount of uric acid excreted, and that it does not produce any other very marked effect upon the distribution of the nitrogen in the different nitrogenous components of the urine.

SUMMARY FOR NOS. 1 AND 2.

It is convenient, for purposes of comparison, to consider first the data for Nos. 1 and 2, they having received salicylic acid, then those for Nos. 11 and 12, they having received equivalent amounts of salicylate 
of soda, and afterwards a summary of the four subjects. In discussing. these summaries attention will be directed mainly to the important points of the disturbance of the nitrogen metabolism, the influence of the preservative upon the amount of uric acid and the other principal nitrogenous bodies of the urine excreted, and the distribution of the nitrogen among the nitrogenous elements of the urine.

Viewing Nos. 1 and 2 together, it is found that there is a slight decrease in the volume of the urine during the preservative period, and a decrease again occurs during the after period. The total solids in the urine are almost the same in the fore and preservative periods, and are somewhat diminished in the after period. The total weight of the nitrogen in the urine is slightly diminished in the preservative period, and this diminution is still more marked in the after period. The quantity of urea nitrogen excreted in the preservative period is slightly less than that in the fore period, and this deficiency is very marked in the case of the after period.

There is a tendency shown to diminish the amount of nitrogen excreted as uric acid in the preservative period. There is a slight increase in the amount of nitrogen excreted as kreatinin during the preservative period, while there is a slight decrease in the amount of nitrogen excreted as ammonia.

The total quantity of urea excreted is less in the preservative period and is still further diminished in the after period. The weight of the uric acid is slightly diminished during the preservative period and remains unchanged in the after period.

The total weight of kreatinin is greater in the preservative period than in the fore period and less in the after period than in the fore period. There is less ammonia excreted during the preservative period, while in the after period the amount is almost the same as in the fore period. Of the nitrogen ingested 84.84 per cent is excreted in the urine in the fore period, 81.87 per cent in the preservative period, and 74.42 per cent in the after period.

In regard to the distribution of the nitrogen among the various nitrogenous elements of the urine, it is noticed that there is no appreciable disturbance in the percentage appearing as urea, there being 92.6 per cent in the fore period, 92.3 per cent in the preservative period, and 92.3 per cent in the after period. The percentage of nitrogen appearing as uric acid is slightly less in the preservative period. In the after period it is the same as in the fore period. A somewhat larger percentage of nitrogen is found as kreatinin in the preservative period and a slightly less percentage as ammonia. 
SUMMARY FOR NOS. 11 AND 12.

In the case of Nos. 11 and 12 there is an increase in the volume of urine in the preservative period and a slight tendency to decrease in the after period. In regard to the total solids excreted in the urine, the amount is found to be considerably greater in the preservative period, amounting to 55.3 grams as compared with 50.3 grams in the fore period. The amount in the after period falls to 51.3 grams, only slightly greater than in the fore period. By reason of an increase in the bread ration of No. 12 the amount of nitrogen ingested in the food is greater in the preservative period and the after period than in the fore period. The total weight of the nitrogen excreted in the urine during the fore period is 12.52 grams daily, during the preservative period 12.75 grams, and in the after period 13.05 grams, showing a gradual increase in the nitrogen in the urine. As urea, 11.32 grams of nitrogen were excreted in the fore period, 11.47 grams in the preservative period, and 11.90 grams in the after period. In the case of uric acid there is again a decrease in the nitrogen excreted as uric acid in the preservative period and a slight increase in the after period over that of the fore period. There is an increase in the amount of nitrogen excreted as kreatinin in the preservative period, from 0.47 gram to 0.51 gram, dropping back to 0.48 gram in the after period. There is a slight decrease in the amount of ammonia excreted during the preservative period.

Of the nitrogen in the food, 84 per cent is excreted in the urine during the fore period, 82.4 in the preservative period, and 84.2 in the after period, thus showing a decrease of 1.6 per cent in the amount of nitrogen metabolized in the preservative period. In regard to the percentage of nitrogen excreted in the various constituents it is seen that there is but little influence of the preservative upon the percentage excreted as urea, it being 90.4 per cent in the fore period, 90.0 per cent in the preservative period, and 91.3 per cent in the after period. There is a decrease in the percentage of nitrogen excreted as uric acid in the preservative period and a slight increase in the percentage of nitrogen excreted as kreatinin, while that excreted as ammonia is slightly less in the preservative period.

GENERAL SUMMARY.

In the general summary of the four men the average daily volume of urine is $1075 \mathrm{cc}$ in the fore period, $1084 \mathrm{cc}$ in the preservative period, and $1032 \mathrm{cc}$ in the after period. The total solids of the urine are 57.1 grams for the fore period, 59.3 grams for the preservative period, and 54.4 grams for the after period, these data showing an increased excretion in the preservative period and a markedly decreased excretion of total solids in the after period. 
There are excreted 14.12 grams of total nitrogen daily in the urine for the fore period, 13.96 grams in the preservative period, and 13.41 grams in the after period, showing a gradual decrease in the total amount of nitrogen excreted.

The average daily amount of nitrogen excreted as urea in the fore period is 12.94 grams, in the preservative period 12.73 grams, and in the after period 12.31 grams, showing a progressive decrease in the amount excreted.

In the case of uric acid, the amount excreted in the preservative period is less than in the fore period, and returns to the amount of the fore period in the after period.

Respecting the quantity of the kreatinin, the amount excreted in the preservative period is slightly increased both over that of the fore period and the after period, while in the case of ammonia the amount of nitrogen excreted is less in the preservative period than in either of the other periods.

In regard to the quantity of the various nitrogenous constituents, it is seen that 27.69 grams of urea are excreted daily in the fore period, 27.26 grams in the preservative period, and 26.36 grams in the after period.

In the case of uric acid, less is excreted in the preservative period than in the fore period, and also less than in the after period.

The quantity of kreatinin is remarkably constant throughout, but is slightly increased in the preservative period over both the fore and the after periods.

In regard to the percentage of excretion of the nitrogen, it is seen that of the total nitrogen in the food 84.4 per cent appears in the urine in the fore period, 82.1 per cent in the preservative period, and 78.9 per cent in the after period.

The percentage of nitrogen excreted as urea does not appear to be appreciably affected by the administration of the preservative.

The percentage of nitrogen appearing in the preservative period as uric acid is slightly less than in the fore period, and also less than in the after period.

The percentage of nitrogen excreted in the urine as kreatinin is increased in the preservative period over both the fore and the after periods.

In the case of ammonia, the percentage of nitrogen excreted is decreased in the preservative period as compared with both the fore and the after periods.

The most important points brought out in the preceding discussion are as follows:

1. Salicylic acid and salicylates tend not only to diminish the quantity of uric acid eliminated, but also to decrease its relative percentage 
of the total nitrogen eliminated in the urine. These interesting observations therefore indicate that the general opinion that has been held respecting the influence of salicylic acid and salicylates in increasing the excretion of uric acid is fallacious, the contrary effect being produced.

2. The preservatives exercised a slightly inhibiting effect upon nitrogen metabolism in so far as the urinary excretion is concerned, and in this the results differ from those of Series VI, which being longer continued and including a greater number of men must be given more weight in the final conclusions. The data indicate a. slight but persistent disturbance of nitrogen metabolism, which can only be regarded as having a prejudicial effect upon health. 


\begin{tabular}{|c|c|c|c|c|c|c|c|c|}
\hline$\Xi$ & 'pәu!̣шхәәәри & $\stackrel{\dot{\sigma}}{\dot{a}}$ & a & -i & & S & i & -1 \\
\hline $\begin{array}{l}\text { ज्ञ } \\
\text { 펴 } 1\end{array}$ & 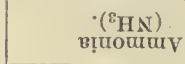 & $\stackrel{-1-}{2}$ & $-i$ & i & ? & i & $\exists$ & $\stackrel{102}{-1}$ \\
\hline 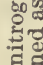 & 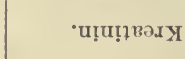 & $\dot{\circlearrowright}^{-1}$ & is & $\dot{0}$ & $=$ & $\stackrel{\leftrightarrow}{*}$ & $\stackrel{10}{\infty}$ & कर \\
\hline 焉 & 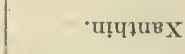 & & & & $\because$ & 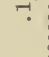 & $\because$ & $\because$ \\
\hline$\vec{~}$ & 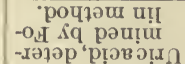 & & $\stackrel{7}{-i}$ & - & $\stackrel{\circ}{-}$ & 。 & & 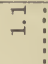 \\
\hline 范 & -вว $x_{\Omega}$ & & ธิં & g่ & si & เึ่ & $\stackrel{\infty}{\infty}$ & \&્ \\
\hline & 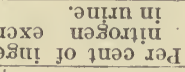 & & $\begin{array}{l}\sim \\
\infty \\
\infty\end{array}$ & $\ddot{\infty}$ & $\underset{\infty}{n}$ & $\begin{array}{l}\infty \\
118\end{array}$ & $\stackrel{0}{0}$ & $\begin{array}{l}\infty \\
\infty \\
\infty\end{array}$ \\
\hline$\stackrel{g}{g}$ & 'в!ุ & हैं స్ & సี. & ה. & เ? & 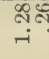 & ตำ & ลูก \\
\hline క & 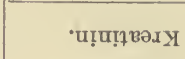 & & $\begin{array}{l}\text { E2 } \\
\text { की }\end{array}$ & $\stackrel{\substack{\infty \\
-1 \\
=}}{-1}$ & 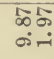 & 해요 & 5\% & $\begin{array}{l}\text { ลิลี } \\
\text { ఏन }\end{array}$ \\
\hline : & 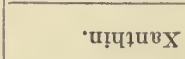 & 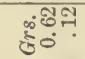 & हृं & $\stackrel{\infty}{\circ} ?$ & 융ㅇํ & b. & 잉ำ & mః \\
\hline 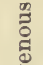 & 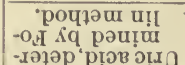 & 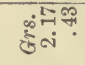 & बु. & 艧? & สูแ & 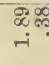 & $\begin{array}{l}\text { कू\% } \\
\text { i. }\end{array}$ & की \\
\hline 宽 & 'вәх & & $\begin{array}{l}\text { कే: } \\
\text { कीं }\end{array}$ & 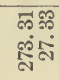 & 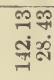 & 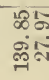 & 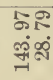 & 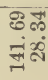 \\
\hline & 'рәи!шхәұәри & & 상. & बूสస్ & ผุष & tim & 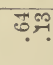 & ஜृ. \\
\hline & $\begin{array}{c}\cdot\left({ }^{8} \mathrm{HN}\right) \\
\text { в!̣นoưus } \mathrm{SV}\end{array}$ & & ్ㅏㅇ. & 고․ & 8.8 & : & 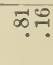 & 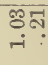 \\
\hline 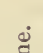 & 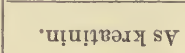 & 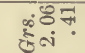 & $\underset{\pi}{\pi}+\underset{7}{4}$ & สิ? & in & 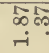 & $\begin{array}{l}\text { हैं } \\
\text { i. }\end{array}$ & लुकी \\
\hline$\stackrel{\Xi}{\Xi}$ & 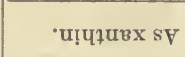 & 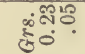 & क्त. & कू० & $\because 8$ & 78 & 구융 & 국용. \\
\hline 总 & 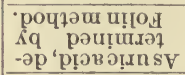 & & ㄱำ & कृ: & $18 \div$ & $\mathscr{8}$ & $R=7$ & 里里 \\
\hline 2 & 'вәдn SY & & : & $\begin{array}{l}\text { tô } \\
\text { ลำ }\end{array}$ & 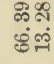 & 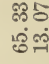 & $\begin{array}{l}919 \\
890 \\
699\end{array}$ & $\begin{array}{l}\text { 고 } \\
\stackrel{4}{\sharp 9}\end{array}$ \\
\hline & '[870工 & & 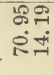 & $\begin{array}{l}\pi \infty \\
\pi \infty \\
\infty \\
\infty \\
\pi\end{array}$ & 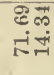 & 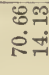 & 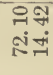 & $\begin{array}{l}\text { अా० } \\
\text { संख्र }\end{array}$ \\
\hline -u! & 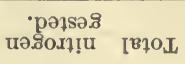 & 我 & $\vdots \stackrel{0}{\bullet}$ & : & : & $\begin{array}{l}0 \\
\vdots \\
\vdots\end{array}$ & : & : \\
\hline & น! sp! & 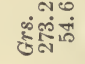 & 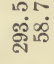 & छั. & 焉吕 & 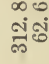 & वृ. & 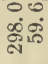 \\
\hline
\end{tabular}

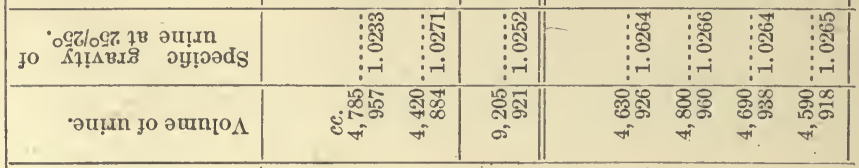

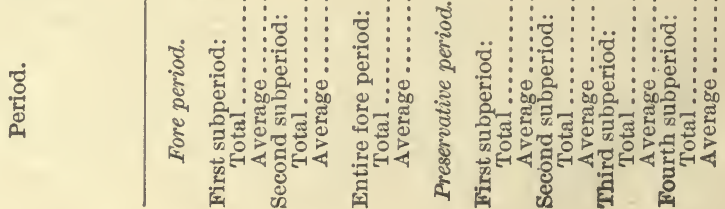




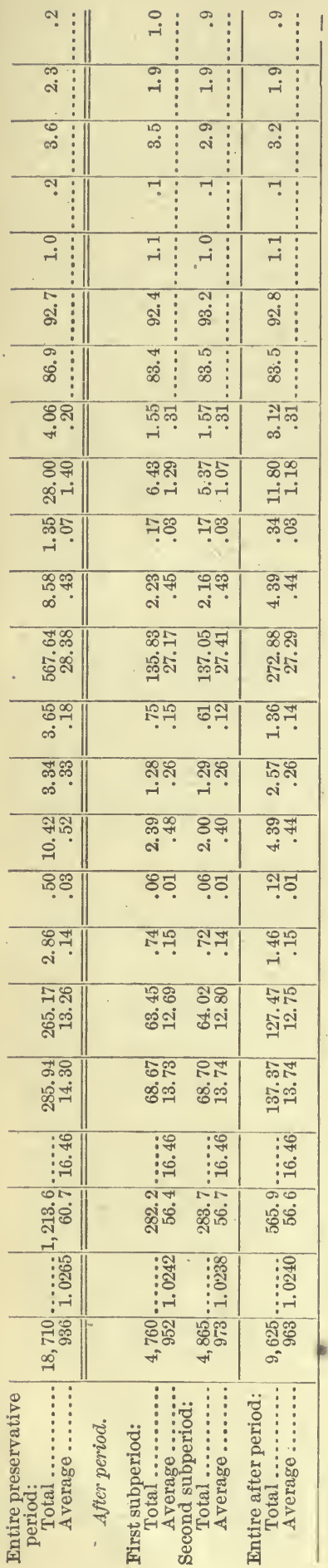




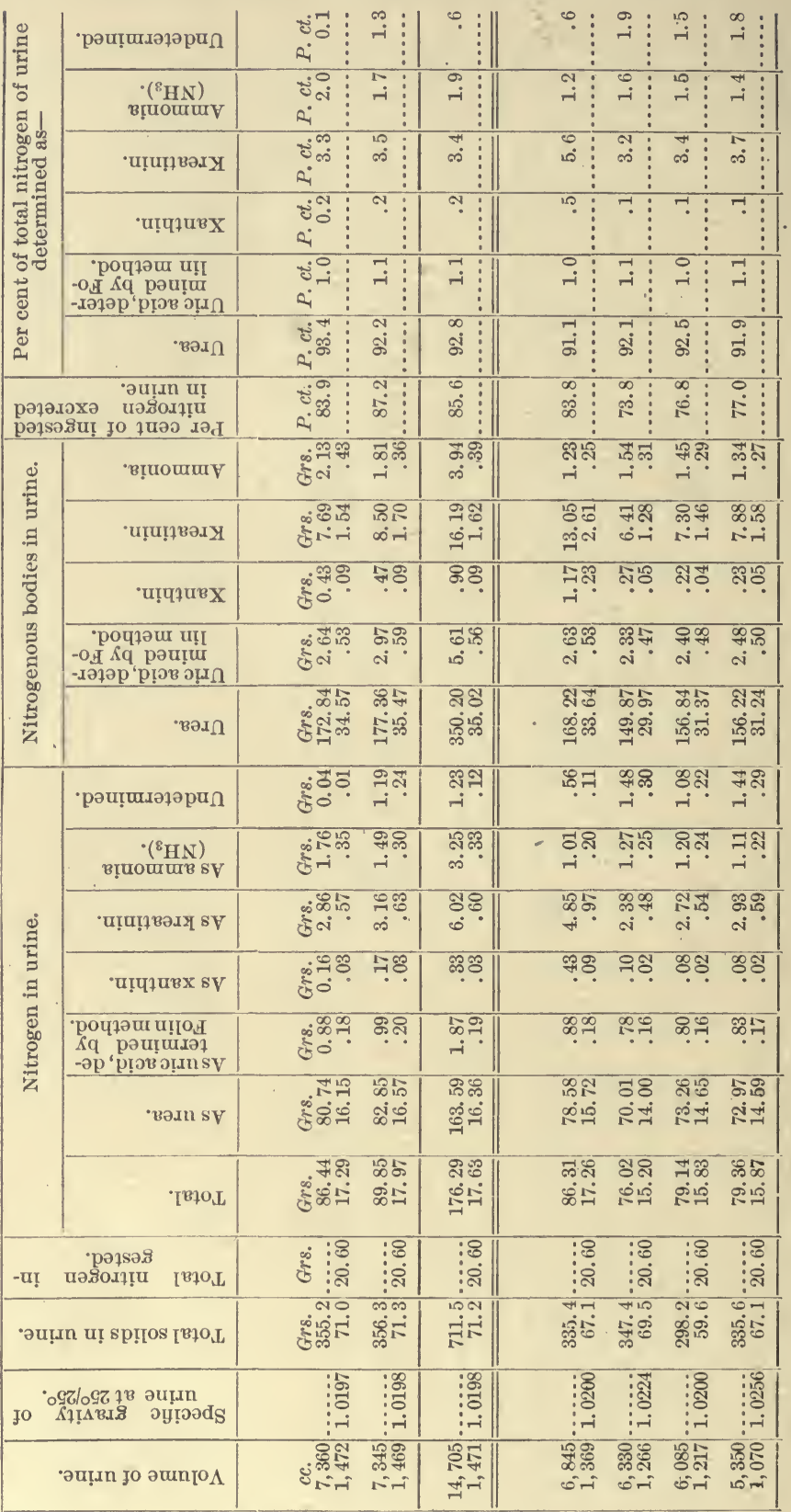

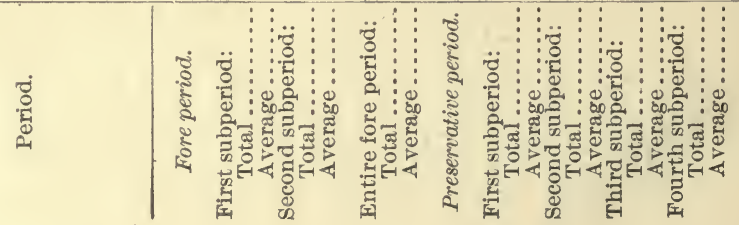




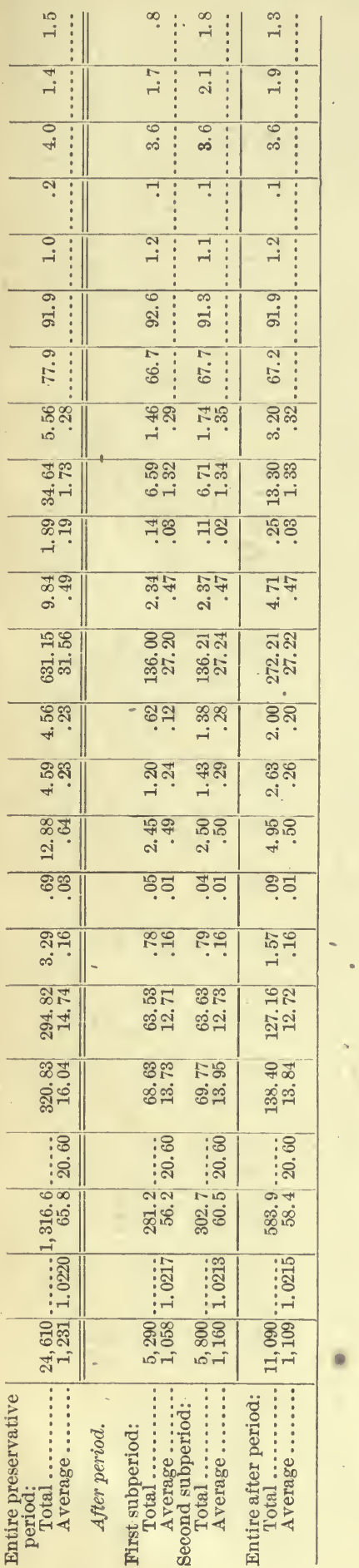




\begin{tabular}{|c|c|c|c|c|c|c|c|c|}
\hline & ' рәи!̣шәәәри & $\overrightarrow{\tilde{J}}_{-j}^{N-1} \vdots$ & $\stackrel{0}{-i}$ & $\stackrel{2}{4}$ & - & i & $\stackrel{\varphi}{\infty}$ & $\stackrel{0}{0}$ \\
\hline & $\begin{array}{c}\cdot\left({ }^{8} \mathrm{HN}\right) \\
\text { в!̣uouruy }\end{array}$ & $\tilde{\Xi}^{\circ}$ & $\stackrel{\infty}{-1}$ & aj & $\pi$ & i & $\stackrel{\sim}{\sim}$ & \\
\hline & 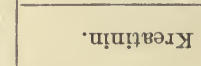 & $\dot{s}^{\infty}$ & $\stackrel{+7}{+4}$ & $\infty$ & $\stackrel{10}{+1}$ & & $\infty$ & का \\
\hline & 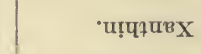 & & & & & & & \\
\hline & 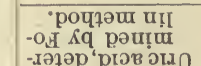 & & $\stackrel{\pi}{\pi}$ & $\begin{array}{l}20 \\
-1\end{array}$ & $\stackrel{\infty}{-\infty}$ & तi & a & m: \\
\hline & •вәх & & ¿: & $\begin{array}{l}8: \\
8:\end{array}$ & $\begin{array}{l}\infty \\
8\end{array}$ & के: & $\begin{array}{ll}\infty \\
\infty\end{array}$ & $\begin{array}{ll}10 \\
\stackrel{\infty}{\infty}:\end{array}$ \\
\hline & 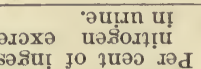 & & $\ddot{0}$ & $\stackrel{\circ}{\circ}$ & & & 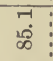 & $\begin{array}{l}\infty \\
\frac{\infty}{\infty}\end{array}$ \\
\hline & 'в!̣uourur & 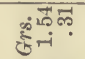 & تৈף & $\begin{array}{l}\text { कु్ } \\
\text { बi }\end{array}$ & क़ष & ్ำ. & $\mathscr{8}$ & ל. \\
\hline & чиฺирввәу & . & $\begin{array}{l}50= \\
10-1\end{array}$ & i: & 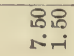 & कू' & ज़न & $\begin{array}{l}\text { का } \\
\text { की }\end{array}$ \\
\hline & ' иเ̣पұนвX & क्षक & 角: & 용요 & चु국 & कू⿻ & छढ: & 겨으 \\
\hline & 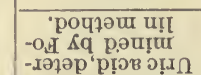 & & 용 & 8 둔. & 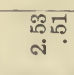 & की? & 때유. & 点 \\
\hline & "вәх口 & 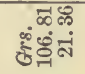 & 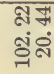 & 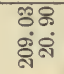 & 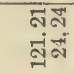 & $\begin{array}{l}\text { कำ } \\
\text { ㅇำ }\end{array}$ & 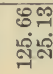 & 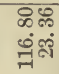 \\
\hline & 'рәи!ฺшзәдәри & 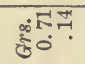 & $\infty$ & : & సুㅇ․ & హ్ & $\begin{array}{l}\operatorname{lot} 5 \\
\text { कi }\end{array}$ & Fis. \\
\hline & $\begin{array}{c}\cdot\left({ }^{8} \mathrm{HN}\right) \\
\text { в!นошسв SY }\end{array}$ & & 휴:? & $\begin{array}{l}\text { הत्: } \\
\text { הi }\end{array}$ & छ․ & कㄴ. & S包 & $\infty \approx$ \\
\hline & 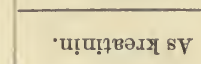 & 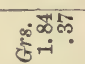 & 유 & $8 \%$ & $\begin{array}{l}\text { Ro } \\
\text { i. } \\
\text { a. }\end{array}$ & 용유. & 英. & 춘 \\
\hline & 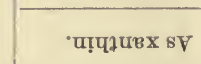 & & ศิธ & कृ & क्ष०० & तेत्ठ & สํํㅇ & 品ठ \\
\hline & 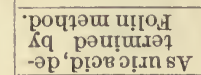 & & EJ & if: & कात & N & No & 里疋 \\
\hline & 'вәдn sy & & $\begin{array}{l}1018 \\
1-10 \\
1-10\end{array}$ & $\begin{array}{l}\text { की } \\
\text { s.0 }\end{array}$ & 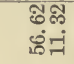 & 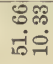 & $\begin{array}{l}\text { RI } \\
\text { ón- }\end{array}$ & 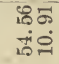 \\
\hline & 'Гв7оL L & 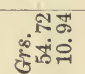 & 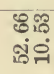 & 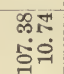 & 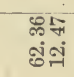 & 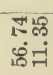 & $\begin{array}{l}\text { ॠ5 } \\
\text { 10 }\end{array}$ & 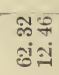 \\
\hline & 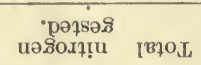 & \&. & : & : & : & : & $\begin{array}{l}10 \\
\vdots \\
\vdots \\
10\end{array}$ & 焗 \\
\hline & 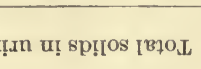 & $\begin{array}{l}\infty 0 \\
\infty \\
\text { केंगें }\end{array}$ & สู่ษ & $\begin{array}{l}\dot{\theta+n} \\
\dot{y} \not \dot{y}\end{array}$ & 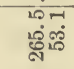 & 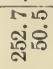 & ลูก & 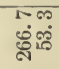 \\
\hline & 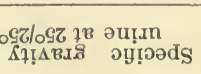 & & !: & & : & : & : & \\
\hline & 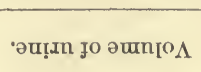 & 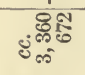 & 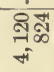 & 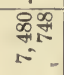 & 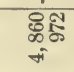 & 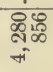 & 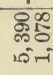 & $\begin{array}{l}\text { क्षेत्ठ } \\
\text { + }\end{array}$ \\
\hline
\end{tabular}

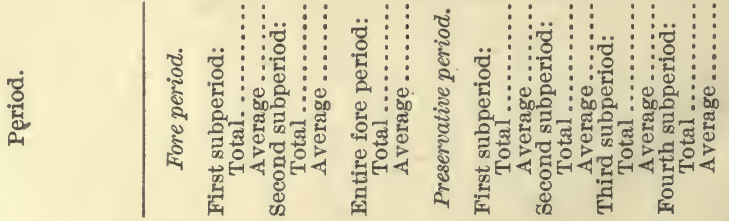




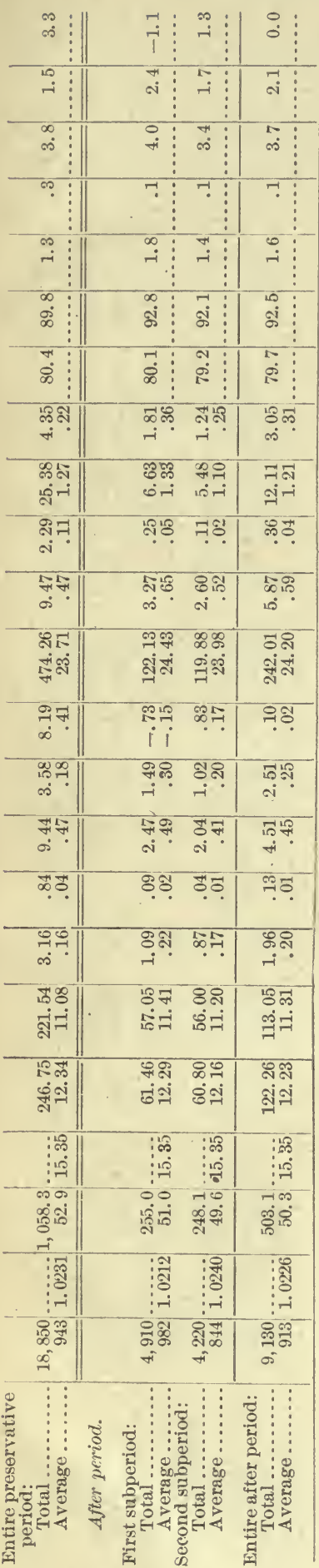

$7656-$ No. 84 , pt $2-06-18$ 


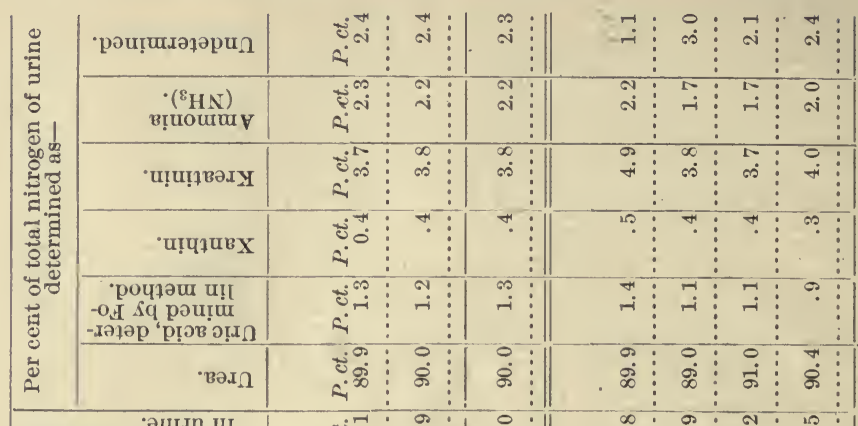

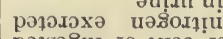

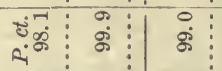

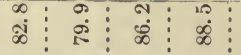

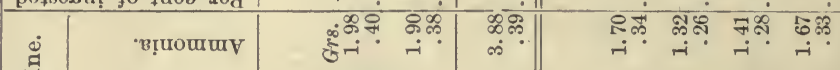

rempor
8. 8 is

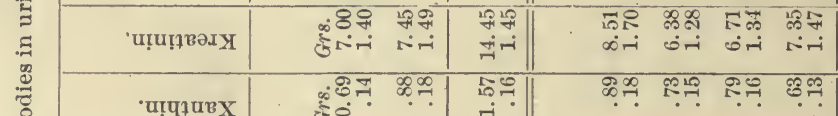

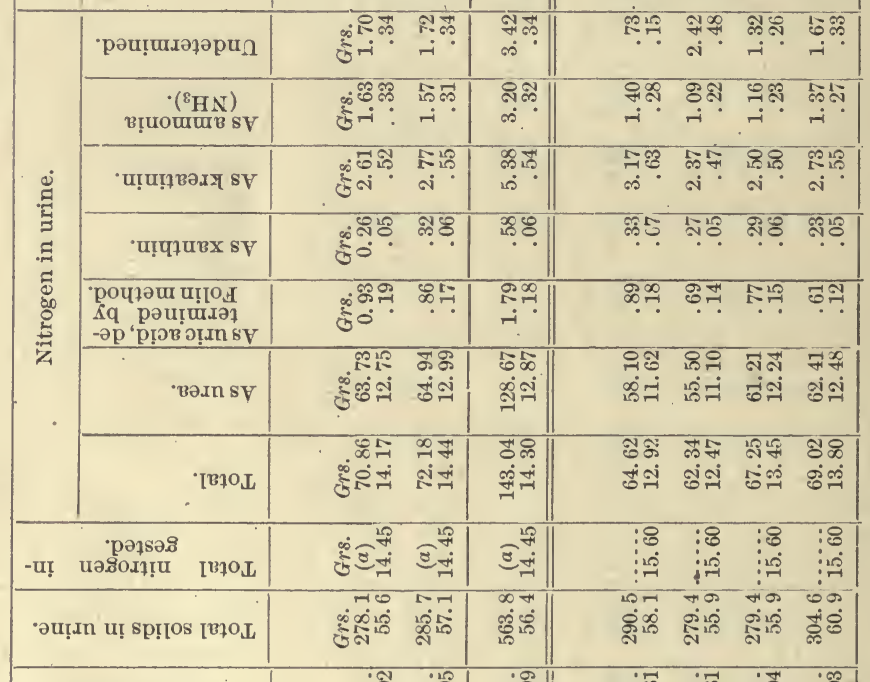

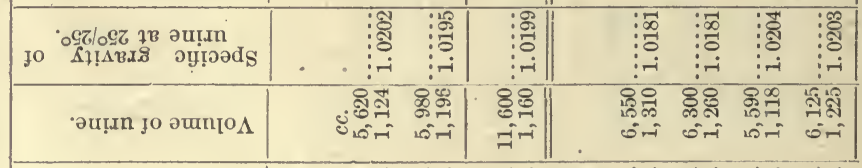

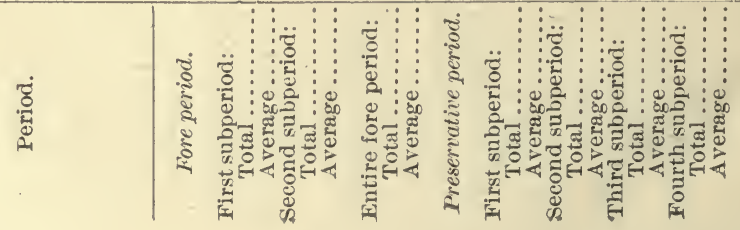




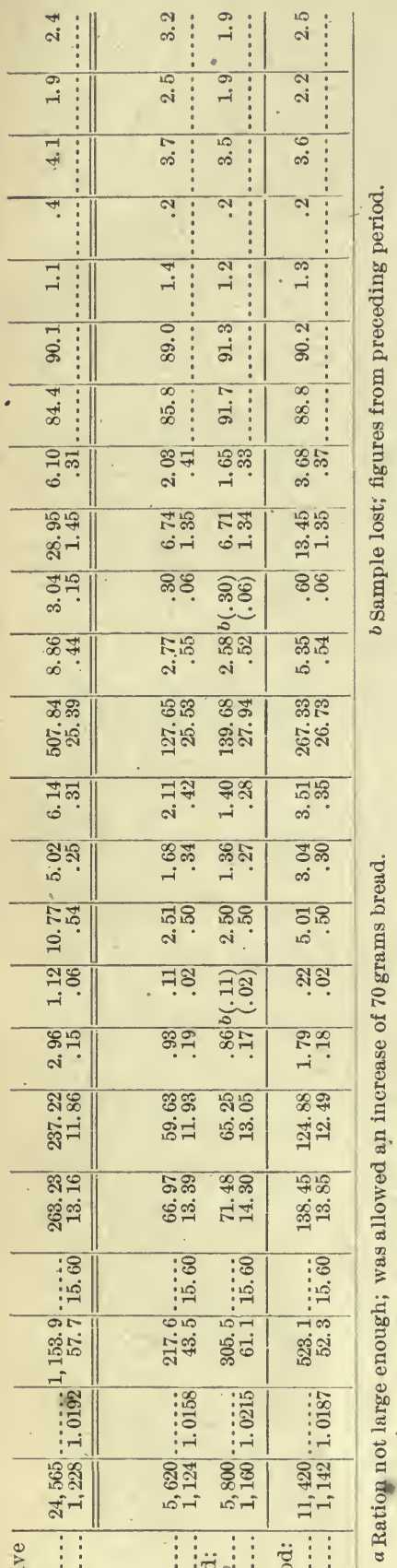

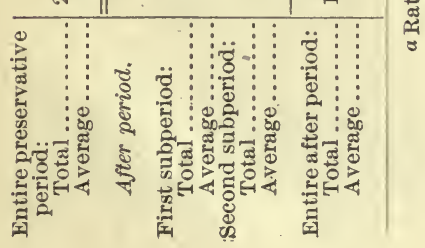




\begin{tabular}{|c|c|c|c|c|c|c|c|c|}
\hline$\Xi$ & 'рәичฺшгәұәри & & & & & i & & $\dot{r}$ \\
\hline 0 & 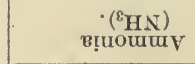 & & 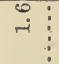 & $-i$ & & $\stackrel{6}{-}$ & & $\vec{i}$ \\
\hline$\underbrace{}_{0}$ & 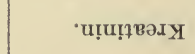 & & & $\infty$ & is & i & $\infty$ & $\infty$ \\
\hline 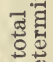 & 'แ!̣чวนвX & & & & ㄱ: & 7 & & $?$ \\
\hline $\begin{array}{l}0 \\
\overrightarrow{0}\end{array}$ & 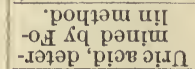 & - تं & $\exists$ & تं & & $\stackrel{\circ}{-}$ & & 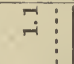 \\
\hline 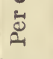 & 'вәхи & & बิં & هి & $\begin{array}{l}\infty \\
\text { 5 }\end{array}$ & $\begin{array}{l}\text { ఇ } \\
\text { si }\end{array}$ & बิ่ & 今ึ \\
\hline рәұә & 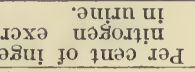 & $\begin{array}{l}\dot{0} \infty \\
\dot{0} \infty \\
\dot{\infty} \infty\end{array}$ & 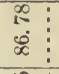 & \begin{tabular}{l|l}
$\overrightarrow{5}$ & $\vdots$ \\
$\vec{\infty}$ & $\vdots$ \\
\end{tabular} & $\begin{array}{l}5 \\
\text { A } \\
\text { an } \\
\infty \\
\infty\end{array}$ & 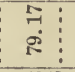 & $\begin{array}{l:l}8 & \\
-\infty & \\
& \end{array}$ & 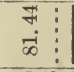 \\
\hline & 'в!̣ounury & 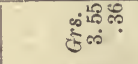 & $\begin{array}{l}\text { म8न } \\
\text { का }\end{array}$ & 迎 & 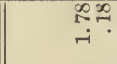 & 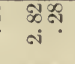 & $\begin{array}{l}\text { ॠ } \\
\text { i }\end{array}$ & $\begin{array}{l}\text { B.? } \\
\text { ai } \\
\text { ai }\end{array}$ \\
\hline$\stackrel{E}{\Xi}$ & 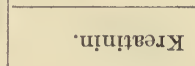 & 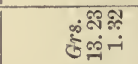 & जָ & 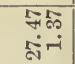 & $\begin{array}{l}\text { कूक } \\
\text { ลู่ }\end{array}$ & $\begin{array}{l}\Re=1 \\
=-1\end{array}$ & ה9 & $\begin{array}{l}8=7 \\
\dot{I}-i\end{array}$ \\
\hline$\stackrel{5}{0}$ & 'แ!ฺेयвX & & $\infty \%$ & 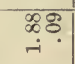 & 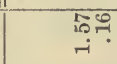 & 108 & .10\% & $0 \%$ \\
\hline 产 & 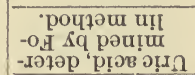 & - है & 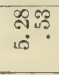 & $\begin{array}{l}8.50 \\
\stackrel{0}{0}^{\circ}\end{array}$ & क? & สูง & $\stackrel{\infty}{\infty}$ if & $\stackrel{\substack{\infty \\
\rightarrow}}{\rightarrow}$ \\
\hline 总 & ${ }^{\cdot в ә \boldsymbol{\alpha}_{\Lambda}}$ & 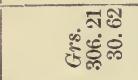 & 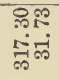 & 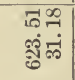 & $\begin{array}{l}\text { की. } \\
\text { कें } \\
\text { लें }\end{array}$ & 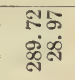 & 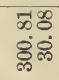 & 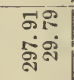 \\
\hline & 'рәи!шнәәәри $\Omega$ & 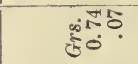 & $\begin{array}{l}\text { EST } \\
\text { ล }\end{array}$ & $\begin{array}{ll}1912 \\
\infty\end{array}$ & मळ\% & $\begin{array}{l}200 \\
\infty\end{array}$ & 존 & $\begin{array}{l}\text { ถูง } \\
\text { สิ }\end{array}$ \\
\hline & $\begin{array}{c}\cdot\left({ }^{8} \mathrm{HN}\right) \\
\text { в!ุoumur }\end{array}$ & 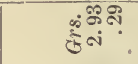 & 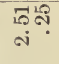 & 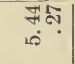 & भin & $\begin{array}{l}\text { कूळ } \\
\text { iे }\end{array}$ & $\begin{array}{l}\text { కై } \\
\text { i. }\end{array}$ & $\begin{array}{l}\text { ก } \\
\text { ลี }\end{array}$ \\
\hline g & 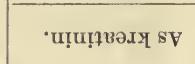 & & $\begin{array}{l}80 \\
\text { in } \\
\text { is }\end{array}$ & ลิ & $\begin{array}{l}\text { 개요 } \\
\infty^{\circ}\end{array}$ & 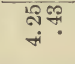 & $\begin{array}{l}\text { Sich } \\
\text { in }\end{array}$ & $\begin{array}{l}\text { ส. } \\
\text { is }\end{array}$ \\
\hline$\stackrel{\Xi}{\Xi}$ & 'u!̣प‡uвx sV & 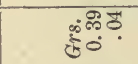 & & कृष & 188 & नत्ड & त्रे & त्रक्ष \\
\hline 总 & 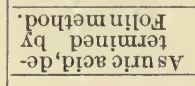 & & in & 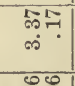 & $\stackrel{90}{-1}$ & $\underset{-i}{F}$ & .5.? & $\begin{array}{l}-6 \% \\
-i \\
0\end{array}$ \\
\hline & 'вә.n sV & 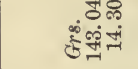 & 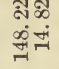 & 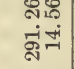 & $\begin{array}{l}5.0 \\
\text { Jỉ }\end{array}$ & 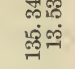 & 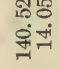 & ๑ळ \\
\hline & "I870I & 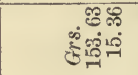 & 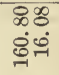 & 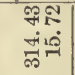 & 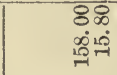 & 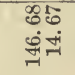 & 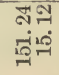 & $\begin{array}{l}180 \\
08 \\
019\end{array}$ \\
\hline & 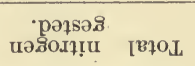 & $\begin{array}{l:l}\infty \\
0 \\
0\end{array}$ & $:$\begin{tabular}{l}
$\infty_{0}^{\circ}$ \\
$\vdots$ \\
\hdashline
\end{tabular} & \begin{tabular}{l}
$\vdots$ \\
$\vdots$ \\
$: \infty$ \\
\hdashline
\end{tabular} & 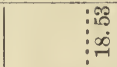 & : & $: \begin{array}{l}: \infty 0 \\
\vdots \\
\vdots \\
0\end{array}$ & : \\
\hline 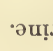 & In u!̣ spṭos [вłOL & జํํㅇํํ & झुं 18 & 象 & मृं & 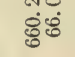 & हुं & 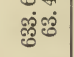 \\
\hline jo ${ }^{\circ}$ & 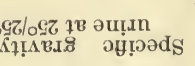 & तี่ & : & : & : & : & : & 荡 \\
\hline & 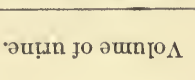 & 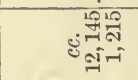 & 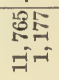 & $\begin{array}{l}\text { केष } \\
\text { कึస }\end{array}$ & 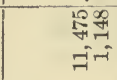 & 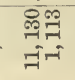 & $\begin{array}{l}18 x \\
150 \\
0-1\end{array}$ & $\begin{array}{l}\text { 융해 } \\
\text { क }\end{array}$ \\
\hline
\end{tabular}

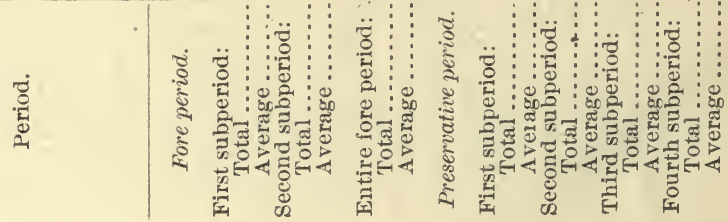




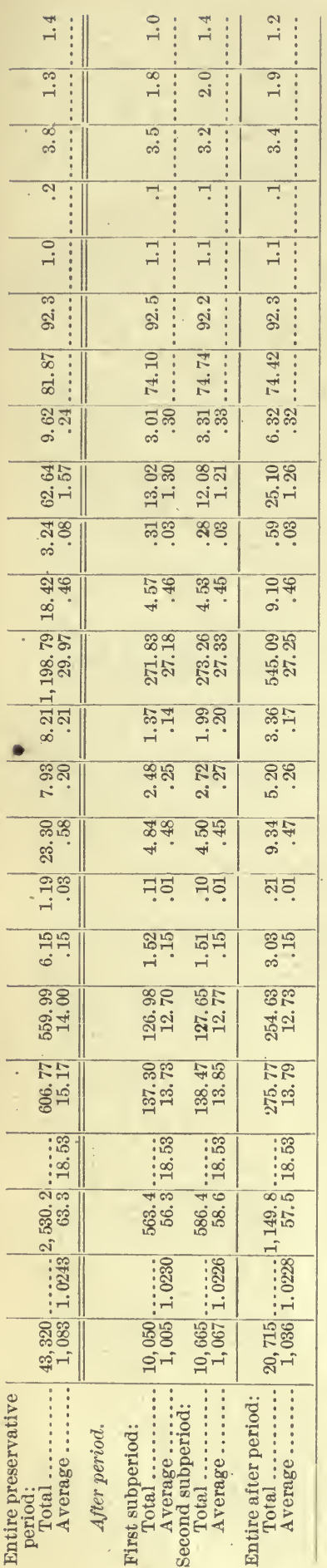




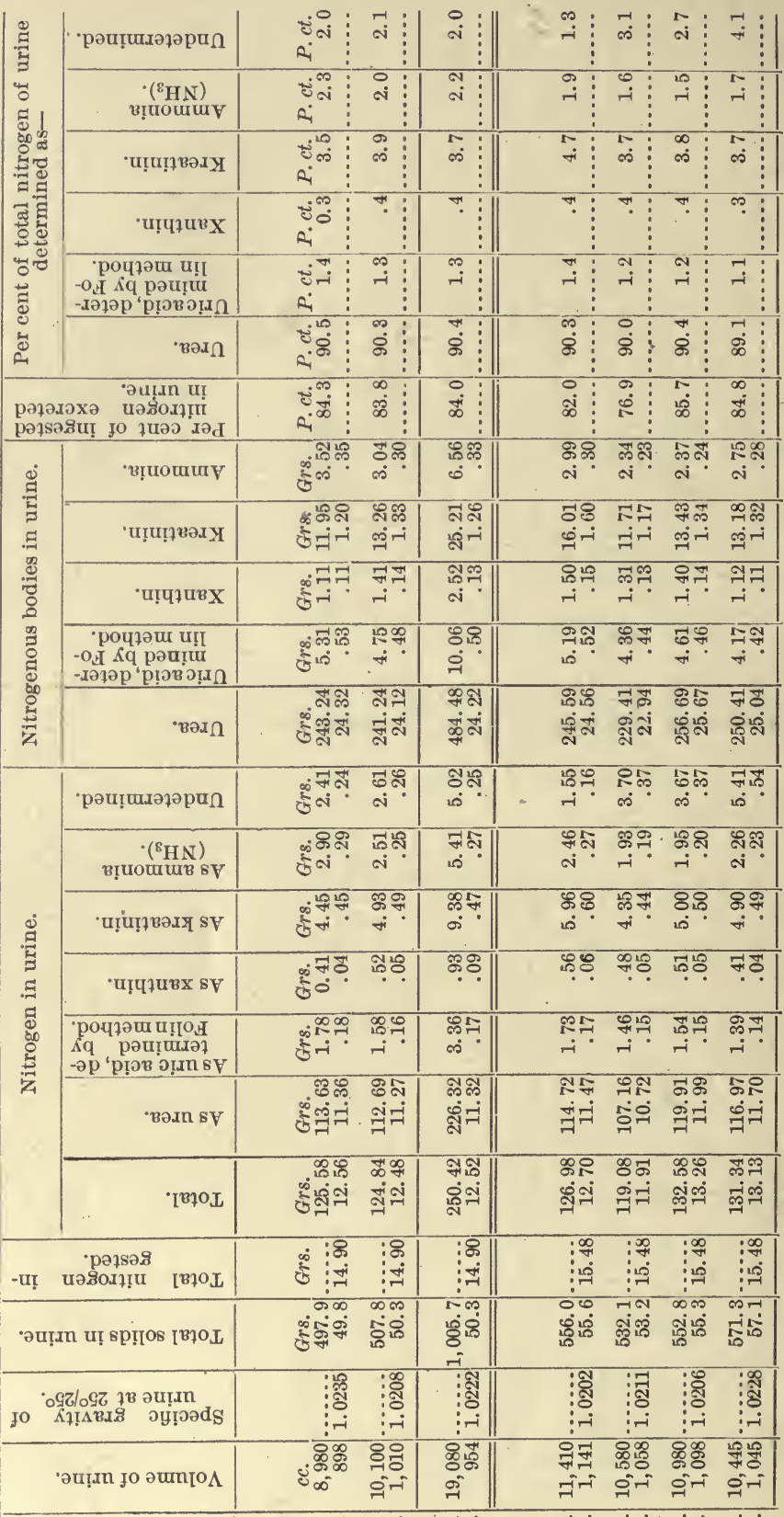

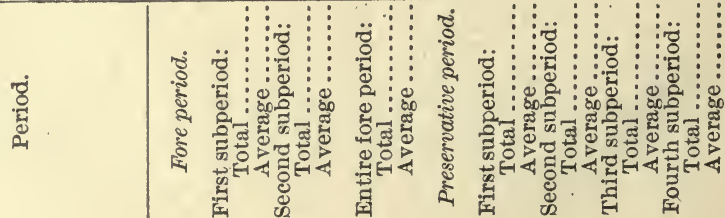




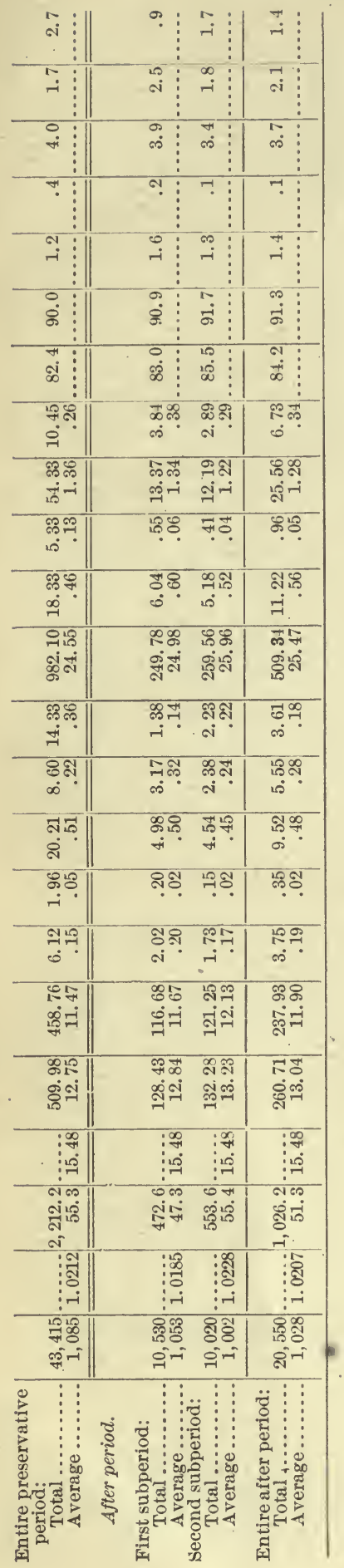




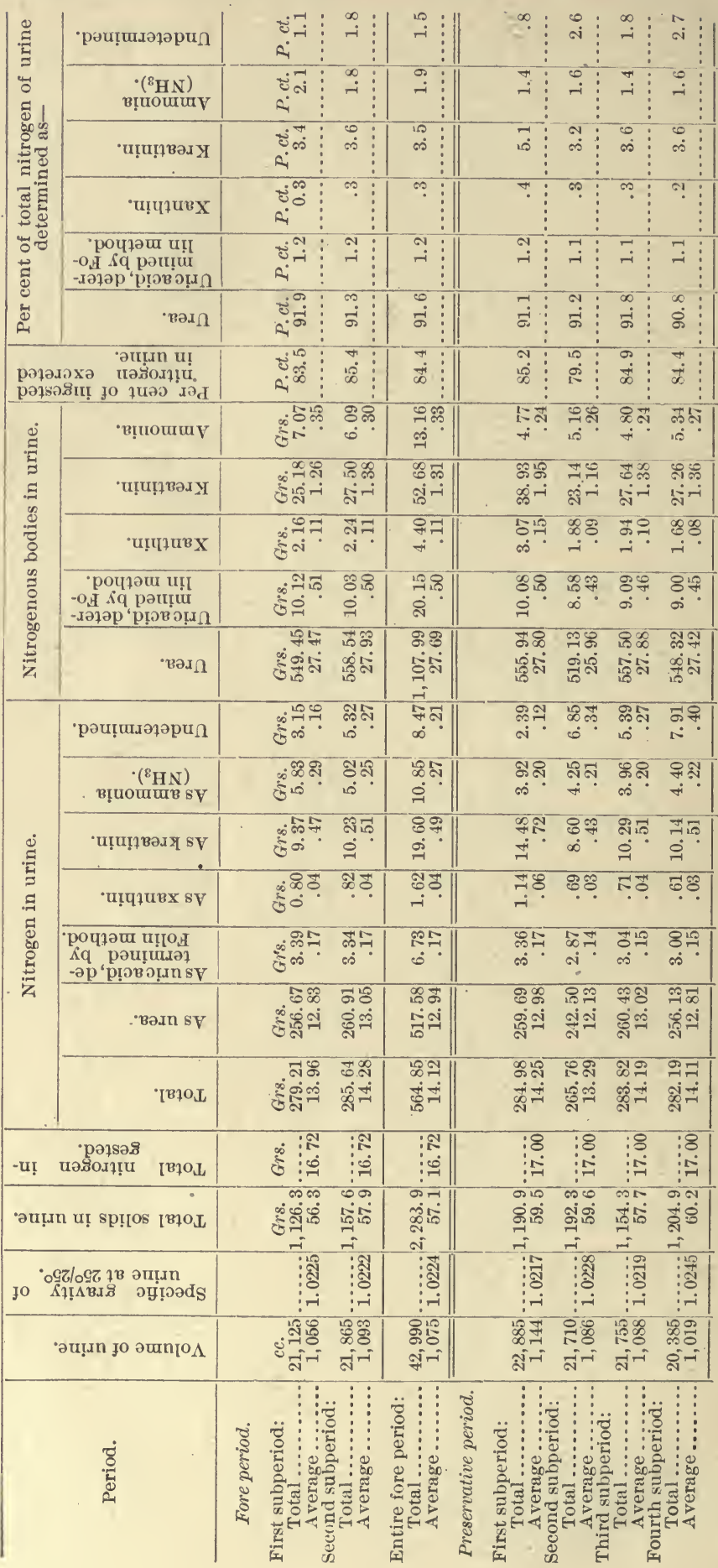




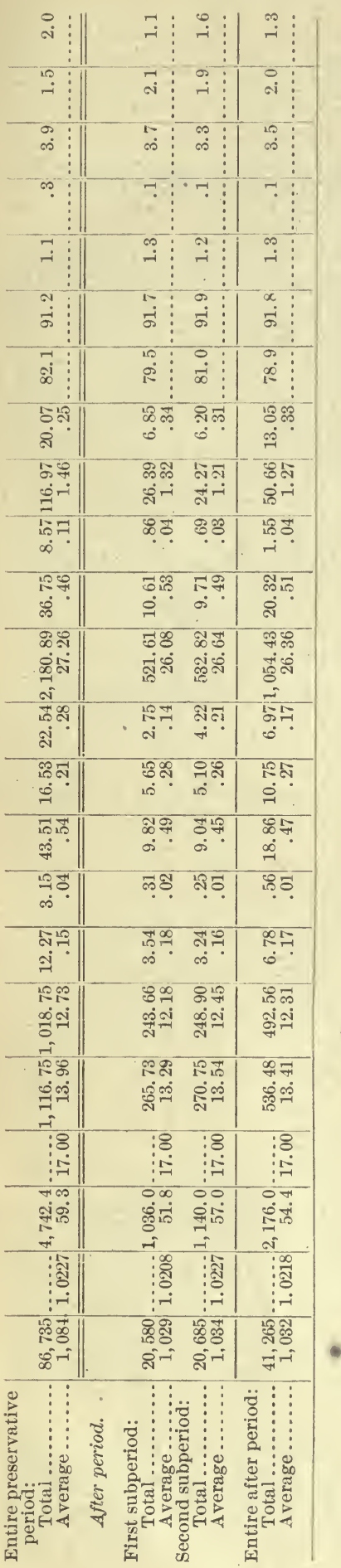




\section{THE USE OF SMALL QUANTITIES OF THE PRESERVATIVE.}

The arguments which have been advanced in excuse of the use of preservatives, when used in minute quantities, have perhaps been more vigorously urged for salicylic acid than for almost any other substance. Since the publication of Part I of this bulletin this argument has been urged with such vigor and such ingenuity that a further reference may not be out of place in these general conclusions. The principle which is laid down is that a substance which is injurious to health when added to foods, if not a natural constituent thereof, or if not added for condimental purposes, does not lose its power of injury to health because it is diluted or given in small quantities. The only.

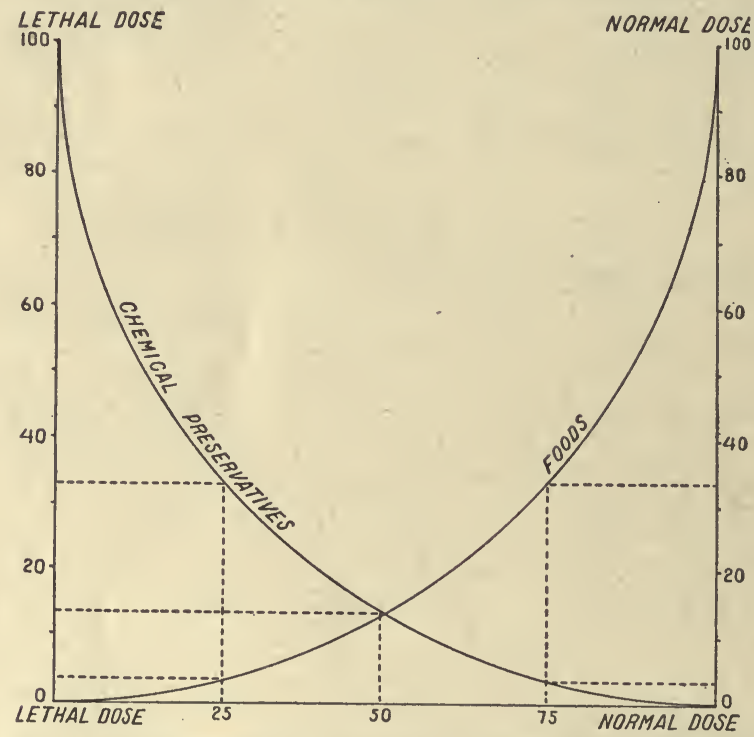

FIG. 3.-Graphic chart, representing the comparative influences of foods and preservatives.

change which is made is to mask the injurious effects produced, to make them more difficult of ascertainment and impossible of measurement. This subject was fully discussed in the hearings before the House Committee on Interstate and Foreign Commerce in February, 1906.

The fallacy of the argument that small quantities of an injurious substance are not injurious may perhaps be best represented graphically. The chart which accompanies this discussion shows theoretically the 
normal and lethal dose of a food and a drug, or, as in this case, a chemical preservative. The chart shows two curves, one representing a chemical preservative and one representing a food. The normal dose of a food is that quantity of food which maintains a healthy adult body in equilibrium. It is represented on the right of the chart by the number 100. If the quantity of food necessary to maintain the equilibrium in a healthy adult body is slightly diminished, no apparent change is at first experienced and possibly even no discomfort. If, however, the quantity of food be still further diminished progressively, as indicated by following the curve down to the left, the point is finally reached when no food is given at all and death ensues, represented by zero on the left-hand of the diagram designated "lethal dose." As the curve begins to deviate from the perpendicular on the right the degree of injury is very readily noticed and starvation or symptoms of starvation are set up. Thus, if you follow the perpendicular on the right downward to the point 80 , the divergence of the corresponding point of the curve is already measurable. As you descend to zero the magnitude of the measurement increases. It requires but very little further illustration to show how easily the effect of diminishing the normal dose of a food can be measured immediately after the curve begins to vary appreciably from the perpendicular on the right.

Let us now consider the perpendicular on the left, which is marked at the top under the term "lethal dose," viz, a quantity of the added preservative sufficient to destroy life. The normal dose of such an added chemical preservative is 0 , and is shown at the base line to the right marked "normal dose." If you add a very minute quantity of a chemical preservative, the curve representing it varies so slightly from the horizontal base as to be impossible of measurement by ordinary means. If we follow along to the number 75 , on the horizontal base, we see the deviation of the curve is sufficiently great to measure. At 50 it is still greater, at 25 still greater, while at the left of the basic line it is a maximum, extending from 0 to 100 , or the lethal dose. It is easy to show by mathematical data that no matter how small the quantity of an injurious substance or preservative is, it will still produce an injurious effect, which may be infinitely small if the dose be infinitely small. It follows then, as a mathematical demonstration, that any quantity of an injurious substance added to a food product must of necessity be injurious, provided it is in the nature of a drug and the body is in a perfectly healthy normal condition.

Hence the argument which has been so persistently urged in favor of a chemical preservative that if in small quantities it is harmless is shown to be wholly untenable. Where there is no necessity for the addition of a harmful substance, where no particular benefit is secured thereby, and where there is no disturbance of the normal state of 
health there can be no possible excuse of a valid nature to offer for the exhibition of even minute quantities. That these minute quantities would not be dangerous, in so far as producing any fatal effect is concerned, is conceded, but that, in the end, they do not produce an injury, even in these small quantities, is certainly to be denied.

The course of safety, therefore, in all these cases is to guard the opening of the door. If the use of small quantities is permitted, then there can never be any agreement among experts or others respecting the magnitude of the "small quantity," and continued litigation and disagreement must follow. On the other hand, when the harmfulness of any substance which it is proposed to add to food is established and no reason for its use can be given other than the convenience, carelessness, or indifference of the manufacturer, the exclusion of such bodies entirely from food products follows as a logical sequence and a hygienic necessity. 


\section{GENERAL CONCLUSIONS.}

In the conclusions based upon the general observations the same conservatism must be observed and the same general reservations made as are found in Part I concerning boric acid and borax. While, as described in the borax report, the attempt has been made to control, as far as possible, all the conditions of the experimental work, the difficulties attending the task are so enormous that it is not possible that complete success should be secured. There has, however, been no attempt made to discriminate in the choice of data, all the observations being recorded and the discussion of the individual data based upon the tabular statements being given without prejudice and without bias. The general assumption has been made, as in the previous case, that, by reason of the regular habits of life which were imposed upon the subjects, the amount of energy developed and the quantity of nourishment expended therein are reasonably constant throughout the experimental period. If these factors vary, as they necessarily must to a certain degree, it is evident that they vary uniformly above or below the average, and hence these variations could not possibly produce any notable effect upon the final result.

There has been a general consensus of opinion among scientific men, including the medical profession, that salicylic acid and its compounds are very harmful substances, and the prejudice against this particular form of preservative is perhaps greater than against any other material used for preserving foods. This is due not only to the belief in the injurious character of salicylic acid, but perhaps is especially due to the fact that it has in the past been so generally used as an antiseptic. That salicylic acid should be singled out especially for condemnation among preservatives does not seem to be justified by the data which are presented and discussed in this bulletin. That it is a harmful substance, however, seems to be well established by the data taken as a whole, but it appears to be a harmful substance of less virulence than has been generally supposed. There is no doubt of the fact that salicylic acid is a drug which is often indicated in diseases well established and also perhaps in certain conditions which, while verging on disease, might still be regarded as a state of health. But the administration of salicylic acid as a medicine should be controlled exclusively by the medical profession, and while it is a remedy well established in the Pharmacopœia and especially 
prized for its effect upon rheumatism and gout, it does not seem that there should be any warrant in this fact for its promiscuous use in foods, even if it were harmless.

The data show very clearly that, salicylic acid and salicylates appear to exert an exciting influence upon the activities which take place in the alimentary canal, stimulating the organs to greater effort, and this stimulation leads at first to increased solubility and absorption of the foods which are introduced into the stomach. In the light of the data which are exhibited salicylic acid may be said to increase the solubility and absorption of the food in the alimentary canal, so that larger parts of the nutrients taken into the stomach actually enter-the circulation.

The data which show the effects just noted also indicate that the general effect upon the system is depressing, in that the tissues are broken down more rapidly than they are built up, and thus the normal metabolic processes are interfered with in a harmful way. The administration of the salicylic acid is attended by a gradual decrease in the weight of the subjects, although the quantity of food elements administered during the preservative and after periods is slightly increased, which fact, together with the greater degree of absorption of the food elements, should have resulted in a slight increase in weight. This increase in weight, however, does not occur, and the disturbing influence of the salicylic acid upon metabolism, although not very great, is specifically demonstrated.

The final conclusion in this matter, therefore, is that the unenviable position which salicylic acid has heretofore held among preservatives, in being regarded as the most injurious of all, is to a certain extent undeserved. Like other ordinary preservatives, it is not one which can be classed as a poison in the usual sense of the word. When used as a medicine in many cases of derangement of health it is like the other chemical preservatives, often highly beneficial when properly prescribed by a competent physician. It is when used in the food at first an apparent stimulant, increasing the absorption and solubility of the common food elements from the alimentary canal. It soon, however, loses its stimulating properties and becomes a depressant, tending to break down the tissues of the body more rapidly than they are built up. It disturbs the metabolic processes, in most cases producing conditions which are not normal and which, apparently, are not beneficial. It has a tendency to diminish the weight of the body and to produce a feeling of discomfort and malaise, which, while not marked, is distinctly indicative of injury. In some cases these symptoms of malaise approach illness, and while not always diagnostic are sufficiently common to point unmistakably to the salicylic acid as their origin. It places upon the excretory organs, especially the kidneys, an additional burden which they are not able to bear and which can 
not possibly result in any good, but on the contrary must necessarily finally result in injury, though perhaps with the use of very small quantities of the preservative these organs would continue to perform their function for many years before finally breaking down.

This work is offered as an unbiased study of all the data recorded, both of those which appear to be in favor of the use of salicylic acid and those which appear to be against its use, and leads to the inevitable conclusion that salicylic acid is a substance which, when added to foods even in small quantities, exerts a depressing and harmful influence upon the digestion and health and the general metabolic activities of the body. Further, there appears to be no necessity for its use, as food can be preserved in unobjectionable ways without its aid. Its indiscriminate use would tend to carelessness in the quantities employed, thus increasing the dangers to which the consumer is subjected. Also, its use in the preservation of foods tends to induce carelessness and indifference on the part of the manufacturer, as when a chemical antiseptic is employed many of the processes necessary to the proper selection, cleaning, and preservation of foods may be omitted.

The addition of salicylic acid and salicylates to foods is therefore a process which is reprehensible in every respect, and leads to injury to the consumer, which, though in many cases not easily measured, must finally be productive of great harm.

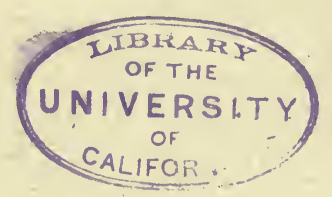




\title{
LIST OF TABLES.
}

\author{
Selies VI
}

TABLE I. Dates of periods and subieriods ........................ 481

II. Schedule of administration of preservative ................ 481

III. Salicylic acid ingested and recovered in urine............... 484

IV. Anount of moist and dry food consumed, expressed as percentage of body weight ..................................

V. Summary for nine men, by periods, showing average daily ratio of food weight to body weight.

VI. Averages, by periods, of corpuscles and hemoglobin in the blood..

VII. Summary of weight and water content of feces, by periods........

VIII. Urine determinations-volume, specific gravity, and total solids ..

IX. Urine determinations-ratio of sulphur, sulphates, and phosphates to nitrogen ..........................................

X. Urine determinations-ratio of preformed sulphates to ethereal

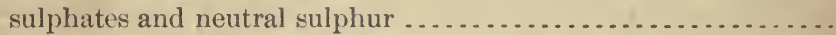

XI. Microscopical examination of the urine .....................

XII. Nitrogen summary, by periods, for nine men . . . . . . . . . . . . .

XIII. Nitrogen balances....................................

XIV. Phosphoric acid summary, by periods, for nine inen...........

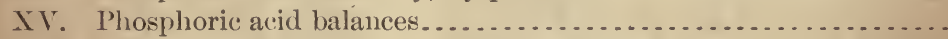

XVI. Sulphur summary, by periods, for nine men .................

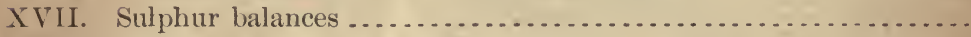

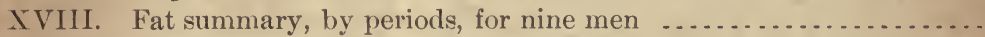

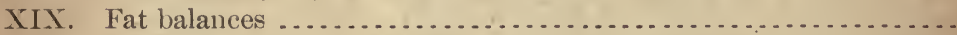

XX. Calories summary, by periods, for nine men .................

XXI. Calories balances .......................................

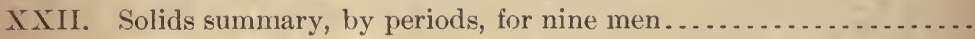

XXIII. Solids balances

\section{SERies XI.}

THE EFFECT OF SALICYLIC ACID AND SALICYLATES UPON THE NITROGENOUS BODIES IN THE URINE.

TABLE I. Daily ration, showing amount of food and quantity of nitrogen ingested in the preliminary experiment ....................

II. Urea and uric acid eliminated in urine, preliminary study ....... . .

III. Comparison of the two methods for the determination of urea ....

IV. Schedule of administration of salicylic acid and salicylates for special study on four men .................................

V. Reaction of the urine and presence of albumin, special study .....

VI. Nitrogenous constituents of the urine, special study 



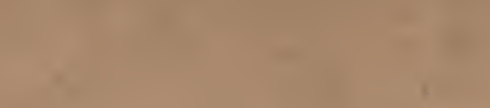

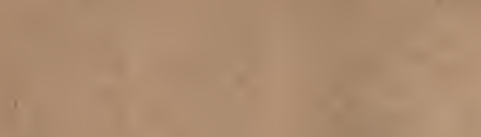

.

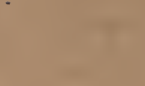

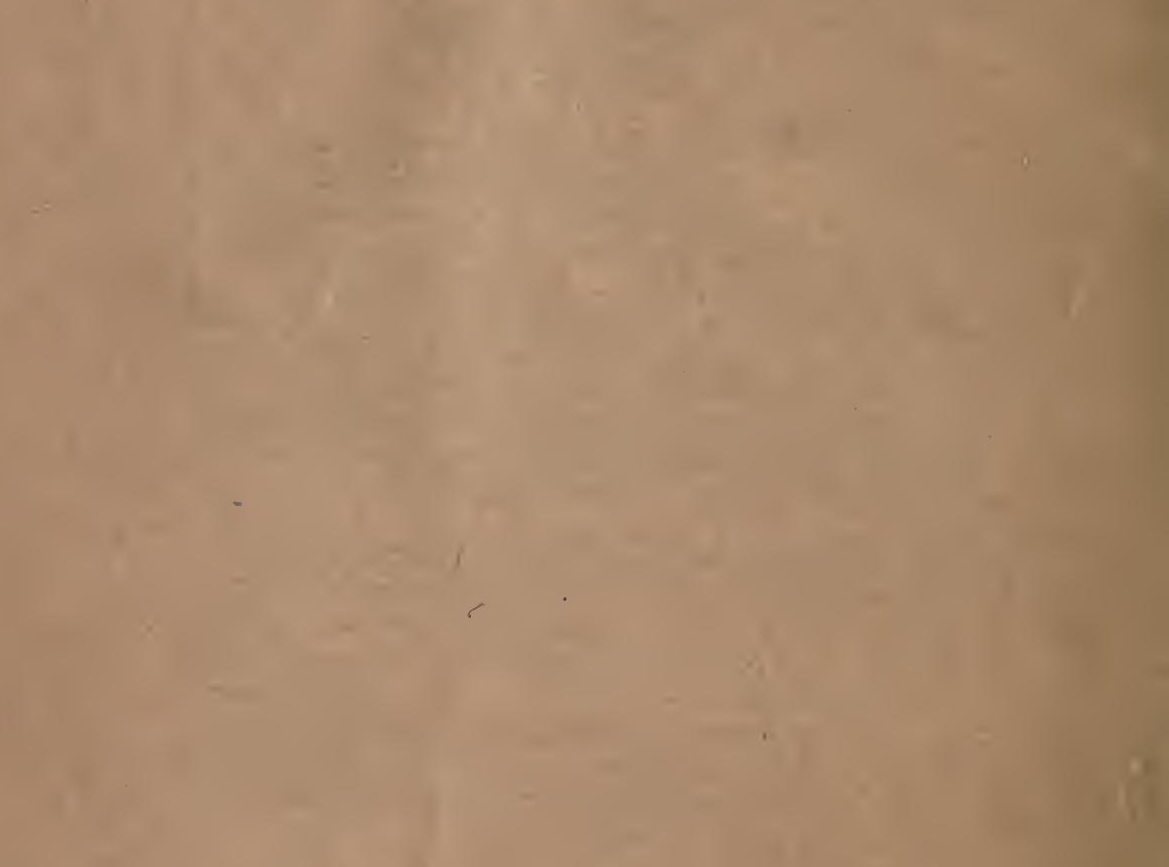

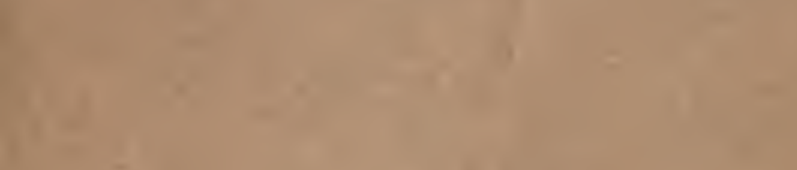

14)

$1=$

45

$$
\text { . }
$$





\section{HOME USE CIRCULATION DEPARTMENT MAIN LIBRARY}

This book is due on the last date stamped below. 1-month loans may be renewed by calling 642-3405. 6-month loans may be recharged by bringing books to Circulation Desk.

Renewals and recharges may be made 4 days prior

ALL BOOKS ARE SUBJECT TO RECALL 7 DAYS AFTER DATE CHECKED OUT.
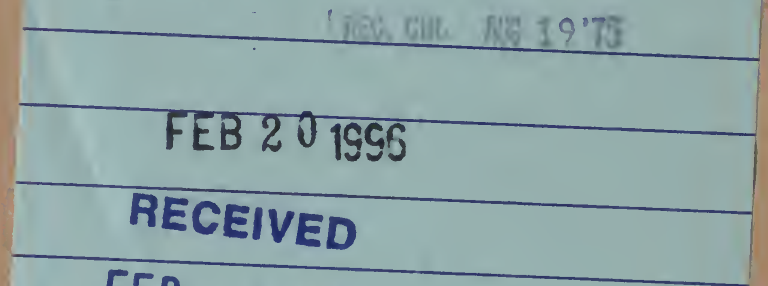

FEB : 21996

CIRCULATION DEPT.

LD21-A-40m-12,'74

(S2700L)

General Library

University of California Berkeley 
U. C. BERKELEY LIBRARIES | || || ||

C056068642

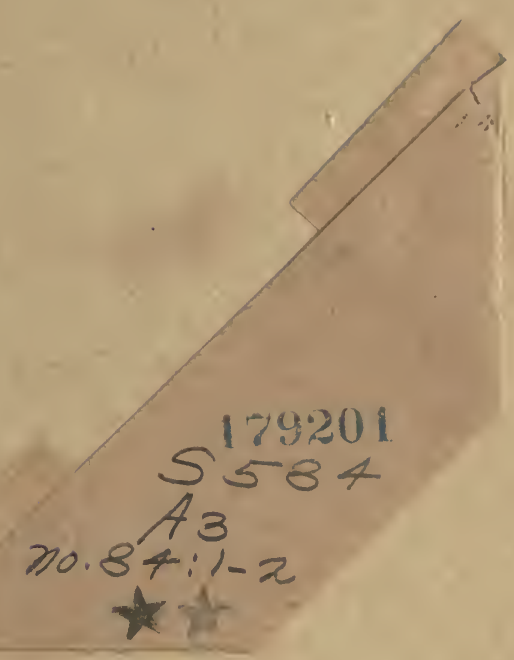


